\title{
Prognosemodelle für ausgewählte Holzqualitätsmerkmale wichtiger Baumarten
}

\author{
Dissertation zur Erlangung des Doktorgrades \\ der Fakultät für Forstwissenschaften und Waldökologie \\ der Georg-August-Universität Göttingen
}

\author{
vorgelegt von \\ Matthias Schmidt \\ geboren in Wunstorf / Luthe
}

Göttingen, im Juni 2001 
1. Berichterstatter: Prof. Dr. Dr. h.c. Klaus von Gadow

2. Berichterstatter: PD Dr. Jürgen Nagel

3. Berichterstatter: Prof. Dr. Dr. h.c. Gero Becker

Tag der mündlichen Prüfung: 10. August 2001 


\section{Vorwort}

Der Wechsel zur Abteilung Waldwachstum der Niedersächsischen Forstlichen Versuchsanstalt vom Institut für Forsteinrichtung und Ertragskunde der Universität Göttingen im Jahr 1998 bedeutete für mich zugleich einen inhaltlichen Wechsel meiner Forschungstätigkeit. Lag der bisherige Schwerpunkt im Bereich der Bestandesinventur und Sortimentsschätzung, so bestand die neue Aufgabe in der Entwicklung von Ansätzen zur Beschreibung der Holzqualität. Später stellte sich heraus, daß das Vorwissen zur Schaftformmodellierung bzw. Sortimentsprognose sehr hilfreich für die Integration der erarbeiteten Qualitätsmodelle in ein Modul zur kombinierten Güte- und Stärkeklassenprognose war. Auch die bereits während der Zeit am Institut für Forsteinrichtung und Ertragskunde erworbenen Programmier- und Statistikkenntnisse verhalfen mir zu einem "fliegenden Start" in das neue Projekt.

Das von mir anfangs mit etwas Skepsis betrachtete Projekt zur Qualitätsprognose weckte mehr und mehr mein Interesse, als ich nach abgeschlossenem Literaturstudium feststellte, daß es auch für diesen Bereich Möglichkeiten zu einer statistischen Problembearbeitung gab. Eine interessante und anspruchsvolle Tätigkeit war damit gesichert. Allerdings bedeutete die Entwicklung statistischer Modelle einen nicht unerheblichen Aufwand bei der Erfassung neuartiger Daten wie z. B. Astdurchmessern und -positionen.

Herrn PD Dr. Jürgen Nagel und Herrn FD Dr. Hermann Spellmann danke ich für das Vertrauen, mir bei der Bearbeitung des Projektes einen großen Spielraum zuzugestehen. Macht doch das kreative Arbeiten in vorgegebenen Forschungsbahnen den besonderen Reiz einer wissenschaftlichen Tätigkeit aus. Gleichzeitig sorgten die Prämissen, die aus der Kombination der entwickelten Modelle mit dem bereits im Einsatz befindlichen Wachstumssimulator BWINPro entstanden, für eine ständige Überprüfung der Praxisrelevanz der Forschungsergebnisse. Dabei war die angestrebte Verwendung der Ergebnisse in der niedersächsischen Forstwirtschaft eine zusätzliche Motivation für mich. Die Diskussionen mit Herrn PD Dr. Jürgen Nagel und Herrn Dr. Matthias Albert erleichterten mir die Lösung waldwachstumskundlicher und programmiertechnischer Probleme sehr.

Herrn Prof. Dr. Klaus von Gadow (Institut für Forsteinrichtung und Ertragskunde der Universität Göttingen) danke ich dafür, daß er mein Interesse an der Waldwachstumskunde geweckt hat und daß das Erlernen des Programmierens von ihm gefördert und gefordert wird. Nur die Beherrschung dieser Arbeitstechnik erlaubte mir ein eigenständiges und kreatives Arbeiten. Herrn Prof. Dr. Walter Zucchini (Institut für Statistik und Ökonometrie der Universität Göttingen) danke ich für die Beratung bei der Entwicklung statistischer Modelle und die Möglichkeiten, im Rahmen von Workshops und Einzeltreffen mein statistisches Wissen zu erweitern. Herrn Dr. Matthias Albert verdanke ich die größte Anzahl an informellen Kurzge- 
sprächen zu aktuellen forstlichen und nicht-forstlichen Problemstellungen sowie eine freundschaftliche Zusammenarbeit innerhalb und außerhalb des Forschungsprojektes. Frau Dipl.Stat. Almuth Wameling danke ich für Hinweise und Vorschläge bei der Bewältigung statistischer Probleme. Meine Arbeitskollegen und Freunde Dipl.-Forstw. Kai Staupendahl, Prof. Dr. Janna Puumalainen, Dr. Jörg Schröder und Dipl-Forstw. Pirrka Pogoda aus mehr oder weniger langen gemeinsamen Zeiten am Institut für Forsteinrichtung und Ertragskunde sorgten vor und nach meinem Wechsel zur Versuchsanstalt bei gemeinsamen Mittagessen in der Nordmensa und nach Feierabend für die Auflockerung des Forschungsalltages.

Für das Korrekturlesen der doch recht umfangreichen Arbeit möchte ich mich bei Frau Christina Hansen M.A., Herrn Dr. Matthias Albert, Herrn Dipl.-Forstw. Michael Wallusch und Herrn Dipl.-Forstw. Kai Staupendahl bedanken. Bei den Außenaufnahmen unterstützten mich Frau Christina Hansen M.A., Frau Dipl.-Biol. Heike Pfirrmann, Herr Dipl.-Forstw. Christian Roschak, Herr Dipl.-Forstw. Michael Hillmann, Herr Dipl.-Forstw. Wolf Hertkorn und Herr FOI Andreas Richter. Herr Jochen Eggemann brachte meinen zeitweise "bockigen“" Computer auch bei schwerwiegenderen Problemen immer wieder zum Laufen. Allen nicht namentlich genannten Arbeitskolleginnen und Arbeitskollegen aus der Abteilung A der Niedersächsischen Forstlichen Versuchsanstalt und dem Institut für Forsteinrichtung und Ertragskunde der Universität Göttingen sei für die angenehme Zusammenarbeit gedankt.

Die langjährige Unterstützung meiner Eltern in allen Bereichen hat diese Promotion überhaupt erst ermöglicht. Ihnen gebührt ein besonderer Dank. 


\section{Inhaltsverzeichnis}

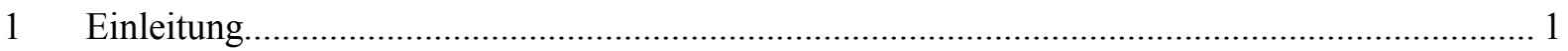

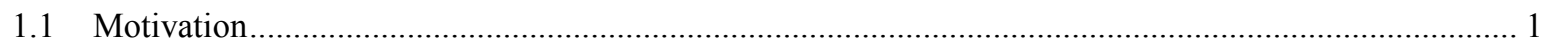

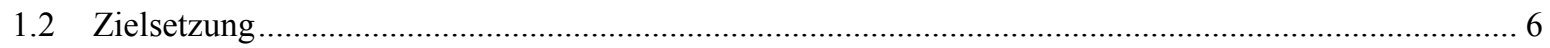

2 Datengrundlage und Modellübersicht für die Beschreibung von Gütemerkmalen ..................... 13

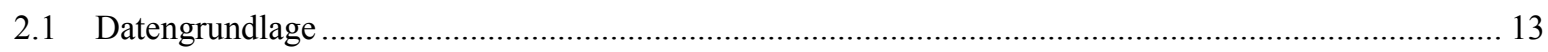

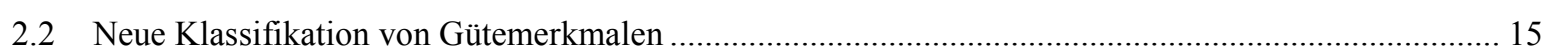

2.3 Schätzung der Kronenansatzhöhe ................................................................................................... 18

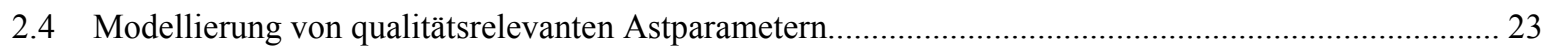

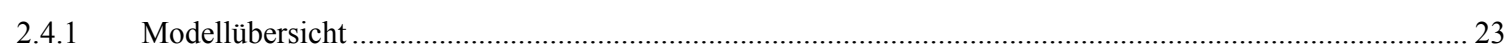

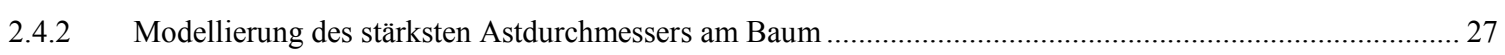

2.4.3 Durchmesser der stärksten Quirläste in der gesamten Krone eines Baumes ............................................ 30

2.4.4 Modellierung der Durchmesser und der Anzahl aller Quirläste eines Baumes ........................................... 33

2.4.5 Modellierung der Durchmesser, der Anzahl und der vertikalen Position aller Äste eines Baumes............... 36

2.4.6 Modellierung der Horizontal- und Vertikalwinkel von Ästen.............................................................. 37

2.4.7 Modellierung des Astabsterbezeitpunktes und der natürlichen Astreinigung ............................................. 37

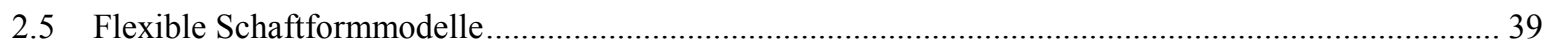

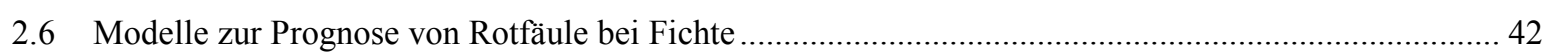

3 Entwicklung neuer Ansätze zur Qualitätsprognose .............................................................. 45

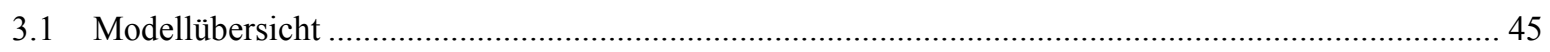

3.1.1 Nadelholz

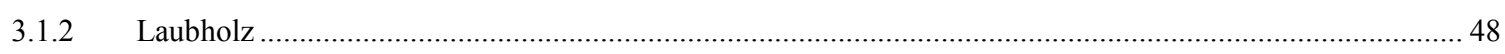

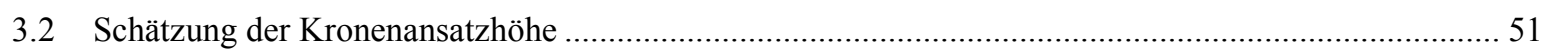

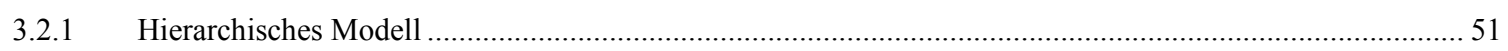

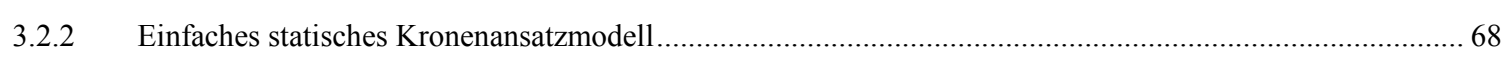

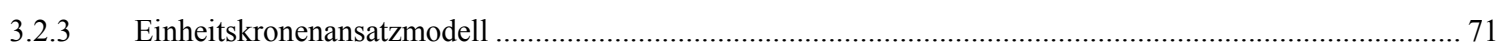

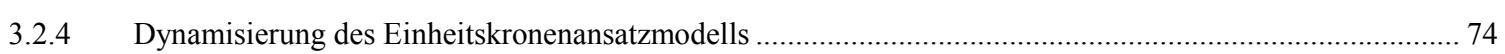

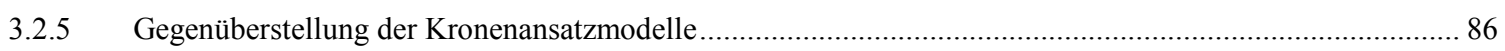

3.3 Modelle zur Durchmesserschätzung aller Quirläste bei Nadelholzarten................................................. 90

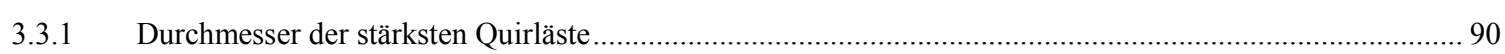

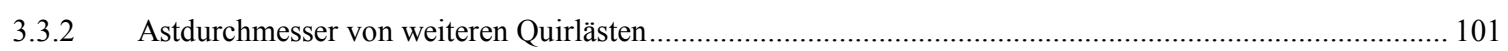

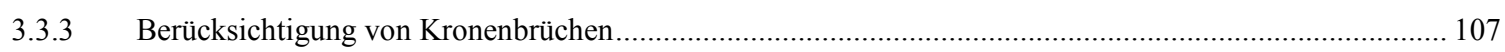

3.4 Modelle zur Beschreibung von Astparametern bei der Baumart Buche ............................................. 110

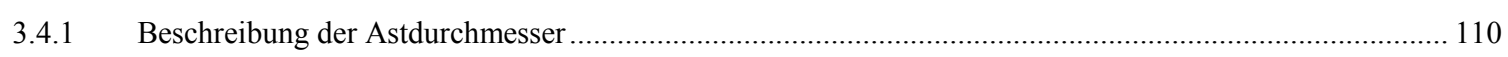

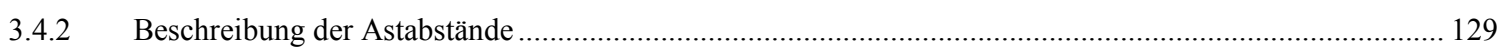

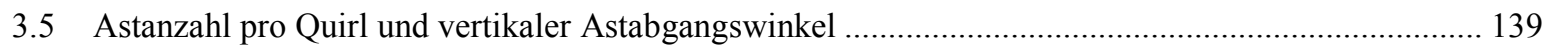

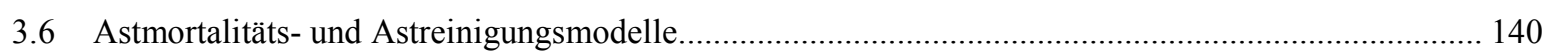

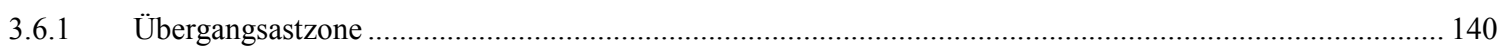

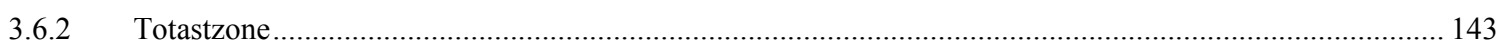

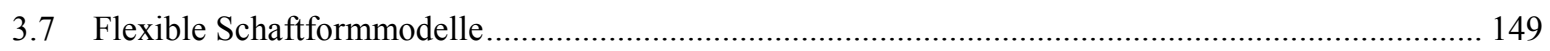

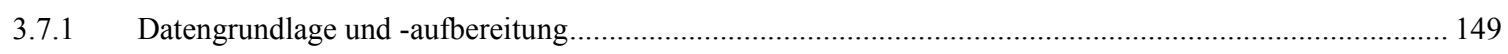

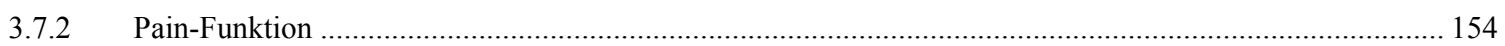

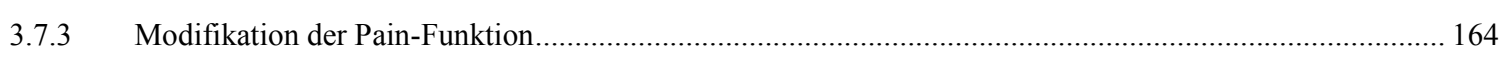

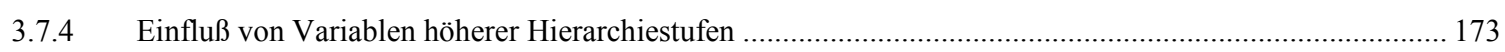




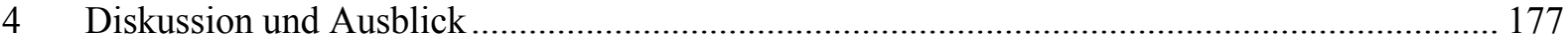

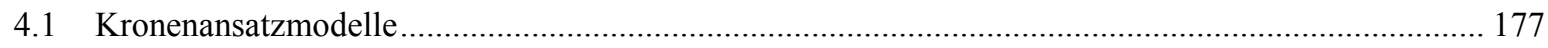

4.1.1 Hierarchisches Modell und einfaches statisches Modell ....................................................................... 177

4.1.2 Einheitskronenansatzmodell / Dynamisierung des Einheitskronenansatzmodells .................................... 183

4.2 Astparametermodelle (Nadelholzarten und Laubholzarten mit starker Apikalkontrolle) ..................... 184

4.2.1 Astdurchmessermodell für die Quirläste in der grünen Krone ................................................................. 184

4.2.2 Astanzahlen pro Quirl und vertikaler Astabgangswinkel .................................................................. 190

4.3 Astparametermodelle (Laubholzarten ohne starke Apikalkontrolle) ........................................... 191

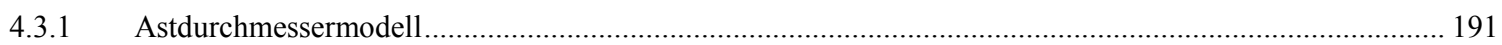

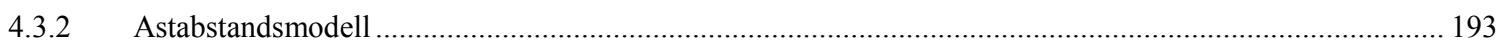

4.4 Modelle zur Beschreibung des Astzustandes ....................................................................... 194

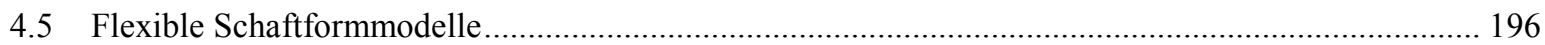

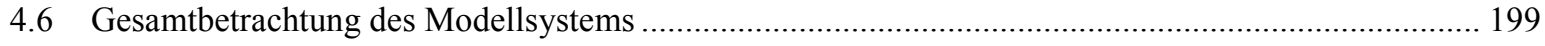

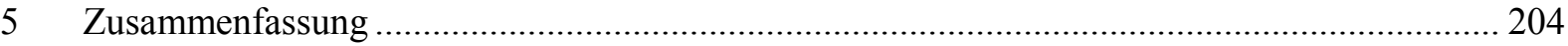

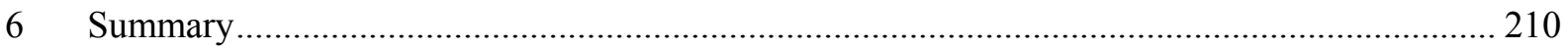

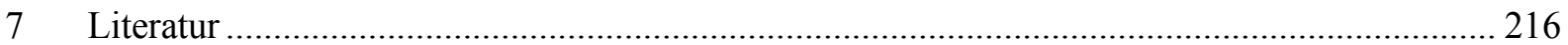

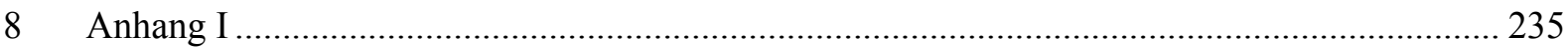

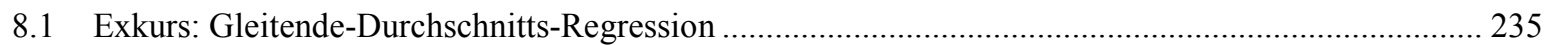

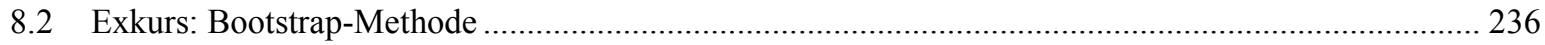

8.3 Exkurs: Modellierung von Dichteverteilungen mit Hilfe nicht-parametrischer Dichte-Funktionen ..... 237

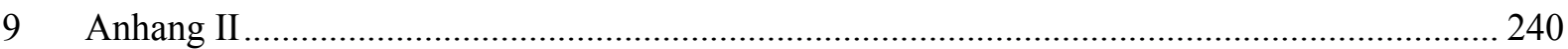

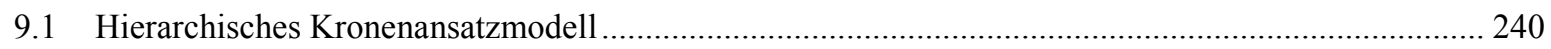

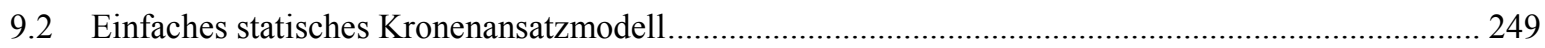

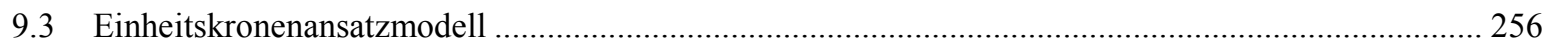

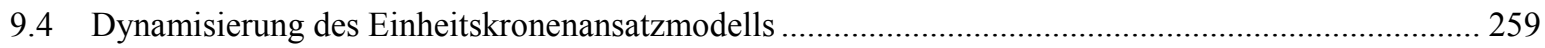

9.5 Astdurchmesser der stärksten Quirläste _....................................................................................... 270

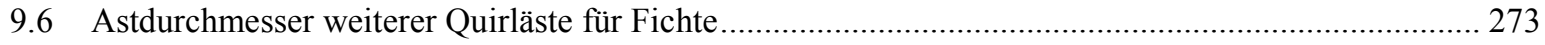

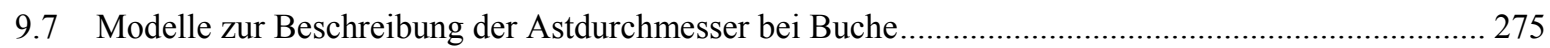

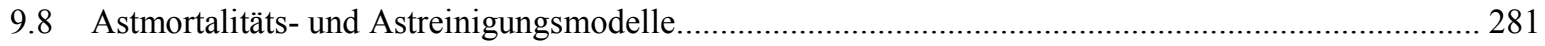

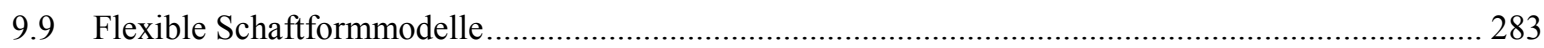




\section{$1 \quad$ Einleitung}

\subsection{Motivation}

In den 1990er Jahren haben in den deutschsprachigen Ländern Europas eine Reihe von statistischen Einzelbaumwachstumssimulatoren einen Entwicklungsstand erreicht, der ihren Einsatz in der forstlichen Praxis zuläßt (Hasenauer, 1994; Sterba, 1995; Pretzsch U. KahN, 1998; NAGEL, 1999 a). Die Notwendigkeit zur Entwicklung von Einzelbaumsimulatoren ergibt sich aus der angestrebten Abkehr vom Altersklassenwald und dem Ziel ungleichaltriger Mischwälder. Der Aufbau von ungleichaltrigen Mischwäldern mit einem hohen Anteil standortsgemäßer heimischer Baumarten, unter Bevorzugung von Naturverjüngung sowie der Anwendung der Zielstärkennutzung ist als naturnaher Waldbau in den Waldbau-Konzepten der Länder der Bundesrepublik Deutschland manifestiert (ОTTO, 1989 u. 1991; GRIESEL U. GAdow, 1995; SPELlmanN, 1995 u. 1997). Der naturnahe Waldbau führt gegenüber der schlagweisen Hochwaldwirtschaft zu einer Vielzahl unterschiedlicher Waldaufbauformen, welche die für die Planung der Waldentwicklung notwendige Informationsbereitstellung erschwert (PRETZSCH ET AL., 2000). So ist die Prognose der Entwicklung strukturreicher Wälder und insbesondere von Überführungsbeständen weder mit Ertragstafel- noch mit Bestandesoder Verteilungsmodellen zu leisten (PRETZSCH, 1992). Unterschiedlichste Waldaufbauformen, insbesondere die in den nächsten Jahrzehnten stark zunehmenden Überführungsbestände, erfordern eine Auflösung der Bestände in Einzelbäume und die Prognose des Bestandeswachstums als Aggregation des Einzelbaumwachstums (PRETZSCH ET AL., 2000). Ökophysiologische Wachstumsmodelle, die teilweise ebenfalls eine Auflösung bis auf die Einzelbaumebene aufweisen (z. B. Bossel, 1996), sind von einer praktischen Anwendung im Rahmen der forstlichen Planung noch relativ weit entfernt. Nach HASENAUER (2001) besteht ein Problem darin, die auf Bestandesebene beschriebenen Stoffkreisläufe auf Einzelbäume aufzuteilen. Weiterhin erfordern ökophysiologische Modelle komplexe Eingangsinformationen, die nur in Einzelfällen zur Verfügung stehen (WHITE ET AL., 2000). Die nicht ausreichende Quantifizierung aller relevanten physiologischen Prozesse (WHITE ET AL., 2000) sowie die fehlende Integration der Auswirkungen forstlicher Eingriffe (HASENAUER, 2001) führt zu einer momentan noch unzureichenden Sensitivität dieser Modelle bei der Prognose unterschiedlicher waldbaulicher Szenarien.

Mit der Erhöhung der Modellkomplexität sind im allgemeinen höhere Anforderungen an die Auflösung der Eingangsdaten verbunden, die für die Erzeugung von Startwerten für die Prognose des Waldwachstums benötigt werden (PrETzSCH ET AL., 2000). Soll das Wachstum unter Berücksichtigung der spezifischen Wachstumsbedingungen des Einzelbaumes modelliert werden, so müssen auch die Startwerte einer Simulation verschiedene Einzelbaumattri- 
bute beinhalten. Unterschiedliche Anforderungen an die Datenstruktur ergeben sich aus der Konzeption des Einzelbaumsimulators als abstandsabhängiges oder -unabhängiges Modell. Erfolgt die Prognose des Einzelbaumwachstums distanzabhängig, so müssen die Koordinaten der Baumfußpunkte aller Einzelbäume bekannt sein. Aber auch beim Einsatz abstandsunabhängiger Einzelbaumsimulatoren werden in den seltensten Fällen alle für die Prognose des Wachstums benötigten Informationen zur Verfügung stehen. Daher sind dem eigentlichen Wachstumsmodul weitere Module zur Ergänzung fehlender Informationen vorgeschaltet.

Der Strukturgenerator Strugen (PRETZSCH, 1993) ermöglicht die Generierung eines Baumverteilungsplanes auf der Basis einer nach Baumarten getrennten BaumzahlDurchmesserverteilung unter Berücksichtigung unterschiedlicher Mischungsformen. Eine weitere Möglichkeit zur Generierung von Baumverteilungsplänen stellen LEWANDOWSKI und GADOw (1997) vor. Der von ihnen entwickelte Generierungsalgorithmus benötigt neben der Durchmesserverteilung allerdings die Verteilungen verschiedener strukturbeschreibender Variablen. Einen Ansatz zur Reproduktion von Baumverteilungsmustern, der die in Kontrollstichprobenpunkten enthaltenen Strukturinformationen mit Algorithmen des Strukturgenerators von PRETZSCH (1993) kombiniert, beschreibt POMMERENING (1998). Für die Anwendung in der forstlichen Praxis müssen ferner Algorithmen zur Generierung von Durchmesserverteilungen (NAgEl U. Biging, 1995), Höhenwerten (Hui U. GAdOw, 1993; SlobodA ET AL., 1993; AlBERT, 2000) und Kronenparametern (NAGEL, 1999 a, S. 30 ff.) vorhanden sein, um auch Bestände, für die nur Informationen über Bestandesmittelwerte vorliegen, fortschreiben zu können.

Die relativ hohe Auflösung der von Einzelbaumsimulatoren benötigten Eingangsinformationen erfordert, wie beschrieben, mehr oder weniger umfangreiche Datenergänzungsroutinen, wenn aus Inventuren lediglich stärker aggregierte Kennwerte vorliegen. Gleichzeitig bieten Einzelbaumsimulatoren dadurch jedoch erst die Möglichkeit, hochwertige Ausgangsinformationen, wie sie im Rahmen von modernen Stichprobeninventuren (SCHMID-HAAS ET AL., 1993; AKৎ̧A, 1993; KÖHL ET AL., 1995; BÖCKMANN ET AL., 1998; GADOW U. SCHMIDT, 1998) erhoben werden, adäquat bei der Prognose zu berücksichtigen. Auch die überwiegend der Zielvereinbarung im praktischen Forstbetrieb und der Simulation von waldbaulichen Behandlungsszenarien dienenden Weiserflächen (BÖCKMANN U. HÜSING, 1999) sollten aufgrund ihrer hohen Datenqualität nur mit Einzelbaumsimulatoren fortgeschrieben werden. Die Simulation von Behandlungsszenarien bildet die Grundlage für die Optimierung einer multifunktionalen Waldbewirtschaftung (VALSTA, 1992; GOVE U. FAIRWEATHER, 1992; ANDERSON U. BARE, 1994) und die Definition von waldbaulichen Handlungsräumen (GADOW U. PuUmalainen, 1998).

Zusammenfassend kann die Motivation für die Entwicklung von Einzelbaumsimulatoren vor allem auf die Veränderung der Waldaufbauformen und die Einführung moderner 
Stichprobeninventuren zurückgeführt werden. Weiterer Anwendungsbereich ist der Einsatz im Rahmen von Schulungen und in der Umwelterziehung (INSTITUT FÜR DEN WISSENSCHAFTLICHEN FILM, 1998). Standortssensitive Simulatoren können darüber hinaus zur Prognose der Auswirkungen von Klimaänderungen auf das Waldwachstum eingesetzt werden (KAHN, 1995). Weiterhin sind Spezialauswertungen wie beispielsweise die Analyse der Struktur- und Wuchsdynamik von Naturwäldern zu nennen (MEYER, 2001).

Neben den Möglichkeiten zur Fortschreibung jeder denkbaren Bestandesstruktur, der Berücksichtigung der Auswirkungen unterschiedlicher Eingriffsfolgen und der Verwertung von Eingangsinformationen mit hoher Auflösung besteht ein weiterer Vorteil der Einzelbaumsimulatoren bei der Ausgabe von Informationen. Da jeder Einzelbaum fortgeschrieben wird, können die Ergebnisse sowohl aggregiert in Form von Bestandesmittelwerten als auch in Form von Baumlisten für die fortgeschriebenen Attribute ausgegeben werden (NAGEL ET AL., 2001). Für die Bewertung von Eingriffsfolgen im Rahmen von waldbaulichen Szenarienvergleichen sind Bestandesmittelwerte nur sehr begrenzt geeignet. So orientiert sich die Bewertung der Nutzfunktion eines Bestandes heutzutage nicht mehr an der reinen Massenleistung, sondern an der Häufigkeitsverteilung der zu Marktpreisen bewerteten Sortimente. Für Sortimentsschätzungen, d. h. Anzahl- und Volumenverteilungen über Sortimentsklassen, werden Verteilungen der naturalen Baumattribute Durchmesser und Höhe benötigt. Eine Ausnahme stellen Sortentafeln dar, die jedoch in ihrer Konzeption und ihrem starren Anwendungsbereich mit den Ertragstafeln gleichzusetzen sind (SCHÖPFER U. DAUBER, 1989). Die Bewertung der Entwicklung der Erholungsfunktion und der biologischen Vielfalt von Waldbeständen erfordert ebenfalls eine Betrachtung der Einzelbaumebene, wenn zu ihrer Beurteilung komplexe, objektiv nachvollziehbare Struktur- und Diversitätsindizes verwendet werden sollen (Clark U. Evans, 1954; Albert U. GAdow, 1998; Gadow ET AL., 1998).

Zur Bewertung der zeitlichen Entwicklung der Nutzfunktion von Waldbeständen im Rahmen von waldbaulichen Szenariensimulationen und der mittelfristigen Planung der Forsteinrichtung werden Einzelbaumsimulatoren häufig mit Programmen zur rechnerischen Sortimentierung kombiniert. So verfügen sowohl der Einzelbaumsimulator Silva (PRETzSCH U. KAHN, 1998) als auch der Einzelbaumsimulator Bwinpro (NAGEL ET AL., 2001) über Schnittstellen zur Ausgabe von naturalen Einzelbaumdaten an das Sortimentierungsprogramm Holzernte $^{1}$ (Kublin U. Scharnagl, 1988). Das Programm Holzernte erlaubt somit eine Bewertung der Nutzfunktion von unterschiedlichen waldbaulichen Behandlungsszenarien auf der Basis von Stärkeklassenverteilungen, was gegenüber einer Bewertung auf der Basis von Durchmesserverteilungen oder sogar Bestandesmittelwerten als großer Fortschritt zu beurtei-

\footnotetext{
${ }^{1}$ Das Programm Holzernte ist ein auf der Grundlage des BWI-Unterprogrammes BDAT entwickeltes Anwenderprogramm. Herausgeber des Programmes ist die Forstliche Versuchs- und Forschungsanstalt BadenWürttemberg, Wonnhaldestraße 4, 79100 Freiburg.
} 
len ist. Häufig wird die Bewertung der Nutzfunktion jedoch stärker durch die Güteklassen- als durch die Stärkeklassenverteilung beeinflußt. Zur Verdeutlichung ist in Abb. 1 der Einfluß der Holzqualität auf den Stammholzwert über dem BHD in einem Buchenbestand dargestellt. Verglichen werden die Bewertung des $8 \mathrm{~m}$ Erdstammstücks zu B- und C-Holzpreisen. Ein zweites Stammstück bis zum Kronenansatz wurde einheitlich mit C-Holzpreisen bewertet. Die unterlegten Preise sind Durchschnittswerte des Winters 2000/2001 für BuchenStammholz bzw. -Parkett. Im Rahmen der Vor- und Nachkalkulation von forstlichen Eingriffen können im Programm Holzernte daher Anteile an verschiedenen Güteklassenkombinationen baumartenspezifisch vorgegeben werden.

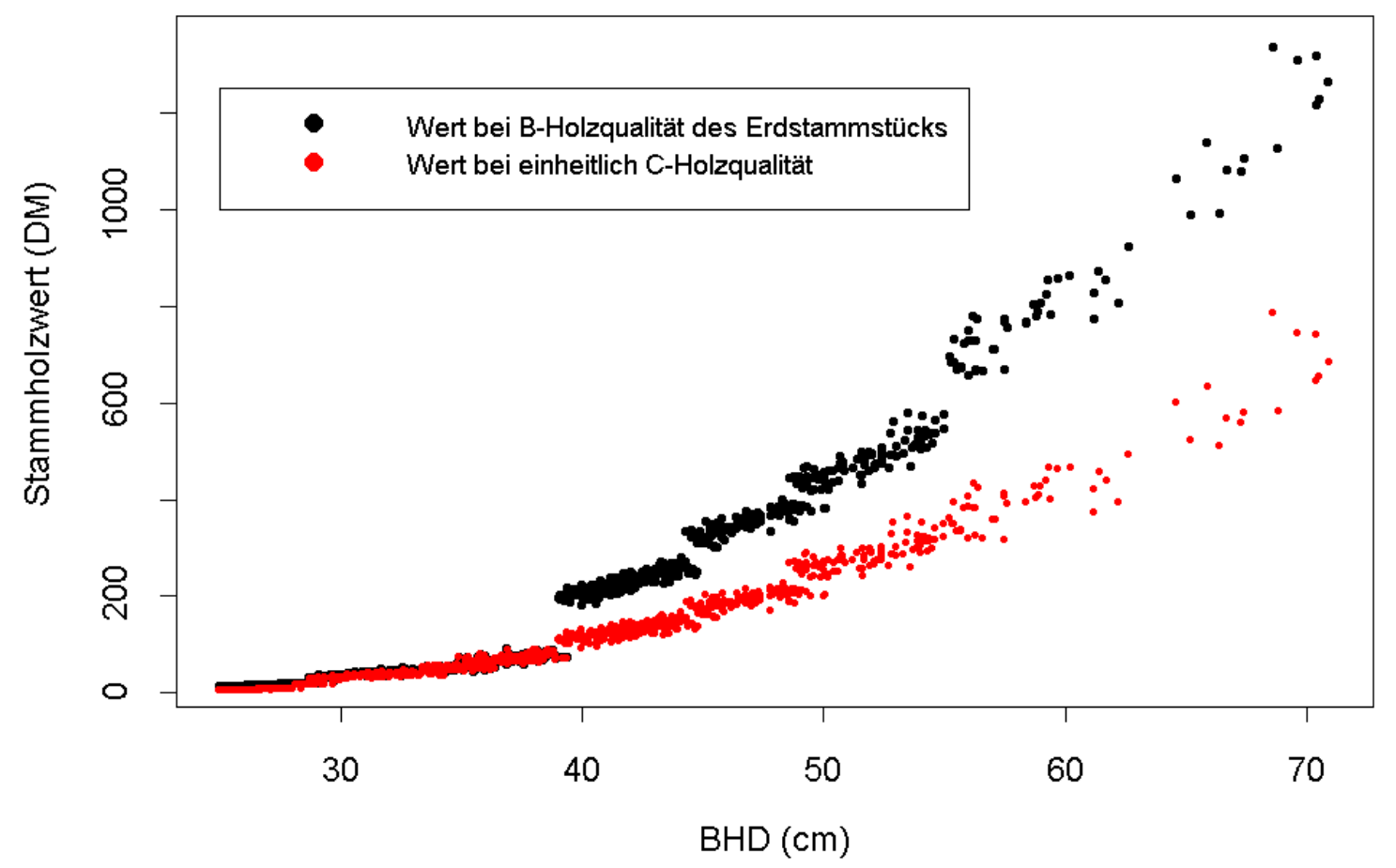

Abb. 1: Einfluß der Bewertung des $8 \mathrm{~m}$ Erdstammstücks auf den gesamten Stammholzwert über dem BHD in einem Buchenbestand.

Im Rahmen der Wachstumsprognose ist es jedoch sinnvoll, Kriterien der Holzqualität, die zumindest teilweise als das Resultat von Wachstums- und Konkurrenzbedingungen betrachtet werden können, in Abhängigkeit von Variablen zu beschreiben, die die Wachstumsbedingungen eines Baumes quantifizieren oder selbst durch die Wachstumsbedingungen beeinflußt werden. Typische Beispiele für derartige Gütemerkmale sind die Aststärke, der Astzustand (Grün- oder Totast) und die Schaftform (voll- oder abholzig). Aber auch Qualitätskriterien, die weniger eng mit den Wachstumsbedingungen eines Baumes korreliert sind, sollten in die Wachstumssimulation integriert werden. Die Modellierung von Qualitätskriterien schließt eine Ergänzung mit Häufigkeitsschätzungen von weiteren nicht modellierten Qualitätskriterien, die beispielsweise aufgrund von Ergebnissen aus dem Holzeinschlag bekannt sind, nicht aus. 
Im naturnahen Waldbau wird aufgrund der Mechanismen der sogenannten "biologischen Automation“ langfristig eine Anhebung der mittleren Holzqualität erwartet (DELORME, 1999; ScHÜTZ, 1999, S. 58 ff.; KNOKE, 1999). In Überführungsbeständen kann es auch zu einem Absinken der Holzqualität kommen, da die Erziehung stabiler und vitaler Einzelbäume in einem Gegensatz zur Erziehung von Bäumen mit hoher Holzqualität stehen kann. Durch die Zunahme der Strukturvielfalt bei der Anwendung der Prinzipien des naturnahen Waldbaus und in der Überführungsphase ist zudem eine größere Variabilität der Holzqualitäten der Einzelbäume zu erwarten. Zur Abschätzung der Auswirkungen des naturnahen Waldbaus auf die Holzqualität sind daher starre Modelle wie Sortentafeln, die auf der Basis gleichaltriger Reinbestände entwickelt wurden, ungeeignet. Vielmehr ergibt sich auch hier die Notwendigkeit der Entwicklung von Modellen zur Prognose der Holzqualität, die flexibel auf veränderte Konkurrenz- und Wachstumsbedingungen reagieren. Beispielsweise muß es zur Berücksichtigung der biologischen Automation möglich sein, den günstigen Einfluß einer Überschirmung auf die Aststärke zu beschreiben. Zusammenfassend kann gesagt werden, daß die flexible Modellierung der Holzqualität für beliebige Waldaufbauformen ebenso wie die Prognose des Waldwachstums eine Auflösung bis auf die Einzelbaumebene erfordert.

Statistische Modelle zur Prognose der Holzqualität im Rahmen von waldwachstumskundlichen Simulationen sind trotz der entscheidenden Bedeutung für die Bewertung der Nutzfunktion von Waldbeständen in Deutschland bisher kaum entwickelt worden. Demgegenüber existiert speziell in Nordamerika, Skandinavien und Frankreich eine große Anzahl an quantitativen Modellen unterschiedlicher Komplexität zur Prognose der Holzqualität (Kapitel 2). Viele dieser Modelle sind als Module zur Kombination mit Einzelbaumsimulatoren konzipiert worden. Grundsätzliche Unterschiede ergeben sich für die Qualitätsmodelle daraus, ob das zugehörige Wachstumsmodell zu den statistischen oder ökophysiologischen Modellen zu rechnen ist (Kapitel 2.4). Die zahlreichen Untersuchungen der forstlichen Forschung in Deutschland zur Erfassung der Zusammenhänge zwischen waldbaulichen Maßnahmen und ihren Auswirkungen auf die Holzqualität sind fast ausnahmslos eher deskriptiver Ausrichtung und überwiegend auf der Grundlage einzelner Bestandesobjekte mit mehreren Behandlungsund/oder Begründungsvarianten durchgeführt worden. Nach Baumarten getrennt sollen folgende Beispiele genannt werden:

- Buche: Mahler U. Höwecke, 1991; SeEling, 1991; Beimgraben et Al., 1998;

- Eiche: Sachsse u. Grünebaum, 1990; Grünebaum et AL., 1993; Peters u. BecKer, 1995

- Douglasie: Hapla, 1981; Hecker u. Becker, 1997; Pelz u. Sauter, 1998;

- Fichte: Kramer et AL., 1971; KenK, 1990; SAUTER U. FAHRBACH, 1993;

- Kiefer: Kramer, 1977; Thren, 1983; Hapla et AL., 1987; SchmaltZ, 1989; SPellmanN U. NAGEL, 1992. 
Es muß allerdings betont werden, daß große Unterschiede bezüglich der Modellierbarkeit der verschiedenen Gütemerkmale bestehen (Kapitel 1.2 u. 2.2). Daher ist für eine Anzahl von Gütemerkmalen zur Zeit noch keine ausreichend genaue Prognose mit Hilfe von Modellen möglich, wenn lediglich Eingangsinformationen aus praxisüblichen Inventuren zur Verfügung stehen. Auch ist die Modellierung der Rohholzfehler bzw. eine rechnerische Güteklassensortierung für Rohholz weitaus einfacher als die Modellierung der Qualität von mechanisch verformtem oder chemisch aufgeschlossenem Holz bzw. der Gütesortierung von verarbeitetem Holz. Während die Modellierung der Rohholzqualität eine Prognose visuell erkennbarer Gütemerkmale des Rohholzes voraussetzt (z. B. MAGUIRE ET AL., 1994; MeILBY, 1999; s. auch Kapitel 1.2 sowie 2.4, 2.5 u. 2.6), müssen für die Modellierung der Qualität von verarbeitetem Holz unter Umständen Eigenschaften des Holzes auf Zell- und Faserebene sowie die Prozesse der Holzverwendung modelliert werden (z. B. DAHLBLOM ET AL., 1999; Cown ET AL., 1999; LIU ET AL., 1999; Bos ET AL., 1999; s. auch Kapitel 1.2). Die bereits existierenden Modelle zur Prognose spezieller Holzeigenschaften können aufgrund der erläuterten Komplexität zur Zeit nur eine Ergänzung zu rein deskriptiven Untersuchungen sein. Auch im Bereich der Modellierung der Rohholzqualität können nicht alle Gütemerkmale bereits mit ausreichender Genauigkeit geschätzt werden, so daß auch in diesem Bereich weiterhin Untersuchungen mit deskriptiver Ausrichtung benötigt werden. Dagegen erreichen statistische Modelle für eine Reihe von Gütemerkmalen, insbesondere Merkmale, die zwingend aus dem Wachstum bzw. den Wuchsbedingungen eines Baumes resultieren, schon eine Genauigkeit, die ihre Anwendung in Waldwachstumsmodellen zweckmäßig erscheinen läßt (Kapitel 2.4 u. 2.5).

Für die quantitative Modellierung, d. h. die Entwicklung von allgemeingültigen Modellen zur Prognose von Teilaspekten der Holzqualität, ist es daher angebracht, den Themenbereich stärker als bisher mit waldwachstumskundlichen und biometrischen Methoden zu bearbeiten.

\subsection{Zielsetzung}

Im Rahmen dieser Arbeit sollen flexible, statistische Modelle zur Prognose der Holzqualität auf einer breiten Datenbasis entwickelt werden, die als Eingangsinformationen lediglich Variablen benötigen, die von statistischen Einzelbaumsimulatoren zur Verfügung gestellt werden. Die Datenbasis muß sowohl eine möglichst hohe regionale als auch bestandesstrukturelle Repräsentativität aufweisen, um die Allgemeingültigkeit der Modelle zu gewährleisten. Die Modelle sollen zusätzlich zu einer rechnerischen Stärkeklassensortimentierung eine Güteklassensortimentierung unter Berücksichtigung ausgewählter Qualitätskriterien ermöglichen. Neben einer deutlich verbesserten Bewertung der Nutzfunktion von Waldbeständen sollen die in einem Modul integrierten Modelle zur kombinierten Stärke- und Güteklassenprognose auch die Prognose der Auswirkungen waldbaulicher Maßnahmen auf Teilaspekte der Holzverwen- 
dung zulassen. Unter Berücksichtigung dieser Zielsetzungen muß die Modellierung der Holzqualität über die Beschreibung der einzelnen Gütemerkmale erfolgen, die die Holzqualität beeinflussen. Demgegenüber hat eine direkte Modellierung der Güteklassen, welche die Ausprägungen der einzelnen Gütemerkmale aggregieren, mehrere Nachteile:

1. Die Abgrenzung der Güteklassen ist nicht immer trennscharf.

2. Bei zeitlichen Veränderungen und regionalen Unterschieden der Anforderungen an die Rohholzqualität ist eine direkte Modellierung der Güteklasse zu unflexibel.

3. Für eine an speziellen Holzverwendungen orientierte Gütesortierung ist die direkte Modellierung der Güteklasse zu unflexibel.

4. Die direkte Modellierung der Güteklasse liefert keine Eingangsinformationen für komplexe Auswertungen.

5. Es ist keine Berücksichtigung der Unterschiede der einzelnen Gütemerkmale bezüglich ihrer Modellierbarkeit möglich.

\section{zu Punkt 1.:}

Die lange Zeit als gesetzliche Grundlage der Holzsortierung dienende Verordnung über gesetzliche Handelsklassen für Rohholz (HKLVO) (BML, 1969) weist lediglich unscharfe Definitionen zur Abgrenzung der Güteklassen auf. Um eine objektiv nachvollziehbare Grundlage der Güteklassensortierung zu schaffen, entwickelten ScHULZ (1959), LÖFFLER (1965) und KNIGGE (1970) trennscharfe Grenzwerte für die Güteklassensortierung auf der Basis der unscharfen Vorgaben der Anlage zu $\S 1$ der HKLVO (HKS). Veränderte Anforderungen an die Qualität des Rohholzes und die Notwendigkeit einer Vereinheitlichung der Rohholzsortierung in Europa sollen zukünftig durch die Europäische Normung zur Rundholzsortierung berücksichtigt werden (DEUTSChES InSTITUT FÜR NORMUNG, 1997 a, b, c, d; RITTER, 1998; DeUtSChes InSTITUT FÜR NORMUNG, 1999 a, b, c). Die Europäische Normung der Rundholzsortierung gibt exakte Grenzwerte und Definitionen vor, die allerdings teilweise noch unverbindlich sind, da die Normen erst den Charakter sogenannter Vornormen haben. Inwieweit die veränderten und trennscharfen Grenzwerte zu Verschiebungen der Anteile in den verschiedenen Güteklassen führen werden, ist bereits anhand von Vergleichssortierungen prognostiziert worden (SCHUMACHER ET AL., 1997 a, b; WALTER ET AL., 1997). WALTER ET AL. (1997) erwarten beispielsweise, daß die neue Normung bei der Baumart Kiefer bei annähernd gleichbleibenden B-Holz-Anteilen zu einer Verminderung des C-Holz-Anteils und einem Anstieg des A-Holz-Anteils führen wird. RITTER (1998) prognostiziert für die Baumart Fichte ein Absinken des B-Holz-Anteils und einen Anstieg des C- und D-Holz-Anteils. Ferner wird darauf hingewiesen, daß der Aufwand für den Sortiervorgang durch die notwendige Überprüfung präziser Grenzwerte ansteigen wird. 


\section{zu Punkt 2.:}

Wie an dem Vergleich von HKS und Rundholzsortierung in der Europäischen Normung deutlich wird, sind die Anforderungen an die Qualität des Rundholzes einem zeitlichen Wandel unterworfen, der u. a. durch den technischen Fortschritt in der holzverarbeitenden Industrie verursacht wird. Weiterhin bestehen auch regionale Unterschiede in den Anforderungen an die Rohholzqualität. Eine flexible Güteklassenmodellierung muß daher die Ausprägung der Qualitätskriterien selbst beschreiben. Eine direkte Modellierung der Güteklassen ist demgegenüber in dem Moment veraltet, in dem die Grenzwerte, die bei der Entwicklung des Modells unterstellt wurden, nicht mehr gültig sind. Die Überlegungen zur flexiblen Konzeption der Qualitätsmodellierung ähneln den Ansätzen der Wertinventur nach WIEGARD (1998). Im Gegensatz zu anderen Wertanspracheverfahren (SPEIDEL, 1955; DüSER, 1978) erfolgt bei der Wertansprache nach WIEGARD (1998) eine separate Ansprache der einzelnen Holzfehler und keine gutachterliche Einschätzung der Güteklasse.

\section{zu Punkt 3.:}

Eine an der Holzverwendung orientierte Qualitätsmodellierung muß auch eine von den Grenzwerten einer mittleren Güteklassensortierung, wie sie die Europäische Normung der Rundholzsortierung darstellt, losgelöste Prognose erlauben. Vielfach bestehen spezielle Anforderungen und Bewertungen von Qualitätskriterien, die aus der jeweiligen Holzverwendung resultieren und die von den mittleren Grenzwerten abweichen. Die Baumart Douglasie eignet sich gut zur Verdeutlichung einer an der Holzverwendung orientierten Gütesortierung, da das Spektrum der potentiellen Verwendungsmöglichkeiten dieser Baumart sehr breit ist (SACHSSE, 1991). So bestehen beispielsweise Unterschiede je nachdem, ob Douglasienschnittholz zu Konstruktionszwecken oder optisch orientierten Zwecken verwendet werden soll (PElz U. SAUTER, 1998), was auch in der Anwendung unterschiedlicher Normen (DIN 4074 bzw. DIN EN 1611, Deutsches Institut FÜr NoRMUng, 1989 bzw. 1999 d) zum Ausdruck kommt. Eine weitere abweichende Bewertung der Qualitätskriterien findet bei der Holzverwendung der Douglasie für die Furnierherstellung statt (HECKER U. BECKER, 1997).

\section{zu Punkt 4.:}

Langfristig kann eine kombinierte Stärke- und Güteklassenmodellierung auch Eingangsinformationen für detailliertere Auswertungen wie die Optimierung der Rohholzsortimentierung (Forster u. Callahan, 1968; Briggs, 1980; Mendoza u. Bare, 1986; Sessions et Al., 1989; Punmalainen, 1997) und die Optimierung und Visualisierung der anfallenden Schnittholzsortimente für unterschiedliche Ausgangsqualitäten des Rohholzes und bei Variation des Einschnittmusters liefern (BARBOUR ET AL., 1999; NePVEU ET AL., 1999; USENIUS, 1999; Seeling U. Daquitaine, 1999). Noch weitergehende Einsatzmöglichkeiten bestehen in 
der Modellierung mechanischer und physikalischer Holzeigenschaften ${ }^{2}$ wie beispielsweise dem Elastizitätsmodul, der Zug-, Druck- und Biegefestigkeit von Schnittholz oder der Oberflächen-Rauhigkeit von Furnieren in Abhängigkeit von der Rohholzqualität und der Art der Holzverwendung. DAHLBlom ET AL. (1999) beschrieben das longitudinale Elastizitätsmodul und die durch Trocknungsvorgänge verursachten Deformationen von Fichtenbrettern in Abhängigkeit von der Faserstruktur. COWN ET AL. (1999) modellierten die räumliche Verteilung der Holzdichte, der Faserstruktur, der Reaktionsholzanteile und des Winkels der Mikrofibrillen im Stamm und schätzten auf der Basis dieser Verteilungsmuster das Elastizitätsmodul und die Biegefestigkeit. LIU ET AL. (1999) konnten Zusammenhänge zwischen Eigenschaften von Furnieren und Sperrhölzern von Pappelarten aus Plantagen in Abhängigkeit von mechanischen und physikalischen Holzeigenschaften (u. a. Faserstärke und -länge, Elastizitätsmodul) mit Hilfe multipler Regressionen modellieren. Die vollständige Erfassung spezieller mechanischer und physikalischer Holzeigenschaften ohne die Verwendung ergänzender empirischer Daten stellt die weitaus höchsten Anforderungen an die Modellkomplexität und -genauigkeit. Neben der vollständigen Beschreibung von Gütemerkmalen, die für eine visuelle Sortierung relevant sind, müssen räumliche Verteilungsmuster von Holzeigenschaften auf Zell- und Faserebene erfaßt werden. Zusätzlich muß ein Modell für die realitätsnahe Simulation des Prozesses der Holzverwendung entwickelt werden. Sollen Holzeigenschaften wie z. B. die Biegefestigkeit prognostiziert werden, die standardmäßig in genormten Versuchen ermittelt wird (z. B. Hapla, 1986; Hapla U. SAuter, 1987; Grammel, 1990; SeEling, 1995), so müssen auch diese Eigenschaften modelliert werden (DAHLBLOM ET AL., 1999). Aufgrund der Komplexität der gesamten Kette von aufeinander aufbauenden Modellen werden bisher überwiegend Teilbereiche modelliert, die als Eingangsinformationen empirisch erhobene Daten verwenden. Aus den genannten Gründen können die Modelle zur Prognose spezieller physikalischer und mechanischer Holzeigenschaften rein deskriptive Untersuchungen bisher nicht ersetzen.

\section{zu Punkt 5.:}

In Kapitel 2 wird anhand einer Klassifikation der die Holzqualität bestimmenden Gütemerkmale und der Literaturübersicht für ausgewählte Qualitätskriterien deutlich, daß große Unterschiede bezüglich der Modellierbarkeit der einzelnen Merkmale bestehen. Vor allem unterscheiden sich die Gütemerkmale darin, inwieweit sie sich in Abhängigkeit von den Eigenschaften eines Baumes beschreiben lassen. So besteht bei konstanter Entfernung von der Baumspitze ein enger Zusammenhang zwischen der Aststärke von Quirlästen und

\footnotetext{
${ }^{2}$ Zur Klassifikation der Holzeigenschaften siehe Grammel (1989, S. 29). Obwohl die Mechanik ein Teilgebiet der Physik ist, ist in der Forstbenutzung die Unterscheidung in mechanische und physikalische Eigenschaften üblich.
} 
verschiedenen Einzelbaumparametern (COLIN U. HOULLIER, 1991; MAGUIRE ET AL., 1994; VESTÖL ET AL., 1996). Demgegenüber bestehen für die Anteile an wurzelbürtiger Rotfäule (Heterobasidion annosum (Fr.) Cooke) in Fichtenbeständen eher Zusammenhänge zum Standort (ZYCHA U. KATÓ, 1967, S. 56 u. 77 ff.). Die Schaftform (Krümmung) der Baumarten Kiefer und Europäische Lärche wird in erheblichem Ausmaß von der Provenienz beeinflußt (Schober in Kramer, 1988, S. 131 bzw. 141). Während die für die Modellierung der Aststärke notwendigen unabhängigen Variablen in jedem Fall vom Wuchsmodell zur Verfügung gestellt werden, kann es vorkommen, daß keine Informationen über den Standort oder die Provenienz der jeweiligen Baumart vorliegen. Zusätzlich bestehen gravierende Unterschiede darin, wie eng die Ausprägung der verschiedenen Gütemerkmale überhaupt mit beschreibenden Variablen korreliert ist. Es erscheint daher auch aus modellkonzeptionellen Überlegungen sinnvoll, Gütemerkmale getrennt zu modellieren, um jedes Gütemerkmal entsprechend der jeweiligen Informationslage optimal einschätzen und beschreiben zu können.

Die im Rahmen dieser Arbeit entwickelten Modelle umfassen die Modellierung der Schaftform (Abholzigkeit), die Modellierung der Aststärke und die Modellierung des Astzustandes (Grünast, Totast und natürliche Astreinigung), da diese Gütekriterien im Vergleich zu anderen Gütekriterien eine relativ enge Korrelation zu den Wachstumsbedingungen und der Dimension eines Baumes sowie der Bestandesstruktur aufweisen. Die ausgewählten Kriterien lassen sich daher sehr gut auf der Basis der naturalen Resultate eines Einzelbaumsimulators prognostizieren. Im Gegensatz zu den genannten Qualitätskriterien gibt es eine Vielzahl weiterer Qualitätskriterien, die nicht direkt aus dem Wachstum bzw. deren Ausprägung kaum aus den Wuchsbedingungen eines Baumes resultieren. Auch für diese Qualitätskriterien, für die als Beispiele die Rotfäule bei Fichte oder der Buchenrotkern genannt werden können, sind bereits Modelle entwickelt worden (Rotfäule: STEnLID, 1987; CHAdOEUf ET AL., 1993; VollBReCHT U. Agestam, 1995; Vollbrecht ET AL., 1995; Buchenrotkern: KremPl U. MARK, 1962; BÖRNER, 1997). Die zur Einschätzung dieser Qualitätskriterien benötigten Variablen sind allerdings häufig keine Variablen, die von Einzelbaumsimulatoren als Resultat von Wachstumsprozessen bereitgestellt werden. Zusätzlich sind die Identifizierung von beschreibenden Variablen und die Modellierung grundsätzlich schwieriger, wenn ein Qualitätskriterium nicht zwingend aus den Wachstumsprozessen eines Baumes resultiert. Die Unterschiede in der Modellierbarkeit werden in Kapitel 2.2 dazu verwendet, eine neue Klassifikation für Qualitätskriterien nach dem Kriterium der Modellierbarkeit zu entwickeln. Die Einordnung eines Qualitätskriteriums in die Klassifikation dient der Verdeutlichung, welche hierarchischen Ebenen bei der Modellierung zu berücksichtigen sind. Die Qualitätskriterien, die sich nicht zwingend aus den Wachstumsprozessen eines Baumes ergeben, werden in der neuen Klassifikation als fakultative Qualitätskriterien bezeichnet. Im Gegensatz dazu werden die Qualitätskriterien, die 
aus dem Wachstum resultieren bzw. deren Ausprägungen überwiegend durch die Wachstumsbedingungen bestimmt werden, als obligate Qualitätskriterien definiert.

In Kapitel 2.6 wird eine Übersicht über bereits entwickelte Modelle zur Prognose der Rotfäule bei Fichte als Beispiel für die Modellierung eines fakultativen Qualitätskriteriums gegeben. Im Vergleich mit bereits bestehenden Ansätzen für Astmodelle, Schaftformmodelle sowie Modelle zur Einschätzung der Kronenansatzhöhe werden die größeren Schwierigkeiten bei der Modellierung der fakultativen Qualitätskriterien verdeutlicht.

Die Konzentration auf die Entwicklung von Modellen zur Prognose obligater Qualitätsmerkmale im Rahmen dieser Arbeit ist damit in den engeren Zusammenhängen zum Einzelbaumwachstum, in einer leichteren Modellierbarkeit und Entwicklung von allgemeingültigen Modellen begründet. Ein weiterer Grund für die getroffene Auswahl ist die Relevanz des jeweiligen Kriteriums für die Gütesortierung von Rohholz bzw. die Holzverwendung:

\section{Schaftform (Abholzigkeit):}

Die Anwendung oder Entwicklung eines Schaftformmodells ist in jedem Fall notwendig, da die Modellierung der Holzqualität nur in Kombination mit einer rechnerischen Stärkeklassensortierung und flexiblen Volumenschätzung von beliebigen Stammabschnitten praxistaugliche Schätzungen erlaubt. Neben der Holzqualität wird die Bewertung von Rohholz maßgeblich von der Dimension bestimmt (PETERS U. BECKER, 1995). Auch ist nach der Rundholzsortierung in der Europäischen Normung für die Baumarten Buche und Eiche eine Aushaltung bestimmter Güteklassen erst ab einem bestimmten Mindestmittendurchmesser möglich (DEUTSCHES InSTITUT FÜR NORMUNG, 1997 a). Für die Baumarten Fichte, Tanne und Kiefer bestehen variable Grenzwerte bei der Definition von zulässigen Werten für Risse, Abholzigkeit und Krümmung in Abhängigkeit von der Dimension des Stammes (DEUTSCHES INSTITUT FÜR NORMUNG, 1999 a und b).

Im Rahmen waldbaulicher Szenariensimulationen liegen keine Messungen von oberen Stammdurchmessern wie der $\mathrm{d}_{7}$-Messung vor. Es soll daher ein Modell entwickelt werden, das die behandlungsabhängigen Variationen der Schaftform mit Hilfe von Variablen beschreibt, die ihrerseits sensitiv auf forstliche Eingriffe und Unterschiede im Ausgangsverband reagieren bzw. die die Konkurrenzsituation eines Baumes beschreiben. Ein derartiges flexibles Schaftformmodell ermöglicht damit über eine reine Stärkeklassensortierung hinaus die Beschreibung des Gütemerkmals "Abholzigkeit" ohne die Verwendung eines weiteren oberen Durchmessers (HuI U. GADOW, 1997).

Schaftformmodelle können auch dazu eingesetzt werden, die Gütemerkmale "Anteil des juvenilen Holzes“" und "Anteil des Kernholzes" von obligaten Kernholzbildnern einzuschätzen (COWn, 1973; Hapla U. Saborowski, 1994; OjansuU U. Maltamo, 1995; s. Kapitel 2.5). Für flexible Schätzungen, d. h. zur Verallgemeinerung eines Modells, müssen allerdings 
Zusammenhänge zwischen den Ausprägungen dieser Gütemerkmale und beschreibenden Variablen identifiziert werden können. Die Kombination mit Rindenfunktionen erlaubt eine Prognose von Stammholzvolumina ohne Rinde (AltherR ET AL., 1978).

\section{Astigkeit:}

Als weiteres zu modellierendes Gütemerkmal wurde die Astigkeit ausgewählt, da hier einerseits enge Korrelationen zu Einzelbaumvariablen bestehen (z. B. COLIN U. Houllier, 1991; Maguire ET AL., 1994; Grace U. PonT, 1997) und gleichzeitig die Astigkeit für die meisten Baumarten das Gütemerkmal mit der höchsten Relevanz bei der Rohholz- und Schnittholzsortierung ist (BurSCHEl, 1981; HAPLA, 1986; SAUTER U. FAHRBACH, 1993; BeCKER U. SEELING, 1998) (s. auch Kapitel 2.4). Da neben der Aststärke auch der Zustand eines Astes (Grünast, Totast) von Bedeutung für die Gütesortierung ist (DEUTSCHES INSTITUT FÜR Normung, 1997 a, b, c und 1999 a, b, c), sollen neben Aststärkemodellen auch Modelle zur Beschreibung der Astmortalität und Astreinigung entwickelt werden. Für die vollständige Modellierung der Astigkeit sind zusätzlich Modelle für die Prognose von Asthäufigkeiten und die räumliche Anordnung der Äste im Holz von Bedeutung (Kapitel 2.4). Einen Einfluß auf die Holzqualität hat zusätzlich der vertikale Abgangswinkel eines Astes (Kapitel 2.4.6, Abb. 8; Colin U. Houllier, 1992). Die Modellierung des Knot Area Ratio (KAR), einem Kriterium der Schnittholzsortierung (vgl. GRAMMEL, 1989, S. 108), erfordert eine derartig umfassende Beschreibung der Astigkeit.

\section{Kronenansatzhöhe, Höhe des untersten Grünastes, Höhe des untersten Totastes:}

Da die Aststärke von der Position eines Astes in der Krone abhängig ist bzw. der Zustand eines Astes (Grünast, Totast, Astbeule und -narbe) davon bestimmt wird, in welcher Relation sich die Höhe des Astes zur Kronenansatzhöhe, zur Höhe des untersten Grünastes und zur Höhe des untersten Totastes befindet, soll im Rahmen dieser Arbeit eine verbesserte Schätzung der Kronenansatzhöhe entwickelt werden. Aufgrund der unterschiedlichen Informationen aus verschiedenen Inventuren, die zur Schätzung der Kronenansatzhöhe verwendet werden können, sollen ferner Modelle entwickelt werden, die eine optimale Ausnutzung der jeweiligen Informationsqualität gewährleisten.

Die Ansätze zur Modellierung der Astmortalität sowie zur Modellierung der Astreinigung berücksichtigen aus modelltechnischen Gründen die Schätzung der Kronenansatzhöhe (Kapitel 3.6). Neben der Gewährleistung von biologisch plausiblen Schätzungen sollen damit die Verbesserungen bei der Schätzung der Kronenansatzhöhe auch in den Modellen zur Beschreibung der Astmortalität und Astreinigung berücksichtigt werden. 


\section{Datengrundlage und Modellübersicht für die Beschreibung von Gü- temerkmalen}

\subsection{Datengrundlage}

Die Modelle zur Prognose der aufgeführten Gütemerkmale wurden für folgende Baumarten entwickelt:

- Kronenansatzmodelle: Fichte (Picea abies, (L.) Karst.), Kiefer (Pinus sylvestris, L.), Europäische Lärche (Larix decidua, L.), Douglasie (Pseudotsuga menziesii, (Mirb.) Franco), Buche (Fagus sylvatica, L.), Eiche (Quercus petraea, Liebl. und Quercus robur, L.);

- Astmodelle: Fichte, Kiefer, Douglasie, Buche;

- Schaftformmodelle: Fichte, Kiefer, Douglasie, Buche, Eiche.

Dabei unterscheiden sich die Geltungsbereiche der zur Verfügung stehenden Daten für die einzelnen Modelle deutlich. Für die Entwicklung der Kronenansatz- und der Schaftformmodelle konnte auf die umfangreiche Datenbank der Niedersächsischen Forstlichen Versuchsanstalt zurückgegriffen werden, während die Modellentwicklung zur Prognose der Astigkeit spezielle Datenerhebungen erforderte. Das ertragskundliche Versuchsflächennetz erstreckt sich über die Bundesländer Schleswig-Holstein, Niedersachsen, Nordrhein-Westfalen, Hessen, Rheinland-Pfalz und das Saarland (SPELlmANn ET AL., 1996). Damit ist für die Kronenansatzmodelle eine breite regionale Repräsentativität gewährleistet. Die erfaßten Bestandesstrukturen repräsentieren bisher überwiegend gleichaltrige Reinbestände, wobei allerdings ein breites Spektrum an Altersklassen und Behandlungsvarianten abgedeckt wird. Für die Entwicklung der Kronenansatzmodelle wurden alle Einzelbäume verwendet, für die Messungen des Brusthöhendurchmessers (BHD), der Höhe und der Kronenansatzhöhe vorlagen. Da bei der Parametrisierung auch abgeleitete Variablen wie die Kronenschirmfläche des Gesamtbestandes sowie die Spitzenhöhe und der mittlere BHD einer Baumart im Bestand verwendet wurden, waren teilweise vorgeschaltete Datenergänzungen notwendig. Dabei wurden für Bäume, für die lediglich der BHD und die Baumart bekannt waren, Höhen über Bestandeshöhenkurven geschätzt (NAGEL, 1999 a, S. 72). Die Ergänzung der Einzelbaumhöhen ermöglicht anschließend die Berechnung der Spitzenhöhe und des mittleren H/D-Wertes einer Baumart im Bestand. Für die Berechnung der Bestandeskronenschirmfläche müssen zusätzlich Kronenbreiten bzw. Kronenschirmflächen für Einzelbäume berechnet und aufsummiert werden (SCHMIDT ET AL., 2001). Die Kronenansatzhöhe ist bei den Nadelholzarten als die Höhe des untersten Quirls mit mindestens drei grünen Ästen und bei den Laubholzarten als die Höhe des untersten Primärastes definiert (NiEDERSÄChSISCHE ForstLiChE VersuchSANSTALT, 1996). Bestimmte neu entwickelte Teilmodelle wie ein dynamisches Kronenansatzmodell erforderten eine weitere Reduzierung der Datengrundlage, da nur Versuchsflächen mit mindestens einer Wiederholungsaufnahme verwendet werden konnten. Genaue Angaben über die 
Anzahl der verwendeten Einzelbaumdatensätze, Parzellenaufnahmen, sowie Häufigkeitsverteilungen der beschreibenden Variablen sind für die entwickelten Modelle jeweils vor der Modellbeschreibung in Kapitel 3.2 (Baumart Fichte) bzw. in Kapitel 9.1 und 9.2 (übrige Baumarten) aufgeführt.

Für die Entwicklung der Schaftformmodelle konnte ebenfalls auf umfangreiche Sektionsmessungen der Niedersächsischen Forstlichen Versuchsanstalt zurückgegriffen werden, womit ein Nordwestdeutschland umfassender regionaler Geltungsbereich gewährleistet ist. Auch hier stammen die Messungen überwiegend aus gleichaltrigen Reinbeständen mit einer großen Alters- bzw. Dimensionsspanne. Ein die Entwicklung von Schaftformmodellen einschränkender Aspekt in der Datenstruktur besteht darin, daß die Sektionsmessungen keinen Versuchsparzellen zugeordnet werden können. Eine Zuordnung ist lediglich bis auf die Ebene des Forstortes bzw. der Unterabteilung möglich. Die Bäume, an denen die Sektionsmessungen erfolgten, wurden häufig nicht direkt auf Versuchsparzellen entnommen, um andere Versuchszwecke nicht zu beeinträchtigen. Damit ist eine Überprüfung des Einflusses von Bestandesvariablen auf die Schaftform nicht möglich. Der Einfluß von Standortsvariablen kann demgegenüber durch die Zuordnung zu einer Unterabteilung erfaßt werden. Detaillierte Angaben über die Anzahl der verwendeten Sektionsmessungen, der zugehörigen Bäume und Versuchsparzellen werden getrennt nach Baumarten in Kapitel 3.7 (Baumart Fichte) bzw. in Kapitel 9.9 (übrige Baumarten) gemacht. Zusätzlich werden Häufigkeitsverteilungen der zur Beschreibung der Schaftform verwendeten Variablen angegeben. Die Sektionsmessungen wurden visuell und rechnerisch auf unplausible Werte überprüft. Eine genaue Beschreibung der rechnerischen Plausibilitätsprüfung erfolgt in Kapitel 3.7.1.

Im Gegensatz zu den Kronenansatz- und Sektionsmessungen enthielt die Datenbank der Niedersächsischen Forstlichen Versuchsanstalt keine Messungen von Astdurchmessern, die für eine Modellierung der vertikalen Astdurchmesserstruktur von Primärästen in der Krone von Waldbäumen geeignet sind. Auch lagen keine Messungen der Höhe des untersten Grünastes und der Höhe des untersten Totastes vor, mit deren Hilfe Modelle für die Astmortalität und -reinigung entwickelt werden können. Da die Datenerhebungen zur Erfassung von Astparametern an gefällten Bäumen durchgeführt werden mußten und diese sehr zeitaufwendig sind, ist der Parametrisierungsbereich der entwickelten Astmodelle weniger umfassend als bei den Kronenansatz- und Schaftformmodellen. So liegen die Aufnahmeflächen für die Astdurchmessermessungen und die Höhe des untersten Grünastes für die Baumart Fichte ausschließlich im Bereich des Harzes, des Sollings und des Reinhardswaldes (Südniedersachsen und Nordhessen) und umfassen lediglich bodensaure Standorte. Da andere Versuchszwecke durch die destruktive Gewinnung der Astparameter nicht beeinträchtigt werden sollten, wurden auch Bäume außerhalb von Versuchsflächen entnommen. Damit wurde auf die Überprüfung des Einflusses von Bestandesvariablen und Konkurrenzindizes auf die untersuchten Ast- 
parameter verzichtet. Anhand bereits entwickelter Modelle zur Schätzung des Astdurchmessers und weiterer Astparameter (Kapitel 2.4) wird jedoch deutlich, daß Bestandesvariablen und Konkurrenzindizes häufig keinen signifikanten Einfluß auf die Ausprägung von Astparametern haben, wenn bestimmte Einzelbaumvariablen bereits im Modell verwendet werden. Die Auswahl von Bäumen außerhalb von Versuchsflächen ermöglichte jedoch vor allem durch die Verwendung von solitärähnlichen Bäumen die Erweiterung des Parametrisierungsbereiches für die verwendeten Einzelbaumvariablen. Auch konnte so gewährleistet werden, daß ein breiter Dimensionsbereich der Einzelbäume und unterschiedliche Bestandesstrukturen berücksichtigt wurden. So sind mit Ausnahme der Baumart Kiefer alle Baumarten auch durch Messungen aus Mischbeständen bzw. den Mischbestandswuchsreihen der Niedersächsischen Forstlichen Versuchsanstalt repräsentiert. Astparameter von unterständigen Bäumen wurden im Rahmen der Arbeit nicht erfaßt. Für die Baumart Douglasie erfolgten mit Ausnahme von drei Forstorten im nordwestdeutschen Tiefland alle Astmessungen im Bereich des Sollings. Die Datenbasis der Baumart Kiefer weist eine breite regionale Repräsentativität für das nordwestdeutsche Tiefland auf und erfaßt Standorte, die durch unterschiedlich nährstoffversorgte Sande geprägt sind. Die Baumart Buche wird überwiegend durch Flächen im Bereich des Sollings und des Harzes und bodensaure Standorte repräsentiert. Eine einzelne Fläche liegt im Bereich des Göttinger Waldes auf einem Kalkstandort. Für die Höhe des untersten Totastes lagen für die Baumarten Fichte, Douglasie und Buche zusätzliche Messungen aus weiteren Mischbestandsversuchen vor. Genaue Angaben über die Art und Anzahl der gemessenen Astparameter, die Anzahl der zugehörigen Bäume und die Lage der Versuchsflächen sind in den Kapiteln 3.3 (Fichte) und 3.4 (Buche) vor der jeweiligen Modellbeschreibung sowie für die Baumarten Douglasie und Kiefer im Anhang (Kapitel 9.5) angegeben. Zusätzlich sind Häufigkeitsverteilungen der beschreibenden Variablen erstellt worden, die in den verschiedenen Astmodellen verwendet werden.

\subsection{Neue Klassifikation von Gütemerkmalen}

Vor der Darstellung der Methoden, die in dieser Arbeit zur Modellierung von ausgewählten Qualitätsmerkmalen verwendet werden, wird ein Überblick über bereits vorhandene Ansätze und Verfahren gegeben. Unter Qualitätsmerkmalen werden hier sowohl Holzeigenschaften als auch Holzfehler und -schäden zusammengefaßt. Aufgrund der spezifischen Eigenschaften der verschiedenen Qualitätsmerkmale bieten sich unterschiedliche Methoden und Modelltypen zu ihrer Beschreibung an. Im folgenden werden daher die zwei übergeordneten Gruppen der obligaten und fakultativen Qualitätsmerkmale definiert, wobei grundsätzliche Unterschiede der Modellierung als Klassifizierungskriterien verwendet werden. Die Klassifikation wurde im Rahmen dieser Arbeit neu entwickelt, da sie die Probleme bzw. Fragestellungen, die bei der Modellierung verschiedener Gütemerkmale auftreten, systematisiert und somit die Entwick- 
lung spezifischer Modelle erleichtert. Dadurch ergibt sich eine Abweichung zu den in der Forstbenutzung üblicherweise verwendeten Klassifikationen (z. B. STEUER, 1985). Die abweichende Klassifikation läßt sich durch die Ausrichtung der Arbeit auf die Modellentwicklung begründen.

In Abb. 2 wird die Einordnung eines Qualitätsmerkmals in die Gruppe der obligaten bzw. der fakultativen Qualitätsmerkmale verdeutlicht. Die Zuordnung eines Merkmals erfolgt hypothetisch, d. h. unabhängig davon, ob bereits Modelle für dieses Merkmal existieren oder ob eine Modellierung überhaupt möglich erscheint.

\begin{tabular}{|c|c|c|c|}
\hline 1. Ebene & $\begin{array}{c}\text { 2. Ebene } \\
\text { steigende Auflösung }\end{array}$ & 3. Ebene & Klassifikation \\
\hline \multirow[t]{2}{*}{$\begin{array}{c}\text { Modellierung des } \\
\text { Qualitätsmerkmals } \\
\text { auf Bestandesebene } \\
\text { notwendig? } \\
\text { JA }\end{array}$} & $\begin{array}{c}\text { Modellierung des } \\
\text { Auftretens des } \\
\text { Qualitätsmerkmals am } \\
\text { Einzelbaum notwendig? } \\
\text { JA }\end{array}$ & $\begin{array}{l}\text { Modellierung } \\
\text { der Ausprägung } \\
\text { am Einzelbaum! }\end{array}$ & \multirow{2}{*}{$\begin{array}{l}\text { Fakultative } \\
\text { Qualitätsmerkmale } \\
\text { z B.: } \\
\text { Stammkrümmungen, } \\
\text { Buchenrotkern, } \\
\text { Rotfäule, } \\
\text { Drehwuchs, } \\
\text { Stammrisse, } \\
\text { Schleimfluß }\end{array}$} \\
\hline & & & \\
\hline $\begin{array}{c}\text { Modellierung des } \\
\text { Qualitätsmerkmals } \\
\text { auf Bestandesebene } \\
\text { notwendig? } \\
\text { NEIN }\end{array}$ & $\begin{array}{c}\text { Modellierung des } \\
\text { Auftretens des } \\
\text { Qualitätsmerkmals am } \\
\text { Einzelbaum notwendig? } \\
\text { NEIN }\end{array}$ & $\begin{array}{l}\text { Modellierung } \\
\text { der Ausprägung } \\
\text { am Einzelbaum! }\end{array}$ & $\begin{array}{l}\text { Obligate } \\
\text { Qualitätsmerkmale } \\
\text { z. B.: } \\
\text { Astigkeit, } \\
\text { Stammform, } \\
\text { Holzfarbe }\end{array}$ \\
\hline
\end{tabular}

Abb. 2: Klassifizierung von Qualitätsmerkmalen nach der Anzahl der notwendigen Modellierungsebenen (fakultative und obligate Qualitätsmerkmale).

Dabei wird die Notwendigkeit einer Modellierung auf drei Hierarchieebenen beurteilt. Obligate und fakultative Qualitätsmerkmale lassen sich dadurch unterscheiden, ob eine Modellierung auf der 1. und 2. Ebene notwendig ist. Die 1. Ebene erfaßt die summarische Modellierung auf der Bestandesebene. Hier wird die Frage geklärt, ob ein Qualitätsmerkmal überhaupt und mit welchen Anteilen es innerhalb des Bestandeskollektivs auftritt. Typische Beispiele sind die Einschätzung des Auftretens und der Anteile von Buchenrotkern, von Rotfäule sowie von Anteilen an drehwüchsigen und krummen Stammformen im Bestand. Im engeren Sinne ließe sich auch diese Ebene noch einmal in die Ebenen Modellierung des Auftretens und der Ausprägung im Bestand unterteilen (Abb. 2).

Wird ein Auftreten auf der Bestandesebene prognostiziert, muß auf der 2. Ebene entschieden werden, ob das Qualitätsmerkmal am Einzelbaum auftritt oder nicht. Beispielsweise muß eingeschätzt werden, ob ein bestimmter Baum in einem Bestand, in dem Rotfäule auftritt, von Rotfäule befallen ist oder nicht. Für die fakultativen Qualitätsmerkmale ist die Mo- 
dellierung sowohl auf der 1. als auch auf der 2. Ebene notwendig, da sich diese Merkmalsgruppe nicht aus den Wachstumsprozessen des Einzelbaumes ableiten läßt. Zu den fakultativen Qualitätskriterien werden hier auch Merkmale gezählt, die zwar an jedem Baum vorkommen, die aber so schwache Ausprägungen haben können, daß sie nicht als Qualitätsmerkmal berücksichtigt werden (Risse, Krümmungen). Damit muß für diese Merkmale modelliert werden, ob ihre Ausprägung einen Grenzwert überschreitet, der das Merkmal zu einem relevanten Qualitätsmerkmal werden läßt. Im Gegensatz dazu entfällt für die Gruppe der obligaten Qualitätsmerkmale die Modellierung für die 1. und 2. Ebene, da diese Merkmale zwingend aus den Wachstumsprozessen des Einzelbaumes resultieren. Zu dieser Merkmalsgruppe zählen die Astigkeit, die Stammform (Abholzigkeit), juveniles Holz, Kernholzanteile bei obligaten Kernholzbildnern und die Jahrringbreite.

Auf der 3. Ebene erfolgt die Einschätzung der Ausprägung am Einzelbaum für obligate und fakultative Qualitätsmerkmale. Typische Ausprägungen für die obligaten Qualitätsmerkmale sind der Astdurchmesser, Astanzahlen, Kernholzanteile bei obligaten Kernholzarten, Formigkeit (Abholzigkeit) etc. Beispiele für fakultative Qualitätsmerkmale sind Rotkernbzw. Rotfäuledurchmesser, Stärke der Krümmung oder des Drehwuchses. Die Schwierigkeiten der Modellierung nehmen von den obligaten zu den fakultativen Qualitätsmerkmalen hin zu, weil für die obligaten Merkmale nur die Ausprägung am Einzelbaum modelliert werden muß. Auch ergeben sich für die obligaten Qualitätsmerkmale häufig engere Zusammenhänge zwischen der Ausprägung und den Wuchsbedingungen oder der Dimension des Baumes, was eine Einschätzung erleichtert.

Die 2. Ebene kann auch als Sonderfall der 3. Ebene (Ausprägung am Einzelbaum) angesehen werden. Tritt das fakultative Merkmal nicht am Baum auf, hätte die Ausprägung den Wert Null. Im Gegensatz dazu können obligate Qualitätsmerkmale nicht den Wert Null annehmen, da sie in jedem Fall vorhanden sind. Die Unterteilung in eine Modellierung des Auftretens und der Ausprägung des Merkmals am Einzelbaum ist jedoch sinnvoll, um Probleme bei der Modellierung zu interpretieren. Eventuell ergeben sich für die Einschätzung der Ausprägung eines fakultativen Merkmals enge Zusammenhänge mit bestimmten Variablen, nicht aber für die Einschätzung des Auftretens.

Abgesehen von der notwendigen Anzahl an Modellebenen, die für die Modellierung von Gütemerkmalen notwendig sind, bestehen auch Unterschiede zwischen den Modelltypen, die für die Modellierung der fakultativen und obligaten Qualitätsmerkmale verwendet werden können. Durch die Unterscheidung bei der Modellierung bezüglich Ausprägung und Auftreten von fakultativen Qualitätsmerkmalen müssen für diese Merkmale nicht nur direkt meßbare Variablen sondern auch Wahrscheinlichkeiten für das Auftreten geschätzt werden. Bei den obligaten Qualitätsmerkmalen sind Modelle zur Schätzung von Wahrscheinlichkeiten eher die Ausnahme. Ein Beispiel hierfür ist die Modellierung von vertikalen Verteilungsmustern von 
Internodalästen im Jahrestrieb ${ }^{3}$ für Douglasie (MAGUIRE ET AL., 1994). Aufgrund der leichteren Modellierbarkeit der obligaten Qualitätsmerkmale übertrifft die Modellanzahl für diese Merkmalsgruppe deutlich die Anzahl der Modelle für fakultative Qualitätsmerkmale. Allerdings ist bei diesem Vergleich auch die unterschiedliche Relevanz der Qualitätsmerkmale zu berücksichtigen. Zusammenfassend gilt, daß für die Modellierung eines Qualitätsmerkmals für jede der drei Hierarchieebenen entschieden werden muß, ob eine Modellierung notwendig ist, ob eine Modellierung möglich ist und wie die Modellierung erfolgen soll.

Die folgende Literaturübersicht (Kapitel 2.3 bis 2.6) befaßt sich mit den Qualitätsmerkmalen, für die in dieser Arbeit neue Modellansätze entwickelt werden. Zur Gruppe der obligaten Qualitätsmerkmale zählen die Astmodelle und flexiblen Schaftformmodelle. Für die Gruppe der fakultativen Qualitätskriterien wird eine Übersicht über Modelle zur Beschreibung der Rotfäule bei Fichte gegeben. Im Rahmen dieser Arbeit wird für dieses Merkmal kein Modell entwickelt. Die Literaturübersicht soll jedoch die neu entwickelte Klassifikation veranschaulichen. Ein zusätzliches Unterkapitel entfällt auf die Beschreibung von Modellen zur Schätzung der Kronenansatzhöhe. Durch die Höhe des Kronenansatzes und des untersten Grünastes wird der Übergang von der Grünast- zur Totastzone definiert (Kapitel 3.6). Auch begrenzt die Höhe des untersten Grünastes den Geltungsbereich des neu entwickelten Astdurchmessermodells (Kapitel 3.3). Die Modellierung der Kronenansatzhöhe beeinflußt somit indirekt die Prognose der Astigkeit. Die Verbesserung bestehender statischer Kronenansatzmodelle läßt daher auch eine Erhöhung der Genauigkeit für die Modellierung der Astigkeit erwarten.

\subsection{Schätzung der Kronenansatzhöhe}

Einzelbaumorientierte Wachstumssimulatoren verfügen im allgemeinen über Modelle zur Schätzung der Kronenansatzhöhe und ihrer Entwicklung im Rahmen von Wachstumsprognosen. Wenn zur Modellierung der Kronenform auf einfache geometrische Formen wie z. B. Paraboloide (Lichtkrone) und Kegelstümpfe (Schattenkrone) zurückgegriffen wird, bestimmen ausschließlich die Höhe des Kronenansatzes, die Kronenbreite sowie die Baumhöhe die Dimension der Baumkrone (Burger, 1939; KÄndler, 1986; PretzSCH, 1992; HanN, 1999; HUSSEIN, 2001). Viele statistische Wachstumssimulatoren verwenden zur Schätzung des Höhen- und Durchmesserzuwachses des Einzelbaumes auch abgeleitete Variablen, wie die Kronenmantelfläche und Konkurrenzindizes, die auf der Kronendimension basieren (Hasenauer, 1994; Pretzsch U. Kahn, 1998; Nagel, 1999 a).

\footnotetext{
${ }^{3}$ Im engeren Sinne müßte anstelle von Jahrestrieb von Internodium gesprochen werden, da durch Syllepsis und Prolepsis auch zwei Internodien pro Wachstumsperiode angelegt werden können (ROLOFF, 1986, S. 72). In dieser Arbeit wird das Internodium mit dem Jahrestrieb gleichgesetzt, da eine Modellierung von Syllepsis bzw. Prolepsis in statistischen Wachstumssimulatoren bisher nicht möglich ist.
} 
Die Verwendung der Kronendimension und der an ihr orientierten Konkurrenzindizes hat gegenüber durchmesser- und baumhöhenbasierten Konkurrenzindizes mehrere Vorteile. Da die Baumarten bei gleichem Durchmesser unterschiedliche Kronenbreiten und Kronenansatzhöhen aufweisen, wird durch die Verwendung von an Kronendimensionen orientierten $\mathrm{Zu}$ wachsfunktionen die Gültigkeit der Wuchsprognose auch für Mischbestände erreicht. Dabei wird unterstellt, daß Baumarten, die zu einem bestimmten Durchmesser, einer bestimmten Höhe und Kronenansatzhöhe größere Kronendimensionen als andere Baumarten aufweisen, auch einen größeren Konkurrenzdruck ausüben und einen größeren Assimilationsapparat besitzen. Die unterschiedlichen Konkurrenzeigenschaften der Baumarten können im Modell durch die Verwendung von sogenannten Transmissionskoeffizienten weiter modifiziert werden (Ellenberg, 1986; Pretzsch U. Kahn, 1998). Die Kronendimension ist weiterhin ein Maß für die Entwicklungsgeschichte eines Baumes bzw. die Entwicklung seiner Konkurrenzsituation, die Einfluß auf den momentanen Zuwachs des Baumes hat. Aus diesen Gründen hat die Verwendung von Kronendimensionen bzw. von aus ihnen abgeleiteten Konkurrenzindizes zu höheren Bestimmtheitsmaßen bei der Prognose des Einzelbaumwachstums gegenüber der Verwendung von Konkurrenzindizes auf der Basis von Durchmesser und Höhe geführt (Biging U. DobBertin, 1995).

Die Vorteile, die sich aus der Berücksichtigung der geschichtlichen Konkurrenzsituation über die Kronendimension ergeben, erfordern allerdings Modelle zur Schätzung der Kronenansatzhöhe und -breite, die sensitiv auf unterschiedliche Konkurrenzsituationen reagieren und eventuell vorhandene Dimensionstrends ${ }^{4}$ berücksichtigen. Die Mischbestandseignung ergibt sich dagegen bereits aus den baumartenspezifischen Kronenbreiten und Kronenansatzhöhen zu gegebenem Durchmesser und/oder gegebener Baumhöhe.

Im Rahmen der Modellierung der Holzqualität kommt der möglichst genauen Schätzung der Kronenansatzhöhe eine mindestens ebenso wichtige Bedeutung zu, wie bei der konkurrenzsensitiven Schätzung von Einzelbaumzuwächsen. Durch die Kronenansatzhöhe wird annähernd der Übergang von der Grünast- zur Totastzone definiert. D. h. die Kronenansatzhöhe definiert die Grenze, ab der ein Ast kein Dickenwachstum mehr hat und ab welcher der Ast abgestorben in den Stamm einwächst. Aus waldwachstumskundlicher Sicht wird mit der Kronenansatzhöhe die untere Grenze des Photosyntheseapparates eines Baumes festgelegt. Eine möglichst genaue Modellierung des Überganges von Grünast- zu Totastzone als eines Bereiches, der zur Primärproduktion eines Baumes nur einen marginalen Beitrag liefert, ist für eine Wachstumsprognose unbedeutend. Unter der Modellierung des Überganges von Grünast- zu Totastzone wird hier die Beschreibung der Verteilung von lebenden und toten

\footnotetext{
${ }^{4}$ Unter Dimensionstrends werden in dieser Arbeit ausschließlich Änderungen der unabhängigen Variablen verstanden, die durch die Einzelbaumdimension und/oder die Entwicklungsstufe des Bestandes beeinflußt werden.
} 
Ästen zwischen der Kronenansatzhöhe und der Höhe des untersten Grünastes verstanden. Aufgrund der Definition zur Festlegung der Kronenansatzhöhe als Höhe des untersten Quirls mit mindestens drei grünen Ästen (NIEDERSÄChsische ForstLiCHE VERSUCHSANSTALt, 1996) können sich bereits auf der Höhe des Kronenansatzes Totäste befinden. Gleichzeitig treten unterschiedlich lange Übergangsbereiche von der Grün- zur Totastzone auf. Für die Qualitätsmodellierung ist die Beschreibung der Übergangszone zusätzlich zur Kronenansatzschätzung von größerer Bedeutung, da ein deutlicher Unterschied in der Bewertung des Einflusses von Tot- und Grünästen auf die Holzqualität besteht. So liegen die zulässigen Grenzwerte zwischen zwei Güteklassen für Grünäste in der Regel über den Werten für Totäste (Deutsches Institut FÜr Normung, 1999 a, b, c und 1997 a). Die Modellierung der Kronenansatzhöhe bzw. des Übergangsbereiches beeinflußt die Holzqualität damit sowohl über die Festlegung des Astzustandes (lebend/tot) als auch über den Astdurchmesser, der bis zum Zeitpunkt des Absterbens erreicht wird. Im Rahmen von Waldwachstumssimulationen ist es daher auch für die Qualitätsmodellierung von Bedeutung, die Kronenansatzhöhe unter Berücksichtigung der Konkurrenzsituation bzw. unterschiedlicher waldbaulicher Behandlungen zu schätzen.

Um im Rahmen von Qualitätsmodellierungen den Übergangsbereich von Grün- und Totastzone möglichst genau zu beschreiben, verwenden KELLOMÄKI und VÄISÄNEN (1988) ein Populationsmodell, das für ein bestimmtes Astalter die Zahl überlebender bzw. toter Äste auf Bestandes- oder Einzelbaumebene (Mittelwert) prognostiziert. Da es sich um ein Populationsmodell handelt, lassen sich Parameter wie die maximale Astpopulationswachstumsrate, die maximale Tragfähigkeit an Ästen (Kapazität) auf Bestandes- und Einzelbaumebene (Mittelwert) und eine dimensionslose Größe zur Quantifizierung der Beeinflußung der Bäume für unterschiedliche Standortstypen berechnen. Das Modell wurde von KELLOMÄKI ET AL. (1989) weiterentwickelt, indem ein zweistufiges Populationsmodell für die Anzahl lebender Quirle und für die Anzahl lebender Äste in den Quirlen verwendet wurde. Soll für einen einzelnen Ast der Absterbezeitpunkt und der Zeitpunkt der natürlichen Astreinigung prognostiziert werden, muß das Populationsmodell um einfache Regeln ergänzt werden. KELLOMÄKI ET AL. (1989) formulierten, daß die prognostizierte Sterberate für einen Quirl, beginnend beim schwächsten Ast, erfüllt wird. Der maximale Astreinigungszeitraum beträgt 9 Jahre und die Astreinigung folgt in derselben Reihenfolge, in der die Äste abgestorben sind.

Die notwendige Datenerfassung für das beschriebene Populationsmodell ist als sehr umfangreich zu beurteilen. Trotzdem müssen die empirisch abgesicherten Regeln zusätzlich durch weitere Annahmen ergänzt werden, für die keine Daten zur Verfügung stehen. So müßten für die Absterbereihenfolge innerhalb eines Quirls eigentlich periodische Aufnahmen erfolgen, was jedoch bei der destruktiven Gewinnung von Astdaten nicht möglich ist. 
Im Gegensatz zum beschriebenen Populationsmodell werden in den meisten Wachstumsmodellen einfache statistische Funktionen zur Schätzung der Kronenansatzhöhe verwendet. Dabei ähneln sich die Funktionstypen unabhängig davon, ob die Prognose neben der eigentlichen Wachstumsprognose auch die Einschätzung der Holzqualität umfassen soll oder nicht. Liegt ein Schwerpunkt auf der Modellierung der Holzqualität, wird das Modell zur Prognose der Kronenansatzhöhe häufig durch ein Modell zur Schätzung der Höhe des untersten lebenden Astes ergänzt (COLIN U. Houllier, 1992). Durch die Schätzung beider Höhenwerte lassen sich Grünastzone, obere Grenze der Totastzone und der Übergangsbereich beider Zonen ebenfalls einschätzen. Im Gegensatz zum Populationsmodell von KELLOMÄKI ET AL. (1989) kann, wie bereits erläutert wurde, die genaue Zusammensetzung der Quirle aus toten und lebenden Ästen nicht direkt beschrieben werden. Allerdings ließen sich für die statistischen Modelle ebenfalls einfache Regeln wie beispielsweise die Festlegung der Absterbereihenfolge der Äste in der Übergangszone formulieren. Damit ließen sich biologisch plausiblere Verteilungsmuster von Grün- und Totästen beschreiben.

Die statistischen Modelle zur Prognose der Kronenansatzhöhe lassen sich in statische und dynamische Modelle untergliedern. Statische Modelle schätzen die Kronenansatzhöhe direkt aus anderen Einzelbaumvariablen wie z. B. dem Durchmesser und der Höhe. Der Vorteil dieses Ansatzes besteht darin, daß zur Parametrisierung keine Wiederholungsaufnahmen benötigt werden. Die Genauigkeit der Schätzung hängt u. a. von der Genauigkeit der Prognose des Durchmessers bzw. anderer unabhängiger Variablen ab, die zur Beschreibung der Kronenansatzhöhe verwendet werden. Als größten Nachteil beurteilt HASENAUER (1994), daß sich die Auswirkungen von veränderten Konkurrenzbedingungen, wie sie durch Durchforstungen verursacht werden, nicht abbilden lassen. Wird ein Baum beispielsweise stark freigestellt, so ist es plausibel, daß sich seine Kronenansatzhöhe für einen bestimmten Zeitaum nicht verändern wird. Ein statisches Modell, das die Kronenansatzhöhe nur aus dem Durchmesser herleitet, wird zu einer weiteren Verschiebung des Kronenansatzes führen, da dem Dimensionstrend folgend dickere Bäume im Mittel höhere Kronenansätze haben (KRAMER, 1988, S. 21, Abb. 7; s. auch Fußnote 4). Hierbei stellt sich jedoch die Frage, ob statische Kronenansatzmodelle, die über weitere unabhängige Variablen verfügen, nicht zumindest theoretisch auch in der Lage sind, Kronenansatzhöhen sensitiver als nur dem allgemeinen Dimensionstrend folgend zu modellieren. Beinhalten statische Modelle Variablen, die die Konkurrenzsituation beschreiben oder Variablen, die ihrerseits auf eine veränderte Konkurrenzsituation reagieren, wäre eine konkurrenzsensitive Modellierung der Kronenansatzhöhe prinzipiell möglich. Voruntersuchungen im Rahmen dieser Arbeit haben jedoch gezeigt, daß derartige Variablen, die die Kronenansatzhöhe kurzfristig beeinflussen, im allgemeinen zu keiner signifikanten Verbesserung statischer Modelle führen. 
Als Beispiele für den statischen Modellansatz werden hier fünf unterschiedlich komplexe Modelle genannt, die alle bereits in Simulationssoftware implementiert wurden. Über der jeweiligen Formel ist der Wachstumssimulator angegeben, in dem das Modell verwendet wird.

NAGEL (1999) schätzt den Kronenansatz ausschließlich mit Hilfe der unabhängigen Variablen Höhe und H/D-Wert bzw. das Kronenansatzverhältnis nur mit Hilfe des H/D-Wertes ${ }^{5}$ : in Bwin:

$$
K A=H *\left[1-e^{-\left(\alpha+\beta^{*} H / D\right)^{2}}\right]
$$

PRETZSCH und KAHN (1998) verwendeten einen ähnlichen Ansatz, wobei als weitere Variable zur Schätzung des Kronenansatzverhältnisses der Brusthöhendurchmesser benutzt wird. Als problematisch ist zu beurteilen, daß positive Werte im Exponenten stehen können, was zu negativen Kronenansatzverhältnissen führt. In der tatsächlichen Anwendung sind diese biologisch unplausiblen Fälle allerdings sehr selten.

in Silva:

$$
K A=H *\left[1-e^{-\left(\alpha+\beta^{*} H / D+\gamma^{*} D\right)}\right]
$$

EK (1974) entwickelte ein statisches Kronenansatzmodell auf der Basis der unabhängigen Variablen BHD und Baumhöhe.

in Forest:

$$
K A=H * e^{\alpha^{*} H^{\beta}(D+\gamma)} \delta
$$

Das Modell (3) wurde von HASENAUER (1994) vereinfacht, da je nach Baumart nur zwei bis drei Koeffizienten bei einer Irrtumswahrscheinlichkeit von 5\% signifikant waren.

in Forest (modifiziert):

$$
K A=H * e^{\alpha^{*} H} \beta * D^{\delta}
$$

Das von COLIN und HoulliER (1992) entwickelte Modell beinhaltet die unabhängigen Variablen Alter, H/D-Wert und Baumhöhe. Wie bei dem Modellansatz von PRETZSCH und KAHN (1998) kann es theoretisch zu unplausiblen Schätzungen von Kronenansatzverhältnissen mit Werten größer 1 und kleiner 0 kommen.

\footnotetext{
${ }^{5}$ Unter Kronenansatzverhältnis wird hier das Komplement des Kronenverhältnisses zu 1, d. h. die relative Höhe bis zum Kronenansatz verstanden.
} 
in $C E P$ :

$$
\left.K A=H *\left[1-\delta-e^{-\left(\alpha^{*}\right. \text { Alter }}{ }^{1.5}\right)-\beta * H / D-\gamma^{*} H^{2}\right]
$$

wobei für alle Modelle gilt:

$K A=$ Kronenansatzhöhe $(\mathrm{m})$;

$H \quad=$ Baumhöhe (m);

$D \quad=$ Brusthöhendurchmesser $(\mathrm{cm})$;

Alter $=$ Alter $(\mathrm{J})$.

Die Modelle werden mit Ausnahme des Originalmodells von EK (1974) im Kapitel 3 "Entwicklung neuer Ansätze zur Qualitätsprognose“ als Vergleichsmodelle genutzt, wobei neben der Gegenüberstellung von statistischen Kenngrößen auch eine Interpretation der Wirkungsrichtung der unabhängigen Variablen erfolgt.

\subsection{Modellierung von qualitätsrelevanten Astparametern}

\subsubsection{Modellübersicht}

Die neu entwickelten Astmodelle sollen neben den flexiblen Schaftformmodellen als Grundlage für die Entwicklung eines Qualitätsmoduls für das Waldwachstumsmodell Bwinpro dienen. Die Astigkeit nimmt unter den die Holzqualität beeinflussenden Kriterien eine herausragende Rolle ein (Sauter U. Fahrbach, 1993; Becker U. Seeling, 1998). Burschel (1981) bezeichnet die Astigkeit und die Einzelstammdimension als wichtigste Qualitätskriterien für Fichtenstammholz. In den Untersuchungen von HAPLA (1986) war die innere Astigkeit zu 98.3\% das für die Schnittholzsortierung von Douglasie ausschlaggebende Kriterium. Speziell für die Qualitätsbeurteilung von Nadelstamm- bzw. Nadelschnittholz ist die Astigkeit das herausragende Qualitätskriterium. Auch bei der Sortierung von Laubstammholz spielt die Astigkeit eine wichtige Rolle. Jedoch nimmt die Bedeutung weiterer Qualitätsmerkmale von den Massenholzarten (Fichte und Weißtanne) über die Nadelholzarten mit Wertholzpotential (Kiefer und Douglasie) zu den Laubholzarten mit Wertholzpotential (Buche und Eiche) zu.

An Astmodelle, die in Waldwachstumsmodellen eingesetzt werden, muß - wie für die Schätzung der Kronenansatzhöhe und die Modellierung der Schaftform - die Forderung gestellt werden, daß eine Sensitivität gegenüber unterschiedlichen Behandlungskonzepten besteht. Diese Sensitivität kann gewährleistet werden, indem die Astparameter als Funktionen von Variablen geschätzt werden, die die Bestandesbehandlung beschreiben (Dichte- und Konkurrenzindizes). Eine weitere Möglichkeit besteht in der Verwendung von Variablen, die ihrerseits auf unterschiedliche waldbauliche Behandlungen reagieren (z. B. H/D-Wert).

Astmodelle können in verschiedene Modellgruppen unterteilt werden. Die grundsätzlich übliche Unterscheidung von Wuchsmodellen in statistische Modelle und Prozeßmodelle läßt sich auch auf Astmodelle übertragen, da diese als Wuchsmodelle für ein spezielles Baum- 
kompartiment aufgefaßt werden können (Abb. 3). Allerdings handelt es sich bei Astmodellen in den meisten Fällen um sogenannte statische Modelle, d. h. es werden die interessierenden Astgrößen direkt und nicht über ihre Veränderungsraten geschätzt.

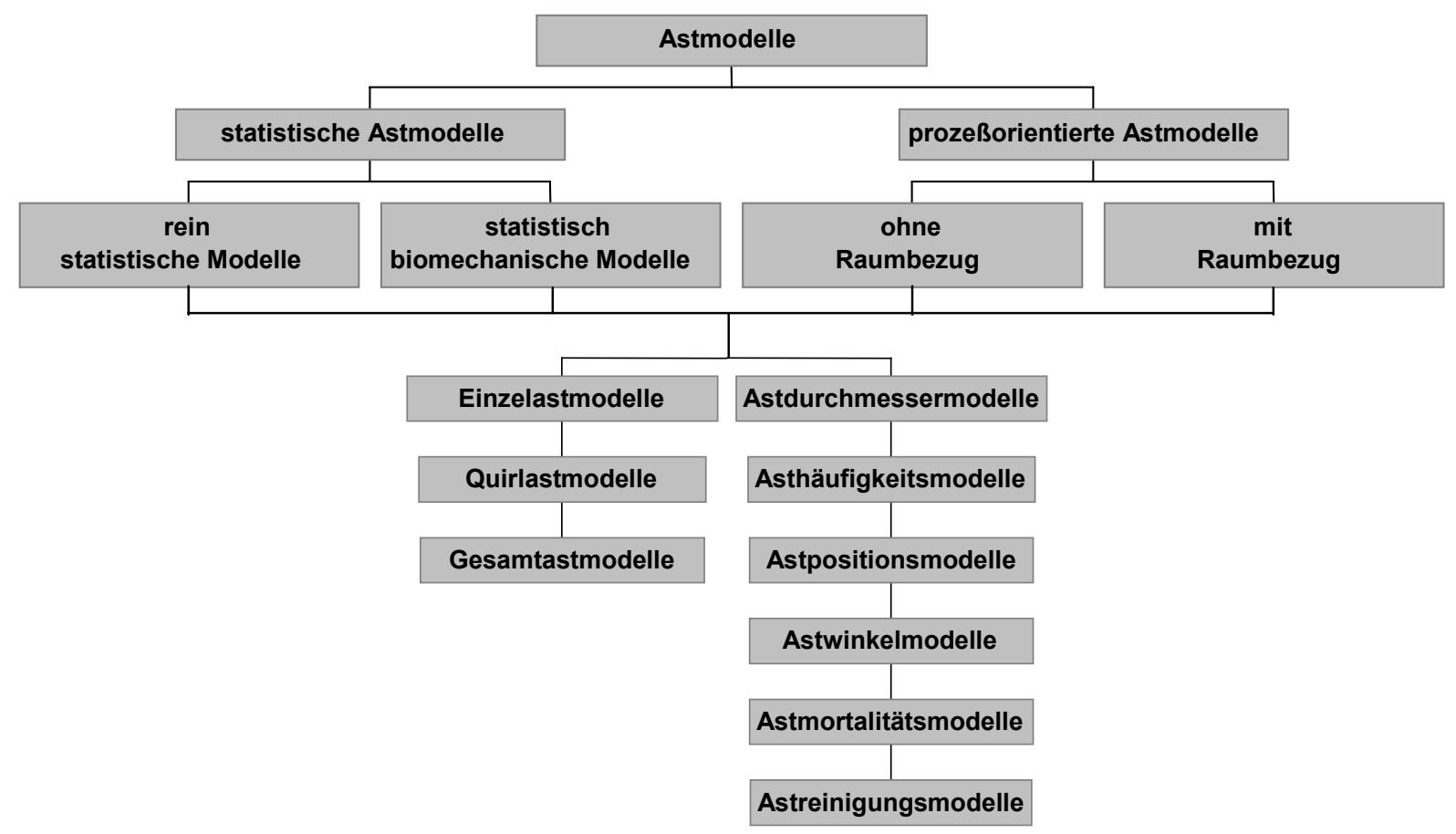

Abb. 3: Systematische Übersicht verschiedener Typen von Astmodellen. Beispiele für die verschiedenen Typen von Astmodellen sind im folgenden Text aufgeführt.

Statistische Astmodelle lassen sich weiter in rein statistische und statistisch biomechanische Modelle untergliedern. Diese Unterteilung kann man mit der Einteilung von positionsabhängigen und positionsunabhängigen Einzelbaumwuchsmodellen vergleichen. Statistisch biomechanische Astmodelle berücksichtigen neben statistischen Zusammenhängen auf Einzelbaumebene auch die individuelle räumliche Wuchskonstellation des Einzelbaumes. Durch die Berücksichtigung der Baumpositionen kann die laterale Kronendynamik als Resultat der Konkurrenzsituation des Baumes dargestellt werden. Unterschiedlicher seitlicher Konkurrenzdruck eines Baumes führt zur Förderung oder Stagnation des Astwachstums in der jeweiligen Richtung. Diese Kronendynamik kann prinzipiell als Verlagerung von Kronenschnitten auf der Höhe von Quirlen dargestellt werden (SEIFERT, 1999). Da die Längen und Durchmesser von Ästen eng korreliert sind, können über die Schätzung von Kronenbreiten bzw. Astlängen auch die zugehörigen Astdurchmesser geschätzt werden (MEILBY, 1999; SEIFERT, 1999). Die resultierenden Astdurchmesser innerhalb eines Quirls weisen in Richtung geringen Konkurrenzdrucks höhere Werte auf als in Richtung hohen Konkurrenzdrucks. Im Gegensatz zu rein statistischen Astmodellen kann die jeweilige kleinräumige Bestandesstruktur bei den statistisch biomechanischen Modellen kausal berücksichtigt werden. Als problematisch muß der hohe Aufwand bei der Datenerhebung für diesen Modelltyp beurteilt werden. Ein Vorteil er- 
gibt sich aber nur dann, wenn die Raumstruktur des modellierten Bestandes wirklichkeitsnah abgebildet werden kann ${ }^{6}$.

Ökophysiologische Prozeßmodelle zeichnen sich durch eine noch stärkere Kausalität bei der Prognose des Wachstums aus, wobei physiologische Gesetzmäßigkeiten mit Verteilungsregeln für die Biomasse verknüpft werden. Bei den Prozeßmodellen wird die räumliche Struktur von Waldbeständen ebenfalls unterschiedlich detailliert berücksichtigt (PRETZSCH, 1992). Prozeßmodelle beinhalten durch die Schätzung von Photosyntheseprodukten und deren Verteilung auf die einzelnen Baumkompartimente in den meisten Fällen bereits Astmodelle unterschiedlicher Komplexität (KellomäKI, 1981; SlobOdA U. Pfreundt, 1989). In den letzten Jahren sind jedoch Prozeßmodelle vermehrt dazu eingesetzt worden, speziell die Astigkeit, die Astreinigung und deren Auswirkungen auf die Holzqualität einzuschätzen (OKer BlOM ET AL., 1988; KellomäKI ET AL., 1989; COURBAUd ET AL., 1996; FourCAUd ET AL., 1996; MÄKELÄ ET AL., 1996).

Eine andere Möglichkeit, Astmodelle zu klassifizieren, besteht in der Einteilung nach der Komplexität des modellierten Objektes (Abb. 3). Die Komplexität steigt von Modelltypen, die die Eigenschaften spezieller einzelner Äste am Baum modellieren, über die Modellierung bestimmter Äste jedes Astquirls und die Modellierung aller Quirläste bis zur Modellierung aller Äste 1. Ordnung eines Baumes. Im Gegensatz zu den Astmodellen, die die Eigenschaften von Ästen 1. Ordnung prognostizieren, bilden Kronenverzweigungsmodelle die Architektur der Baumkrone als Gefüge der Einzeläste unter Berücksichtigung ihrer Eigenschaften ab (RolofF, 1986; Gruber, 1987; KURTH, 1994; KranigK, 1995). Für die vollständige Modellierung der Astigkeit als Gütemerkmal bei der Rohholz- und Schnittholzsortierung ${ }^{7}$ reicht allerdings die Prognose der Durchmesser, der Anzahl, der vertikalen Position am Stamm, der Horizontalwinkel und der Abgangswinkel (Vertikalwinkel) der Äste 1. Ordnung eines Baumes aus. Diese Modelle müssen mit solchen zur Einschätzung des Mortalitäts- und Astreinigungszeitpunktes ergänzt werden. Bei der Qualitätsmodellierung kann daher auf die Nachbildung der Kronenarchitektur verzichtet werden.

Astmodelle sind bisher vor allem für Nadelholzarten entwickelt worden, weil durch das streng orthotrope Wachstum einer eindeutigen Zentralachse und die stärker ausgeprägte Apikalkontrolle eine systematischere Kronenarchitektur vorgegeben ist (Abb. 4) ${ }^{8}$.

\footnotetext{
${ }^{6}$ Algorithmen zur Erzeugung der Raumstruktur wurden u. a. von PRETZSCH (1995), LEWANDOWSKI U. GADOW (1997), POMMERENING (1998) sowie BIBER (1999) entwickelt.

${ }^{7}$ Für die Rohholzsortierung vgl. DEUTSCHES InSTITUT FÜR NORMUNG (1997 a, b, c, d und 1999 a, b, c), für die Schnittholzsortierung vgl. DEUTSCHES INSTITUT FÜR NORMUNG (1989).

${ }^{8}$ GRUBER (1987, S. 185) spricht bei fehlender Zentralachse bzw. mehreren steilen Mischachsen von einer decurrenten und bei vorhandener Zentralachse mit mehr oder weniger horizontal abzweigenden Seitenachsen von einer excurrenten Kronenform.
} 


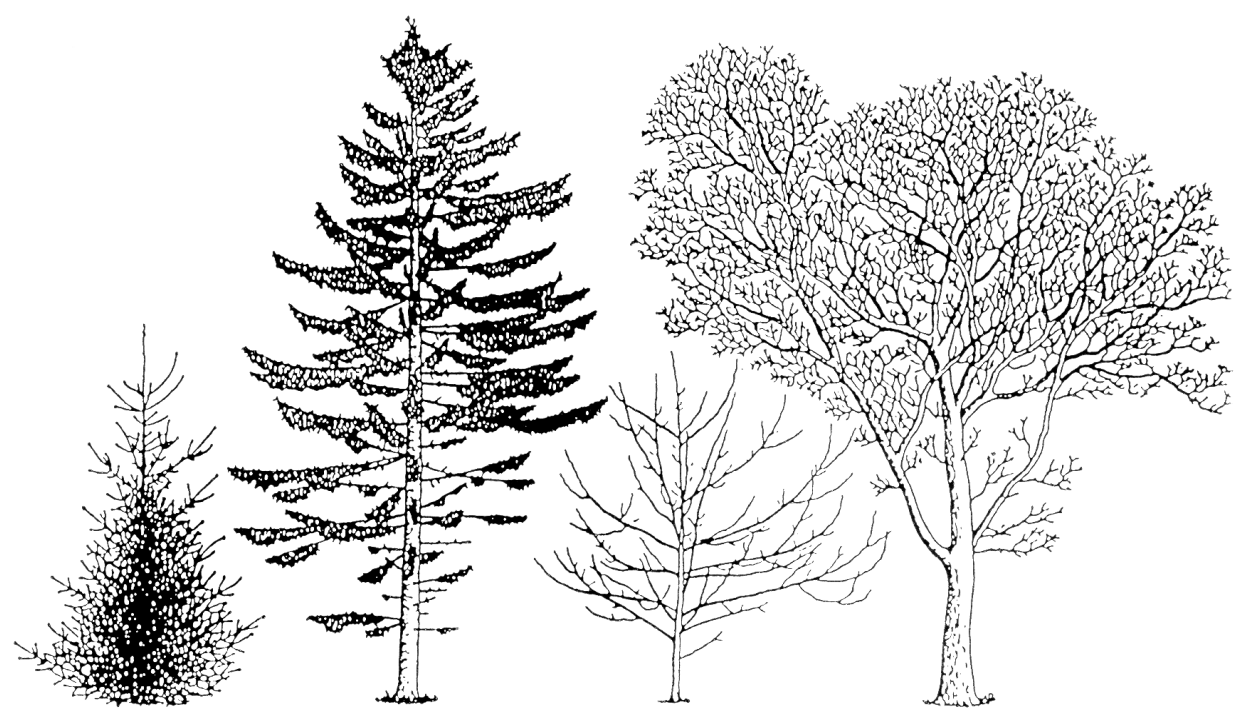

Apikaldominanz

stark

stark

Apikalkontrolle

stark

schwach

Abb. 4: Durch Unterschiede in der Apikalkontrolle geprägte Verzweigungsmuster bei Nadelund Laubholzarten (aus BARTELS, 1993, verändert).

Im folgenden sollen einfache statistische Modelle unterschiedlicher Komplexität anhand konkreter Beispiele erläutert werden. Die Beispiele beschränken sich auf Modelle für Nadelholzarten, da die Literatur fast ausschließlich diese Modellgruppe beschreibt. Als Gründe sind die einheitlichere Kronenarchitektur der Nadelholzarten und die gegenüber anderen Gütemerkmalen dominierende Bedeutung der Astigkeit bei der Qualitätsbeurteilung von Nadelholzarten zu nennen.

Aufgrund der schwächer ausgeprägten Apikalkontrolle, eines weniger stark ausgeprägten orthotropen Wachstums der Sproßachse, einer fehlenden Anordnung der Äste 1. Ordnung in Quirlen sowie der Veränderung des Verzweigungsmusters durch natürliche Astreinigungsprozesse innerhalb der grünen Krone ergeben sich für die Laubbaumarten deutlich heterogenere Verzweigungsmuster (decurrente Kronenformen) als für die Nadelholzarten. Auch ist das Wachstum der Äste 1. Ordnung nicht streng plagiotrop. Statt dessen kommen alle Variationen von Steilästen bis zu senkrecht zur Sproßachse stehenden Ästen vor. Der Astabgangswinkel der Nadelholzarten ist zwar in geringerem Maß ebenfalls variabel, jedoch ist diese Variabilität mit der Variablen Tiefe in der Krone (TINK) korreliert (Kapitel 2.4.6; ColIN U. Houllier, 1992). Eine Ausnahme stellen Steilastbildungen aufgrund von traumatischen Ereignissen dar (GrUBER, 1987, S. 58). Die Variable Tiefe in der Krone ist die Entfernung eines Astes von der Baumspitze.

Hinzu kommen innerhalb der Laubholzarten unterschiedliche Verzweigungssysteme (BRAUn, 1988, S. 25). So weisen beispielsweise die Eichen, die Rotbuche, die Esche und der Bergahorn ein monopodiales Wachstum wie die Nadelholzarten auf. D. h., die Seitenzweige 
bleiben in ihrem Wachstum stets der Hauptachse untergeordnet. Demgegenüber stirbt die Endknospe der Sproßachse bei den sympodial wachsenden Baumarten bereits nach einem Jahr ab, und die oberste Seitenknospe übernimmt den Aufbau der Sproßachse. Typische Baumarten dieses Verzweigungstyps sind die Linden und die Hainbuche.

\subsubsection{Modellierung des stärksten Astdurchmessers am Baum}

Als einfache Modellierung der sortierrelevanten Astigkeit bietet sich die Schätzung des stärksten Astes am Baum nach Maguire ET AL. (1991) an (Formel (6), Abb. 5):

$\ln (A D)=b_{0}+b_{1} \ln (T I N K)+b_{2} \ln (D)+b_{3} \ln (R D)+b_{4} S I$

wobei:

$A D=$ Mittlerer maximaler Astdurchmesser innerhalb eines Quirls nahe dem Kronenansatz (inch);

TINK $=$ Position des Astes, beschrieben als Tiefe in der Krone (Entfernung) von der Baumspitze aus gemessen ( $\mathrm{ft}$ );

$D=$ Brusthöhendurchmesser (inch);

$R D=$ Relative Dichte (CURTIS, 1982);

$S I=$ Site Index;

$\mathrm{b}_{0}, \mathrm{~b}_{1}, \mathrm{~b}_{2}, \mathrm{~b}_{3}, \mathrm{~b}_{4}=$ Parameter.

Direkt modellierter Astdurchmesser des stärksten Astes am Baum

(MAGUIRE ET AL., 1991)
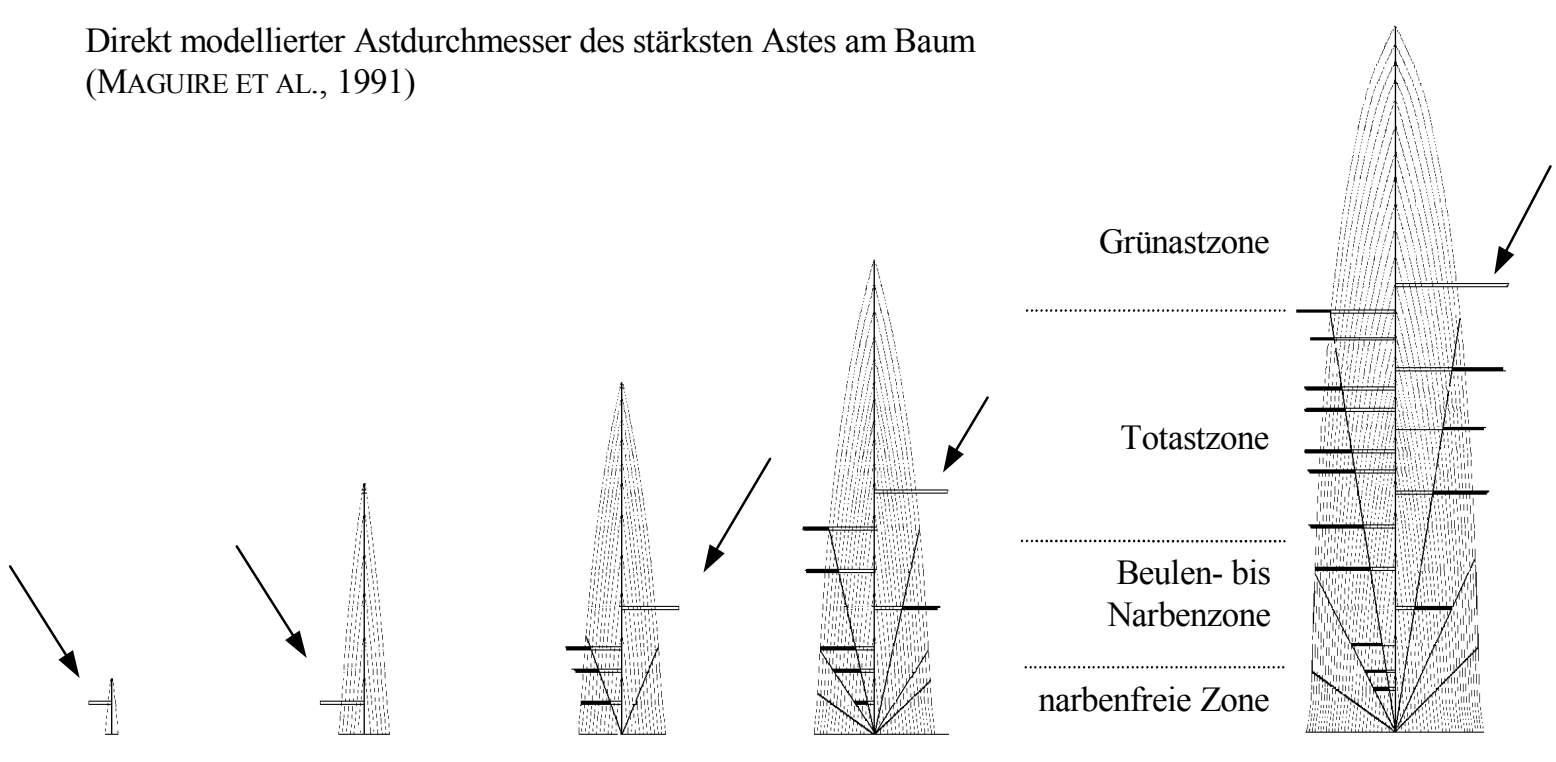

Abb. 5: Schematische Darstellung der Modellierung des stärksten Astdurchmessers eines Baumes im Bereich des unteren Kronendrittels (durch Äste dargestellt; nur die mit Pfeilen gekennzeichneten Äste werden direkt geschätzt). Die Prozesse Astmortalität, Astreinigung bzw. die Entstehung von astfreier Zone, Narbenzone, Totastzone und Lebend-astzone können durch die Kombination mit einem Wuchsmodell beschrieben und dargestellt werden.

Dieser Modelltyp ist ein Spezialfall des nächst komplexeren Modelltyps zur Schätzung der Durchmesser der stärksten Quirläste eines Baumes. Hierbei wird unterstellt, daß sich der stärkste Ast pro Baum in einem Quirl im unteren Kronendrittel befindet, so daß sich der notwendige Datenumfang durch eine lokal begrenzte Parametrisierung des Modells stark redu- 
zieren läßt. Der Durchmesser dieses stärksten Astes wird dadurch beeinflußt, wie tief er sich in der Krone befindet. Eine Vorgabe, die dieser Modelltyp aus Sicht biologischer Plausibilität erfüllen muß, ist die stetige monotone Zunahme des Astdurchmessers mit der Zunahme der Tiefe in der lebenden Krone. Um die Datengrundlage zu erweitern, können weitere stärkste Quirläste aus dem unteren Kronendrittel für die Parametrisierung der Beziehung zwischen Astdurchmesser und der Position in der Krone genutzt werden. Je mehr dieser stärksten Quirläste in Richtung der Baumspitze verwendet werden, desto ähnlicher wird dieser Modelltyp demjenigen zur Beschreibung aller stärksten Quirläste für den Bereich der lebenden Krone (Kapitel 2.4.3). Allerdings schätzen die Modelle zur Prognose der stärksten Äste je Quirl im allgemeinen sowohl den Verlauf in der lebenden Krone als auch in der Totastzone (Maguire et Al., 1994; Colin U. Houllier, 1991).

Werden nur wenige oder nur der stärkste Quirlast aus dem unteren Kronendrittel für die Parametrisierung verwendet, können nur begrenzt Aussagen über die Struktur der Astdurchmesser der stärksten Quirläste innerhalb der lebenden Krone gemacht werden. Bei der Schätzung von Astdurchmessern im mittleren und oberen Bereich der Krone muß beachtet werden, daß es sich um Extrapolationen handelt. Im Zusammenhang mit den komplexeren Astmodellen wird noch erläutert, daß die Zunahme des Astdurchmessers mit zunehmender Tiefe in der Krone nur für den Vergleich der stärksten Äste verschiedener Bäume gilt. Das bedeutet, daß im Vergleich unterschiedlich tief bekronter Bäume gleicher Dimension lang bekronte Bäume größere maximale Aststärken haben als kurz bekronte Bäume. Innerhalb einer Krone wird die maximale Aststärke jedoch deutlich oberhalb des Kronenansatzes erreicht. Zur Beschreibung der Astdurchmesserstruktur der stärksten Quirläste innerhalb der gesamten Krone muß daher ein anderer Modelltyp verwendet werden (MAGuiRE ET AL. 1994; Colin U. Houllier, 1991).

Um die Schaftqualität eines hiebsreifen Baumes ausreichend genau einschätzen zu können, reicht es in vielen Fällen, die Astigkeit des Stammes bis zum Kronenansatz zu prognostizieren. Für die stärksten Quirläste unterhalb des stärksten Astes des Baumes können die Durchmesser zum Zeitpunkt des Absterbens durch Kombination des Astmodells mit Wachstumssimulationen geschätzt werden (Abb.5). Mit dem zum Absterbezeitpunkt erreichten Durchmesser wächst der stärkste Quirlast des jeweiligen Quirls durch das Dickenwachstum des Baumes in den Stamm ein. Dabei wird unterstellt, daß der Durchmesser des stärksten Astes am Baum nach dem Zeitpunkt der Schätzung mit Hilfe des Modells (6) nicht weiter zunimmt. Diese Annahme wird durch das Ergebnis einer Untersuchung von KERSHAW ET AL. (1990) unterstützt, daß bei den untersuchten Douglasien im Mittel während 34\% der Lebensdauer keine Jahrringe gebildet wurden. Allerdings war keine Zunahme des Anteils fehlender Jahrringe mit steigendem Astalter zu beobachten.

Da der Zeitpunkt des Absterbens bzw. die Tiefe in der lebenden Krone einen großen Einfluß auf den Astdurchmesser haben, ist dieser Modelltyp auf eine möglichst exakte Pro- 
gnose der Kronenansatzentwicklung angewiesen. Als weitere, die Aststärke beeinflussende Faktoren wurden von MAGUIRE ET AL. (1991) die Einzelbaumdimension (BHD, positiver Effekt), die Bestandesdichte (Relative Dichte (CURTIS, 1982), negativer Effekt) und der Standort (Site-Index, negativer Effekt) identifiziert. Die Wirkungsrichtungen der unabhängigen Variablen stimmen mit den von LÄMSÄ ET AL. (1991) für die Kiefer beschriebenen Untersuchungen überein, wobei in diesem Modell die Tiefe in der Krone nicht als unabhängige Variable verwendet wurde. Der negative Trend bei steigender Standortsgüte erscheint nicht plausibel. Auch existieren Beispiele für einen positiven Effekt auf den Astdurchmesser durch Düngung, d. h. Standortsaufwertung (WILL, 1971). MAGUIRE ET AL. (1994) führen den negativen Effekt auf einen Scheinzusammenhang zurück, da die Standortsgüte in den von ihnen untersuchten Daten eng mit der Baumhöhe korreliert war. Daraus folgt, daß die Baumhöhe bei konstantem BHD einen negativen Effekt auf den Astdurchmesser hat.

Die genannten unabhängigen Variablen, die die Konkurrenzsituation auf Bestandesebene, die Einzelbaumdimension und die Wuchsbedingungen beschreiben, haben allerdings einen relativ geringen Einfluß, wenn gleichzeitig die Position des stärksten Astes in der lebenden Krone (Tiefe in der Krone) als unabhängige Variable verwendet wird (MAGUIRE ET AL., 1994). Die Position eines Astes in der lebenden Krone kann als das Resultat des Höhenwachstums des Baumes und der Kronenrezession durch das Absterben der untersten grünen Äste seit der Enstehung des Astes betrachtet werden. Das Höhenwachstum und die Kronenrezession werden $u$. a. durch den Standort und die Konkurrenzsituation bestimmt. Die Tiefe in der Krone des stärksten Astes im unteren Drittel der lebenden Krone ist daher eine Variable, die viele, das Astwachstum beeinflussende Variablen aggregiert. Diese Aggregation mehrerer unabhängiger Variablen zusätzlich zur engen Korrelation mit dem Alter des Astes erklärt den großen Einfluß der Variablen Tiefe in der Krone auf den Astdurchmesser.

Im Gegensatz zu den durch die Variable Tiefe in der Krone mehr oder weniger ersetzbaren Variablen wird in allen Astmodellen die absolute Einzelbaumdimension (BHD, Höhe) als weitere unabhängige Variable verwendet (positiver Effekt) (MAGUIRE ET AL., 1991; MAGUIRE ET AL., 1994; COLIN U. Houllier, 1991). Der Einfluß der absoluten Einzelbaumdimension auf den Astdurchmesser von Bäumen mit sonst gleichen Kronenparametern kann mit der veränderten Kronendynamik erklärt werden. In jungen Beständen steigt der Wert der Variablen Tiefe in der Krone eines Astes infolge des relativ raschen Höhenwachstums schnell. Durch die dynamischere Kronenrezession nach Kronenschluß ist die Lebensdauer der Äste geringer als in Altbeständen, in denen sich das Höhenwachstum und die Kronenrezession verlangsamen. In Altbeständen und speziell in der Oberschicht von Plenterwäldern ist auch eine minimale oder gar keine vertikale Kronendynamik möglich. Bei einem Astdurchmessermodell, das nur durch die Tiefe in der lebenden Krone gesteuert wird, würde der Stillstand der vertikalen Kronendynamik in einem Stillstand des Astdurchmesserwachstums resultieren. Auch 
wenn stagnierendes Durchmesserwachstum von lebenden Ästen bei vielen Baumarten typisch ist (KERSHAW ET AL., 1990; FUJIMORI, 1975), kann nicht davon ausgegangen werden, daß ein Stillstand der Kronendynamik automatisch mit einer Stagnation des Astwachstums verbunden ist. Durch die Verwendung des absoluten Durchmessers als unabhängige Variable können Bäume mit Durchmesserwachstum, aber stagnierender Kronendynamik, durchaus ein Astdurchmesserwachstum aufweisen.

Der Modellansatz von MAGUIRE ET AL. (1991) schätzt den stärksten Astdurchmesser eines Baumes im Bereich einer fixierten relativen Tiefe in der Krone (unteres Kronendrittel). Durch die Kombination mit Wuchsmodellen kann dieser Ansatz, der eigentlich nur eine punktuelle Astigkeit prognostiziert, für eine Prognose der Astigkeit des gesamten Stammstücks unterhalb des Kronenansatzes verwendet werden. Im Rahmen der Auswertung von Verbandsversuchen sind demgegenüber Gleichungen für die Schätzung von mittleren maximalen Reihen- und Gassenästen unterschiedlicher Standraumverbände in fixierten absoluten Höhen entwickelt worden (THREN, 1983). Da dieser Ansatz nur ein Bestandesmodell auf der Ebene von Verbandsversuchen darstellt, ist grundsätzlich nur eine sehr grobe Beurteilung der Astigkeit möglich. Allerdings wäre auch bei Berücksichtigung von unabhängigen Einzelbaumvariablen die Modellierung der Astigkeit einzelner Stammstücke nicht möglich, da bei der Kombination mit einem Wuchsmodell die Prognose für eine fixierte absolute Höhe ungeeignet ist. MAGUIRE ET AL. (1991) parametrisierten ihr Modell mit Daten von 519 Douglasien aus Südwest-Oregon (USA) mit einem BHD von 0.9 bis 44.2 inch $(2.3$ bis $112.3 \mathrm{~cm}$ ) und einem Alter von 12 bis 127 Jahren.

\subsubsection{Durchmesser der stärksten Quirläste in der gesamten Krone eines Baumes}

Wie bereits beschrieben, können die Modelle zur Schätzung der Durchmesser aller stärksten Quirläste in der lebenden Krone eines Baumes als allgemeine Form des Modells zur Schätzung des stärksten Astes am Baum betrachtet werden (Abb. 6). Ein Unterschied besteht lediglich im Bereich der zur Parametrisierung verwendeten Daten und einiger, die biologische Plausibilität berücksichtigenden Modelleigenschaften. Um Aussagen über die Entwicklung der stärksten Quirläste in der lebenden Krone machen zu können, muß die Parametrisierung für den gesamten Bereich der lebenden Krone erfolgen. Die Astdurchmesser der Totastzone können dagegen mit Hilfe von Wachstumssimulationen indirekt über die Schätzung des Astdurchmessers zum Absterbezeitpunkt geschätzt werden. Sofern für die Parametrisierung der Modelle auch Astdurchmesser aus der Totastzone verwendet werden, lassen sich diese direkt ohne Wachstumssimulationen schätzen. Allerdings nimmt mit zunehmendem Abstand des Totastes zum Kronenansatz der biologische bzw. statistische Zusammenhang des Astdurchmessers mit den verwendeten unabhängigen Variablen ab. 


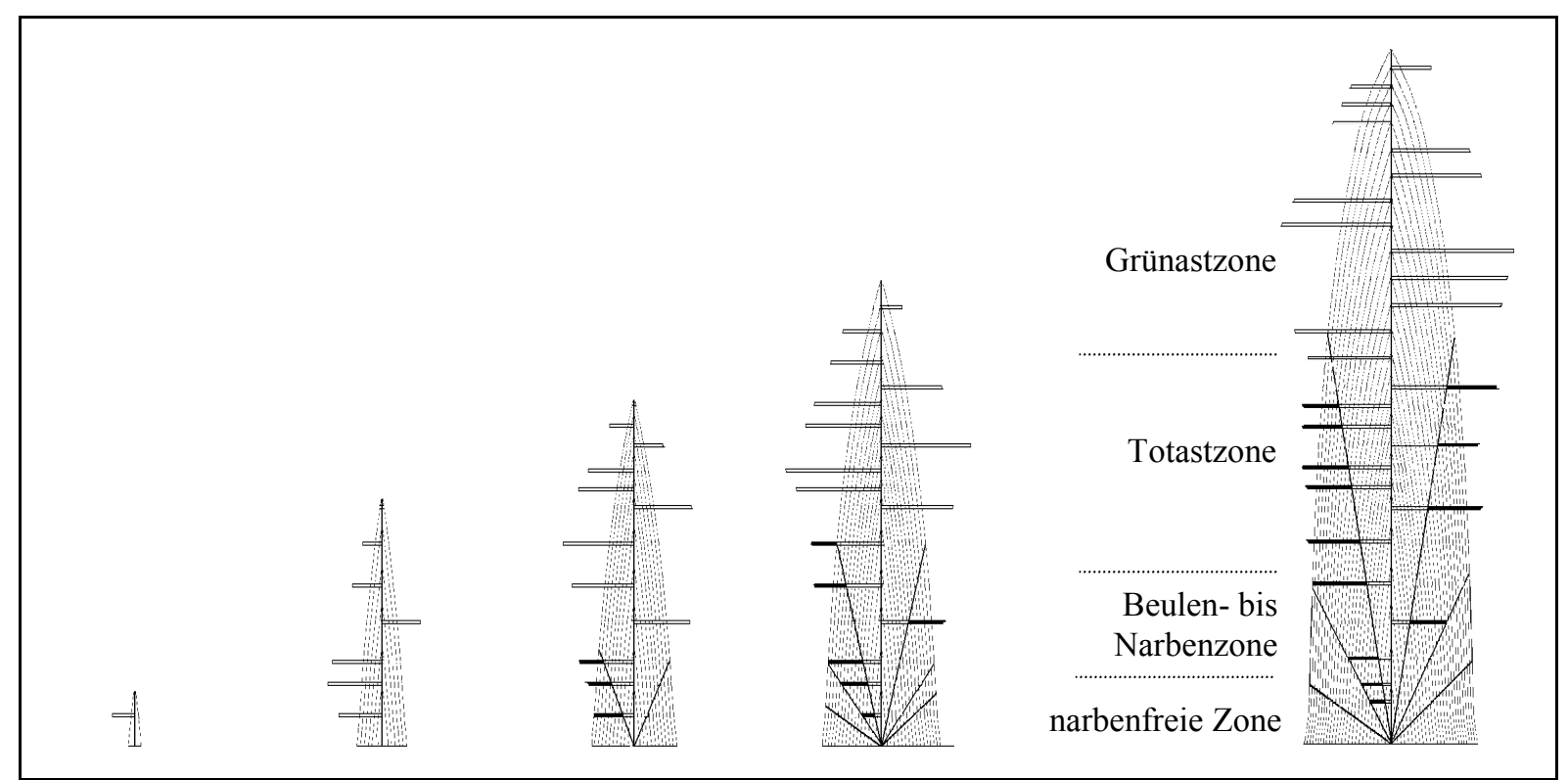

Abb. 6: Schematische Darstellung der Modellierung aller stärksten Quirlastdurchmesser (durch Äste dargestellt). Je nach Konzeption und Parametrisierungsgrundlage werden nur die lebenden stärksten Quirläste oder alle stärksten Quirläste modelliert. Die Prozesse Astmortalität, Astreinigung bzw. die Entstehung von astfreier Zone, Narbenzone, Totastzone und Lebendastzone können durch die Kombination mit einem Wuchsmodell beschrieben und dargestellt werden.

Für die Beschreibung des Verlaufs der Astdurchmesser in der Krone hat sich der Funktionstyp zur Modellierung der maximalen Aststärke eines Baumes, der eine stetig monotone Zunahme der Astdurchmesser in der Krone unterstellt (Formel (6)), als ungeeignet erwiesen (Maguire et AL., 1994; Colin U. Houllier, 1991). Ursache dafür ist, daß die Höhe des maximalen Astdurchmessers eines Baumes im allgemeinen deutlich über dem Kronenansatz liegt. Für die Modellierung der Astdurchmesser der Krone bzw. der gesamten Astzone sind daher Modelle entwickelt worden, die ein Maximum oberhalb des Kronenansatzes beschreiben können (Maguire et AL., 1990; MaguiRe ET AL., 1994; Colin U. Houllier, 1991; VESTÖL ET AL., 1996). Nach dem Erreichen des Durchmessermaximums nehmen die Astdurchmesser im allgemeinen mit steigender Tiefe in der Krone mehr oder weniger schnell ab. Der Kronenansatz als obere Grenze des Übergangsbereichs von lebender Krone zur Totastzone ist im Verlauf der Astdurchmesserstruktur entlang des Stammes nicht zu erkennen. Ein Grund ist vermutlich die von KERSHAW ET AL. (1990) beobachtete, zeitweilige Wachstumsstagnation von lebenden Ästen, insbesondere im unteren Kronenbereich. Funktionstypen, die die Modellierung eines Astdurchmessermaximums innerhalb der lebenden Krone ermöglichen, sind Polynomialgleichungen in segmentierter (MAGUIRE ET AL., 1994; COLIN U. HoulliER, 1991) und nicht segmentierter Form (VESTÖL ET AL., 1996). Die Aststärkenentwicklung wird bei Verwendung von segmentierten Modellen in einem oberen und unteren Stammbereich durch unterschiedliche Funktionen beschrieben. Der Übergangspunkt, an dem 
beide Funktionen einen gemeinsamen Funktionswert aufweisen, wird bei CoLIN und Houllier (1991) an der Stelle des maximalen Astdurchmessers fixiert. MAGUIRE ET AL. (1994) bestimmten den Übergangspunkt iterativ. Sie wählten den Kronenlängenanteil, der über alle Datensätze den geringsten RMSE $^{9}$ aufwies. Stellvertretend für den Modelltyp der segmentierten Polynomialgleichung wird in Formel (7) das von MAGUIRE ET AL. (1994) entwickelte Modell dargestellt:

$$
\begin{aligned}
B D_{\max }= & b_{0}+b_{1} * X_{1}+b_{2} * D * X_{1}+b_{3} * D * X_{2} \\
& +b_{4} * H * X_{1}+b_{5} * H * X_{2}+b_{6} * \operatorname{TINK}_{\text {rel }} * X_{3}
\end{aligned}
$$

wobei:

$B D_{\max }=$ Durchmesser des stärksten Quirlastes im Quirl (cm);

$X_{1}=$ Tiefe in der Krone (Entfernung des Astes von der Baumspitze), wobei sich der Parametrisierungsbereich auch in die Totastzone erstreckt (TINK) (m);

$T I N K_{\text {rel }}=T I N K$ in Relation zur Gesamtkronenlänge;

$X_{2}=T I N K^{2}$;

$X_{3} \quad=I^{*}\left(K^{2}-2 * K^{*} T I N K+T I N K^{2}\right)$;

$D=$ Brusthöhendurchmesser $(\mathrm{cm})$;

$H=$ Baumhöhe $(\mathrm{m})$;

$K=0.1$ *Kronenlänge und $\mathrm{I}=0$ wenn $T I N K \leq K$ und $\mathrm{I}=1$ wenn $T I N K>K$.

für Douglasie mit:

$\mathrm{b}_{0}=4.585672, \mathrm{~b}_{1}=6.417612, \mathrm{~b}_{2}=0.904437, \mathrm{~b}_{3}=-0.095010, \mathrm{~b}_{4}=-1.548851$, $\mathrm{b}_{5}=0.162199, \mathrm{~b}_{6}=-0.673175$.

Die Parameterwerte beziehen sich auf die von MAGUIRE ET AL. (1994) verwendeten Douglasiendaten mit einem Brusthöhenalter von maximal 7 Jahren und einem BHD von 5.1 bis $14.7 \mathrm{~cm}$. Insgesamt wurden 206 Bäume aus Versuchsflächen in Oregon und Washington westlich der Kaskaden untersucht.

Die Lage des gemeinsamen Funktionswertes beider Funktionssegmente wurde für die untersuchten Douglasien bei einer Tiefe in der Krone von 10\% der lebenden Krone definiert. Wie in Modell (6) haben neben den Variablen Tiefe in der Krone bzw. relative Tiefe in der Krone auch Dimensionsvariablen wie der BHD und die Höhe Einfluß auf den Astdurchmesser. Allerdings beeinflußt die Tiefe in der Krone auch den Trend innerhalb eines Baumes und nicht wie in Modell (6) nur die Unterschiede zwischen Bäumen. Im Gegensatz zu Modell (6) sind weitere Variablen wie beispielsweise die Bestandesdichte und der Standort nicht signifikant. Als Ursache ist der bereits beschriebene Einfluß der aggregierenden Variablen Tiefe in der Krone zu nennen. Die im Modell enthaltene Kronenlänge sowie die Kombination von BHD und Höhe beinhalten ebenfalls Informationen über die Bestandesbehandlung und unterschiedliche Pflanzverbände. Auch COLIN und HoulLIER (1991) integrierten neben Einzel-

\footnotetext{
${ }^{9}$ Im weiteren Text wird für die Wurzel des mittleren quadratischen Fehler bzw. englisch Root Mean Square Error die Abkürzung (RMSE) verwendet.
} 
baum- bzw. Astvariablen wie BHD, Alter, Kronenverhältnis bzw. relative Tiefe in der Krone keine weiteren übergeordneten Bestandesvariablen in ihr Modell.

Als Nachteil beider Modelle wird hier angesehen, daß durch die Verwendung von Polynomialgleichungen eine biologische Interpretation der Wirkungsrichtungen der unabhängigen Variablen schwierig ist. Auch sind unplausible Kombinationen von Vorzeichen unvermeidlich, da nur so der Verlauf der Astdurchmesserstruktur beschrieben werden kann. Es ist beispielsweise nicht sinnvoll, daß die Einzelbaumvariablen BHD und Höhe innerhalb des Modells von MAGUIRE ET AL. (1994) sowohl einen positiven als auch einen negativen Einfluß auf den Astdurchmesser haben. Auch ist die Wirkungsrichtung der Vielzahl von BHD- und Höhen-Kombinationen schwieriger zu interpretieren als die Verwendung des H/D-Wertes. Die Verwendung des Alters im Modell von Colin und HoulLIER (1991) erschwert eine Anwendung in ungleichaltrigen Beständen.

HÖIBÖ ET AL. (1996) und Vestöl ET AL. (1996) haben in ihren Modellansätzen die Tiefe in der Krone, die mittlere Jahrringbreite der ersten 40 Lebensjahre, die mittlere periodische Höhenwachstumsrate des Baumes bzw. die Tiefe in der Krone und die soziale Stellung des Baumes als unabhängige Variablen verwendet. Während MAGUIRE ET AL. (1994) sowie COLIN und Houllier (1991; 1992) in ihren Modellen ausschließlich Zustandsvariablen als unabhängige Variablen einsetzen, verwenden HöIBÖ ET AL. (1996) mit der mittleren Jahrringbreite und dem mittleren Höhenwachstum periodische Zuwachsgrößen. Damit ist ein Einsatz dieser Modelle an die Kombination mit Wachstumsmodellen gebunden, da Zuwachsgrößen nicht wie Zustandsvariablen im Rahmen von einmaligen Inventuren geschätzt werden können. Auch hängt die Schätzgenauigkeit der Astdurchmessermodelle von der Schätzgenauigkeit der Wachstumsmodelle bzw. der periodischen Inventuren bezüglich der Wachstumsraten ab.

Das Modell von Colin und Houllier (1991) ist mit Daten von Fichten mit einem Alter zwischen 30 und 180 Jahren und einem BHD von 16 bis $44 \mathrm{~cm}$ aus dem Bereich der Vogesen parametrisiert worden. Die Modelle von HöIBÖ ET AL. (1996) und VESTÖL ET AL. (1996) sind als Fallbeispiele, nicht aber als regionalisierte Modelle zu beurteilen. Das von MAGUIRE ET AL. (1994) entwickelte Modell dient in Kapitel 3 als Vergleichsbasis für die neu entwickelten Astdurchmessermodelle. Der Einfluß von Behandlungsvarianten ist in den beschriebenen Modellen weniger klar zu interpretieren, da er aus dem Zusammenspiel der in mehreren Termen enthaltenen Variablen BHD und Höhe resultiert.

\subsubsection{Modellierung der Durchmesser und der Anzahl aller Quirläste eines Baumes}

Eine Unterscheidung der Modelltypen "Modellierung aller Quirläste" und "Modellierung aller Äste“ wird vor allem wegen der unterschiedlichen Kronenarchitektur der verschiedenen Nadelholzarten vorgenommen. Die Kronenarchitektur kann danach unterschieden werden, ob an einem Jahrestrieb neben den Quirlästen (Nodalästen) auch Internodaläste vorkommen. Fichte 
und Douglasie bilden im Gegensatz zur Kiefer auch Internodaläste aus. Bei der Kiefer ist damit die Modellierung aller Quirläste gleichbedeutend mit der Modellierung aller Äste eines Baumes.

Das Phänomen der Akrotonie (GRUBER, 1987, S. 21) führt bei den Nadelholzarten, die Internodaläste bilden, zu bedeutend stärkeren Dimensionen der Quirläste gegenüber den Internodalästen und innerhalb des Jahrestriebes zu einem schnellen Absinken der Astanzahl mit steigender Entfernung zum Quirl. Daher sind die Quirläste für eine Einschätzung der qualitätsbestimmenden Astigkeit von größerer Bedeutung als die Internodaläste.

Während bei den Modelltypen zur Beschreibung der stärksten Quirläste bzw. ihrer Durchmesser die Astanzahl durch die Anzahl der existierenden Quirle festgelegt war, muß beim Modelltyp zur Beschreibung aller Quirläste neben den Astdurchmessern auch die Anzahl der Äste eines Quirls geschätzt werden (Abb. 7).

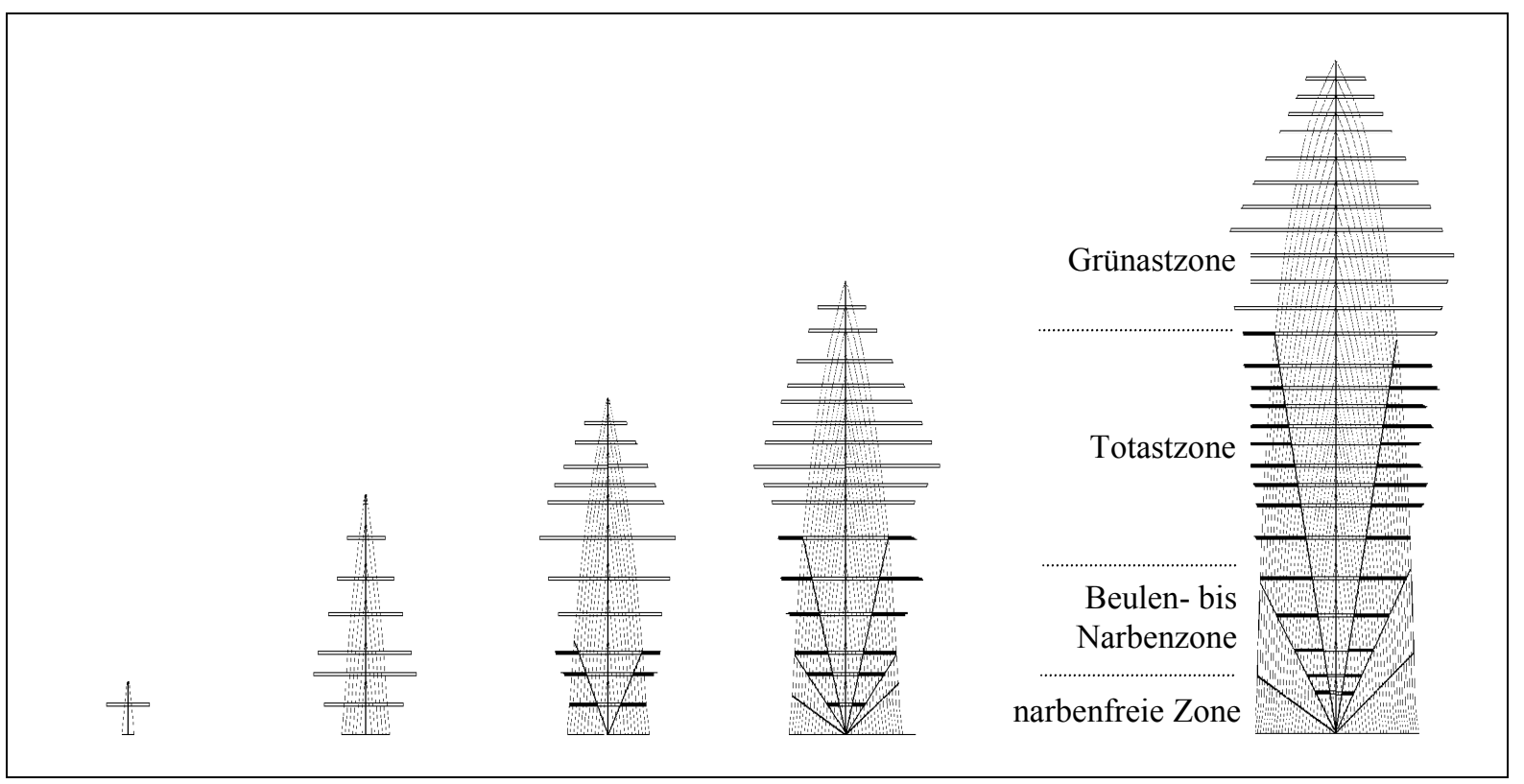

Abb. 7: Schematische Darstellung der Modellierung aller Quirlastdurchmesser (durch Äste dargestellt). Je nach Konzeption und Parametrisierungsgrundlage werden nur die lebenden Quirlastdurchmesser oder alle Quirlastdurchmesser modelliert. Die Prozesse Astmortalität, Astreinigung bzw. die Entstehung von astfreier Zone, Narbenzone, Totastzone und Lebendastzone können durch die Kombination mit einem Wuchsmodell beschrieben und dargestellt werden.

Die vertikale Position des jeweiligen Astes ist in beiden Modelltypen näherungsweise durch die vertikale Position des Astquirls festgelegt. In Kombination mit einem Wuchsmodell werden die Positionen der Astquirle aus dem prognostizierten Höhenwachstum abgeleitet. COLIN und Houllier (1992) stellten fest, daß bei Fichte die Entfernung der Quirläste vom Beginn des Jahrestriebes maximal 20\% der Jahrestrieblänge betrug. Bei jungen Douglasien befanden sich die Quirläste sogar in den obersten 10\% der Jahrestrieblänge (MAGUIRE ET AL., 1994). Für den einfachsten Modelltyp zur Schätzung des Durchmessers des stärksten Astes am Baum 
entfällt die Schätzung der Anzahl der Quirläste, und die vertikale Position ist lediglich unscharf auf das untere Kronendrittel fixiert.

Beispiele für die Modellierung der Anzahl und Durchmesser aller Quirläste für die Kiefer sind die Ansätze von KellomÄKI ET AL. (1989) sowie KellomäKI und VÄISÄNEN (1988). Im Unterschied zu den bisher genannten mathematisch statistischen Modellen verwenden sie ein Populationsmodell, das die Äste eines Baumes als Individuen betrachtet. Da eine Zu- bzw. Abwanderung von Ästen ausgeschlossen ist, wird die Entwicklung der "Gesamtpopulation" durch die Neubildung von Ästen und die Astmortalität bestimmt. Für die Schätzung der Gesamtanzahl an Quirlen, Ästen je Quirl sowie Anteilen an lebenden und toten Ästen in den einzelnen Quirlen werden einfache Regeln auf der Basis empirischer Daten formuliert. Die Gesamtanzahl an lebenden Quirlen nimmt bis zum Zeitpunkt, bei dem erstmals oder nach Durchforstungen wieder Kronenschluß erreicht wird, jährlich um den Wert 1 zu. Nach Eintreten des Kronenschlusses bleibt die Anzahl der lebenden Quirle konstant und der maximale Zeitraum der natürlichen Astreinigung beträgt neun Jahre. Es werden konstant fünf Äste in einem neuen Quirl gebildet und der Anteil an lebenden Ästen in einem Quirl wird vom Alter des Quirls und der Gesamtzahl an lebenden Quirlen bestimmt.

In Anlehnung an Populationsmodelle können standortsabhängige Kapazitäten (pro ha oder pro Mittelstamm) an lebenden Ästen nach Erreichen des Kronenschlusses definiert werden, die eine weitere Erhöhung der Anzahl an lebenden Ästen beschränken bzw. ein Gleichgewicht bei übereinstimmender Neubildungs- und Mortalitätsrate der Äste definieren (KELLOMÄKI U. VÄISÄNEN, 1988). Die Astdurchmesser werden nicht direkt geschätzt, sondern aus der Quirl- bzw. Astbiomasse berechnet, die über ein Allokationsmodell aus der Produktionsrate der Gesamtbiomasse des Baumes abgeleitet wird. Die Verteilung der Gesamtbiomasseproduktion auf die Quirle erfolgt über eine am Alter des Quirls orientierte Regel. Für die Verteilung der Quirlbiomasse auf die Äste wird Normalverteilung mit dem Mittelwert von einem fünftel der Quirlbiomasse und einem Variationskoeffizienten von 0.25 unterstellt. Für die Anwendung muß das Astdurchmessermodell im Gegensatz zu den bisher beschriebenen Modellen mit einem prozeßorientierten oder mathematisch statistischen Biomassemodell kombiniert werden (KELLOMÄKI ET AL., 1989). Statistisch mathematische Modelle sind für die Beschreibung der Dynamik von Kronenansatzhöhe und Höhe des untersten Totastes nicht notwendig, weil das Absterben und die natürliche Astreinigung der Einzeläste durch die genannten Regeln bestimmt werden.

Eine Möglichkeit zur Beschreibung der Durchmesser weiterer Quirläste mit statistischen Modellen besteht darin, die zur Schätzung der stärksten Quirläste verwendeten Ansätze (Kapitel 2.4.3) für den schwächsten Ast, den Median der Quirlastdurchmesser etc. zu parametrisieren. Colin und Houllier (1992) haben dieses Verfahren zur Modellierung des mittleren Astdurchmessers je Quirl eingesetzt. 


\subsubsection{Modellierung der Durchmesser, der Anzahl und der vertikalen Position aller Äste eines Baumes}

Für den komplexesten Modelltyp, die Modellierung aller Äste von Nadelholzarten, die Quirlund Internodaläste ausbilden, müssen zusätzlich zu den Eigenschaften der Quirläste auch die Astdurchmesser, die Anzahl und die vertikale Position der Internodaläste geschätzt werden. Der von MaguiRe ET AL. (1994) entwickelte Ansatz modelliert die Anzahl der Äste innerhalb eines Jahrestriebes in Abhängigkeit von der Länge des Jahrestriebes, der Tiefe des Jahrestriebes in der Krone und des relativen BHD (in Relation zum stärksten Baum im Bestand).

Die vertikale Verteilung der Äste im Quirl kann in Form von Dichteverteilungen über relativen Abschnitten eines Jahrestriebes beschrieben werden. Für junge Douglasien wurde innerhalb des Jahrestriebes ein sehr schneller Abfall der Häufigkeit der Astanzahl mit zunehmender Entfernung vom Quirl beobachtet (MAGUIRE ET AL., 1994). In der Mitte des Jahrestriebes trat ein weiteres, allerdings sehr schwach ausgeprägtes Maximum auf. Dieser Verlauf der relativen Häufigkeiten konnte mit keiner der untersuchten parametrischen Dichtefunktionen befriedigend erfaßt werden. MAGUIRE ET AL. (1994) verzichteten deshalb auf eine Modellierung der Asthäufigkeiten und verwendeten die mittlere empirische Häufigkeitsverteilung aller untersuchten Jahrestriebe zur Schätzung der vertikalen Positionen der Internodaläste.

Zur Modellierung der Durchmesser der Internodaläste eines Jahrestriebes relativierten MaGuiRE ET AL. (1994) die Durchmesser mit dem stärksten (Quirl-)Astdurchmesser des Jahrestriebes. Als geeignete Methode zur Modellierung der resultierenden Struktur relativer Durchmesser über der relativen Tiefe im Jahrestrieb verwendeten sie die reverse Verteilungsfunktion der 3-parametrigen Weibullfunktion (WEIBULL, 1951). Durch die Modellierung des Skalenparameters in Abhängigkeit vom Jahrestriebalter und der relativen Tiefe des Jahrestriebes in der Krone konnte ein unterschiedlich schnelles Abfallen der relativen Durchmesserwerte von Internodalästen beschrieben werden.

COLIN und Houllier (1992) schätzten in ihrem Modell nur die Anzahl der Äste pro Jahrestrieb, nicht aber deren vertikale Position und Durchmesserstruktur im Jahrestrieb. Als unabhängige Variablen verwendeten sie wie MAGUIRE ET AL. (1994) die Länge des Jahrestriebes und zusätzlich das Alter des Jahrestriebes. Der Informationsgehalt der Schätzung nimmt zu, wenn das Modell auch für nach Astdurchmesserstärke stratifizierten Astanzahlen parametrisiert wird.

Ein einfacher Ansatz zur Beschreibung der Gesamtastigkeit der lebenden Krone eines Baumes besteht in der Schätzung der kumulativen Astquerschnittsfläche in Abhängigkeit von der Stammquerschnittsfläche entlang des Stammes (LAASASENAHO, 1987). Durch die Kombination des Modells mit Schaftformfunktionen kann für jede beliebige Höhe die kumulative Astquerschnittsfläche oder durch Differenzenbildung die Astquerschnittsfläche eines Stammabschnittes berechnet werden. Auch wenn das Modell zum Modelltyp der Beschrei- 
bung der Gesamtastigkeit aller Grünäste 1. Ordnung zu rechnen ist, ist die Auflösung relativ gering, da keine Aussagen zu Einzelastvariablen gemacht werden können. Dafür wäre eine Kombination mit Allokationsmodellen notwendig, wie sie von KELLOMÄKI ET AL. (1989) verwendet werden.

\subsubsection{Modellierung der Horizontal- und Vertikalwinkel von Ästen}

Für eine noch umfassendere Beschreibung der Astigkeit als Qualitätsmerkmal sind Angaben zum Vertikalwinkel der Quirläste (Winkel zur Stammachse) und eventuell noch Angaben zum Horizontalwinkel der Quirläste notwendig (Abb. 8). Insbesondere steile Vertikalwinkel führen zu stärkeren Störungen der Jahrringstruktur im Überwallungsbereich und bei bestimmten Einschnittsmustern zu sogenannten Flügelästen (KNIGGE U. SchUlz, 1996, S. 176). ColiN und HoulliER (1992) haben für die Schätzung des Vertikalwinkels ein Modell vorgeschlagen, das als unabhängige Variable das Alter des Jahrestriebes verwendet. Für Fichte beobachteten sie eine Zunahme des vertikalen Astwinkels mit dem Alter des Quirls.

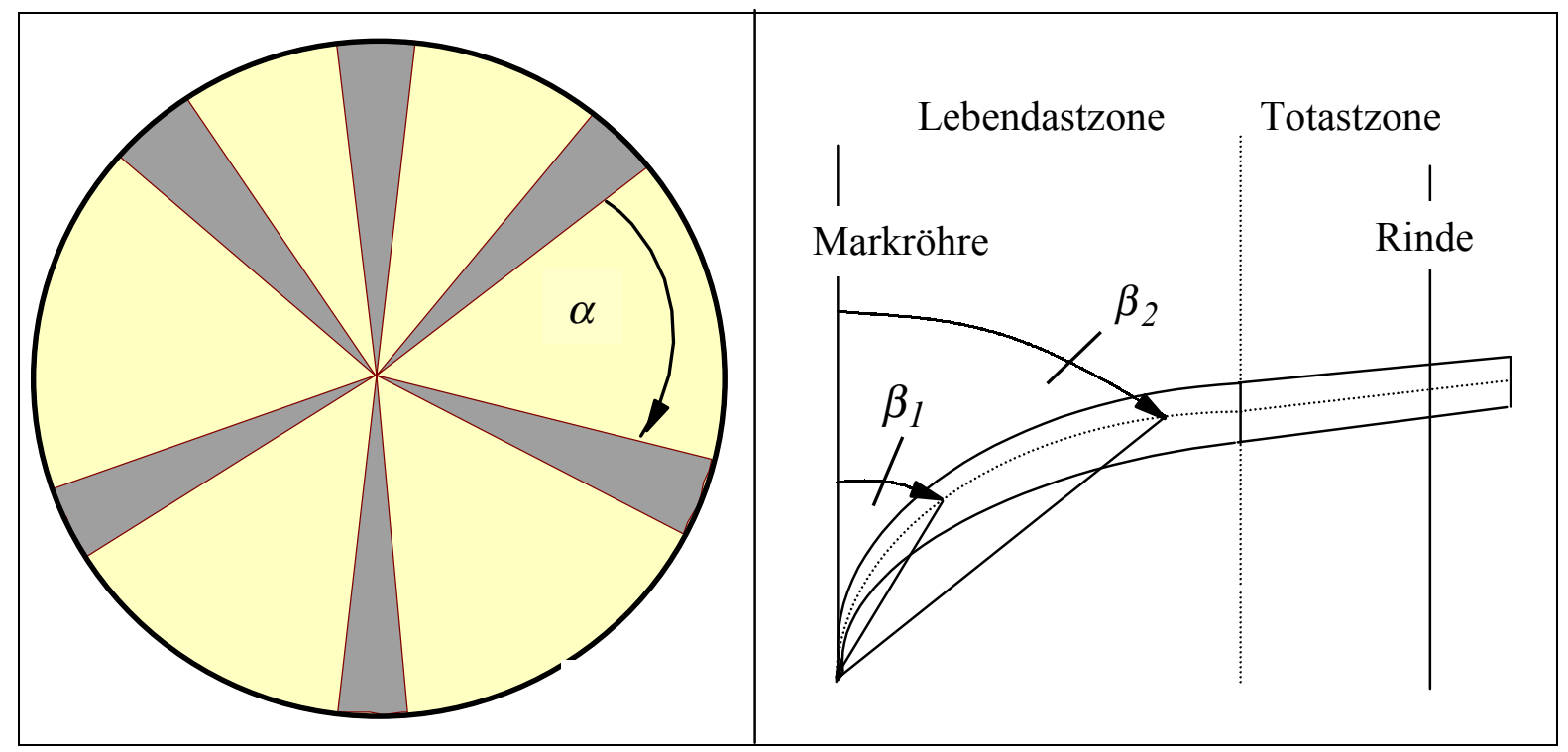

Abb. 8: Darstellung des Asthorizontalwinkels $\alpha$ im Querschnitt eines Stammes auf der Ebene eines Quirls (links) und des Astvertikalwinkels $\beta$ im Radialschnitt (rechts). Der Astvertikalwinkel nimmt in radialer Richtung zu $\left(\boldsymbol{\beta}_{1}<\boldsymbol{\beta}_{2}\right)$ (LEBAN ET AL., 1996, verändert).

\subsubsection{Modellierung des Astabsterbezeitpunktes und der natürlichen Astreinigung}

Durch die Kombination der Astmodelle mit Einzelbaumwuchsmodellen, die neben dem Höhenwachstum auch die Veränderung der Kronenansatzhöhe beschreiben, ist es im allgemeinen nicht notwendig, den Absterbezeitpunkt eines Astes gesondert zu schätzen. Eine Ausnahme stellt beispielsweise das Populationsmodell von KELLOMÄKI und VÄISÄNEN (1988) dar, bei dem der Absterbezeitpunkt für jeden Ast direkt geschätzt wird. Ein Problem bei der Definition des Absterbezeitpunktes auf der Basis der Dynamik der Kronenansatzhöhe ist, daß der Kronenansatz zu einem gewissen Anteil noch lebende Äste aufweist. D. h., daß der Übergang 
von der lebenden Krone zur Totastzone nicht scharf begrenzt ist, sondern durch eine mehr oder weniger lange Übergangszone charakterisiert ist. Diese Übergangszone wird an der oberen Grenze näherungsweise durch den Kronenansatz und an der unteren Grenze eindeutig durch die Höhe des untersten grünen Astes begrenzt. Colin und Houllier (1992) haben ein Modell zur Schätzung der Höhe des untersten Grünastes vorgestellt, dessen Funktionsgleichungstyp mit der Schätzung der Kronenansatzhöhe identisch ist. Ein Ast stirbt demnach nicht direkt, wenn seine Höhe am Stamm mit der Kronenansatzhöhe identisch ist, sondern innerhalb der Übergangszone. Die Wahrscheinlichkeit, daß ein Ast bereits abgestorben ist, erhöht sich mit zunehmender Tiefe in der Krone innerhalb der Übergangszone.

Um die Dynamik der natürlichen Astreinigung einschätzen zu können, haben COLIN und Houllier (1992) auch ein Modell für die Höhe des untersten Totastes entwickelt. Mit Hilfe der Prognose der Höhenentwicklung des untersten Totastes kann der Zeitpunkt bestimmt werden, ab dem spätestens die Überwallung eines Aststumpfes einsetzt. Die Astreinigung setzt wie der Absterbeprozeß nicht an einer scharfen Grenze, sondern innerhalb einer Übergangszone ein. Die Wahrscheinlichkeit, daß ein Ast infolge von Zersetzungsprozessen vom Stamm abgefallen ist, steigt mit der Länge des Zeitraumes, der seit dem Absterben vergangen ist, bzw. mit der Tiefe seiner Position in der Absterbezone.

Bei den Ansätzen von COLIN und Houllier (1992) handelt es sich um statische Modelle, die die Höhe des Kronenansatzes, des untersten Grünastes und des untersten Totastes schätzen. Die Schätzung der Kronenansatzhöhe kann auch über dynamische Modelle erfolgen. Die Kronenansatzhöhe ergibt sich dann aus der Kronenansatzhöhe zum Beginn einer Prognoseperiode plus der Veränderungsrate innerhalb der Prognoseperiode (MAGUIRE U. HANN, 1990). Für die Veränderung der Höhe des untersten Grünastes könnten bei ausreichender Datengrundlage ebenfalls dynamische Modelle parametrisiert werden. Neben dem Nachteil, daß im Gegensatz zu statischen Modellen mindestens zwei Folgeaufnahmen für die Parametrisierung benötigt werden, haben dynamische Modelle vor allem den Vorteil, daß die Einflüsse von forstlichen Eingriffen auf die Veränderung der Höhen von Kronenansatz und unterstem Grünast beschrieben werden können. SHORT und BURKHART (1992) verwendeten neben Variablen für die Beschreibung der Konkurrenzsituation des Baumes eine weitere Variable für die Quantifizierung des Eingriffs. HASENAUER (1994) hat gezeigt, daß ein nach einem Eingriff ermittelter Konkurrenzindex für die Berücksichtigung von Eingriffen ausreicht und nicht durch eine Durchforstungsvariable ergänzt werden muß. Die Höhe des untersten Totastes hängt, da dieser Ast bereits abgestorben ist, nicht mehr von den Konkurrenzbedingungen des Baumes ab, so daß ein statisches Modell für die Prognose als ausreichend erscheint. 


\subsection{Flexible Schaftformmodelle}

Die Modellierung der Schaftform zur quantitativen und qualitativen Beschreibung von Sortimenten baut wie die Astmodelle auf der Prognose der Naturaldaten eines Wachstumssimulators auf. Sie dient, ähnlich wie die Aststärkenmodellierung, direkt der Erfassung eines Gütemerkmals und ist daher ein Bestandteil des eigentlichen Qualitätsmoduls im Waldwachstumssimulator Bwinpro.

Im Bereich der Simulation von Waldwachstumsszenarien ist die Schätzung von Sortimenten für eine Bewertung der Nutzfunktion alternativer waldbaulicher Strategien unerläßlich. Die Stehendsortimentierung erfolgt im allgemeinen mit Hilfe sogenannter Schaftformfunktionen, mit denen sich die Stammform von Waldbäumen beschreiben läßt. Schaftformfunktionen finden weiterhin bei einer Vielzahl von überwiegend qualitätsrelevanten Spezialauswertungen Verwendung, wie z. B. bei der Schätzung des Totholzvolumens stehender Baumstümpfe in Naturwäldern (NAGEL, 1999 b) und bei der Schätzung von astfreien Stammholzanteilen (HoULLIER ET AL., 1995). Schaftformfunktionen können dazu verwendet werden, Stammholzvolumina ohne Rinde, Kernholzvolumina bzw. resultierende Splintholzvolumina und Volumina des juvenilen Holzes zu schätzen. Die Schätzung von Kernholzvolumina ist bisher auf Nadelholzarten beschränkt, die einen obligaten Kern bilden (OJANSUU U. Maltamo, 1995; Hapla U. Saborowski, 1994; Cown, 1973). Die Kernholzvolumina sind bei Nadelhölzern infolge des monopodialen Wachstums mit größerer Genauigkeit einzuschätzen als bei Laubhölzern. Da die obligaten Kernholzbaumarten eine stetige Abnahme der Kernholzradien mit der Höhe am Stamm aufweisen, können zur Modellierung die gleichen Typen von Funktionsgleichungen verwendet werden wie für die Beschreibung der Schaftform. Für die Modellierung der Kernholzvolumina von fakultativen Kernholzbildnern eignen sich Schaftformfunktionen nicht, da uneinheitliche Kernholzformen ausgebildet werden (HAPLA U. SABOROWSKI, 1994). Auch liegt nicht immer eine Verjüngung der Kernholzradien mit zunehmender Höhe am Stamm vor. RACZ, ScHUlZ und KNIGGE (1961) haben in ihren Untersuchungen zum Buchenrotkern festgestellt, daß neben dem sogenannten Kegeltyp mit stetiger Verjüngung der Kernholzradien auch sehr häufig ein Spindeltyp mit einem Maximum des Kernradius' deutlich oberhalb des BHD auftritt. Die prozentualen Kernflächenanteile weisen zumindest in jungen Nadelholzbeständen beträchtliche Schwankungen auf, wobei CowN (1973) für Monterey-Kiefer (Pinus radiata D. Don) keinen Zusammenhang mit der Durchforstungsstärke feststellen konnte. Wie der Kernholzanteil beeinflußt auch der Anteil an juvenilem Holz die Möglichkeiten der Holzverwendung. Da die Grenze von juvenilem zu adultem Holz näherungsweise über ein bestimmtes Radialalter geschätzt werden kann, eignen sich Schaftformfunktionen auch zur Beschreibung des Volumens an juvenilem Holz. Wie bei den Schätzungen der Kernholzvolumina wird die Genauigkeit der Schätzung weniger durch die 
Schaftformfunktion als vielmehr dadurch bestimmt, wie sich variierende Anteile an juvenilem Holz erklären lassen.

MitCHELL (1975) hat ein nicht auf Schaftformfunktionen basierendes Modell entwikkelt, welches das Durchmesser- bzw. das Grundflächenwachstum in jeder beliebigen Höhe am Stamm schätzt. Als Grundlage dient das Pressler'sche Wachstumsgesetz, das unterstellt, daß der Grundflächenzuwachs in einer Höhe proportional zur kumulativen Nadelbiomasse oberhalb dieser Höhe ist. Daraus folgt, daß der resultierende Durchmesserzuwachs von der Baumspitze in Richtung des Kronenansatzes zu- und danach wieder abnimmt. Für den Verlauf des Grundflächenzuwachses im Kronenbereich ist eine Modellierung der Blattbiomasse notwendig. Da der Verlauf des Durchmessers bzw. des Grundflächenwachstums entlang des Stammes durch die Kronenansatzhöhe entscheidend beeinflußt wird, werden die Konkurrenzverhältnisse des Baumes bei der Modellierung der Schaftform berücksichtigt.

Aufgrund der großen Bedeutung der Stehendsortimentierung ist eine Vielzahl von Schaftformfunktionen entwickelt worden, die sich in mehrere Typen untergliedern läßt. HuI und GADOW (1997) unterscheiden parameterkarge Schaftformfunktionen von Schaftformfunktionen mit einer hohen Anzahl an Parametern. Zur letzteren Gruppe sind Polynomialgleichungen (LAASASENAHO, 1982), Spline-Funktionen (SABOROWSKI ET AL., 1981; KUBLIN U. SCHARNAGL, 1988) und lineare Schaftformmodelle (GAFFREY, 1996; SLOBODA ET AL., 1998) zu rechnen. Die Verwendung einer hohen Anzahl von Parametern erhöht die Flexibilität der Modelle und ermöglicht eine sehr genaue Beschreibung einzelner Bäume, für die Sektionsmessungen vorliegen. Allerdings sind die Modelle dieser Gruppe gerade aufgrund ihrer hohen Parameterzahl nur begrenzt dazu in der Lage, Unterschiede im Formenspektrum von Baumarten zu beschreiben, wenn als Eingangsvariablen nur der BHD und die Höhe zur Verfügung stehen und Parameter-Schätz-Methoden zur Flexibilisierung der Schaftform verwendet werden (Kublin U. Scharnagl, 1988, S. 2; Hui U. GAdOw, 1997). Bei der Verwendung von vielparametrigen Schaftformmodellen ist neben einer visuellen Überprüfung des Datenmaterials teilweise eine vorhergehende Glättung der Daten mit Hilfe von z. B. SplineFunktionen notwendig, da die hohe Flexibilität der Modelle auch ein für ein mittleres Modell unplausibles punktuelles Ansteigen des Schaftdurchmessers mit steigender Schafthöhe erlauben würde.

$\mathrm{Zu}$ den parameterkargen Schaftformfunktionen zählen beispielsweise Modelle von BrinK und GADOw (1986), ReAL und MOORE (1987), PAIN und BOYER (1996) und Bi (2000). Diese Modellgruppe verzichtet auf höchste Genauigkeit bei der Anpassung an die Sektionsdaten einzelner Bäume. Die eingeschränkte Flexibilität bietet jedoch die Möglichkeit, die Funktionsparameter in Abhängigkeit von Wuchsgebiets-, Bestandes- und/oder Einzelbaumvariablen zu schätzen. Im Gegensatz zu vielparametrigen Schaftformfunktionen können somit Formunterschiede von Einzelbäumen auf der Basis von Variablen unterschiedlicher Hierar- 
chiestufen beschrieben werden. So können beispielsweise der H/D-Wert oder Variablen, die die Bestandesdichte beschreiben, dazu genutzt werden, den Einfluß der Durchforstungsart und -stärke auf die Schaftform zu berücksichtigen. Unterschiede zwischen Wuchsgebieten können über die Höhenlage, mittlere Windgeschwindigkeiten oder aggregierende Klimaindizes erfaßt werden. Damit wird eine Flexibilisierung der Schaftform bzw. die Entwicklung eines Einheitsschaftformmodells ohne die Verwendung eines weiteren gemessenen Schaftdurchmessers ermöglicht. Unter Einheitsschaftformmodellen werden hier Schaftformmodelle verstanden, die die Beschreibung von Formunterschieden in Abhängigkeit von Einzelbaum-, Bestandesvariablen und/oder Variablen höherer Hierarchieebenen wie z. B. Geländehöhenstufen oder Breitengraden (KORHONEN, 1993) ermöglichen. Vielparametrige Schaftformmodelle bieten demgegenüber bei der ausschließlichen Verwendung von BHD und Baumhöhe nur die Möglichkeit, nach Straten getrennte Schaftformfunktionen zu parametrisieren (HRADETZKY, 1981; GAFFREY U. NAGEL, 1998). Bei mehreren unabhängigen Variablen können dabei jedoch sehr viele Straten entstehen, für die jeweils eine ausreichende Datengrundlage zur Verfügung stehen muß. Durch die Parametrisierung getrennter Modelle besteht weiterhin die Gefahr von nicht konsistenten Schaftformen der Straten untereinander (Kublin U. ScHARNAGL, 1988, S. 7).

Nach HuI und GADOW (1997) ist eine weitere Einteilung nach dem Geltungsbereich des Schaftformmodells möglich. Danach werden Einzelbaumschaftformmodelle, Bestandesschaftformmodelle und Einheitsschaftformmodelle unterschieden. Einzelbaumschaftformmodelle beschreiben das Schaftprofil eines einzelnen Baumes auf der Basis einer ausreichenden Anzahl von Sektionsmessungen. Bestandesschaftformmodelle beschreiben die Schaftprofile von Bäumen eines bestimmten Bestandes, wobei das Modell in der Lage sein muß, Formunterschiede der Einzelbäume in Abhängigkeit von Einzelbaum- und/oder Bestandesvariablen zu beschreiben. Ein mittleres Einzelbaumschaftformmodell, das lediglich das mittlere Schaftprofil eines Kollektivs beschreibt, wird hier nicht als Bestandesschaftformmodell, sondern als Einzelbaumschaftformmodell verstanden, da das Modell die Erfassung der Formvariabilität nicht beinhaltet. Sogenannte Einheitsschaftformmodelle beschreiben die Schaftprofile von Einzelbäumen in mehr oder weniger großen Gebieten. Auch diese Modelle müssen in der Lage sein, Formunterschiede der Einzelbäume in Abhängigkeit von Einzelbaum- und/oder Bestandesvariablen sowie Variablen höherer Hierarchiestufen (Klimaindizes, Höhenlage, geographische Koordinaten) zu beschreiben. Dabei ist es nicht zwingend notwendig, daß alle Hierarchiestufen im Modell berücksichtigt werden, wenn nachgewiesen werden kann, daß die Variablen bestimmter Hierarchiestufen keinen signifikanten Einfluß auf die Schaftform haben.

Letztlich kann ein ausschließlich mit Hilfe von Einzelbaumvariablen flexibilisiertes Schaftformmodell nur dann als Einheitsschaftformmodell gelten, wenn Variablen höherer 
Hierarchiestufen auf ihren Einfluß bezüglich der Schaftform überprüft und als nicht signifikant identifiziert worden sind. Im weiteren wird jedoch auch ohne diese Überprüfung bei erfolgreicher Flexibilisierung mit Hilfe von Einzelbaumvariablen von einem Einheitsschaftformmodell gesprochen.

\subsection{Modelle zur Prognose von Rotfäule bei Fichte}

Anhand des Gütemerkmals Stammfäule bei Fichte sollen die Modellebenen, die bei der Klassifikation von Gütemerkmalen nach dem Kriterium der Modellierbarkeit beschriebenen wurden (Abb. 2), auch für ein fakultatives Gütemerkmal erläutert und der Unterschied zu den obligaten Kriterien verdeutlicht werden. Als Beispiel dient die Stammfäule (Wundfäule, Kernfäule) der Fichte, da bei diesem Merkmal für alle drei Klassifikationsebenen Modelle entwickelt oder zumindest Zusammenhänge identifiziert werden konnten. ZYCHA und KAT (1967) grenzen die Wundfäule von der wurzelbürtigen Kernfäule ab, weil für das Auftreten unterschiedliche Ursachen ausschlaggebend sind. Für die Kernfäule der Fichte wird im allgemeinen der Wurzelschwamm (Heterobasidion annosum (Fr.) Cooke) verantwortlich gemacht, jedoch konnten ZYCHA und KAT (1967) weitere primäre, Kernfäule verursachende Pilze wie Armillaria mellea (Vahl ex Fr.) Kumm., Coryne sarcoides (Jacq.) Tul. und Polyporus abietinus (Anon.) ex Fr. isolieren, wenn auch zu deutlich geringeren Anteilen. Das Auftreten unterschiedlicher Pilze mit ihren spezifischen ökologischen Amplituden erschwert die Modellierung der nicht nach Pilzarten differenzierten Kernfäule in Abhängigkeit von den Standortseigenschaften.

\section{Modellebene zur Prognose des Auftretens und der Anteile an befallenen Stämmen auf Bestandesebene:}

ZYCHA und KAT (1967, S. 77 ff.) konnten positive Zusammenhänge zwischen dem durch Heterobasidion annosum verursachten Stammzahlprozent fauler Stämme (Faulstammprozent) und vorhandener Wechselfeuchtigkeit, sonnenseitiger Exposition, intensiver Bodenbearbeitung und Düngung bei Erstaufforstungen sowie Basenreichtum identifizieren. Ein statistisches Modell, insbesondere zur Prognose der nicht nach Pilzarten differenzierten Kernfäule, wurde jedoch nicht entwickelt. VollBRECHT und AGESTAM (1995) entwickelten ein statistisches Modell zur Prognose des Faulstammprozentes für Fichte in Südschweden, das sowohl Kernals auch Wundfäule berücksichtigt. Das Faulstammprozent wies einen Zusammenhang mit der Art der vorherigen Nutzung auf, wobei der Anteil von Laub- über Nadelwald zu ehemaliger Acker- und Grünlandnutzung hin anstieg. Die Berücksichtigung der Nutzungsform im Modell erfolgte über die Integration einer mit dem Faulstammprozent der vorhergehenden Durchforstung gewichteten kategorischen Variablen. Der Site-Index hatte einen negativen Einfluß (Verringerung), während die zur Quantifizierung des Einflusses forstlicher Eingriffe verwendeten Variablen kumulierte Grundfläche entnommener Bäume seit 1950 (ausschließ- 
lich des letzten Eingriffs), die Anzahl der entnommenen Bäume im vorletzten Eingriff und der Zeitraum seit dem vorletzten Eingriff einen positiven Einfluß aufwiesen. SCHÖNHAR (1994) stellte für den Bereich der Schwäbischen Alb fest, daß das Infektionsrisiko durch Kernfäule von der 1. Generation Fichte nach Laubholz über Erstaufforstungen zur 2. Generation Fichte nach Laubholz hin ansteigt. Bei Erstaufforstungen spielt das Fehlen von Antagonisten bei der Besiedlung von frischen Stubben nach Durchforstungen eine Rolle, während in der 2. Generation nach Laubholz alte Stubben eine Infektionsquelle darstellen (SCHÖNHAR, 1988). Ein Modell wurde auf der Basis der identifizierten Zusammenhänge nicht entwickelt.

\section{Modellebene zur Prognose des Auftretens von Stammfäule am Einzelbaum:}

STENLID (1987) entwickelte ein Modell, das die Wahrscheinlichkeit der Infektion einer Fichte mit Heterobasidion annosum in Abhängigkeit von der Anzahl der Infektionsquellen in bestimmten kritischen Abständen vom jeweiligen Einzelbaum schätzt. Dabei wurden unterschiedliche Infektionsrisiken für verschiedene Infektionsquellen ermittelt (alter Stubben, frischer Stubben sowie bereits infizierter lebender Nachbarbaum). Durch Multiplikation der Infektionsrisiken der einzelnen Infektionsquellen kann das Gesamtrisiko, das von beliebigen Kombinationen der Infektionsquellen ausgeht, bestimmt werden. Das Modell von STENLID (1987) geht damit über eine Prognose des Faulstammprozents auf Bestandesebene hinaus und schätzt individuelle Wahrscheinlichkeiten für Einzelbäume unter Berücksichtigung der Raumstruktur von Infektionsquellen und betrachtetem Baum. Für eine praxisnahe Anwendung besteht das Problem, daß die beschreibenden Variablen nicht mit praxisüblichen Inventuren erhoben werden können. Für eine Analyse der Infektionswege von Stammfäule liefert das Modell jedoch sehr gute Prognosen. CHADOEUf ET AL. (1993) beschrieben mit Hilfe eines Markov-Punkt-Prozesses das Infektionsrisiko für Einzelbäume in Gummibaumplantagen in Abhängigkeit davon, ob bereits abgetötete Bäume in der Nähe standen oder nicht. Zusätzlich konnten sie räumliche Interaktionen zwischen zwei unterschiedlichen pathogenen Pilzarten nachweisen. Die biologische Interpretierbarkeit der Parameter des Markov-Punkt-Prozesses ermöglichte weiterhin, zeitliche Verläufe der Epidimie und den Einfluß verschiedener Behandlungen auf den Epidimieverlauf zu beschreiben.

\section{Modellebene zur Beschreibung der Ausprägung der Stammfäule am Einzelbaum:}

ZYCHA und KAT (1967, S. 22 ff.) stellten fest, daß der Fäuledurchmesser und der Stammdurchmesser auf ähnlichen Standorten in einem fast proportionalen Verhältnis zueinander stehen. D. h., daß der prozentuale Anteil des Fäuledurchmessers am gesamten Stammdurchmesser auf dem gleichen Standort konstant ist. Dieser Zusammenhang gilt für einen Stammdurchmesserbereich von 20 bis $50 \mathrm{~cm}$, während bei sehr starken Dimensionen größere Schwankungen zu beobachten sind. Auch wurde das Verhältnis nicht davon beeinflußt, in 
welcher Höhe am Stamm die Messung erfolgte und ob Bäume eines Bestandes oder aus unterschiedlichen Beständen verglichen wurden. 


\section{Entwicklung neuer Ansätze zur Qualitätsprognose}

\subsection{Modellübersicht}

Um das Verständnis für das System und das Zusammenwirken der im Rahmen dieser Arbeit entwickelten Modelle zur Beschreibung von Gütemerkmalen zu erleichtern, soll einleitend anhand der Abb. 9 und Abb. 10 erläutert werden, welcher Teilaspekt von welchem Modelltyp beschrieben wird.

\subsubsection{Nadelholz}

Die sortier- und verwendungsrelevante äußere Astigkeit bei Nadelholzarten wird über ein Modell zur Prognose der Astdurchmesser aller Quirläste in der grünen Krone bis zum untersten Grünast beschrieben (Kapitel 3.3.1 für den stärksten Quirlast und Kapitel 3.3.2 für weitere Quirläste) (Abb. 9 (a)). Das Modell soll gegenüber vergleichbaren Ansätzen eine größere Sensitivität bei der Berücksichtigung forstlicher Eingriffe gewährleisten. Eine Sonderuntersuchung dient der Quantifizierung des Einflusses von Kronenbrüchen auf die Astdurchmesserstruktur bei Fichte (Kapitel 3.3.3). Auf eine Beschreibung von Internodalästen wurde verzichtet, da sie die qualitätsrelevante Astigkeit nur wenig beeinflussen. Zusätzlich müssen für eine vollständige Erfassung der äußeren qualitätsrelevanten Astigkeit die Astanzahlen pro Quirl (Kapitel 3.5) und der Quirlabstand geschätzt werden ${ }^{10}$. Die Astdurchmesser in der Totastzone werden über den zum Absterbezeitpunkt erreichten Astdurchmesser beschrieben. Die Modellierung der Astdurchmesser in der Totastzone erfordert damit die Kombination mit einem Wachstumssimulator, denn ein Ast wird immer dann zum Totast, wenn er sich unterhalb der im Simulationsmodell fortgeschriebenen Kronenansatzhöhe befindet. Eine Ausnahme stellt die Prognose der Astdurchmesserstruktur zum Simulationsbeginn dar. Hier muß die Prognose in der Totastzone direkt mit Hilfe des Astdurchmessermodells erfolgen, wobei auf den gleichen Funktionstyp mit einer um die Totastdurchmesser erweiterten Parametrisierungsgrundlage zurückgegriffen wird.

Neben den Astgrößenparametern spielt die Art des Astes eine entscheidende Rolle bei der Einschätzung der qualitätsrelevanten Astigkeit, da sich die Grenzwerte zwischen den Güteklassen für Grün- und Totäste unterscheiden. Die Modelle zur Prognose der Astdurchmesser müssen daher für eine realitätsnahe Prognose der Holzqualität um ein Modell zur Beschreibung des Absterbezeitpunktes von Einzelästen ergänzt werden. Um auch die Anteile an hochwertigem Stammholz einschätzen zu können, bedarf es zusätzlich eines Modells zur Beschreibung der natürlichen Astreinigung. Die Beschreibung der Länge der grünen Krone (Ka-

\footnotetext{
${ }^{10}$ Der Quirlabstand wird über das Höhenwachstumsmodell des Wachstumssimulators Bwinpro (NAGEL ET. AL, 2001) geschätzt, wobei unterstellt wird, daß die jährliche Höhenwachstumsrate mit dem Quirlabstand übereinstimmt.
} 
pitel 3.2), der Übergangsastzone, in der lebende und tote Äste gemeinsam auftreten (Kapitel 3.6.1), der Totastzone (Kapitel 3.6.2) und der astfreien Zone (resultiert aus den übrigen Schätzungen) dient der Prognose, ob ein Ast lebend, tot oder außerhalb des Stammes nicht mehr vorhanden ist (Abb. 9 (a)).

Die Kronenansatzschätzung wirkt sich über die Prognose der verschiedenen Astzonen direkt auf den Astzustand aus. Zusätzlich geht die Kronenansatzhöhe indirekt in die Schätzung der Astdurchmesser ein, da der baumspezifische Maximalwert für die Tiefe in der Krone (Kapitel 2.4.1) über die Kronenansatzhöhe bzw. die Höhe des untersten Grünastes festgelegt wird. Aufgrund der großen Bedeutung bei der Prognose der Astigkeit und der zusätzlichen Auswirkungen auf das Einzelbaumwachstum soll ein System von mehreren Modellen zur Schätzung der Kronenansatzhöhe entwickelt werden, das sich dadurch auszeichnet, daß der Informationsgehalt unterschiedlicher Eingangsdaten besser ausgenutzt wird (Kapitel 3.2).

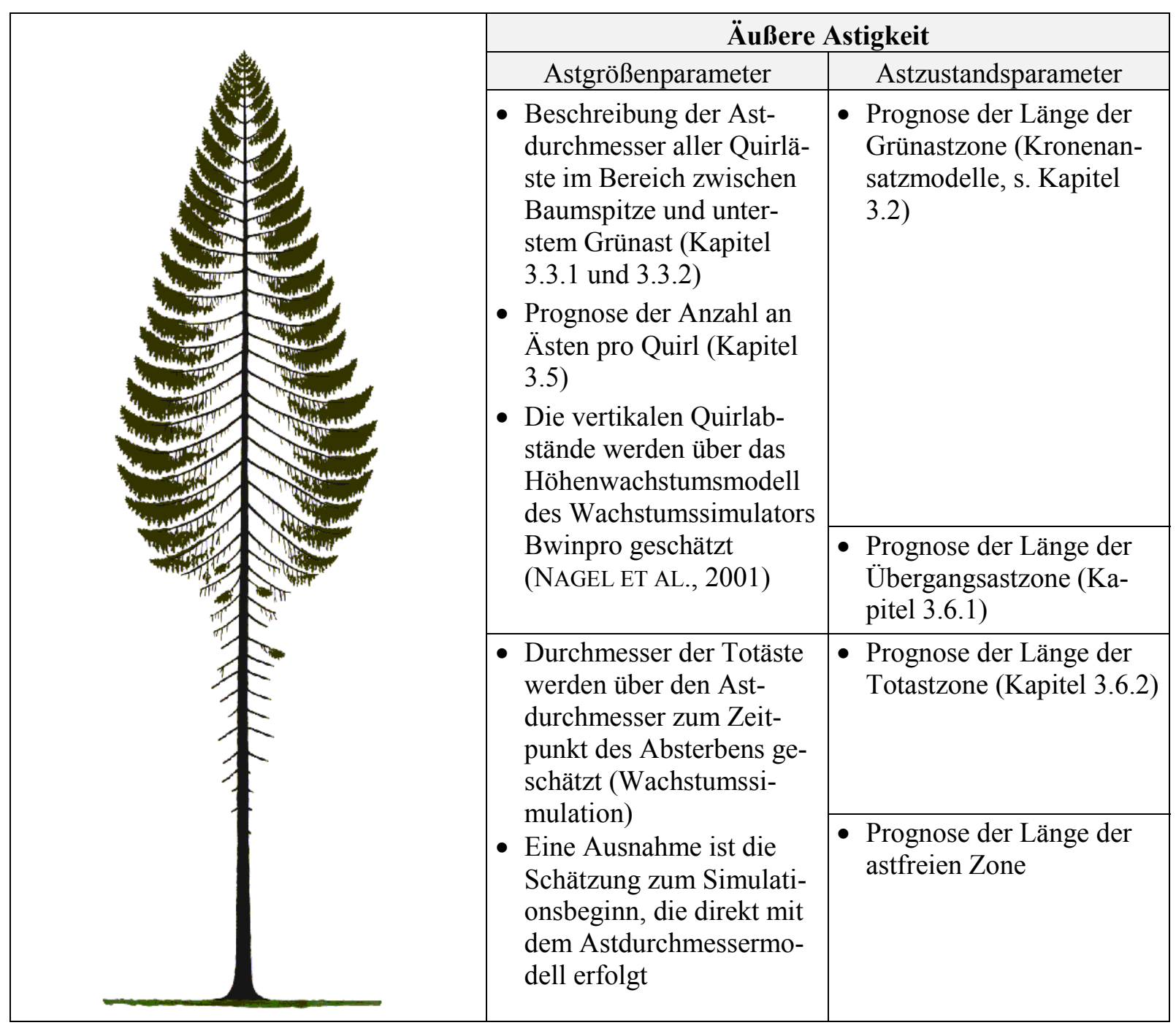

Abb. 9 (a): Modelle zur Beschreibung der äußeren qualitätsrelevanten Astigkeit von Nadelholzbaumarten und Darstellung ihres Geltungsbereiches am Baum. 
Zwei Modelltypen, die den gleichen bzw. sogar einen niedrigeren Informationsbedarf voraussetzen als die statischen Modelle von NAGEL (1999), PRETZSCH und KAHN (1998), COLIN und Houllier (1991) sowie HASENAUER (1994) (Kapitel 2.3), führen durch die Berücksichtigung des bestandesübergreifenden Dimensionstrends (Fußnote 4) zu einer Erhöhung der Schätzgenauigkeit (Kapitel 3.2.1 u. 3.2.2). Weiterhin wird ein Modell vorgestellt, das es ermöglicht, eine höhere Datenqualität, wie einzelne gemessene Kronenansatzhöhen oder Schätzungen des Kronenverhältnisses eines Mittelstammes, zu berücksichtigen (Einheitskronenansatzmodell, s. Kapitel 3.2.3). Das Modell führt bei gleichzeitig höheren Anforderungen an die Datenqualität zu einer weiteren Verbesserung der Schätzung der Kronenansatzhöhe. Für dieses Modell wird eine Methode zur Dynamisierung vorgestellt, die eine Berücksichtigung hochwertiger Eingangsinformationen über den Simulationsbeginn hinaus ermöglicht (Kapitel 3.2.4).

Eine Prognose der inneren Astigkeit erfordert eine Kombination der Astparametermodelle mit einem Schaftformmodell und einem Wachstumssimulator (Abb.9 (b) u. Abb. 10 (b)).

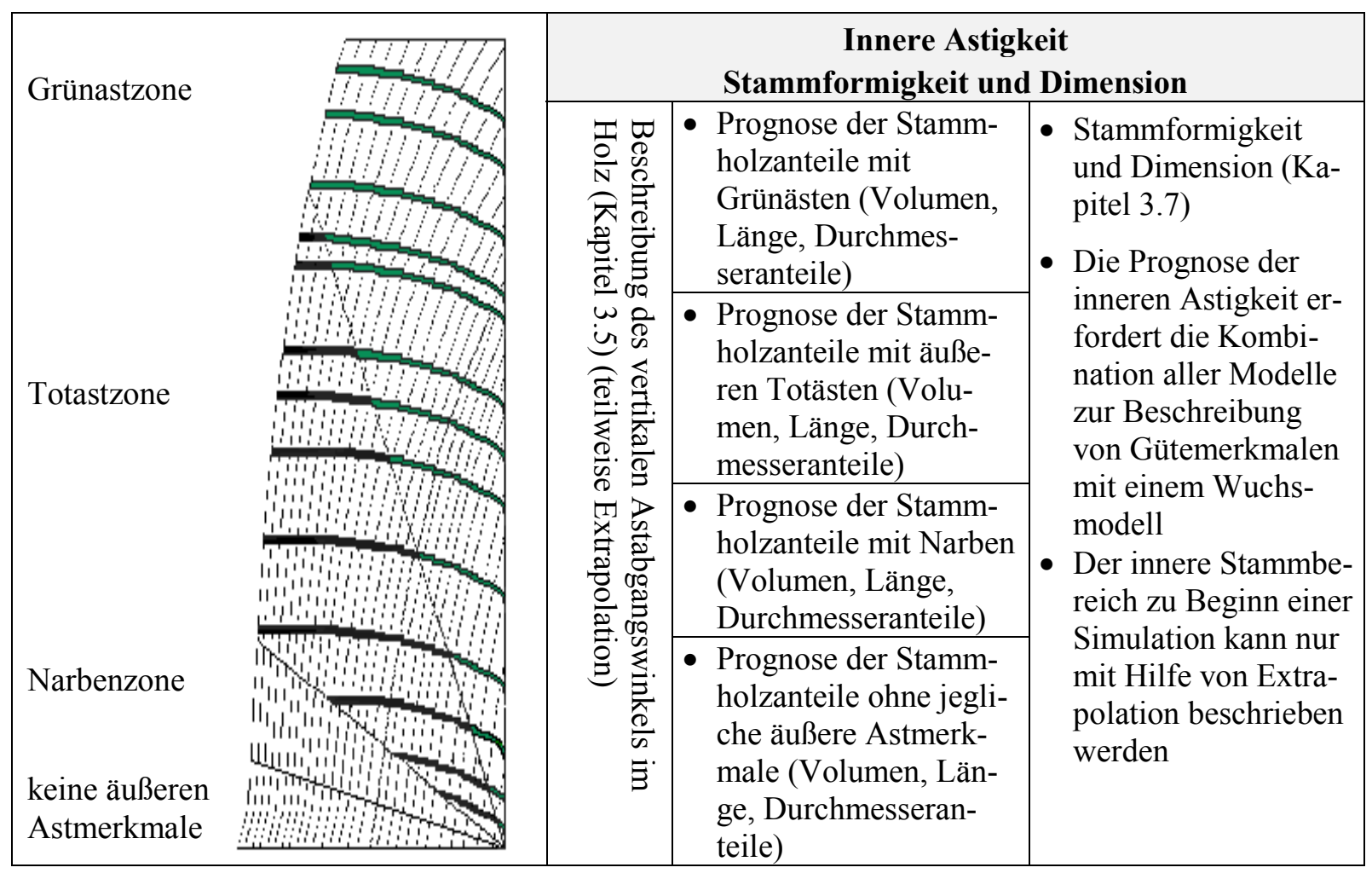

Abb. 9 (b): Modelle zur Beschreibung der inneren qualitätsrelevanten Astigkeit von Nadelholzbaumarten (Totasterhalter).

Da die Formigkeit selbst ein Gütemerkmal ist und wie die Dimension Einfluß auf die Bewertung der Stammgüte hat, soll ein Schaftformmodell entwickelt werden, das die Auswirkungen von forstlichen Eingriffen auf die Abholzigkeit erfaßt (Kapitel 3.7). Für die Störung der Faserstruktur im Radialschnitt muß prognostiziert werden, wie der vertikale Astabgangswinkel (Kapitel 2.4.6) mit steigendem Alter des Astes zunimmt (Kapitel 3.5). Durch die Kombination 
mit einem Wachstumssimulator kann weiterhin die Verjüngung des Astdurchmessers von der Rinde zum Mark in radialer Richtung dargestellt werden (Abb. 8).

Durch die Verlagerung der verschiedenen Astzustandszonen im Rahmen einer Wachstumssimulation kann der Ast im Radialschnitt in einen Bereich, in dem er als Totast nicht mit dem Stammholz verwachsen ist, und in einen Bereich, in dem er als Gesundast mit dem Stammholz verwachsen ist, unterteilt werden.

Die Prognose der inneren Astigkeit des zu Simulationsbeginn bereits existierenden Stammes erfordert eine Extrapolation des Astdurchmessers und des vertikalen Astabgangswinkels nach innen. Die Extrapolation des Astabgangswinkels ist dabei unproblematisch, da nur eine starre, lediglich von der Quirlrangfolge abhängige Schätzung erfolgt (Kapitel 3.5). Auch können eventuell auftretende Übergänge von Gesund- zu Totastbereich in radialer Richtung (Abb. 9 (b)) nicht direkt aus der Simulation geschätzt werden, wenn sie in diesem inneren Schaftformbereich liegen.

\subsubsection{Laubholz}

Für die Laubholzarten ist ein streng orthotropes Wachstum einer eindeutigen Zentralachse (Wipfelschäftigkeit) über die gesamte Lebensdauer die Ausnahme (für die Buche vgl. z. B. RolofF, 1986, S. 90 ff.). Bei der häufig auftretenden Auflösung des Stammes in zwei oder mehrere Schaftachsen kann eine Astdurchmesserbeschreibung nur im Stammbereich erfolgen (Abb. 10 (a), grün markierte Äste). Im Gegensatz zu den Nadelholzarten werden für die Buche daher nur die Astdurchmesser der vier untersten lebenden Äste im Kronenansatzbereich geschätzt (Kapitel 3.4.1). Die Anzahl vier ergibt sich daraus, daß zur Parametrisierung des Modells im Mittel vier Astdurchmesser pro Baum verwendet wurden. Für die Baumart Buche wird zusätzlich ein Modell zur Schätzung der vertikalen Astabstände der vier untersten lebenden Äste beschrieben, da die Äste nicht in Quirlen angeordnet sind (Kapitel 3.4.2). Eine Modellierung der qualitätsrelevanten Astdurchmessersummen erfordert jedoch neben der Beschreibung der Astdurchmesser auch eine Erfassung der Astdichte. Die Astdurchmesser von Totästen werden wie bei den Nadelholzarten über den Astdurchmesser geschätzt, den das Astdurchmessermodell zum Zeitpunkt des Absterbens prognostiziert. Dies setzt eine Kombination mit einem Wachstumsmodell voraus. Die äußere Astigkeit wird damit nur für den Kronenansatzbereich und die Totastzone beschrieben.

Auch kann die Totastzone zu Simulationsbeginn im Gegensatz zu den Nadelholzarten nur eingeschränkt (d. h. nur für kurze Totastzonenlängen) beschrieben werden, da das Durchmessermodell auf der Basis von Grünästen aus dem Kronenansatzbereich parametrisiert wurde. Die Buche weist jedoch überwiegend kurze oder keine Totastzonen auf (Kapitel 3.6.2), so daß die Anwendung des Modells auch für die initiale Prognose der Astdurchmesser in der Totastzone möglich erscheint. Im Gegensatz zu den Nadelholzarten wird der Kronenan- 
satz bei den Laubholzarten durch den untersten lebenden Primärast definiert (Kapitel 2.1). Geht man wie bei den Nadelholzarten davon aus, daß sich oberhalb des Kronenansatzes keine Totäste befinden, so entfällt die Prognose einer Übergangsastzone. Sekundäräste können mit dem Astdurchmessermodell nicht prognostiziert werden.

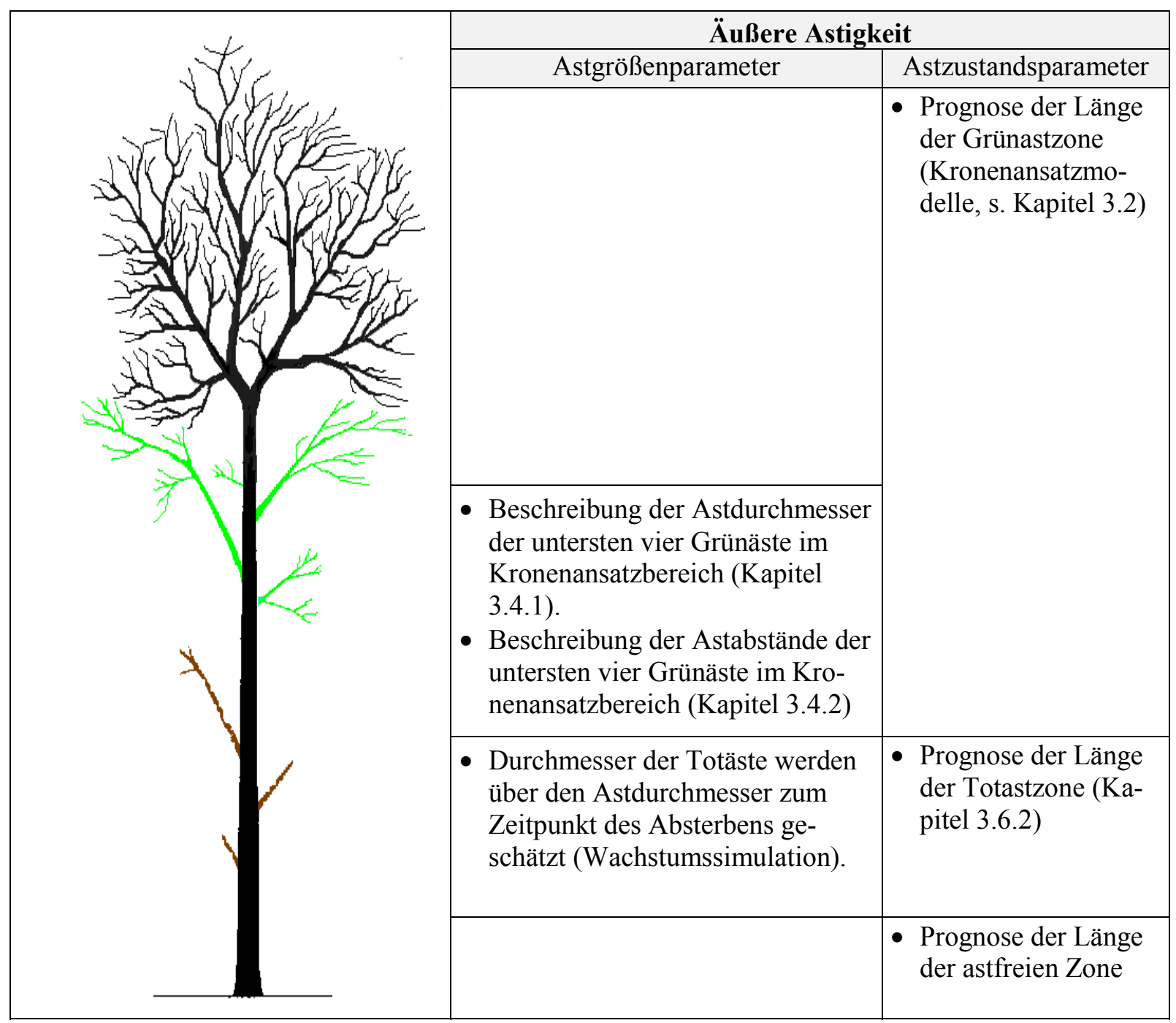

Abb. 10 (a): Modelle zur Beschreibung der äußeren qualitätsrelevanten Astigkeit von Laubholzbaumarten und Darstellung ihres Geltungsbereiches am Baum.

Die Modellierung der inneren Astigkeit erfolgt wie bei den Nadelholzbaumarten durch die Kombination der Modelle zur Beschreibung von Gütemerkmalen mit Schaftformmodellen und einem Wachstumssimulator.

Für die Beschreibung der inneren Astigkeit des gesamten Stammbereiches ist für die Buche ein Simulationsbeginn bei einem möglichst niedrigen physiologischen Alter entscheidender als bei den Nadelholzarten, da durch die kurzen Totastzonen die Ausgangsdurchmesser für die Extrapolation in radialer Richtung fehlen. 


\begin{tabular}{|c|c|c|c|}
\hline \multirow{2}{*}{\multicolumn{2}{|c|}{$\begin{array}{l}\text { Grünastzone } \\
\text { Totastzone }\end{array}$}} & \multicolumn{2}{|c|}{$\begin{array}{c}\text { Innere Astigkeit } \\
\text { Stammformigkeit und Dimension }\end{array}$} \\
\hline & & $\begin{array}{l}\text { - Prognose der Stamm- } \\
\text { holzanteile mit Grünästen } \\
\text { (Volumen, Länge, } \\
\text { Durchmesseranteile) }\end{array}$ & $\begin{array}{l}\text { - Stammformigkeit und } \\
\text { Dimension (Kapitel 3.7) } \\
\text { - Die Prognose der inne- } \\
\text { ren Astigkeit erfordert }\end{array}$ \\
\hline Narbenzone & 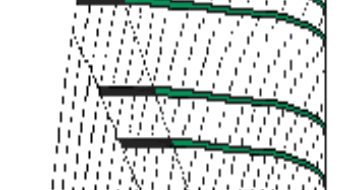 & $\begin{array}{l}\text { Prognose der Stamm- } \\
\text { holzanteile mit äußeren } \\
\text { Totästen (Volumen, Län- } \\
\text { ge, Durchmesseranteile) }\end{array}$ & $\begin{array}{l}\text { die Kombination aller } \\
\text { Modelle zur Beschrei- } \\
\text { bung von Gütemerk- } \\
\text { malen mit einem }\end{array}$ \\
\hline & 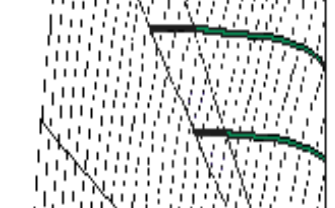 & $\begin{array}{l}\text { - Prognose der Stamm- } \\
\text { holzanteile mit Narben } \\
\text { (Volumen, Länge, } \\
\text { Durchmesseranteile) }\end{array}$ & Wuchsmodell \\
\hline $\begin{array}{l}\text { keine äußeren } \\
\text { Astmerkmale }\end{array}$ & 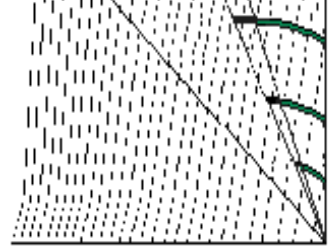 & $\begin{array}{l}\text { Prognose der Stamm- } \\
\text { holzanteile ohne jegliche } \\
\text { äußere Astmerkmale } \\
\text { (Volumen, Länge, } \\
\text { Durchmesseranteile) }\end{array}$ & \\
\hline
\end{tabular}

Abb. 10 (b): Modelle zur Beschreibung der inneren qualitätsrelevanten Astigkeit von Laubholzbaumarten .

Die Kronenansatzmodelle werden für die Baumarten Fichte, Douglasie, Kiefer, Europäische Lärche, Buche und Eiche parametrisiert. Astdurchmesser- und Astmortalitätsmodelle sowie Modelle für die natürliche Astreinigung werden für die genannten Baumarten mit Ausnahme der Eiche und der Europäischen Lärche entwickelt. Flexible Schaftformmodelle liegen für die Baumarten Fichte, Douglasie, Kiefer, Buche und Eiche vor, wobei für die Nadelholzarten deutlichere Verbesserungen gegenüber der Verwendung einer mittleren Schaftform erreicht werden als für die Laubholzarten. Die allgemeingültigen Überlegungen zur Modellentwicklung im Rahmen dieser Arbeit werden anhand der Baumart Fichte erläutert. Baumartenspezifische Parameterwerte und statistische Kennwerte sowie von den Modellen der Baumart Fichte abweichende Ansätze werden in Kapitel 9 aufgeführt. Das Astabstandsmodell wird für die Baumart Buche beschrieben, weil die Modellierung dieses Astparameters für die Nadelholzarten entfällt. Zusätzlich werden in Kapitel 3 Validierungen für die neu entwickelten Modelle mit Hilfe von Vergleichen mit bestehenden Modellen, mit Hilfe von Diagnosegrafiken sowie Prognosen für Modellbäume durchgeführt. 


\subsection{Schätzung der Kronenansatzhöhe}

\subsubsection{Hierarchisches Modell}

In Tab. 1 sind die Parameterschätzungen und der mittlere Quadratische Fehler (RMSE) der vier in Kapitel 2.3 vorgestellten Modelle für ein Kollektiv von 19060 Kronenansatzhöhen für die Baumart Fichte aufgeführt. Die Modelle werden in der weiteren Arbeit nach dem Wachstumssimulator bezeichnet, in dem sie implementiert sind (Bwin (NAGEL, 1999 a); Silva (Pretzsch U. KAhN, 1998); Forest (modifiziert) (HaSENAUer, 1994); CEP (COLIN U. Houllier, 1992)). Die Einzelmessungen verteilen sich auf 456 Versuchsparzellenaufnahmen, wobei auch Wiederholungsaufnahmen berücksichtigt wurden. Verwendet wurden nur Flächen mit mehr als 20 gemessenen Kronenansatzhöhen. Die Versuchsparzellen repräsentieren die unterschiedlichen Wuchsbedingungen in ganz Nordwestdeutschland. Abb. 11 zeigt die Verteilungen der unabhängigen Variablen, die in die neu entwickelten Modelle zur Schätzung der Kronenansatzhöhe eingehen.
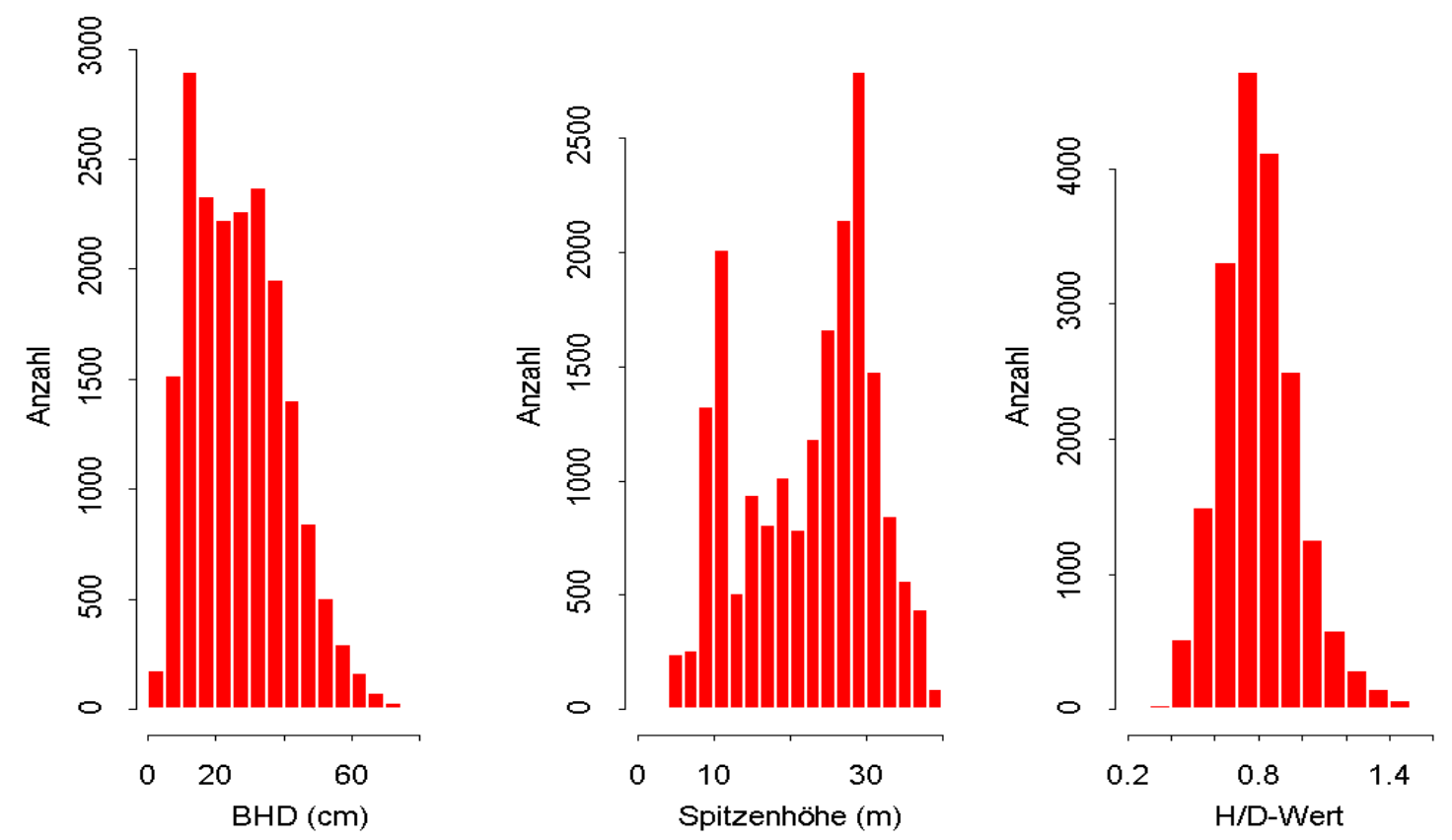

Abb. 11: Verteilungen der Variablen, die in den neu entwickelten Modellen zur Schätzung des Kronenansatzes verwendet werden für die Baumart Fichte.

Neben den deutlichen Unterschieden in der Schätzgenauigkeit stimmen die vier Vergleichsmodelle darin überein, daß ausschließlich Einzelbaumvariablen zur Schätzung verwendet werden. Beim Vergleich der an den hier verwendeten Datensätzen parametrisierten Modelle mit den in der Literatur für Fichte angegebenen Modellkoeffizienten stimmen die Vorzeichen in ihren Wirkungsrichtungen für die Kronenansatzmodelle in Bwin, Forest (modifiziert) sowie CEP (Ausnahme: Parameter $\delta$ ) überein. Beim Kronenansatzmodell in Bwin führen einheitlich positive oder negative Koeffizientenkombinationen durch die Quadrierung des Exponenten zum gleichen Ergebnis. Der Koeffizient $\delta$ im Kronenansatzmodell in CEP drückt nicht die 
Wirkungsrichtung einer Variablen aus, so daß der Unterschied hier nicht zu biologischen Unplausibilitäten führt. Für Fichte geben PRETZSCH und KAHN (1998, S. 70) für alle drei Koeffizienten negative Vorzeichen an, was in jedem Fall zu unplausiblen Kronenansatzhöhen mit negativem Vorzeichen führt. Im weiteren wird daher auf die hier geschätzten Koeffizienten Bezug genommen.

\begin{tabular}{|c|c|c|c|r|}
\hline Parameter & $\begin{array}{c}\text { Bwin } \\
\text { Formel (1) }\end{array}$ & $\begin{array}{c}\text { Silva } \\
\text { Formel (2) }\end{array}$ & $\begin{array}{c}\text { Forest (modifiziert) } \\
\text { Formel (4) }\end{array}$ & $\begin{array}{c}\text { CEP } \\
\text { Formel (5) }\end{array}$ \\
\hline$\alpha$ & -0.573 & -1.0613 & -5.283 & 0.0000108 \\
\hline$\beta$ & -0.446 & 1.714 & -1.824 & -42.524 \\
\hline$\gamma$ & & 0.0164 & & -0.000189 \\
\hline$\delta$ & & & 1.0417 & -0.0874 \\
\hline RMSE (m) & 2.678 & 2.227 & 2.047 & 2.313 \\
\hline
\end{tabular}

Tab. 1: Koeffizienten und RMSE (m) für vier statische Kronenansatzmodelle für die Baumart Fichte.

Bei der Interpretation der Wirkungsrichtungen der unabhängigen Variablen sollen hier zwei Ebenen unterschieden werden. Auf der übergeordneten Ebene wird die Wirkungsrichtung einer Variablen im bestandesübergreifenden allgemeinen Dimensionstrend (vgl. Kapitel 2.3, s. Fußnote 4) betrachtet, auf der untergeordneten Ebene wird die Wirkungsrichtung der Variablen innerhalb von Beständen betrachtet. Diese Unterscheidung ist notwendig, da für einige Variablen umgekehrte Wirkungsrichtungen auf der über- und untergeordneten Ebene auftreten, die von Wachstumsmodellen, die für die Wachstumsprognose von Beständen entwickelt werden, berücksichtigt werden sollten. Es wird im weiteren gezeigt, daß der BHD im Gegensatz zum H/D-Wert auf über- und untergeordneter Ebene in vielen Bestandesstrukturen entgegengesetzte Wirkungsrichtungen aufweist.

Zur Darstellung der hierarchischen Strukturen der Kronenansatzdaten bietet sich eine einzelbestandsweise Untersuchung an. An die Daten jedes Bestandes wurde ein lineares Regressionsmodell erster Ordnung angepaßt, wobei als unabhängige Variable der BHD und als abhängige Variable das Kronenverhältnis verwendet wurde (Formel (8), Modell "Geradengleichung $\left.{ }^{6}\right)$.

$K V=\alpha+\beta * D$

wobei:

$K V=$ Kronenverhältnis;

$D=$ Brusthöhendurchmesser (cm);

$\alpha=$ Ordinatenabschnitt der Geradengleichung;

$\beta=$ Steigung der Geradengleichung.

Die Beschränkung auf Bestände mit mehr als 20 gemessenen Höhen diente einer sicheren Schätzung der Funktionsparameter. 

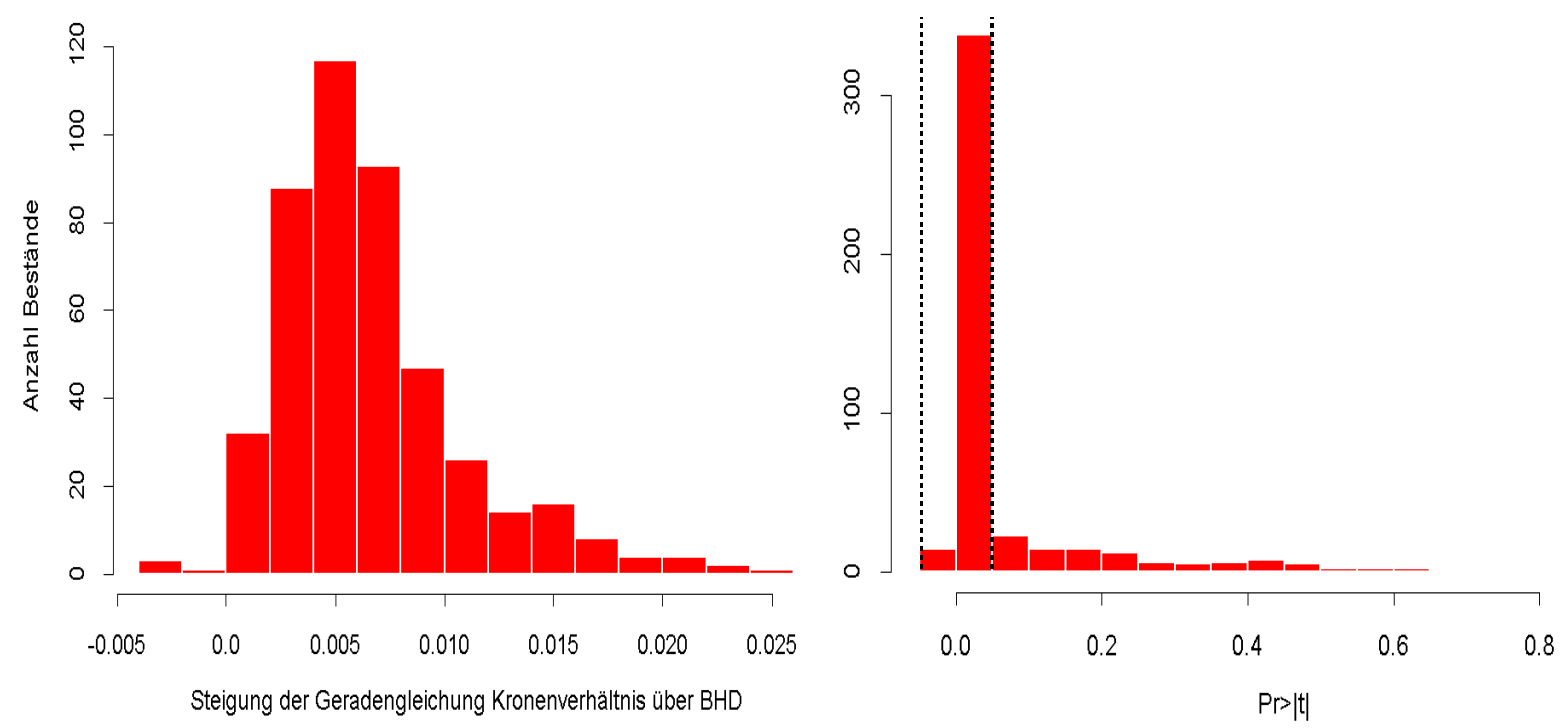

Abb. 12: Häufigkeitsverteilung des Stei- Abb. 13: Häufigkeitsverteilung der zu den tgungskoeffizienten für 456 einzelbestandsweise Anpassungen des Kronenansatzmodells "GeradenWerten zugehörigen kumulativen Wahrscheinlichkeiten (Komplement zu 1) der $t$ Verteilung des Steigungskoeffizienten für gleichung" (Formel (8)) mit der undas Kronenansatzmodell "Geradengleichung" mit der unabhängigen Variablen BHD (Formel (8)) bei einzelbestandsweiser Anpassung an 456 Bestände (Fichtenbestände und Fichtenanteile in Mischbeständen). Innerhalb der gestrichelten Linien befinden sich die Bestände, in denen der BHD einen signifikanten Einfluß auf das Kronenverhältnis bei 5\% Irrtumswahrscheinlichkeit hat.

Wie in Abb. 12 deutlich wird, treten nur vier Bestände auf, in denen der Zusammenhang zwischen dem BHD und dem Kronenverhältnis negativ ist. D. h. innerhalb von Beständen steigt das Kronenverhältnis mit steigendem BHD, was den gegenläufigen Trend zur übergeordneten Ebene belegt, da es als allgemeingültig angesehen werden kann, daß bestandesübergreifend das Kronenverhältnis mit steigendem BHD bzw. steigender Baumhöhe abnimmt (KRAMER, 1988, S. 21). Abb. 13 zeigt die zu den einzelbestandsweise berechneten $t$-Werten der Variablen BHD zugehörigen kumulativen Wahrscheinlichkeiten (Komplement $\mathrm{zu} 1$ ) einer $t$ Verteilung mit den entsprechenden Freiheitsgraden. In 352 von 456 Beständen hat der BHD einen signifikanten Einfluß auf das Kronenverhältnis bei einer Irrtumswahrscheinlichkeit von $5 \%$.

Wie aufgrund der engen Korrelation von BHD und Höhe zu erwarten ist, treten innerhalb von Beständen kaum negative Zusammenhänge zwischen dem Kronenverhältnis und der Einzelbaumhöhe auf (11 von 456 Beständen weisen einen negativen Steigungskoeffizienten auf (Abb. 14); 354 von 456 geschätzten Koeffizienten sind signifikant zum 5\% Niveau). 
Wird der H/D-Wert als einzige Variable zur Schätzung des Kronenverhältnisses innerhalb von Beständen verwendet, so ergibt sich, daß in 439 von 456 Beständen der H/D-Wert einen negativen Einfluß auf das Kronenverhältnis hat (Abb. 15). 275 der 439 negativen Steigungskoeffizienten sind signifikant zum 5\% Niveau.

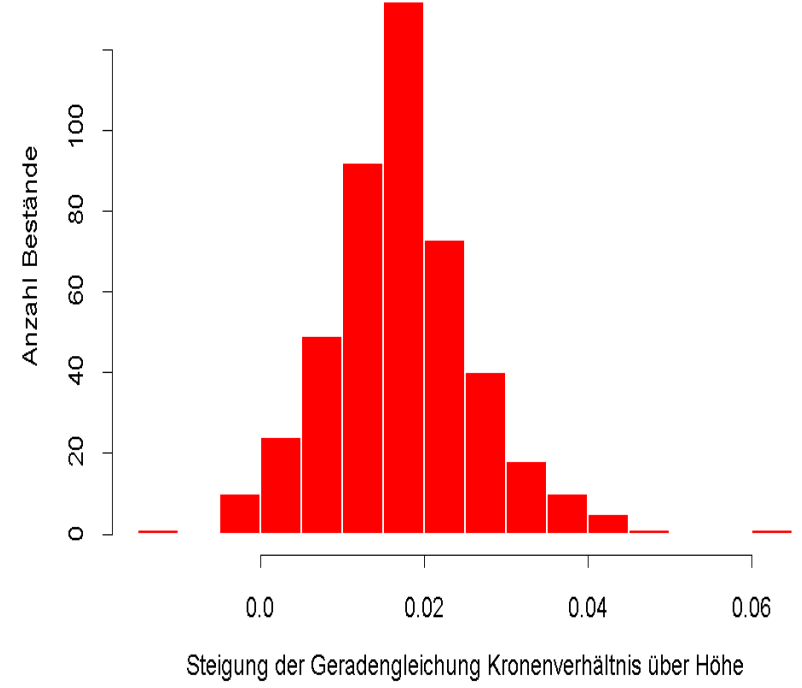

Abb. 14: Häufigkeitsverteilung des Steigungskoeffizienten für 456 einzelbestandsweise Anpassungen des Kronenansatzmodells "Geradengleichung" mit der unabhängigen Variablen Einzelbaumhöhe für die Baumart Fichte.

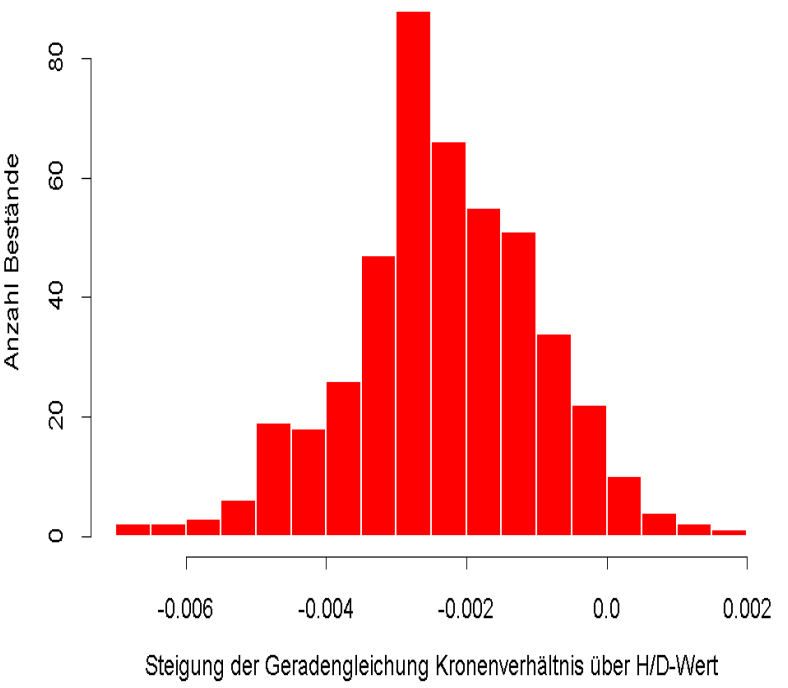

Abb. 15: Häufigkeitsverteilung des Steigungskoeffizienten für 456 einzelbestandsweise Anpassungen des Kronenansatzmodells "Geradengleichung" mit der unabhängigen Variablen H/D-Wert für die Baumart Fichte.

Weiterhin wurde untersucht, inwieweit der H/D-Wert innerhalb von Beständen, zusätzlich zum Einfluß des BHD, einen signifikanten Einfluß auf das Kronenverhältnis hat. In Abb. 16 sind die einzelbestandsweise berechneten kumulativen Wahrscheinlichkeiten (Komplement zu 1) der $t$-Werte der unabhängigen Variablen H/D-Wert dargestellt. In nur 78 von 456 Beständen hat der H/D-Wert zusätzlich zum BHD einen signifikanten Einfluß auf das Kronenverhältnis bei einer Irrtumswahrscheinlichkeit von 5\%.

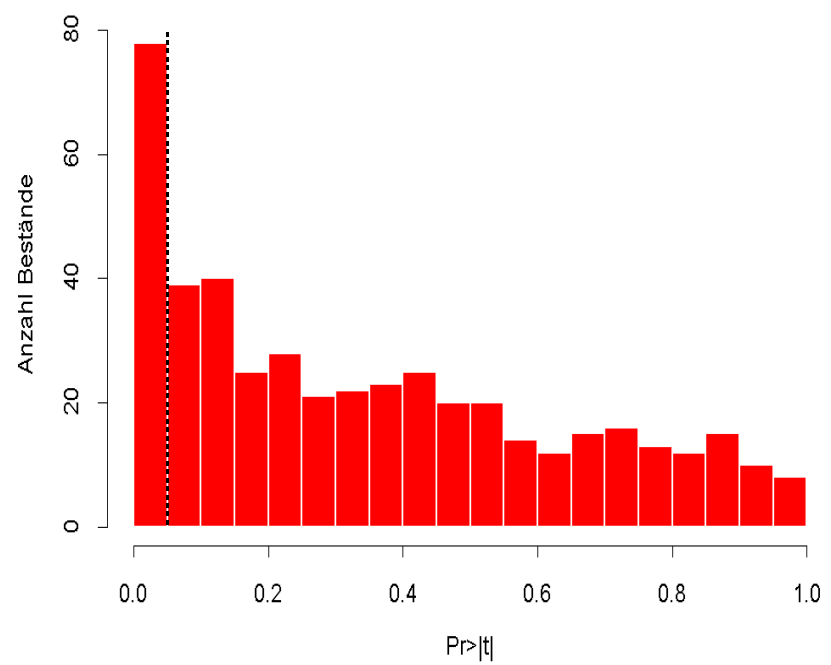

Abb. 16: Häufigkeitsverteilung der zu den $t$-Werten zugehörigen kumulativen Wahrscheinlichkeiten (Komplement zu 1) der t-Verteilung des Koeffizienten der Variablen H/D-Wert für das Kronenansatzmodell "multiple lineare Regression" mit den unabhängigen Variablen BHD und H/DWert bei einzelbestandsweiser Anpassung an 456 Bestände (Fichtenreinbestände und Fichtenanteile in Mischbeständen)(zur Erläuterung der gestrichelten Linie siehe Abb. 13). 
Zusammenfassend kann aufgrund der Datenanalyse festgestellt werden, daß innerhalb von fast allen untersuchten Beständen (untergeordnete Ebene) ein gegenläufiger Trend zum allgemeinen bestandesübergreifenden Zusammenhang (übergeordnete Ebene) zwischen der Einzelbaumdimension (BHD, Höhe) und dem Kronenverhältnis besteht. Es besteht folglich überwiegend ein positiver Zusammenhang zwischen dem BHD und dem Kronenverhältnis innerhalb von Beständen im Gegensatz zum negativen Zusammenhang auf der übergeordneten Ebene. Weiterhin hat der H/D-Wert, zusätzlich zum BHD, nur in etwa 17\% der Bestände einen signifikanten Einfluß auf das Kronenverhältnis zum Signifikanzniveau 5\%. Der H/DWert hat sowohl bei bestandesübergreifender Betrachtung als auch auf der untergeordneten Ebene einen negativen Einfluß auf das Kronenverhältnis. Der Trend kehrt sich damit, im Gegensatz zu den Dimensionsvariablen, nicht um.

Nach diesen Voruntersuchungen können die Koeffizienten der beschriebenen Modelle auf ihre Wirkungsrichtung hin untersucht werden. Für das Kronenansatzmodell in Bwin ist unerheblich, welches Vorzeichen der Parameter $\beta$ hat, da der Ausdruck im Exponenten quadriert wird und kein Zusammenwirken mit anderen unabhängigen Variablen auftritt. Mit steigendem H/D-Wert steigt das Kronenansatzverhältnis, was biologisch plausibel ist (KRAMER, 1988, S. 27). Gegenläufige Tendenzen treten für den H/D-Wert innerhalb beider betrachteter Ebenen nicht auf. Somit werden sowohl bei einer Bestandessimulation als auch bei der Simulation auf einer bestandesübergreifenden Ebene biologisch plausible Werte geschätzt. Der H/D-Wert ist daher eine Variable, die gleichzeitig die Trends auf über- und untergeordeneter Ebene beschreibt. Allerdings weist das Kronenansatzmodell in Bwin den höchsten Standardfehler aller untersuchten Modelle auf (Tab. 1).

Für das Kronenansatzmodell in Silva gilt, daß die Variablen BHD und H/D-Wert die gleiche Wirkungsrichtung aufweisen und daß steigende Werte für beide Variablen zu größeren Kronenansatzverhältnissen führen. Es wird davon ausgegangen, daß der Gesamtausdruck im Exponenten negativ bleibt, damit es nicht zu den bereits erwähnten unplausiblen negativen Kronenansatzhöhenschätzungen kommt. Weiterhin ist es ebenfalls plausibel, daß - einem allgemeinen bestandesübergreifenden Dimensionstrend folgend - mit steigendem BHD auch das Kronenansatzverhältnis steigt. Problematisch ist allerdings, daß sich diese Beziehung innerhalb von Beständen häufig umkehrt (Abb. 12). Bäume mit größerem BHD weisen vor allem in mehr oder weniger einschichtigen Kollektiven größere Kronenverhältnisse bzw. geringere Kronenansatzverhältnisse auf als Bäume mit geringerem BHD. Trotzdem können auch für Einzelbestände plausible Kronenverhältnisse geschätzt werden, da der Trend innerhalb von Beständen durch die Verwendung der Variablen H/D-Wert erfaßt werden kann. Die stärkeren Bäume eines gleichaltrigen Bestandes werden in der Regel niedrigere H/D-Werte aufweisen als die schwächeren. Im Vergleich zum Kronenansatzmodell in Bwin führt die Verwendung einer weiteren Einzelbaumvariablen (BHD), die aufgrund ihres Vorzeichens dahingehend 
interpretiert werden muß, daß sie den bestandesübergreifenden allgemeinen Dimensionstrend beschreibt, zu einer deutlich genaueren Schätzung (Tab. 1).

Das Kronenansatzmodell in CEP weist mit dem Alter eine Variable auf, die in gleichaltrigen Beständen auch als Bestandesvariable aufgefaßt werden kann. Mit der Verwendung der Variablen Alter besteht damit die Möglichkeit, den bestandesübergreifenden Dimensionstrend zu erfassen. Die Wirkungsrichtung im Modell führt mit steigendem Alter zu größeren Kronenansatzverhältnissen bzw. Kronenansatzhöhen, was biologisch plausibel ist. Die Wirkungsrichtung des H/D-Wertes und der Baumhöhe führen bei steigenden Werten ebenfalls zu gröBeren Kronenansatzverhältnissen. Was für den H/D-Wert sowohl im allgemeinen Trend als auch im Einzelbestand plausibel erscheint, gilt für die Höhe uneingeschränkt nur im allgemeinen bestandesübergreifenden Trend. Wie beim BHD kehrt sich der Zusammenhang zwischen der Höhe und dem Kronenverhältnis im Einzelbestand häufig um (Abb. 14). Diese Aussage ist nur für mehr oder weniger einschichtige Kollektive nicht aber für Femel- oder Plenterwälder gültig, die den allgemeinen Dimensionstrend durch die kleinräumige Mischung unterschiedlichster Baumdimensionen zumindest zeitweise innerhalb eines Bestandes aufweisen. Für das Kronenansatzmodell in CEP kann festgestellt werden, daß die Einzelbaumvariable Baumhöhe weiterhin den allgemeinen Dimensionstrend beschreibt (Vorzeichen), obwohl zusätzlich die Variable Alter verwendet wird. Plausible Schätzungen von Kronenansatzverhältnissen innerhalb von Beständen sind ebenfalls möglich, da die beschriebenen Trends mit Hilfe der Variablen H/D-Wert wie beim Kronenansatzmodell in Silva abgebildet werden können.

Beim Kronenansatzmodell in Forest (modifiziert) nimmt mit steigender Einzelbaumhöhe, entsprechend dem allgemeinen Alterstrend, das Kronenansatzverhältnis zu, während der BHD genau gegenläufig wirkt. Obwohl beide Variablen Einzelbaumvariablen sind, muß das Modell so interpretiert werden, daß die Einzelbaumhöhe den allgemeinen bestandesübergreifenden Dimensionstrend und der BHD den Einfluß der Baumdimension innerhalb eines Bestandes beschreibt.

Zusammenfassend kann gesagt werden, daß sich die untersuchten Modelle in der Wirkungsrichtung von Einzelbaumvariablen, die die Baumdimension beschreiben, unterscheiden. Dabei ist entscheidend, ob diese Variablen (BHD, Baumhöhe) den Einfluß des bestandesübergreifenden Dimensionstrends oder die Datenstruktur innerhalb von Beständen beschreiben. Bei der Variablen H/D-Wert treten diese Unterschiede nicht auf, da die Wirkungsrichtung innerhalb beider Ebenen gleichgerichtet ist.

\section{Bestandesindividuelles Kronenansatzmodell:}

Aufgrund der untersuchten Datenstrukturen wurde ein Modell zur Schätzung des Kronenansatzverhältnisses entwickelt, das die hierarchische Struktur der Daten widerspiegelt. Ziel ist es, Einzelbaumvariablen mit der Wirkungsrichtung zu integrieren, die sie innerhalb von Be- 
ständen aufweisen. Zu diesem Zweck wurden, wie bei den Voruntersuchungen, Modelle für alle 456 Einzelbestände parametrisiert und die Struktur der Koeffizienten dieser Modelle untersucht. Unter Verwendung von Bestandesvariablen wurden anschließend Funktionen zur Schätzung dieser Koeffizienten entwickelt.

Bei der Wahl des bestandesindividuellen Kronenansatzmodells wurden unterschiedliche Kombinationen von unabhängigen Variablen in multiplen linearen Modellen untersucht. Beispielsweise führt die Verwendung des H/D-Wertes zusätzlich zum BHD innerhalb von Beständen nur in seltenen Fällen zu einer Verbesserung der Schätzung des Kronenverhältnisses. Wie gezeigt wurde, war nur in ca. 17\% der Bestände der H/D-Wert zusätzlich zum BHD signifikant (Abb. 16). In Tab. 2 sind die Standardfehler bei Verwendung unterschiedlicher Kombinationen von unabhängigen Variablen für das bestandesindividuelle Kronenansatzmodell und bei bestandesweiser Parametrisierung aufgeführt. Keine der Kombinationen führt zu einer deutlichen Verringerung des Standardfehlers, der für alle Kombinationen um 6\% schwankt.

\begin{tabular}{|c|c|c|r|r|r|r|r|}
\hline $\begin{array}{c}\text { Unabhängige } \\
\text { Variablen }\end{array}$ & BHD & Höhe & H/D-Wert & $\begin{array}{c}\text { BHD und } \\
\text { Höhe }\end{array}$ & $\begin{array}{c}\text { BHD und } \\
\text { H/D-Wert }\end{array}$ & $\begin{array}{c}\text { BHD und } \\
\text { C }_{66}\end{array}$ & $\begin{array}{c}\text { BHD und } \\
\text { KSFL }\end{array}$ \\
\hline RMSE & 0.0619 & 0.0609 & 0.0659 & 0.05966 & 0.0608 & 0.0555 & 0.0612 \\
\hline
\end{tabular}

Tab. 2: RMSE der Schätzung des Kronenverhältnisses für verschiedene Kombinationen von unabhängigen Variablen bei einzelbestandsweiser Anpassung von multiplen und einfachen linearen Regressionsmodellen für die Baumart Fichte $\left(C_{66}\right.$ : Im Waldwachstumssimulator Bwin verwendeter Konkurrenzindex (NAGEL, 1999 a); KSFL: Summe der Kronenschirmflächen der Bäume mit einem BHD, der größer ist als der BHD des Bezugsbaumes).

Wie zu erkennen ist, unterscheiden sich die Modelle im Vergleich zur alleinigen Verwendung der unabhängigen Variablen BHD in den meisten Fällen nur unwesentlich. Der höchste Fehler tritt bei alleiniger Verwendung des H/D-Wertes und der niedrigste Fehler bei der Kombination von $\mathrm{BHD}$ und dem Konkurrenzindex $\mathrm{C}_{66}$ auf. Allerdings liegt bei Verwendung des $\mathrm{C}_{66^{-}}$ Index ‘ ein Scheinzusammenhang vor, da die Höhe des Kronenansatzes und die Baumhöhe in die Berechnung des $\mathrm{C}_{66}$-Index ‘ eingehen.

Es kann davon ausgegangen werden, daß die Variable BHD im allgemeinen die höchste Datenqualität aufweist. Das gilt sowohl für Versuchsflächen, für die häufig Vollaufnahmen der Variablen BHD vorliegen, als auch für Anwendungen, in denen alle Einzelbaumvariablen mit Hilfe spezieller Algorithmen ergänzt werden müssen. Daher wurde das geringfügig bessere Modell mit der Höhe als unabhängiger Variablen nicht verwendet. Die Vorteile der Modelle mit der Kombination von BHD und Höhe, der Kombination von BHD und H/D-Wert und der Kombination von BHD und KSFL (Summe der Kronenschirmflächen der Bäume mit einem größeren BHD als der BHD des Bezugsbaums) sind geringfügig. Infolgedessen wurde abschließend als Modell der untergeordneten Ebene eine Geradengleichung mit der unabhän- 
gigen Variablen BHD gewählt (Formel (8); Abb. 17). Da die Koeffizienten in dem einfachen Modell statistisch unabhängig voneinander sind, ist ohne weitere Umformungen eine Voraussetzung für die effiziente Koeffizientenschätzung mit Hilfe von Bestandesvariablen erfüllt.

Für die Koeffizientenschätzung wird das Modell zur Beschreibung des Kronenverhältnisses, wie es in Formel (8) dargestellt ist, umgeformt (Formel (9)).

$$
\begin{aligned}
& K V=\alpha+\beta * D \\
& K V=\overline{K V}+\beta *(D-\bar{D})
\end{aligned}
$$

wobei:

$$
\begin{array}{ll}
K V & =\text { Kronenverhältnis; } \\
\bar{D} & =\text { Brusthöhendurchmesser }(\mathrm{cm}) ; \\
\overline{K V} & =\text { Mittleres Kronenverhältnis; } \\
\bar{D} & =\text { Arithmetischer Mitteldurchmesser }(\mathrm{cm}) ; \\
\alpha, \beta & =\text { Ordinatenabschnitt und Steigung der Geradengleichung. }
\end{array}
$$

mit folgenden Werten für das Beispiel in Abb. 17, einen Fichtenbestand:

$\alpha=0.0386 ; \beta=0.0179 ; \overline{K V}=0.302 ; \bar{D}=14.69$.

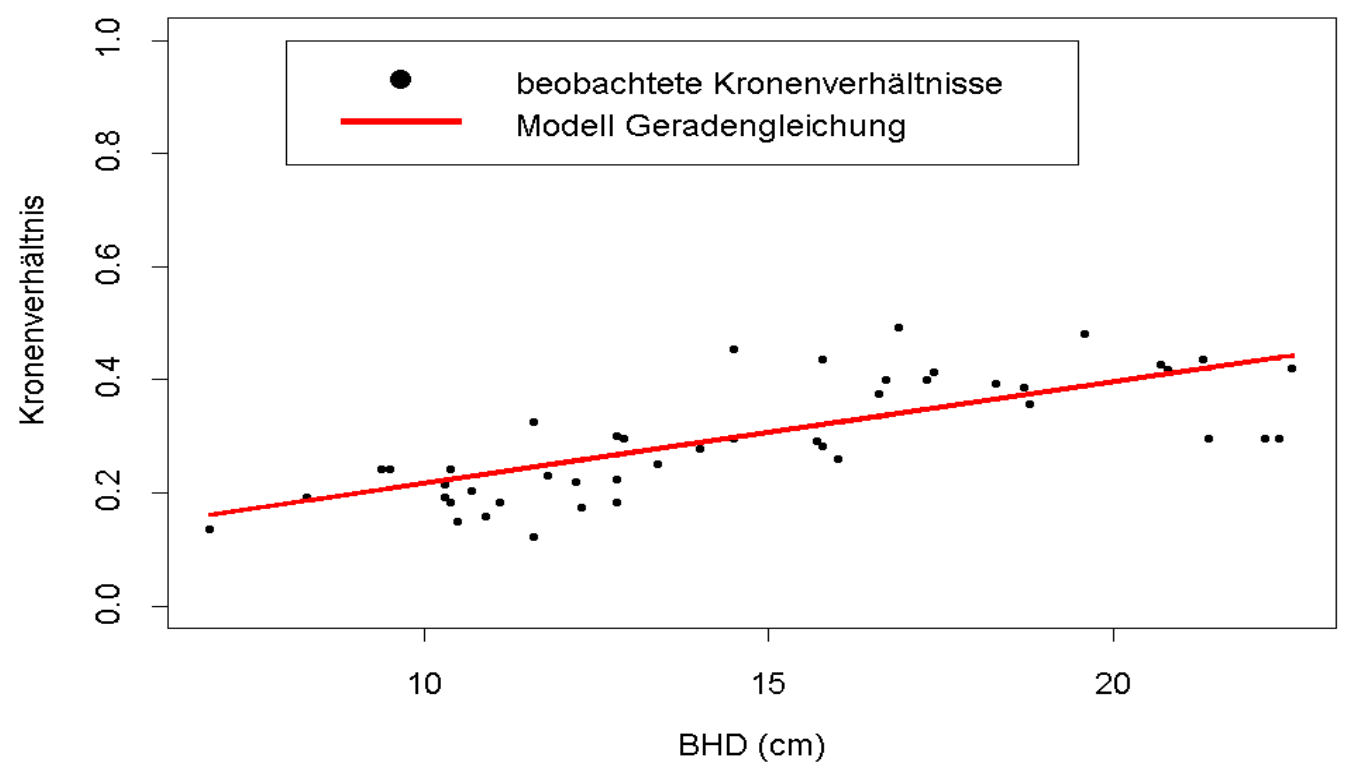

Abb. 17: Einzelbestandsweise Anpassung des Kronenansatzmodells "Geradengleichung" mit der unabhängigen Variablen BHD in einem Beispielbestand der Baumart Fichte (Formel (8)).

Gegenüber dem Modell (8) weist das Modell (9) einen Vorteil auf. Dieser Vorteil besteht darin, daß das mittlere Kronenverhältnis bei der begrenzten Anzahl an Datenpunkten mit größerer statistischer Sicherheit geschätzt werden kann als der Ordinatenabschnitt. Weiterhin ist das mittlere Kronenverhältnis biologisch sinnvoll zu interpretieren, während der Ordinatenabschnitt als Kronenverhältnis eines Baumes mit $0 \mathrm{~cm}$ BHD interpretiert werden müßte. Im weiteren wird daher das Modell (9) als Modell zur Schätzung des Kronenverhältnisses innerhalb von Beständen verwendet. 


\section{Verallgemeinerung des bestandesindividuellen Einzelbaummodells:}

Nach der Auswahl des bestandesindividuellen Modells wurde das Modell (9) an jeden der 456 Fichtenbestände angepaßt und die Struktur der Koeffizienten untersucht (Abb. 18).

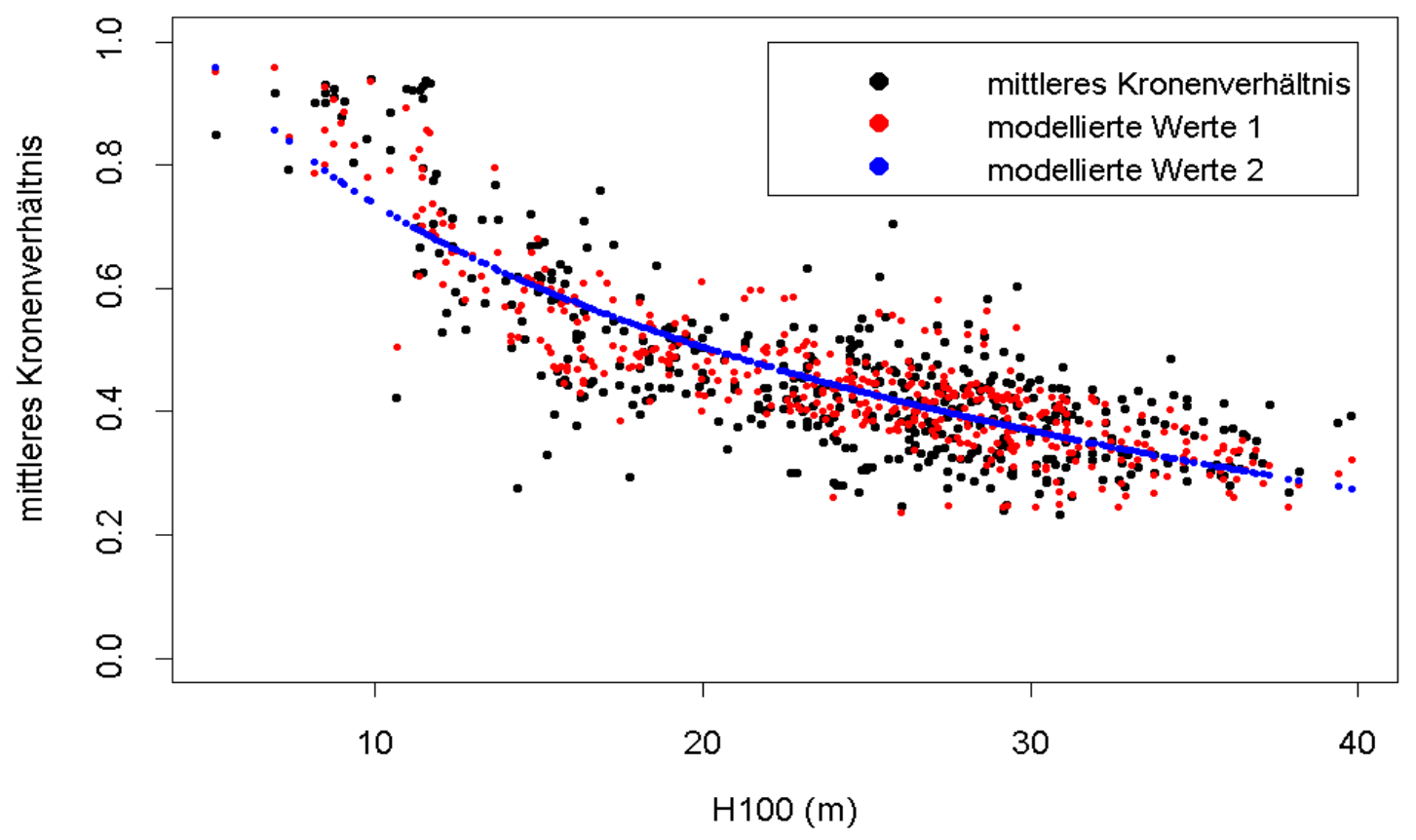

Abb. 18: Koeffizient "mittleres Kronenverhältnis" für 456 einzelbestandsweise Modellanpassungen (Formel (9)) und die Schätzung der Koeffizienten mit Hilfe der unabhängigen Variablen: natürlicher Logarithmus der Spitzenhöhe der Baumart im Bestand, mittlerer H/D-Wert der Baumart im Bestand, natürlicher Logarithmus der Kronenschirmfläche des Gesamtbestandes (modellierte Werte 1; Formel (10)) sowie bei ausschließlicher Verwendung der unabhängigen Variablen natürlicher Logarithmus der Spitzenhöhe der Baumart im Bestand (modellierte Werte 2). Verwendet wurden Fichtenreinbestände und der Fichtenanteil aus Fichtenmischbeständen.

Das Modell zur Schätzung des mittleren Kronenverhältnisses wurde mittels einer schrittweisen multiplen Regressionsanalyse ermittelt (Formel (10)). Die ausgewählten unabhängigen Variablen sind der mittlere H/D-Wert der Baumart im Bestand sowie der natürliche Logarithmus der Spitzenhöhe der Baumart im Bestand und der Kronenschirmfläche des Gesamtbestandes.

$\overline{K V}=\alpha+\beta * \ln (H 100)+\lambda * \overline{H D}+\delta * \ln (K S F)$

wobei für Formel (10)-(12):

$\begin{aligned} \overline{K V}= & \text { mittleres Kronenverhältnis der Bäume einer Baumart im Bestand; } \\ \overline{H D}= & \text { mittlerer H/D-Wert der Bäume der Baumart im Bestand }(\text { BHD }(\mathrm{cm}), \\ & H(m)) ; \\ \ln \left(H_{100)}\right)= & \text { natürlicher Logarithmus der Spitzenhöhe der Baumart im Bestand }(\mathrm{m}) ; \\ \ln (K S F)= & \text { natürlicher Logarithmus der Kronenschirmfläche des Gesamtbestandes } \\ & \left(\mathrm{m}^{2}\right)^{11} .\end{aligned}$

${ }^{11}$ Die Kronenschirmfläche wurde mit Hilfe eines Modells zur Schätzung der Kronenbreite aus dem BHD für jeden Einzelbaum berechnet und anschließend für alle Bäume der Versuchsfläche aufsummiert bzw. auf Hektarwerte hochgerechnet. Zur Schätzung der Kronenbreite siehe SCHMIDT ET AL. (2001). 
für Formel (10) mit:

\begin{tabular}{|c|c|c|c|c|}
\hline Koeffizienten & Wert & Std. Fehler & $\mathrm{t}$-Wert & $\operatorname{Pr}(>|t|)$ \\
\hline$\alpha$ & 2.347 & 0.0886 & 26.506 & 0.0000 \\
\hline$\beta$ & -0.316 & 0.0087 & -36.338 & 0.0000 \\
\hline$\lambda$ & -0.439 & 0.0245 & -17.943 & 0.0000 \\
\hline$\delta$ & -0.0595 & 0.0111 & -5.374 & 0.0000 \\
\hline \multicolumn{2}{|c|}{ Residual Std. Fehler } & \multicolumn{3}{|c|}{0.0607 bei 452 Freiheitsgrader } \\
\hline \multicolumn{2}{|c|}{ Multiples Bestimmtheitsmaß } & \multicolumn{3}{|r|}{0.828} \\
\hline \multicolumn{2}{|r|}{ F-Statistik } & \multicolumn{3}{|c|}{725.2 bei 3 und 452 Freiheitsgraden, $p$-Wert $=0$} \\
\hline
\end{tabular}

Tab. 3: Koeffizienten und statistische Kenngrößen zur Schätzung des Koeffizienten "mittleres Kronenverhältnis " einer Baumart im Bestand (Formel (9)) mit Hilfe des Modells (10) für die Baumart Fichte.

(10) wurde zu (11) vereinfacht:

$\overline{K V}=\alpha+\beta * \ln (H 100)+\lambda * \overline{H D}$

mit:

\begin{tabular}{|c|c|c|c|c|}
\hline Koeffizienten & Wert & Std. Fehler & $\mathrm{t}$-Wert & $\operatorname{Pr}(>|t|)$ \\
\hline$\alpha$ & 1.903 & 0.0326 & 58.353 & 0.0000 \\
\hline$\beta$ & -0.334 & 0.0083 & -40.002 & 0.0000 \\
\hline$\lambda$ & -0.481 & 0.0239 & -20.126 & 0.0000 \\
\hline \multicolumn{2}{|c|}{ Residual Std. Fehler } & \multicolumn{3}{|c|}{0.0625 bei 453 Freiheitsgraden } \\
\hline \multicolumn{2}{|c|}{ Multiples Bestimmtheitsmaß } & \multicolumn{3}{|r|}{0.817} \\
\hline \multicolumn{2}{|r|}{ F-Statistik } & \multicolumn{3}{|c|}{1011 bei 2 und 453 Freiheitsgraden, $p$-Wert $=0$} \\
\hline
\end{tabular}

Tab. 4: Koeffizienten und statistische Kenngrößen zur Schätzung des Koeffizienten "mittleres Kronenverhältnis" einer Baumart im Bestand (Formel (9)) mit Hilfe des Modells (11) für die Baumart Fichte.

Ein wesentlicher Vorteil der Vereinfachung sind die geringeren Anforderungen an die Eingangsvariablen, bei annähernd gleichbleibender Schätzgenauigkeit. So lassen sich die Spitzenhöhe und der mittlere H/D-Wert im Rahmen eines Waldbeganges schätzen, während die Kronenschirmflächenberechnung in den meisten Anwendungsfällen umfangreiche Datenergänzungsroutinen voraussetzt. Der Vorteil der Verwendung der Kronenschirmfläche ist die Integration einer unabhängigen Variablen, die zumindest teilweise die Beschreibung der Eingriffsstärke von Durchforstungen erlaubt. Auf die Problematik, daß kurzfristig wirksame Veränderungen der Dichte prinzipiell besser in dynamischen als in statischen Modellen integriert werden können, wurde in Kapitel 2.3 hingewiesen.

Abb. 19 zeigt, daß der Koeffizient "mittleres Kronenverhältnis" gegenüber dem Koeffizienten "Ordinatenabschnitt" eine deutlich geringere Streuung aufweist, was sich in der höhe- 
ren Schätzgenauigkeit bei der Verallgemeinerung des Modells (9) gegenüber der des Modells (8) auswirkt.

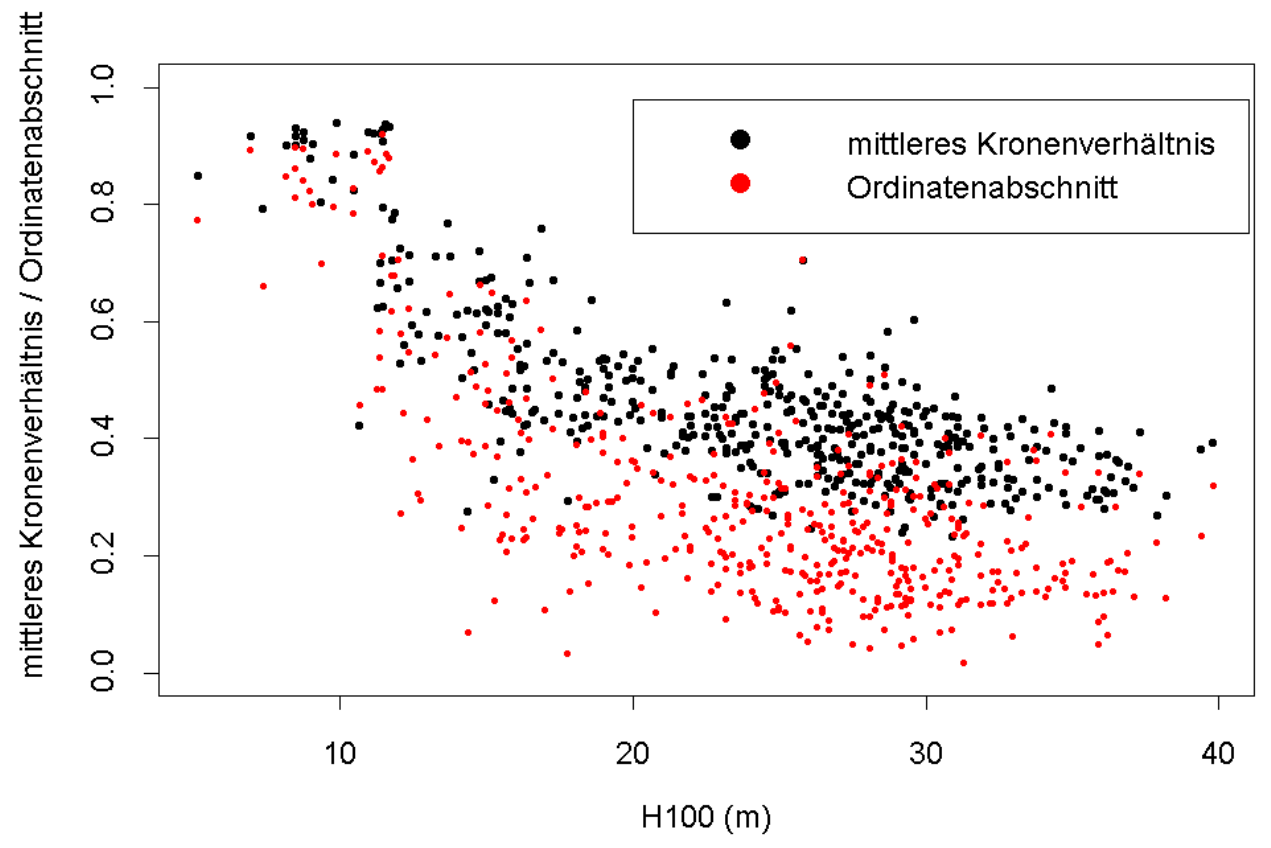

Abb. 19: Vergleich der Koeffizienten "Ordinatenabschnitt" (Formel (8)) und "mittleres Kronenverhältnis" (Formel (9)) der einzelbestandsweisen Anpassung an 456 Fichtenbestände bzw. Fichtenanteile in Fichtenmischbeständen.

Die Struktur des Steigungskoeffizienten des Modells (9) bei einzelbestandsweiser Anpassung an die 456 Bestände ist in Abb. 20 dargestellt.

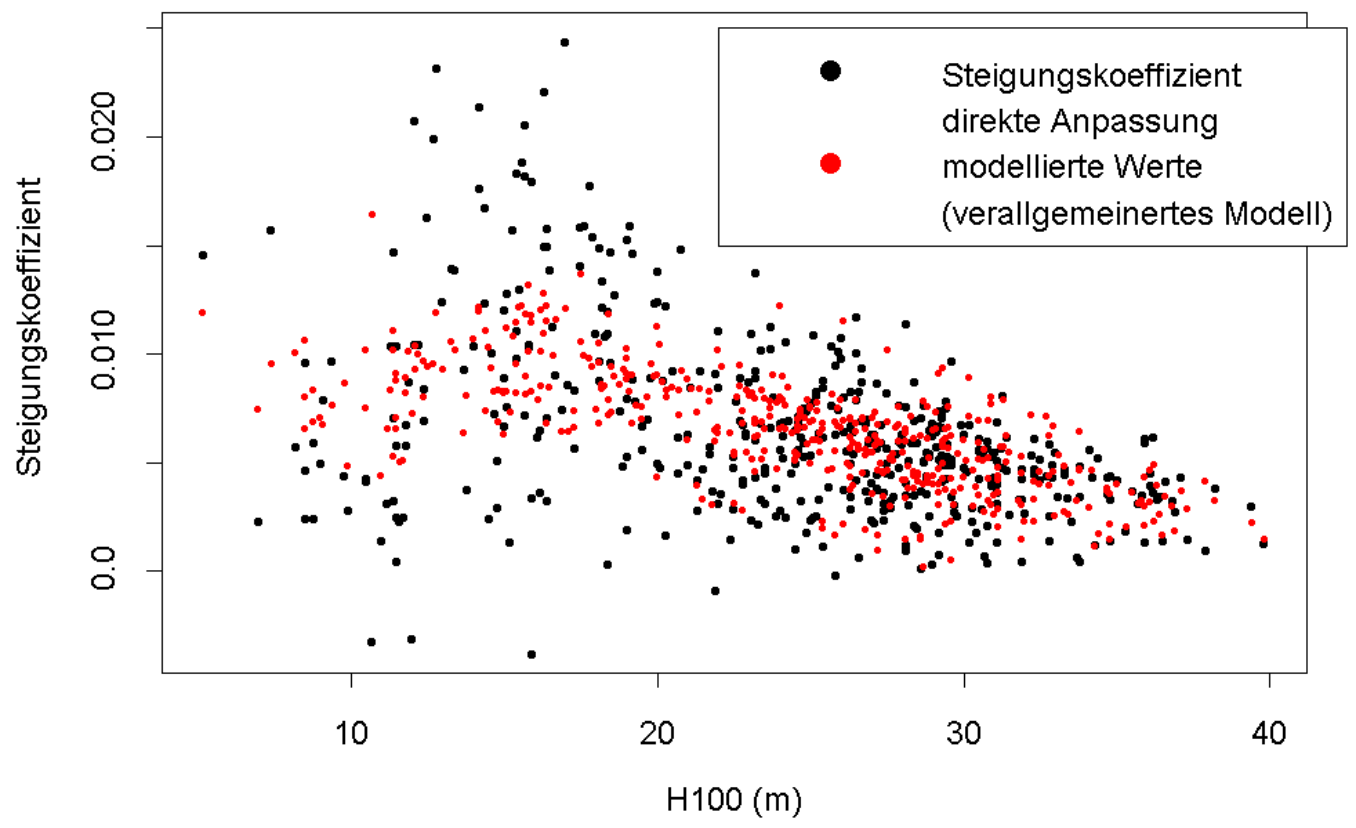

Abb. 20: Steigungskoeffizient für 456 einzelbestandsweise Modellanpassungen (Formel (9)) und die Schätzung der Koeffizienten mit Hilfe der unabhängigen Variablen Spitzenhöhe und mittlerer H/D-Wert der Bäume einer Baumart im Bestand (modellierte Werte; Formel (12)). Verwendet wurden Fichtenreinbestände und der Fichtenanteil aus Fichtenmischbeständen. 
Zur Schätzung des Steigungskoeffizienten für die Bäume einer Baumart im Bestand wurde als Modelltyp ein lineares multiples Regressionsmodell verwendet (Formel (12)).

Steigungskoeffizient $=\alpha * H 100+\beta * \overline{H D}$

mit:

\begin{tabular}{|c|c|c|c|c|}
\hline Koeffizienten & Wert & Std. Fehler & $\mathrm{t}$-Wert & $\operatorname{Pr}(>|t|)$ \\
\hline$\alpha$ & -0.000248 & 0.0000 & -12.943 & 0.0000 \\
\hline$\beta$ & 0.0155 & 0.0005 & 26.307 & 0.0000 \\
\hline \multicolumn{2}{|c|}{ Residual Std. Fehler } & \multicolumn{3}{|c|}{0.00334 bei 454 Freiheitsgraden } \\
\hline \multicolumn{2}{|c|}{ Multiples Bestimmtheitsmaß } & \multicolumn{3}{|r|}{0.8206} \\
\hline \multicolumn{2}{|r|}{ F-Statistik } & \multicolumn{3}{|c|}{1039 bei 2 und 454 Freiheitsgraden, $p$-Wert $=0$} \\
\hline
\end{tabular}

Tab. 5: Koeffizienten und statistische Kenngrößen zur Schätzung des Steigungskoeffizienten (Formel (9)) mit Hilfe des Modells (12) für die Baumart Fichte.

Bei der Schätzung des mittleren Kronenverhältnisses wird bei der Baumart Kiefer nur das vereinfachte Modell (Formel (11)) angegeben, da die Kronenschirmfläche keinen signifikanten Einfluß zum 5\% Niveau auf das mittlere Kronenverhältnis hat (Kapitel 9.1). Bei der Baumart Europäische Lärche wurde die Schätzung des mittleren Kronenverhältnisses deutlich verbessert, indem anstelle der Spitzenhöhe das Bestandesalter verwendet wurde. Für die Baumarten Buche und Eiche konnten keine signifikanten Zusammenhänge zwischen den Koeffizienten des Modells innerhalb von Beständen und Bestandesvariablen ermittelt werden.

\section{Validierung und Bewertung des hierarchischen Kronenansatzmodells:}

In Abb. 21 ist der Einfluß der Spitzenhöhe auf das Modell zur Schätzung des Kronenverhältnisses in Beständen bei konstanten Werten (Mittelwerte über alle Bestände) für den mittleren H/D-Wert und die Kronenschirmfläche bei Anwendung der Gleichungen (10) und (12) für die Baumart Fichte dargestellt. Es wird deutlich, daß die Spitzenhöhe als Variable zur Erfassung des bestandesübergreifenden Dimensionstrends (Kapitel 2.3, Fußnote 4) eine herausragende Bedeutung bei der Schätzung des Kronenverhältnisses hat. Dabei beeinflußt die Spitzenhöhe nicht nur das mittlere Kronenverhältnis, sondern auch die Steigung des Modells innerhalb von Beständen. Durch den hierarchischen Aufbau des Modells ist zudem gewährleistet, daß die Einzelbaumvariable BHD einen positiven Einfluß auf das Kronenverhältnis hat. 


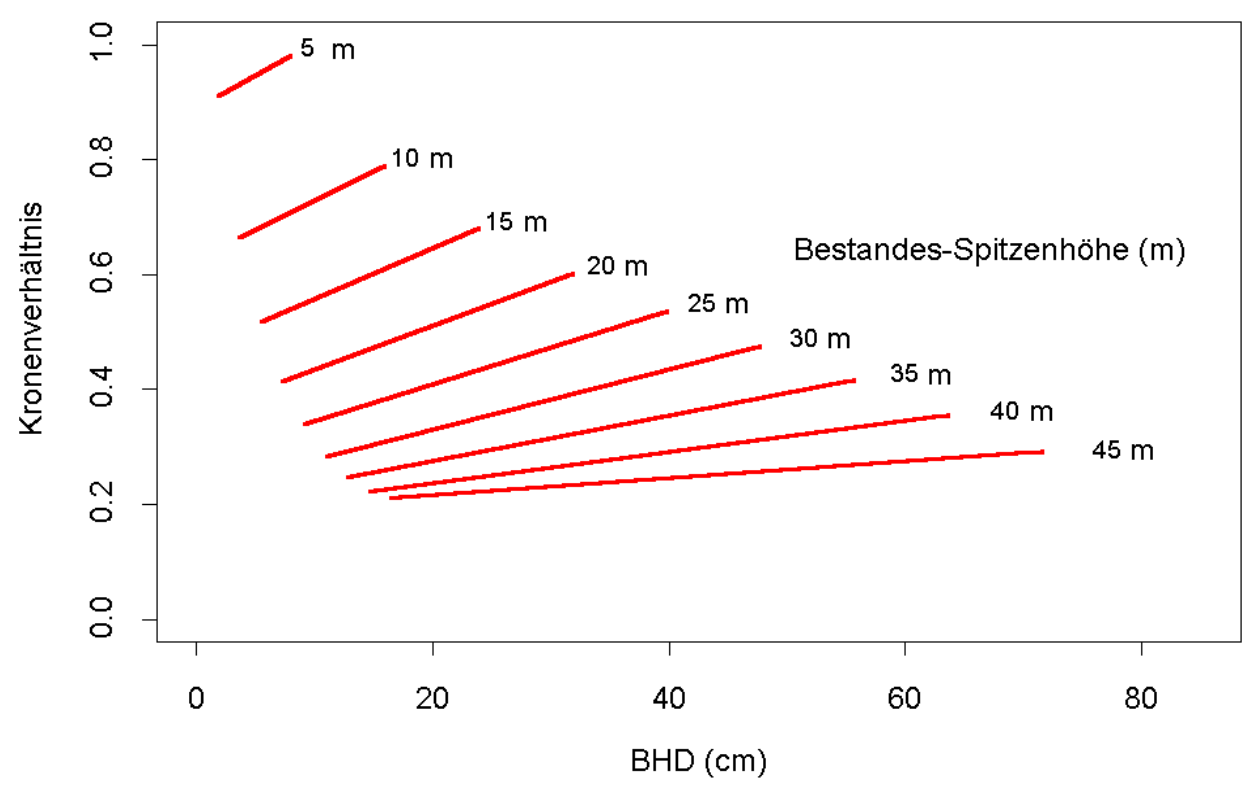

Abb. 21: Einfluß der Spitzenhöhe $\left(H_{100}\right)$ auf Lage und Steigung des Kronenverhältnismodells innerhalb von Beständen. Die weiteren unabhängigen Variablen mittlerer H/D-Wert und Kronenschirmfläche sind konstant auf die Mittelwerte der 456 verwendeten Fichtenbestände gesetzt $\left(0.82 \mathrm{bzw} .8055 \mathrm{~m}^{2} / \mathrm{ha}\right)$. Die Durchmesserwerte als Eingangswerte für das Kronenverhältnismodell wurden jeweils im Intervall 30-130\% des $D_{100}$-Wertes erzeugt. Die $D_{100}$-Werte wurden über die variierenden $H_{100}$-Werte und den konstanten mittleren H/D-Wert berechnet.

In Abb. 22 ist der Einfluß des mittleren H/D-Wertes der Bäume einer Baumart im Bestand auf die Lage und Steigung des Kronenverhältnismodells innerhalb von Beständen dargestellt.

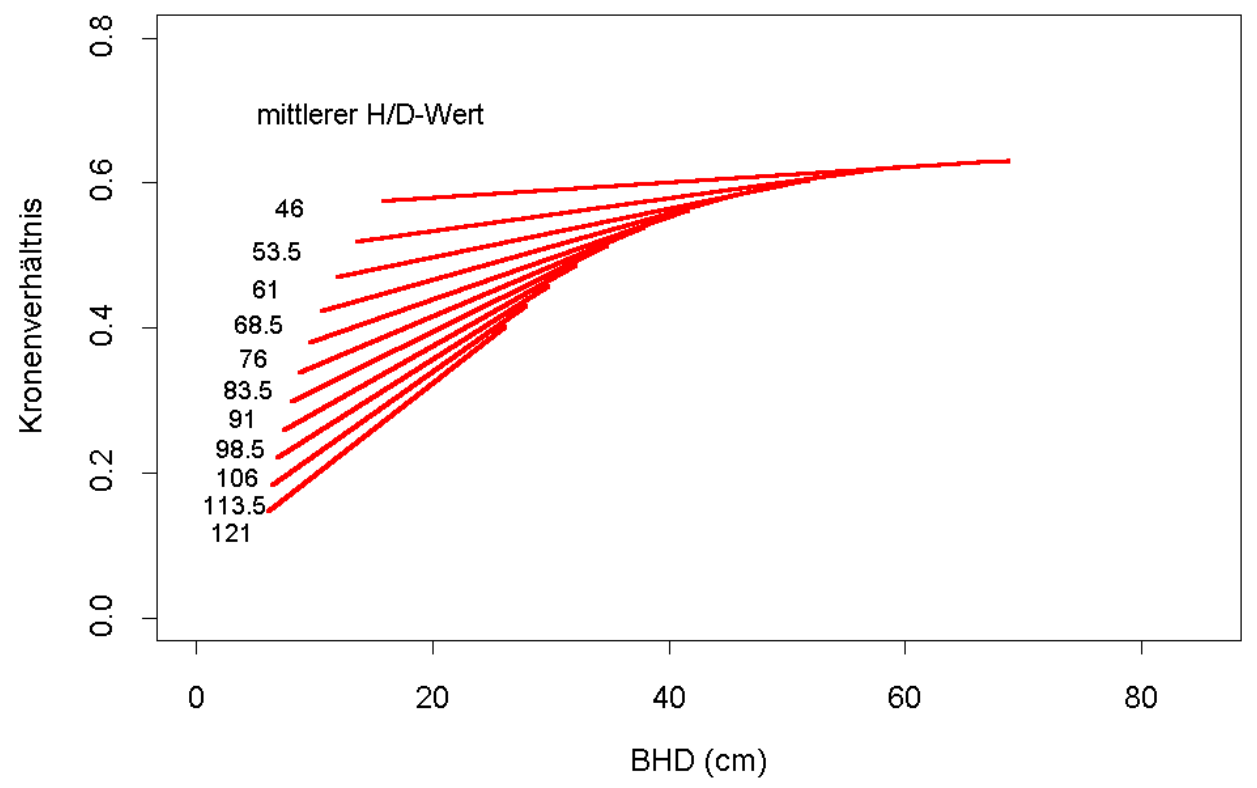

Abb. 22: Einfluß des mittleren H/D-Wertes im Bestand auf Lage und Steigung des Kronenverhältnismodells innerhalb von Beständen. Die weiteren unabhängigen Variablen $H_{100}$ und Kronenschirmfläche sind konstant auf die Mittelwerte der 456 verwendeten Fichtenbestände gesetzt (24.3 m bzw. $\left.8055 \mathrm{~m}^{2} / \mathrm{ha}\right)$. Die Durchmesserwerte als Eingangswerte für das Kronenverhältnismodell wurden jeweils im Intervall 30-130\% des $D_{100^{-}}$ Wertes erzeugt. Die $D_{100}$-Werte wurden über den konstanten $H_{100}$-Wert und die variierenden mittleren $H / D$-Werte berechnet. 
In Tab. 6 sind die Standardfehler für die Schätzung des Kronenansatzes und des Kronenverhältnisses angegeben. Das hierarchische Modell (hierarchisches Modell I; Formeln (10) u. (12)) weist deutlich geringere Standardfehler als die eingangs beschriebenen Kronenansatzmodelle in Bwin, Silva, CEP und Forest (modifiziert) auf. Als weiterer Vorteil kann genannt werden, daß das hierarchische Modell in der jetzigen Form als Einzelbaumvariablen nur den BHD benötigt, wenn anstelle der Formel (10) mit minimalen Genauigkeitsverlusten Formel (11) verwendet wird. Die unabhängigen Variablen Spitzenhöhe und mittlerer H/D-Wert in den Formeln (11) und (12) lassen sich mit wenigen orientierenden Messungen im Rahmen eines Waldbeganges schätzen. Der Vorteil der Verwendung des hierarchischen Modells I nach Formel (9) gegenüber dem hierarchischen Modell II nach Formel (8) wird beim Vergleich der mittleren RMSE deutlich.

\begin{tabular}{|l|c|c|c|c|c|}
\hline & Bwin & Silva & Bwinpro & EKVK & EKVK (Kor.) \\
\hline RMSE $(\mathrm{m})$ & $\mathbf{2 . 6 7 7 4 7 0 0}$ & $\mathbf{2 . 2 2 6 9 2 0}$ & 1.81704000 & 1.39911000 & 1.3896320 \\
\hline RMSE & $\mathbf{0 . 2 1 3 9 4 7 7}$ & $\mathbf{0 . 1 3 7 1 8 9}$ & 0.09263013 & 0.07304272 & 0.0672913 \\
\hline
\end{tabular}

\begin{tabular}{|l|c|c|c|c|c|}
\hline & $\begin{array}{c}\text { Dyn. } \\
\text { EKVK (Kor.) }\end{array}$ & $\begin{array}{c}\text { Dyn. } \\
\text { EKVK (Kor.) }<=15\end{array}$ & $\begin{array}{c}\text { Dyn. } \\
\text { EKVK (Kor.) }<=5\end{array}$ & $\begin{array}{c}\text { hierarchisches } \\
\text { Modell I }\end{array}$ & $\begin{array}{c}\text { hierarchisches } \\
\text { Modell II }\end{array}$ \\
\hline RMSE $(\mathrm{m})$ & 1.87040200 & 1.67173700 & 1.59195600 & $\mathbf{1 . 8 0 5 9 8 7 0 0}$ & $\mathbf{2 . 1 9 6 5 6 7 0}$ \\
\hline RMSE & 0.08419242 & 0.07922634 & 0.07050255 & $\mathbf{0 . 0 9 0 3 9 3 5 8}$ & $\mathbf{0 . 1 0 2 3 5 3 4}$ \\
\hline
\end{tabular}

\begin{tabular}{|l|c|r|r|}
\hline & $\begin{array}{c}\text { Direkte } \\
\text { Anpassung } \\
\text { an die Daten }\end{array}$ & $\begin{array}{c}\text { Forest } \\
\text { modifiziert }\end{array}$ & \multicolumn{1}{c|}{ CEP } \\
\hline RMSE $(\mathrm{m})$ & 1.31423400 & $\mathbf{2 . 0 4 6 7 5}$ & $\mathbf{2 . 3 1 2 8 2}$ \\
\hline RMSE & 0.06043807 & $\mathbf{0 . 1 1 4 4 1 5}$ & $\mathbf{0 . 1 5 7 9 1 2 8}$ \\
\hline
\end{tabular}

Tab. 6: Standardfehler der Kronenansatzschätzung (m) und der Kronenverhältnisschätzung für verschiedene Kronenansatzmodelle für die Baumart Fichte. Fett markiert sind die bis hier im Text beschriebenen Modelle. Das neu entwickelte hierarchische Modell (Formel (9)) bzw. Formeln (10) u. (12)) wird hier hierarchisches Modell I genannt. Verwendet man ein hierarchisches Modell, daß auf Formel (8) basiert (hierarchisches Modell II), so verschlechtern sich die Schätzungen gegenüber dem hierarchischen Modell I.

Demgegenüber benötigen die übrigen Modelle entweder direkt oder indirekt aufgrund der Verwendung von Einzelbaum-H/D-Werten Höhen, die häufig über Einheits- oder Bestandeshöhenkurven ergänzt werden müssen. Die dabei vernachlässigte Bestandeshöhenstreuung führt dazu, daß die Baumhöhe bzw. der H/D-Wert in funktionalem Zusammenhang zum BHD stehen und wenig zusätzliches Erklärungspotential aufweisen. Noch deutlicher wird der Vorteil des hierarchischen Modells, wenn anstelle des mittleren RMSE die Residuen der Kronenansatzschätzung über der Variablen $\mathrm{H}_{100}$ dargestellt werden (Abb. 23). 


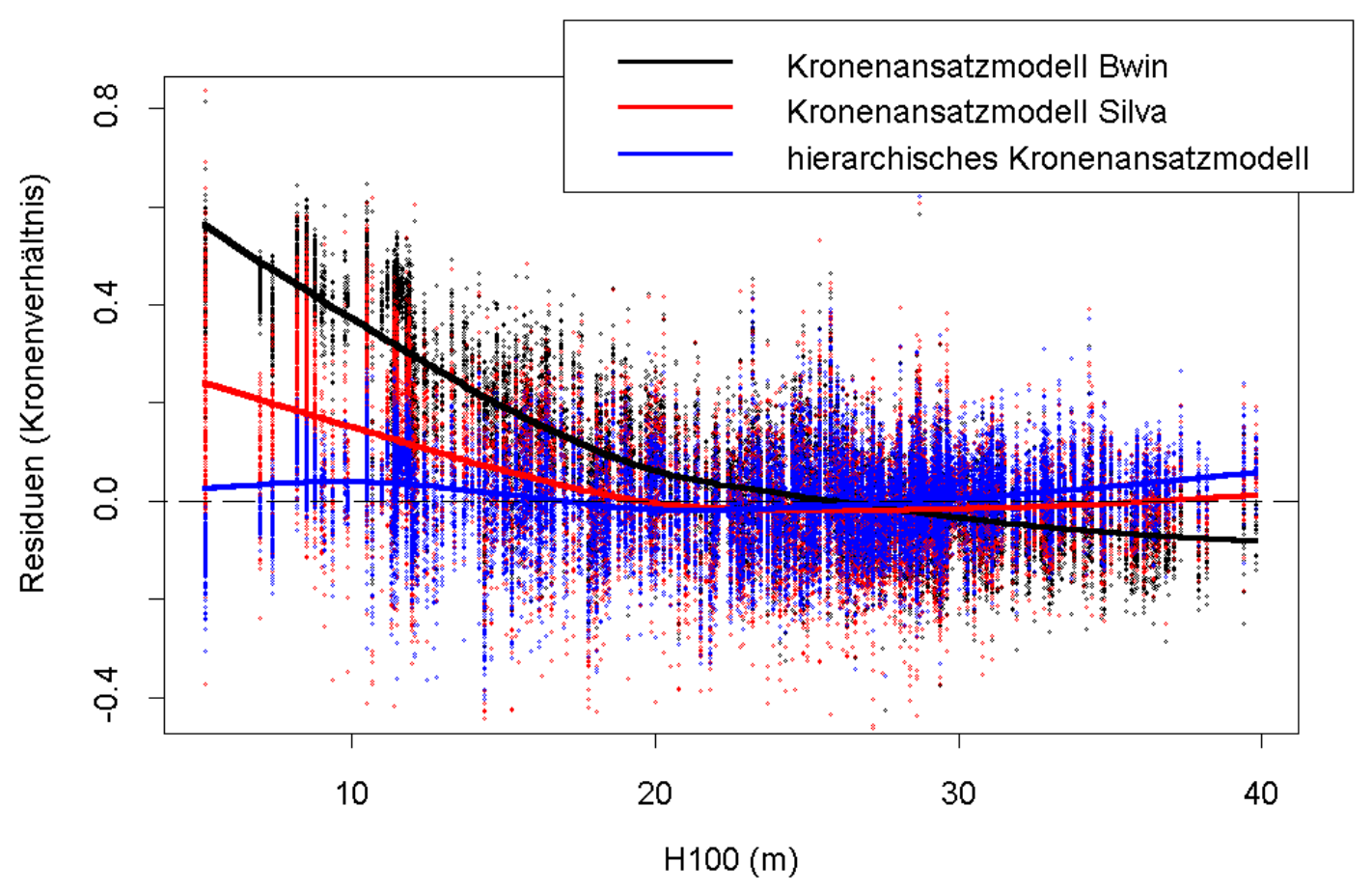

Abb. 23: Residuen der Kronenverhältnisschätzung mit Hilfe der Kronenansatzmodelle in Bwin, in Silva und mit dem neu entwickelten hierarchischen Modell für die Baumart Fichte. Die Linien sind die zugehörigen Funktionswerte, die mittels eines GleitendenDurchschnitts-Modells geschätzt wurden (Kapitel 8.1).

In Abb. 23 sind die den einzelnen Modellen zugehörigen Residuen mit Hilfe eines Gleitenden-Durchschnitts-Modells ausgeglichen worden, um systematische Schätzfehler in einzelnen Bereichen von $\mathrm{H}_{100}$-Werten $\mathrm{zu}$ verdeutlichen. Die bedingten mittleren Residuen des hierarchischen Modells weisen kaum Abweichungen von Null auf. Demgegenüber weisen die Kronenansatzmodelle in Bwin und in Silva im Bereich von Spitzenhöhen von unter ca. $22 \mathrm{~m}$ systematische Unterschätzungen auf. Die systematischen Fehler steigen mit sinkender Spitzenhöhe stark an, wobei das Kronenansatzmodell in Bwin deutlich höhere Abweichungen aufweist als das Kronenansatzmodell in Silva. Als Ursache ist zu vermuten, daß mit der Verwendung der Variablen BHD zusätzlich zum H/D-Wert ein Teil des starken Dimensionstrends in der Kronenverhältnisentwicklung erfaßt wird. Abb. 24 verdeutlicht anhand von quadrierten Residuen der Kronenverhältnisschätzung, in welchen Bereichen große Gesamtfehler auftreten. Wie zu erwarten war, treten die höchsten Gesamtfehler der Kronenansatzmodelle in Bwin und in Silva im Bereich niedriger Spitzenhöhen auf, die auch die höchsten systematischen Fehler aufwiesen. 


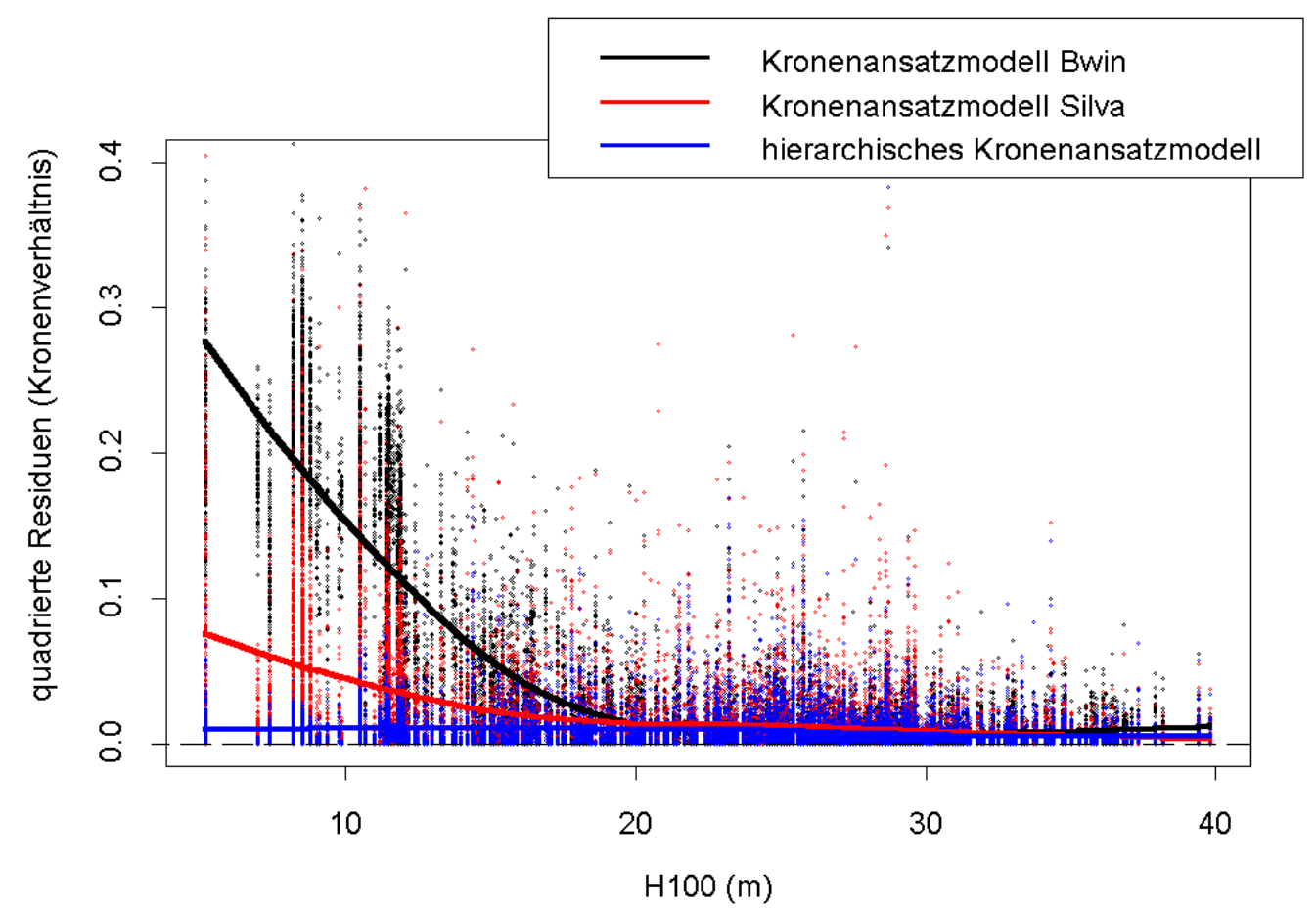

Abb. 24: Quadrierte Residuen der Kronenverhältnisschätzung mit Hilfe der Kronenansatzmodelle in Bwin, in Silva und mit dem neu entwickelten hierarchischen Modell für die Baumart Fichte. Die Linien sind die zugehörigen Funktionswerte, die mittels eines Gleitenden-Durchschnitts-Modells geschätzt wurden (Kapitel 8.1).

Die Datenstruktur, auf der die Parametrisierung der Modelle basiert, kann zum jetzigen Zeitpunkt noch dadurch charakterisiert werden, daß die Kronenansatzmessungen zum überwiegenden Teil aus mehr oder weniger einschichtigen Rein- und Mischbeständen stammen. Durch die Zunahme strukturreicher Mischbestände wird sich in Zukunft auch die Datenstruktur ändern und die Integration weiterer Einzelbaumvariablen in das bestandesindividuelle Kronenverhältnismodell könnte erforderlich werden.

Wie in den Voruntersuchungen deutlich wurde, war der H/D-Wert in nur 17\% der Bestände zusätzlich zum BHD signifikant (Abb. 16). Der Standardfehler über alle Bestände bei direkter Anpassung des Modells (8) wurde bei zusätzlicher Verwendung des H/D-Wertes nur unwesentlich verringert (Tab. 2). Bestandesstrukturen mit ähnlichen Dimensionen der Einzelbäume, in denen der H/D-Wert zusätzlich zum BHD einen signifikanten Einfluß hat, müßten eine sehr starke Bestandeshöhenstreuung aufgrund von Dichteunterschieden aufweisen. D. h., daß beispielsweise solitärähnliche Plenterwaldbäume mit Bäumen, die in gleichaltrigen Gruppen erwachsen sind, in einem Bestand gemeinsam auftreten müßten.

Die Berücksichtigung des H/D-Wertes innerhalb von Beständen erscheint nur in dem beschriebenen Ausnahmefall notwendig. Problematischer ist die Anwendung der beschriebenen Modellkonzeption auf Bestände mit einer Mischung stark unterschiedlicher Dimensionen, beispielsweise in einem Femel- oder Plenterwald. In diesen Beständen tritt der eingangs beschriebene allgemeine Dimensionstrend innerhalb eines Bestandes auf. 
In Abb. 25 ist die Anwendung des hierarchischen Modells auf einen Femelbestand dargestellt. Der Femelbestand ist aus zwei unterschiedlichen gleichaltrigen Beständen erzeugt worden, weil ein derartiger Bestand innerhalb der 456 Bestände nicht zur Verfügung stand. Während die Kronenverhältnisse des Oberstandes relativ gut beschrieben werden, ist die Schätzung für die Bäume des Femelkegels vollkommen ungeeignet (Abb. 25, hierarchisches Modell). Als Grund ist zu nennen, daß der Unterstand in keiner Beziehung zu den Variablen Spitzenhöhe und mittlerer H/D-Wert des Hauptbestandes steht. Eine direkte Anpassung an die Bestandesdaten mit Hilfe der unabhängigen Variablen BHD und Höhe durch multiple Regression liefert eine sehr viel bessere Schätzung (Abb. 25, direkte Anpassung: BHD und Höhe). Im Gegensatz zur Datenstruktur der 456 Fichtenbestände verbessert sich im künstlichen Femelbestand die Schätzung stark, wenn die Einzelbaumhöhe zusätzlich zum BHD als unabhängige Variable verwendet wird. Als Problem der Integration einer weiteren Einzelbaumvariablen in das bestandesindividuelle Modell ist zu nennen, daß die Koeffizienten des Modells nicht mehr unabhängig voneinander wären. Damit ist zu vermuten, daß eine effektive Verallgemeinerung des bestandesindividuellen Modells bei Verwendung von zwei unabhängigen Einzelbaumvariablen nur eingeschränkt möglich sein wird bzw. weitere Verfahren zur Gewährleistung der statistischen Unabhängigkeit der Koeffizienten, wie eine Umformung in Hauptkomponenten, getestet werden müßten (ZUCCHINI, 2001: mündliche Mitteilung).

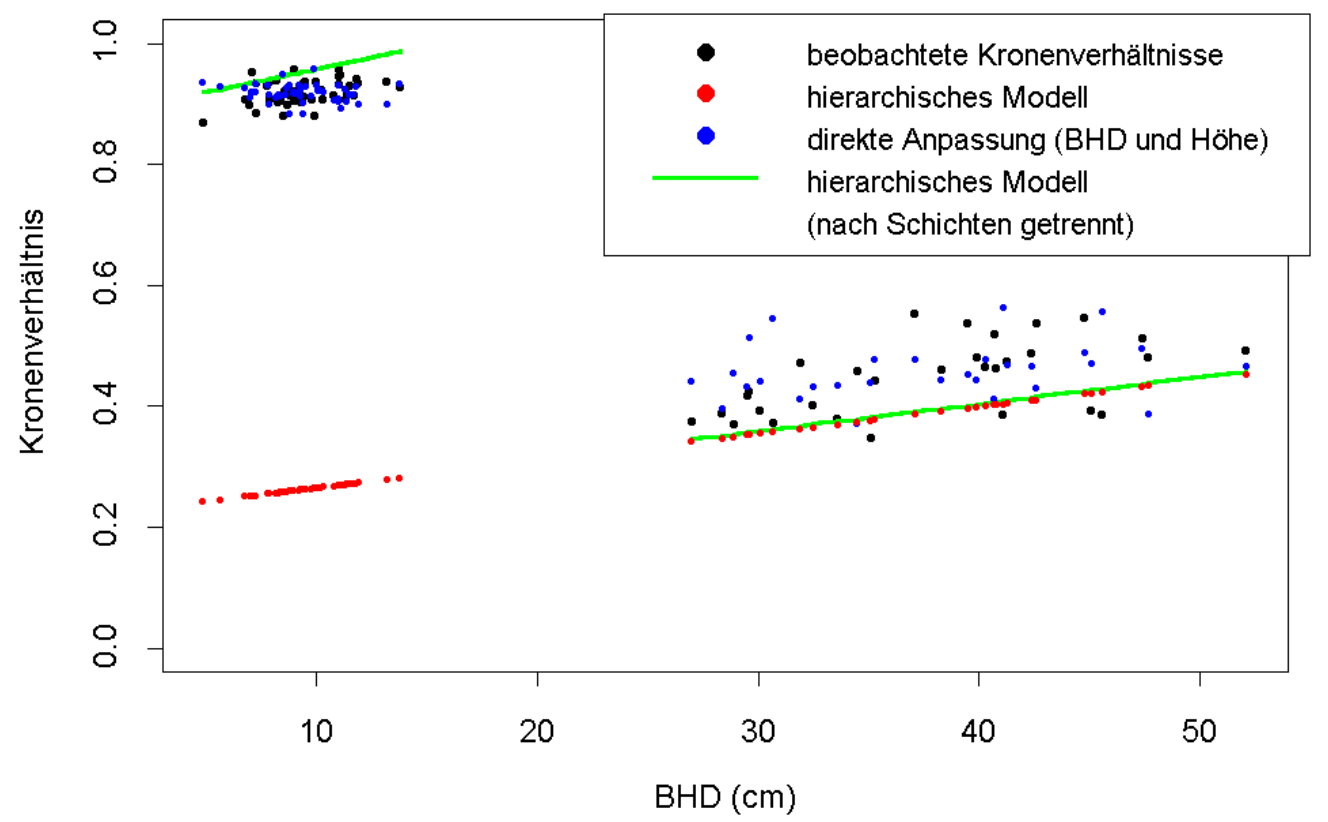

Abb. 25: Anpassungen verschiedener im Text erläuterter Kronenansatzmodelle an die Daten eines aus zwei realen einschichtigen Beständen erzeugten Fichtenfemelbestandes.

Das entwickelte hierarchische Modell kann allerdings auch in Femelwäldern eingesetzt werden, wenn die Spitzenhöhe $\mathrm{H}_{100}$ getrennt für Ober- und Unterschicht erfaßt, der mittlere H/DWert getrennt berechnet und das Modell quasi für zwei getrennte Bestände angepaßt wird (Abb. 25, hierarchisches Modell: nach Schichten getrennt). 
Im Gegensatz zu den Einschränkungen in strukturierten Beständen ist ein Einsatz in mehr oder weniger einschichtigen Mischbeständen unproblematisch, da die unabhängigen Variablen Spitzenhöhe und mittlerer H/D-Wert baumartenweise berechnet werden. Bei den auch im Datenmaterial vorhandenen Mischbeständen wurde auf diese Weise verfahren. Es muß darauf hingewiesen werden, daß trotz der teilweise erheblichen Verbesserungen der Schätzgenauigkeit gegenüber bekannten statischen Modellen eine direkte Sensitivität gegenüber forstlichen Eingriffen nicht besteht. Versuche, ein dynamisches Kronenansatzmodell zu entwickeln, um bisher unberücksichtigte Durchforstungseffekte abzubilden, wiesen nur sehr geringe Bestimmtheitsmaße auf. Als Ursachen können die große Datenheterogenität und die problematische Messung der Kronenansatzhöhe bzw. ihrer Veränderungsrate vermutet werden.

Als Vorteile des Modells gegenüber bisher verwendeten statischen Modellen sind zusammenfassend die höhere Schätzgenauigkeit bei gleichzeitig geringerer Anforderung an die Eingangsdaten zu nennen. Weiterhin wird durch den hierarchischen Aufbau gewährleistet, daß die Variable BHD, die für den Großteil der untersuchten Bestände zur Beschreibung des Kronenverhältnisses innerhalb von Beständen ausreicht, die Wirkungsrichtung (Vorzeichen) aufweist, die bei einer bestandesweisen Parametrisierung zu beobachten ist.

\subsubsection{Einfaches statisches Kronenansatzmodell}

Zusätzlich zum beschriebenen hierarchischen Modell wird untersucht, inwieweit eine Erweiterung des Modellansatzes von PRETZSCH und KAHN (1998) um die unabhängige Variable Spitzenhöhe ebenfalls zu einer Verbesserung der Schätzgenauigkeit führt. Als unabhängige Variablen werden die Einzelbaumvariablen BHD und H/D-Wert und die Bestandesvariable Spitzenhöhe verwendet (Formel (13)). Im Vergleich mit dem hierarchischen Modellansatz kann daher untersucht werden, wie sich die Verwendung der unabhängigen Variablen Einzelbaum-H/D-Wert im Gegensatz zum mittleren H/D-Wert (Formeln (10) u. (12)) auf die Schätzgenauigkeit auswirkt. Gleichzeitig steigt dadurch aber auch der Anspruch an die Datenqualität, da für die Berechnung von Einzelbaum-H/D-Werten in den meisten Anwendungsfällen Höhenergänzungsalgorithmen eingesetzt werden müssen.

Wie in Kapitel 3.2.1 dargestellt wurde, ist der Einfluß des Einzelbaum-H/D-Wertes bei einer bestandesweisen Parametrisierung von Kronenansatzmodellen zusätzlich zum BHD nur in einem Bruchteil der Bestände signifikant. Das einfache statische Modell kann deshalb besser als der hierarchische Modellansatz dazu eingesetzt werden, den Einfluß des EinzelbaumH/D-Wertes auf das Kronenverhältnis von Bäumen mit ähnlichem BHD und ähnlicher zugehöriger Spitzenhöhe zu untersuchen, die nicht innerhalb des gleichen Bestandes vorkommen. Zusätzlich erschien ein hierarchischer Modellansatz aufgrund der erläuterten Koeffizientenabhängigkeiten (Kapitel 3.2.1, Validierung und Bewertung), die auftreten, wenn mehrere unabhängige Variablen im bestandesindividuellen Modell verwendet werden, als weniger geeig- 
net zur Überprüfung des Einflusses des Einzelbaum-H/D-Wertes. Das Modell wird als einfach statisch bezeichnet, da es im Gegensatz zum zweistufigen Aufbau des hierarchischen Modells, keine Unterscheidung in eine Einzelbaum- und Bestandesebene aufweist.

Im Gegensatz zum Kronenansatzmodell in Silva (Formel (2)) wird der Betrag des linearen Ausdrucks im Exponenten gebildet, um die Schätzung von unplausiblen negativen Kronenansatzhöhen zu verhindern. Die im Kronenansatzmodell in Bwin (Formel (1)) angewendete Quadrierung des Exponenten führte zu etwas höheren Schätzfehlern gegenüber der Verwendung des Modells (13). Durch die Bildung des Betrages des Exponenten kehrt sich die Wirkungsrichtung der unabhängigen Variablen bei Überschreitung des Wertes Null für den Exponenten um. Diese für die Interpretation nachteilige Eigenschaft wurde jedoch toleriert, um unplausible Schätzungen zu verhindern.

in Bwinpro:

$$
K A=H^{*}\left(1-e^{-a b s\left(\alpha+\beta^{*} H / D+\gamma^{*} D+\delta^{*} \ln (H 100)\right)}\right)
$$

wobei:

$K A=$ Kronenansatzhöhe $(\mathrm{m})$;

$H=$ Baumhöhe $(\mathrm{m})$;

$D=$ Brusthöhendurchmesser $(\mathrm{cm})$;

$\ln \left(H_{100}\right)=$ natürlicher Logarithmus der Spitzenhöhe $(\mathrm{m})$.

mit:

\begin{tabular}{|c|c|c|c|}
\hline Koeffizienten & Wert & Std. Fehler & t-Wert \\
\hline$\alpha$ & 2.042 & 0.0135 & 151.737 \\
\hline$\beta$ & -0.334 & 0.0163 & -20.467 \\
\hline$\gamma$ & 0.00906 & 0.000262 & 34.571 \\
\hline$\delta$ & -0.900 & 0.00802 & -112.221 \\
\hline \multicolumn{2}{|c|}{ Residual Std. Fehler (m) } & \multicolumn{2}{|c|}{1.817 bei 19056 Freiheitsgraden } \\
\hline
\end{tabular}

Tab. 7: Koeffizienten und statistische Kenngrößen zur Kronenansatzschätzung mit Hilfe des Modells (13) für die Baumart Fichte.

Die Ergebnisse der Kronenansatz- und Kronenverhältnisschätzungen für die Baumarten Fichte, Douglasie, Kiefer und Europäische Lärche sind in Kapitel 9.1 unter der Bezeichnung Bwinpro angegeben, da dieser Modellansatz im Wachstumssimulator Bwinpro (NAGEL ET. AL., 2001) als Standardmodell verwendet wird. In Kapitel 9.1 sind weiterhin die Koeffizienten für die Baumarten Douglasie, Kiefer und Europäische Lärche aufgeführt sowie geringfügige Abweichungen vom Ausgangsmodell der Fichte (Formel (13)) angegeben.

\section{Validierung und Bewertung des einfachen statischen Kronenansatzmodells:}

Es fällt auf, daß das Modell (13) fast exakt die Fehlerwerte aufweist, wie das hierarchische Modell. Die Verwendung der Spitzenhöhe hat somit in diesem Modellansatz zu etwa der gleichen Verbesserung gegenüber den Vergleichsmodellen geführt. Die Verwendung von Einzel- 
baum-H/D-Werten gegenüber der Verwendung des mittleren H/D-Wertes der Baumart im Bestand verbessert die Schätzung nicht. In Tab. 7 sind die Koeffizienten und die zugehörigen statistischen Kenngrößen für die Baumart Fichte angegeben. Die Wirkungsrichtung der unabhängigen Variablen kann aufgrund der Betragsbildung nur in Relation zueinander beschrieben werden. So wirken die Spitzenhöhe und der H/D-Wert in die gleiche Richtung, während der BHD entgegengesetzt wirkt. Solange der lineare Ausdruck im Exponenten vor der Betragsbildung positiv ist, hat eine steigende Spitzenhöhe ein sinkendes Kronenverhältnis zur Folge. Die Wirkungsrichtung des BHD ist entgegengesetzt. Damit ist eine biologisch plausible Wirkungsrichtung im Zusammenspiel der unabhängigen Variablen gegeben, die den Zusammenhängen in der Datenstruktur entspricht (Kapitel 3.2.1).

In Abb. 26 sind die Vorteile des neuen statischen Kronenansatzmodells (in Bwinpro) gegenüber den Kronenansatzmodellen in Bwin und in Silva in Form von Streudiagrammen der gemessenen über den geschätzten Kronenverhältnissen verdeutlicht. Die vierte Grafik zeigt das Ergebnis der Schätzung des in Kapitel 3.2.3 beschriebenen Einheitskronenansatzmodells $(E K V K($ Kor. $))$.
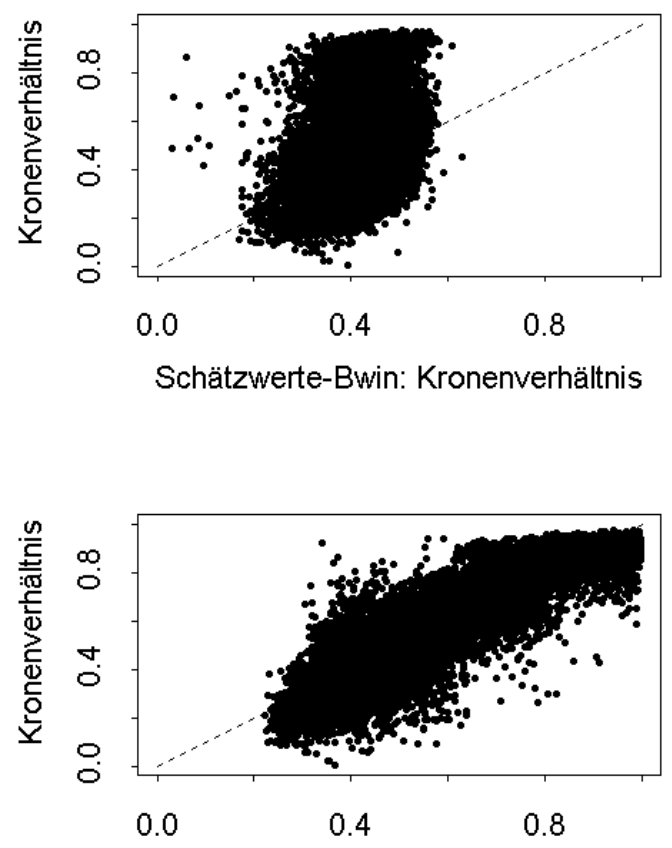

Schätzwerte-Bwinpro: Kronenverhältnis
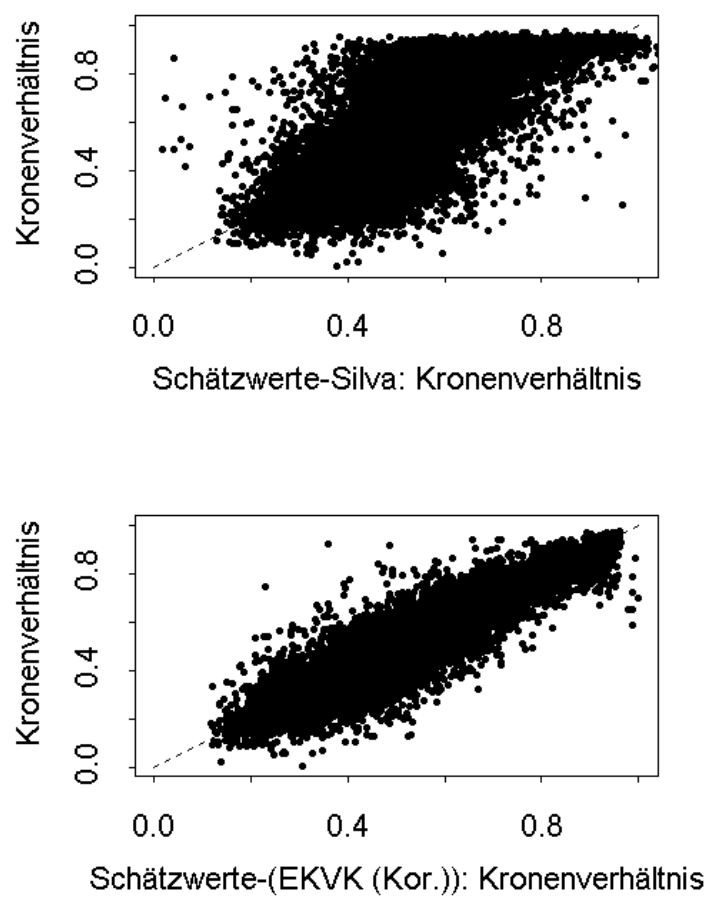

Abb. 26: Darstellung der gemessenen über den geschätzten Kronenverhältnissen der Schätzung der Kronenansatzmodelle in Bwin, in Silva, des neuen statischen Modells (in Bwinpro) und des in Kapitel 3.2.3 beschriebenen Einheitskronenansatzmodells mit Korrektur durch eine Begrenzungsfunktion (EKVK (Kor.)) für die Baumart Fichte.

Die zugehörigen Abbildungen der übrigen Baumarten sind in Kapitel 9.2 aufgeführt. Während für die Buche in Übereinstimmung mit dem hierarchischen Modellansatz keine Verbesserung gegenüber den Vergleichsmodellen erreicht wurde (Kapitel 9.2), konnte die Schätzung für die Eiche leicht verbessert werden (Kapitel 9.2). 


\subsubsection{Einheitskronenansatzmodell}

Das vorgestellte hierarchische Modell verwendet lediglich den BHD, den mittleren H/D-Wert und die Spitzenhöhe der Baumart im Bestand als unabhängige Variablen, wenn Formel (10) zu Formel (11) vereinfacht wird. Für das einfache statische Kronenansatzmodell (13) erhöht sich die Anforderung an die Datenqualität, da für Einzelbaum-H/D-Werte im allgemeinen eine Ergänzung der Baumhöhen wenn möglich unter Berücksichtigung der bedingten Höhenstreuung notwendig ist (SCHMIDT U. GADOW, 1999; ALBERT, 2000). Im folgenden wird untersucht, inwieweit sich die Güte der Kronenansatzschätzung erhöht, wenn eine Schätzung für das Kronenverhältnis eines bestimmten Bestandesmittelstammes, in diesem Fall den Spitzenhöhenstamm $\left(K V_{H 100}\right)$ vorliegt. Diese Schätzung kann im Rahmen eines Waldbeganges mittels einiger Messungen an den Bäumen erfolgen, an denen für die Schätzung der Spitzenhöhe auch die Höhe gemessen wird. Die Variable $K V_{H 100}$ wird wie bei einer Einheitshöhenkurve zur Einhängung einer Funktion genutzt, die das Kronenverhältnis eines Baumes innerhalb des Bestandes in Abhängigkeit von der Differenz des BHD des Baumes vom BHD des $\mathrm{D}_{100^{-}}$ Stammes beschreibt (Formel (14)). Die Modellkonzeption orientiert sich an dem von SLOBODA ET AL. (1993, vgl. auch GAFFREY, 1996) entwickelten Einheitshöhenkurvenmodell. Neben dem Kronenverhältnis des $\mathrm{D}_{100}$-Stammes ist damit der BHD des $\mathrm{D}_{100}$-Stammes als unabhängige Variable notwendig, der jedoch auch im Rahmen des Waldbeganges oder aus der BHD-Verteilung des jeweiligen Simulationsbestandes geschätzt werden kann. Die Verwendung des Einzelbaum-H/D-Wertes als weitere unabhängige Variable bedeutet über die Verwendung des Kronenverhältnisses und des BHD des Spitzenhöhenstammes hinaus keine weitere Erhöhung der Anforderung an die Datenqualität gegebenüber dem einfachen statischen Modell (13). Das Modell wird ebenfalls im Waldwachstumssimulator Bwinpro verwendet und ersetzt das einfache statische Kronenansatzmodell (13) wenn die höheren Anforderungen an die Eingangsdaten erfüllt werden:

Bwinpro-EKVK:

$$
K A=H *\left(1-K V_{H 100}\right) * e^{-\left(\alpha+\beta * \ln (H 100)+\gamma^{*} H / D\right) *(1 / D-1 / D 100)}
$$

wobei:

$K A=$ Kronenansatzhöhe $(m)$;

$H \quad=$ Baumhöhe $(\mathrm{m})$;

$D=$ Brusthöhendurchmesser $(\mathrm{cm})$;

$\ln \left(H_{100}\right) \quad=$ natürlicher Logarithmus der Spitzenhöhe $(\mathrm{m})$;

$D_{100}=$ BHD des Spitzenhöhenstammes $(\mathrm{cm})$;

$K V_{H 100}=$ Kronenverhältnis des Bestandesspitzenhöhenstammes. 
für Formel (14) mit:

\begin{tabular}{|c|c|c|c|}
\hline Koeffizienten & Wert & Std. Fehler & t-Wert \\
\hline$\alpha$ & -3.401 & 0.717 & -4.747 \\
\hline$\beta$ & -3.431 & 0.200 & -17.196 \\
\hline$\gamma$ & 6.559 & 0.272 & 24.133 \\
\hline \multicolumn{2}{|c|}{ Residual Std. Fehler (m) } & \multicolumn{2}{|c|}{1.399 bei 19057 Freiheitsgraden } \\
\hline
\end{tabular}

Tab. 8: Koeffizienten und statistische Kenngrößen zur Kronenansatzschätzung mit Hilfe des Modells (14) für die Baumart Fichte.

Das Einheitskronenansatzmodell wurde am gleichen Datenmaterial wie die bereits beschriebenen Modelle parametrisiert. Zur Schätzung der Variablen $K V_{H 100}$ wurde der Durchmesser des Spitzenhöhenstammes in Formel (8) eingesetzt und $K V_{H 100}$ getrennt nach Parzellenaufnahmen geschätzt. In Tab. 8 sind die Koeffizienten und statistischen Kennziffern des Modells für die Baumart Fichte dargestellt.

\section{Validierung und Bewertung des Einheitskronenansatzmodells:}

Der Standardfehler des Einheitskronenansatzmodells ist in Tab. 6 unter EKVK für die Kronenansatzschätzung und das Kronenverhältnis angegeben. Durch die Orientierung des Modells am Kronenverhältnis des Spitzenhöhenstammes kann der Fehler gegenüber dem hierarchischen Modellansatz und dem einfachen statischen Modell (13) um ca. $40 \mathrm{~cm}$ bzw. 2\% gesenkt werden. Die hohe Genauigkeit des Modells kommt auch bei einem Vergleich mit der einzelbestandsweisen Anpassung von Modell (8) zum Ausdruck. Die Fehlerwerte für diese Anpassung sind in Tab. 6 unter "Direkte Anpassung an die Daten" aufgeführt und liegen nur knapp unterhalb der Werte für das Einheitskronenansatzmodell.

Das Einheitskronenansatzmodell schätzt bei extremen Kombinationen von unabhängigen Variablen Kronenansatzhöhen, die größer als die zugehörige Baumhöhe sind. Eine einfache Begrenzung durch die Betragsbildung im Exponenten ist nicht sinnvoll. Um unplausible Schätzungen zu verhindern, wurde daher eine sogenannte Begrenzungsfunktion parametrisiert: Dafür wurde in $1 \mathrm{~m}$ Höhenstufen das 99.5\%-Quantil der Kronenansatzhöhen berechnet. Anschließend wurden diese Werte über der Höhe ausgeglichen. Als Ausgleichsfunktion wurde eine modifizierte Verteilungsfunktion der 2-parametrigen Weibull-Verteilung verwendet (Kozak und Yang, 1978; Formel (15); Tab. 9).

KA.995-Quantil $=\alpha *\left(1-e^{-(H / \beta)^{\delta}}\right)$

wobei:

$$
\begin{array}{ll}
\text { KA.995-Quantil } & =\text { bedingte } 99.5 \% \text { Quantile der Kronenansatzhöhe (m); } \\
H & =\text { Baumhöhe }(\mathrm{m}) .
\end{array}
$$


für Formel (15) mit:

\begin{tabular}{|c|r|r|r|}
\hline Koeffizienten & Wert & Std. Fehler & t-Wert \\
\hline$\alpha$ & 29.762 & 1.334 & 22.304 \\
\hline$\beta$ & 21.542 & 1.389 & 15.515 \\
\hline$\delta$ & 1.536 & 0.107 & 14.310 \\
\hline \multicolumn{2}{|c|}{ Residual Std. Fehler (m) } & \multicolumn{2}{|c|}{1.362 bei 40 Freiheitsgraden } \\
\hline
\end{tabular}

Tab. 9: Koeffizienten und statistische Kenngrößen der Begrenzungsfunktion (2-parametrige Verteilungsfunktion der Weibull-Verteilung; Formel (15)) der Kronenansatzschätzung mit Hilfe des Modells (14) für die Baumart Fichte.

In Abb. 27 sind die Auswirkungen der Begrenzungsfunktion auf die Kronenansatzschätzungen dargestellt. Im Bereich niedriger Baumhöhen wurden durch das Modell (14) einige Kronenansatzhöhen geschätzt, die größer als die zugehörige Baumhöhe sind. Diese Werte und alle Schätzungen, die größer als die über die Begrenzungsfunktion berechneten Werte sind, wurden durch die jeweiligen Werte der Begrenzungsfunktion ersetzt.
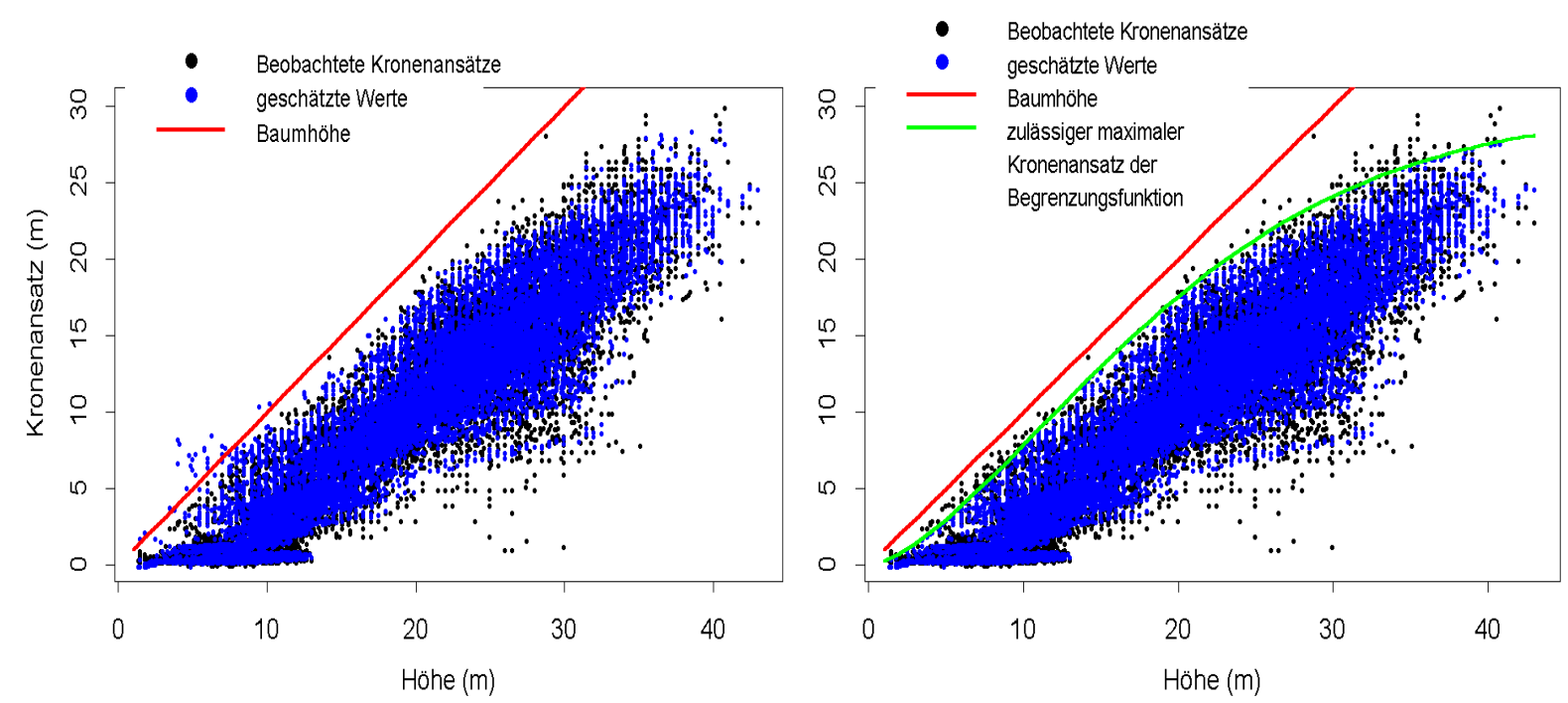

Abb. 27: Auswirkungen der Begrenzungsfunktion (15) zur Schätzung der maximalen Kronenansatzhöhe auf die Kronenansatzschätzungen durch das Modell (14) für die Baumart Fichte.

Durch die Korrektur mit Hilfe der Begrenzungsfunktion wird der Fehler der Kronenansatzschätzung geringfügig verringert (Tab. 6; EKVK (Kor.)). Dabei sinkt der Fehler der Kronenverhältnisschätzung stärker als der Fehler der Schätzung der Kronenansatzhöhe, da die von der Begrenzungsfunktion beeinflußten Bäume überwiegend niedrige Höhen aufweisen. Auch in den Streudiagrammen der beobachteten über den geschätzten Kronenverhältnissen (Abb. 26) wird die höhere Schätzgenauigkeit des Einheitskronenansatzmodells (mit Korrektur) gegenüber den Kronenansatzmodellen in Bwin, in Silva und dem einfachen statischen Kronenansatzmodell (in Bwinpro) deutlich. 
Der Schätzfehler für das Einheitskronenansatzmodell mit und ohne Korrektur ist für die Baumarten Douglasie, Kiefer, Europäische Lärche in Kapitel 9.1 und die Baumarten Eiche und Buche in Kapitel 9.2 angegeben. Es fällt die deutliche Erhöhung der Schätzgenauigkeit für alle Baumarten gegenüber den untersuchten statischen Modellen ohne Verwendung des Kronenverhältnisses des Spitzenhöhenstammes auf. Wie bei Fichte werden für alle Baumarten Schätzgenauigkeiten im Bereich der einzelbestandsweisen Parametrisierung von Kronenansatzmodellen erreicht (Formel (8); "Direkte Anpassung an die Daten"). Die Koeffizienten und statistischen Kennziffern für die Baumarten Douglasie, Kiefer, Europäische Lärche, Eiche und Buche für das Einheitskronenansatzmodell und die Begrenzungsfunktion sind in Kapitel 9.3 aufgeführt. Wenn leichte Veränderungen gegenüber dem Standardmodell (14) notwendig waren, sind die entsprechenden Formeln dargestellt (gilt für die Baumarten: Kiefer, Europäische Lärche, Buche). In Kapitel 9.2 sind die Vorteile des korregierten Einheitskronenansatzmodells gegenüber den Kronenansatzmodellen in Bwin, in Silva und dem einfachen statischen Kronenansatzmodell (13) anhand von Streudiagrammen der gemessenen über den geschätzten Werten verdeutlicht.

Es muß darauf hingewiesen werden, daß Probleme, wie sie für das hierarchische Modell bei der Anwendung in mehrschichtigen Beständen erläutert wurden (Abb. 25), auch beim Einheitskronenansatzmodell und dem einfachen statischen Kronenansatzmodell (13) auftreten können. Die Kronenverhältnisschätzung des Spitzenhöhenstammes bzw. die Spitzenhöhe bedeuten nur für die Schätzung von Bäumen in der Oberschicht - inklusive unterdrückter Individuen - einen zusätzlichen Informationsgewinn. Die Kronenverhältnisse von Bäumen aus Verjüngungskegeln mit sehr hohen Kronenverhältnissen werden wie beim hierarchischen Modellansatz systematisch unterschätzt. Als Alternative bietet sich wie beim hierarchischen Modell eine nach Schichten getrennte Betrachtung an. Da eine Kronenverhältnisschätzung des "Spitzenhöhenstammes der Unterschicht" nicht zur Verfügung steht, sollte die Schätzung für die Verjüngung mit Hilfe des neu entwickelten statischen Modellansatzes (13) unter Berücksichtigung der "Spitzenhöhe der Unterschichtbäume" erfolgen. Es wird nochmals darauf hingewiesen, daß das Einheitskronenansatzmodell und das neu entwickelte statische Modell (13) ohne Modifizierung auch für unterdrückte zwischenständige Bäume geeignet sind.

\subsubsection{Dynamisierung des Einheitskronenansatzmodells}

Wenn es gelingt, ein dynamisches Kronenansatzmodell zu parametrisieren, ergeben sich Vorteile gegenüber statischen Kronenansatzmodellen. So lassen sich Veränderungen der Bestandesdichte als kurzfristig wirkende Einflußgrößen im allgemeinen nur in dynamischen Modellen integrieren. Ein weiterer Vorteil dynamischer Modelle ergibt sich, wenn im Rahmen waldbaulicher Szenariensimulationen Ausgangsdaten mit hoher Qualität zur Verfügung stehen. Denkbar sind eine Anzahl an gemessenen Kronenansatzhöhen oder das Kronenver- 
hältnis des Spitzenhöhenstammes (Kapitel 3.2.3). Das Einheitskronenansatzmodell (14) ermöglicht gegenüber den statischen Modellansätzen ohne Schätzung des Kronenverhältnisses eines Mittelstammes (z. B. Formeln (1) - (5), (13)) die Ausnutzung der höheren Datenqualität. Dieser Vorteil besteht jedoch nur zum Aufnahmezeitpunkt, da im Rahmen einer Simulation schon nach der ersten Prognoseperiode keine Schätzung des Kronenverhältnisses des Spitzenhöhenstammes (Waldbegang) bzw. keine gemessenen Kronenansatzhöhen mehr zur Verfügung stehen. Bei Verwendung eines statischen Modellansatzes muß die Kronenansatzschätzung damit schon nach der ersten Prognoseperiode wieder ohne die Berücksichtigung der hohen Datenqualität des Ausgangszustandes erfolgen. Um unplausible Sprünge in der Entwicklung des Kronenansatzes zu verhindern, kann die Angleichung an das statische Modell über mehrere Prognoseschritte verteilt durch Interpolation durchgeführt werden.

Die hohe Qualität der Ausgangsinformation wird bei der schrittweisen Interpolation nur sehr unvollständig genutzt, da die folgende Entwicklung nicht über die erklärenden Variablen eines dynamischen Modellansatzes, sondern nur vom Abstand zum statischen Modell bestimmt wird. In Kapitel 3.2.1 wurde darauf hingewiesen, daß keine Entwicklung von dynamischen Modellansätzen mit ausreichender Modellgüte für die untersuchten Einzelbaumdaten möglich ist. Im folgenden wird daher ein Modellansatz zur Schätzung der Veränderungsrate des Kronenverhältnisses des Spitzenhöhenstammes vorgestellt, der anschließend eine Verwendung des Einheitskronenansatzmodells erlaubt. Es kann nur von einer Veränderungsrate nicht aber von einer Wachstumsrate gesprochen werden, da die Veränderung des Kronenverhältnisses des Spitzenhöhenstammes auch durch rechnerische Verschiebung infolge von Eingriffen beeinflußt wird. Das in Formel (16) dargestellte Modell wurde mittels schrittweiser linearer Regression ermittelt.

$$
i_{-} K V_{H 100}=\alpha+\beta * \overline{K S F / D 100}+\lambda * \overline{H 100}+\delta * K V_{H 100}
$$

wobei:
i_K $K V_{H 100}$
$=$ durchschnittliche jährliche Veränderungsrate des Kronenverhältnisses des Spitzenhöhenstammes der Baumart im Bestand während der Progno- seperiode;
$\overline{K S F / D 100}=$ mittlere normierte Bestandeskronenschirmfläche in der Prognoseperiode;
$\overline{H 100}=$ mittlere Höhe des Spitzenhöhenstammes der Baumart im Bestand in der Prognoseperiode (m);
$K V_{H 100}$
$=$ Kronenverhältnis des Spitzenhöhenstammes der Baumart im Bestand zu Beginn der Prognoseperiode. 
für Formel (16) mit:

\begin{tabular}{|c|c|c|c|c|}
\hline Koeffizienten & Wert & Std. Fehler & t-Wert & $\operatorname{Pr}(>|t|)$ \\
\hline$\alpha$ & 0.0628 & 0.0063 & 9.901 & 0.0000 \\
\hline$\beta$ & -0.0000207 & 0.00000499 & -4.139 & 0.0000 \\
\hline$\lambda$ & -0.000976 & 0.0001 & -7.360 & 0.0000 \\
\hline$\delta$ & -0.0719 & 0.0058 & -12.478 & 0.0000 \\
\hline \multicolumn{2}{|c|}{ Residual Std. Fehler } & \multicolumn{3}{|c|}{0.00736 bei 226 Freiheitsgraden } \\
\hline \multicolumn{2}{|c|}{ Multiples Bestimmtheitsmaß } & \multicolumn{3}{|r|}{0.4555} \\
\hline \multirow{2}{*}{\multicolumn{2}{|c|}{ F-Statistik }} & \multicolumn{3}{|c|}{63.01 bei 3 und 226 Freiheitsgraden, $p$-Wert $=0$} \\
\hline & & \multicolumn{3}{|c|}{ Periodenlänge weniger als 11 Jahre } \\
\hline \multicolumn{2}{|c|}{ Residual Std. Fehler } & \multicolumn{3}{|c|}{0.00772 bei 185 Freiheitsgraden } \\
\hline \multicolumn{2}{|c|}{ Multiples Bestimmtheitsmaß } & \multicolumn{3}{|r|}{0.4883} \\
\hline \multicolumn{2}{|r|}{ F-Statistik } & \multicolumn{3}{|c|}{58.85 bei 3 und 185 Freiheitsgraden, $p$-Wert $=0$} \\
\hline
\end{tabular}

Tab. 10: Koeffizienten und statistische Kenngrößen für das Modell zur Schätzung der Kronenverhältnisveränderungsrate des Spitzenhöhenstammes für die Baumart Fichte. Die statistischen Kenngrößen sind für die Berechnung bei Berücksichtigung aller Wachstumsperioden und bei Berücksichtigung der Wachstumsperioden mit einer Periodenlänge von weniger als 11 Jahren angegeben.

Als erklärende Variablen wurden das Ausgangskronenverhältnis des Spitzenhöhenstammes, die mittlere Spitzenhöhe der Prognoseperiode sowie der mittlere Quotient aus Bestandeskronenschirmfläche zum BHD des Spitzenhöhenstammes der Prognoseperiode ermittelt. Die Kronenschirmfläche gilt immer für den Gesamtbestand während der BHD, die Höhe und das Ausgangskronenverhältnis des Spitzenhöhenstammes baumartenweise berechnet werden. Als unabhängige Variablen führten Periodenmittelwerte der normierten Kronenschirmfläche und der Spitzenhöhe gegenüber Periodenanfangswerten zu höheren Bestimmtheitsmaßen des Modells. Periodenmittelwerte beinhalten im Gegensatz zu Anfangswerten wachstums- und eingriffsbedingte Veränderungen der unabhängigen Variablen. Sie charakterisieren damit den Zustand eines Bestandes während der Prognoseperiode besser als die Werte des verbleibenden Bestandes zu Beginn der Periode, speziell wenn längere Wachstumsperioden zur Parametrisierung verwendet werden.

Validierung und Bewertung der Dynamisierung des Einheitskronenansatzmodells:

Vergleicht man das Modell mit dem dynamischen Kronenansatzmodell auf Einzelbaumbasis von HASENAUER (1994; Formel (17)) so fällt die Ähnlichkeit der Variablentypen auf. Beim Vergleich des Ansatzes von HASENAUER mit dem Modell (16) kann dem Konkurrenzindex von EK und MONSERUD (1974) das Bestandesdichtemaß Quotient aus Kronenschirmfläche zu BHD des Spitzenhöhenstammes zugeordnet werden. Beide Variablen beschreiben den Einfluß der Dichte auf die Kronenansatz- bzw. Kronenverhältnisveränderungsrate und unterscheiden 
sich letztlich nur in der Betrachtungsebene Einzelbaum bzw. Bestandesmittelstamm. Die Kronenschirmfläche wurde mit dem zugehörigen Durchmesser des Spitzenhöhenstammes normiert, um ein vom Dimensionstrend (Kapitel 2.3, Fußnote 4) unabhängiges Dichtemaß zu erhalten. Als Dimensionstrends werden Trends in der Entwicklung der Bestandeskronenschirmfläche mit sich verändernden Baumdimensionen verstanden.

$i_{-} H K A=\alpha_{0} * h^{\alpha_{1}} * e^{\left(\alpha_{2}{ }^{*} K V^{0.5}+\alpha_{3} / C I+\alpha_{4}{ }^{*} B H D\right)}$

wobei:

$i_{-} H K A=$ jährliche Kronenansatzveränderungsrate;

$\bar{B} H D=$ Brusthöhendurchmesser;

$h=$ Baumhöhe;

$K V=$ Kronenverhältnis;

CI = abstandsabhängiger Konkurrenzindex von EK und MONSERUD (1974) nach erfolgter Freistellung.

Der Einzelbaumdimensionsvariablen BHD im Modell von HASENAUER (1994) kann die Bestandesdimensionsvariable Spitzenhöhe zugeordnet werden. Weitere Übereinstimmung besteht in der Verwendung des Ausgangskronenverhältnisses des Einzelbaums bzw. des Spitzenhöhenstammes. Als jährliche Kronenverhältnisveränderungsrate wurde die durchschnittliche jährliche Veränderungsrate während der betrachteten Wachstumsperiode verwendet.

Es kann davon ausgegangen werden, daß die unabhängigen Variablen, die den Bestand während der Zuwachsperiode beschreiben, mit fortschreitender Periodenlänge an Einfluß auf die Kronenverhältnisveränderungsrate verlieren. Daher wurde untersucht, wie sich das Bestimmtheitsmaß des Modells (16) verändert, wenn anstatt aller Wachstumsperioden nur Perioden in die Regression eingehen, die weniger als 11 Jahre umfassen (Tab. 10). Da das Bestimmtheitsmaß lediglich um ca. 3\% steigt, wenn eine Periodenlänge von 10 Jahren als Maximalwert verwendet wird, wurde in den weitergehenden Untersuchungen das Modell auf Basis aller Wachstumsperioden getestet. Für alle drei unabhängigen Variablen gilt, daß mit steigenden Werten die Kronenverhältniszunahmen geringer werden und es schließlich zu einer Abnahme des Kronenverhältnisses kommt.

Im Gegensatz zu negativen Änderungen der Kronenansatzhöhe sind positive Veränderungen des Kronenverhältnisses biologisch plausibel, da es zu einer Zunahme des Kronenverhältnisses kommt, wenn das Verhältnis aus Höhenwachstumsrate zur Veränderungsrate des Kronenansatzes größer ist als das Verhältnis von Ausgangshöhe zu Ausgangskronenansatzhöhe.

Der negative Einfluß der Spitzenhöhe erklärt sich dadurch, daß nach Überschreiten des maximalen laufenden Höhenzuwachses in jungem Alter der Höhenzuwachs stetig abnimmt. Wenn man davon ausgeht, daß die Veränderungsrate des Kronenansatzes von der Dimension 
unabhängig ist, muß mit steigender Spitzenhöhe die Kronenverhältnisveränderungsrate langsamer zu- und schließlich abnehmen.

Mit steigender Bestandesdichte, beschrieben durch den mittleren Quotienten aus Kronenschirmfläche und BHD des Spitzenhöhenstammes, kommt es zu einem schnelleren Absterben von Grünästen im Kronenansatzbereich. Wenn man davon ausgeht, daß das Höhenwachstum durch die Erhöhung der Bestandesdichte nicht in gleichem Maße gefördert wie die Verlagerung des Kronenansatzes beeinflußt wird, ergibt sich eine negative Kronenverhältnisveränderungsrate.

Bei hohen Ausgangskronenverhältnissen muß für eine Veränderungsrate von 0 das Verhältnis von Höhenveränderungsrate zu Kronenansatzveränderungsrate sehr groß sein, so daß sich schon bei geringfügigen Verlagerungen der Kronenansatzhöhe negative Kronenverhältnisveränderungsraten ergeben.

Zur Validierung des Modellansatzes bietet sich ein Vergleich mit einem statischen Modellansatz an. Der Vergleich kann nur anhand von Versuchsflächen erfolgen, für die mehrere Aufnahmen zur Verfügung stehen. Für diese Flächen können mit einem statischen Modellansatz Kronenansatzhöhen ohne Berücksichtigung von Informationen aus der vorhergehenden Aufnahme geschätzt werden. Im Gegensatz dazu kann mit Hilfe des dynamischen Modells (16) das Kronenverhältnis des Spitzenhöhenstammes unter Berücksichtigung dieser Informationen geschätzt werden. Auf Basis dieser Schätzung kann das Einheitskronenansatzmodell (14) auch für Flächen angewendet werden, für die bei einer vorhergehenden Aufnahme das Kronenverhältnis des Spitzenhöhenstammes geschätzt wurde. Das dynamische Modell erlaubt damit neben der potentiell integrierbaren Behandlungssensitivität eine Berücksichtigung von qualitativ hochwertigen Eingangsinformationen über den Zeitpunkt der Erhebung dieser Informationen hinaus.

In Abb. 28 sind die je Parzelle und Aufnahme berechneten Differenzen der mittleren Fehler der Kronenansatzhöhenschätzung mit Hilfe des dynamisierten Einheitskronenansatzmodells (14) bzw. (16) und des einfachen statischen Modellansatzes (13) über der Länge der Prognoseperiode dargestellt. Positive Werte repräsentieren Bestände, in denen der dynamische Modellansatz günstiger als das statische Modell ist. Es fällt auf, daß ab einem Prognosezeitraum von ca. 11 Jahren das statische Modell günstiger ist, wobei die geringe Datengrundlage für Bestände mit großen Prognosezeiträumen berücksichtigt werden muß.

Die Schätzung erfolgte beim dynamischen Ansatz in einem Schritt, ohne daß eine laufende Aktualisierung der unabhängigen Variablen mit Hilfe einer Wachstumssimulation in beispielsweise 1-Jahresschritten durchgeführt wurde. Verwendet wurde das gleiche Datenmaterial wie bei allen vorhergehenden Parametrisierungen ausschließlich der Parzellenaufnahmen, für die keine früheren Aufnahmen vorlagen. Die Schätzungen mit Hilfe des dynamisierten Modellansatzes wurden, wie beim Einheitskronenansatzmodell beschrieben, mit Hilfe 
der Begrenzungsfunktion (15) auf biologische Plausibilität überprüft und gegebenenfalls korregiert.

In Abb. 28 ist weiterhin ein Modell (Formel (18); Tab. 11) dargestellt, daß eine Schätzung der Über- bzw. Unterlegenheit (Differenz der Schätzfehler des dynamischen und des statischen Modells) in Abhängigkeit vom Prognosezeitraum zuläßt (rote Ausgleichskurve). Das Modell wurde in einem zweiten Schritt nur für Bestände parametrisiert, bei denen das Einheitskronenansatzmodell im Ausgangsbestand eine höhere Genauigkeit als das statische Modell aufwies, d. h. in denen auch wirklich Vorteile bezüglich des Startpunktes vorlagen (blaue Ausgleichskurve).

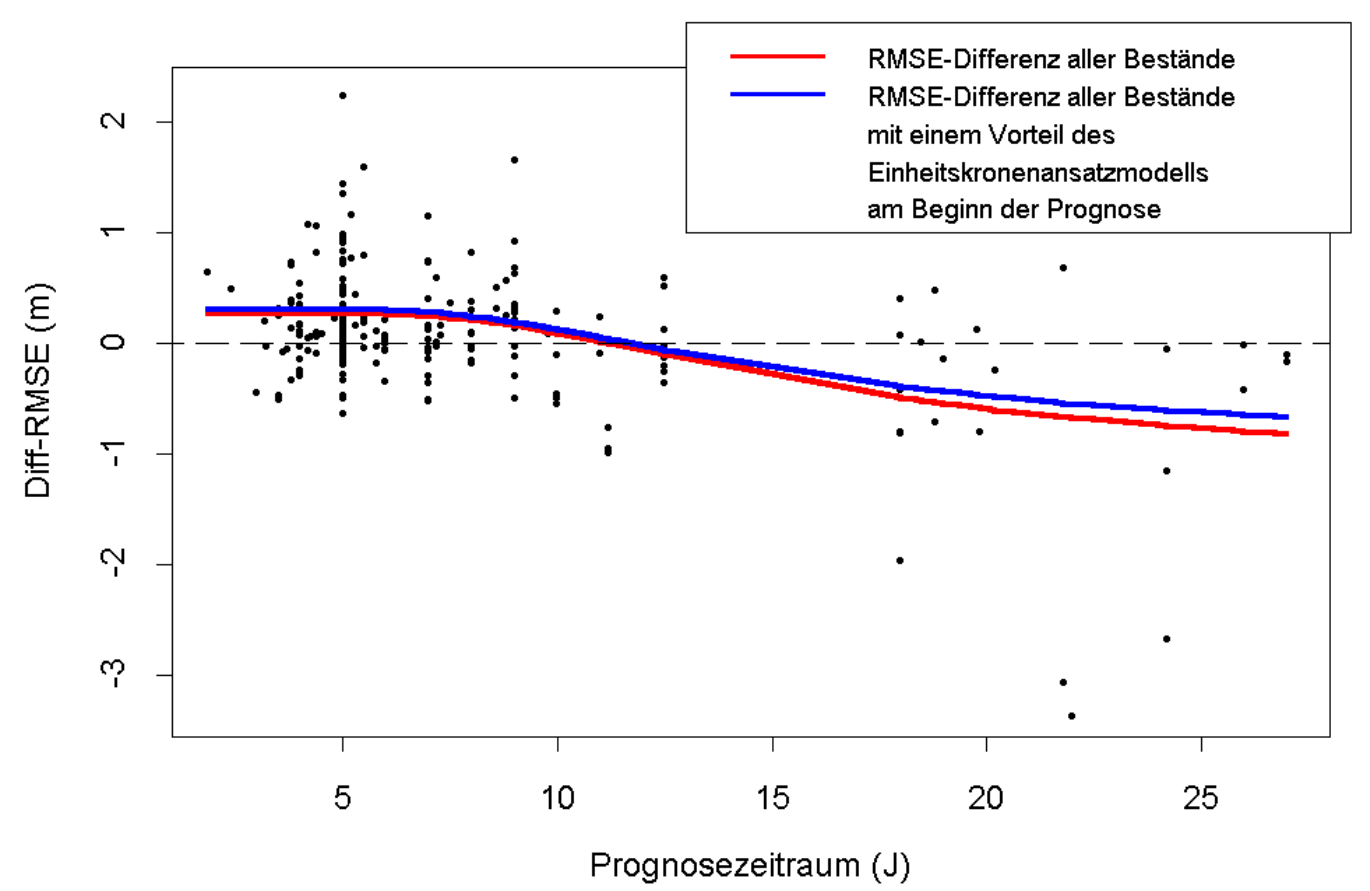

Abb. 28: Fehlerdifferenz der nach Parzellen und Aufnahmezeitpunkten berechneten RMSE des statischen und dynamischen Modellansatzes zur Kronenansatzschätzung in Abhängigkeit vom Prognosezeitraum für die Baumart Fichte. Die dynamische Schätzung wurde in einem Schritt ohne Zwischenberechnungen der unabhängigen Variablen durchgeführt. Das zum Ausgleich verwendete Modell ist in Formel (18) dargestellt.

Als Ursachen der sich ändernden Differenzen der Schätzgenauigkeit der Modellansätze kommen mehrere Faktoren in Frage. Erstens entfernt man sich bei Anwendung des dynamischen Ansatzes mit größer werdendem Prognosezeitraum von der Kronenverhältnisschätzung auf Basis der hochwertigen Information "geschätztes Kronenverhältnis des Spitzenhöhenstammes“. Darüber hinaus spielt die Schätzgenauigkeit der Veränderungsrate des Kronenverhältnisses des Spitzenhöhenstammes eine Rolle. Aufgrund des Verlaufs der Fehlerdifferenz kann vermutet werden, daß das dynamische Modell zumindest bei langen Prognoseperioden eine geringere Schätzgenauigkeit der Veränderungsrate der Einzelbaumkronenansätze aufweist als das statische Modell. Es ist auch denkbar, daß das dynamische Modell unabhängig von der 
Prognoselänge geringere Schätzgenauigkeiten der Veränderungsrate aufweist, daß jedoch der Vorteil der hochwertigen Eingangsinformation bzw. des besseren Startpunktes erst ab einem bestimmten Prognosezeitraum vom statischen Modell kompensiert wird. Nach Abb. 28 kann ausgeschlossen werden, daß das dynamische Modell für den gesamten Bereich von Prognoselängen höhere oder gleichbleibende Schätzgenauigkeiten der Veränderungsraten aufweist. In diesen Fällen müßte das dynamische Modell über den gesamten Bereich einen Vorteil aufweisen bzw. die mittlere Differenz müßte sich mit steigender Prognoselänge dem Wert Null nähern. Es wird noch einmal darauf hingewiesen, daß sich die Ergebnisse und daraus abgeleitete Schlußfolgerungen für den dynamischen Modellansatz nur auf die Berechnung der Kronenverhältnisveränderungsrate in einem einzigen Simulationsschritt beziehen. Die in Abb. 28 dargestellten Fehlerdifferenzen wurden mit folgendem Modellansatz ausgeglichen:

$$
R M S E_{-} \text {Differenz }=\alpha-\alpha * e^{-(\beta / t \operatorname{Pr} o g)^{2}} * \lambda
$$

wobei:

$$
\begin{aligned}
\text { RMSE_Differenz }= & \text { Differenz der getrennt nach Parzellen und Aufnahmezeitpunkten } \\
& \text { berechneten RMSE von dynamischem und statischem Modellansatz (m); } \\
= & \text { Länge des Prognosezeitraumes (Jahr). }
\end{aligned}
$$

mit:

\begin{tabular}{|r|r|r|r|}
\hline \multicolumn{1}{|c|}{ Koeffizienten } & \multicolumn{1}{|c|}{ Wert } & Std. Fehler & t-Wert \\
\hline$\alpha$ & 0.262 & 0.0446 & 5.875 \\
\hline$\beta$ & 14.645 & 2.297 & 6.376 \\
\hline$\lambda$ & 5.586 & 1.573 & 3.551 \\
\hline \multicolumn{2}{|c|}{ Residual Std. Fehler (m) } & \multicolumn{2}{|c|}{0.542 bei 227 Freiheitsgraden } \\
\hline
\end{tabular}

Nur Bestände mit Vorteilen des Einheitskronenansatzmodells gegenüber dem statischen Modell für den Ausgangsbestand (Startpunkt der Wachstumsprognose)

\begin{tabular}{|r|r|r|r|}
\hline Koeffizienten & \multicolumn{1}{|c|}{ Wert } & Std. Fehler & t-Wert \\
\hline$\alpha$ & 0.308 & 0.0461 & 5.688 \\
\hline$\beta$ & 13.854 & 2.428 & 3.705 \\
\hline$\lambda$ & 4.125 & 1.1392 & 3.621 \\
\hline \multicolumn{2}{|c|}{ Residual Std. Fehler (m) } & \multicolumn{2}{|c|}{0.523 bei 197 Freiheitsgraden } \\
\hline
\end{tabular}

Tab. 11: Koeffizienten und statistische Kenngrößen des Modells zur Schätzung des Vorteils von statischem (13) und dynamischem Modellansatz (14) und (16) der Kronenansatzschätzung für die Baumart Fichte. Das Modell wurde für alle Parzellenaufnahmen parametrisiert, für die eine vorhergehende Aufnahme vorlag. In einem zweiten Schritt wurde das Modell nur für die Parzellenaufnahmen parametrisiert, bei denen das Einheitskronenansatzmodell eine höhere Genauigkeit als das statische Modell bei der vorhergehenden Aufnahme aufwies. 
Anhand der Funktionsverläufe in Abb. 28 wird ersichtlich, daß die Beschränkung auf günstige Startpunkte nur zu geringfügigen Verschiebungen beim Vergleich von dynamischem und statischem Ansatz führt. Mit Hilfe der Modelle zur Abschätzung des Modellvorteils in Abhängigkeit vom Prognosezeitraum lassen sich die Zeitpunkte (kritischer Prognosezeitraum) ermitteln, an denen beide Modellansätze die gleiche Schätzgenauigkeit aufweisen (Tab. 12). Weiterhin beschreibt der Parameter $\alpha$ in Formel (18) den maximalen Vorteil des dynamischen Modellansatzes im Durchschnitt über alle Bestände. In Tab. 12 sind diese durchschnittlichen maximalen Vorteile und kritischen Prognosezeiträume für alle Bestände und für das Kollektiv von Beständen mit günstigem Startpunkt (Definition s. o.) für die Kronenansatzschätzung und die Kronenverhältnisschätzung angegeben.

\begin{tabular}{|l|r|rr|}
\hline & Alle Bestände & \multicolumn{2}{|c|}{$\begin{array}{l}\text { Nur Bestände mit guter Anpassung des } \\
\text { Einheitskronenansatzmodells } \\
\text { (Startpunktvorteil besteht) }\end{array}$} \\
\hline $\begin{array}{l}\text { Kritischer Prognosezeitraum der Kro- } \\
\text { nenansatzschätzung (Jahre) }\end{array}$ & 11.1 & 11.6 \\
\hline $\begin{array}{l}\text { Maximaler Modellvorteil (m) im } \\
\text { Durchschnitt über alle Bestände }\end{array}$ & 0.262 & & 13.0 \\
\hline $\begin{array}{l}\text { Kritischer Prognosezeitraum der Kro- } \\
\text { nenverhältnisschätzung (Jahre) }\end{array}$ & 12.0 & & 0.0130 \\
\hline $\begin{array}{l}\text { Maximaler Modellvorteil im Durch- } \\
\text { schnitt über alle Bestände (Kronen- } \\
\text { verhältnis) }\end{array}$ & 0.0106 & \\
\hline
\end{tabular}

Tab. 12: Kritische Prognosezeiträume und durchschnittliche maximale Vorteile (aller Prognosen und der Prognosen mit günstigem Startpunkt) des dynamischen Kronenansatzmodells für die Baumart Fichte. Die Werte wurden über das Modell zur Einschätzung der Unterschiede der Genauigkeit von statischem (13) und dynamischem Modellansatz (14) und (16) bestimmt (Formel (18)). Die Berechnung erfolgte anhand der Fehlerdifferenzen für die Kronenansatz- und die Kronenverhältnisschätzung.

Für den Vergleich beider Modelle ist in erster Linie der Modellvorteil in Abhängigkeit vom Prognosezeitraum unabhängig von den Ursachen entscheidend. Dennoch ist es wichtig, die einzelnen Einflüsse besser voneinander zu trennen. Wenn beispielsweise festgestellt werden kann, daß die Schätzgenauigkeit der Veränderungsrate beim dynamischen Modellansatz von dem in einem Simulationsintervall beschriebenen Prognosezeitraum beeinflußt wird, müssen die einzelnen Simulationsschritte möglichst kurz gewählt werden. Es kann davon ausgegangen werden, daß der Bestandeszustand bei kurzen Simulationsschritten durch die schrittweise neu berechneten unabhängigen Variablen (Formel (16)) besser beschrieben wird, da es sich um Periodenmittel- bzw. -anfangswerte handelt.

Um den Vorteil des dynamischen Modells vom Einfluß des günstigeren Startpunktes zu bereinigen, wurden die RMSE der periodischen Veränderungsraten der Einzelbaumkronenansätze je Versuchsparzelle und Aufnahme verglichen. Die Veränderungsraten des statischen 
Modells ergeben sich aus der Differenz der Schätzung der Einzelbaumkronenansätze zum Ende bzw. Anfang der Prognoseperiode. Für den dynamischen Modellansatz werden die Kronenverhältnisse am Ende der Prognoseperiode über die Kombination der Formeln (14) und (16) ermittelt. Die Kronenverhältnisse am Anfang der Prognoseperiode werden über Formel (14) geschätzt. Zum Vergleich steht die Veränderungsrate zwischen zwei gemessenen Werten zur Verfügung. In Abb. 29 ist der getrennt nach Parzellen und Aufnahmezeitpunkten berechnete mittlere RMSE der periodischen Kronenansatzveränderungsrate (gemittelte Einzelbaumwerte) für den statischen und den dynamischen Modellansatz dargestellt.

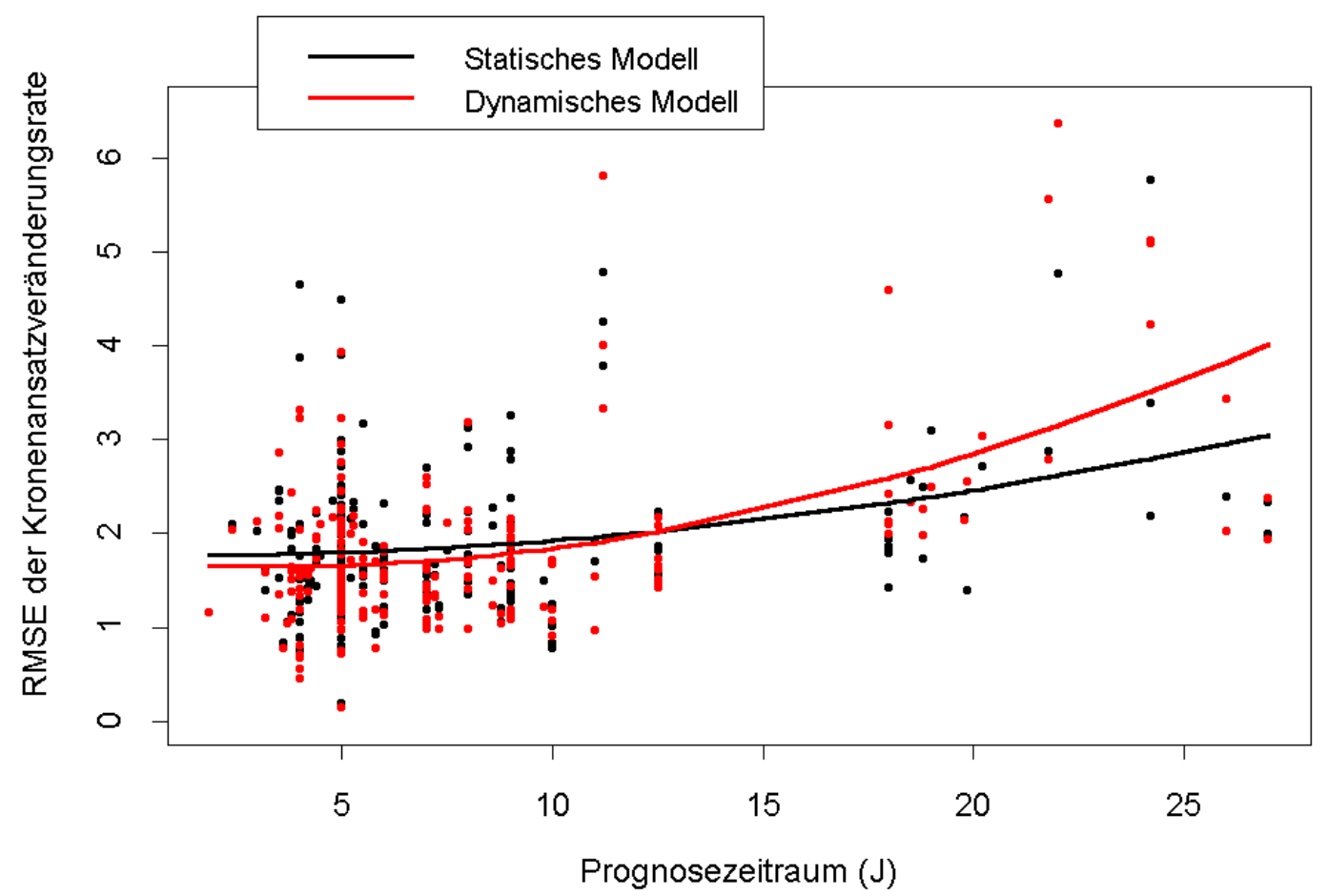

Abb. 29: Getrennt nach Parzellen und Aufnahmezeitpunkten berechnete Fehler (m) der Schätzung der periodischen Kronenansatzhöhenveränderung (gemittelte Einzelbaumwerte) für den statischen und dynamischen Modellansatz. Zur Verdeutlichung der Unterschiede wurden die Fehlerwerte beider Modelle mit einem Gleitenden-Durchschnitts-Modell über der Länge des Prognosezeitraumes (J) ausgeglichen (Kapitel 8.1).

Bei der Beurteilung von dynamischen Kronenansatzmodellen ist die problematische Messung der Kronenansatzhöhe zu beachten, die bei kurzen Aufnahmeintervallen größere Meßfehler als die zu messende Veränderungsrate aufweisen kann. Wenn man eine von der Länge der Wachstumsperiode unabhängige Höhe des Meßfehlers unterstellt, muß der Meßfehleranteil bei der Berechnung durchschnittlicher jährlicher Veränderungsraten mit sinkender Wachstumsperiodenlänge steigen. Als Resultat verdoppeln sich die Fehlerwerte mit verdoppelnder 
Prognoseperiode nicht einmal bzw. die jährlichen Fehlerraten steigen mit sinkender Prognosedauer stark an (Abb. 29) ${ }^{12}$.

Infolge der unterschiedlich hohen Meßfehleranteile an den gemessenen Veränderungsraten ist die absolute Höhe der Schätzfehler der Modellansätze für einen Vergleich unterschiedlicher Längen von in einem Simulationsschritt beschriebenen Prognosezeiträumen ungeeignet. Da von einem zufälligen Meßfehler ausgegangen wird, kann jedoch die Relation der Schätzfehler von statischem und dynamischem Modellansatz interpretiert werden. Das dynamische Modell weist bis zu einem Prognosezeitraum von ca. 13 Jahren einen geringfügigen Vorteil bei der Schätzung der Kronenansatzveränderungsrate auf. Der Schätzfehler steigt jedoch mit steigendem Prognosezeitraum deutlich schneller an als beim statischen Modellansatz. Auf dieser Basis können die in Abb. 28 und Tab. 12 dargestellten Ergebnisse weitergehend interpretiert werden. Damit setzt sich der Vorteil des dynamischen gegenüber dem statischen Modell bis zu einem Prognosezeitraum von ca. 12 Jahren sowohl aus dem günstigeren Startpunkt als auch der geringfügig genauer geschätzten Veränderungsrate für kurze Prognosezeiträume zusammen. Für das statische Modell kann angenommen werden, daß die Schätzgenauigkeit der jährlichen Veränderungsrate von der Periodenlänge unabhängig ist. Trotzdem weist der Schätzfehler der periodischen Veränderungsrate keinen proportionalen Zusammenhang zur Prognoselänge auf, da die beobachteten Veränderungsraten einen vom Prognosezeitraum unabhängigen fixen Meßfehler aufweisen (Abb. 29).

Weil die Entwicklung des Fehlers der periodischen Veränderungsrate des dynamischen Modells vom Verlauf des statischen Modells abweicht, muß von einem Zusammenhang der in einem Simulationsschritt beschriebenen Prognoselänge und der durchschnittlichen jährlichen Kronenansatzveränderungsrate ausgegangen werden. Daraus kann abgeleitet werden, daß eine weitere Verbesserung des dynamischen Modellansatzes erreicht werden kann, wenn Prognosezeiträume in möglichst kurzen Simulationsschritten fortgeschrieben werden, wobei jeweils die zur Schätzung verwendeten unabhängigen Variablen (Formel (16)) nach jedem Simulationsschritt neu berechnet werden. Die Fehlerentwicklung der Schätzung der Kronenansatzveränderungsrate würde somit nur noch indirekt von der Prognoselänge abhängen, da davon auszugehen ist, daß sich die Schätzfehler der unabhängigen Variablen (Formel (16)) mit steigender Prognoselänge erhöhen werden. Wenn diese Fehler bei der Schätzung von unabhängigen Variablen für das dynamische und statische Modell vernachlässigt werden, resultiert daraus, daß das dynamische Modell bei Anwendung von kurzen Simulationsschritten von z. B. fünf Jahren über den gesamten Bereich von Prognosezeiträumen einen Vorteil bei der Schätzung der Kronenansatzhöhe gegenüber dem statischen Modell aufweisen müßte. Dieser Vorteil

\footnotetext{
${ }^{12}$ In die Parametrisierung des Modells zur Schätzung der Kronenverhältnisveränderungsrate des Spitzenhöhenstammes wurden jedoch auch die Parzellenaufnahmen mit sehr kurzen Wachstumsperioden einbezogen, da der Meßfehler als zufällig betrachtet wird.
} 
würde allerdings mit steigender Prognoselänge durch den nachlassenden Einfluß des günstigen Startpunktes sehr gering werden.

In Tab. 6 sind die Fehler der Kronenansatz- und Kronenverhältnisschätzung für den dynamischen Modellansatz aufgeführt (Dyn. EKVK (Kor.)). Eine weitere Unterteilung erfolgte nach Obergrenzen der betrachteten Wachstumsperiodenlänge, wobei immer zu beachten ist, daß nur die Werte der Kronenansatzschätzung des Spitzenhöhenstammes, nicht aber die unabhängigen Variablen fortgeschrieben wurden (keine Wachstumssimulation). Als unabhängige Variablen (Formeln (13) bzw. (14) u. (16)) wurden immer Meßwerte bzw. aus ihnen berechnete Werte der Aufnahmen zu Beginn und Ende der Wachstumsperiode verwendet. Die gesamte Wachstumsperiode wurde somit immer in einem Simulationsschritt fortgeschrieben.

Die Unterteilung erfolgte neben der Berücksichtigung aller Wachstumsperioden nach Prognoselängen von maximal 5 und 15 Jahren (Dyn. EKVK (Kor.) <=5, Dyn. EKVK (Kor.) $<=15$ ). Mit abnehmendem Prognosezeitraum nimmt die Residualstreuung der Kronenverhältnisschätzung der Einzelbäume ab, wobei auch das dynamische Modell ohne Zeitraumbegrenzung einen leichten Vorteil gegenüber dem statischen Modell (in Bwinpro) aufweist (Abb. 30).
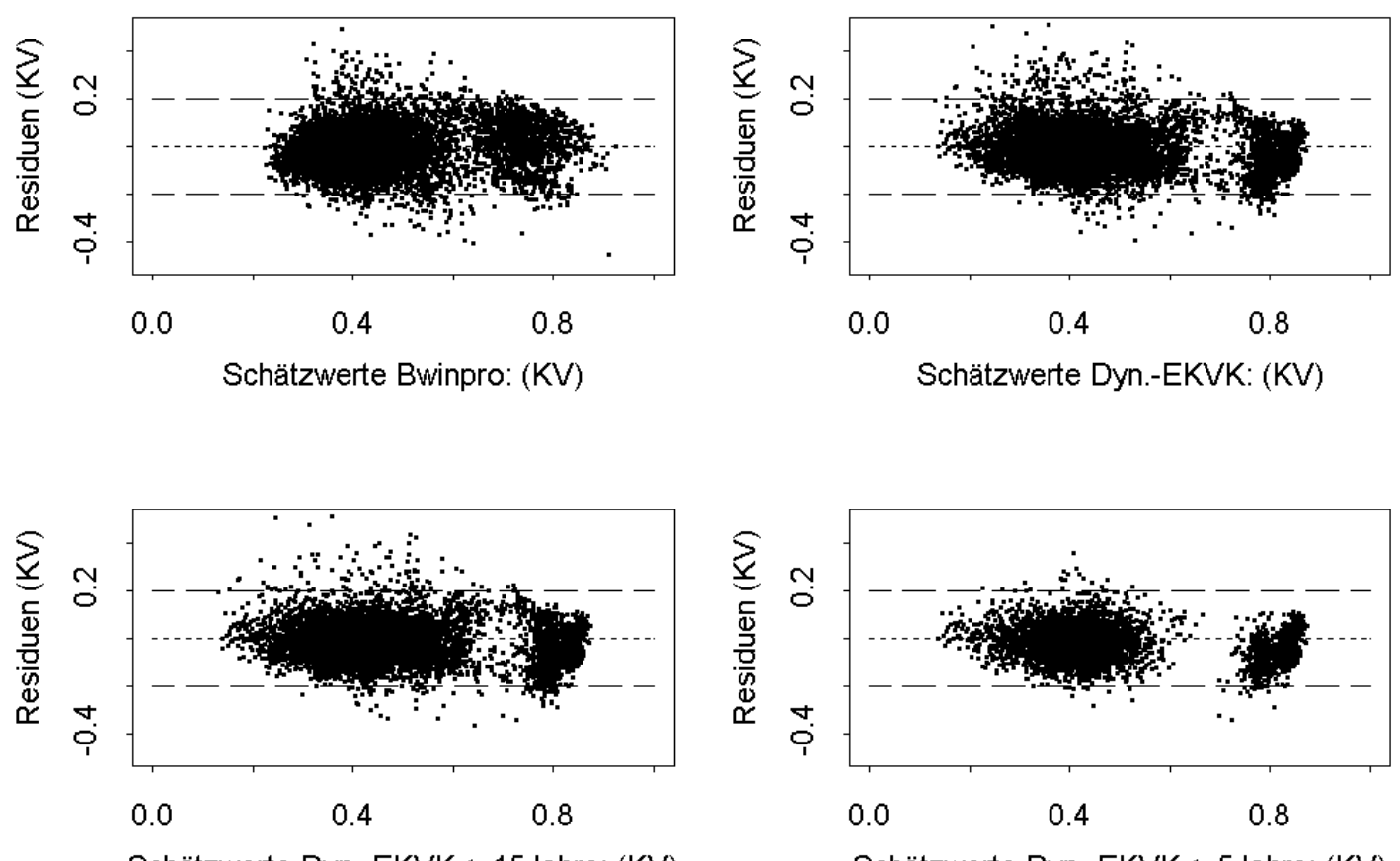

Abb. 30: Residuenplots der Kronenverhältnisschätzung mit Hilfe des neu entwickelten, einfachen statischen Modells (in Bwinpro, Formel (13)) und des dynamischen Modellansatzes (14) und (16) für die Baumart Fichte. Für den dynamischen Modellansatz sind neben den Ergebnissen der Schätzung für alle Prognosezeiträume auch die Ergebnisse der Begrenzung der Prognosezeiträume auf maximal 15 und 5 Jahre dargestellt (Dyn. EKVK, Dyn. EKVK <=15, Dyn. EKVK <=5). Die Schätzungen des dynamischen Modells sind mit Hilfe der Begrenzungsfunktion (15) überprüft und gegebenenfalls korrigiert worden. 
Infolge seiner Überlegenheit bis zu einem Prognosezeitraum von ca. 12 Jahren, der in einem Simulationsschritt beschrieben wird, sollte das dynamische Modell (14) und (16) insbesondere im Rahmen der mittelfristigen Planung dem statischen Modell (13) vorgezogen werden. Bei Verkürzung des Zeitintervalls der Simulationsschritte kann davon ausgegangen werden, daß das dynamische Modell über den gesamten Bereich von Prognosezeiträumen einen Vorteil gegenüber dem statischen Modell aufweisen wird. Durch den nachlassenden Einfluß des günstigen Startpunktes ist ein deutlicher Vorteil jedoch nur in den ersten ca. 15 Jahren der Simulation zu erwarten. Somit ist der Ansatz prädestiniert für einen Einsatz in der mittelfristigen Planung, wobei allerdings die Schätzung des Kronenverhältnisses des Spitzenhöhenstammes anhand von orientierenden Messungen im Rahmen des Waldbeganges notwendig ist.

Die Fehlerwerte der Kronenansatz- und Kronenverhältnisschätzungen für die Baumarten Douglasie, Kiefer, Europäische Lärche, sind in Kapitel 9.1 und für die Baumarten Eiche und Buche in Kapitel 9.2 dargestellt. Die Tabellen, die die Fehlerwerte enthaltenen, entsprechen Tab. 6 der Baumart Fichte und auch die Bezeichnungen der zugehörigen Spalten orientieren sich an der Baumart Fichte (Dyn. EKVK (Kor.), Dyn. EKVK (Kor.) <=5, Dyn. EKVK (Kor.) <=15). Die Darstellung der Modellunterschiede von dynamischem und statischem Modellansatz ist in Kapitel 9.4 wiedergegeben. Die Schätzungen der kritischen Prognosezeiträume und maximalen Vorteile des dynamischen Modellansatzes sind ebenfalls in Kapitel 9.4 aufgeführt. Die Herleitung von kritischen Prognosezeiträumen war für die Baumart Europäische Lärche nicht möglich, da nur eine Wachstumsperiode mit mehr als 12 Jahren zur Verfügung stand. Die Vergleiche der Residualstreuung von statischem und dynamischem Modellansatz sowie die Auswirkungen des Prognosezeitraumes auf die Residualstreuung sind in Kapitel 9.4 verdeutlicht. Im Gegensatz zu den Nadelholzarten tritt bei Eiche und Buche bei der Anwendung des dynamischen Modellansatzes speziell bei langen Prognosezeiträumen, die durch einen Simulationsschritt beschrieben werden, ein deutlicher Bias der Kronenansatzschätzung auf. Die Zerlegung des Fehlers der Kronenansatzschätzung des dynamischen Modells in den Fehler des Startpunktes und den Fehler der Kronenansatzveränderungsrate wäre für eine genauere Interpretation unbedingt notwendig. Im Rahmen dieser Arbeit konnte jedoch nur für die Baumart Fichte die Vorgehensweise dargestellt bzw. eine umfassendere Interpretation durchgeführt werden.

Für die Baumarten Eiche und Buche konnten aufgrund des großen Datenumfanges mit Hilfe von Bootstrap-Simulationen (Kapitel 8.2) zusätzlich Genauigkeitsrahmen der Regressionsfunktion zur Schätzung der Modellunterschiede von dynamischem und statischem Modellansatz ermittelt werden (Kapitel 9.4, Abb. 104 u. Abb. 108). Auf diesen Untersuchungen basierend wurden anschließend Verteilungen der kritischen Prognosezeiträume und maximalen Vorteile geschätzt. 


\subsubsection{Gegenüberstellung der Kronenansatzmodelle}

Anhand von Abb. 31 soll eine abschließende Gegenüberstellung der neu entwickelten Kronenansatzmodelle untereinander und gegenüber den statischen Vergleichsmodellen von NAGel (1999 a), Pretzsch und KAHN (1998), COLIN und Houllier (1991) sowie HASENAUER (1994) erfolgen. Die Anforderungen an die Qualität der Eingangsdaten liegen für das in Bwinpro verwendete, einfache statische Modell (13) auf dem Niveau der vier statischen Vergleichsmodelle. Das einfache statische Modell (13) weist jedoch für alle untersuchten Nadelholzarten höhere Schätzgenauigkeiten als alle statischen Vergleichsmodelle auf. Dabei ist die Rangfolge der statischen Vergleichsmodelle von Baumart zu Baumart unterschiedlich. Für die Baumart Eiche erreicht das einfache statische Modell ebenfalls höhere Schätzgenauigkeiten, wobei die Verbesserungen allerdings weniger deutlich sind. Für die Baumart Buche werden mit Hilfe des neuen statischen Modells keine Verbesserungen gegenüber den Vergleichsmodellen erreicht.

Der bestandesübergreifende Dimensionstrend (Kapitel 2.3, Fußnote 4), daß Bäume in jungen Beständen im Mittel größere Kronenverhältnisse aufweisen als in alten Beständen, kann in ausgeprägter Form nur für die Nadelholzarten beobachtet werden. Der Vorteil des neu entwickelten statischen Modells beruht darauf, daß dieser bestandesübergreifende Trend über die Integration der Spitzenhöhe im Modell berücksichtigt wird. Gibt es nur einen schwach ausgeprägten bzw. keinen bestandesübergreifenden Dimensionstrend, so ist auch die Verbesserung mit Hilfe des neuen Kronenansatzmodells gering oder nicht vorhanden.

Das hierarchische Kronenansatzmodell weist für die untersuchten Baumarten fast die identischen Schätzgenauigkeiten auf, wie das einfache statische Kronenansatzmodell. Da das Modell jedoch den BHD als einzige Einzelbaumvariable benötigt, ist der Anspruch an die Datenqualität geringer als bei beim einfachen statischen Modell und den vier Vergleichsmodellen. Neben dem BHD werden die Bestandesvariablen Spitzenhöhe und mittlerer H/D-Wert als unabhängige Variablen zur Definition eines Einheitsmodells bzw. zur Verallgemeinerung verwendet.

Ein weiterer Vorteil der beiden neu entwickelten Modelle sind biologisch plausible Wirkungsrichtungen der verwendeten unabhängigen Variablen. Im Gegensatz zur Wirkungsrichtung des BHD in einigen Vergleichsmodellen, weist der BHD bei bestandesweiser Parametrisierung innerhalb der untersuchten Bestände häufig einen positiven Zusammenhang zum Kronenverhältnis auf. Da der bestandesübergreifende Dimensionstrend bereits durch die Verwendung der Spitzenhöhe berücksichtigt wird, hat der BHD in den neu entwickelten Modellen die positive Wirkungsrichtung, die innerhalb von Beständen zu beobachten ist. In den Vergleichsmodellen mit Ausnahme des Kronenansatzmodells in Forest (modifiziert) übernimmt die Einzelbaumvariable BHD die Beschreibung des allgemeinen Dimensionstrends. Der negative Zusammenhang zwischen BHD und Kronenverhältnis, der von diesen Modellen 
unterstellt wird, entspricht nicht dem Zusammenhang der bei bestandesweiser Betrachtung zu beobachten ist. Die Zusammenhänge innerhalb von Beständen werden bei diesen Modellen stellvertretend u. a. durch den H/D-Wert beschrieben, der jedoch bei einzelbestandsweiser Betrachtung eine deutlich geringere Korrelation zum Kronenverhältnis aufweist als der BHD.

Im Waldwachstumssimulator Bwinpro wird trotz der etwas höheren Anforderungen an die Qualität der Eingangsdaten das neu entwickelte, einfache statische Kronenansatzmodell (13) anstelle des hierarchischen Modells verwendet. Als Grund ist der zukünftig zu erwartende größere Anteil von vertikal stark strukturierten Beständen zu nennen. Die derzeitige Datenstruktur ist dadurch gekennzeichnet, daß aufgrund einer relativ schwach ausgeprägten Strukturierung der Bestände die Variable BHD bei bestandesweiser Betrachtung für die Kronenverhältnisschätzung ausreicht.

Wie in Kapitel 3.2.1 anhand eines künstlich erzeugten Femelbestandes dargestellt, kann die Beschreibung komplizierterer Bestandesstrukturen die Integration einer weiteren Einzelbaumvariablen im bestandesindividuellen Modell erfordern. Die Schätzung der Koeffizienten für einen speziellen Bestand mit Hilfe von Bestandesvariablen ist bei Verwendung von zwei Variablen innerhalb des bestandesindividuellen Modells durch statistische Abhängigkeiten der Koeffizienten erschwert. Dagegen können in das neu entwickelte, einfache statische Modell (13) über den BHD hinaus leicht weitere Einzelbaumvariablen integriert werden. In der jetzigen Modellform ist mit dem H/D-Wert bereits eine weitere Einzelbaumvariable integriert. Zum derzeitigen Zeitpunkt ist die Erhöhung der Schätzgenauigkeit, die durch die Integration erreicht wird, geringfügig. Das Modell ist aber bereits durch die Verwendung von zwei Einzelbaumvariablen so konzipiert, daß theoretisch auch stärker strukturierte Bestände beschrieben werden können.

Das einfache statische Modell weist zusätzlich den Vorteil auf, daß der Einfluß weiterer Einzelbaumvariablen mit Hilfe von Bäumen mit ähnlichem BHD und ähnlicher zugehöriger Spitzenhöhe, die nicht aus den gleichen Beständen stammen, besser untersucht werden kann. Bei der skizzierten Datenstruktur hat beispielsweise der H/D-Wert innerhalb von Beständen zusätzlich zum BHD in der Mehrzahl der Fälle keinen signifikanten Einfluß. Bei gesamthafter Betrachtung von Beständen mit sehr unterschiedlichen waldbaulichen Behandlungsmodellen, die in sich jedoch relativ homogen sind, kann der H/D-Wert dagegen einen signifikanten Einfluß aufweisen. Eine Ausdehnung der Anwendung auf stark strukturierte Bestände setzt allerdings voraus, daß eine große Anzahl derartiger Bestände im zur Parametrisierung verwendeten Datenmaterial enthalten ist. Da stark strukturierte Bestände im Datenmaterial fehlen, können das einfache statische Modell und vor allem das hierarchische Modell zum jetzigen Zeitpunkt nur theoretisch für eine Anwendung in strukturierten Beständen weiterentwickelt werden (Kapitel 4). 
Das dritte neu entwickelte Kronenansatzmodell dient einer besseren Ausnutzung von hochwertigen Eingangsinformationen. In vielen Anwendungsfällen von Wachstumsmodellen, wie dem Vergleich von waldbaulichen Szenarien, steht zumindest am Beginn der Simulation eine Anzahl an gemessenen Kronenansatzhöhen zur Verfügung, wenn auf Versuchs- oder Weiserflächen zurückgegriffen wird. Aber auch im Rahmen der Forsteinrichtung wäre es denkbar, bei den Höhenmessungen, die der Bonitierung eines Bestandes dienen, gleichzeitig die zugehörigen Kronenansatzhöhen zu messen. Das neu entwickelte Einheitskronenansatzmodell erfordert zusätzlich zu den bereits beschriebenen Modellen den Durchmesser und das Kronenverhältnis des Spitzenhöhenstammes als unabhängige Variablen. Das Modell kann Informationen wie eine Anzahl gemessener Kronenansatzhöhen oder das Kronenverhältnis des Spitzenhöhenstammes, das mit Hilfe von Messungen oder okularen Schätzungen bestimmt wurde, bei der Schätzung für alle Bäume eines Bestandes berücksichtigen. Liegt jedoch eine ausreichend große Anzahl von gemessenen Kronenansatzhöhen vor, so sollte die Schätzung mit Hilfe eines bestandesindividuell parametrisierten Regressionsmodells erfolgen. Das Einheitskronenansatzmodell kommt in allen Fällen zur Anwendung, in denen für ein solches Modell zu wenig Kronenansatzhöhen bekannt sind, jedoch mehr Informationen vorhanden sind, als für die Anwendung der Standardmodelle (einfaches statisches Modell (13), hierarchisches Modell, statische Vergleichsmodelle) benötigt werden.

Mit Hilfe des Einheitskronenansatzmodells lassen sich die Schätzungen der Kronenansatzhöhe gegenüber dem einfachen statischen Modell (13) und dem hierarchischen Modell noch einmal deutlich verbessern. Das gilt sowohl für die untersuchten Nadel- als auch besonders die Laubholzarten, für die nur geringe bzw. keine Verbesserungen mit Hilfe der neu entwickelten Standardmodelle erreicht wurden. Die Schätzfehler des Einheitskronenansatzmodells liegen für die untersuchten Baumarten nur geringfügig über den Schätzfehlern einer bestandesweisen Anpassung eines linearen Modells, das das Kronenverhältnis in Abhängigkeit vom BHD beschreibt.

Zusätzlich zum Einheitskronenansatzmodell wurde ein Modell zur dynamischen Fortschreibung des Kronenverhältnisses des Spitzenhöhenstammes entwickelt, um die hochwertigen Eingangsinformationen, die die Anwendung des Einheitskronenansatzmodells ermöglichen, über den Startpunkt einer Simulation hinaus berücksichtigen zu können. Die Fortschreibung des Kronenverhältnisses des Spitzenhöhenstammes ermöglicht somit eine Einhängung des Einheitskronenansatzmodells auch zu späteren Zeitpunkten. Für kurz- und mittelfristige Fortschreibungszeiträume bis ca. 11 Jahren wies das dynamisierte Einheitskronenansatzmodell höhere Genauigkeiten auf als das einfache statische Modell (13). Es konnte nachgewiesen werden, daß sich dieser Vorteil vor allem aus der hochwertigen Ausgangsinformation aber auch aus einer geringfügig besseren Schätzung der Veränderungsrate zusammensetzt. Bezüglich der Anforderungen an die Datenqualität wird das dynamisierte Einheitskronenansatzmo- 
dell zwischen den einfachen statischen Modellen und dem Einheitskronenansatzmodell eingestuft. Das Einheitskronenansatzmodell (14) benötigt zu jedem Zeitpunkt Informationen über das Kronenverhältnis des Spitzenhöhenstammes $\left(K V_{H 100}\right)$, während die übrigen statischen Modelle (1)- (5) sowie (13) diese Variable nicht verwenden. Das dynamisierte Einheitskronenansatzmodell benötigt zum Zeitpunkt der Kronenansatzhöhenschätzung selbst keine direkt erhobene Information über das Kronenverhältnis des Spitzenhöhenstammes, jedoch muß diese Information in einem kurz- bis mittelfristigen Zeitraum vor dem Zeitpunkt der Schätzung erhoben worden sein.

Im dynamisierten Einheitskronenansatzmodell sollten die unabhängigen Variablen, die die Schätzung der Veränderungsrate des Kronenverhältnisses des Spitzenhöhenstammes beeinflussen, in kurzen Simulationsschritten aktualisiert werden. Simulationszeiträume von über 10 Jahren, die in einem Schritt ohne Aktualisierung der unabhängigen Variablen berechnet werden, führen dazu, daß das dynamisierte Einheitskronenansatzmodell in diesen Fällen deutlich größere Ungenauigkeiten aufweist als das einfache statische Modell (13). Genauere Untersuchungen der Zusammenhänge, die die Schätzgenauigkeit des dynamisierten Einheitsschaftformmodells beeinflussen, wurden nur für Baumart Fichte durchgeführt.

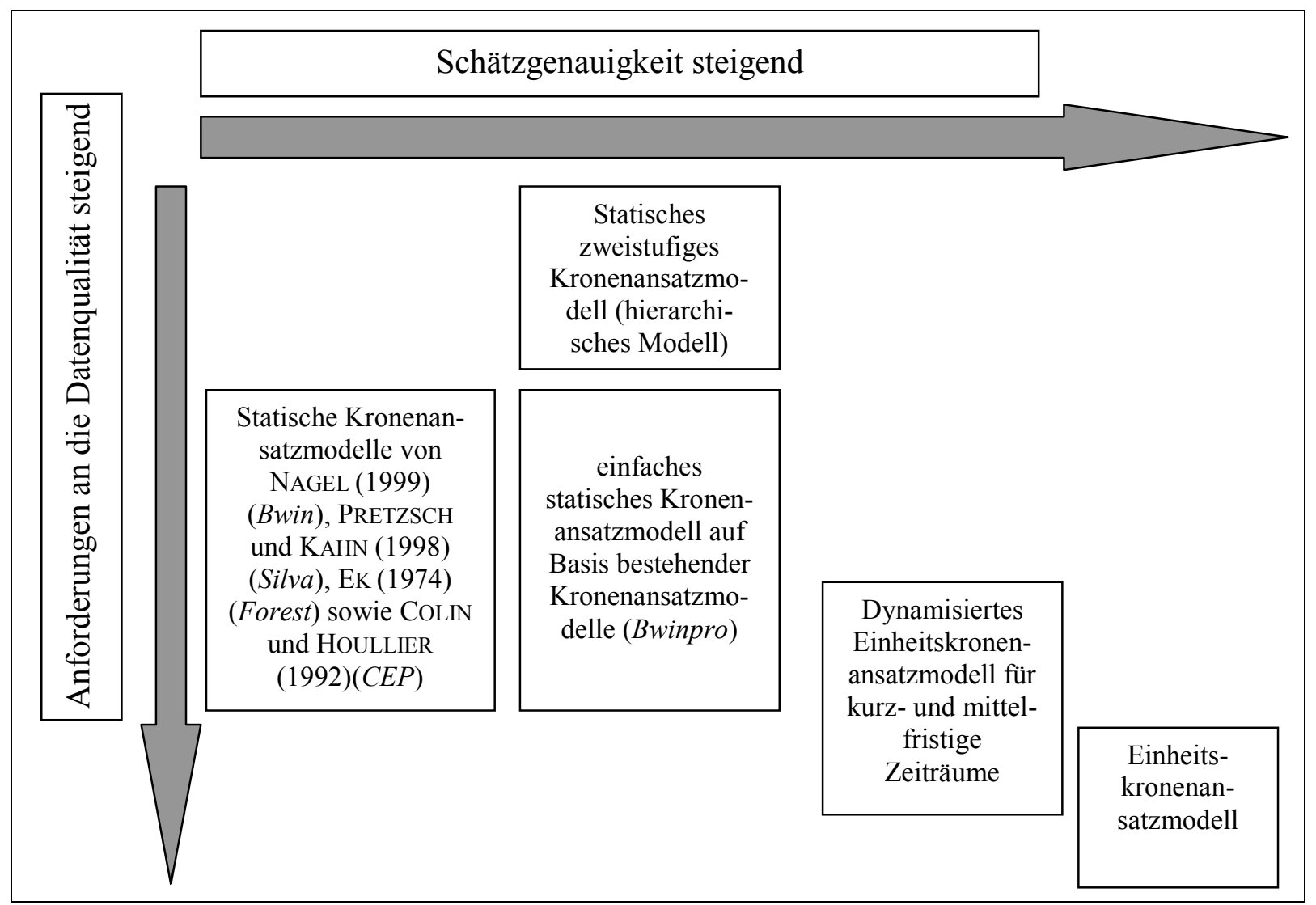

Abb. 31: Einordnung neu entwickelter Modelle und statischer Vergleichsmodelle zur Schätzung der Kronenansatzhöhe unter Berücksichtigung der Datenqualität und der Schätzgenauigkeit. 


\subsection{Modelle zur Durchmesserschätzung aller Quirläste bei Nadelholzarten}

\subsubsection{Durchmesser der stärksten Quirläste}

Wie bereits in Kapitel 2.4.3 erläutert wurde, haben die zur Beschreibung aller Quirläste eines Baumes häufig eingesetzten segmentierten und nicht-segmentierten Polynomialgleichungen den Nachteil, daß die Wirkungsrichtung der verwendeten unabhängigen Variablen nicht eindeutig interpretierbar ist. Weiterhin müßte untersucht werden, inwieweit die statischen Astdurchmessermodelle im Rahmen von Wachstumssimulationen konsistente Werte schätzen, d. h. ob sich biologisch plausible Zeitreihenwerte für einen Ast ergeben. Auch unter dieser Fragestellung sind die nicht eindeutigen Wirkungsrichtungen und Interaktionen der unabhängigen Variablen als nachteilig zu beurteilen.

Die beschriebenen Modelle betrachten die einzelnen Beobachtungen als unabhängig, obwohl sie untereinander korreliert sind, da es sich um Clusterstichproben handelt. So stammen immer Gruppen von Beobachtungen von einem Baum und es kommen überwiegend mehrere Bäume aus einem Bestand. In Abb. 32 ist dargestellt, wie sich für die Baumart Fichte die Durchmessermessungen der stärksten Quirläste innerhalb der grünen Krone auf verschiedene Versuchsflächen aufteilen ${ }^{13}$. Die Messungen stammen fast ausschließlich aus dem Bereich des Sollings und des Harzes.

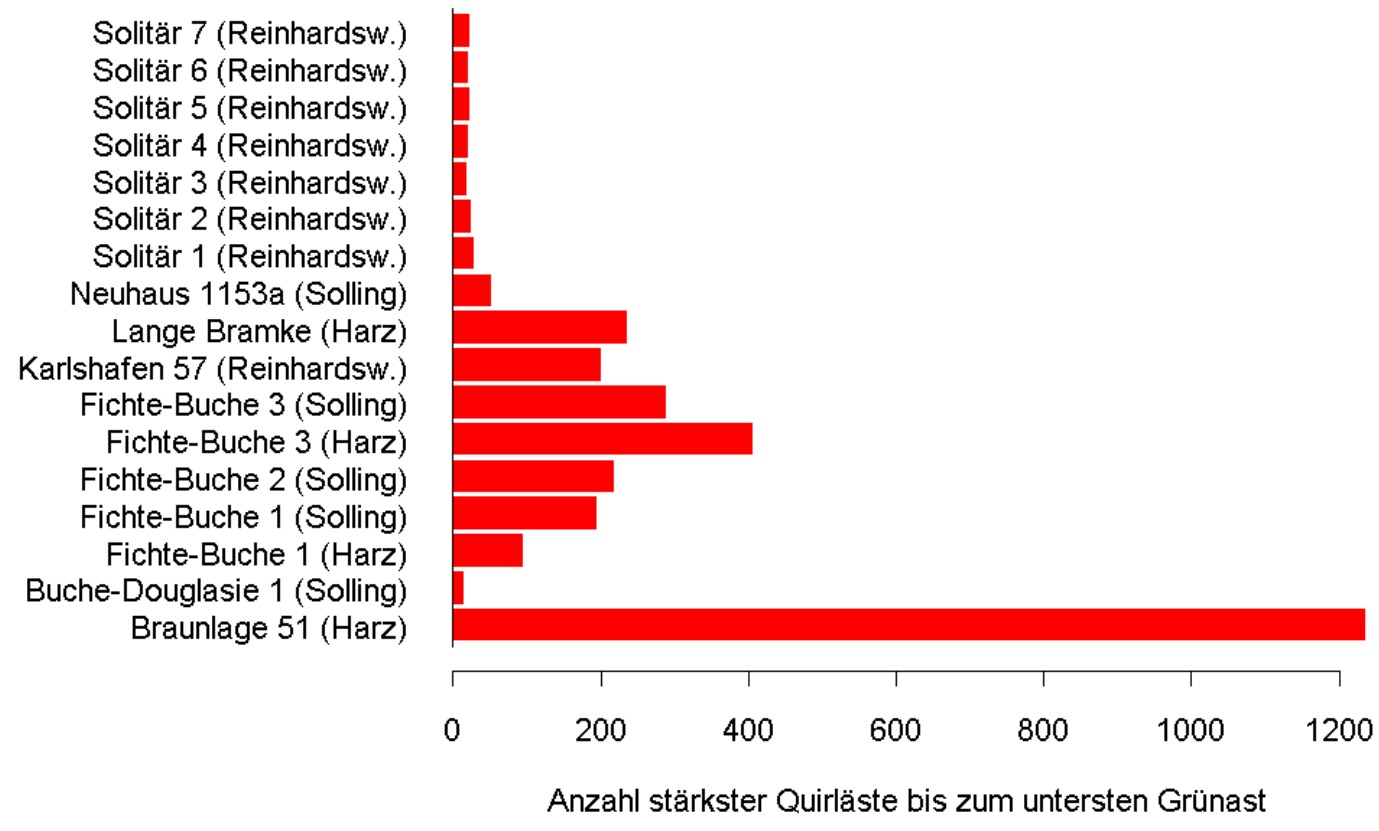

Abb. 32: Verteilung der Astdurchmessermessungen der stärksten Quirläste innerhalb der grünen Krone gegliedert nach Versuchsflächen bzw. Abteilungen für die Baumart Fichte (bei den Solitären handelt es sich um Einzelbäume, die keinen Versuchsflächen zugeordnet werden können).

\footnotetext{
${ }^{13}$ Für die Fläche Braunlage 51 wurde keine Untergliederung nach Versuchsparzellen vorgenommen.
} 
Die Astdurchmesser weisen daher eine Datenstruktur auf, die derjenigen der Sektionsmessungen (Kapitel 3.7) sehr ähnlich ist. Hinzu kommt bei beiden Datenarten die enge Korrelation zwischen den Beobachtungen an einem Baum, die in der Ausbildung von bestimmten $\mathrm{Mu}-$ stern, wie den stetig monoton fallenden Schaftdurchmesserwerten mit steigender Baumhöhe und dem Ansteigen der Astdurchmesser bis zu einem Maximum und dem anschließenden Absinken der Astdurchmesser mit steigender Tiefe in der Krone ${ }^{14}$ deutlich wird. Dabei sind Abweichungen von bestimmten Datenmustern bei der Struktur der Astdurchmesser eines Baumes allerdings in deutlich stärkerem Maße als bei den Sektionsmessungen möglich. Zusätzlich weisen die Astdurchmesser eine höhere Residualstreuung um die wahren bedingten Mittelwerte über der Tiefe in der Krone auf (Abb. 33).

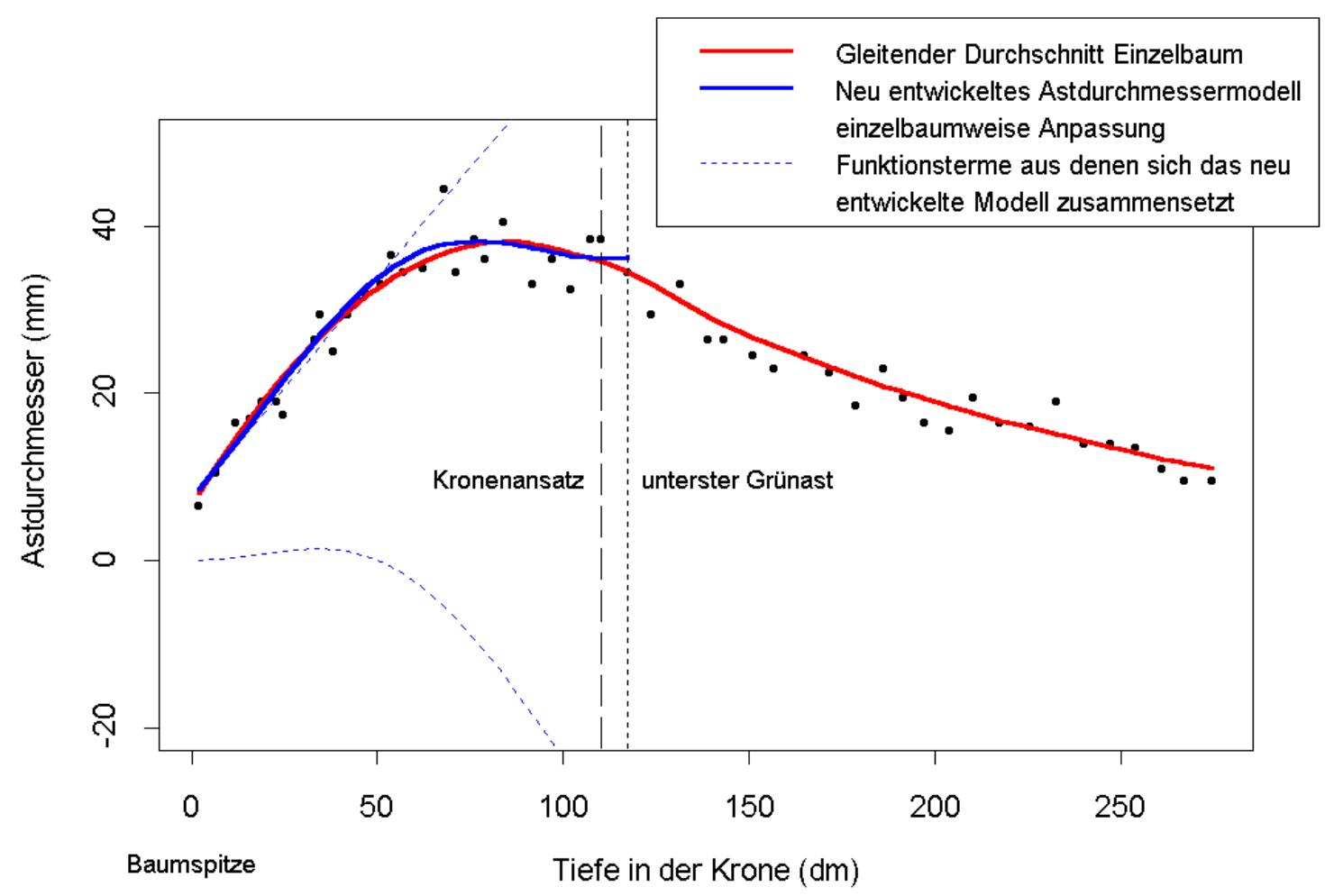

Abb. 33: Verlauf der Astdurchmesser der stärksten Quirläste einer Fichte mit einem BHD von $30.5 \mathrm{~cm}$ und einer Höhe von $29.6 \mathrm{~m}$ über der Tiefe in der Krone. Zur Verdeutlichung des mittleren Verlaufs und der Residualstreung wurde ein Gleitendes-DurchschnittsModell verwendet (rot) (Kapitel 8.1). Die schwarz gestrichelten Linien markieren die Position des Kronenansatzes und des untersten Grünastes. Zusätzlich ist die einzelbaumweise Anpassung des neu entwickelten Astdurchmessermodells (19) (blau) und die Zerlegung der Funktion in die Terme, aus denen sie sich zusammensetzt, dargestellt (blau-gestrichelt).

Aufgrund der Ähnlichkeit der Datenstruktur von Astdurchmessern und Sektionsmessungen wurde ein neuer Modelltyp für die Baumarten Fichte, Douglasie und Kiefer entwickelt und

\footnotetext{
${ }^{14}$ Die bereits in Kapitel 2.4 beschriebene Variable Tiefe in der Krone drückt den vertikalen Abstand eines Quirles bzw. Astes von der Baumspitze aus.
} 
parametrisiert, der den in Kapitel 3.7 verwendeten parameterkargen Schaftformfunktionen ähnlich ist. Insbesondere mit den unterschiedlichen Varianten der Pain-Funktion bestehen große Übereinstimmungen. Das neue Modell hat den Vorteil, daß die Wirkungsrichtung der unabhängigen Variablen eindeutig interpretierbar ist. Im Gegensatz zu den Polynomialgleichungen wird dabei der grundsätzliche Verlauf der Astdurchmesserstruktur durch die Wahl der Funktion vorgegeben (Formel (19)) und damit die Autokorrelation der Messungen eines Baumes berücksichtigt.

$$
\begin{aligned}
B D_{\text {max }}= & \left(a_{0}+a_{1} * \text { TINK }\right)+\left(a_{2}+a_{3} * \text { TINK }\right) \\
& *\left(1-\left(1-\text { TINK }_{\text {rel }}\right)^{\left.a_{4} * T_{N} K_{\text {rel }}\right)}\right.
\end{aligned}
$$

wobei:

$B D_{\max }=$ mittlerer Durchmesser des stärksten Quirlastes $(\mathrm{mm})$;

TINK = Tiefe in der Krone, wobei sich der Parametrisierungsbereich nur bis zum untersten Grünast erstreckt (dm);

$T I N K_{\text {rel }}=T I N K$ in Relation zur Gesamtkronenlänge (bis zum untersten Grünast).

Eine einzelbaumweise Anpassung der Funktion an gemessene Astdurchmesser ist in Abhängigkeit von den unabhängigen Variablen Tiefe in der Krone und relativer Tiefe in der Krone möglich. Die Flexibilisierung bzw. die Beschreibung für unterschiedliche Einzelbäume erfolgt, indem die Modellkoeffizienten als Funktion von Einzelbaumvariablen geschätzt werden (Verallgemeinerung des Modells). Da nur einem Teil der Datensätze Bestandesvariablen zugeordnet werden konnten und aufgrund der Erfahrungen, daß Bestandes- und Standortsvariablen häufig keinen signifikanten Einfluß auf die Astdurchmesserstruktur haben, wenn die aggregierende Variable Tiefe in der Krone verwendet wird (Kapitel 2.4.3, MAGUIRE ET AL. 1991), wurde der Einfluß von Variablen höherer Hierarchiestufen nicht untersucht (Kapitel 3.7.4). Außerdem wären für derartige Untersuchungen auch bedeutend größere Datenmengen aus einer Vielzahl von Beständen mit unterschiedlichen waldbaulichen Behandlungen und Standortseigenschaften notwendig gewesen.

Alle neu entwickelten Astmodelle werden anhand der Baumart Fichte beschrieben. Für die Baumarten Douglasie und Kiefer werden für einen Teil der Modelle die zugehörigen Ergebnisse in Kapitel 9.5 erläutert. In Abb. 33 ist die einzelbaumweise Anpassung des Modells (19) dargestellt. Aufgrund der Modellform erlaubt das Modell nur die Schätzung von Werten, die kleiner oder gleich dem Wert sind, der zur Normierung der Variablen Tiefe in der Krone verwendet wurde (hier: Tiefe in der Krone bis zum untersten Grünast). Im Gegensatz zu den unterschiedlichen Varianten der Pain-Funktion wies nur ein Teil der Parameter $\left(\mathrm{a}_{0}\right.$ und $\left.\mathrm{a}_{2}\right)$ der Grundfunktion (19) eine Variation über den Einzelbaumvariablen BHD und H/D-Wert auf. Modell (20) entsteht aus (19), indem die Parameter $\mathrm{a}_{0}$ und $\mathrm{a}_{2}$ über die Einzelbaumvariablen BHD und H/D-Wert geschätzt werden. Parameter des Modells (19), die nicht als Funktion 
von Einzelbaumvariablen ausgedrückt wurden, sind weiterhin mit dem Buchstaben $a$ plus Index bezeichnet.

$$
\begin{aligned}
B D_{\text {max }}= & \left(b_{0} * D+a_{1} * T I N K\right)+\left(b_{2} * D+b_{3} *(1 / H D)+a_{3} * \text { TINK }\right) \\
& *\left(1-\left(1-\text { TINK }_{\text {rel }}\right)^{\left.a_{4} * T K_{\text {rel }}\right)}\right.
\end{aligned}
$$

wobei:

$B D_{\max }=$ Durchmesser des stärksten Quirlastes (mm);

TINK = Tiefe in der Krone, wobei sich der Parametrisierungsbereich nur bis zum untersten Grünast erstreckt (dm);

$T I N K_{\text {rel }}=T I N K$ in Relation zur Gesamtkronenlänge (bis zum untersten Grünast);

$D \quad=$ Brusthöhendurchmesser $(\mathrm{mm})$;

$H \quad=$ Baumhöhe $(\mathrm{dm})$;

$H D=\mathrm{H} / \mathrm{D}-$ Wert.

\begin{tabular}{|c|c|c|c|}
\hline Koeffizient & Wert & Std. Fehler & t-Wert \\
\hline$a_{1}$ & 0.431 & 0.00854 & 50.459 \\
\hline$a_{3}$ & -0.447 & 0.00754 & -59.272 \\
\hline$a_{4}$ & 1.844 & 0.0875 & 21.079 \\
\hline$b_{0}$ & 0.0277 & 0.000903 & 30.660 \\
\hline$b_{2}$ & 0.0399 & 0.00245 & 16.320 \\
\hline$b_{3}$ & 10.352 & 0.508 & 20.388 \\
\hline Residual Std. Fehle & \multicolumn{3}{|c|}{3.821 bei 3072 Freiheitsgraden } \\
\hline
\end{tabular}

mit:

Tab. 13: Koeffizienten und statistische Kenngrößen zur Durchmesserschätzung der stärksten Quirläste innerhalb der grünen Krone mit Hilfe des neu entwickelten Modells (20) für die Baumart Fichte.

\section{Validierung und Bewertung des Modells zur Beschreibung der Durchmesser der stärk- sten Quirläste:}

Auf eine direkte Modellierung der Totastzone, wie sie mit den Modellen von MAGUIRE ET AL. (1994) sowie COLIN und HoULLIER (1991) möglich ist, wurde bewußt verzichtet. Es wird davon ausgegangen, daß in erster Linie die Astdurchmesserstruktur innerhalb der Grünastzone in einem Zusammenhang mit den aktuellen Einzelbaumvariablen steht. Je nach den Wuchsbedingungen des Baumes in der Vergangenheit, sind unterschiedliche Strukturen der Durchmesser in der Totastzone bei den gleichen aktuellen Einzelbaumparametern denkbar. Eine direkte Modellierung der Durchmesser in der Totastzone vernachlässigt somit Unterschiede in der Entwicklung der Wuchsbedingungen von Bäumen, die heute dieselben oder ähnliche Eigenschaften aufweisen. Zur Modellierung der Totastzone im Rahmen von waldbaulichen Szenariensimulationen mit Hilfe des neu entwickelten Astdurchmessermodells erhält jeder Totast den Durchmesser, den er zum Zeitpunkt des Absterbens aufwies. Durch diese Vorgehensweise wird berücksichtigt, daß die Astdurchmesser in der Totastzone durch die zum Zeitpunkt 
des Absterbens aktuellen Baumeigenschaften bestimmt werden. Die in Abb. 8 (rechts) dargestellte Verjüngung des Astdurchmessers in Richtung des Marks kann durch die in 1Jahresschritten berechneten Astdurchmesser im Rahmen einer Waldwachstumssimulation beschrieben werden. Wie bei allen statischen Modellen ist hierbei zu überprüfen, inwieweit auch im Rahmen einer dynamischen Anwendung plausible Werte geschätzt werden. D. h., ein Astdurchmesser kann mit steigendem radialen Alter höchstens konstant bleiben nicht aber abnehmen.

In Abb. 34 sind die Streudiagramme der Residuen und beobachteten Astdurchmesser über geschätzten Astdurchmessern für das neu entwickelte Astdurchmessermodell dargestellt. Abgesehen von einer leichten Überschätzung im Bereich starker Äste weist das Modell keine systematischen Schätzfehler auf. Bei der Beurteilung der systematischen Überschätzung im Bereich starker Äste ist die geringe Datengrundlage für diesen Bereich zu berücksichtigen.
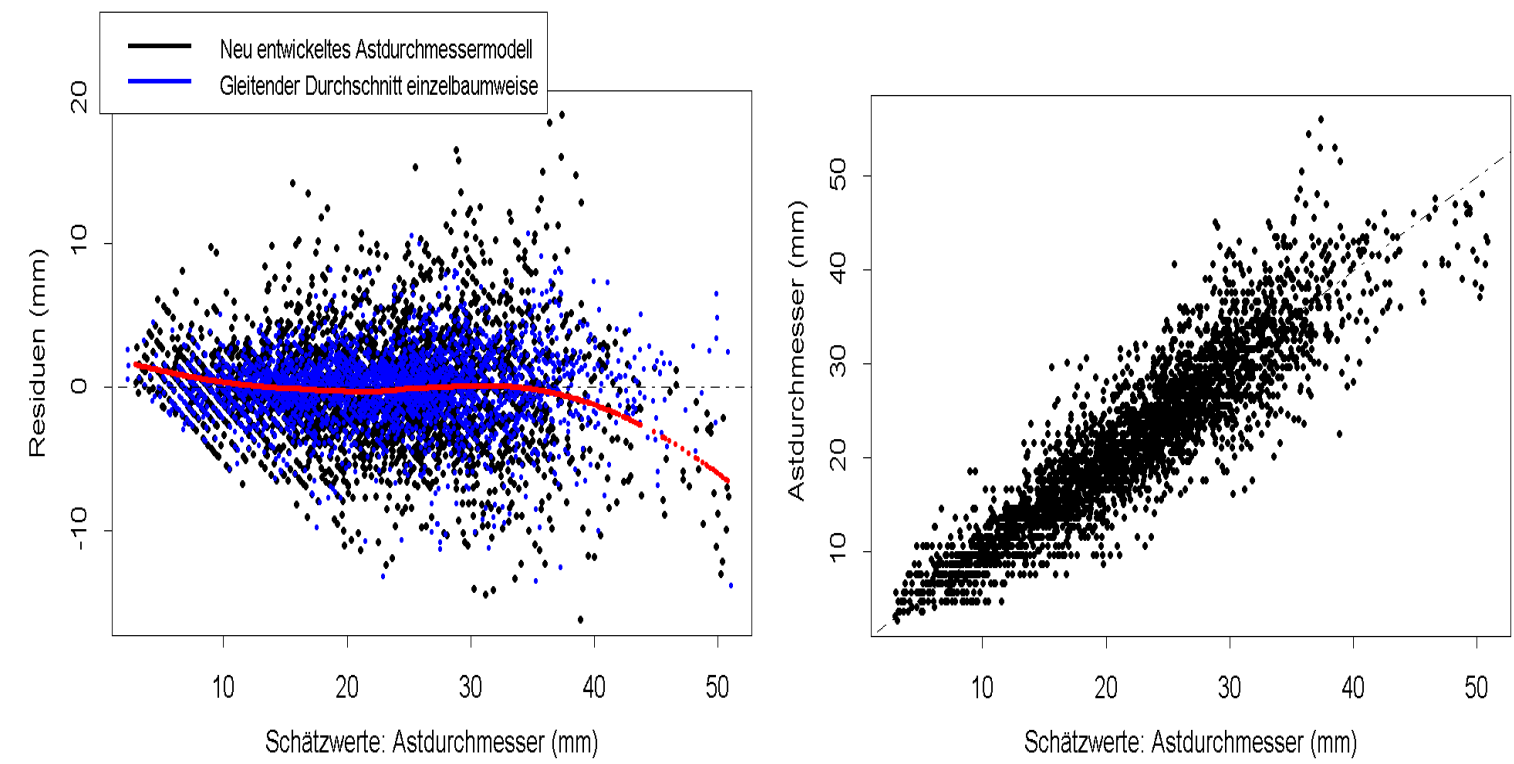

Abb. 34: Validierungsplots für das neu entwickelte Astdurchmessermodell. Im Residuenplot sind zusätzlich die Vergleichswerte bei einzelbaumweiser Anpassung eines GleitendenDurchschnitts-Modells dargestellt (Kapitel 8.1). Im Residuenplot ist zur Verdeutlichung von systematischen Fehlern eine Trendlinie (rot) für das neu entwickelte Astdurchmessermodell mit Hilfe eines Gleitenden-Durchschnitts-Modells geschätzt worden.

Im Gegensatz zu den beschriebenen Polynomialgleichungen haben die Variablen BHD und H/D-Wert eindeutige Wirkungsrichtungen innerhalb des Modells. Bei gleicher Tiefe in der Krone und gleicher relativer Tiefe in der Krone ist ein stärkster Quirlast umso stärker je größer der BHD des Baumes und je niedriger der H/D-Wert des Baumes ist. Die Variable Tiefe in der Krone hat im ersten Term, der die Astdurchmesserschätzung bis zum Maximum dominiert, einen positiven Einfluß auf den Astdurchmesser. Im zweiten Term der Funktion, der die Lage des Astdurchmessermaximums und das Absinken der Astdurchmesser nach dem Erreichen des Maximalwertes beeinflußt, hat die Variable Tiefe in der Krone einen negativen Ein- 
fluß auf den Astdurchmesser. Dabei wird der Punkt des maximalen Astdurchmessers bei einer umso größeren Tiefe in der Krone erreicht, je größer der BHD und je niedriger der H/D-Wert ist. Mit der Berücksichtigung des H/D-Wertes ist gewährleistet, daß das Astdurchmessermodell behandlungssensitiv reagiert. Da weite Ausgangsverbände und/oder starke Eingriffe zu einer Förderung des Durchmesserwachstums führen, ist bei derartigen waldbaulichen Behandlungsszenarien mit niedrigeren H/D-Werten als in eng begründeten und/oder schwach durchforsteten Beständen zu rechnen. Weitständig erzogene Bäume weisen stärkere Astdurchmesser als engständig erzogene auf. Da der H/D-Wert innerhalb des Modells einen positiven Einfluß auf den Astdurchmesser hat, ist eine biologisch plausible Behandlungssensitivität gewährleistet. Eine Voraussetzung für eine behandlungssensitive Prognose ist weiterhin, daß das verwendete Wachstumsmodell möglichst behandlungssensitive Zuwachsfunktionen für das Durchmesser- und das Höhenwachstum besitzt.

Abb. 35 zeigt die Verteilungen der zur Flexibilisierung verwendeten Variablen BHD, H/D-Wert und Kronenlänge der 155 Fichten, die zur Parametrisierung des Astdurchmessermodells verwendet wurden.
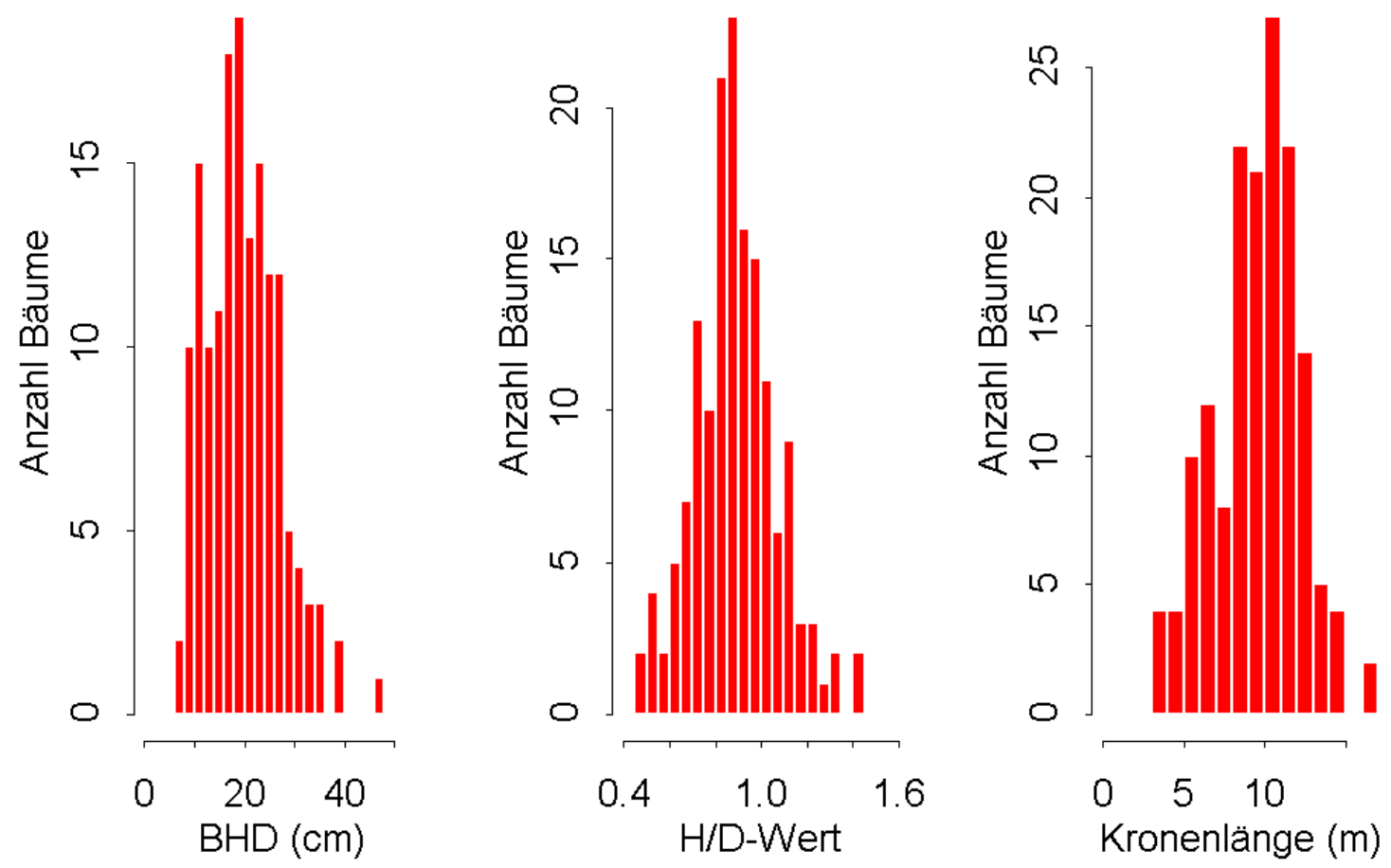

Abb. 35: BHD-, H/D-Wert- und Kronenlängenverteilungen für 155 Fichten, die zur Entwicklung des Modells zur Durchmesserschätzung der stärksten Quirläste verwendet wurden.

In Abb. 36 wird der Einfluß des BHD und des H/D-Wertes auf die Astdurchmesserstruktur der stärksten Quirläste innerhalb der grünen Krone anhand von Beispielkombinationen der unabhängigen Variablen verdeutlicht. Um die Wirkungsrichtungen und die Einflußstärke der einzelnen unabhängigen Variablen darzustellen, werden der BHD bzw. der H/D-Wert variiert und die übrigen unabhängingen Variablen konstant belassen. Für die Kombination BHD 
$15 \mathrm{~cm}$ und H/D-Wert 0.4 und der resultierenden Baumhöhe von $6 \mathrm{~m}$ ist nur eine maximale Kronenlänge von $6 \mathrm{~m}$ möglich. Wie bei den flexiblen Schaftformmodellen gilt, daß das Astdurchmessermodell nicht im Extrapolationsbereich eingesetzt werden sollte. Trotzdem erscheinen die Schätzungen für die Beispielkombinationen mit einem BHD von $60 \mathrm{~cm}$ bzw. einem H/D-Wert von 0.4, die außerhalb des zur Parametrisierung verwendeten Datenbereiches liegen, vom Trend her (qualitativ) biologisch plausibel. Für den Extrapolationsbereich kann jedoch nicht beurteilt werden, inwieweit die Schätzwerte vom Betrag her (quantitativ) eine realitätsnahe Prognose darstellen.

Für die Abgrenzung des Extrapolationsbereiches müssen bei der Verwendung mehrerer unabhängiger Variablen auch die vorhandenen und nicht vorhandenen Kombinationen der Variablen innerhalb des Datenmaterials betrachtet werden.

In Abb. 37 sind die Auswirkungen unterschiedlicher Kronenlängen bei verschiedenen BHD-H/D-Wert-Kombinationen dargestellt. Dabei beeinflußt die Kronenlänge bei sonst gleichen Baumvariablen auch den Astdurchmesserverlauf im oberen Kronenbereich. Diese Aussage gilt vor allem für Kombinationen mit großen BHD- und/oder niedrigen H/D-Werten. So weisen derartige Kombinationen bei kürzeren Kronen einen deutlich steileren Anstieg der Astdurchmesser auf als bei längeren Kronen. $\mathrm{Ob}$ es sich bei diesen, durch das neu entwickelte Modell beschriebenen, Unterschieden um real zu beobachtende, biologisch plausible Verläufe handelt, kann in dieser Untersuchung aufgrund der geringen bzw. nicht vorhandenen Datengrundlage für derartige Variablen-Kombinationen noch nicht endgültig geklärt werden. Die durch das Datenmaterial gut repräsentierten schwächeren BHD-Bereiche bis $30 \mathrm{~cm}$ und die mittleren H/D-Wert-Bereiche weisen die durch unterschiedliche Kronenlängen verursachten Unterschiede im Astdurchmesserverlauf im oberen Kronenbereich überhaupt nicht oder nur schwach ausgeprägt auf (Abb. 37). Daher kann vermutet werden, daß diese Unterschiede des Astdurchmesserverlaufs zumindest zum Teil durch die Anwendung des Modells im Extrapolationsbereich verursacht werden. 


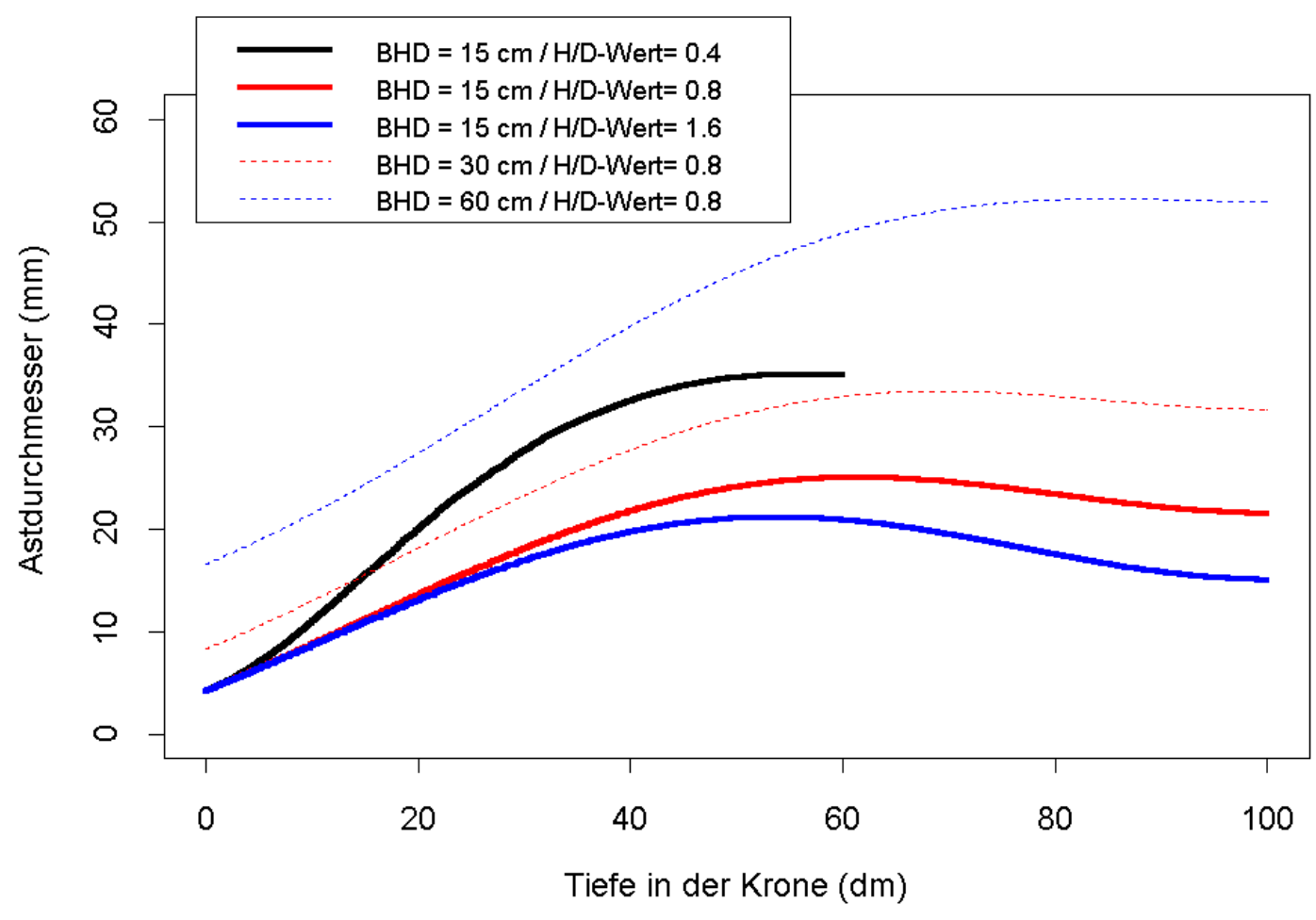

Abb. 36: Einflu $\beta$ der unabhängigen Variablen BHD und H/D-Wert auf die Astdurchmesserstruktur der stärksten Quirläste in der grünen Krone von Fichten mit konstanten Kronenlänge von $10 \mathrm{~m}$ bzw. $6 \mathrm{~m}$ (bis zum untersten Grünast).

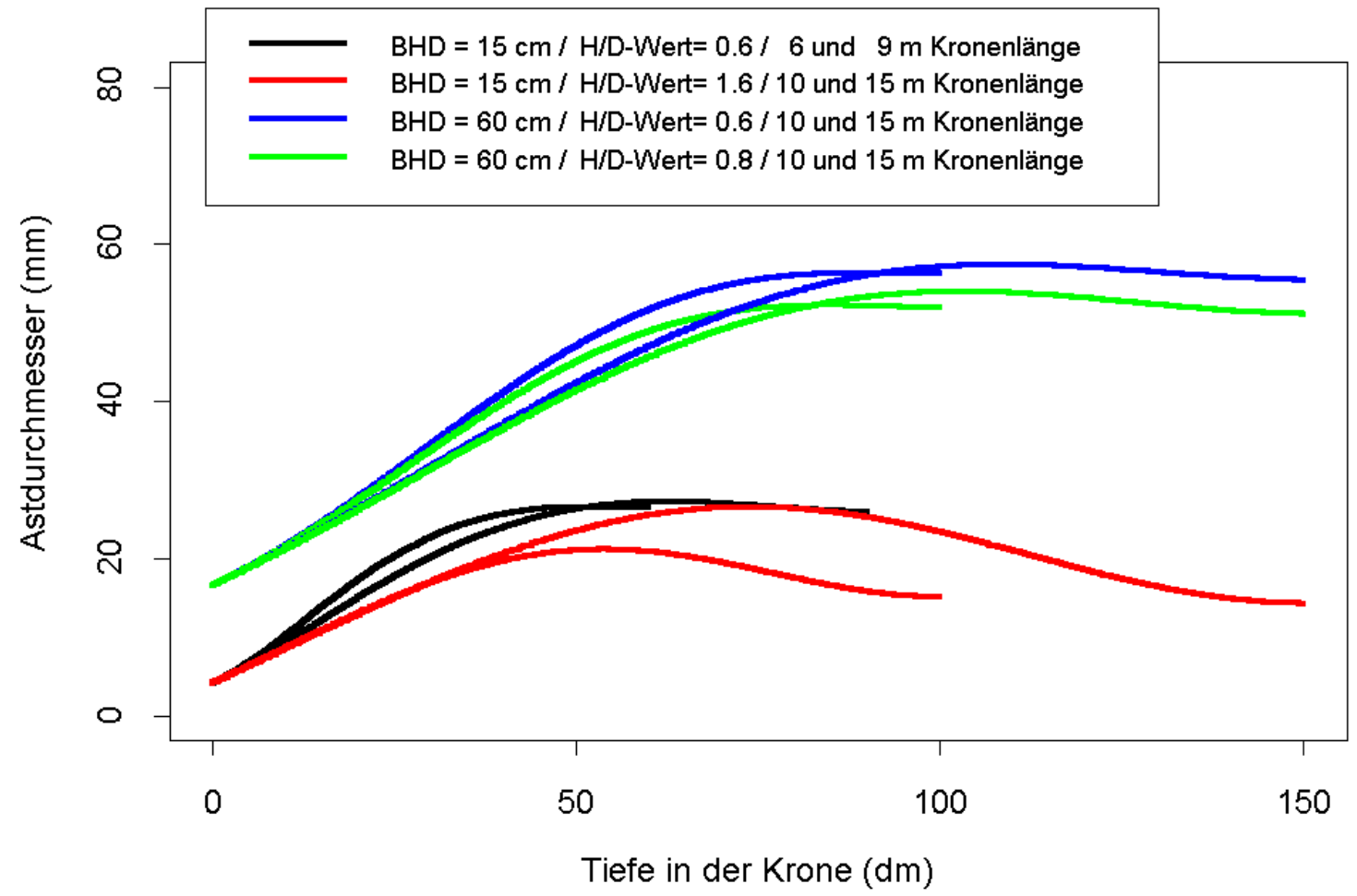

Abb. 37: Einfluß der unabhängigen Variablen Kronenlänge (10 und $15 \mathrm{mbzw} .6$ und $9 \mathrm{~m}$ ) auf die Astdurchmesserstruktur der stärksten Quirläste in der grünen Krone (bis zum untersten Grünast) von Fichten mit unterschiedlichen BHD-H/D-Wert-Kombinationen. 
Zum Vergleich bzw. zur Validierung des neu entwickelten Modells wurde das von MAGUIRE ET AL. (1994) entwickelte Modell ebenfalls mit Hilfe der Fichtenastdaten parametrisiert (Formel (7)). Zusätzlich wurde einzelbaumweise ein Gleitendes-Durchschnitts-Modell als optimales Referenzmodell angepaßt (Kapitel 8.1). Die Koeffizientenschätzungen und statistischen Kenngrößen sind für beide Vergleichsmodelle in Tab. 14 angegeben.

\begin{tabular}{|c|c|c|c|}
\hline Koeffizient & Wert & Std. Fehler & t-Wert \\
\hline$b_{0}$ & 7.129 & $1.887 * 10^{-1}$ & 37.772 \\
\hline$b_{1}$ & $1.494 * 10^{-1}$ & $8.114 * 10^{-3}$ & 18.411 \\
\hline$b_{2}$ & $1.278^{*} 10^{-3}$ & $7.537 * 10^{-5}$ & 16.959 \\
\hline$b_{3}$ & $-1.440 * 10^{-6}$ & $7.197 * 10^{-7}$ & -2.000 \\
\hline$b_{4}$ & $2.553 * 10^{-4}$ & $1.072 * 10^{-4}$ & 2.382 \\
\hline$b_{5}$ & $-8.060 * 10^{-6}$ & $1.096 * 10^{-6}$ & -7.355 \\
\hline$b_{6}$ & $-1.699 * 10^{-5}$ & $1.288 * 10^{-6}$ & -13.198 \\
\hline \multicolumn{2}{|c|}{ Residual Std. Fehler (mm) } & \multicolumn{2}{|c|}{4.007 bei 3071 Freiheitsgraden } \\
\hline \multicolumn{2}{|c|}{$\begin{array}{r}\text { Residual Std. Fehler bei einzelbaumweiser Anpassung mit } \\
\text { einem Gleitenden-Durchschnitts-Modell (mm) }\end{array}$} & \multicolumn{2}{|r|}{2.501} \\
\hline
\end{tabular}

Tab. 14: Koeffizienten und statistische Kenngrößen zur Durchmesserschätzung der stärksten Quirläste innerhalb der grünen Krone mit Hilfe des von MAGUIRE ET AL. (1994) entwikkelten Modells (7) und der einzelbaumweisen Anpassung mit Hilfe eines GleitendenDurchschnitts-Modells für die Baumart Fichte. Der iterativ ermittelte gemeinsame Funktionswert beider Segmente des Modells von MAGUIRE ET AL. liegt bei einer Tiefe in der Krone von $20 \%$ der Kronenlänge (vgl. Kapitel 2.4.3).

Das von MaguiRe ET AL. (1994) entwickelte Modell weist gegenüber dem neu entwickelten Astdurchmessermodell einen etwas höheren mittleren Fehler auf. Der Fehler des einzelbaumweise angepaßten Gleitenden-Durchschnitts-Modells kann als Schätzung für den Minimalwert des Fehlers interpretiert werden, den ein Verallgemeinertes Modell erreichen kann.

Bei den Astdurchmessermessungen handelt es sich, wie bei den Sektionsmessungen, um Daten, die gruppenweise einzelnen Bäumen zugeordnet werden können. Daher bietet sich zusätzlich zu den üblicherweise verwendeten Validierungskriterien eine Berechnung des RMSE getrennt nach Einzelbäumen an. Wie bei den Sektionsmessungen wurden die Residuen vor der Berechnung des jeweiligen Einzelbaumfehlers mit dem wahren Astdurchmesser normiert, um Bäume verschiedener Dimensionen besser miteinander vergleichen zu können (Abb. 38). Es wird deutlich, daß das neu entwickelte Astdurchmessermodell an den Randbereichen der Verteilungen der zur Flexibilisierung verwendeten Variablen BHD und H/D-Wert eine höhere Schätzgenauigkeit als das Modell von MAGUIRE ET. AL. (1994) aufweist. Bei der Beurteilung der Fehlerwerte ist zu beachten, daß speziell der Bereich von BHD-Werten oberhalb von $30 \mathrm{~cm}$ und der Bereich von H/D-Werten oberhalb von 1.2 nur schlecht in den Parametrisierungsdaten repräsentiert ist. Da davon ausgegangen werden kann, daß das einzelbaumweise parametrisierte Gleitende-Durchschnitts-Modell keine Verzerrung für bestimmte 
BHD- und H/D-Wert-Bereiche aufweist, muß die Ursache für den nicht abzissen-parallelen Verlauf des bedingten mittleren Fehlers in der Struktur der Residuen liegen. Abweichungen von einem nicht abzissen-parallelen Verlauf sind - abgesehen von Verzerrungen - auch dadurch zu erklären, daß die mit dem wahren Astdurchmesser normierten Residuen eine uneinheitliche Streuung in den unterschiedlichen BHD- und H/D-Wert-Bereichen aufweisen. Das neu entwickelte Astdurchmessermodell weist in den durch die Daten gut repräsentierten BHD- und H/D-Wert-Bereichen einen zum einzelbaumweise angepaßten Modell fast parallelen Verlauf des bedingten mittleren Fehlers auf. Der nicht abszissen-parallele Verlauf des mittleren bedingten Fehlers des neu entwickelten Modells kann daher ebenfalls auf die uneinheitliche Streuung der normierten Residuen zurückgeführt werden. Verzerrte Schätzungen für Teilbereiche der BHD- und H/D-Wert-Verteilungen liegen erst dann vor, wenn der mittlere bedingte Schätzfehler nicht mehr oder weniger parallel zum Fehler des unverzerrten einzelbaumweise angepaßten Modells verläuft. Danach weist das von MAGUIRE ET AL. (1994) entwickelte Modell im Bereich schwacher BHD und hoher H/D-Werte systematische Schätzfehler auf.
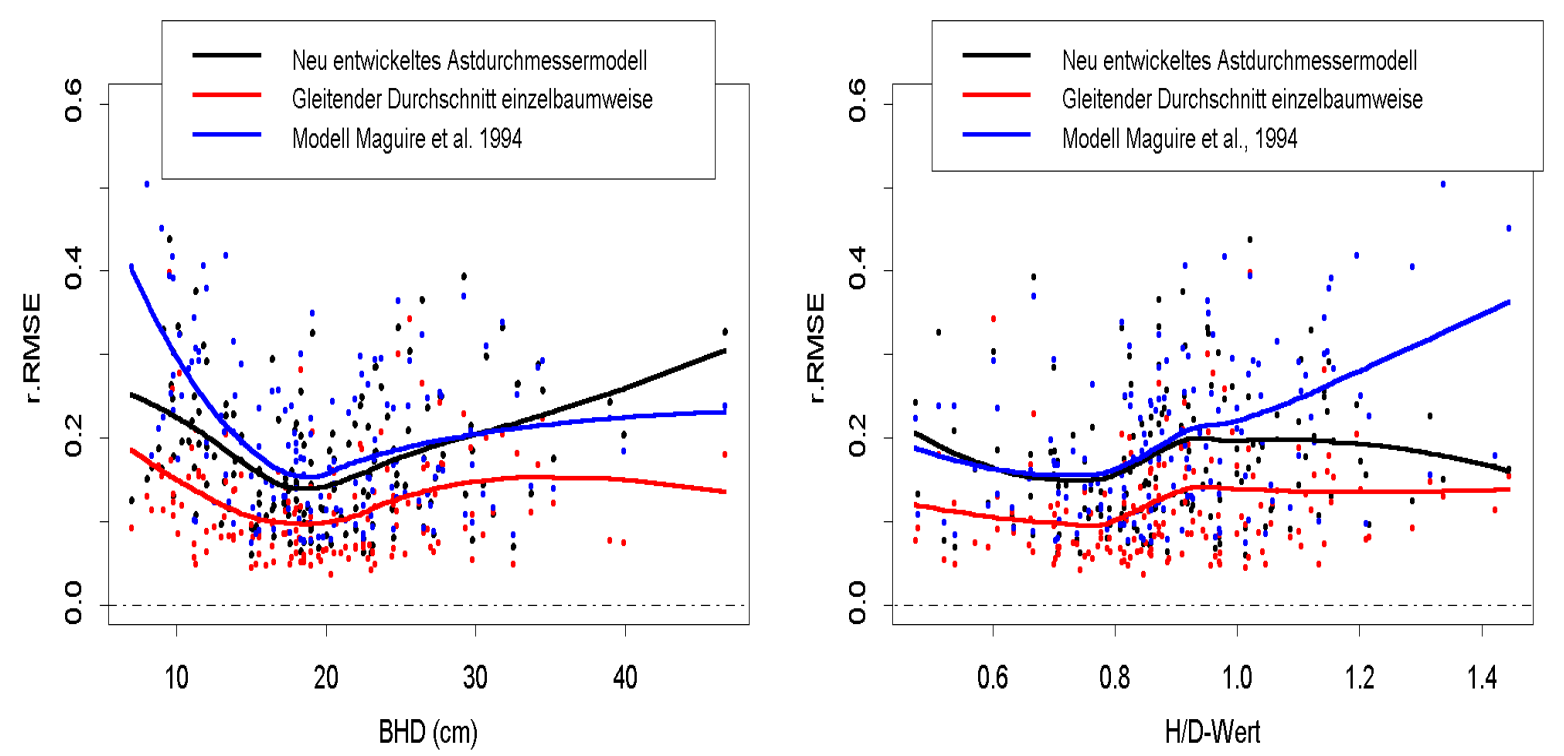

Abb. 38: Einzelbaumweise berechnete relative RMSE über den zugehörigen BHD und H/DWerten der Einzelbäume für das neu entwickelte Astdurchmessermodell, für ein einzelbaumweise parametrisiertes Modell (Gleitender Durchschnitt) und für ein von MAGUIRE ET AL. (1994) entwickeltes Modell für die Baumart Fichte. Der Ausgleich der Fehlerwerte erfolgte mit einem Gleitenden-Durchschnitts-Modell (Kapitel 8.1).

In Abb. 39 und Abb. 40 sind die mit Hilfe des neu entwickelten Modells prognostizierten Verläufe der stärksten Quirläste innerhalb der grünen Krone im Vergleich der Baumarten Fichte, Douglasie und Kiefer dargestellt. Die Prognose erfolgte für einen Baum mit $30 \mathrm{~cm}$ BHD und 10 m Kronenlänge und einen Baum mit $15 \mathrm{~cm}$ BHD und 6 m Kronenlänge. Um den Einfluß einzelner Variablen auf den Verlauf des Astdurchmessers zu verdeutlichen, ergeben 
sich teilweise untypische Kombinationen der unabhängigen Variablen. Einige Kombinationen liegen im Extrapolationsbereich der zur Parametrisierung verwendeten Daten. Trotz dieser Einschränkungen werden auch im Vergleich der Baumarten plausible Verläufe der Durchmesser der stärksten Quirläste prognostiziert.

BHD $30 \mathrm{~cm}, \mathrm{H} / \mathrm{D}-$ Wert 0.4 (oberer Verlauf) und 1.6 (unterer Verlauf)

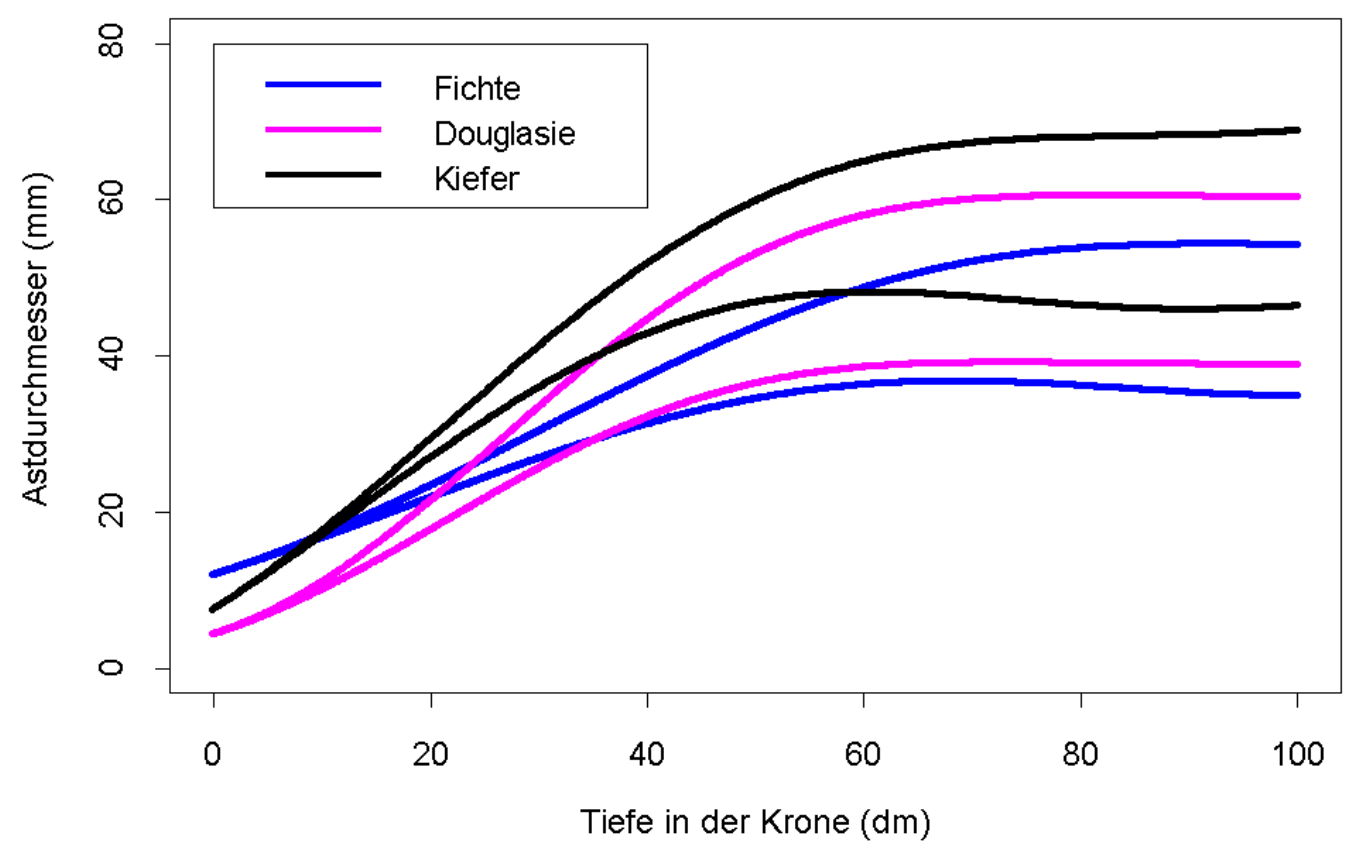

Abb. 39: Prognostizierter Verlauf des Durchmessers der stärksten Quirläste innerhalb der grünen Krone für die Baumarten Fichte, Douglasie und Kiefer bei einem BHD von $30 \mathrm{~cm}$, einem H/D-Wert von 0.4 bzw. 1.6 und einer Kronenlänge bis zum untersten Grünast von $10 \mathrm{~m}$.

So nimmt der Gradient der Astdurchmesserzunahme mit der Tiefe in der Krone von der Lichtbaumart Kiefer über die Douglasie zur Halbschattbaumart Fichte ab. Auch sind die Unterschiede im Astdurchmesserverlauf für Bäume mit gleichem BHD und gleicher Kronenlänge aber unterschiedlichen H/D-Werten bei der Kiefer und Douglasie stärker ausgeprägt als bei der Fichte. Es wurde schon beschrieben, daß über die Berücksichtigung des H/D-Wertes eine Sensitivität des Modells gegenüber unterschiedlichen waldbaulichen Behandlungen erreicht wird. Daher ist es plausibel, daß die Lichtbaumart Kiefer und die Douglasie sehr viel stärker auf ein Ansteigen des H/D-Wertes bzw. auf die Erhöhung der Konkurrenz reagieren als die Halbschattbaumart Fichte. Es scheint daher auch kein Problem darin zu bestehen, das Modell auch auf unterständige Bäume in mehrschichtigen Beständen anzuwenden. Das vor allem bei unterständigen Douglasien und Kiefern zu beobachtende stark reduzierte Durchmesserwachstum bei gleichzeitig nur schwach oder gar nicht reduziertem Höhenwachstum führt zu sehr hohen H/D-Werten und damit zu sehr schwachen Astdurchmessern. Diese Prognose deckt sich mit den in der Natur zu beobachtenden Aststärken bei unterständigen Douglasien 
und Kiefern. Selbstverständlich setzt eine Anwendung auf unterständige Bäume eine Erweiterung des zur Parametrisierung verwendeten Datenmaterials auf entsprechende Bäume voraus. Die Schwierigkeiten einer Qualitätsprognose von unterständigen Bäumen bestehen momentan weniger in der eigentlichen Astdurchmessermodellierung als vielmehr in der genauen Modellierung des Durchmesser- und Höhenwachstums.

\section{BHD $15 \mathrm{~cm}, \mathrm{H} / \mathrm{D}-$ Wert 0.4 (oberer Verlauf) und 1.6 (unterer Verlauf)}

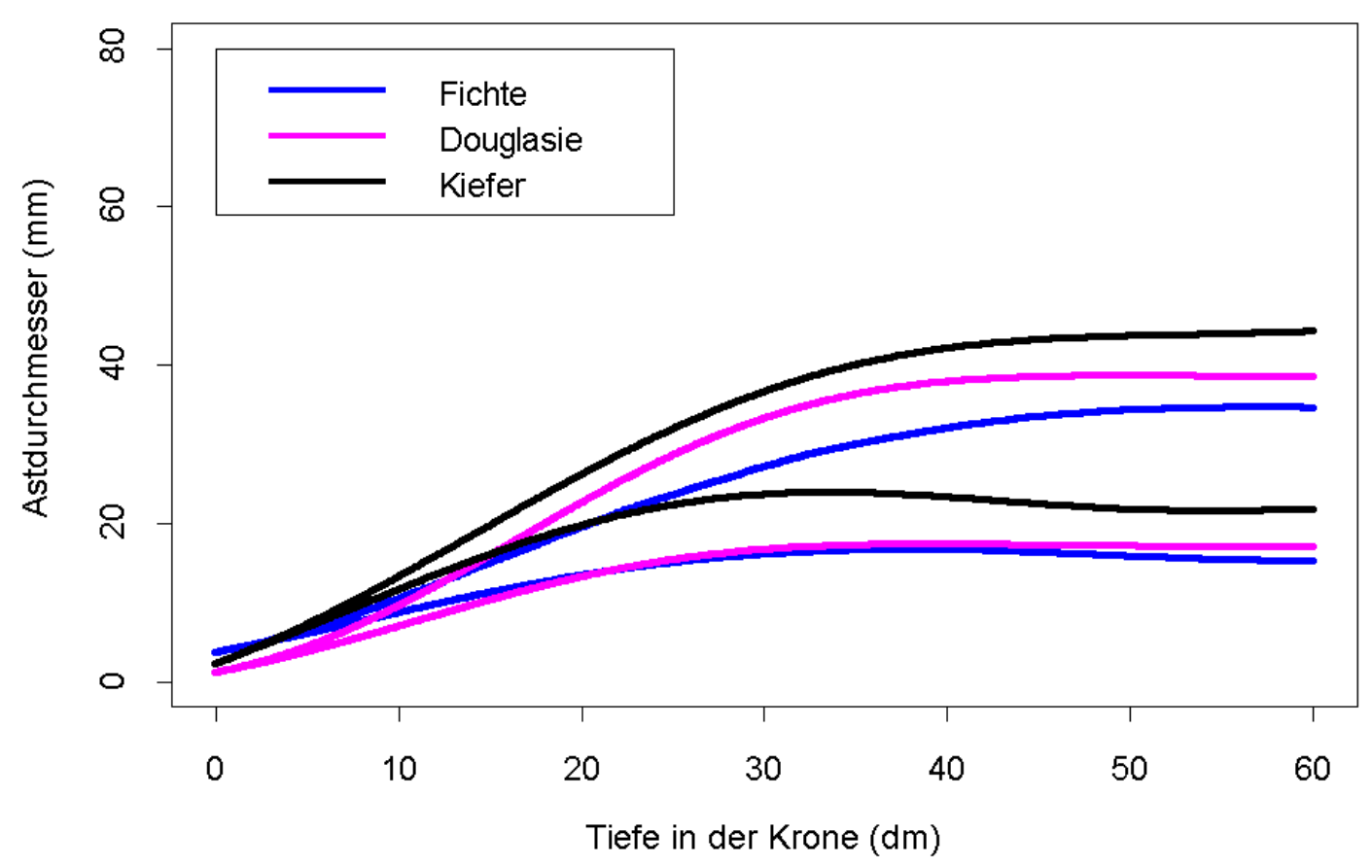

Abb. 40: Prognostizierter Verlauf des Durchmessers der stärksten Quirläste innerhalb der grünen Krone für die Baumarten Fichte, Douglasie und Kiefer bei einem BHD von $15 \mathrm{~cm}$, einem H/D-Wert von 0.4 bzw. 1.6 und einer Kronenlänge bis zum untersten Grünast von $6 \mathrm{~m}$.

\subsubsection{Astdurchmesser von weiteren Quirlästen}

Eine naheliegende Möglichkeit, die Astdurchmesser weiterer schwächerer Quirläste zu schätzen, ist die Parametrisierung eines Modells, das zur Prognose der stärksten Quirläste verwendet wird, mit Astdurchmesserdaten der 2.-, 3.-, usw. stärksten Quirläste. COLIN und HoULLIER (1992) verwenden beispielsweise den gleichen Modelltyp für die Prognose der Durchmesser der stärksten Quirläste und des mittleren Astdurchmessers je Quirl. Ein Problem besteht darin, daß bei einer nach Straten getrennten Parametrisierung, wie bei der Einteilung der Quirläste entsprechend ihrer Durchmesserrangfolge, für bestimmte Kombinationen von unabhängigen Variablen Überschneidungen der Regressionsfunktionen möglich sind. Überschneidungen würden aber bedeuten, daß beispielsweise für den 2.-stärksten Quirlastdurchmesser ein stärkerer Durchmesser prognostiziert würde, als für den stärksten Quirlastdurchmesser. Daher werden auch in diesem Zusammenhang segmentierte und nicht-segmentierte Polynomialglei- 
chungen als nachteilig beurteilt, da sie durch die vielfachen Kombinationen der unabhängigen Variablen im Extrapolationsbereich sehr instabil sind. Es kann gezeigt werden, daß das neu entwickelte Astdurchmessermodell bei der Schätzung der Astdurchmesserverläufe der fünf stärksten Quirläste sehr stabile Verläufe prognostiziert. Es kommt selbst im Extrapolationsbereich - abgesehen von geringfügigen Ausnahmen - zu keinen Überschneidungen der Regressionsfunktionen (Abb. 41 und Abb. 42).

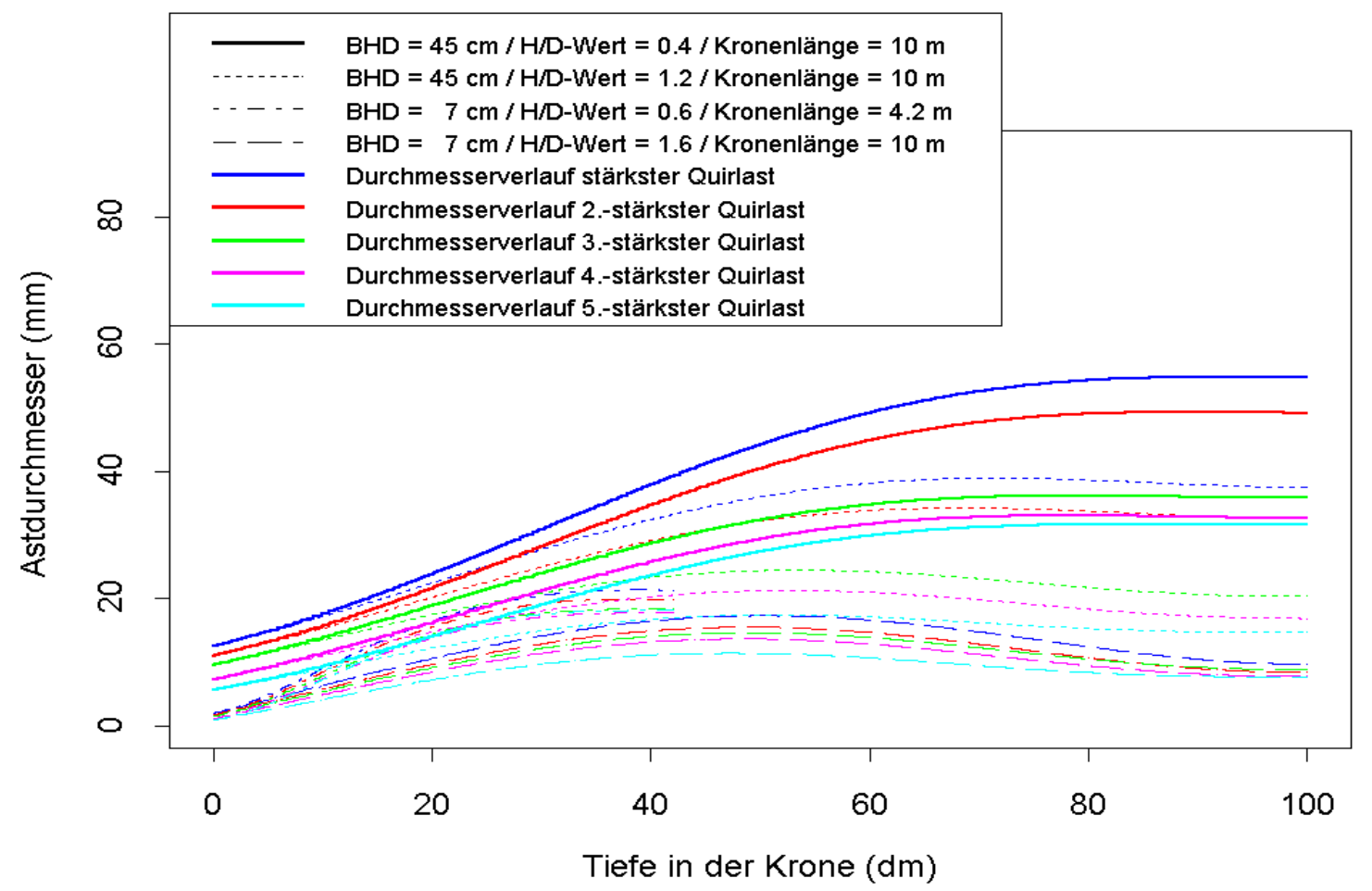

Abb. 41: Prognostizierter Verlauf des Durchmessers des stärksten bis 5.-stärksten Quirlastes innerhalb der grünen Krone für die Baumart Fichte bei einem BHD von 7 und $45 \mathrm{~cm}$ und verschiedenen $H / D$-Wert- und Kronenlängenkombinationen.

Solche Ausnahmen sind beispielsweise die Schätzungen für den Baum mit $7 \mathrm{~cm}$ BHD und einem H/D-Wert von 1.6 für den unteren Kronenbereich und für den Baum mit $7 \mathrm{~cm}$ BHD und einem H/D-Wert von 0.4 für den gesamten Kronenbereich (Abb. 41). Um auch für Bäume schwacher Dimension plausible Astdurchmesserverläufe zu prognostizieren, wurden die Koeffizienten der Modelle zur Schätzung der Astdurchmesserverläufe über der Aststärkenrangfolge ausgeglichen (Abb. 43). Durch den Koeffizientenausgleich mit Hilfe einer einfachen linearen Regression (21) werden in jedem Bereich der Kombination von unabhängigen Variablen zueinander plausible Astdurchmesserverläufe prognostiziert (Abb. 44). Durch den Koeffizientenausgleich wird unterstellt, daß die Funktionsparameter äquidistante Abstände zwischen den Aststärkenrängen aufweisen. 


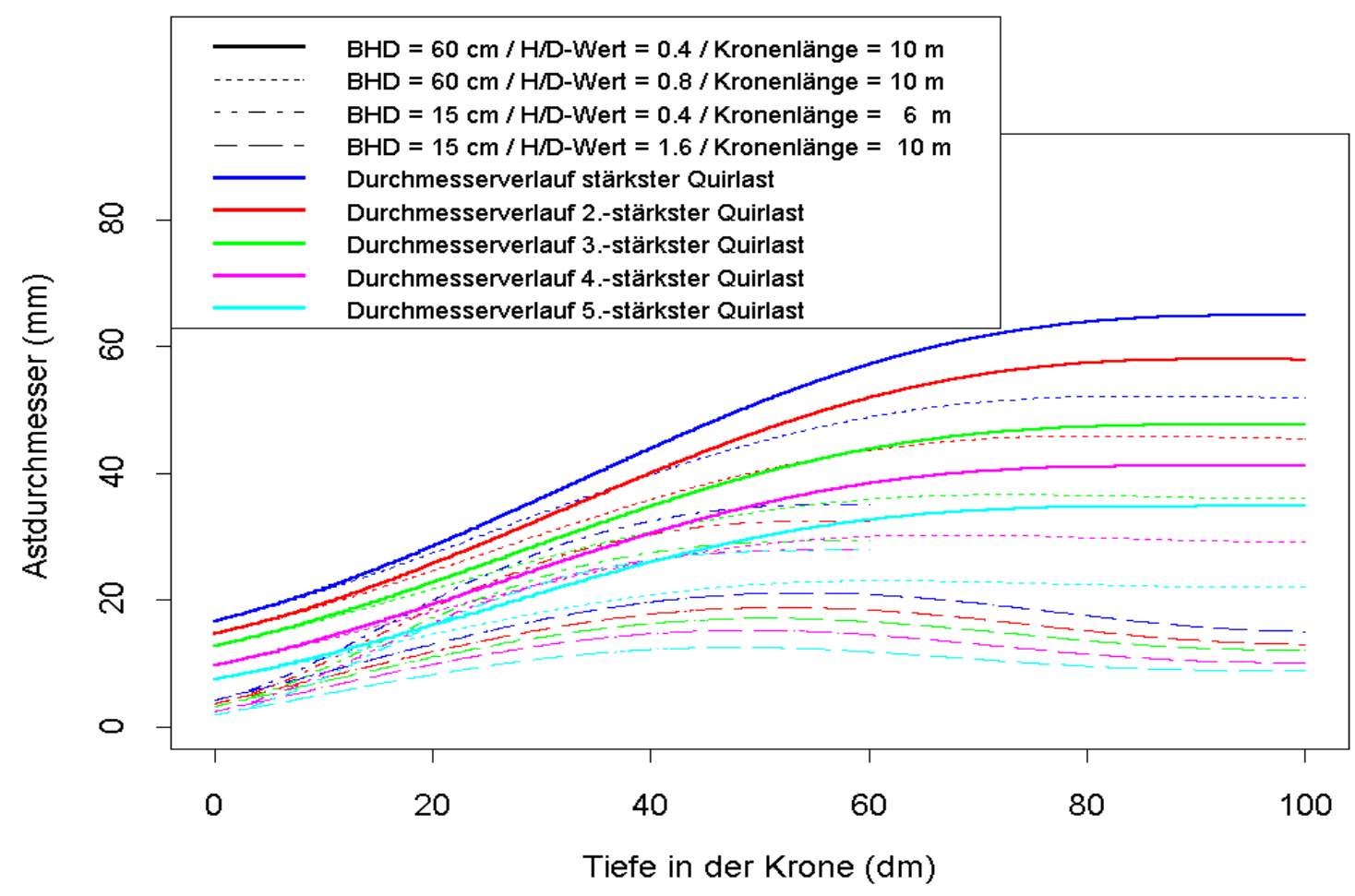

Abb. 42: Prognostizierter Verlauf des Durchmessers des stärksten bis 5.-stärksten Quirlastes innerhalb der grünen Krone für die Baumart Fichte bei einem BHD von 15 und $60 \mathrm{~cm}$ und verschiedenen $H / D$-Wert- und Kronenlängenkombinationen.

Bei der Betrachtung der Struktur der direkt geschätzten Parameter fällt der lineare Zusammenhang der Parameter auf, wenn diese Parameter über der Aststärkenrangfolge dargestellt werden (Abb. 43). Eine Ausnahme bildet der Parameter $b_{3}$ für den 2.- und 4.-stärksten Quirlast. Die mehr oder weniger starken Abweichungen der einzelnen Parameterwerte von einer Geraden können als zufällige Schwankungen betrachtet werden, die dadurch möglich sind, daß der Zusammenhang zwischen den Ausprägungen eines Parameters bei einer stratenweisen Parametrisierung nicht berücksichtigt wird. Auch sind bei sechs Funktionsparametern mehrere Kombinationen der Parameter mit annähernd gleicher Schätzgenauigkeit denkbar. Da bei einer stratenweisen Parametrisierung bestimmte Randbedingungen wie die nicht zulässige Überscheidung der Regressionsfunktionen nicht berücksichtigt werden können, wird eventuell eine Parameterkombination geschätzt, die diese Randbedingungen zumindest im Extrapolationsbereich verletzt. Mit Hilfe des Koeffizientenausgleichs läßt sich für das neu entwickelte Modell eine Parameterkombination ermitteln, die bei annähernd gleicher Schätzgenauigkeit (Tab. 15) auch die Randbedingung der konsistenten Astdurchmesserverläufe erfüllt. 

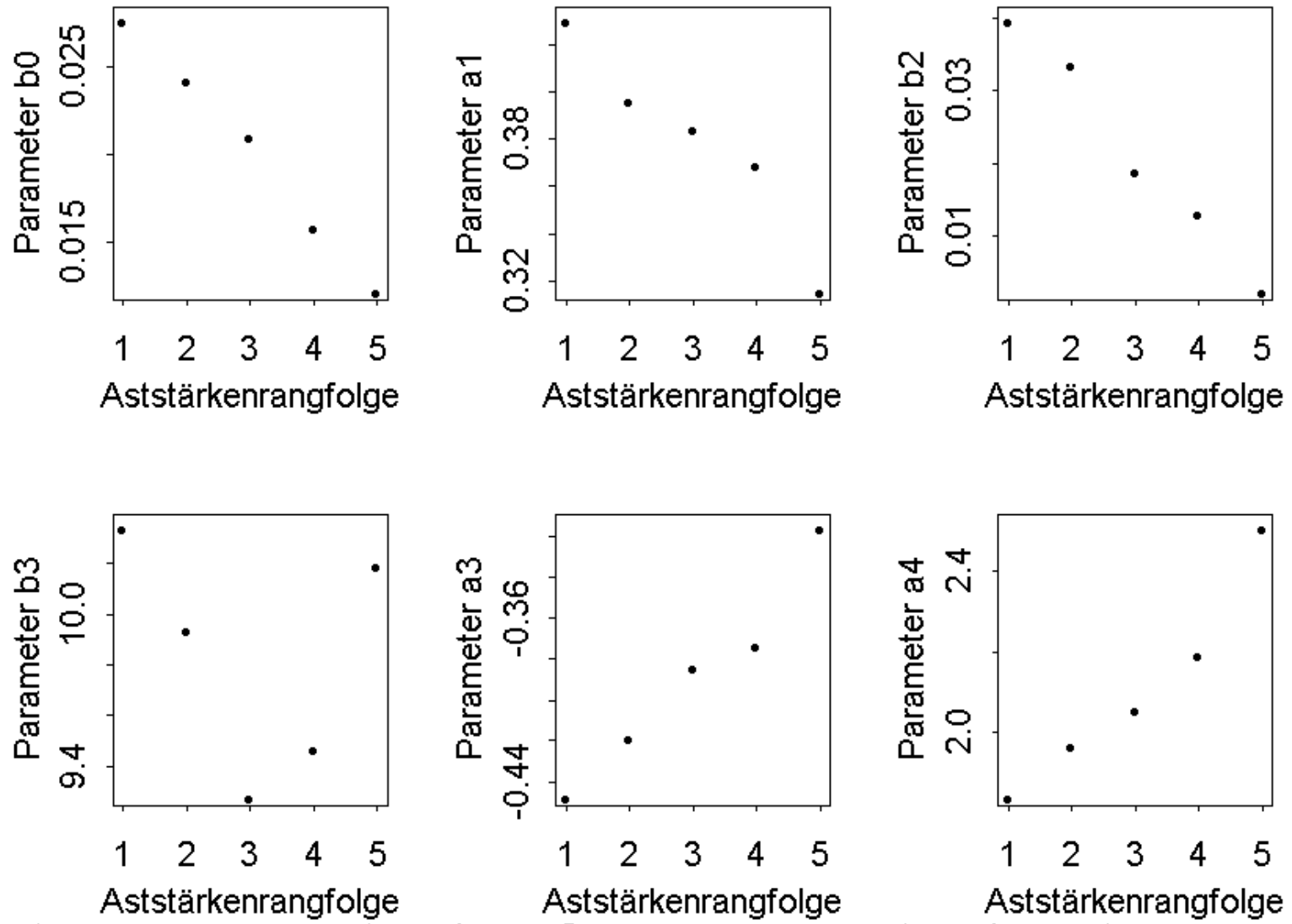

Abb. 43: Parameterschätzungen für das neu entwickelte Astdurchmessermodell über der Aststärkenrangfolge.

$a_{l_{i}}=\alpha+\beta *$ Aststärkenrang $_{i}$,

$a_{3_{i}}=\alpha+\beta *$ Aststärkenrang ${ }_{i}$,

$a_{4_{i}}=\alpha+\beta *$ Aststärkenrang ${ }_{i}$,

$b_{0_{i}}=\alpha+\beta *$ Aststärkenrang ${ }_{i}$,

$b_{2_{i}}=\alpha+\beta *$ Aststärkenrang ${ }_{i}$,

$b_{3_{i}}=\alpha+\beta *$ Aststärkenrang ${ }_{i}$, wobei $\alpha, \beta$ Parameter des Koeffizientenausgleichs für den Parameter $a_{1}$. (i=1 bis 5); wobei $\alpha, \beta$ Parameter des Koeffizientenausgleichs für den Parameter $a_{3}$. (i=1 bis 5); wobei $\alpha, \beta$ Parameter des Koeffizientenausgleichs für den Parameter $a_{4}$. ( $\mathrm{i}=1$ bis 5); wobei $\alpha, \beta$ Parameter des Koeffizientenausgleichs für den Parameter $b_{0}$. ( $\mathrm{i}=1$ bis 5 ); wobei $\alpha, \beta$ Parameter des Koeffizientenausgleichs für den Parameter $b_{2}$. ( $\mathrm{i}=1$ bis 5); wobei $\alpha, \beta$ Parameter des Koeffizientenausgleichs für den Parameter $b_{3}$. (i=1 bis 5).

Das neue Modell mit Parameterausgleich prognostiziert dabei auch im extremen Extrapolationsbereich plausible Verläufe (Abb. 44). Bei einer Erweiterung der Datenbasis muß erneut geprüft werden, inwieweit der Koeffizientenausgleich weiterhin notwendig ist, um für alle biologisch plausiblen Kombinationen von unabhängigen Variablen konsistente Durchmesserverläufe der Quirläste zu schätzen. Die Parameter für die Modelle des Koeffizientenausgleichs sowie die Parameter zur Schätzung der Astdurchmesserverläufe für alle Quirläste mit Hilfe von Modell (20) mit und ohne Koeffizientenausgleich sind in Kapitel 9.6 aufgeführt. 
Abschließend wird das neu entwickelte Modell, speziell in der Version mit Koeffizientenausgleich, als sehr geeignet zur Prognose konsistenter Astdurchmesserverläufe aller Quirläste beurteilt. Als Ursache für die auch im Extrapolationsbereich stabilen Prognosen ist der verwendete Modelltyp zu nennen, der die grundsätzliche Struktur der Astdurchmesser bereits über den Funktionstyp berücksichtigt und lediglich eine Variation dieser Grundform in Abhängigkeit von den verwendeten Einzelbaumvariablen zuläßt.

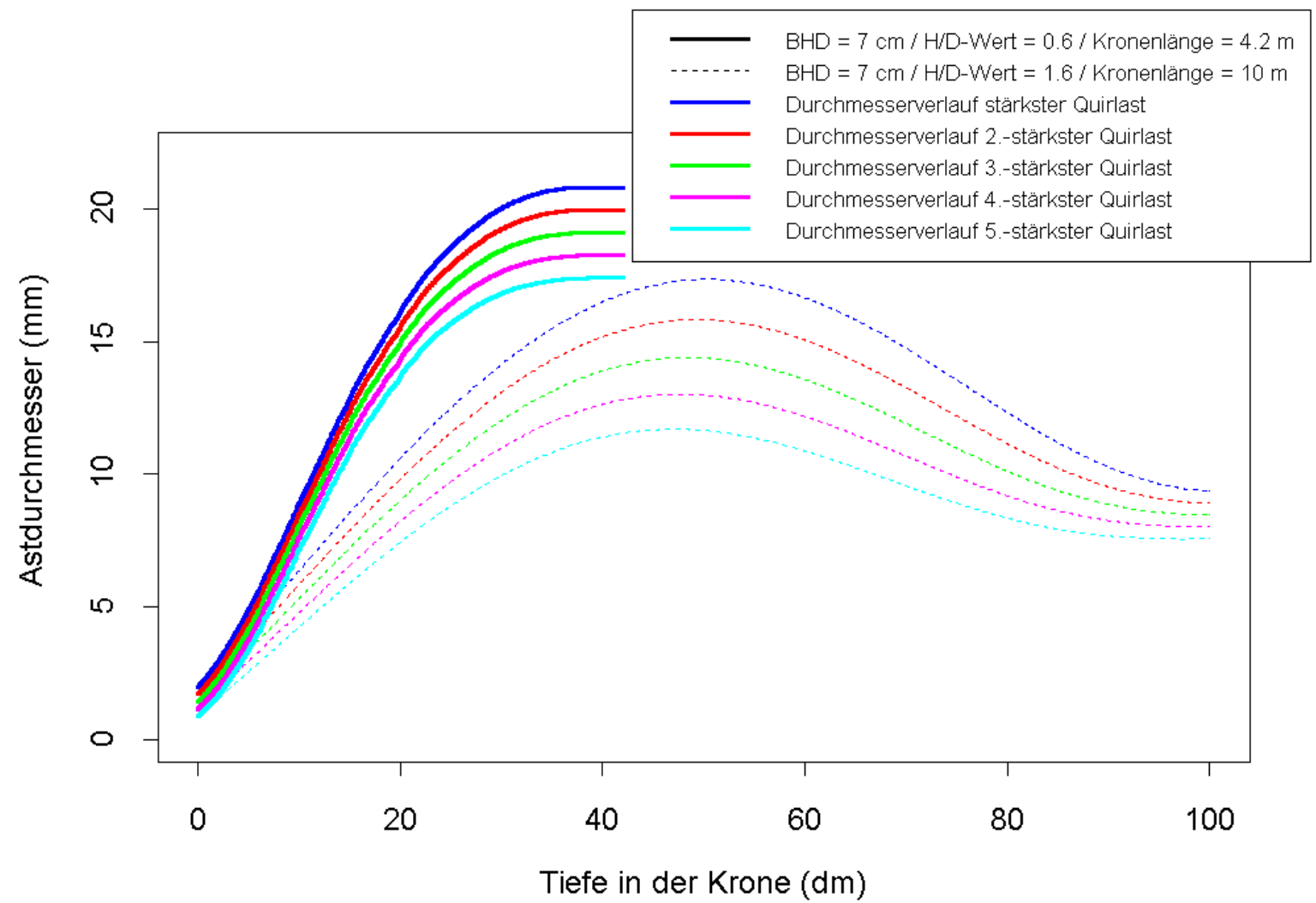

Abb. 44: Prognose der Astdurchmesserverläufe für zwei Fichten mit einem BHD von $7 \mathrm{~cm}$ und den H/D-Werten 0.6 und 1.6 mit dem neuen Modell zur Beschreibung von Astdurchmessern mit Koeffizientenausgleich.

\begin{tabular}{|l|r|r|r|r|r|}
\hline & stärkster & $\begin{array}{c}2 .- \\
\text { Qü̈rster } \\
\text { Quirlast }\end{array}$ & $\begin{array}{c}\text { 3.- } \\
\text { Qüarlast }\end{array}$ & $\begin{array}{c}\text { 4.- } \\
\text { Quirlast } \\
\text { stärkster } \\
\text { Quirlast }\end{array}$ & $\begin{array}{c}\text { 5.- } \\
\text { stärkster } \\
\text { Quirlast }\end{array}$ \\
\cline { 2 - 7 } & \multicolumn{6}{|c|}{ RMSE (mm) } \\
\hline Parameter werden direkt geschätzt; Formel (19) & 3.817 & 3.686 & 3.776 & 3.894 & 4.028 \\
\hline $\begin{array}{l}\text { Parameter werden über Koeffizientenausgleich } \\
\text { ermittelt; Formeln (21) bzw. (19) }\end{array}$ & 3.821 & 3.688 & 3.781 & 3.900 & 4.034 \\
\hline
\end{tabular}

Tab. 15: Vergleich der Schätzgenauigkeit des neu entwickelten Modells zur Beschreibung der Astdurchmesserverläufe mit direkter Schätzung aller Parameter und der Variante mit Koeffizientenausgleich über der Aststärkenrangfolge für die Baumart Fichte.

Aus streng statischer Sichtweise ist der Koeffizientenausgleich problematisch, da die Parameter des Modells zur Beschreibung des Astdurchmesserverlaufs in Abhängigkeit von einer 
ordinal skalierten Variablen geschätzt werden (Astrangfolge). Aufgrund der guten Resultate ist das Modell mit Koeffizientenausgleich trotzdem als heuristischer Ansatz mit hoher Praxistauglichkeit bei der behandlungssensitiven Prognose aller Quirlastdurchmesser der Baumart Fichte zu beurteilen.

Ein weiterer Modellansatz zur Prognose aller Quirlastdurchmesser von Nadelholzarten soll hier nur kurz theoretisch skizziert werden. Nach der Schätzung der Durchmesser der stärksten Quirläste können die weiteren Quirlastdurchmesserwerte in absoluter oder relativer Form mit Hilfe einer Dichtefunktion beschrieben werden. Da diese Durchmesserwerte größer als Null sowie kleiner oder gleich dem Durchmesser des stärksten Quirlastes sein müssen, d. h. auf ein endliches Intervall beschränkt sind, bietet sich zu ihrer Beschreibung die BetaVerteilung an. Die Beta-Verteilung wird häufig für die Darstellung von Prozessen mit natürlichen unteren und oberen Grenzen verwendet. Beispiele zur Anwendung der Beta-Verteilung findet man in HAHN und SHAPIRO (1967). Ein Ansatz zur Modellierung der Astdurchmesser bei der Baumart Buche und weitere forstliche Anwendungsbeispiele werden in Kapitel 3.4.1 beschrieben. Um Unterschiede in der Form der Verteilungen für bestimmte Kronenbereiche sowie unterschiedliche BHD- und H/D-Werte erfassen zu können, bietet sich die Relativierung der Astdurchmesser mit dem stärksten Quirlastdurchmesser und ihre Beschreibung mit Hilfe der standardisierten Beta-Verteilung an. Anschließend können eventuell bestehende Zusammenhänge zwischen der Form der Beta-Verteilung bzw. den Parametern der BetaVerteilung und Einzelbaumvariablen ermittel werden. Mit Hilfe von Regressionen zur Schätzung der Formparameter der Beta-Verteilung in Abhängigkeit von Einzelbaumvariablen können Quirlastdurchmesserverteilungen für beliebige Bäume prognostiziert werden. Konkrete relative oder absolute Quirlastdurchmesser können durch Einsetzen von im Intervall [0,1] gleichverteilten Zahlen in die invertierte Verteilungsfunktion (Quantilsfunktion) der jeweiligen Beta-Verteilung geschätzt werden. Durch dieses Verfahren ist ausgeschlossen, daß es zu inkonsistenten Schätzungen in der Astrangfolge von Quirlästen kommt. 


\subsubsection{Berücksichtigung von Kronenbrüchen}

Im Datenmaterial der Baumart Fichte sind auch Astmessungen von Bäumen mit Kronenbrüchen enthalten. Es fällt auf, daß die Äste unterhalb der Höhe des Kronenbruchs häufig stärkere Durchmesser aufweisen als die entsprechenden Äste von Bäumen ohne Kronenbruch mit ansonsten aber übereinstimmenden Einzelbaumeigenschaften. Das neu entwickelte Modell zur Schätzung der Durchmesser der stärksten Quirläste (20) wurde aufgrund dieser Beobachtung weiterentwickelt, indem zusätzlich eine Dummyvariable mit den beiden Ausprägungen "Kronenbruch" und "kein Kronenbruch" in das Modell integriert wurde (Formel (22)).

$$
\begin{aligned}
B D_{\text {max }}= & \left(b_{0} * D+a_{1} * \text { TINK }+b_{4} * \text { Kronenbruch }\right)+\left(b_{2} * D+b_{3} *(1 / H D)\right. \\
& \left.+a_{3} * \text { TINK }\right) *\left(1-\left(1-\text { TINK }_{\text {rel }}\right)^{\left.a_{4} * T I N K_{\text {rel }}\right)}\right.
\end{aligned}
$$

wobei:

$B D_{\max }=$ Durchmesser des stärksten Quirlastes (mm);

TINK = Tiefe in der Krone, wobei sich der Parametrisierungsbereich nur bis zum untersten Grünast erstreckt (dm);

$T I N K_{\text {rel }}=$ TINK in Relation zur Gesamtkronenlänge (bis zum untersten Grünast);

$D \quad=$ Brusthöhendurchmesser (mm);

$H \quad=$ Baumhöhe $(\mathrm{dm})$;

$H D=\mathrm{H} / \mathrm{D}-\mathrm{Wert}$;

Kronen = Dummyvariable mit der Ausprägung 0 für "kein Kronenbruch" und -bruch 1 für "Kronenbruch".

mit:

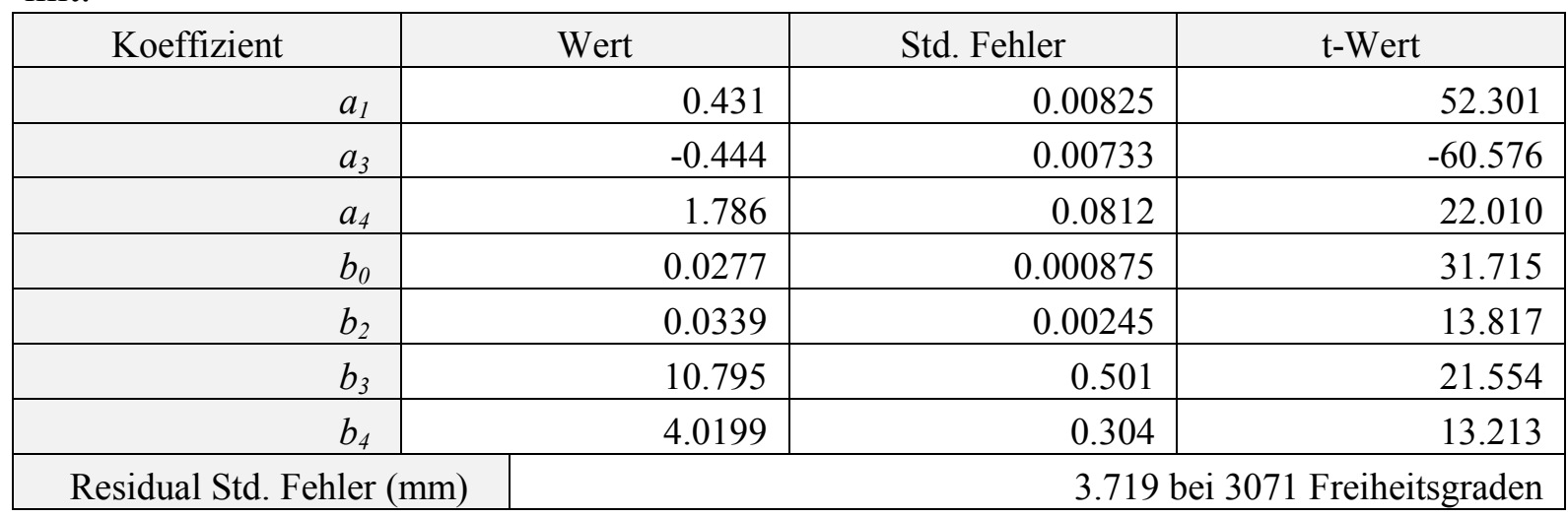

Tab. 16: Koeffizienten und statistische Kenngrößen zur Durchmesserschätzung der stärksten Quirläste innerhalb der grünen Krone mit Hilfe des neu entwickelten Modells (22) für die Baumart Fichte.

Der Parameter $b_{4}$ ist biologisch interpretierbar und gibt an, um wieviel stärker ein Astdurchmesser (mm) ist, wenn sich der Ast bei sonst gleichen Einzelbaumvariablen in einem Quirl unterhalb eines Kronenbruchs befindet. Die Reduktion des mittleren Fehlers gegenüber dem Modell ohne Berücksichtigung von Kronenbrüchen (20) erscheint gering. Bei der Interpretation des mittleren Fehlers muß allerdings auch hier die Datenstruktur berücksichtigt werden, die überwiegend Bäume ohne Kronenbrüche enthält. Auch ist zu erwarten, daß das Modell 
mit Berücksichtigung von Kronenbrüchen nur für die Äste, die sich unterhalb eines Kronenbruches befinden, eine Verbesserung der Schätzgenauigkeit liefert. Es wurde daher ein Vergleich der Modelle nur für die Äste, die sich unterhalb eines Kronenbruches befinden, und für alle übrigen Äste vorgenommen (Tab. 17).

\begin{tabular}{|l|c|c|c|r|}
\hline & \multicolumn{3}{|c|}{$\begin{array}{c}\text { Modell ohne Berücksichtigung von } \\
\text { Kronenbrüchen (20) }\end{array}$} & \multicolumn{2}{c|}{$\begin{array}{c}\text { Modell mit Berücksichtigung von } \\
\text { Kronenbrüchen (22) }\end{array}$} \\
\hline Astkollektiv & Kollektiv I & Kollektiv II & Kollektiv I & \multicolumn{1}{|c|}{ Kollektiv II } \\
\hline Residual Std. Fehler (mm) & 6.127 & 3.636 & 4.880 & 3.642 \\
\hline
\end{tabular}

Tab. 17: Standardfehler für die Astdurchmessermodelle mit (22) und ohne (20) Berücksichtigung von Kronenbrüchen für die Kollektive: I. Ast befindet sich in einem Quirl unterhalb eines Kronenbruchs; II. Ast befindet sich oberhalb eines Kronenbruchs oder gehört zu einem Baum ohne Kronenbruch.

Beim Vergleich der beiden Modelle zeigt sich eine deutliche Verbesserung der Schätzung für das Kollektiv I (Äste in Quirlen unterhalb eines Kronenbruchs), wenn das Modell mit der Berücksichtigung von Kronenbrüchen (22) verwendet wird. Beim Vergleich der beiden Modelle ist weiterhin zu erkennen, daß sich die Schätzungen für das Kollektiv II (Äste in Quirlen oberhalb von Kronenbrüchen und Äste von Bäumen ohne Kronenbrüche) durch die Anwendung des Modells mit Berücksichtigung von Kronenbrüchen nicht verschlechtern.

Die Anwendung des Modells mit Berücksichtigung von Kronenbrüchen setzt voraus, daß die Zeitpunkte von Schneebruchereignissen für einen Bestand bekannt sind. Dabei ist für einen Baum das zeitlich am weitesten zurückliegende Schneebruchereignis interessant, da nur der unterhalb des Kronenbruchs liegende Stammabschnitt für eine höherwertige Verwendung in Frage kommt. Die Prognose der Aststärke von Ästen aus Quirlen oberhalb des ersten Kronenbruchs ist mit den entwickelten Modellen möglich, jedoch von keinem wirtschaftlichen Interesse. Neben der Kenntnis des Zeitpunktes von Schneebruchereignissen muß die Anzahl der Bäume geschätzt werden, die einen entscheidenden - d. h. den ersten - Schneebruchschaden erleiden. In waldbaulichen Szenariensimulationen können unterschiedliche Zeitpunkte von Schneebruchereignissen und unterschiedlich starke Schneebruchereignisse vorgegeben werden bzw. ihre Auswirkungen auf die Holzqualität geprüft werden. Geht es darum, die Auswirkungen von Schneebruchereignissen in realen Beständen zu erfassen, ist man auf Aufzeichnungen der Zeitpunkte von Schneebruchereignissen und die Einschätzung der Anteile an erstmals geschädigten Bäumen angewiesen. Neben der Modellierung der Astigkeit können derartige Prognosen dazu verwendet werden, die Verluste an Stammholzvolumen durch ein Schneebruchereignis zu quantifizieren.

Ein komplexeres Modell zur Berücksichtigung von Kronenbrüchen, das einen nachlassenden Einfluß des Kronenbruchs auf den Astdurchmesser mit größerer Entfernung zur Höhe des Kronenbruchs unterstellt, führte zu keinen Verbesserungen. Allerdings bedarf es auch hier 
weiterer Untersuchungen auf umfangreicherer Datenbasis, da nur 17 der 155 untersuchten Fichten einen Kronenbruch aufwiesen. In Abb. 45 sind die Unterschiede der Modelle zur Astdurchmesserschätzung mit und ohne Berücksichtigung von Kronenbrüchen bei der Anwendung auf einen realen Einzelbaum mit einem Kronenbruch bei ca. $7 \mathrm{~m}$ Kronentiefe dargestellt. Die Astdurchmessermodelle (20) und (22) verwenden nur die Einzelbaumvariablen BHD, H/D-Wert, Tiefe in der Krone bzw. zusätzlich die Höhe des Kronenbruchs. Als Referenzmodell wurde ein Gleitendes-Durchschnitts-Modell direkt mit den Astdurchmessern parametrisiert. Es wurde bewußt ein Baum ausgewählt, für den beide neu entwickelten Astdurchmessermodelle eine gute Anpassung aufweisen, um den Vorteil der Berücksichtigung der Information Kronenbruchhöhe zu verdeutlichen. Unterhalb der Höhe des Kronenbruchs weist das Modell mit Berücksichtigung des Kronenbruchs (grüne Linie) deutliche Vorteile gegenüber dem Modell ohne Berücksichtigung des Kronenbruchs auf (blaue Linie).

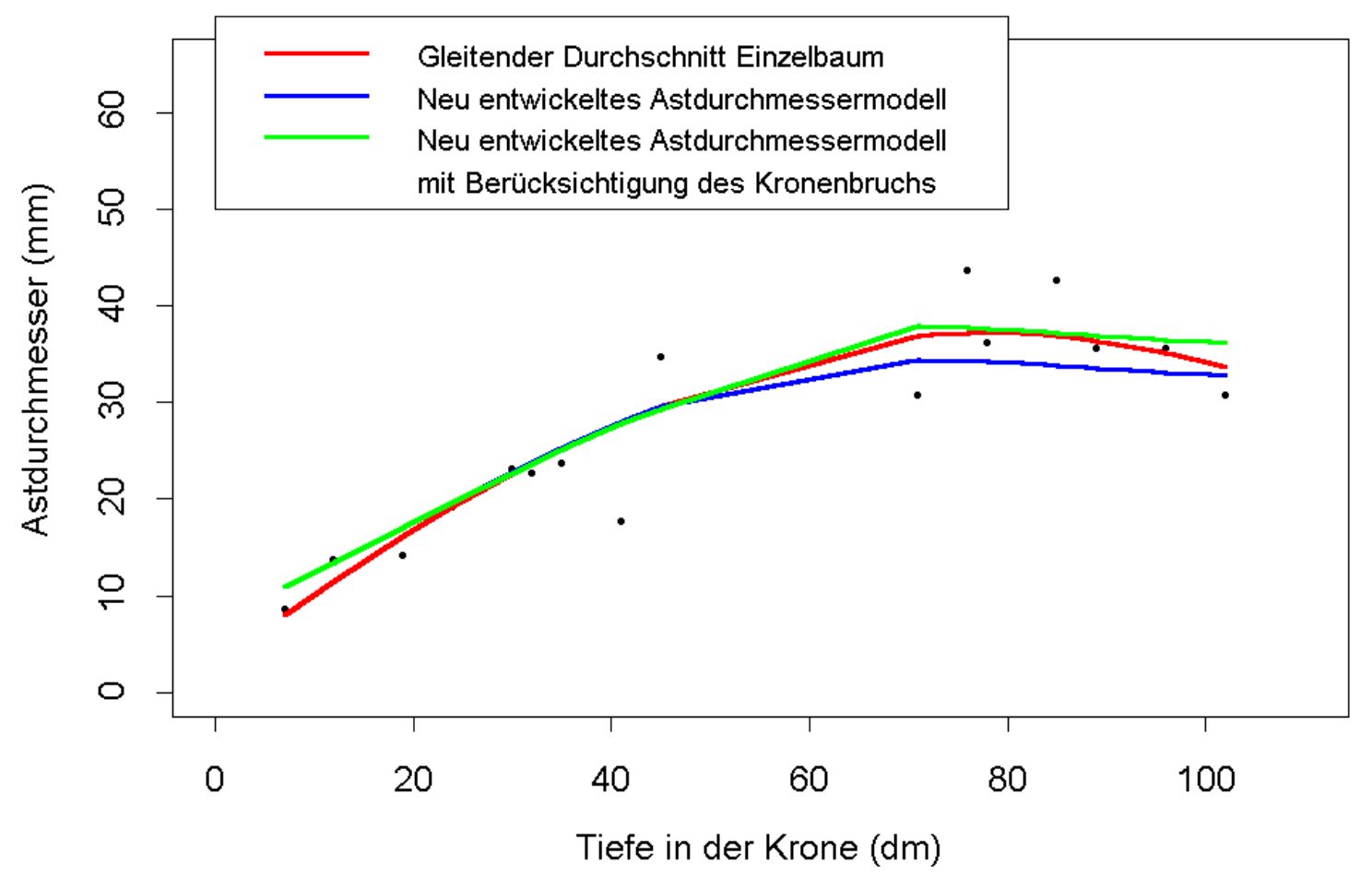

Abb. 45: Vergleich der mit Hilfe des neu entwickelten Astdurchmessermodells (20) (blau) und mit Hilfe des neu entwickelten Astdurchmessermodells mit Berücksichtigung von Kronenbrüchen (22) (grün) geschätzten Verläufe der stärksten Quirläste in der grünen Krone für eine Fichte mit einem Kronenbruch bei ca. 7 m Tiefe in der Krone. Als Referenz für eine optimale Anpassung ist ein direkt an die Einzelbaumdaten angepaßtes Gleitendes-Durchschnitts-Modell dargestellt (rote Linie) (Kapitel 8.1). 


\subsection{Modelle zur Beschreibung von Astparametern bei der Baumart Buche}

\subsubsection{Beschreibung der Astdurchmesser}

In Kapitel 2.4.1 wurde bereits darauf hingewiesen, daß die in der Literatur beschriebenen Modelle zur Prognose der qualitätsrelevanten Astigkeit fast ausschließlich für Nadelholzarten entwickelt wurden. Als eine Ursache wurde der komplexere und stärker von einer einheitlichen Struktur abweichende Kronenaufbau der Laubholzarten genannt. Beispielsweise unterscheidet RolofF (1983) bei der Buche die drei Kronenformen wipfelschäftiger Typ, Besenund Zwieseltyp. Dagegen treten Auflösungen der Sproßachse in zwei oder mehrere gleichstarke Achsen bei den Nadelholzarten sehr selten und in der Regel nur nach traumatischen Ereignissen auf (GRUBER, 1987, S. 58). Die Laubholzarten weisen demgegenüber nur geringe Anteile an Individuen mit einer einheitlichen Zentralachse (Wipfelschäftigkeit) während der gesamten Lebensdauer des Baumes auf.

Aufgrund dieser Eigenschaften scheiden die für die Nadelholzarten aufgeführten Modellansätze zur Beschreibung der Astdurchmesserstruktur der stärksten Quirläste im gesamten Kronenbereich (Kapitel 2.4 u. 3.3) für die Modellierung der meisten Laubholzarten aus. Eine Ausnahme stellen z. B. die Erle, sowie die Esche und die Ahornarten im jungen Alter bzw. bei geringen Dimensionen dar, da bei diesen Baumarten zumindest bis zu bestimmten Dimensionen ein großer Anteil von Individuen mit streng orthotropen Wachstum einer eindeutigen Zentralachse und starker Apikalkontrolle (Abb.4) auftritt. Das streng orthotrope Wachstum einer eindeutigen Zentralachse und eine starke Apikalkontrolle sind als die wichtigsten Voraussetzungen für eine Modellierung der Astdurchmesserstruktur der Äste 1. Ordnung für den gesamten Kronenbereich mit einfachen statistischen Modellen zu nennen. Komplexere Modelltypen wie die von KURTH (1994) entwickelten Wuchsgrammatiken können die gesamte Kronenarchitektur auch für Laubholzarten abbilden. Jedoch ist bei sehr komplexen Modellen zu hinterfragen, inwieweit sie infolge der hohen Detailgenauigkeit noch eine behandlungssensitive Beschreibung der qualitätsrelevanten Astigkeit gewährleisten können und nicht nur eine Beschreibung der "mittleren" Kronenarchitektur einer Baumart darstellen.

Für die Beschreibung der Durchmesser von Ästen 1. Ordnung bei der Buche wurde ein Modell gewählt, das dem einfachsten Ansatz zur Modellierung der Astigkeit von Nadelholzarten ähnelt (Kapitel 2.4.2). Dieser von MAGUIRE ET AL. (1991) entwickelte Modellansatz beschreibt den Durchmesser des stärksten Astes eines Baumes in einem Quirl etwas oberhalb des Kronenansatzes. Hierbei werden die Durchmesser einer variablen Anzahl von Ästen, die sich im Bereich bzw. direkt oberhalb des Kronenansatzes befinden, geschätzt. Durch diese Vorgehensweise konnten auch Bäume mit sich auflösender Schaftachse, einer Wuchsform, die für Buche häufiger als die Wipfelschäftigkeit ist (ROLOFF, 1986, S. 90), mit in die Untersuchung einbezogen werden. Die Entscheidung, wann eine Auflösung der Schaftachse vorlag, 
wurde in dieser Arbeit bei der Datenerhebung im Gelände getroffen. Ein Baum wies nur bis zu der Stammhöhe eine einheitliche Zentralachse auf, solange noch eine Aushaltung von Stammholz möglich erschien. Bei Bäumen, die noch keine Stammholzdimensionen erreicht hatten, lag eine Auflösung der Stammachse vor, wenn ein Astdurchmesser auftrat, der zukünftig die Aushaltung von Stammholz verhindern wird. Ein fester Grenzwert für den Astdurchmesser, beispielsweise ein bestimmter Prozentsatz des BHD oder des Schaftdurchmessers auf der Höhe des jeweiligen Astes, erschien nicht sinnvoll. Es bestehen nämlich Unterschiede in Abhängigkeit von der Baumdimension, ab wann ein Ast eine Aushaltung von Stammholz nicht mehr zuläßt bzw. zukünftig nicht zulassen wird. Die Prognose, zu welchem Zeitpunkt sich bei einem Baum die Stammachse auflöst, kann mit dem entwickelten Modell zur Schätzung von Astdurchmessern im Kronenansatzbereich von Buchen nicht erfolgen.

Die Astdurchmessermessungen erfolgten im Kronenansatzbereich unterhalb der Stammhöhen, an denen sich die Stammachse auflöst (Abb. 10 (a)). Für wipfelschäftige Bäume wurden ebenfalls einige Äste im Bereich des Kronenansatzes gemessen. In Abb. 46 sind die Brusthöhendurchmesser von 211 Buchen, an denen Astdurchmesser im Kronenansatzbereich gemessen wurden, und die Tiefe in der Krone, das ist die Entfernung von der Baumspitze, für die einzelnen Äste angegeben. Diese Variablen haben sich in Voruntersuchungen als die einzigen Größen erwiesen, die einen nennenswerten Einfluß auf den Durchmesser von Ästen im Kronenansatzbereich hatten.
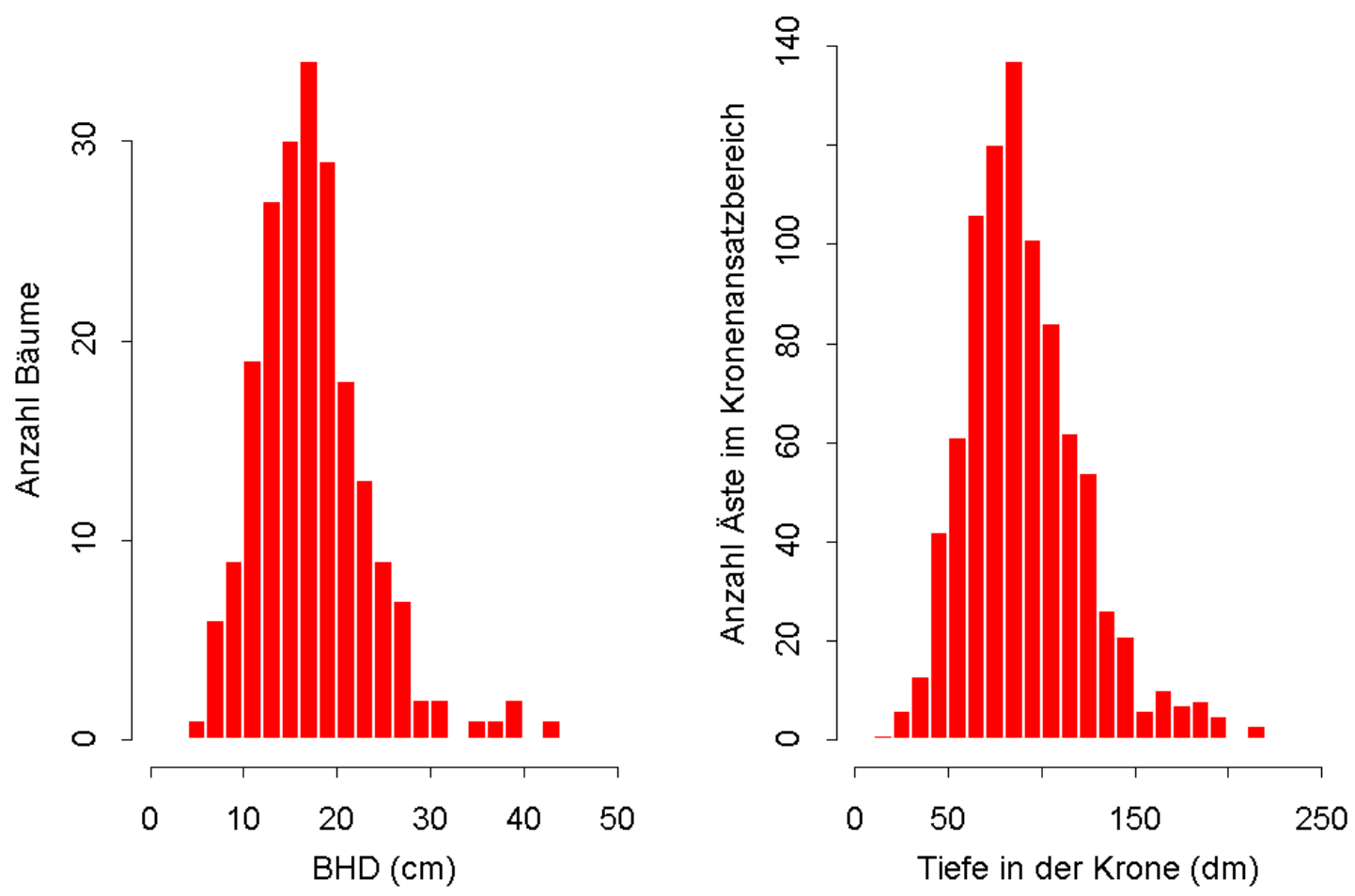

Abb. 46: Häufigkeitsverteilungen der Variablen BHD und Tiefe in der Krone, die den Astdurchmesser von Ästen im Kronenansatzbereich bei der Baumart Buche beeinflussen. 
Im Gegensatz zu den Nadelholzbaumarten stammen alle untersuchten Buchen aus Mischoder Reinbestandsversuchsflächen. Diese Flächen liegen fast ausschließlich im Solling und im Harz (Abb. 47).

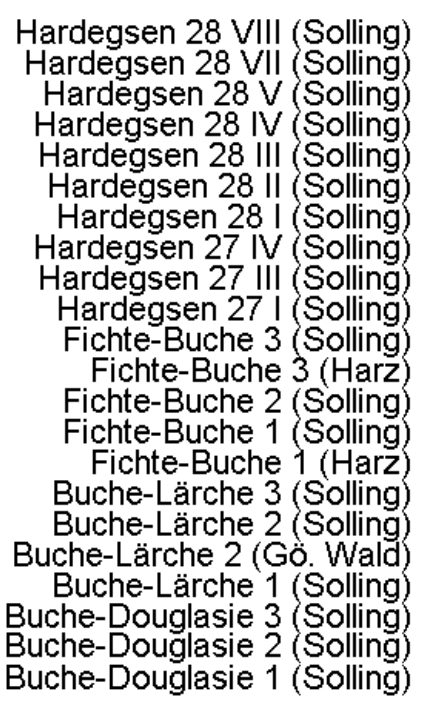

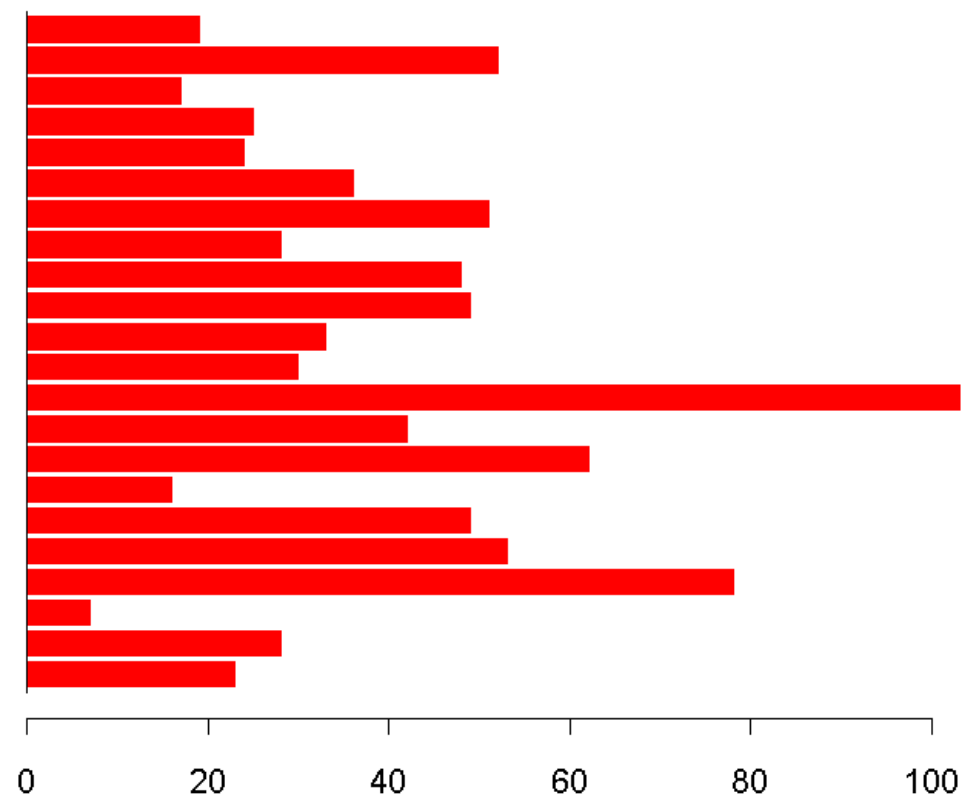

Anzahl Astdurchmesser aus dem Kronenansatzbereich

Abb. 47: Verteilung der Durchmessermessungen von Ästen aus dem Kronenansatzbereich gegliedert nach Versuchsflächen für die Baumart Buche.

Da für alle Versuchsflächen mindestens zwei aufeinander folgende Datenerhebungen mit Stammverteilungsplänen vorlagen, konnte der kleinräumige $\mathrm{C}_{66}$-Konkurrenzindex (NAGEL, 1999 a, S. 20) zum Zeitpunkt vor der Durchforstung für die letzte Aufnahme (bei der die untersuchten Buchen entnommen wurden) und zu den Zeitpunkten vor und nach der Durchforstung für die vorletzte Aufnahme berechnet werden. Als Einflußradius für die Berechnung des kleinräumigen $\mathrm{C}_{66}$-Index wurde für alle Bäume ein einheitlicher Radius von $6 \mathrm{~m}$ gewählt. Mit Hilfe des $\mathrm{C}_{66}$-Konkurrenzindex wurde untersucht, ob die Konkurrenzsituation der untersuchten Buchen während der letzten ca. 10 Jahre einen Einfluß auf die Astdurchmesser im Kronenansatzbereich hat. Dabei wurde davon ausgegangen, daß der $\mathrm{C}_{66}$-Konkurrenzindex, der vor dem Eingriff bei der vorletzten Aufnahme berechnet wurde, zur Beschreibung der Konkurrenzsituation in den letzten Jahren vor diesem Eingriff geeignet ist.

Es konnten jedoch keinerlei Beziehungen zwischen den zu den beschriebenen Zeitpunkten berechneten Werten des kleinräumigen $\mathrm{C}_{66}$-Konkurrenzindex bzw. dem zugehörigen Periodenmittelwert und den Astdurchmessern identifiziert werden. Als eine Ursache kann vermutet werden, daß durch die unterschiedlichen Astabgangswinkel der Baumart Buche auch unter hohem Konkurrenzdruck starke Astdurchmesser gebildet werden können. Beispielsweise können Steilastbildungen teilweise auf hohen Konkurrenzdruck zurückgeführt werden, 
gleichzeitig aber ein langes Durchmesserwachstum der Äste ermöglichen. Der Zeitraum von etwa 10 Jahren, für den die Konkurrenzsituation erfaßt werden konnte, wird dagegen als ausreichend für die Untersuchungen beurteilt.

In Abb. 48 sind die normierten Astdurchmesser der untersuchten Buchen über dem BHD dargestellt. Durch die Normierung der Astdurchmesser mit dem BHD kann überprüft werden, inwieweit über den Zusammenhang hinaus, daß stärkere Bäume im Mittel auch stärkere Astdurchmesser aufweisen als schwächere Bäume, Zusammenhänge zwischen dem Astdurchmesser und der Einzelbaumdimension bzw. der Tiefe in der Krone bestehen.

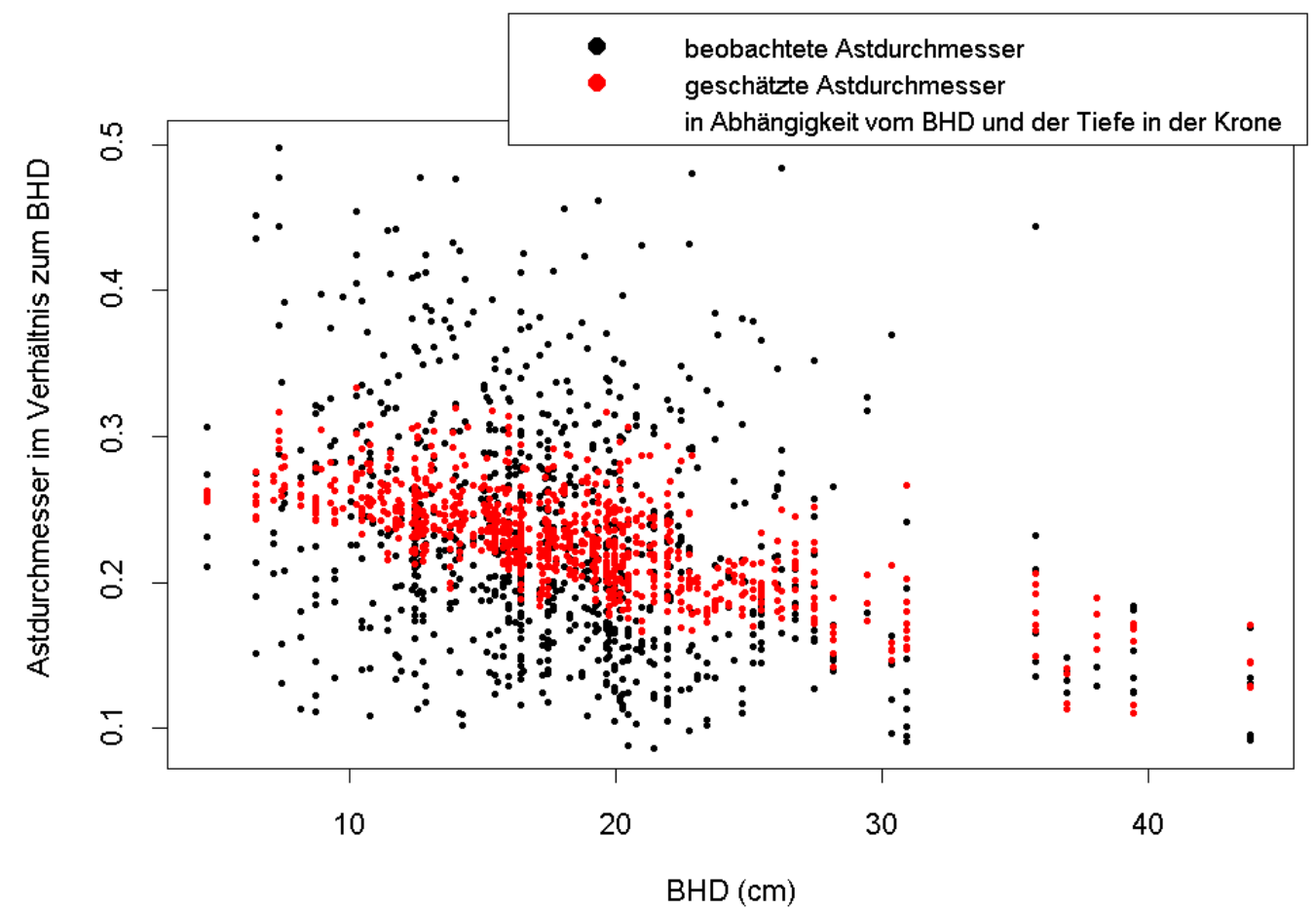

Abb. 48: Normierte Durchmesser von Ästen aus dem Bereich des Kronenansatzes der Baumart Buche und die zugehörigen Schätzungen mit Hilfe eines multiplen linearen Modells in Abhängigkeit vom BHD und der Tiefe in der Krone des Astes.

Es wird deutlich, daß über den Dimensionseffekt hinaus Zusammenhänge zwischen den Astdurchmessern und den unabhängigen Variablen BHD und Tiefe in der Krone bestehen. Das zur Schätzung der normierten Astdurchmesser verwendete Modell ist in Formel (23) dargestellt.

$A D=\alpha_{0}+\alpha_{1} * D+\alpha_{2} * T I N K$

wobei:

$A D=$ Durchmesser eines Astes im Kronenansatzbereich (mm);

$D \quad=$ Brusthöhendurchmesser (mm);

$T I N K=$ Tiefe in der Krone (dm). 
für Formel (23) mit:

\begin{tabular}{|c|c|c|c|c|}
\hline Koeffizient & Wert & Std. Fehler & t-Wert & $\operatorname{Pr}(>|t|)$ \\
\hline$\alpha_{0}$ & 0.272 & 0.0078 & 34.743 & 0.0000 \\
\hline$\alpha_{1}$ & -0.0008 & 0.0001 & -14.038 & 0.0000 \\
\hline$\alpha_{2}$ & 0.0012 & 0.0001 & 10.015 & 0.0000 \\
\hline Residual Std. Fehler & \multicolumn{4}{|c|}{0.0717 bei 870 Freiheitsgraden } \\
\hline Multiples Bestimmtheitsmaß & \multirow{2}{*}{\multicolumn{4}{|c|}{$\begin{array}{r}0.185 \\
98.75 \text { bei } 2 \text { und } 870 \text { Freiheitsgraden, } p \text {-Wert }=0\end{array}$}} \\
\hline F-Statistik & & & & \\
\hline
\end{tabular}

Tab. 18: Koeffizienten und statistische Kenngrößen zur Schätzung von normierten Durchmessern von Ästen aus dem Kronenansatzbereich von Buchen mit Hilfe eines multiplen linearen Modells (23).

Es wird deutlich, daß bei gegebenem BHD und gegebener Tiefe in der Krone nach der Schätzung eine sehr große unerklärte Streuung verbleibt. Bei den Nadelholzarten wurde durch die stratenweise (Aststärkenrangfolge) Parametrisierung eine Reduktion der unerklärten Streuung für die einzelnen Stratenversionen des Modells erreicht. Die Gesamtheit der Stratenmodelle wiederum erlaubt eine gute Beschreibung der bedingten Streuung der Astdurchmesser eines Quirls bei gegebenem BHD, H/D-Wert und gegebener Tiefe in der Krone. Bei den Laubholzarten erscheint eine Stratifizierung nach der Aststärkenrangfolge als Mittel zur Erfassung der vom Modell nicht erklärten Reststreuung ungeeignet, da die Äste nicht in Quirlen angeordnet sind und den Ästen nicht eindeutig ein Rang zugeordnet werden kann. Weiterhin besteht für die nach Straten parametrisierten Modelle das bereits bei den Nadelholzarten skizzierte Problem, daß im Vergleich der Modelle inkonsistente Schätzungen möglich sind (Kapitel 3.3.2).

Zur Berücksichtigung der nicht erklärten Streuung der normierten Astdurchmesser bei gegebenem BHD und gegebener Tiefe in der Krone wurde daher ein verallgemeinertes Verteilungsmodell auf der Basis der Beta-Verteilung entwickelt. Dabei wurden für Straten von Ästen mit ähnlichen Kombinationen der Variablen BHD und mittlere Tiefe in der Krone BetaVerteilungen angepaßt. Die mittlere Tiefe in der Krone ist der Mittelwert der betrachteten Äste im Kronenansatzbereich eines Baumes. Da die Variablen BHD und Tiefe in der Krone die normierten Astdurchmesser beeinflussen, kann davon ausgegangen werden, daß die empirischen Astdurchmesserverteilungen in den verschiedenen Straten unterschiedliche Eigenschaften aufweisen. Die Eigenschaften der empirischen Verteilungen werden durch die Anpassung der Dichtefunktionen modellhaft erfaßt und kommen in den Ausprägungen der Formparameter der Dichtefunktion zum Ausdruck. In einem weiteren Schritt wurde untersucht, inwieweit sich die Formparameter mit Hilfe der Stratenmittelwerte für den BHD und die mittlere Tiefe in der Krone beschreiben lassen. Lassen sich die Formparameter der Dichtefunktion in Abhängigkeit vom BHD eines Baumes und der mittleren Tiefe in der Krone der betrachteten Äste beschreiben, können Astdurchmesser für beliebige Bäume unter Berück- 
sichtigung der zugehörigen Astdurchmesserstruktur geschätzt werden. Die Stratifizierung der Astdurchmesserwerte nach Kombinationen der unabhängigen Variablen BHD und mittlere Tiefe in der Krone war notwendig, da für eine einzelbaumweise Anpassung der Dichtefunktion viel zuwenig Beobachtungen vorlagen. Die verwendeten Klassenbreiten sind unterschiedlich, da an den Randbereichen von Variablenkombinationen weniger Werte zur Verfügung standen und Klassen zusammengefaßt werden mußten. Weiterhin wurden unterschiedlich viele BHD-Klassen in Abhängigkeit von der zugehörigen Klasse der Variablen Tiefe in der Krone zusammengefaßt. In Tab. 19 sind die resultierenden Klassenbesetzungen abgebildet.

\begin{tabular}{|c|r|r|r|r|r|r|r|}
\hline & \multicolumn{7}{|c|}{$\begin{array}{c}\text { Klassen für die Variable mittlere Tiefe in der Krone } \\
\text { (variable Klassenbreiten / dm) }\end{array}$} \\
\hline \multirow{3}{*}{$\begin{array}{c}\text { BHD-Klassen } \\
\text { (variable Klassen- } \\
\text { breiten / mm) }\end{array}$} & & $\begin{array}{r}17.5- \\
53.8\end{array}$ & $\begin{array}{r}53.9- \\
71.9\end{array}$ & $\begin{array}{r}72.0- \\
90.0\end{array}$ & $\begin{array}{r}90.1- \\
108.2\end{array}$ & $\begin{array}{r}108.3- \\
126.3\end{array}$ & $\begin{array}{r}126.4- \\
189.9\end{array}$ \\
\cline { 2 - 9 } & $47.0-125.4$ & & 67 & 22 & & & \\
\cline { 2 - 9 } & $47.0-164.4$ & 60 & & & 26 & & \\
\cline { 2 - 9 } & $125.4-164.4$ & & 47 & 113 & & & 34 \\
\cline { 2 - 9 } & $164.5-203.8$ & & 40 & 100 & 106 & & \\
\cline { 2 - 9 } & $203.9-243.0$ & & & 21 & 53 & 37 & \\
\cline { 2 - 9 } & $243.1-439.0$ & & & & 20 & 39 & 54 \\
\hline
\end{tabular}

Tab. 19: Klasseneinteilung für die Variablen BHD und mittlere Tiefe in der Krone sowie die resultierenden Besetzungen.

Die in Formel (24) dargestellte (standardisierte) Dichtefunktion der Beta-Verteilung ist auf den Wertebereich zwischen 0 und 1 beschränkt und eignet sich daher für die Beschreibung von Verteilungen mit natürlichen unteren und oberen Grenzen. Die Beta-Verteilung ist im forstlichen Kontext vor allem zur Beschreibung und Prognose von Stammdurchmesserverteilungen verwendet worden. Dabei wurden in Vergleichen mit der Weibull-Verteilung sowohl gleichwertige (SwINDEL ET AL., 1987; MALTAMO ET. AL., 1995) als auch schlechtere Anpassungsergebnisse erzielt (VAN LAAR U. MOSANDL, 1989; GADOW, 1984 u. 1987). Für die Anwendung der Beta-Verteilung auf Durchmesserverteilungen ist eine Reskalierung bzw. die Anwendung einer nicht-standardisierten Form notwendig. Für die Reskalierung sind die Angabe eines unteren und oberen Schwellenwertes bzw. eines unteren Schwellenwertes und der Spannweite der Verteilung notwendig. Für eine Regionalisierung bzw. Prognose von Durchmesserverteilungen auf der Basis von Bestandesmittelwerten bedeutet das jedoch, daß Schätzungen für die zur Reskalierung verwendeten Schwellenwerte vorliegen müssen. Damit kommt der Vorteil der standardisierten Beta-Verteilung, die implizit einen unteren (0) und oberen Schwellenwert (1) berücksichtigt, bei der Modellierung von Durchmesserverteilungen nicht zum Tragen. 
Dagegen sind die mit dem BHD normierten Astdurchmesser auf den Wertebereich im Intervall $[0,1]$ begrenzt, wobei Werte über 0.5 als sehr selten zu betrachten sind. Im Gegensatz zur Modellierung von Durchmesserverteilungen können für die Beta-Verteilung bei der Modellierung von Astdurchmesserverteilungen theoretische Vorteile aufgrund der impliziten Schwellenwerte gegenüber anderen Verteilungsfunktionen angeführt werden. Die Dichtefunktion der standardisierten Beta-Verteilung hat die folgende Form:

$f(x ; v, \omega)=\Gamma(v+\omega) /(\Gamma(v) \Gamma(\omega)) * x^{v-1} *(1-x)^{\omega-1}$

$0 \leq x \leq 1$,

$v>1, \omega>1$,

wobei:

$\Gamma(v+\omega) / \Gamma(v) * \Gamma(\omega)$ die Beta-Funktion ist;

$\Gamma=$ die Gamma-Funktion ist;

$\mathrm{x}=$ Zufallsvariable, für die die Dichteverteilung modelliert werden soll, hier der normierte Astdurchmesser;

$v, \omega=$ Formparameter.

Im Rahmen von Anwendungen der Beta-Verteilung auf biologische Phänomene hat sich eine spezielle Methode bei der Schätzung der Formparameter bewährt (ZuCCHINI, 2001: Mündliche Mitteilung $)^{15}$. Dabei werden die Formparameter $v$ und $\omega$ nicht direkt sondern als Kombinationen der Parameter $\mu$ und $\phi$ geschätzt:

$v=\mu^{*}(1-\phi) / \phi$

$\omega=(1-\mu) *(1-\phi) / \phi$

wobei:

$0 \leq \mu \leq 1 ; 0 \leq \phi \leq 1$.

Die Transformation wirkt sich nur unwesentlich auf die Anpassung der Dichtefunktion an eine empirische Verteilung aus. Der Vorteil der Transformation besteht jedoch darin, daß sich der Parameter $\mu$ besser in Abhängigkeit von unabhängigen Variablen schätzen läßt, als die Formparameter $v$ und $\omega$ der Beta-Verteilung. Der Parameter $\phi$ weist dagegen häufig nur sehr geringe Abhängigkeiten zu unabhängigen Variablen auf und wird durch eine Konstante ersetzt. Auch sind die transformierten Parameter $\mu$ und $\phi$ als Mittelwert bzw. Streuungsmaß im Gegensatz zu den Originalparameter biologisch interpretierbar. Nach der Ermittlung der Kon-

\footnotetext{
${ }^{15} \mathrm{Zu}$ Methoden der Transformation der Parameter der Beta-Verteilung siehe KLEINMAN (1973) und KUPPER ET AL. (1986).
} 
stanten, beispielsweise kann der Mittelwert aller Parameter $\phi$ der stratenweisen Anpassungen verwendet werden, erfolgt eine erneute Anpassung der Dichtefunktion der Beta-Verteilung an die empirischen Verteilungen, wobei nur der Parameter $\mu$ variiert und der Parameter $\phi$ konstant belassen wird. Diese Einschränkungen wirken sich über die Formeln (25) und (26) auf die Formparameter $v$ und $\omega$ aus. Bei der Anpassung der Beta-Verteilung ist zu beachten, daß der zulässige Variationsbereich der Parameter $\mu$ und $\phi$ bzw. nur $\mu$ bei konstantem $\phi$ auf das Intervall [0,1] begrenzt ist. Das Statistik-Paket S-PLUS 3.3 (MATHSOFT, 1995) bietet zu diesem Zweck eine Minimierungsfunktion, die eine Angabe von unteren und oberen Grenzen des Variationsbereiches eines Parameters ermöglicht.

Für die im zweiten Anpassungsschritt bei konstantem Parameter $\phi$ ermittelten Werte des Parameters $\mu$ wird anschließend mittels Regressionsanalyse ein Modell entwickelt, das die Schätzung des Parameters in Abhängigkeit von den unabhängigen Variablen BHD und mittlerer Tiefe in der Krone ermöglicht. Zur Anpassung der Beta-Verteilung wurde die MaximumLikelihood-Methode verwendet. Die mit Hilfe der Maximum-Likelihood-Methode ermittelten Werte für die Parameter $v$ und $\omega \mathrm{bzw}$. bei Anwendung der Transformation für die Parameter $\mu$ und $\phi$ sind die Werte, die den folgenden Term maximieren:

$\log$ likelihood, $L(v, \omega) \equiv \sum \log p\left(X_{i} \mid v, \omega\right)$

bei Transformation:

log likelihood, $L(\mu, \phi) \equiv \sum \log p\left(X_{i} \mid \mu, \phi\right)$

bei Transformation und konstantem Parameter $\phi$ :

log likelihood, $L(\mu) \equiv \sum \log p\left(X_{i} \mid \mu\right)$

wobei bei Transformation gilt:

$v=\mu *(1-\phi) / \phi$;

$\omega=(1-\mu) *(1-\phi) / \phi$;

$0 \leq \mu \leq 1 ; 0 \leq \phi \leq 1$;

$p\left(X_{i} \mid\right.$ Parameter $)=$ Dichtefunktion der Beta-Verteilung.

Da das Statistik-Paket S-PLUS 3.3 (MATHSOFT, 1995) nur einen Minimierungsalgorithmus mit der Möglichkeit zur Einschränkung des Variationsbereiches der zu schätzenden Parameter bietet, wird der zu maximierende Term mit -1 multipliziert. In Abb. 115 (Kapitel 9.7) sind die Anpassungen der Dichtefunktion der Beta-Verteilung an die empirischen Verteilungen der normierten Astdurchmesser in den einzelnen Straten dargestellt. Zusätzlich sind die Mittelwerte der Variablen BHD und mittlere Tiefe in der Krone für die Bäume eines Stratums ange- 
geben. Die Formparameter $v$ und $\omega$ wurden nicht direkt, sondern als Kombination der Parameter $\mu$ und $\phi$ geschätzt (Formeln (25) u. (26)).

In Tab. 20 sind die Prüfgrößen und zugehörigen $p$-Werte des Kolmogorov-SmirnovTests für die stratenweise Anpassung der Beta-Verteilung angegeben. Der Test dient der Überprüfung, inwieweit die empirischen Verteilungen in der Tat einer Beta-Verteilung mit den spezifischen Formparametern $v$ und $\omega$ bei einer bestimmten Irrtumswahrscheinlichkeit entsprechen. Es wird deutlich, daß zum Niveau von $\alpha=0.05$ die Nullhypothese, daß die empirische Verteilung beta-verteilt ist, für keines der Straten verworfen werden kann. Die Anordnung der Prüfgrößen und $p$-Werte entspricht der Anordnung der Histogramme in Abb. 115 (Kapitel 9.7).

\begin{tabular}{|r|r|l|r|r|l|}
\hline Prüfgrösse D & $p$-Wert & Signifikanz & Prüfgrösse D & $p$-Wert & Signifikanz \\
\hline 0.1171 & 0.3831 & & 0.0610 & 0.9644 & \\
\hline 0.1106 & 0.9507 & & 0.1149 & 0.5643 & \\
\hline 0.0490 & 0.9494 & & 0.1468 & 0.3551 & \\
\hline 0.0732 & 0.6575 & & 0.2384 & 0.1838 & \\
\hline 0.1041 & 0.9408 & & 0.0697 & 0.6815 & \\
\hline 0.0984 & 0.6833 & & 0.2045 & 0.3732 & \\
\hline 0.2272 & 0.0902 & & 0.1017 & 0.8384 & \\
\hline 0.1188 & 0.6410 & & 0.1033 & 0.8610 & \\
\hline 0.1111 & 0.5178 & & & & \\
& & & &
\end{tabular}

Tab. 20: Prüfgrößen und p-Werte des Kolmogorov-Smirnov-Tests zur Überprüfung, inwieweit die empirischen Astdurchmesserverteilungen Beta-Verteilungen mit den spezifischen Parametern entsprechen (signifikante Unterschiede zwischen empirischer und theoretischer Verteilung *, bei $\alpha=0.05$ ).

Für eine Verallgemeinerung eines Verteilungsmodells muß es möglich sein, die Parameter einer Verteilungsfunktion in Abhängigkeit von unabhängigen Variablen zu schätzen, die leicht meßbar sind. Für die Anwendung im Rahmen von waldbaulichen Szenariensimulationen kommen weiterhin Variablen in Betracht, für die Waldwachstumssimulatoren Schätzungen bereitstellen. Zur Verallgemeinerung des Astdurchmessermodells der Baumart Buche wurde untersucht, inwieweit die zur Stratifizierung verwendeten Variablen BHD und mittlere Tiefe in der Krone die Form bzw. die Parameter der Beta-Verteilungen beeinflussen. In Abb. 49 wird deutlich, daß zwischen den Formparametern $v$ und $\omega$ der Beta-Verteilung und der unabhängigen Variablen BHD nur relativ schwache Zusammenhänge bestehen, die die Entwicklung eines Regressionsmodells zur Verallgemeinerung als schwierig erscheinen lassen. Ein Problem bei der getrennten Schätzung der Parameter von Verteilungsfunktionen mit Hilfe von unabhängigen Variablen können statistische Abhängigkeiten zwischen den zu schätzenden Parametern sein. Wenn mehrere Kombinationen von Parametern ähnliche Ver- 
teilungsformen beschreiben, die Ausprägung eines Parameters aber nicht nur durch unabhängige Variablen, sondern auch durch die Ausprägung anderer Verteilungsparameter beeinflußt wird, sind die Zusammenhänge zu den unabhängigen Variablen überlagert. D. h. jedoch nicht, daß die Zusammenhänge zwischen der Form der Verteilungen und den unabhängigen Variablen schwach ausgeprägt sein müssen.
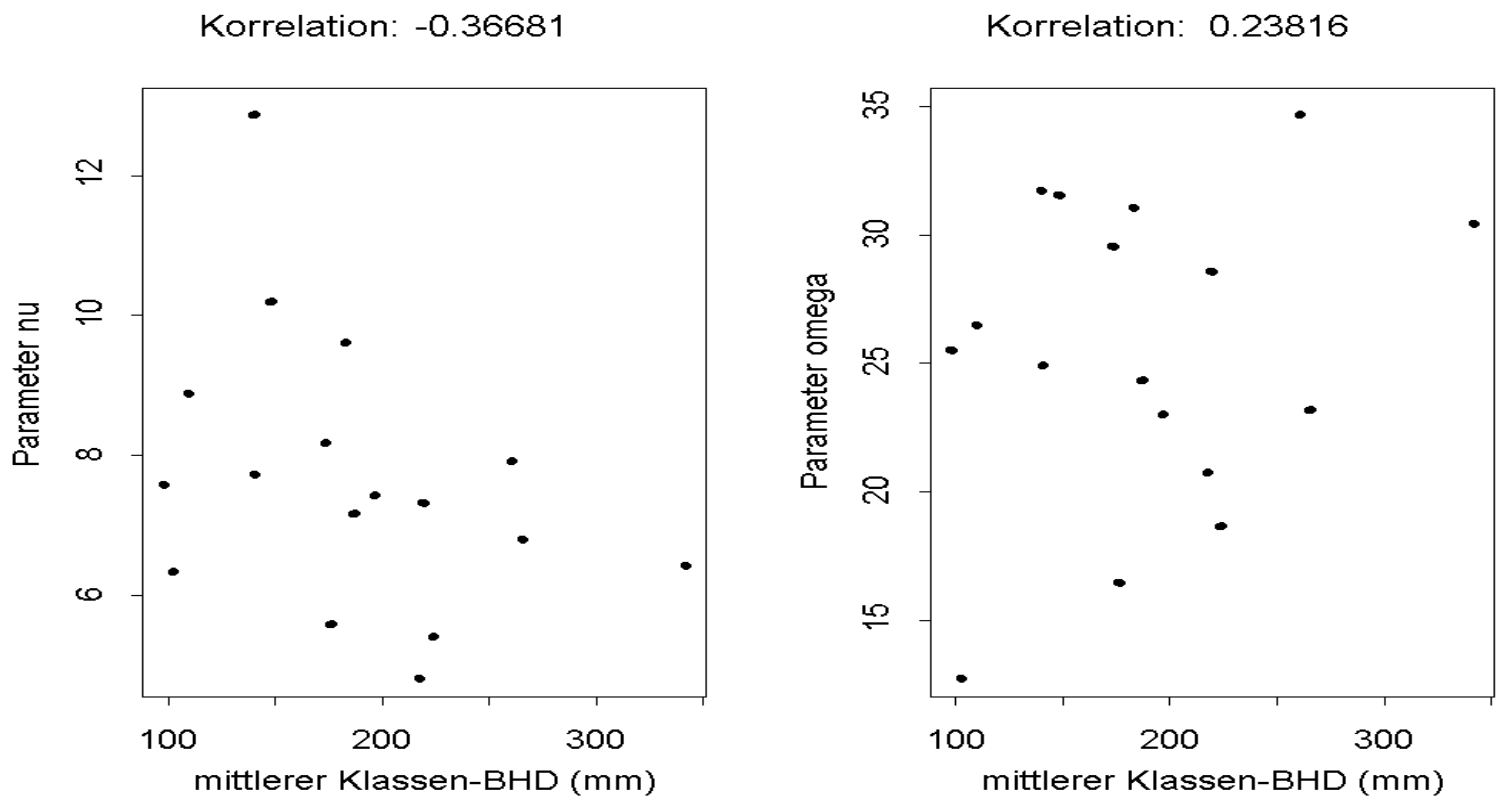

Abb. 49: Zusammenhänge zwischen den Formparametern der Beta-Verteilung und der unabhängigen Variablen BHD. Die Formparameter wurden über die stratenweise Anpassung der Beta-Verteilung ermittelt und der BHD ist der Mittelwert der Bäume des jeweiligen Stratums. Der Korrelationskoeffizient zwischen den Parametern $v$ und $\omega$ beträgt 0.669. Der Korrelationskoeffizient zwischen den Stratenmittelwerten der mittleren Tiefe in der Krone und den Parametern v und $\omega$ beträgt-0.216 bzw. 0.027.

Die in den Formeln (25) und (26) dargestellten Transformationen der Parameter $v$ und $\omega$ dienen der Beseitigung des Anteils der Korrelation zwischen den Parametern, der nicht durch die Struktur der Daten vorgegeben ist, sondern dadurch, daß mehrere Parameterkombinationen ähnliche Verteilungsformen definieren. Dieser Effekt ist in Abb. 50 deutlich zu erkennen. Der Parameter $\mu$ weist eine enge Korrelation zum BHD auf und auch zur unabhängigen Variablen mittlere Tiefe in der Krone besteht ein engerer Zusammenhang als für die ursprünglichen Parameter $v$ und $\omega$. Gleichzeitig hat die Korrelation zwischen den Verteilungsparametern $\mu$ und $\phi$ gegenüber der Korrelation zwischen den Parametern $v$ und $\omega$ abgenommen. Aufgrund der engeren statistischen Zusammenhänge zwischen dem Parameter $\mu$ und den unabhängigen Variablen erscheint die Entwicklung eines Regressionsmodells zur Verallgemeinerung des Astdurchmessermodells möglich. Dies gilt jedoch nicht für den Parameter $\phi$. Besonders hervorzuheben ist, daß die transformierten Parameter biologisch plausibel und gut zu 
interpretieren sind, da zwischen ihnen keine Korrelationen auftreten können, die nicht die Struktur der Daten widerspiegeln.
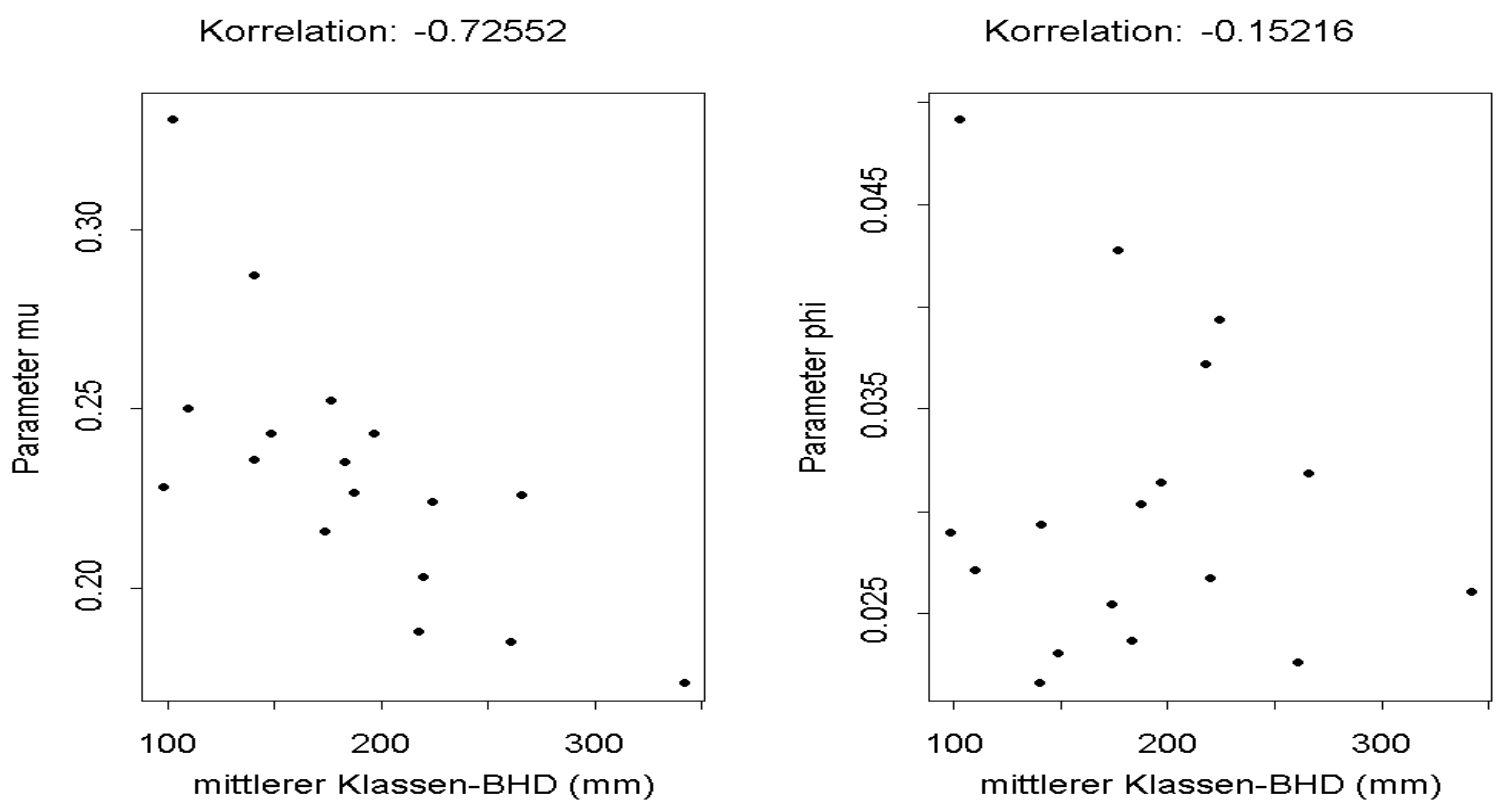

Abb. 50: Zusammenhänge zwischen den zur Transformation der ursprünglichen Parameter $v$ und $\omega$ verwendeten Parameter $\mu$ und $\phi$ und der unabhängigen Variablen BHD. Die Parameter wurden über die stratenweise Anpassung der Beta-Verteilung ermittelt und der BHD ist der Mittelwert der Bäume des jeweiligen Stratums. Der Korrelationskoeffizient zwischen den Parametern $\mu$ und $\phi$ beträgt 0.410. Der Korrelationskoeffizient zwischen den Stratenmittelwerten der mittleren Tiefe in der Krone und den Parametern $\mu$ und $\phi$ beträgt -0.270 bzw. 0.023 .

Dies bestätigt der niedrigere Korrelationskoeffizient zwischen den Parametern $\mu$ und $\phi$ gegenüber den Parametern $v$ und $\omega$. Da der Parameter $\phi$ aber nicht aus den unabhängigen Variablen geschätzt werden kann, wurde untersucht, inwieweit die Vorgabe eines konstanten Wertes für den Parameter $\phi$ zu einer weiteren Verbesserung der Schätzung des Parameters $\mu$ führt. Für alle Straten wurde die Beta-Verteilung mit den transformierten Parametern erneut parametrisiert, wobei für den Parameter $\phi$ der Mittelwert aller Ausprägungen der 1. Anpassung verwendet wurde. 
In Tab. 21 sind zur Überprüfung, inwieweit sich die Güte der Anpassung der Beta-Verteilung durch die Fixierung eines Parameters verschlechtert, die Prüfgrößen und $p$-Werte des Kolmogorov-Smirnov-Test aufgeführt.

\begin{tabular}{|r|r|l|r|r|l|}
\hline Prüfgrösse D & $p$-Wert & Signifikanz & Prüfgrösse D & $p$-Wert & Signifikanz \\
\hline 0.1195 & 0.3583 & & 0.0629 & 0.9538 & \\
\hline 0.1717 & 0.5354 & & 0.1170 & 0.5411 & \\
\hline 0.0784 & 0.4916 & & 0.1391 & 0.4214 & \\
\hline 0.0718 & 0.6813 & & 0.2365 & 0.1906 & \\
\hline 0.1247 & 0.8137 & & 0.0697 & 0.6818 & \\
\hline 0.1238 & 0.3911 & & 0.2104 & 0.3387 & \\
\hline 0.2331 & 0.0767 & & 0.091 & 0.9194 & \\
\hline 0.1181 & 0.6483 & & 0.1016 & 0.8740 & \\
\hline 0.1269 & 0.3491 & & & & \\
\cline { 2 - 5 } & & & & & \\
\end{tabular}

Tab. 21: Prüfgrößen und p-Werte des Kolmogorov-Smirnov-Tests zur Überprüfung inwieweit die empirischen Astdurchmesserverteilungen Beta-Verteilungen mit den spezifischen Parametern entsprechen, wenn der Parameter $\phi$ konstant belassen wird (signifikante Unterschiede zwischen empirischer und theoretischer Verteilung *, $\alpha=0.05)$. Die Anordnung der Prüfgrößen und p-Werte entspricht der Anordnung der Histogramme in Abb. 115 (Kapitel 9.7).

Es wird deutlich, daß sich bei der Maximum-Likelihood-Anpassung mit konstantem Parameter $\phi$ im Durchschnitt die Werte der Prüfgrösse erhöhen bzw., daß die $p$-Werte sinken. Aber auch mit konstantem Parameter $\phi$ kann für keine der empirischen Verteilungen die Nullhypothese zum 5\% Niveau verworfen werden, die besagt, daß empirische und theoretische Verteilung übereinstimmen. D. h., die empirischen Verteilungen können in der Tat als betaverteilt mit den jeweiligen Parameterwerten betrachtet werden. In Abb. 51 wird deutlich, daß sich die Korrelation zwischen den unabhängigen Variablen und dem Parameter $\mu$ durch die Fixierung des Parameters $\phi$ kaum erhöht.

Die Transformation der ursprünglichen Formparameter hatte den Zweck der Beseitigung der nicht durch die Datenstruktur bedingten Korrelation zwischen den Formparametern. Insofern war durch die Fixierung des Parameters $\phi$ keine deutliche Erhöhung der Korrelation zu erwarten. Die enge Korrelation ermöglicht jedoch weiterhin die Entwicklung eines Regressionsmodells zur Schätzung des Parameters $\mu$ in Abhängigkeit von den unabhängigen Variablen BHD und mittlerer Tiefe in der Krone. In Abb. 51 sind zusätzlich zu den über die Maximum-Likelihood-Methode auf der Basis der empirischen Verteilungen geschätzten Parameter die mit Hilfe des Modells (27) geschätzten Parameterwerte dargestellt. 

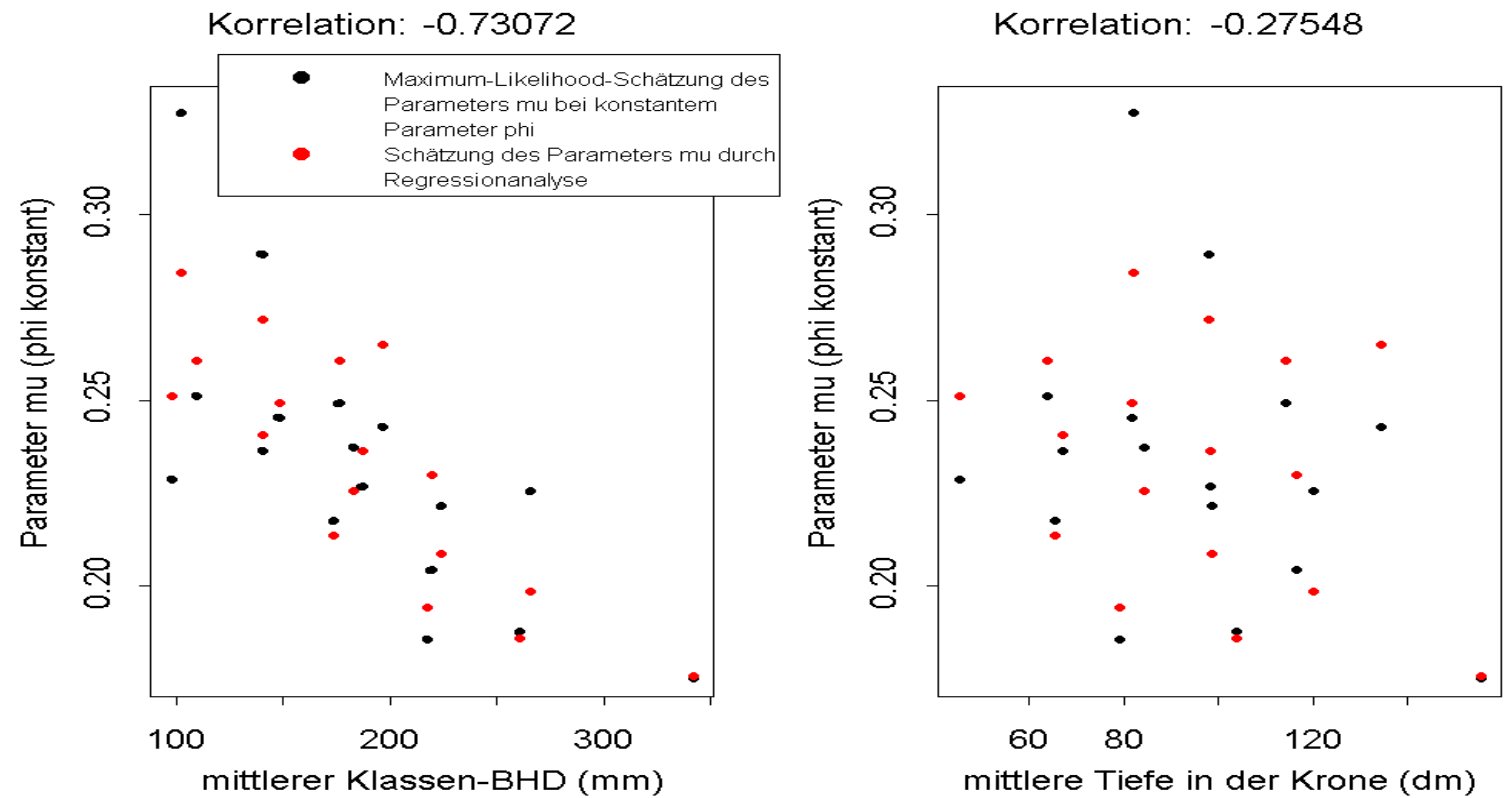

Abb. 51: Zusammenhänge zwischen dem Parameter $\mu$ und den unabhängigen Variablen BHD und mittlere Tiefe in der Krone. Der Parameter $\mu$ wurde mit Hilfe der MaximumLikelihood-Methode geschätzt, wobei der Parameter $\phi$ konstant gehalten wurde. Weiterhin ist eine Schätzung für den Parameter $\mu$ mit Hilfe des in Formel (27) beschriebenen Modells dargestellt. Die unabhängigen Variablen BHD und mittlere Tiefe in der Krone sind Mittelwerte der jeweiligen Astdurchmesserstraten.

Das Modell (27) dient der Verallgemeinerung des Astdurchmesserverteilungsmodells für die Baumart Buche in Abhängigkeit von den Stratenmittelwerten des BHD und der mittleren Tiefe in der Krone.

$\mu=\alpha+\beta^{*} D+\gamma^{*} M_{-} T I N K$

wobei:

$D=$ Brusthöhendurchmesser (mm) (Stratenmittelwert);

$M \_T I N K=$ mittlere Tiefe in der Krone (dm) (Stratenmittelwert);

$\mu \quad=$ zur Transformation der ursprünglichen Formparameter der Beta-Verteilung verwendeter Parameter (Mittelwert);

$\alpha, \beta, \gamma \quad=$ Parameter.

mit:

\begin{tabular}{|c|c|c|c|c|}
\hline Koeffizienten & Wert & Std. Fehler & t-Wert & $\operatorname{Pr}(>|t|)$ \\
\hline$\alpha$ & 0.282 & 0.0172 & 16.395 & 0.0000 \\
\hline$\beta$ & -0.000761 & 0.000121 & -6.279 & 0.0000 \\
\hline$\gamma$ & 0.000998 & 0.000278 & 3.598 & 0.0029 \\
\hline \multicolumn{2}{|c|}{ Residual Std. Fehler } & \multicolumn{3}{|c|}{0.0195 bei 14 Freiheitsgraden } \\
\hline \multicolumn{2}{|c|}{ Multiples Bestimmtheitsmaß } & \multicolumn{3}{|r|}{0.758} \\
\hline \multicolumn{2}{|c|}{ F-Statistik } & \multicolumn{3}{|c|}{21.9 bei 2 und 14 Freiheitsgraden, $p$-Wert $=0$} \\
\hline
\end{tabular}

Tab. 22: Koeffizienten und RMSE für das Modell zur Schätzung des Parameters $\mu$ bzw. zur Verallgemeinerung des Astdurchmessermodells für die Baumart Buche. 


\section{Modellvalidierung und -bewertung:}

Für die Parametrisierung des Modells wurden die Mittelwerte des jeweiligen Astdurchmesserstratums verwendet. Das Modell soll im Rahmen von Waldwachstumssimulationen jedoch für die Prognose der qualitätsrelevanten Astigkeit von Einzelbäumen eingesetzt werden. Die Integration des BHD in das Modell (27) dient der Berücksichtigung des Einflusses der Einzelbaumdimension auf die Astdurchmesserstruktur, die über den reinen Dimensionstrend hinaus geht, daß starke Bäume im Mittel dickere Äste als dünnere Bäume haben. Der reine Dimensionstrend wird bereits dadurch berücksichtigt, daß die Astdurchmesser im Verhältnis zum BHD angegeben werden. Es wird deutlich, daß starke Bäume schwächere normierte Astdurchmesser als dünnere Bäume haben (Formel (27), Abb. 52). Dabei ist zu bedenken, daß die zur Parametrisierung verwendeten Äste Durchmesserwerte nicht überschreiten durften, die zu einer Auflösung der Stammachse führen. Der vom Modell beschriebene Zusammenhang kann daher auch zum Ausdruck bringen, daß bei starken Bäumen bereits niedrigere normierte Astdurchmesser zur Auflösung der Stammachse führen als bei schwächeren Bäumen. Für die Anwendung und Interpretation des Modells muß somit der Geltungsbereich beachtet werden, der nur für Astdurchmesser gilt, die zu keiner Auflösung der Stammachse führen. Die Prognose, ab welcher Höhe sich die Stammachse einer Buche auflöst, ist aufgrund sehr großer statistischer Unsicherheiten problematisch. Vereinfachend kann angenommen werden, daß im Mittel bei Einzelbaumdimensionen, für die Kronenansatzmodelle (Kapitel 2.3 u. 3.2) nur noch geringfügige Verlagerungen der Kronenansatzhöhe prognostizieren, Astdurchmesser erreicht werden, die zu einer Auflösung der Stammachse führen.

Durch die Integration der Kronenlänge in das verallgemeinerte Astdurchmessermodell wird eine Behandlungssensitivität gewährleistet. Es ist davon auszugehen, daß weitständig erwachsene Bäume bzw. Bäume, die aus stark durchforsteten Beständen stammen, längere Kronen aufweisen als Bäume aus dichten Beständen. Bäume mit längeren Kronen werden im Durchschnitt stärkere Äste im Kronenansatzbereich besitzen als Bäume mit kurzen Kronen, da der Zeitraum bis zum Absterben eines Astes länger ist. Demgegenüber ließen die Voruntersuchungen zum Einfluß des kleinräumigen $\mathrm{C}_{66}$-Konkurrenzindex keinen Zusammenhang zur normierten Aststärke erkennen. Es kann jedoch vermutet werden, daß die Kronenlänge für Äste aus dem Kronenansatzbereich ein höheres Erklärungspotential aufweist als der mittlere Konkurrenzindex der letzten 10-Jahresperiode, da sie die Konkurrenzbedingungen eines Baumes in einem längeren, zurückliegenden Zeitraum besser widerspiegelt und mit dem Alter des Astes korreliert ist. 
Die Wirkungsrichtung der Kronenlänge im Modell (27) ist somit biologisch plausibel, da sie für Bäume mit längeren Kronen stärkere normierte Astdurchmesser prognostiziert als für Bäume mit kürzeren Kronen (vgl. Abb. 52). Als problematisch ist allerdings zu beurteilen, daß die entwickelten Kronenansatzmodelle (Kapitel 3.2) trotz der gegenüber den Vergleichsmodellen verbesserten Schätzung keine Behandlungssensitivität aufweisen. Die Baumart Buche wies zudem die größten Standardfehler der untersuchten Baumarten bei der Schätzung der Kronenansatzhöhe auf.

Das Astdurchmessermodell für die Baumart Buche setzt jedoch eine behandlungssensitive Schätzung der Kronenlänge voraus, wenn auch die Aststärke behandlungssensitiv modelliert werden soll. Für Szenariensimulationen sollte daher auf langfristig beobachtete Versuchsflächen zurückgegriffen werden, die über die Integration zusätzlicher Informationen eine genauere Modellierung der Kronenansatzhöhe ermöglichen (Kapitel 3.2.3 u. 3.2.4, Einheitskronenansatzmodell).

In Tab. 23 wird überprüft, inwieweit das verallgemeinerte Astdurchmessermodell noch eine Beschreibung der empirischen Verteilungen in den einzelnen Straten gewährleistet. Es ist ein deutliches Ansteigen der Werte der Prüfgröße bzw. ein Absinken der $p$-Werte gegenüber den beiden direkten Anpassungen mit Hilfe der Maximum-Likelihood-Schätzung zu beobachten (vgl. Tab. 20 u. Tab. 21). Trotzdem muß nur für zwei Straten die Annahme, die empirische Verteilung sei beta-verteilt, abgelehnt werden.

\begin{tabular}{|r|c|c|r|r|l|}
\hline Prüfgrösse $D$ & $p$-Wert & Signifikanz & Prüfgrösse $D$ & $p$-Wert & Signifikanz \\
\hline 0.2392 & 0.0021 & $*$ & 0.1124 & 0.3659 & \\
\hline 0.2840 & 0.0575 & & 0.1394 & 0.3206 & \\
\hline 0.0895 & 0.3261 & & 0.1180 & 0.6334 & \\
\hline 0.1076 & 0.1971 & & 0.2870 & 0.0629 & \\
\hline 0.2012 & 0.2432 & & 0.1216 & 0.0868 & \\
\hline 0.1553 & 0.1552 & & 0.1997 & 0.4020 & \\
\hline 0.2922 & 0.0119 & $*$ & 0.2140 & 0.0675 & \\
\hline 0.1877 & 0.1279 & & 0.2173 & 0.0806 & \\
\hline 0.1299 & 0.3217 & & \multicolumn{2}{|l}{} &
\end{tabular}

Tab. 23: Prüfgrößen und p-Werte des Kolmogorov-Smirnov-Tests zur Überprüfung inwieweit die empirischen Astdurchmesserverteilungen Beta-Verteilungen mit den spezifischen Parametern entsprechen, wenn die Formparameter über unabhängige Variablen geschätzt werden (signifikante Unterschiede zwischen empirischer und theoretischer Verteilung *, $\alpha=0.05)$. Die Anordnung der Prüfgrößen und p-Werte entspricht der Anordnung der Histogramme in Abb. 115 (Kapitel 9.7). 
In Abb. 116 (Kapitel 9.7) wird das Spektrum der Astdurchmesserverteilungen, das über die Maximum-Likelihood-Methode auf der Basis der empirischen Daten geschätzt wurde, mit dem Spektrum des verallgemeinerten Astdurchmessermodells auf der Basis von Formel (27) verglichen. Die direkte Anpassung an den empirischen Daten ist die Variante mit fixiertem Parameter $\phi$. Es wird deutlich, daß für die überwiegende Anzahl der Astdurchmesserstraten nur geringe Unterschiede zwischen den direkt an den empirischen Verteilungen geschätzten Dichtefunktionen der Beta-Verteilung und dem verallgemeinerten Astdurchmessermodell zu beobachten sind. Damit kann das verallgemeinerte Astdurchmessermodell als geeignet zur Prognose der Verteilung der Astdurchmesser im Kronenansatzbereich von Buchen beurteilt werden.

Zur Beurteilung der Astigkeit des gesamten Stammholzbereiches im Rahmen von Waldwachstumssimulationen wird unterstellt, daß Totäste mit dem Durchmesser in den Stamm einwachsen, der zum Zeitpunkt des Absterbens mit Hilfe des verallgemeinerten Modells geschätzt wird. Im Gegensatz zu den Nadelholzarten weist die Baumart Buche jedoch nur sehr kurze Totastzonen auf. Auch eine Prognose der qualitätsrelevanten Astigkeit bzw. Holzqualität, die durch bereits überwallte Äste bestimmt wird, kann erfolgen, indem der Astdurchmesser zum Zeitpunkt der Astreinigung und der Stammradius an astfreiem Holz über dem Ast eingeschätzt werden (Kapitel 3.7). Die Beschreibung der Verjüngung des Astdurchmessers in Richtung des Stammzentrums (Kapitel 2.4.6, Abb. 8) erfolgt wie bei Nadelholzarten, indem für den (lebenden) Ast nach jedem Simulationsintervall ein Durchmesser geschätzt wird. Diese Prognose kann im Gegensatz zu den Nadelholzarten erst ab dem Zeitpunkt erfolgen, ab dem sich der Ast im Kronenansatzbereich des Baumes befindet.

In Abb. 52 ist der Einfluß unterschiedlicher Kombinationen von BHD-Werten und Werten für die mittlere Tiefe in der Krone auf die Verteilungsform der normierten Astdurchmesser dargestellt. Es wird deutlich, daß der Mittelwert und die Spannweite der Verteilungen mit sinkendem BHD und steigender Tiefe in der Krone zunehmen. 

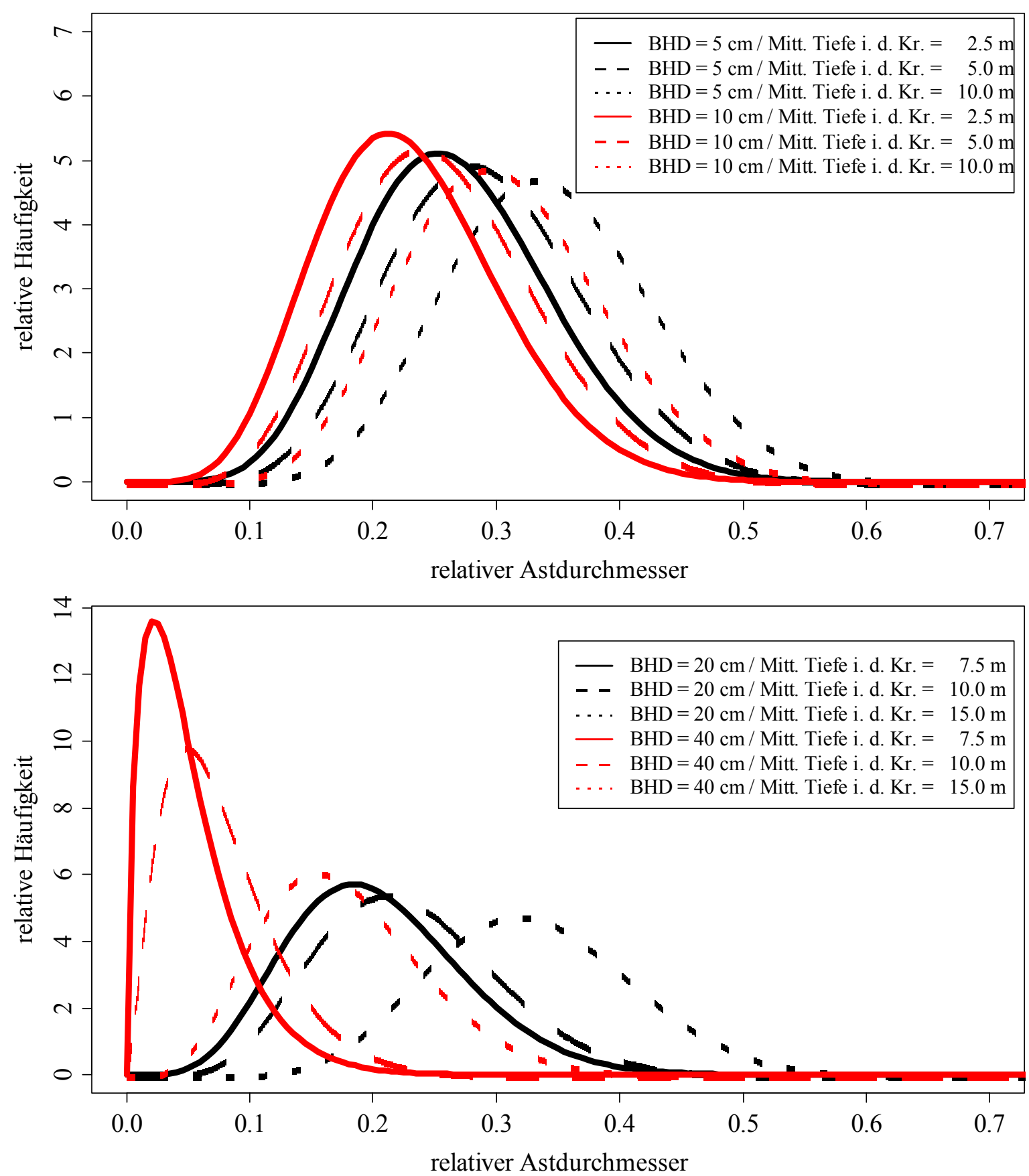

Abb. 52: Verteilungen der normierten Astdurchmesser im Kronenansatzbereich von Buchen mit unterschiedlichen Kombinationen von BHD-Werten und Werten für die mittlere Tiefe in der Krone.

Zur Erzeugung konkreter Astdurchmesser wird, wie bei der Anwendung von Verteilungsmodellen häufig üblich, eine invertierte Verteilungsfunktion (Quantilsfunktion) verwendet, in die im Intervall [0,1] äquidistant verteilte Zahlen oder Zufallszahlen eingesetzt werden (z. B. SCHMIDT U. GADOw, 1999). Mit Hilfe dieses Verfahrens können beliebige Anzahlen an Astdurchmessern für den Kronenansatzbereich von Buchen unter Berücksichtigung der zugehörigen Verteilung prognostiziert werden. In Formel (28) ist die Verteilungsfunktion (kumulative Dichtefunktion) der Beta-Verteilung dargestellt. Die Verteilungsfunktion beschreibt die 
Wahrscheinlichkeit, daß ein zufällig gezogener Wert $X$ kleiner oder gleich einem gegebenen Wert $x$ ist.

$$
F(X \leq x ; v, \omega)=\Gamma(v+\omega) /(\Gamma(v) \Gamma(\omega)) * \int_{0}^{x} x^{v-1} *(1-x)^{\omega-1} d t
$$

wobei:

$0 \leq x \leq 1$,

$v>1, \omega>1$,

wobei:

$\Gamma(v+\omega) / \Gamma(v) * \Gamma(\omega)=$ Beta-Funktion;

$\Gamma=$ Gamma-Funktion;

$\mathrm{X}=$ Zufallsvariable (zufällig gezogener BHD), für die die Wahrscheinlichkeit berechnet wird, daß sie kleiner oder gleich dem Wert $x$ ist;

$v, \omega=$ Formparameter.

In Abb. 53 sind Boxplots für jeweils 1000 generierte Astdurchmesser aus dem Kronenansatzbereich von Buchen mit unterschiedlichen Kombinationen von BHD-Werten und Werten für die mittlere Tiefe in der Krone dargestellt.

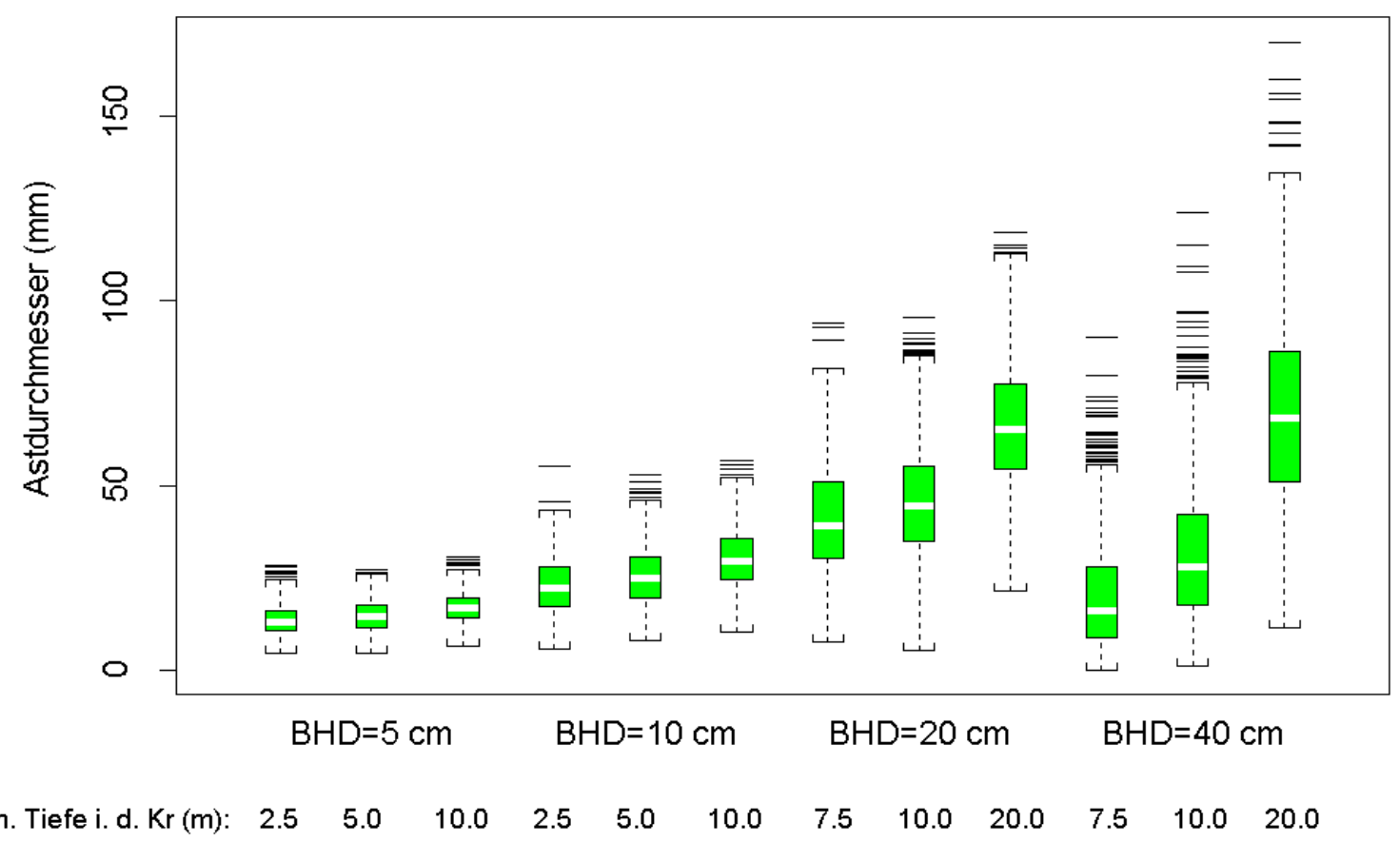

Abb. 53: Boxplots jeweils 1000 generierter Astdurchmesser im Kronenansatzbereich für Buchen mit unterschiedlichen Kombinationen von BHD und mittlerer Tiefe in der Krone. Die Boxplots beinhalten Median, unteres und oberes Quartil sowie unteren und oberen Extremwert ohne Ausreißer. Alle Werte, die außerhalb der 1.5-fachen Inter-QuartilDifferenz liegen, werden als Ausreißer betrachtet. 
Es wird deutlich, daß die Astdurchmesser mit steigenden Werten für die Variable mittlere Tiefe in der Krone bei gleichbleibendem BHD ansteigen. Auch mit steigendem BHD bei konstanten Werten für die Variable mittlere Tiefe in der Krone steigen die absoluten Astdurchmesser im Gegensatz zu den normierten Astdurchmessern an. Eine Ausnahme stellen die Schätzungen für den Baum mit $40 \mathrm{~cm}$ BHD und der mittleren Tiefe in der Krone von 7.5 und $10.0 \mathrm{~m}$ dar. Für diese Kombinationen sinken die Astdurchmesser gegenüber einem Baum mit $20 \mathrm{~cm}$ BHD und denselben Werten für die mittlere Tiefe in der Krone, was biologisch unplausibel erscheint. Die Ursache ist darin zu sehen, daß sich die Wertekombinationen im Extrapolationsbereich befinden. Das Datenmaterial beinhaltet keine Wertepaare, die bei einem BHD von mehr als $30 \mathrm{~cm}$ Werte für die mittlere Tiefe in der Krone von weniger als $11.0 \mathrm{~m}$ aufweisen (Abb. 54 a).

Gleichzeitig fällt auf, daß die Astdurchmesser der Bäume mit einem BHD von mehr als $30 \mathrm{~cm}$ relativ gering sind, was aber bei der geringen Anzahl der Messungen, die von wenigen Bäumen stammen, zufällig sein kann (Abb. 54 b). Das Astdurchmessermodell sollte daher nur für Buchen mit einem BHD von höchstens $30 \mathrm{~cm}$ verwendet werden. Für die praktische Anwendung ergeben sich daraus nur geringe Einschränkungen, da die unteren Stammlängen, die bei der Buche den Großteil des Wertes beinhalten, im allgemeinen bei Erreichen dieser Dimensionen längst astfrei sind. Die Prognose der Astigkeit für untere Stammlängen im Rahmen einer waldwachstumskundlichen Simulation ist daher beim Eintritt in den Extrapolationsbereiches bereits erfolgt, wenn es sich nicht um solitärähnlich erwachse Bäume handelt. Langfristig muß jedoch angestrebt werden, den Geltungsbereich des Modells zu erweitern.
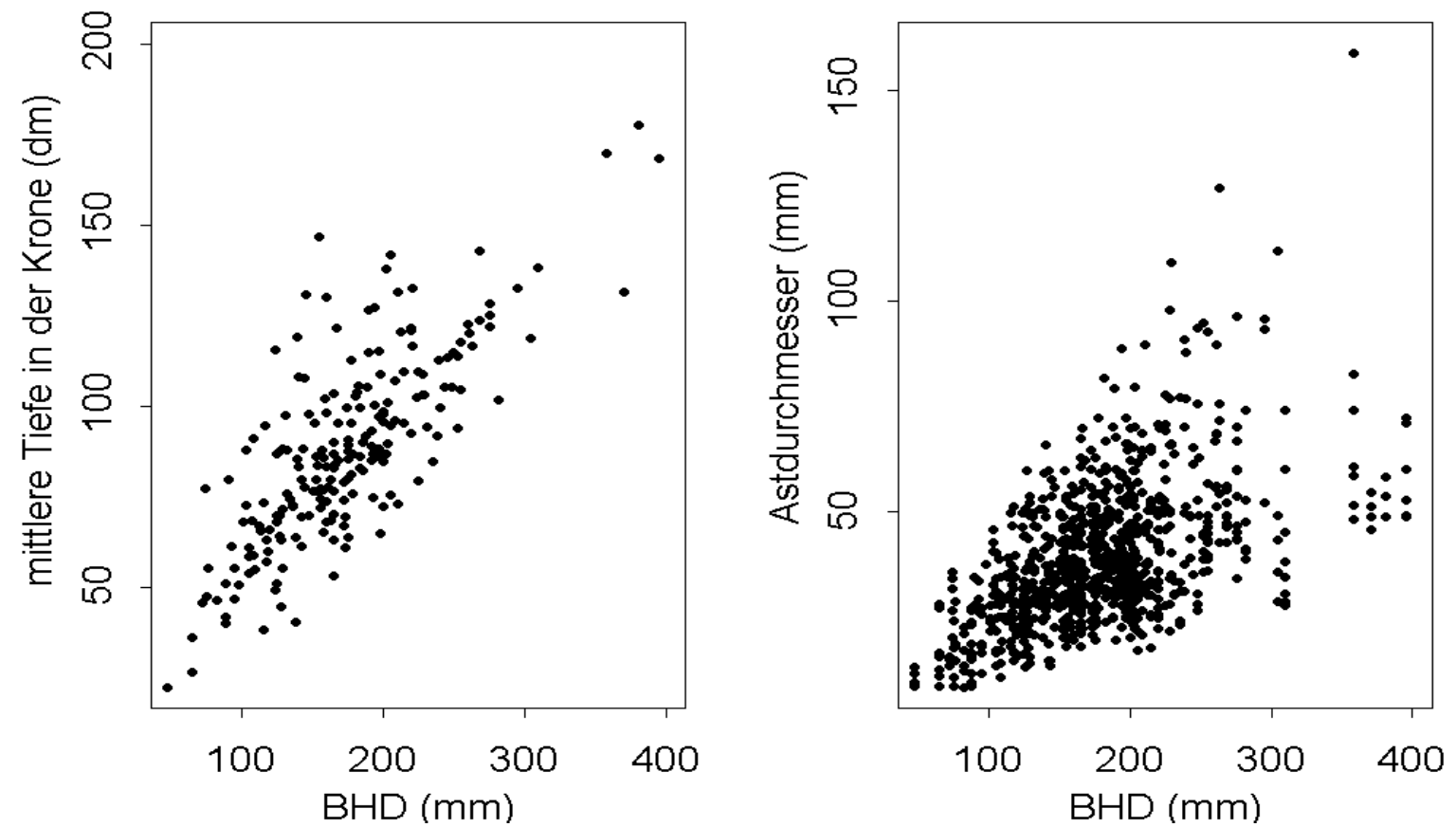

Abb. 54: (a) Zur Parametrisierung des Astdurchmessermodells verwendete Kombinationen von BHD und mittlerer Tiefe in der Krone (links); (b) Astdurchmesserwerte in Abhängigkeit vom BHD (rechts). 


\subsubsection{Beschreibung der Astabstände}

Im Gegensatz zur Modellierung der Quirläste von Nadelholzarten ist die vertikale Anordnung der Äste bei der Baumart Buche nicht durch das jährliche Höhenwachstum der Bäume vorgegeben. Aufgrund der geringfügigen vertikalen Abweichungen vom Ende des Jahrestriebes, die für Quirläste bei den Nadelholzarten zu beobachten sind (Kapitel 2.4.4), kann den Ästen vereinfachend die durch das Höhenwachstum vorgegebene vertikale Position zugeordnet werden. Bei der Baumart Buche werden im Gegensatz zu den Nadelholzarten mit Internodalästen alle Äste 1. Ordnung im Kronenansatzbereich modelliert, da prinzipiell alle Äste eine Stärke erreichen können, die relevant für die Holzqualität ist. Die Modellierung aller Astdurchmesser im Kronenansatzbereich ist nicht mit der Modellierung aller Äste gleichzusetzen, die in diesem Bereich angelegt und initial ausgebildet worden sind. Als Ursache für die Abweichungen von initial ausgebildeter und tatsächlicher Aststruktur im Kronenansatzbereich bei der Buche sind natürliche Astreinigungsprozesse in der grünen Krone zu nennen. D. h., selbst wenn bestimmte Muster wie eine distiche (zweizeilige) Anordnung und bestimmte Astabstände innerhalb eines gerade ausgebildeten Jahrestriebes erkennbar sind (Abb. 55), werden diese durch die schnelle partielle Astreinigung der Buche (ROLOFF, 1986, S.84 ff.) schon in der grünen Krone verändert.

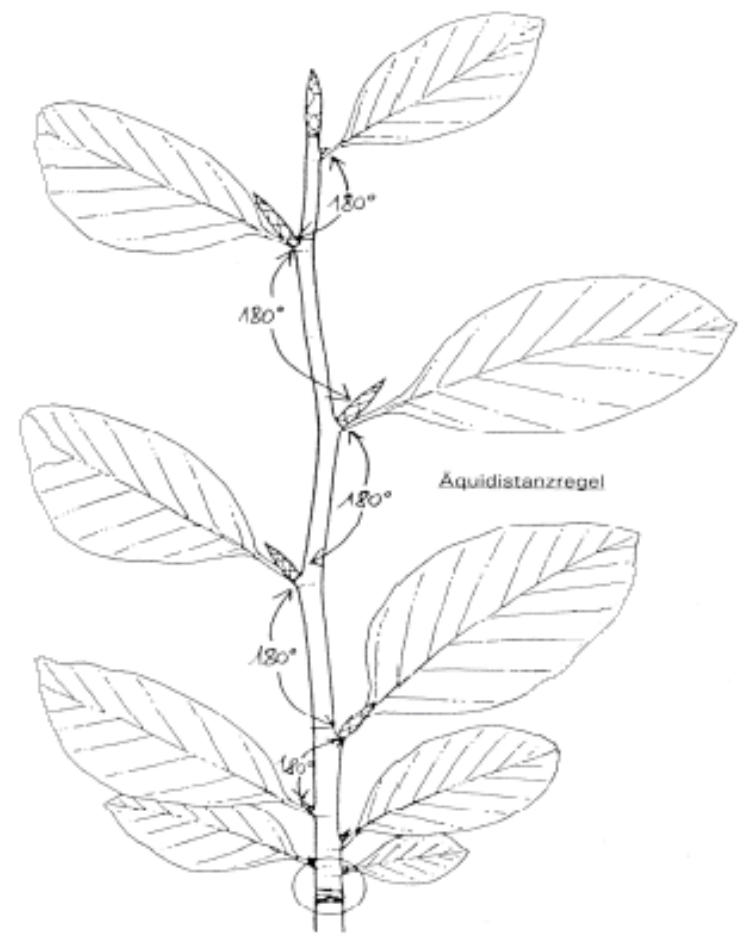

Abb. 55: Initiales distiches Verzweigungsmuster bei der Buche (aus ROLOFF, 1986, S. 67, verändert)

Unter partieller Astreinigung wird hier das Absterben von Ästen verstanden, die eine geringere Tiefe in der Krone aufweisen als der unterste lebende oder tote Ast. Die endgültige Astreinigung bis zu einer bestimmten Stammhöhe tritt bei Astreinigung des untersten Astes ein. 
Aufgrund der durch Akrotonie bedingten spitzenwärtigen Zunahme der Astlänge in einem Jahrestrieb der Tragachse, sinkt die Wahrscheinlichkeit des Absterbens eines Astes, je höher er im Jahrestrieb angeordnet ist (Abb. 56).

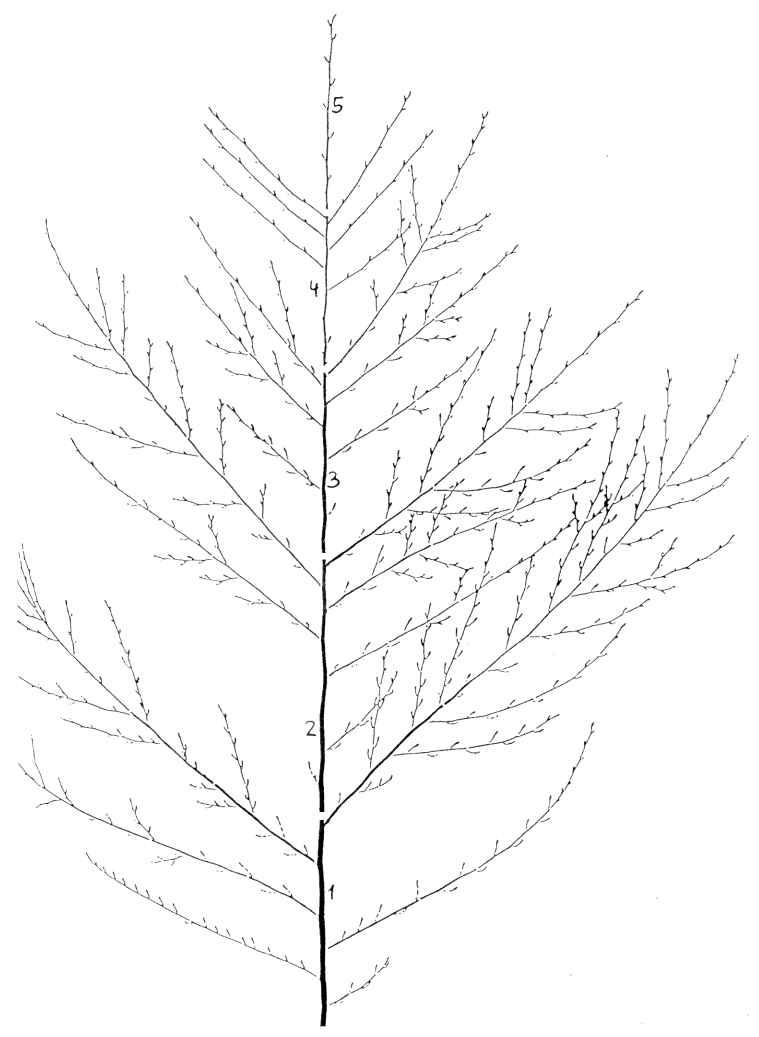

Abb. 56: Durch Akronotonie geprägtes Verzweigungsmuster bei der Buche (aus ROLOFF, 1983, verändert). Die einzelnen Jahrestriebe der Tragachse sind zur Verdeutlichung voneinander getrennt dargestellt.

Datenerhebungen zur Beschreibung der gesamten Kronenmorphologie sind sehr umfangreich, so daß häufig nur wenige Einzelbäume untersucht werden können (ROLOFF, 1986; GRUBER, 1987). Zur Beschreibung der qualitätsrelevanten Astigkeit der Buche erscheint es jedoch als ausreichend, Astdurchmesser und Astabstände im Kronenansatzbereich zu modellieren, da die Sortierung in Güteklassen über Maximaldurchmesser (SCHULZ, 1959; LÖFFLER, 1965; KNIGGE, 1970) oder Astdurchmessersummen (DEUTSCHES INSTITUT FÜR NORMUNG, 1997 a und c; s. Kapitel 1.2) erfolgt. Während das entwickelte Astdurchmessermodell für eine Modellierung der qualitätsrelevanten Astigkeit mit Hilfe des Maximaldurchmessers eines Stammstücks bereits ausreicht, erfordert die Sortierung über Astdurchmessersummen zusätzlich die Modellierung der Astdichte bzw. der Astabstände. In Formel (29) ist ein Modell dargestellt, daß den vertikalen Abstand zwischen zwei Ästen in Abhängigkeit vom Astdurchmesser eines bereits plazierten Astes, der Tiefe in der Krone des bereits plazierten Astes, des BHD des zugehörigen Baumes und der Durchmesserdifferenz zwischem dem bereits plazierten Ast und dem oberhalb zu plazierenden Ast beschreibt. Das Modell ist aus Gründen der 
biologischen Plausibilität so konzipiert, daß nur positive Werte geschätzt werden können ( $\alpha$ muß positiv sein), da definitionsgemäß der Abstand zum nächst höheren Ast geschätzt wird.

$$
\text { Astabst }=\left(\alpha * \text { Astd }_{1}\right) * e^{\beta^{*} T I N K+\delta^{*} D+\gamma^{*} \text { DiffAstd }_{1} \text { Astd }_{2}}
$$

wobei:

Astabst $=$ vertikaler Abstand von einem bereits am Baum plazierten Ast und dem nächst höheren Ast (dm);

Astd $_{1} \quad=$ Durchmesser des bereits am Baum plazierten Astes (mm);

TINK $\quad=$ Tiefe in der Krone des bereits am Baum plazierten Astes (dm);

$D \quad=$ BHD des zugehörigen Baumes $(\mathrm{mm})$;

DiffAstd $_{1}$ Astd $_{2}=$ Durchmesserdifferenz der zwei benachbarten Äste (mm).

mit:

\begin{tabular}{|c|c|c|c|}
\hline Koeffizient & Wert & Std. Fehler & t-Wert \\
\hline$\alpha$ & 0.173 & 0.0167 & 10.339 \\
\hline$\beta$ & 0.0192 & 0.000945 & 20.281 \\
\hline$\delta$ & -0.00854 & 0.000547 & -15.622 \\
\hline$\gamma$ & -0.00611 & 0.00169 & -3.624 \\
\hline Residual Std. F & \multicolumn{3}{|c|}{7.312 bei 657 Freiheitsgraden } \\
\hline
\end{tabular}

Tab. 24: Koeffizienten und statistische Kenngrößen für das Modell zur Schätzung des Abstandes zwischen zwei Ästen im Kronenansatzbereich von Buchen.

\section{Validierung und Bewertung des Modells zur Beschreibung der Astabstände bei Buche:}

Innerhalb des Modells haben der Durchmesser und die Tiefe in der Krone des bereits plazierten Astes einen positiven Einfluß auf den Abstand zum nächst höheren Ast. Diese Wirkungsrichtungen können als biologisch plausibel angesehen werden. Es kann davon ausgegangen werden, daß starke Äste einen großen Konkurrenzdruck auf andere Äste ausüben. Dieser Konkurrenzdruck führt zu Mortalität und natürlicher Astreinigung benachbarter Äste bzw. zu größeren Abständen zu den resultierenden nächsten Nachbarästen. Auch wird ein Ast nur dann einen starken Durchmesser aufweisen, wenn er günstige Wuchsbedingungen hat, $d . h$. nur geringe Konkurrenz durch benachbarte Äste erfährt bzw. größere Abstände zu benachbarten Ästen hat. Auch das durch Akronotonie (Abb. 56) geprägte initiale Astverteilungsmuster könnte einen Einfluß darauf haben, daß oberhalb von starken Ästen größere Abstände zu den nächsten benachbarten Ästen auftreten. Oberhalb starker Äste an der Spitze eines Jahrestriebes sind sehr schwache Äste am unteren Ende des nächsten Jahrestriebes plaziert. Diese schwachen Äste sterben im allgemeinen schnell ab und es entstehen größere Abstände zu den resultierenden Nachbarästen. 
Mit steigender Tiefe in der Krone werden die bereits beschriebenen Astreinigungsprozesse in der grünen Krone immer stärker fortschreiten. Dadurch werden die Abstände eines überlebenden Astes zu den nächsten lebenden Ästen mit steigender Tiefe in der Krone immer größer.

Der BHD des zugehörigen Baumes hat einen negativen Einfluß auf den Astabstand. D. h., daß bei sonst gleichen Voraussetzungen zwei Äste umso dichter plaziert sind, je stärker der zugehörige Baum ist. Als Ursache für diesen Zusammenhang kann vermutet werden, daß durch einen steigenden Stammdurchmesser eine stärkere horizontale Trennung der Äste und damit eine Entspannung der Konkurrenzbedingungen verbunden ist.

Mit steigender Differenz zwischen den Durchmessern zweier Äste bei sonst gleichen Eingangsvariablen sinkt der Abstand zwischen den Ästen. Ein schwacher Ast beeinträchtigt durch den geringen Konkurrenzdruck, den er ausübt, das Wachstum benachbarter Äste nur wenig, sodaß in seiner Nähe starke Äste entstehen können. Gleichzeitig wird ein starker Ast durch seine Konkurrenzkraft das Wachstum nah benachbarter Äste beeinträchtigen. Diese Vorgänge führen dazu, daß benachbarte Äste mit geringen vertikalen Abständen unterschiedliche Durchmesser aufweisen. Sterben schwache Äste infolge der natürlichen Astreinigung ab, wird im Mittel die Durchmesserdifferenz zum neuen benachbarten Ast sinken und der vertikale Abstand ansteigen.

In Abb. 57 sind die beobachteten vertikalen Astabstände und die Ergebnisse der Modellschätzung mit Hilfe von Modell (29) dargestellt.

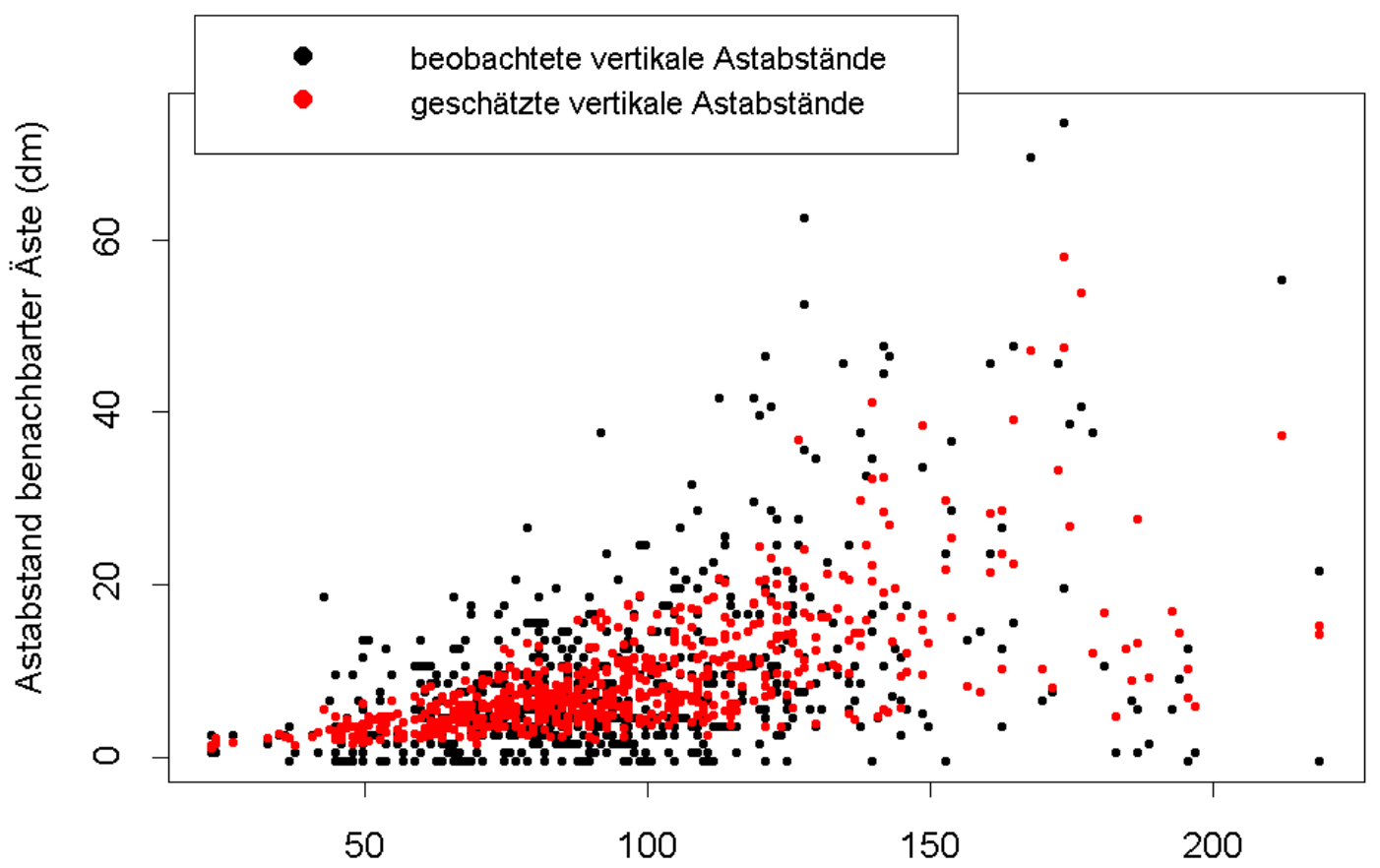

Tiefe in der Kronen des bereits plazierten Astes (dm)

Abb. 57: Beobachtete und prognostizierte vertikale Abstände zwischen einem bereits plazierten Ast und dem nächst höheren Ast für die Baumart Buche. 
Das Streudiagramm der Residuen über geschätzten Astabständen verdeutlicht in Kombination mit einem Gleitenden-Durchschnitts-Modell, daß die Schätzungen des Modells kaum systematische Schätzfehler aufweisen (Abb. 58). Bei der Beurteilung der leichten Unterschätzung im Bereich größerer Astabstände ist der geringe Datenumfang zu beachten.

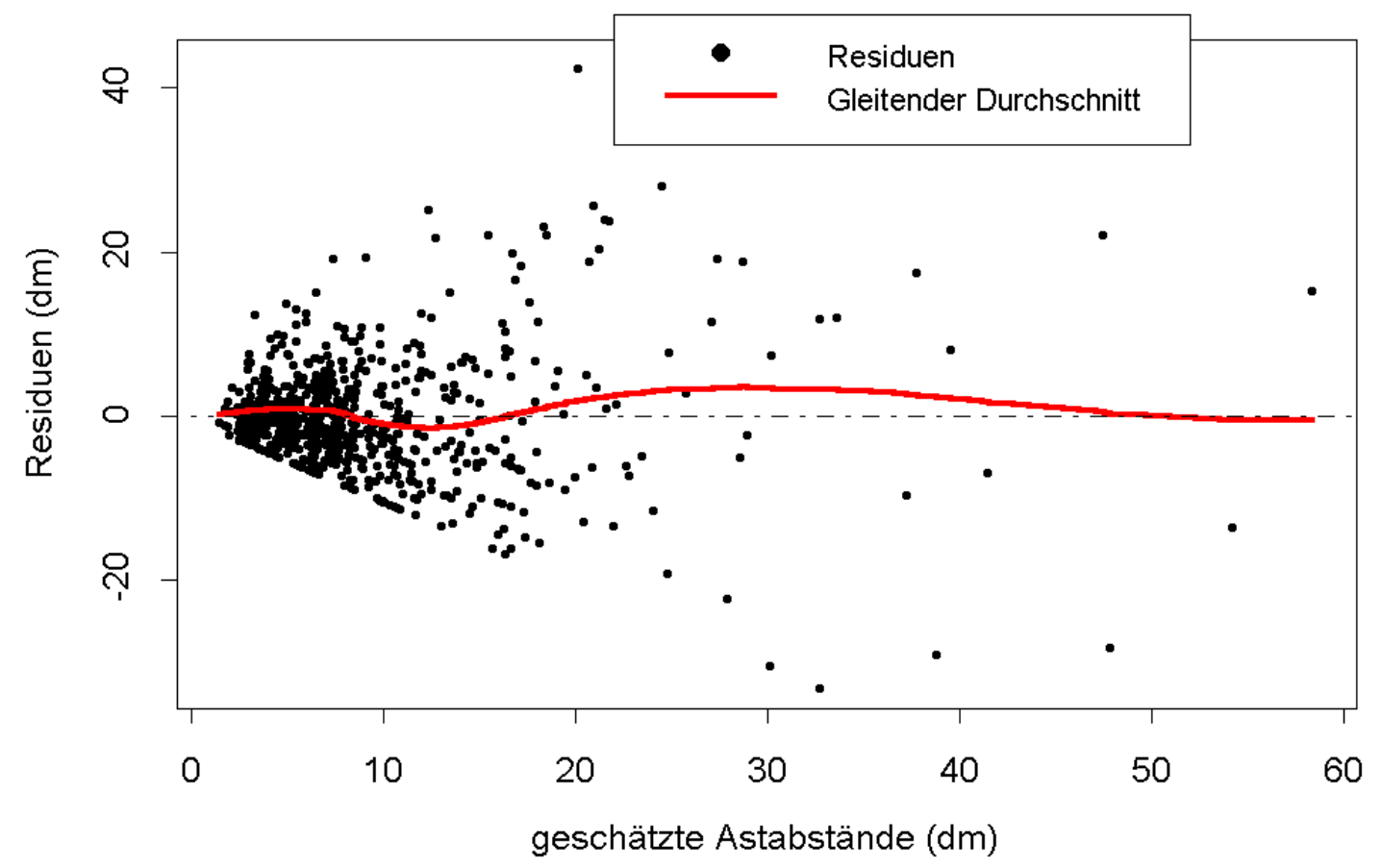

Abb. 58: Residuen über geschätzten Astabständen für das Modell zur Schätzung der Astabstände zwischen einem bereits plazierten Ast und dem nächst höheren Ast. Zur Verdeutlichung von eventuell vorhandenen systematischen Schätzfehlern wurde ein GleitendesDurchschnitts-Modell angepaßt (Kapitel 8.1).

Abschließend soll die Generierung von Astverteilungsmustern für die Baumart Buche im Rahmen von waldbaulichen Szenariensimulationen erläutert werden. Dabei wird erst die statische Prognose der Astdurchmesser und Astabstände im Kronenansatzbereich und anschließend ihre Fortschreibung beschrieben und in Abb. 59 dargestellt. Im Mittel wurden pro Baum vier Äste gemessen, sodaß die bereits beschriebene Generierung von Astdurchmessern für vier Äste im Kronenansatzbereich erfolgt. Die Schätzung der zugehörigen Astdurchmesserverteilung benötigt neben dem BHD auch die mittlere Tiefe in der Krone der Äste, für die die Schätzung erfolgen soll. Die mittlere Tiefe in der Krone kann zum Simulationsbeginn noch nicht geschätzt werden, da die Prognose der Astabstände wiederum die Prognose der Astdurchmesser vorausetzt. Im Gegensatz dazu ist die Tiefe in der Krone des untersten lebenden Astes des Baumes bekannt. Bei der Datenerhebung wurde die Kronenansatzhöhe definitionsgemäß durch die Höhe des untersten Grünastes festgelegt (NIEDERSÄCHSISCHE FORSTLICHE Versuchsanstalt, 1996). Somit stimmt die Tiefe in der Krone des untersten lebenden Astes mit der Kronenansatzhöhe des Baumes überein, die vom Waldwachstumssimulator mit unterschiedlichen Modellen geschätzt wird (Kapitel 3.2 ). Auf der Basis der Information Tiefe in 
der Krone des untersten lebenden Astes und des BHD wurde das in Formel (30) dargestellte Modell zur Schätzung der mittleren Tiefe in der Krone der vier untersten lebenden Äste entwickelt (Abb. 59, Schritt I). Eingangsdaten für die Berechnung der mittleren Tiefe in der Krone waren die untersten vier Äste aller untersuchten Buchen mit mindestens vier gemessenen Astdurchmessern. Das Modell schätzt die mittlere Tiefe in der Krone der vier untersten lebenden Äste nicht direkt, sondern über den Abstand zur Tiefe in der Krone des untersten lebenden Astes. Der verwendete Modelltyp ähnelt dem Modell zur Schätzung der Astabstände (29), da auch hier nur positive Werte plausibel sind. Ein Modell zur direkten Schätzung der mittleren Tiefe in der Krone der vier untersten lebenden Äste wurde nicht entwickelt. Bei getrennter Parametrisierung des Kronenansatzmodells und eines Modells zur direkten Schätzung der mittleren Tiefe in der Krone ist modelltheoretisch nicht gewährleistet, daß die Schätzung der Kronenansatzhöhe in jedem Fall unter der Schätzung der mittleren Tiefe in der Krone liegt, was jedoch aus Plausibilitätsgründen gelten muß.

Auf eine modelltechnische Einschränkung der Maximalwerte der zu schätzenden vertikalen Differenz zwischen der Kronenansatzhöhe und der mittleren Tiefe in der Krone der vier untersten Äste wurde verzichtet, da die Schätzungen maximal 28\% der Kronenlänge erreichen und somit unplausible Schätzungen als extrem unwahrscheinlich gelten müssen. Als unplausibel wären Schätzungen zu bezeichnen, die eine resultierende mittlere Tiefe in der Krone außerhalb des Kronenansatzbereiches oder im Extremfall größer der Baumhöhe aufweisen würden.

$$
\operatorname{Diff}_{M_{-} T I N K}=(\alpha * D) * e^{\beta * B H D+\delta^{*} T I N K} u A s t
$$

wobei:

$$
\begin{array}{ll}
\text { Diff }_{M_{-} \text {TINK }}= & \text { vertikale Differenz zwischen der Kronenansatzhöhe (Tiefe in der Krone des } \\
& \text { untersten lebenden Astes) und der mittleren Tiefe in der Krone der } \\
& \text { untersten vier lebenden Äste }(\mathrm{dm}) ; \\
D & \text { Brusthöhendurchmesser }(\mathrm{mm}) ; \\
T_{I N K_{\text {UAst }}}= & \text { Tiefe in der Krone des untersten lebenden Astes }(\mathrm{dm}) . \\
\text { mit: } &
\end{array}
$$

\begin{tabular}{|c|c|c|c|}
\hline Koeffizient & \multicolumn{2}{|c|}{ Wert } & \\
\hline$\alpha$ & & 0.0927 & 7.031 \\
\hline$\beta$ & & -0.0106 & -11.932 \\
\hline$\delta$ & & 0.0175 & 11.940 \\
\hline \multicolumn{2}{|c|}{ Residual Std. Fehler (dm) } & \multicolumn{2}{|c|}{7.973 bei 143 Freiheitsgraden } \\
\hline
\end{tabular}

Tab. 25: Koeffizienten und statistische Kenngrößen für das Modell zur Schätzung des vertikalen Abstandes zwischen der Tiefe in der Krone des untersten lebenden Astes und der mittleren Tiefe in der Krone der untersten vier lebenden Äste der Baumart Buche. 
Auf der Basis des BHD eines Baumes und der Schätzung der mittleren Tiefe in der Krone der vier untersten lebenden Äste des Baumes können mit Hilfe des verallgemeinerten Modells (27) (Schritt II) und (28) Durchmesser für den Startpunkt einer waldwachstumskundlichen Simulation generiert werden (Kapitel 3.4.1). Für die initiale Erzeugung der Astdurchmesser wird in diesem Fall eine im Intervall [0,1] verteilte Zufallszahl verwendet (Schritt III). Da pro Baum nur vier Astdurchmesser generiert werden, würde die alternative Verwendung von im Intervall $[0,1]$ äquidistant verteilten Zahlen eine Einengung des resultierenden Astdurchmesserbereiches bedeuten. Um zu Simulationsbeginn Durchmesser für die Äste in der Totastzone zu erzeugen, muß ebenfalls das für die Äste im Kronenansatzbereich entwickelte Modell verwendet werden. Da die Buche in der Regel nur kurze Totastzonen aufweist, kann diese Extrapolation jedoch als zulässig beurteilt werden. Zur Prognose der Anzahl Totäste muß zusätzlich eine initiale Schätzung der Totastzonenlänge (Schritt IX) und die Plazierung von Ästen über das Astabstandsmodell erfolgen (Schritt IV). Auch diese Extrapolation des Astabstandmodells am Simulationsbeginn kann als zulässig beurteilt werden.

Über das Modell zur Schätzung der Astabstände von bereits plazierten Ästen zu den nächst höheren Ästen, können die Astdurchmesser im Kronenansatzbereich verteilt werden (Schritt IV). Um Zustandsgrößen wie Kronenansatzhöhen oder Astdurchmesser fortschreiben zu können, muß ein statischer Modellansatz auch im Rahmen von Wachstumssimulationen eingesetzt werden können. Die Fortschreibung erfolgt über die Verknüpfung der statischen Modelle für diese Variablen mit den Zuwachsfunktionen des Wachstumssimulators. Durch das Einzelbaumwachstum verändern sich die Variablen BHD und mittlere Tiefe in der Krone, die die Astdurchmesserverteilung beeinflussen. Nach einem Prognoseintervall müssen den bereits generierten Astdurchmessern somit neue Werte auf der Basis der aktuellen Astdurchmesserverteilung zugeordnet werden. Die Anwendung des statischen Astdurchmessermodells zu verschiedenen Zeitpunkten ersetzt damit eine echte Zuwachsfunktion. Wie bei allen statischen Modellen können Probleme aus den unabhängigen Schätzungen des statischen Modells zu zwei Zeitpunkten entstehen, die bei dynamischen Modellansätzen nicht auftreten.

Ein Beispiel ist das Absinken der Kronenansatzhöhe mit steigenden Einzelbaumdimensionen, das biologisch unplausibel ist, wenn man von der Bildung von Sekundärkronen absieht. Neben diesem allgemeinen Problem muß die Generierung von Astdurchmessern für Äste, für die bereits Astdurchmesser aus der vorhergehenden Periode vorliegen, auf der Basis der Zufallszahlen erfolgen, die für die initiale Astdurchmesserschätzung erzeugt wurden (Schritt V). Nur wenn die gleiche Zufallszahl verwendet wird, wird das "Wachstum" des Astdurchmessers nur von der Verlagerung der Astdurchmesserverteilung bestimmt. Bei der Generierung von Astdurchmessern unter Verwendung neu erzeugter Zufallszahlen sind biologisch unplausible Schätzungen wie ein Absinken oder ein zu starkes Ansteigen des Astdurchmessers möglich. 
Da die Astabstände durch die Astdurchmesser, die Tiefe in der Krone und den BHD beeinflußt werden, stimmen die zum Zeitpunkt der ersten Generierung den Ästen zugewiesenen Astabstände nach einer Simulationsperiode nicht mehr mit den aktuellen Schätzungen des Modells zur Astabstandsschätzung überein (Schritt X). Da jedoch bei der Schätzung der Astabstände eine große nicht erklärte Streuung auftritt (Abb. 58), sind die neu entstandenen Kombinationen von Astabständen und den sie beschreibenden Variablen nicht automatisch unplausibel.

Mit steigender Simulationsdauer beziehungsweise zeitlicher Distanz von der ersten Generierung eines Astes kann die Kombination von Astabständen und den zugehörigen unabhängigen Variablen jedoch immer unwahrscheinlicher werden. Das ist vor allem ab einem Entwicklungsstadium der Fall, ab dem sich die Kronenansatzhöhe nur noch langsam verändert. Die Äste erreichen ein hohes Alter, so daß sich die unabhängigen Variablen, die den Astabstand beeinflussen, stark verändern können. Bei jungen Bäumen mit schneller Veränderung der Kronenansatzhöhe sterben die Äste innerhalb kurzer Simulationszeiträume, so daß sich die unabhängigen Variablen nur relativ geringfügig verändern werden. Im Gegensatz zur Fortschreibung der Astdurchmesser ist eine Veränderung von Astabständen biologisch unplausibel.

Die Vergrößerung von Astabständen erfolgt in der Natur über das Absterben und die partielle Astreinigung von Ästen innerhalb der grünen Krone. Eine Möglichkeit plausible Kombinationen von Astabständen und sie beschreibenden Variablen zu gewährleisten, besteht darin, die partielle Astreinigung in der grünen Krone nachzubilden (Schritt XI). Detaillierte Mortalitätsmodelle für Einzeläste (Kapitel 2.4.4) konnten aufgrund Datenmangels nicht erstellt werden. Es können jedoch biologisch plausible Regeln in Kombination mit dem Modell zur Schätzung von Astabständen dazu genutzt werden, Absterbereihenfolge und -zeitpunkte für einzelne Äste zu definieren. Auf der Grundlage der Standardabweichung der Residuen der Astabstandsschätzung kann ein Grenzwert definiert werden, ab dem Astabstände als unwahrscheinlich beurteilt werden können.

Aufgrund der auftretenden Heteroskedastizität wurden die Residuen mit den zugehörigen geschätzten Abstandswerten (Formel (29)) normiert. Als Grenzwert wurde die einfache Standardabweichung der normierten Residuen der Abstandswerte definiert. Vergleicht man während einer Simulation die ursprünglich zugewiesenen Astabstände mit den Astabständen, die über die aktuellen Variablen geschätzt würden, so kann der Zeitpunkt bestimmt werden, ab dem der ursprünglich zugewiesene Astabstand den Grenzwert unterschreitet. Unterschreitet ein initial zugewiesener Astabstand die aktuelle Schätzung des Astabstandes um mehr als 70\% des aktuellen Schätzwertes (einfache Standardabweichung), so wird der Astabstand als unplausibel bewertet und der schwächere der beiden Äste, für die der Astabstand berechnet wurde, stirbt ab. Da die Tiefe in der Krone des untersten Astes durch das Kronenansatzmodell 
gesteuert wird, stirbt im Falle eines unplausiblen Astabstandes immer der Ast oberhalb des untersten Astes unabhängig von der Aststärke ab. Die Plausibilitätskontrolle wird für alle drei vertikalen Abstände zwischen den untersten vier Ästen durchgeführt. Unterschreiten die zwei Abstände zwischen drei aufeinanderfolgenden Ästen gleichzeitig den Grenzwert, so stirbt in beiden Astpaaren der schwächere der beiden Äste, wobei auch nur ein Ast absterben kann. Die Regel, daß bei unplausiblen Astabständen, an denen der unterste Ast beteiligt ist, immer der Ast oberhalb des untersten Astes stirbt, gilt auch in diesen Fällen. Die Überprüfung der Astabstände erfolgt von unten nach oben.

Mit Hilfe der bisher skizzierten Modelle und Regeln lassen sich die Durchmesser und Abstände für vier Äste im Kronenansatzbereich sowie ihre Veränderung unter Berücksichtigung der partiellen Astreinigung beschreiben. Durch partielle Mortalität ${ }^{16}$ oder die endgültige Mortalität wird die Zahl der modellierten lebenden Äste im Kronenansatzbereich reduziert. Um im Rahmen einer Waldwachstumssimulation eine fortlaufende Modellierung der untersten vier lebenden Äste im Kronenansatzbereich zu ermöglichen, werden bei partieller oder endgültiger Mortalität ein neuer Astdurchmesser und Astabstand generiert (Schritt VII, Schritt VIII, Schritt XII und Schritt XIII). Zur Schätzung des Astdurchmessers wird erneut eine im Intervall $[0,1]$ verteilte Zufallszahl generiert. Für die Prognose der Astdurchmesserverteilung zur initialen Generierung des neuen Astdurchmessers wird vereinfachend die mittlere Tiefe in der Krone der bereits generierten überlebenden Äste verwendet. Der neu erzeugte Ast wird oberhalb des obersten Astes der vier ehemaligen Äste plaziert. Zur Prognose des Astabstandes wird immer der oberste Ast der vier ehemaligen Äste verwendet, unabhängig davon ob er überlebt hat oder nicht.

Die in Abb. 59 zusammengefaßte Abfolge von Astparameterprognosen und einfachen Regeln erlaubt innerhalb von Wachstumssimulationen die Prognose der qualitätsrelevanten Astigkeit für den Kronenansatzbereich und den Stammbereich bis zum Kronenansatz. Die Simulationsschritte VI und IX werden im Text nicht erläutert, da sie inhaltlich zu den Kronenansatzmodellen (Kapitel 3.2) und den Modellen zur Beschreibung der Totastzone (Kapitel 3.6.2 ) gehören.

\footnotetext{
${ }^{16}$ Wie bereits beschrieben, wird hier unter partieller Mortalität und Astreinigung, die Mortalität bzw. Astreinigung von Ästen innerhalb der grünen Krone, d. h. oberhalb des untersten Grünastes verstanden. Die endgültige Mortalität ist das Absterben des untersten grünen Primärastes.
} 


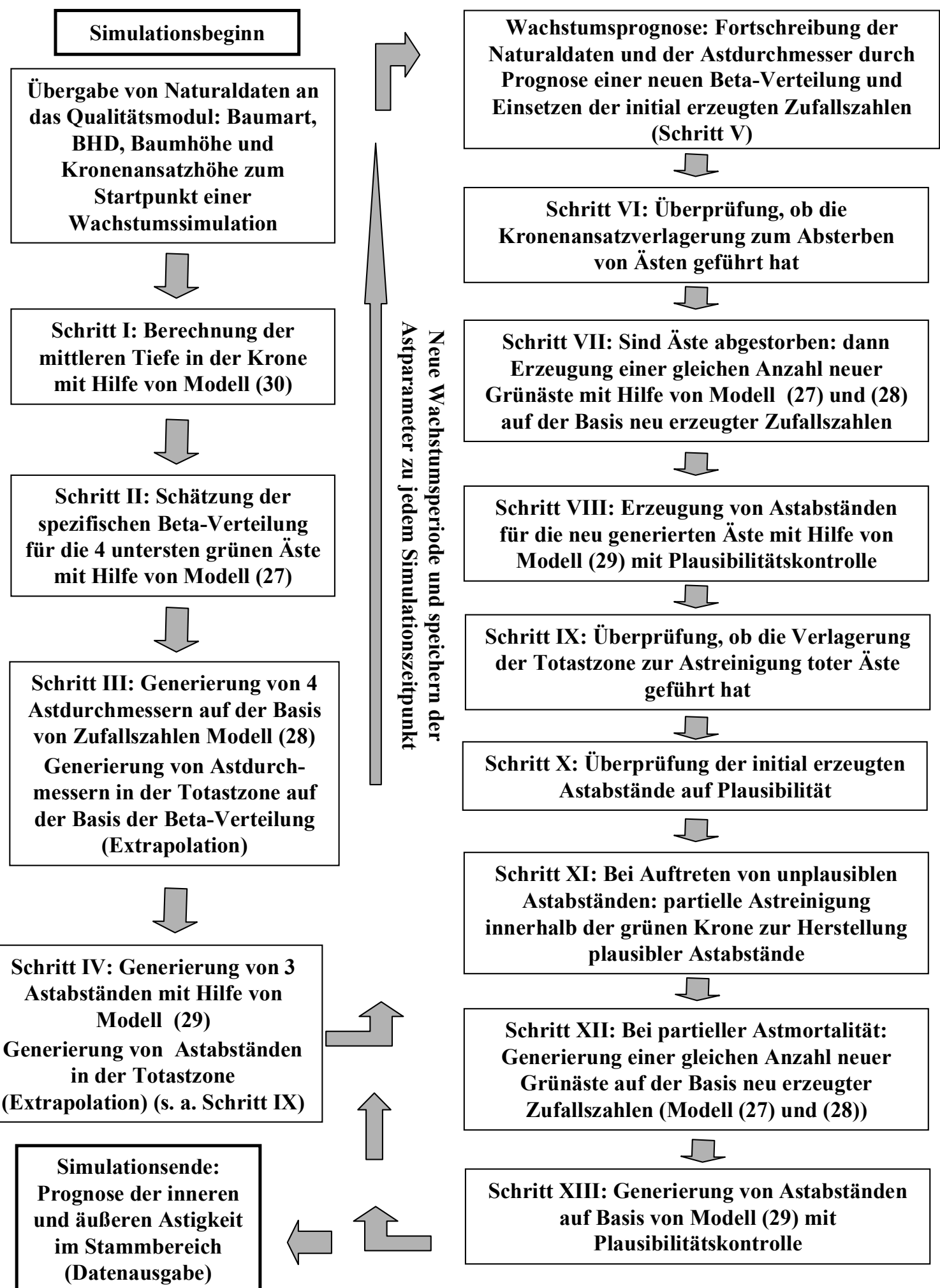

Abb. 59: Einsatz verschiedener statischer Modelle zur Schätzung von Astdurchmessern und -abständen für Äste im Kronenansatzbereich von Buchen im Rahmen einer Wachstumssimulation. 


\subsection{Astanzahl pro Quirl und vertikaler Astabgangswinkel}

Für die Nadelholzarten Fichte, Douglasie und Kiefer wurden im Rahmen dieser Arbeit lediglich Mediane der Astanzahlen pro Quirl für Äste mit einem Durchmesser von mehr als $5 \mathrm{~mm}$ mit Rinde ermittelt. Diese Vorgehensweise deckt sich mit anderen bereits entwickelten Modellen, da häufig nur schwache Zusammenhänge zwischen der Astanzahl pro Quirl und Einzelbaumvariablen identifiziert werden können. So verwendeten KELLOMÄKI ET AL. (1988) für Kiefer einen konstanten Wert von 5 Ästen pro Quirl, solange die natürliche Astreinigung noch nicht eingesetzt hatte. MAGUIRE ET AL. (1994) konnten für Douglasie eine Zunahme der Anzahl aller Äste (Quirl- und Internodaläste) pro Jahrestrieb mit steigender Länge des Jahrestriebes nachweisen. Geringen zusätzlichen Einfluß hatten die Tiefe in der Krone des Jahrestriebes (negativer Einfluß mit steigender Tiefe) und die soziale Stellung des Baumes (negativer Einfluß mit sinkender Stellung). Allerdings kann vermutet werden, daß die Anzahl aller Äste eines Jahrestriebes stärker von den verwendeten Variablen beeinflußt wird, als die Anzahl der Äste pro Quirl. Weiterhin setzt eine Prognose der Länge des Jahrestriebes ein klimasensitives Modell zur Prognose des Höhenwachstums voraus, wenn nicht lediglich der Einfluß des Alterstrends im Höhenwachstum auf die Astanzahl beschrieben werden soll. CoLIN und Houllier (1992) konnten für Fichte auch für die Anzahl der Äste pro Quirl einen Zusammenhang zur Länge des Jahrestriebes und zur Reihenfolge der Quirle, von der Baumspitze aus gezählt, ermitteln. Es scheint daher möglich, die Modellierung der Astanzahl pro Quirl noch zu verbessern. Auch wenn keine Zusammenhänge zu beschreibenden Variablen identifiziert werden können, besteht in jedem Fall die Möglichkeit, die in der Häufigkeitsverteilung der Astanzahl pro Quirl enthaltenen Informationen über eine Verteilungsfunktion im Modell zu integrieren (Kapitel 3.6).

\begin{tabular}{|l|r|r|}
\hline \multicolumn{1}{|c|}{ Baumart } & $\begin{array}{c}\text { Median } \\
\text { (Anzahl) }\end{array}$ & Inter-Quantil Bereich 10-90\% (Anzahl) \\
\hline Fichte & 5 & $3-6$ \\
\hline Douglasie & 6 & $4-9$ \\
\hline Kiefer & 5 & $3-7$ \\
\hline
\end{tabular}

Tab. 26: Mittelwert und Inter-Quantil Bereich (10-90\%) der Astanzahl pro Quirl für die Baumarten Fichte, Douglasie und Kiefer.

Ein Modell zur Beschreibung des vertikalen Astabgangswinkels (Kapitel 2.4.6, Abb. 8 (rechts)) von Quirlästen zur Schaftachse konnte im Rahmen dieser Arbeit nicht entwickelt werden, da die Messung des Winkels aus Zeitgründen nicht erfolgte. Bei einer Modellierung des vertikalen Astabgangswinkels für die Fichte wäre zu überprüfen, inwieweit das Modell von COLIN und Houllier (1992; s. Kapitel 2.4.6) mit identischen Parametern übernommen werden kann, da die einzige beschreibende Variable die Reihenfolge der Quirle von der 
Baumspitze aus ist. Weiterhin ist zu vermuten, daß der vertikale Astabgangswinkel von mechanischen Gesetzmäßigkeiten beeinflußt wird und daher keine regionalen Unterschiede aufweist. Allerdings wäre zu überprüfen, ob genetische Unterschiede bezüglich der Merkmalsausprägung des vertikalen Astabgangswinkels bestehen. Für die horizontale Anordnung der Äste in einem Quirl (Kapitel 2.4.6, Abb. 8 (links)) können für die Verknüpfung der Astparametermodelle in einem Qualitätsmodul vereinfachend gleiche Winkelgrößen zwischen allen Ästen unterstellt werden.

\subsection{Astmortalitäts- und Astreinigungsmodelle}

\subsection{1 Übergangsastzone}

Eine Möglichkeit, den Übergang von der Grünast- zur Totastzone bei den Nadelholzarten zu beschreiben, besteht darin, das Modell zur Schätzung der Kronenansatzhöhe um ein Modell zur Schätzung der Höhe des untersten Grünastes zu ergänzen (Kapitel 2.4.7, CoLIN U. HOULLIER, 1992). Während der Kronenansatz näherungsweise die Grünastzone nach unten begrenzt ${ }^{17}$, wird durch die Höhe des untersten Grünastes die Länge der Übergangsastzone, in der sowohl lebende als auch tote Äste auftreten, und der Beginn der Totastzone definiert. Als Totastzone wird hier im Gegensatz zur Übergangsastzone der Bereich bezeichnet, in dem ausschließlich Totäste auftreten. Zusätzlich muß für die Beschreibung der Totastzonenlänge die Höhe des untersten Totastes modelliert werden.

Um die Reihenfolge des Absterbens von Ästen in der Übergangsastzone und der natürlichen Astreinigung in der Totastzone zu beschreiben, können ergänzend einfache, biologisch plausible Regeln formuliert werden. Beispielsweise kann angenommen werden, daß die Anzahl grüner Äste pro Quirl zwischen dem Kronenansatz und unterstem Grünast linear abnimmt und daß die Äste in der Reihenfolge vom schwächsten zum stärksten Quirlastdurchmesser hin absterben. Eine ähnliche Regel ist für die Astreinigung denkbar, wobei anstelle des Absterbens die Reihenfolge der Astreinigung zwischen unterstem Grünast und unterstem Totast definiert wird.

Abgesehen von der beschriebenen partiellen Astmortalität (Kapitel 3.4.2), die prinzipiell in der gesamten Krone einer Buche auftreten kann, weist die Buche infolge der schnellen natürlichen Astreinigung nur sehr kurze Totastzonen auf. Die Modellierung einer Übergangsastzone unterhalb des Kronenansatzes entfällt, da die Kronenansatzhöhe bei Laubholzarten definitionsgemäß durch die Höhe des untersten Grünastes bestimmt wird (NiEDERSÄCHSISCHE ForstLiCHE VERSUCHSANSTALT, 1996).

\footnotetext{
${ }^{17}$ Die in dieser Arbeit verwendeten Kronenansatzdaten der Nadelholzarten sind als die Höhe des untersten Quirls mit mindestens drei grünen Ästen definiert (NIEDERSÄCHSISCHE FORSTLICHE VERSUCHSANSTALT, 1996).
} 
In Formel (31) ist ein neu entwickeltes Modell zur Schätzung der relativen Höhendifferenz zwischen der Kronenansatzhöhe und der Höhe des untersten Grünastes bei Nadelholzarten dargestellt. Diese Differenz beschreibt die relative Länge der Übergangsastzone:

$$
H D I F F \ddot{U}_{r e l}=\alpha * e^{\beta * K A}
$$

wobei:

$$
\begin{aligned}
H D I F F \ddot{U}_{r e l}= & \text { Höhendifferenz zwischen der Kronenansatzhöhe und der Höhe des untersten } \\
& \text { Grünastes im Verhältnis zur Kronenansatzhöhe (relative Länge der } \\
& \text { Übergangsastzone); } \\
= & \text { Kronenansatzhöhe }(\mathrm{dm}) \\
K A & \text { Koeffizienten. }
\end{aligned}
$$

\begin{tabular}{|c|c|c|c|}
\hline Koeffizient & Wert & Std. Fehler & $\mathrm{t}$-Wert \\
\hline$\alpha$ & 0.359 & 0.0320 & 11.215 \\
\hline$\beta$ & -0.00807 & 0.00143 & -5.656 \\
\hline \multicolumn{2}{|c|}{ Residual Std. Fehler (Fichte) } & \multicolumn{2}{|c|}{0.0888 bei 105 Freiheitsgr. bzw. $6.46 \mathrm{dm}$} \\
\hline \multicolumn{2}{|c|}{ Residual Std. Fehler (Douglasie) } & \multicolumn{2}{|c|}{0.161 bei 87 Freiheitsgr. bzw. $9.65 \mathrm{dm}$} \\
\hline \multicolumn{2}{|c|}{ Residual Std. Fehler (Kiefer) } & \multicolumn{2}{|c|}{0.0987 bei 71 Freiheitsgr. bzw. $6.05 \mathrm{dm}$} \\
\hline
\end{tabular}

mit:

Tab. 27: Koeffizienten und statistische Kenngrößen für das Modell zur Schätzung des Abstandes zwischen der Kronenansatzhöhe und der Höhe des untersten Grünast für die Baumart Fichte (Übergangsastzone). Der Fehler für die relative und absolute Längenschätzung der Übergangsastzone ist auch für die Baumarten Douglasie und Kiefer angegeben.

\section{Bewertung des Modells zur Beschreibung der Länge der Übergangsastzone:}

Die Höhe des untersten Grünastes wird nicht direkt geschätzt (vgl. Colin U. HoulLIER, 1992), um unplausible Schätzungen modelltechnisch ausschließen zu können. Bei getrennter direkter Schätzung der Kronenansatzhöhe und der Höhe des untersten Grünastes können theoretisch Schätzungen erfolgen, bei denen die Höhe des untersten Grünastes über der Kronenansatzhöhe liegt. Derartige Probleme treten überwiegend im Extrapolationsbereich auf und erfordern z. B. zusätzliche Einschränkungen mit Hilfe von Begrenzungsfunktionen (vgl. Kapitel 3.2.3, Formel (15)).

Weiterhin wird die Höhendifferenz im Verhältnis zur Kronansatzhöhe beschrieben und ein Modelltyp gewählt, der eine Begrenzung der Schätzwerte auf das Intervall [0,1] erlaubt. So kann verhindert werden, daß bei sehr niedrigen Kronenansatzhöhen negative Werte für die Höhe des untersten Grünastes geschätzt werden. Ein weiterer Grund für die Schätzung der Höhendifferenz besteht darin, daß das Modell auf den Schätzungen der verschiedenen neu entwickelten Kronenansatzmodelle (Kapitel 3.2) aufsetzt und so die unterschiedliche Genau- 
igkeit der Eingangsinformationen nicht nur bei der Kronenansatzschätzung sondern auch bei der Schätzung der Höhe des untersten Grünastes berücksichtigt werden kann.

Die neu entwickelten statischen Kronenansatzmodelle basieren vor allem auf der Beschreibung des Alters- bzw. Dimensionstrends des Kronenverhältnisses (Kapitel 2.3, Fußnote 4). Bei einer direkten Schätzung der Höhe des untersten Grünastes wären innerhalb der untersuchten Datenstrukturen ähnliche Zusammenhänge wie bei der Schätzung der Kronenansatzhöhe zu erwarten. Für die Schätzung der Höhendifferenz zwischen der Kronenansatzhöhe und der Höhe des untersten Grünastes ist der Dimensionstrend beseitigt, da die Differenz zweier Variablen berechnet wird, die beide einen sehr ähnlichen Verlauf über der Einzelbaumdimension aufweisen.

Für die Höhendifferenz zwischen der Kronenansatzhöhe und der Höhe des untersten Grünastes (Länge der Übergangsastzone) konnten für die untersuchten Baumarten nur sehr schwache statistische Zusammenhänge zu Einzelbaum- und Bestandesvariablen identifiziert werden. Die Problematik der behandlungssensitiven Schätzung von Variablen, die aus den Wuchsbedingungen eines Baumes während einer längeren Wachstumsperiode resultieren, mit Hilfe von aktuellen Zustandsgrößen wurde bereits bei der Entwicklung der Kronenansatzmodelle verdeutlicht (Kapitel 3.2). Wie auch für eine behandlungs- bzw. konkurrenzsensitive Schätzung der Kronenansatzhöhe könnte eine Lösung in der Entwicklung von dynamischen Modellen bestehen (HASENAUER, 1994, Formel (17)). Allerdings konnte im Rahmen dieser Arbeit für keine der untersuchten Baumarten eine zufriedenstellende Modellierung der Veränderungsrate der Kronenansatzhöhe mit Hilfe eines dynamischen Modells erreicht werden (Kapitel 3.2.1).

Infolge der schwachen statistischen Zusammenhänge zwischen der Länge der Übergangsastzone und Einzelbaum- und Bestandesvariablen wird die Übergangsastzone lediglich durch baumartenspezifische Mittelwerte modelliert. Die Funktionalisierung (31) ist aufgrund der vorgenommenen Normierung der Höhendifferenz notwendig. Da die absolute Länge der Übergangsastzone kaum einen Zusammenhang mit der Kronenansatzhöhe aufweist, tritt bei der Normierung mit Hilfe der Kronenansatzhöhe ein abfallender Trend mit steigender Kronenansatzhöhe auf, der mit Hilfe des Modells (31) erfaßt wird.

In Tab. 27 sind neben den Fehlerwerten für die Baumart Fichte auch die entsprechenden Werte für die Baumarten Douglasie und Kiefer angegeben. Der vom Modell geschätzte Fehler der normierten Länge der Übergangsastzone ist wenig aussagekräftig, so daß auch der Fehler für die absoluten Längenwerte angegeben wird. Die zugehörigen Koeffizienten des Modells für die Baumarten Douglasie und Kiefer sind in Kapitel 9.8 aufgeführt. 


\subsubsection{Totastzone}

Die Länge der Totastzone wird durch die Höhe des untersten Totastes und die Höhe des untersten Grünastes bestimmt. Ähnlich wie bei der Beschreibung der Kronenansatzhöhe und der Länge der Übergangsastzone bietet es sich aus modelltechnischen Gründen an, die Totastzone in Relation zu der Größe anzugeben, die sie theoretisch maximal erreichen kann. Während die Kronenansatzhöhe maximal die Gesamthöhe des Baumes erreichen kann und folgerichtig über das Kronenverhältnis modelliert wird, wird die Übergangsastzone in Relation zur Kronenansatzhöhe beschrieben, weil die Kronenansatzhöhe den theoretisch möglichen Maximalwert für die Länge der Übergangsastzone darstellt. Die Totastzone kann maximal den Wert der Höhe des untersten Grünastes erreichen und wird daher in Relation zu diesem Wert ausgedrückt. Da bei der Buche definitionsgemäß keine Übergangsastzone modelliert wird, wird die Länge der Totastzone im Verhältnis zur Kronenansatzhöhe angegeben.

Die Entwicklung der Höhe des untersten Totastes hat bei den Nadelholzarten mit Totasterhaltung einen gänzlich von der Entwicklung der Kronenansatzhöhe bzw. der Höhe des untersten Grünastes abweichenden Verlauf. Bis zu einem Bestandesstadium, in dem sich infolge nachlassender Konkurrenz die Astmortalität stark verlangsamt, wird sich die Länge der Totastzone ständig vergrößern, da die Mortalitätsrate über der Astreinigungsrate liegt. Die Entwicklung der Totastzonenlänge unterscheidet sich somit deutlich von der Entwicklung der Länge der Übergangsastzone. Während für die absolute Totastzonenlänge ein enger Zusammenhang zur Einzelbaumdimension bzw. Baumhöhe besteht, kann mit Hilfe der relativen Totastzonenlänge untersucht werden, inwieweit über den reinen Dimensionstrend hinaus $\mathrm{Zu}-$ sammenhänge zu Einzelbaumvariablen bestehen.

In Abb. 60 ist die relative Totastzonenlänge über der Einzelbaumhöhe für 639 Fichten dargestellt. Für die Totastzonenlänge standen weitaus mehr Messungen zur Verfügung als für die Länge der Übergangsastzone. Da aber die Höhe des untersten Grünastes zur Normierung der Totastzonenlänge notwendig war, wurden die Werte einheitlich über Formel (31) berechnet. Mit Hilfe eines Gleitenden-Durchschnitts-Modells (Kapitel 8.1) kann gezeigt werden, daß mit steigender Gesamthöhe des Baumes die relative Totastzonenlänge abnimmt (Abb. 60).

Wenn man davon ausgeht, daß die untersten Totäste in Bodennähe plaziert sind, wird die relative Totastzonenlänge solange einen Wert von nahe 1 aufweisen, bis die Astreinigung einsetzt. Mit einer Verlangsamung der konkurrenzbedingten Astmortalität und einsetzender Astreinigung verringert sich die relative Totastzonenlänge mit steigender Höhe Aufgrund der Vorüberlegungen wurde ein Modell zur Beschreibung der relativen Totastzone entwickelt, das sich für Bäume mit geringen Höhen dem Wert 1 nähert, wobei der Wert 1 aus Plausibilitätsgründen nicht überschritten wird (Formel (32)). 


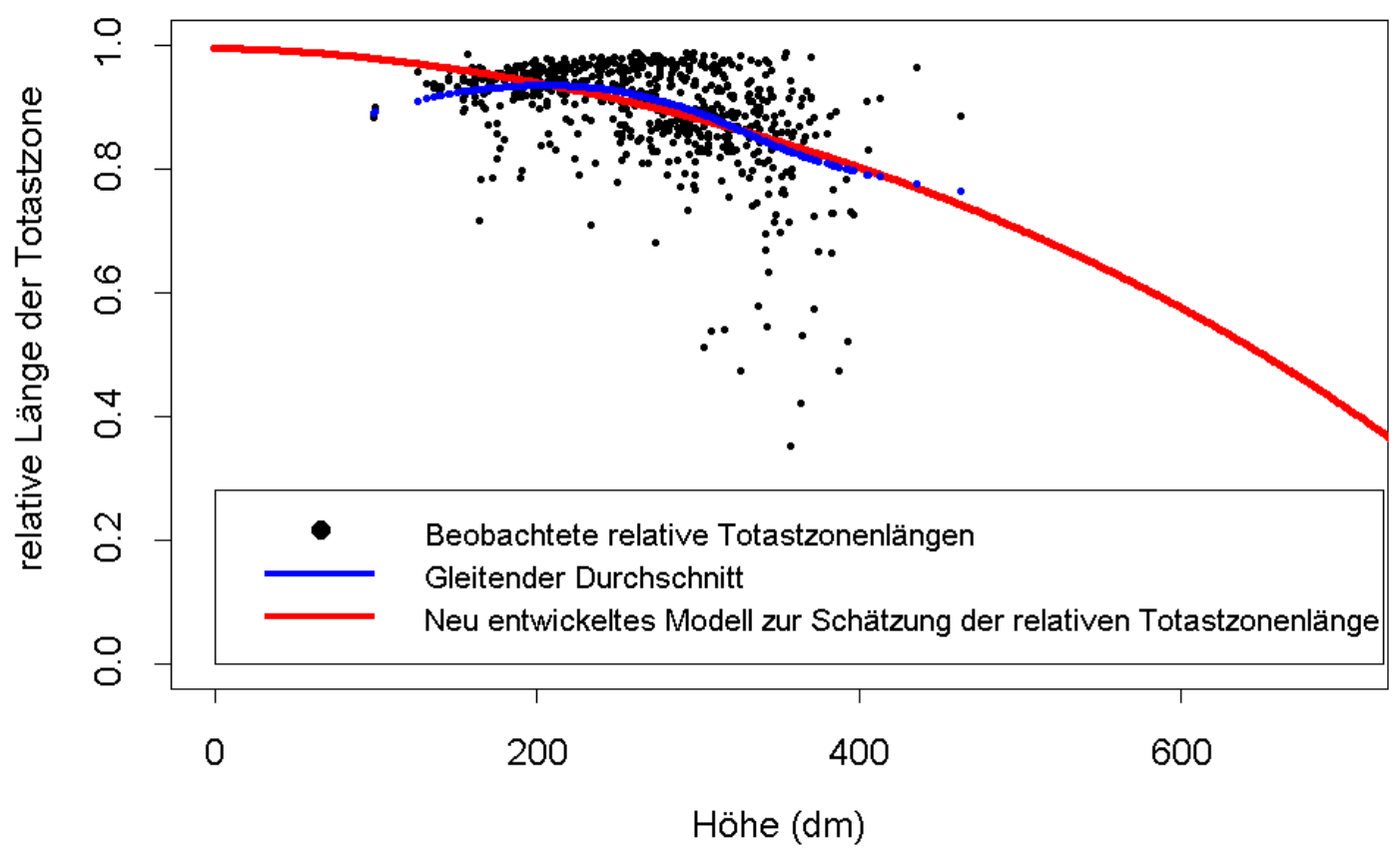

Abb. 60: Modell zur Beschreibung der relativen Länge der Totastzone in Abhängigkeit von der Baumhöhe für die Baumart Fichte. Zur Verdeutlichung des Trends wird ein Gleitendes-Durchschnitts-Modell verwendet (Kapitel 8.1).

Bis zu einer Baumhöhe von 89.7 m werden keine negativen Werte geschätzt, womit auch im Extrapolationsbereich sehr hoher Bäume plausible Werte gewährleistet sind (Formel (32)). Diese Einschränkungen des Modells, die die Schätzung plausibler Werte sicherstellen, ersetzen jedoch nicht die anzustrebende Erweiterung der Datenbasis im Bereich niedriger und großer Höhen.

HDIFFTOT $_{r e l}=2-e^{\left(\alpha^{*} H\right)^{\beta} / 1000}$

wobei:

HDIFFTOT $_{\text {rel }}=$ Höhendifferenz zwischen der Höhe des untersten Grünastes und der des untersten Totastes im Verhältnis zur Höhe des untersten Grünastes (relative Länge der Totastzone);

$H \quad=$ Gesamthöhe des Baumes $(\mathrm{dm})$;

$\alpha, \beta \quad=$ Koeffizienten.

mit:

\begin{tabular}{|c|c|c|c|}
\hline Koeffizient & Wert & Std. Fehler & $\mathrm{t}$-Wert \\
\hline$\alpha$ & 0.0523 & 0.0128 & 4.083 \\
\hline$\beta$ & 1.700 & 0.149 & 11.404 \\
\hline \multicolumn{2}{|c|}{ Residual Std. Fehler (Fichte) } & \multicolumn{2}{|c|}{0.076 bei $637 \mathrm{FG}$ bzw. $13.1 \mathrm{dm}$} \\
\hline \multicolumn{2}{|c|}{ Residual Std. Fehler (Douglasie) } & \multicolumn{2}{|c|}{0.183 bei $180 \mathrm{FG}$ bzw. $33.6 \mathrm{dm}$} \\
\hline
\end{tabular}

Tab. 28: Koeffizienten und statistische Kenngrößen für das Modell zur Schätzung der Totastzonenlänge. Der Fehler für die relative und absolute Längenschätzung ist auch für die Baumart Douglasie angegeben. 


\section{Validierung und Bewertung des Modells zur Beschreibung der Totastzonenlänge:}

Das neu entwickelte Modell zur Prognose der relativen Länge der Totastzone verwendet lediglich die Gesamthöhe des Baumes als beschreibende Variable. Weitere Variablen wurden nicht im Modell berücksichtigt, da ihre Integration keine nennenswerte Erhöhung der Modellgenauigkeit bewirkte. Dabei ist zu bedenken, daß die Schätzung für eine relative Größe erfolgt. Variablen, die wie der H/D-Wert, die Aststärke beeinflussen (Kapitel 3.3) und somit auch einen Einfluß auf die Astreinigung haben könnten, werden schon bei der Schätzung der Kronenansatzhöhe verwendet (Kapitel 3.2). Die Höhe des untersten Grünastes steht wiederum in einem engen Zusammenhang zur Kronenansatzhöhe. Der eventuell vorhandene positive Zusammenhang des H/D-Wertes zur Astreinigungsgeschwindigkeit könnte also bereits über die zur Normierung der Totastzonenlänge verwendete Höhe des untersten Grünastes bei der Prognose berücksichtigt worden sein. Voraussetzung für die beschriebenen Überlegungen ist, daß stärkere Äste eine langsamere Astreinigung aufweisen als dünne Äste.

Das beschriebene Modell konnte nur für die Baumarten Fichte und Douglasie parametrisiert werden. Aufgrund der weniger breiten Datenbasis für die Douglasie mit nur wenigen Höhenmessungen unterhalb von 20 m, war es notwendig, noch weitergehende Annahmen bei der Parametrisierung des Modells als bei der Baumart Fichte zu treffen. So wurde davon ausgegangen, daß der grundsätzliche Verlauf der relativen Totastzonenlänge für die totasterhaltenden Baumarten Fichte und Douglasie übereinstimmt. D. h., daß neben der Beschränkung des Vorhersagebereiches auf das Intervall $[0,1]$ auch bei der Douglasie der konvexe Verlauf der relativen Totastzonenlänge über der Gesamthöhe zu beobachten ist. In Abb. 117 wird mit Hilfe eines Gleitenden-Durchschnitts-Modells verdeutlicht, daß diese Annahme gerechtfertigt ist. Das verwendete Gleitende-Durchschnitts-Modell weist im Gegensatz zu dem in Kapitel 8.1 beschriebenen Ansatz einen variablen Glättungsparameter auf. Der Glättungsparameter wird in Abhängigkeit von der Anzahl der in den unterschiedlichen Datenbereichen zur Verfügung stehenden Messungen geschätzt. Ein variabler Glättungsparameter bietet sich daher bei unterschiedlich repräsentierten Datenbereichen an.

Für die Modellierung eines konvexen Verlaufes der geschätzten relativen Totastzonenlänge war es notwendig, für den Parameter $\beta$ des Modells (32) eine untere Grenze vorzugeben, da der Modellverlauf für die Baumart Douglasie ohne diese Einschränkung von dem als typisch unterstellten konvexen Verlauf abweicht. Als Ursache ist das Fehlen von Datensätzen mit Höhenwerten von unter $20 \mathrm{~m}$ zu vermuten. Werden für Douglasien mit niedrigen Höhen hohe Totastzonenprozente nahe dem Wert 1 unterstellt, so kann auch ein konvexer Gesamtverlauf unterstellt werden. Für einen konvexen Verlauf muß der Parameter $\beta$ einen Wert von größer 1 haben. Um unter den gegebenen Einschränkungen eine optimale Modellanpassung zu gewährleisten, wurde $\beta$ bei der Parametrisierung im Intervall [1.2,1.5] schrittweise um 0.1 erhöht. Der geringste Standardfehler wurde mit dem Wert 1.2 erzielt. Ein Parameterwert 
von 1.1 wurde nicht getestet, da bei diesem Wert nur ein sehr schwach konvexer Verlauf prognostiziert wird. Das Modell schätzt bis zu einer Baumhöhe von $75.6 \mathrm{~m}$ positive Totastzonenprozente, so daß auch im Extrapolationsbereich plausible Werte gewährleistet sind. Wie schon erwähnt, kann die Gewährleistung der biologischen Plausibilität eine Modellparametrisierung auf einer erweiterten Datenbasis nicht ersetzen. Die Koeffizienten und statischen Kenngrößen für die Baumart Douglasie sind in Kapitel 9.8 aufgeführt. Die Douglasie weist innerhalb des durch Daten repräsentierten Bereiches etwas geringere normierte Totastzonenlängen als die Fichte auf.

Für die Baumarten Kiefer und Buche konnte das Modell (32) nicht parametrisiert werden. Während für die Baumart Kiefer lediglich 52 Messungen der Höhe des untersten Totastes bei einem sehr engen Gesamthöhenrahmen vorlagen, konnten für die Baumart Buche nur schwache Zusammenhänge bei gleichzeitig sehr hoher nicht erklärter Reststreuung identifiziert werden.

Im Gegensatz zur normierten Länge der Übergangsastzone, die einen Zusammenhang zur Kronenansatzhöhe aufweist (Formel (31)), treten bei der normierten Totastzonenlänge innerhalb der für die Baumarten Kiefer und Buche zur Verfügung stehenden Daten keine Zusammenhänge zu den untersuchten Einzelbaumvariablen auf. Die Zunahme der absoluten Totastzonenlänge mit steigender Baumhöhe wird bereits durch die Normierung erfaßt.

Es besteht jedoch die Möglichkeit, weitergehende Informationen als nur den Mittelwert bei der Modellierung zu berücksichtigen, indem die baumartenspezifische Verteilung der normierten Totastzonenlänge mit Hilfe einer Dichtefunktion beschrieben wird. Im Gegensatz zur Modellierung der Astdurchmeser bei Buche (Kapitel 3.4.1) wird aufgrund der fehlenden statistischen Zusammenhänge lediglich eine mittlere Verteilung für alle Bäume einer Baumart unterstellt.

In Fällen, in denen eine Verallgemeinerung eines Verteilungsmodells infolge fehlender statistischer Zusammenhänge entfällt, bieten sich nicht-parametrische Verfahren der Dichteschätzung an. Diese auch als Kernel-Schätzungen bekannten Verfahren (SILVERMAN, 1986; HÄRDLE, 1989), weisen gegenüber parametrischen Dichtefunktionen eine größere Flexibilität bzw. die Möglichkeit einer stärkeren Berücksichtigung der Datenstruktur auf. Im Bereich forstlicher Anwendungen werden nicht-parametrische Dichtefunktionen bisher vor allem zur Modellierung von Durchmesserverteilungen eingesetzt. Dabei erfolgt eine Regionalisierung häufig in Kombination mit sogenannten k-nearest-neighbour Regressionen (HAARA ET AL. 1997; Maltamo u. Kangas, 1998; Niggemeyer u. SchmidT, 1999). Auf der Basis der zugehörigen invertierten Verteilungsfunktionen (Quantilsfunktion) können anschließend mit Hilfe von Zufallszahlen oder äquidistant verteilten Zahlen aus dem Intervall $[0,1]$ normierte Längen für die Totastzone generiert werden. 
Im Kapitel 8.3 wird ein Verfahren vorgestellt, mit dem normierte Längen für die Totastzone auf der Basis einer nicht-parametrischen Dichtefunktion generiert werden können. Das Verfahren ist in einzelne Schritte unterteilt, für die das Statistik-Paket S-PLUS 3.3 (MATHSOFT, 1995) spezielle Funktionen zur Verfügung stellt ${ }^{18}$.

In Abb. 61 ist die kalibrierte und nicht kalibrierte Dichtefunktion, die mit Hilfe der Kernel-Schätzung ermittelt wurde, dargestellt. Die Kalibrierung wird in Kapitel 8.3 erläutert und dient der Begrenzung der Funktion auf das Intervall [0,1]. Die normierten Totastzonenlängen der Kiefer haben ähnlich wie bei den Baumarten Fichte und Douglasie überwiegend Werte im Bereich nahe 1. Dies ist aufgrund des engen Höhenrahmens (7.6 bis $14.3 \mathrm{~m}$ ) auch zu erwarten. Für diesen Höhenbereich werden auch für die Baumarten Fichte und Douglasie Werte im Bereich von 1 prognostiziert (Abb. 60 u. Abb. 117).

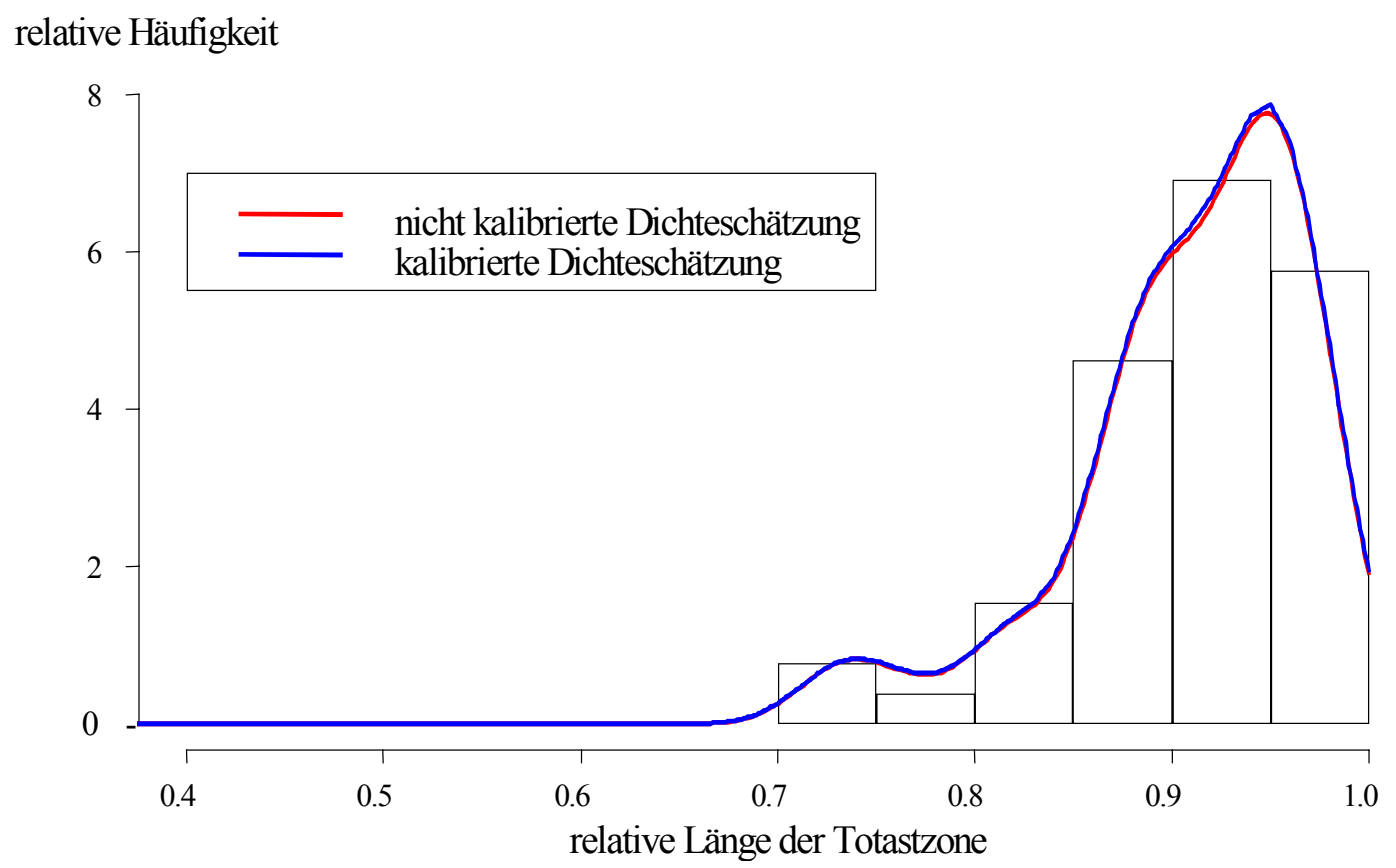

Abb. 61: Modellierung der normierten Länge der Totastzone mit Hilfe einer KernelSchätzung für die Baumart Kiefer. Der Glättungsparameter $h$ wurde über einen von SILVERMAN (1986, S. 45 ff., Formel (44)) vorgeschlagenen Algorithmus berechnet und beträgt 0.02226174 .

Im Unterschied zu den Nadelholzarten weist die Baumart Buche nur in Ausnahmefällen normierte Totastzonenlängen mit einem Wert von 1 auf (Abb. 62). Im Gegensatz zu den totasterhaltenden Baumarten kommt es jedoch vor, daß zwischen einem Totast in niedriger Höhe und dem Kronenansatz nur noch wenige weitere Totäste auftreten. Den höchsten Anteil machen Bäume ohne Totäste oder mit untersten Totästen in unmittelbarer Nähe des Kronenansatzes aus (28\%). Alle anderen Klassen von normierten Totastzonenlängen weisen fast gleiche An-

\footnotetext{
${ }^{18}$ Das erläuterte Verfahren wurde von ZUCCHINI (2001) im Rahmen eines Seminars zur statistischen Datenanalyse des Instituts für Statistik und Ökonometrie der Universität Göttingen vorgestellt.
} 
teile auf. In Abb. 62 wird stärker als bei der Kernel-Schätzung für die Baumart Kiefer deutlich, daß die Begrenzung der Werte auf das Intervall [0,1] eine Kalibrierung der Funktionswerte der Dichtefunktion erfordert. Eine Modellierung der partiellen Astreinigung, wie sie für die Kronenansatzzone möglich war (Kapitel 3.4.2), konnte für die Totastzone nicht durchgeführt werden, weil für diesen Bereich keine Astabstandsmessungen vorlagen.

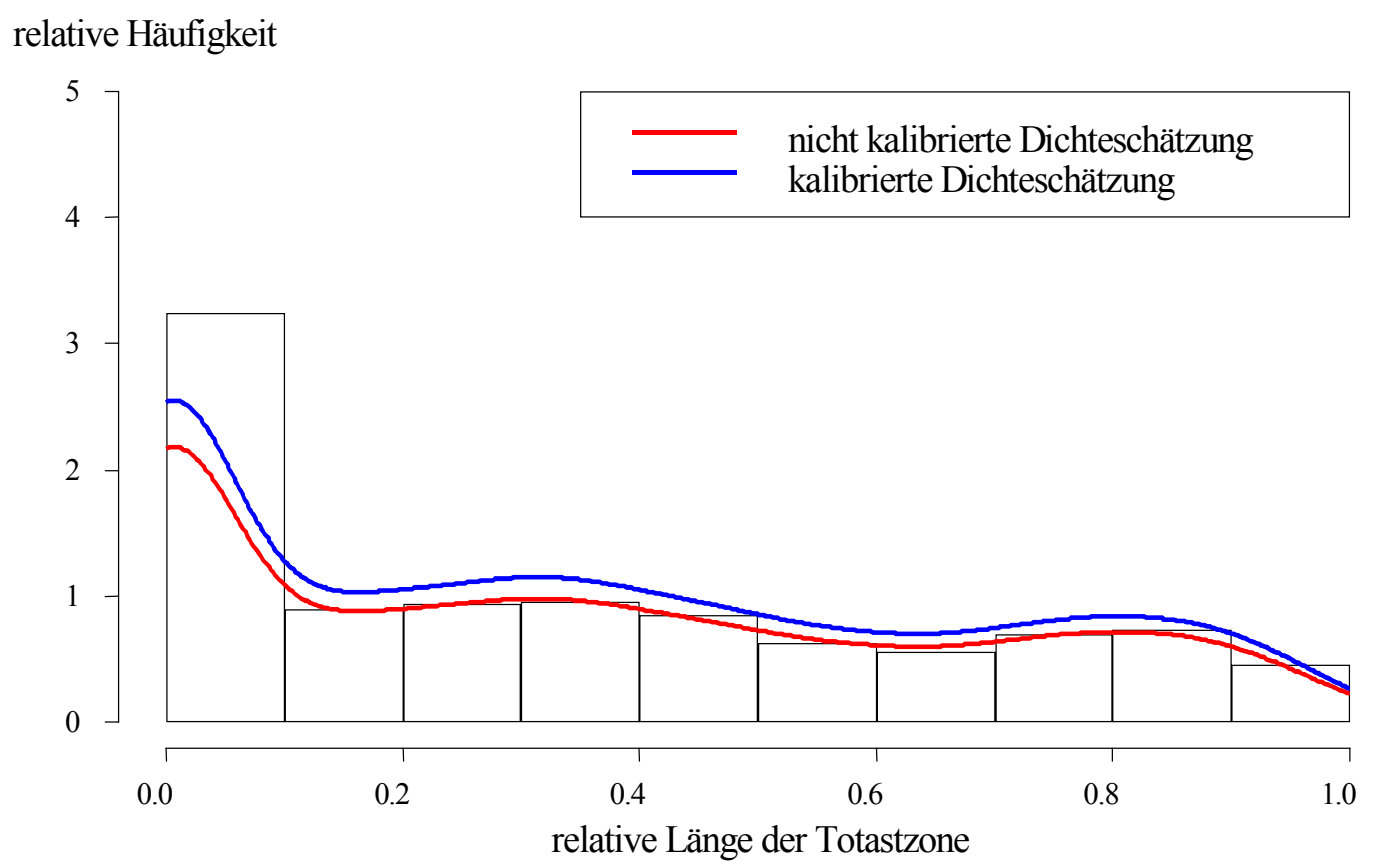

Abb. 62: Modellierung der normierten Länge der Totastzone mit Hilfe einer KernelSchätzung für die Baumart Buche. Der Glättungsparameter $h$ wurde über einen von SILVERMAN (1986, S. 45 ff., Formel (44)) vorgeschlagenen Algorithmus berechnet und beträgt 0.0571079 .

Das Regressionsmodell (32) zur Beschreibung der Totastzonenlänge bei den Baumarten Fichte und insbesondere Douglasie kann aus statistischer Sicht infolge der getroffenen Annahmen und einer extremen Heteroskedastizität kritisch beurteilt werden. Unter Berücksichtigung der geringen Datenbasis erscheinen die entwickelten heuristischen Modelle jedoch für eine stabile Prognose geeignet. Die Entwicklung von Modellen ohne Einschränkungen für die Douglasie und auch die Kiefer erfordert jedoch eine Erweiterung der Datengrundlage. 


\subsection{Flexible Schaftformmodelle}

Im folgenden Kapitel wird nachgewiesen, daß ein von PAIN und BOYER (1996) entwickeltes Schaftformmodell einen Teil der Schaftformvariation von Einzelbäumen für die Baumarten Fichte, Douglasie und Kiefer mit Hilfe der Einzelbaumvariablen BHD, Höhe und H/D-Wert erfassen und beschreiben kann. Der Vorteil dieses Modells wird anhand von zwei Vergleichsmodellen deutlich, die eine einheitliche Form bzw. nur eine geringe Variation der Schaftform beschreiben. Für die Baumarten Buche und Eiche weist das im weiteren als PainFunktion bezeichnete Modell keine Vorteile gegenüber dem Vergleichsmodell modifizierte Brink-Funktion auf (RIEMER ET AL., 1995).

Der Funktionswert der Pain-Funktion in $1.3 \mathrm{~m}$ Baumhöhe stimmt nicht zwingend mit dem Meßwert des BHD überein. Es wurde daher eine Modifikation der Pain-Funktion entwickelt, die die Bedingung erfüllt, daß der Meß- und Funktionswert auf $1.3 \mathrm{~m}$ Höhe übereinstimmen. Diese Modifikation führt durch die Einschränkung der Flexibilität zu geringfügig höheren Schätzfehlern gegenüber der Originalversion der Pain-Funktion. Eine anschließende Segmentierung in zwei Funktionen zur Beschreibung des unteren und oberen Schaftabschnittes führte zur Entwicklung des Schaftformmodells, das für alle untersuchten Baumarten die höchste Genauigkeit aufweist. Mit Hilfe dieses im folgenden als modifizierte Pain-Funktion bezeichneten Modells wurden auch die Schätzungen für die Baumarten Buche und Eiche gegenüber den Vergleichsmodellen verbessert, was mit der Originalversion der Pain-Funktion nicht möglich war.

In Kapitel 3.7.4 wird der Einfluß der kategorischen Variablen Forstort auf die Schaftform für die Baumart Fichte erläutert. Der Forstort aggregiert eine Vielzahl von metrischen Bestandesvariablen und Variablen höherer Hierarchiestufen, die zum jetzigen Zeitpunkt nicht den Versuchsflächen zugeordnet werden können. Es wird nachgewiesen, daß die Beschreibung der Schaftform durch die Integration der Variablen Forstort zusätzlich zu den verwendeten Einzelbaumvariablen verbessert werden kann. Damit liegen die weiteren Möglichkeiten zur Verbesserung von Schaftformmodellen vor allem im Bereich der Indentifikation von Bestandes- und Standortsvariablen, die die Schaftform beeinflussen, sowie ihrer Integration in vorhandene Schaftformmodelle.

\subsubsection{Datengrundlage und -aufbereitung}

Wie in den vorhergehenden Kapiteln werden die einzelnen Arbeitsschritte der Modellparametrisierung bzw. -entwicklung anhand der Baumart Fichte erläutert. Für die Baumarten Douglasie, Kiefer, Buche und Eiche werden die wichtigsten Ergebnisse in Kapitel 9.9 dargestellt. Die Aufbereitung der Rohdaten wird in diesem Kapitel ausführlicher beschrieben, da der Grad der Manipulation bei der Interpretation von Fehlerrahmen zu berücksichtigen ist. Auch werden in der Literatur unterschiedlich starke Veränderungen der Rohdaten beschrie- 
ben. GAFFrey und NAgel (1998) glätten die Sektionsmessungen mit Hilfe von kubischen Spline-Funktionen (nach visueller Kontrolle). Die anschließende Parametrisierung und Validierung erfolgt auf der Basis von gleichverteilten Funktionswerten der Spline-Funktionen. Demgegenüber gibt es auch ausschließlich visuelle Kontrollverfahren, in denen nur offensichtliche Meßfehler, wie ansteigende Sektionsdurchmesser mit steigender Baumhöhe beseitigt oder korregiert werden (TRINCADO ET AL., 1996). Die Parametrisierung und Validierung erfolgt anschließend an bereinigten Rohdaten, nicht aber an Funktionswerten, die durch Glättung ermittelt werden. Beim Vergleich von Fehlerrahmen unterschiedlicher Modelle sind daher eventuell vorhandene Unterschiede in der Datenaufbereitung bzw. Streuungsreduktion zu beachten.

In dieser Arbeit wurde ein Kompromiß bei der Aufbereitung der Daten gewählt: In einem ersten Schritt wurden die Sektionsdaten visuell auf unplausible Meßwerte überprüft, indem nur stetig absteigende Schaftradien mit steigender Schafthöhe zugelassen wurden. Anschließend wurde einzelbaumweise eine von PAIN und BOYER (1996) entwickelte Schaftformfunktion (33) angepaßt und Abweichungen von mehr als 5\% vom Funktionswert durch den Funktionswert ersetzt.

$r\left(h_{r e l}\right)=\alpha\left(1-h_{r e l}{ }^{3}\right)+\beta\left(\ln \left(h_{r e l}\right)\right)$

wobei:

$r\left(h_{r e l}\right)=$ Schaftradius in der mit der Gesamtlänge des Baumes relativierten Schafthöhe $h_{r e l}$ (cm);

$\alpha, \beta=$ dimensions- und formbeschreibende Parameter.

Durch die geringe Anzahl an Parametern reagiert das Modell nur relativ schwach auf einzelne untypische Meßwerte der bereits visuell überprüften Sektionsmessungen und eignet sich daher gut für den zweiten Schritt der Datenkontrolle. Die bereinigten Meßwerte dienten als Basis für die Parametrisierung und Validierung der Schaftformmodelle. Für die Baumarten Douglasie, Fichte, Buche und Eiche konnte auf bereits visuell überprüfte Sektionsmessungen zurückgegriffen werden ${ }^{19}$, für die nur noch der zweite Schritt der Datenkontrolle durchgeführt werden mußte. Die Sektionsmessungen der Baumart Kiefer wurden vollständig im Rahmen dieser Arbeit überprüft.

In Abb. 63 sind die Verteilungen der Variablen BHD und H/D-Wert für 827 Fichten dargestellt. Beide Variablen waren neben der Baumhöhe die beiden Einzelbaumvariablen, die durchgängig für alle Bäume zur Verfügung standen. Die 827 Bäume mit insgesamt 9769 Sek-

\footnotetext{
${ }^{19}$ Die Sektionsmessungen für die Baumart Douglasie wurden von Herrn Dipl.-Forstwirt F. Steingaß, die für die Baumarten Fichte und Buche von Herrn G. Trincado (M.A.) und die für die Baumart Eiche von Herrn Dr. Kh. Hussein visuell überprüft.
} 
tionsmessungen stammen aus 100 unterschiedlichen Unterabteilungen aus dem gesamten Bereich Nordwestdeutschlands.
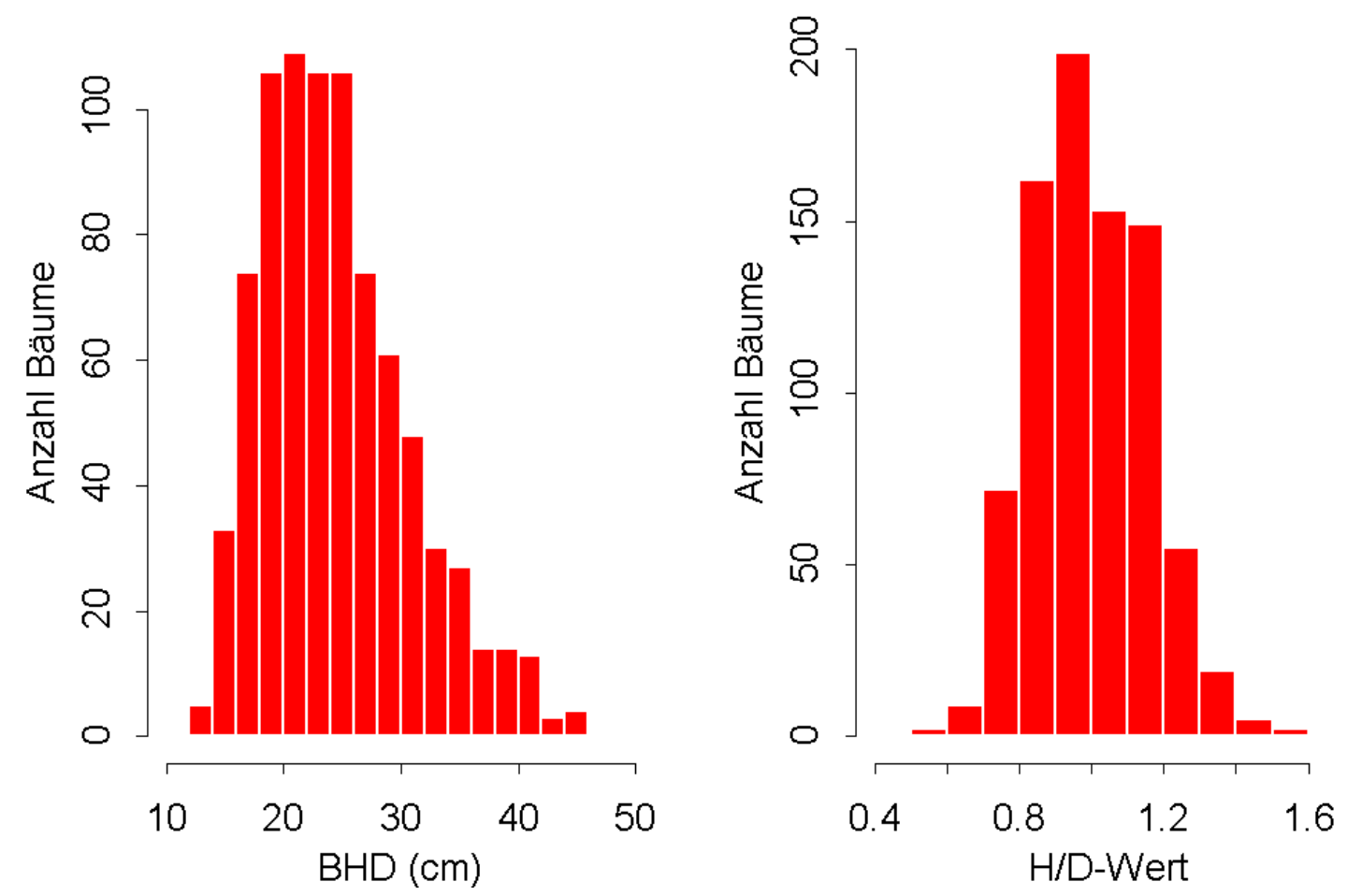

Abb. 63: BHD- und H/D-Wert-Verteilungen für 827 Fichten, die zur Entwicklung eines Einheitsschaftformmodells verwendet wurden.

Bäume mit sehr starken Dimensionen sind im untersuchten Kollektiv nicht enthalten. Die Randbereiche der H/D-Wert-Verteilung mit sehr niedrigen und hohen H/D-Werten sind ebenfalls relativ schwach besetzt.

Vor der eigentlichen Entwicklung flexibler Schaftformmodelle ist es sinnvoll, das Spektrum unterschiedlicher Schaftformprofile innerhalb des untersuchten Kollektivs zu erfassen. KUBLIN und SCHARNAGL (1988) zeigten anhand von Mittelwertpolygonen, daß deutliche Formunterschiede zwischen Einzelbäumen aus unterschiedlichen Wuchsräumen selbst innerhalb der gleichen Durchmesser-Höhen-Stufe auftreten. Das von ihnen entwickelte Sortimentierungsprogramm $B D A T$ greift zur Berücksichtigung dieser Formunterschiede bei der Auswertung von BWI-Daten auf den zusätzlich erfaßten Schaftdurchmesser in $7 \mathrm{~m} \mathrm{Höhe}\left(\mathrm{d}_{7}\right) \mathrm{zu}-$ rück. GAFFREY und NAGEL (1998) konnten für Douglasie nachweisen, daß Formunterschiede beim Vergleich unterschiedlicher Altersklassen bestehen. Die Entwicklung eines Einheitsschaftformmodells führt daher in allen Anwendungsbereichen, in denen keine zusätzlichen Durchmessermessungen zum BHD vorliegen, durch die Verringerung des Bias für bestimmte Teilkollektive zu einer Erhöhung der Schätzgenauigkeit gegenüber Modellen mit einem festen Parametersatz (PAIN U. BOYER, 1996). Hierzu zählen Inventuren ohne zusätzliche Schaftdurchmessermessungen und vor allem waldbauliche Szenariensimulationen. Denkbar ist z. B. 
die Prognose der Auswirkungen von unterschiedlichen Ausgangsverbänden und/oder Durchforstungsstrategien. Bei der Verwendung eines Modells mit festen Parametern besteht die Möglichkeit, daß Szenarien mit geringen Ausgangsstammzahlen oder starken Eingriffen zu günstig bewertet werden, da die Abholzigkeit falsch eingeschätzt wird.

Wenn eine Variabilität der Schaftprofile festgestellt wird, müssen anschließend Variablen identifiziert werden, die diese Formunterschiede beeinflussen. Für beide Voruntersuchungen eignen sich graphische Methoden. In Abb. 64 sind die auf ihre Form reduzierten Schaftprofile eines Teils der 827 Fichten dargestellt. Die Reduktion auf die Form bzw. die Eliminierung der Dimensionsunterschiede der Einzelbäume erfolgte, indem sowohl die Sektionsmessungen mit dem Maximaldurchmesser (in $1 \mathrm{~m}$ Höhe) als auch die zugehörigen Höhenwerte mit der Gesamthöhe des Baumes zwischen 0 und 1 normiert wurden. Abb. 64 verdeutlicht den Einfluß des H/D-Wertes auf die Schaftform. Es sind nur die Schaftprofile der Bäume mit H/D-Werten größer1.2 und kleiner 0.8 dargestellt, weil die Bäume mit mittleren H/D-Werten mittlere Schaftformen aufweisen und daher der Effekt des H/D-Wertes weniger deutlich wird. Es zeigt sich, daß die Bäume mit niedrigen H/D-Werten abholzigere Formen haben als die Bäume mit hohen H/D-Werten.

\section{Fichte}

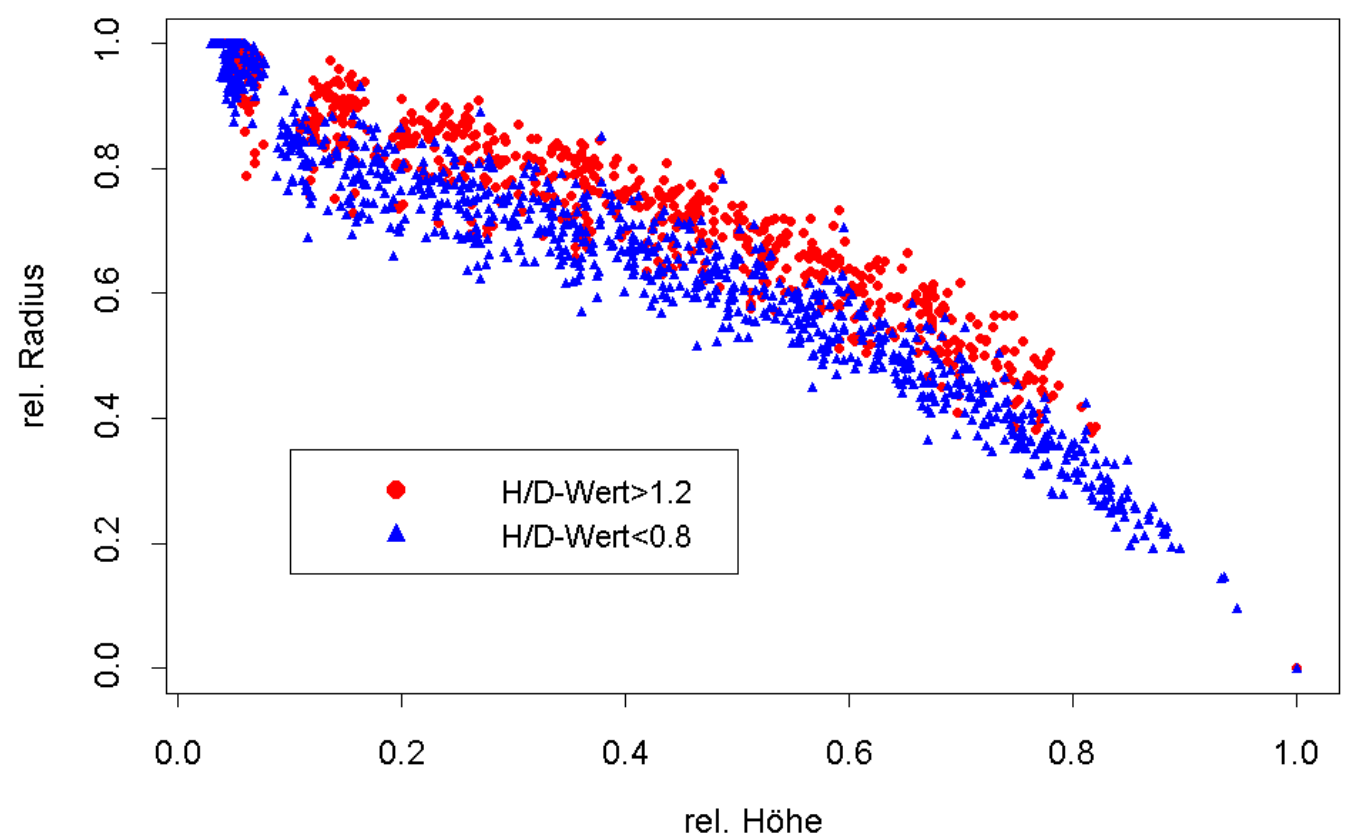

Abb. 64: Normierte Sektionsmessungen für die Teilkollektive von Fichten mit einem H/D-Wert von über 1.2 bzw. unter 0.8 zur Darstellung des Formenspektrums und des Einflusses der Variablen H/D-Wert.

In Abb. 65 sind die auf ihre Form reduzierten Fichten des Kollektivs mit einem BHD von größer als $34 \mathrm{~cm}$ und kleiner als $17 \mathrm{~cm}$ dargestellt. Die deutliche Auftrennung der relativen 
Schaftprofile zeigt, daß auch die Variable BHD einen Einfluß auf die Schaftform hat, wobei die Abholzigkeit von den schwachen zu den starken Dimensionen hin zunimmt.

\section{Fichte}

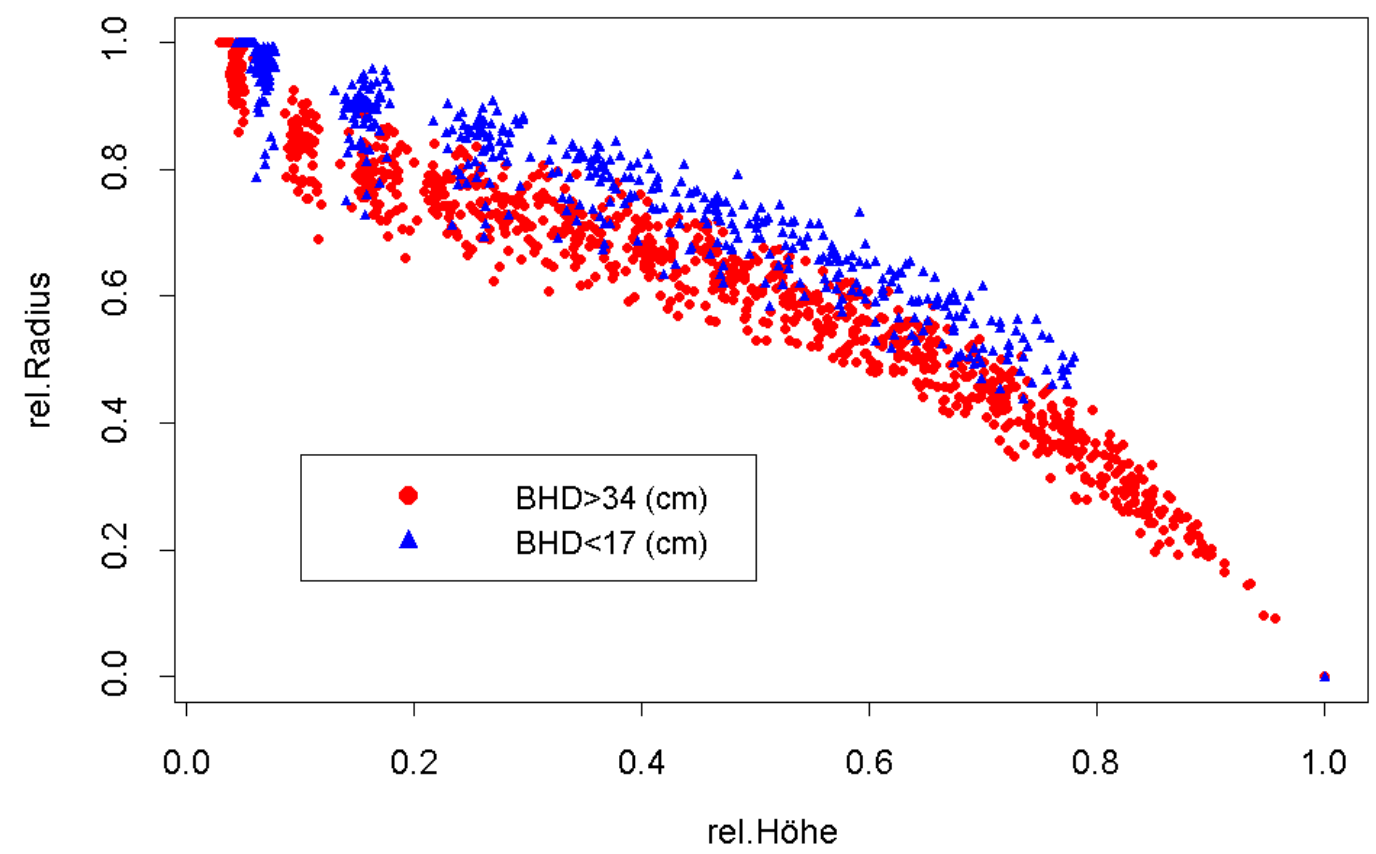

Abb. 65: Normierte Sektionsmessungen für die Teilkollektive von Fichten mit einem BHD von über $34 \mathrm{~cm}$ bzw. unter $17 \mathrm{~cm}$ zur Darstellung des Formenspektrums und des Einflusses der Variablen $B H D$.

Als weitere Variable konnte neben der Einzelbaumhöhe nur noch die Variable Forstort bzw. Unterabteilung bezüglich ihres Einflusses auf die Schaftform geprüft werden. Auf die graphische Darstellung des Einflusses der Einzelbaumhöhe wurde verzichtet, weil eine enge Korrelation mit dem BHD besteht. Da die Zuordnung der Bäume zu einer Versuchsfläche nicht möglich ist, hat der Forstort den Charakter einer kategorischen Variablen, die eine Vielzahl von Bestandes- und Standortsvariablen aggregiert. Auch für diese kategorische Variable konnte geprüft werden, ob sie über den Einfluß der verwendeten Einzelbaumvariablen hinaus signifikant ist bzw. zur Erhöhung der Genauigkeit bei der Beschreibung des Schaftprofils beiträgt. Für die Entwicklung eines in der Praxis anwendbaren Modells ist es jedoch notwendig, die den Forstort beschreibenden metrischen Variablen zu identifizieren bzw. zu integrieren. Diese Möglichkeit bestand bei der vorliegenden Datengrundlage nicht. In einem ersten Schritt wurde daher ein Einheitsschaftformmodell ausschließlich auf der Basis von Einzelbaumvariablen parametrisiert. 


\subsubsection{Pain-Funktion}

Aufgrund der Beschränkung, die Form der Schaftprofile nur in Abhängigkeit von Einzelbaumvariablen beschreiben zu können, wurde das von PAIN und BOYER (1996) für Fichte entwickelte Modell auf seine Eignung zur Beschreibung des Schaftformenspektrums der Baumarten Fichte, Douglasie, Kiefer, Buche und Eiche für den Bereich Nordwestdeutschland überprüft (Formeln (33), (34) u. (35)). Das Modell, das im weiteren als Pain-Funktion bezeichnet wird, hat sich bei der Beschreibung der Schaftform von Fichte für Nordostfrankreich (Vogesen, Jura) unter Berücksichtigung eines Teils der Formvariabilität bewährt. Die dimensions- und formbeschreibenden Parameter $\alpha$ und $\beta$ der Pain-Funktion (33) werden in Abhängigkeit von den formbeeinflussenden Variablen BHD und Höhe wie folgt geschätzt:

$$
\begin{aligned}
& \alpha=a_{0}+a_{1}\left(\frac{1}{\ln \left(H^{1 / D}\right)}\right)+a_{2}\left(\frac{1}{(H / D)^{2}}\right), \\
& \beta=b_{0}+b_{1}\left(\frac{1}{\ln \left(H^{1 / D}\right)}\right)+b_{2}\left(\frac{1}{(H / D)^{2}}\right),
\end{aligned}
$$

wobei:

$\alpha, \beta=$ dimensions- und formbeschreibende Parameter aus Formel (33);

$H=$ Baumhöhe (m);

$D=$ Brusthöhendurchmesser $(\mathrm{cm})$;

$a_{0}, a_{1}, a_{2}, b_{0}, b_{1}, b_{2}=$ Parameter.

Da die Pain-Funktion den Schaftdurchmesser ausschließlich in Abhängigkeit von der relativen Schafthöhe beschreibt, ist eine Kombination mit den in den Formeln (34) und (35) beschriebenen Funktionen zur Parameterschätzung notwendig. Die Funktionen integrieren über die Verwendung der Variablen BHD und Höhe bzw. abgeleiteter Kombinationen der beiden Variablen die Dimension des Baumes in das Schaftformmodell. Soll das Modell darüber hinaus die Entwicklung eines Einheitsschaftformmodells ermöglichen, so müssen die Funktionen zur Parameterschätzung über die Integration der Dimension hinaus auch einen Teil der Formvariabilität beschreiben.

Die Zerlegung der Pain-Funktion in die Funktion (33) und die Funktionen (34) und (35) war im Rahmen der Modellentwicklung notwendig. Nach der einzelbaumweisen Anpassung der Funktion (33) mußten die Zusammenhänge zwischen den form- und dimensionsbeschreibenden Parametern $\alpha$ und $\beta$ und den erklärenden Einzelbaumvariablen erst identifiziert und geeignete Funktionen zur Beschreibung ermittelt werden. Nach der Herleitung der Funktionen zur Parameterschätzung erhält man das Gesamtmodell durch Einsetzen der Funktionen (34) und (35) in die Funktion (33). 
Die Parameterschätzungen und statistischen Kenngrößen für das Gesamtmodell der PainFunktion zur Schaftformbeschreibung der Baumart Fichte sind in Tab. 29 wiedergegeben.

\begin{tabular}{|c|c|c|c|c|}
\hline Koeffizient & Wert & Std. Fehler & t-Wert & $\operatorname{Pr}(>|t|)$ \\
\hline$a_{0}$ & -0.223 & 0.0615 & -3.632 & 0.0003 \\
\hline$a_{1}$ & 1.595 & 0.0138 & 115.608 & 0.0000 \\
\hline$a_{2}$ & -3.155 & 0.0667 & -47.307 & 0.0000 \\
\hline$b_{0}$ & 0.512 & 0.0333 & 15.386 & 0.0000 \\
\hline$b_{1}$ & -0.158 & 0.0075 & -21.042 & 0.0000 \\
\hline$b_{2}$ & -0.502 & 0.0362 & -13.847 & 0.0000 \\
\hline \multicolumn{2}{|c|}{ Residual Std. Fehler (mm) } & \multicolumn{3}{|c|}{0.504 bei 9763 Freiheitsgraden } \\
\hline \multicolumn{2}{|c|}{ Multiples Bestimmtheitsmaß } & \multicolumn{3}{|r|}{0.997} \\
\hline \multicolumn{2}{|r|}{ F-Statistik } & \multicolumn{3}{|c|}{591400 bei 6 und 9763 Freiheitsgraden, $p$-Wert $=0$} \\
\hline
\end{tabular}

Tab. 29: Koeffizienten und statistische Kenngrößen zur Schaftdurchmesserschätzung mit Hilfe des Gesamtmodells der Pain-Funktion für 827 Fichten aus dem Bereich Nordwestdeutschlands.

Für die Untersuchung, inwieweit die Funktionen zur Schätzung der dimensions- und formbeschreibenden Parameter $\alpha$ und $\beta$ wirklich einen Teil der Formvariabilität und nicht nur Dimensionsunterschiede beschreiben, bot sich wie bei der Modellentwicklung die getrennte Betrachtung der Funktionen (34) und (35) an. In Abb. 66 sind die Parameterschätzungen der einzelbaumweisen Anpassungen der Funktion (33) an Sektionsmessungen und die Parameterschätzungen mit Hilfe der Funktionen (34) und (35) abgebildet.
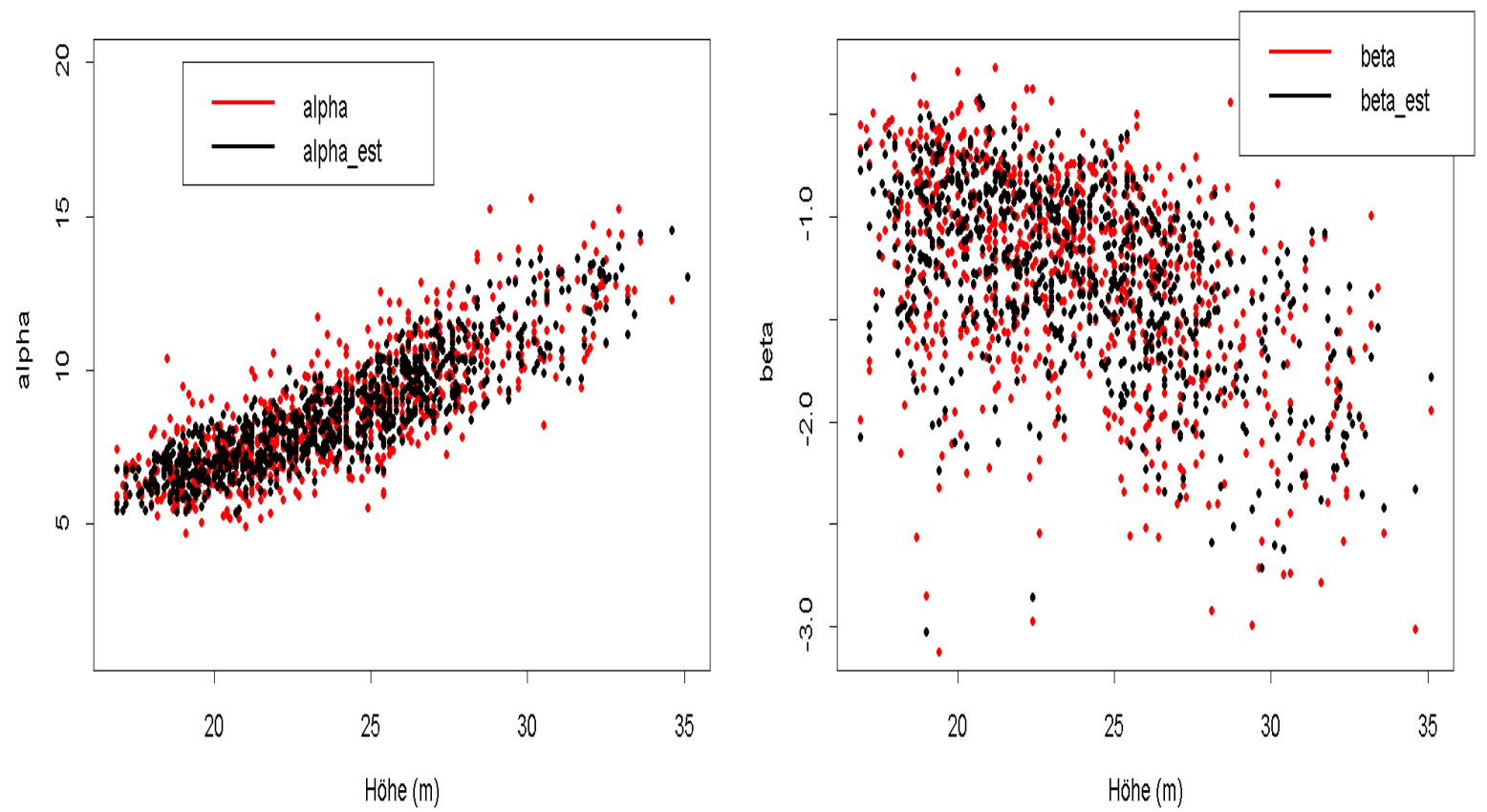

Abb. 66: Zusammenhänge zwischen der Baumhöhe und den dimensions- und formbeschreibenden Koeffizienten $\alpha$ (links) und $\beta$ (rechts) der Pain-Funktion, die über die einzelbaumweise Anpassung von Formel (33) an Sektionsmessungen geschätzt wurden. Die über die Formeln (34) und (35) in Abhängigkeit von Einzelbaumvariablen geschätzten Werte sind mit $\alpha \_$est und $\beta \_$est bezeichnet. 
Insbesondere für den Parameter $\alpha$ wird der enge Zusammenhang mit der Dimensionsvariablen Höhe deutlich. Hierin wird die schon beschriebene Integration der Dimension in das Modell durch das Einsetzen der Funktionen (34) und (35) in das Modell (33) erkennbar. Gleichzeitig verdeutlichen die Schätzungen für $\alpha$ und $\beta$ ( $\alpha \_e s t$ und $\beta \_e s t$ ) mit Bestimmtheitsmaßen von 0.84 und 0.72 , daß ein Großteil der Streuung der Parameter mit Hilfe von Einzelbaumvariablen erklärt werden kann (Tab. 30). Für die Fragestellung, inwieweit die Pain-Funktion Formunterschiede erklärt, ist Abb. 66 nur bedingt geeignet, da nicht erkennbar ist, welcher Anteil der Parameterstreuung dimensions- und welcher formbedingt ist. Damit kann auch nicht geklärt werden, ob die Parameterschätzungen trotz ihrer hohen Bestimmtheitsmaße eventuell nur Dimensionsunterschiede beschreiben.

\begin{tabular}{|c|c|}
\hline $\begin{array}{l}\text { Schätzung des Parameters } \alpha \text { mit Hilfe von } \\
\text { Formel (34) }\end{array}$ & $\begin{array}{c}\text { Schätzung des Parameters } \beta \text { mit Hilfe von } \\
\text { Formel (35) }\end{array}$ \\
\hline $\mathrm{a}_{0}=-0.270$ & $\mathrm{~b}_{0}=0.490$ \\
\hline$a_{1}=1.606$ & $b_{1}=-0.151$ \\
\hline $\mathrm{a}_{2}=-3.198$ & $\mathrm{~b}_{2}=-0.532$ \\
\hline Multiples Bestimmtheitsmaß: 0.839 & Multiples Bestimmtheitsmaß: 0.722 \\
\hline
\end{tabular}

Tab. 30: Funktionsparameter und Bestimmtheitsmaße für die zur Schätzung der dimensionsund formbeschreibenden Parameter $\alpha$ und $\beta$ der Pain-Funktion verwendeten Modelle (34) und (35) für die Baumart Fichte.

Um die formbedingte Streuung der Parameter und den Anteil der mit Hilfe der Modelle (34) und (35) erklärten Streuung zu quantifizieren, wurde die Pain-Funktion (33) dahingehend verändert, daß als unabhängige Variable der normierte Schaftdurchmesser verwendet wurde (Formel (36)).

$r_{r e l}\left(h_{r e l}\right)=\alpha_{r e l}\left(1-h_{r e l}^{3}\right)+\beta_{r e l}\left(\ln \left(h_{r e l}\right)\right)$

wobei:

$h_{\text {rel }}=$ Schafthöhe, mit der Einzelbaumschaftlänge relativiert;

$r_{\text {rel }}=$ mit dem maximalen Stammradius (in $1 \mathrm{~m}$ Höhe) relativierter Schaftradius;

$\alpha_{\text {rel }}, \beta_{\text {rel }}=$ ausschließlich formbeschreibende Parameter.

Das Modell (36) schätzt relative Schaftdurchmesser in Abhängigkeit von der relativen Schafthöhe. Damit ist der Dimensionseinfluß auf die Parameter vollständig aus dem Modell eliminiert. Die Streuung der Parameter $\alpha_{\text {rel }}$, und $\beta_{\text {rel }}$, die durch die einzelbaumweise Anpassung des Modells (36) an die Sektionsdaten geschätzt wurden (Abb. 67), gibt ausschließlich die in den Daten beobachtete Formvariabilität wieder (Abb. 64 und Abb. 65). Das Modell (36) wird im weiteren als Pain-Funktion II bezeichnet. 

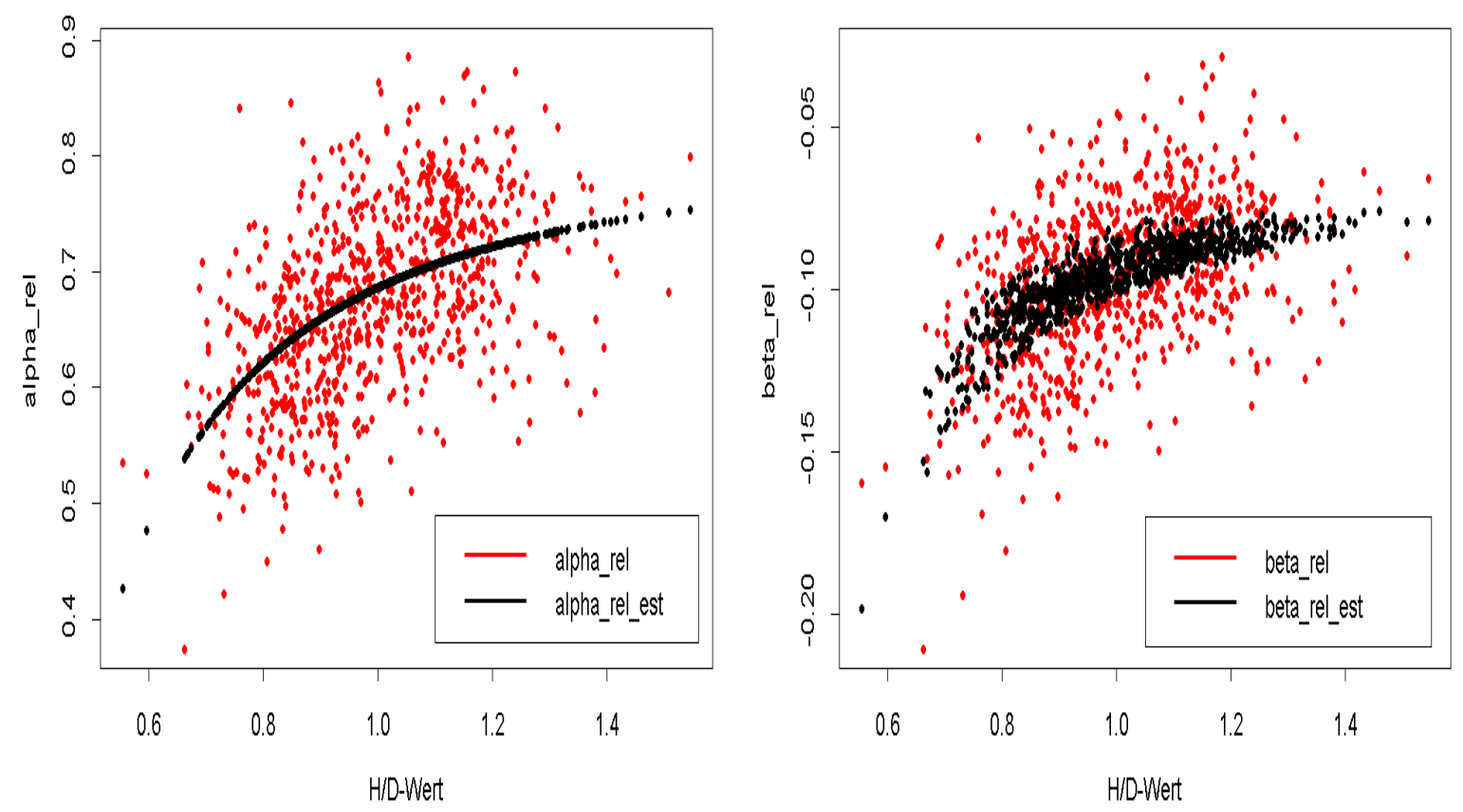

Abb. 67: Zusammenhänge zwischen dem H/D-Wert und den ausschließlich formbeschreibenden Koeffizienten $\alpha_{\text {rel }}$ (links) und $\beta_{\text {rel }}$ (rechts) des Modells Pain-Funktion II, die über die einzelbaumweise Anpassung von Formel (36) an Sektionsdaten geschätzt wurden. Die über die Formeln (34) und (35) (bezogen auf die Parameter der Pain-Funktion II) in Abhängigkeit von Einzelbaumvariablen geschätzten Werte sind mit $\alpha_{\text {rel_est }}$ und $\beta_{\text {rel_est bezeichnet. }}$

Durch den Vergleich der Tab. 31 und Tab. 30 wird deutlich, wie stark die Bestimmtheitsmaße der Parameterschätzung von $\alpha_{\text {rel }}$ und $\beta_{\text {rel }}$ gegenüber den Bestimmtheitsmaßen der Parameterschätzung von $\alpha$ und $\beta$ absinken, da der dimensionsbeschreibende Anteil wegfällt. Die Schätzungen der formbeschreibenden Parameter weisen allerdings noch Bestimmtheitsmaße von 0.28 und 0.30 auf, womit der Nachweis erbracht ist, daß die Pain-Funktion einen Teil der Formvariabilität der Schaftformprofile erfassen und beschreiben kann.

\begin{tabular}{|r|r|}
\hline $\begin{array}{c}\text { Schätzung des Parameters } \alpha_{\text {rel }} \text { mit Hilfe von } \\
\text { Formel (34) }\end{array}$ & $\begin{array}{c}\text { Schätzung des Parameters } \beta_{\text {rel }} \text { mit Hilfe von } \\
\text { Formel (35) }\end{array}$ \\
\hline $\mathrm{a}_{0}=0.806$ & $\mathrm{~b}_{0}=-0.0801$ \\
\hline $\mathrm{a}_{1}$ nicht signifikant & $\mathrm{b}_{1}=0.0061$ \\
\hline $\mathrm{a}_{2}=-0.115$ & $\mathrm{~b}_{2}=-0.0578$ \\
\hline Multiples Bestimmtheitsmaß: 0.277 & Multiples Bestimmtheitsmaß: 0.303 \\
\hline
\end{tabular}

Tab. 31: Funktionsparameter und Bestimmtheitsmaße für die zur Schätzung der ausschließlich formbeschreibenden Parameter $\alpha_{\text {rel }}$ und $\beta_{\text {rel }}$ der Pain-Funktion II verwendeten Modelle (34) und (35).

In Abb. 67 wird der Zusammenhang der formbeschreibenden Parameter $\alpha_{\text {rel }}$ (links) und $\beta_{\text {rel }}$ (rechts) mit dem H/D-Wert deutlich. Die Parameter werden im Gegensatz zu Abb. 66 nicht über der Höhe, sondern über dem H/D-Wert dargestellt, da die formbeschreibenden Parameter 
$\alpha_{\text {rel }}$ und $\beta_{\text {rel }}$ eine sehr viel engere Korrelation zum H/D-Wert aufweisen. So ist in der Funktion (34) zur Schätzung des Parameters $\alpha_{\text {rel }}$ der Einfluß des ersten Terms nicht mehr signifikant (Tab. 31). Dieser erste Term integriert die Einzelbaumdimension als unabhängige Variable in die Schätzung der formbeschreibenden Parameter. Bei der Schätzung des Parameters $\beta_{\text {rel }}$ ist der Einfluß beider Terme in der Funktion (35) signifikant (Tab. 31). D. h., daß mit dem Modell neben der Variation der Schaftprofilform mit sich ändernden H/D-Werten auch eine Veränderung mit sich ändernden Baumdimensionen beschrieben wird. In Abb. 68 ist erkennbar, wie sich das Gesamtschaftprofil der Pain-Funktion aus zwei Einzeltermen zusammensetzt. Der rot markierte Verlauf des Terms $\left(1-\mathrm{h}_{\mathrm{rel}}{ }^{3}\right)$ wird über den Parameter $\alpha$ bzw. $\alpha_{\text {rel }}$ beeinflußt. Daher weist dieser Term nur eine Formvariation über dem H/D-Wert auf. Der Verlauf des blau markierten Terms $\ln \left(\mathrm{h}_{\text {rel }}\right)$ zeigt eine Formvariation sowohl über dem H/D-Wert als auch über der Einzelbaumdimension.

\section{Pain-Funktion}

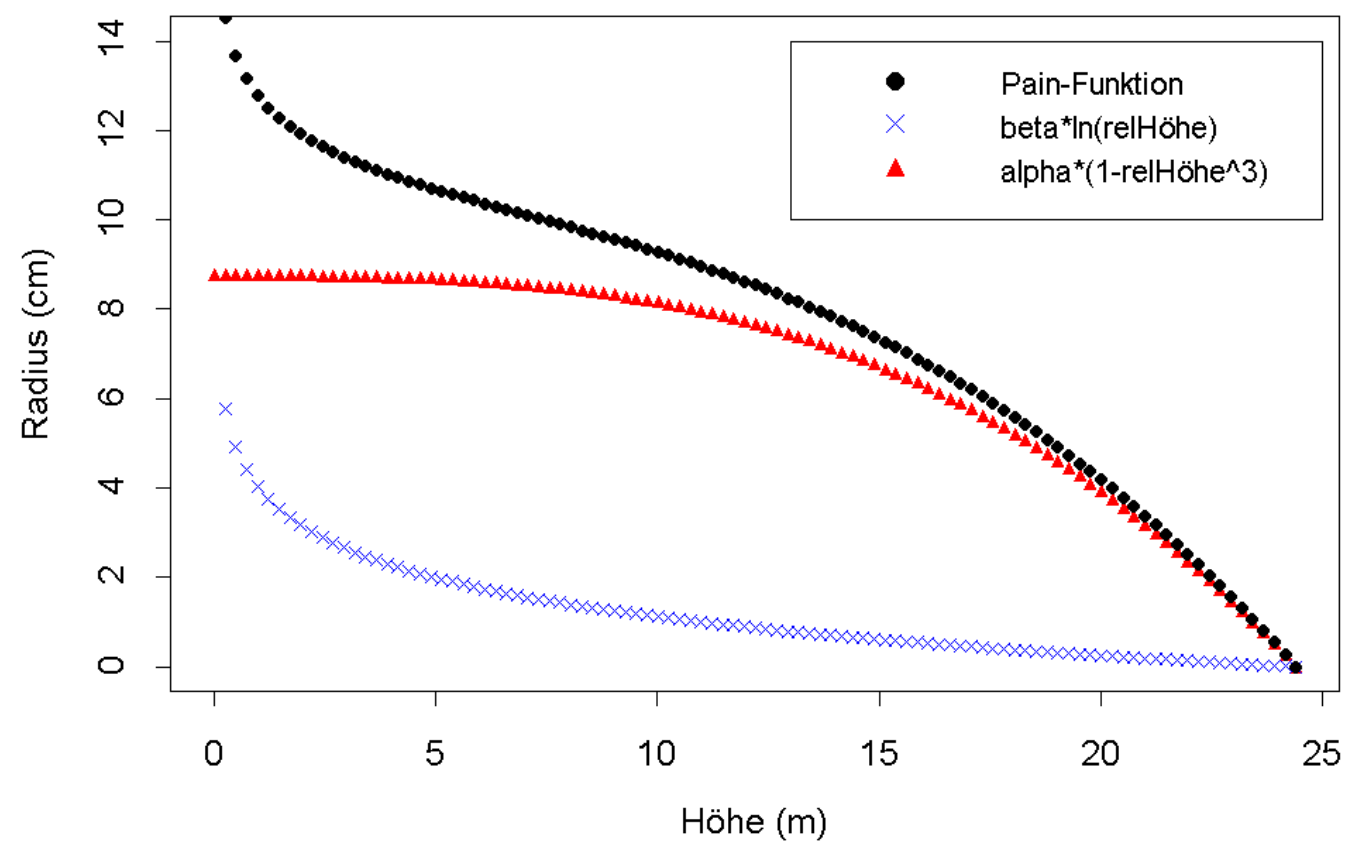

Abb. 68: Verlauf der durch die Pain-Funktion beschriebenen Schaftform einer Fichte mit dem BHD und der Höhe, die den Medianen der BHD- und der Höhenverteilung des untersuchten Datenmaterials entsprechen. Zusätzlich dargestellt sind die beiden Terme, aus denen sich die Pain-Funktion zusammensetzt.

Nachdem gezeigt werden konnte, daß die Streuung der formbeschreibenden Parameter z. T. mit Hilfe von Einzelbaumvariablen erklärt werden kann, sollen nun die Auswirkungen auf die Schätzgenauigkeit des Modells dargestellt werden. Um die Vorteile eines flexiblen Schaftformmodells zu verdeutlichen, bietet sich der Vergleich mit einem mittleren Einzelbaumschaftformmodell an. Ein solches Schaftformmodell beschreibt die mittlere Form des Kollektivs, das zu seiner Parametrisierung verwendet wurde. An den Randbereichen des Formen- 
spektrums kommt es daher zu größeren Schätzfehlern als bei Verwendung eines flexiblen Schaftformmodells. Als Vergleichsmodell bot sich die Pain-Funktion II (36) an, wobei anstelle der Schätzungen der Formparameter (Formeln (34) u. (35)), die Mittelwerte der Parameter $\alpha_{\text {rel }}$ und $\beta_{\text {rel }}$ innerhalb des Kollektivs verwendet wurden. Für die Verwendung eines mittleren Parametersatzes mußte die Pain-Funktion II (36) verwendet werden, da die Parameter der Pain-Funktion (33) auch die Dimension des Baumes berücksichtigen und daher keine Mittelwerte verwendet werden können. Die über die Pain-Funktion II mit einem mittleren Parametersatz geschätzten Schaftradien wurden anschließend wieder in absolute Schaftradien umgerechnet.

Als weiteres Vergleichsmodell wurde die modifizierte Brink-Funktion (RIEMER ET. AL., 1995) mit mittleren Parameterwerten für die Parameter $p$ und $q$ verwendet (Formel (37)). Der Parameter $i$ wird mit hohen Bestimmtheitsmaßen als Funktion des BHD bzw. des Radius in 1.3 m Höhe geschätzt und hat nur einen sehr begrenzten Einfluß auf die Stammform.

$r(h)=u+v \cdot e^{-p h}-w \cdot e^{q h}$

mit: $u=\frac{i}{1-e^{q(1.3-H)}}+\left(r_{1.3}-i\right) *\left(1-\frac{1}{1-e^{p(1.3-H)}}\right) v=\frac{\left(r_{1.3}-i\right) \cdot e^{p \cdot 1.3}}{1-e^{p(1.3-H)}}$ und $w=\frac{i \cdot e^{-q H}}{1-e^{q(1.3-H)}}$ wobei:
$r(h)=$ Baumradius $(\mathrm{cm})$ in Höhe $h(\mathrm{~m})$;
$H \quad=$ Scheitel- bzw. Gesamthöhe des Baumes (m);
$r_{1.3}=$ Baumradius in Brusthöhe $(\mathrm{cm})$;
$i=$ Parameter (gemeinsame Asymptote), mit $i=k * \mathrm{BHD} / 2$;
$p \quad=$ Parameter (unterer Schaftteil);
$q \quad=$ Parameter (oberer Schaftteil).

für Fichte mit:

\begin{tabular}{|c|c|c|c|}
\hline Koeffizient & Wert & Std. Fehler & t-Wert \\
\hline$k$ & 0.563 & 0.0114 & 49.217 \\
\hline$p$ & 0.0683 & 0.00368 & 18.548 \\
\hline$q$ & 0.188 & 0.00473 & 39.792 \\
\hline
\end{tabular}

Tab. 32: Koeffizienten und statistische Kenngrößen zur Schaftdurchmesserschätzung mit Hilfe der modifizierten Brink-Funktion (37) für die Baumart Fichte.

Für einheimische Baumarten ist es bisher nicht gelungen, die Parameter der modifizierten Brink-Funktion in Abhängigkeit von weiteren Einzelbaum- oder Bestandesvariablen zu schätzen (StEINGASS, 1996) ${ }^{20}$. HuI und GADOw (1997) dagegen entwickelten für die Plantagen-

\footnotetext{
${ }^{20}$ Im Rahmen dieser Arbeit sind für die untersuchten Baumarten ebenfalls Tests zur Entwicklung eines flexiblen Schaftformmodells auf der Basis der Brink-Funktion durchgeführt worden.
} 
baumart Cunninghamia lanceolata ein flexibles Schaftformmodell auf der Basis der BrinkFunktion, wobei als erklärende Variablen auch Bestandesvariablen zur Verfügung standen. Allerdings beinhaltet die modifizierte Brink-Funktion, im Gegensatz zum Modell der PainFunktion (33), bereits die beiden Einzelbaumvariablen BHD und Höhe. Daher kann selbst bei konstanten Parametern (Ausnahme $i$ ) nicht davon ausgegangen werden, daß die modifizierte Brink-Funktion lediglich eine mittlere Schaftform beschreibt, da unterschiedliche Kombinationen von BHD und Höhenwerten eine Variation der Form bewirken könnten.

\section{Validierung und Bewertung der Pain-Funktion:}

Beim Vergleich der Standardfehler der Pain-Funktion (Tab. 29, RMSE: $0.5042 \mathrm{~cm}$ ), der PainFunktion II (konstante Parameter) (RMSE: $0.6544 \mathrm{~cm}$ ) und der Brink-Funktion (Tab. 32, RMSE: $0.6440 \mathrm{~cm}$ ) fällt der geringere Fehler des flexiblen Modells auf. Die Differenz der mittleren Fehler der Modelle ist nur schwierig zu interpretieren, da sie stark von der Datenstruktur abhängt. Man kann davon ausgehen, daß das flexible Modell besonders an den Randbereichen der Verteilungen der Einzelbaumvariablen Vorteile aufweist. Da aber Bäume mit mittleren Variablenwerten bzw. Schaftformen häufiger vorkommen, wird der Vorteil des flexiblen Modells bei der Betrachtung von mittleren Fehlern weniger deutlich.

Um die Datenstruktur bei der Validierung zu berücksichtigen, wurden die RMSE getrennt nach Einzelbäumen berechnet und über den Einzelbaumvariablen H/D-Wert und BHD dargestellt (Abb. 69 und Abb. 70). Die Residuen wurden mit dem zugehörigen beobachteten Sektionsdurchmesser relativiert, um die Fehler in unterschiedlichen Bereichen von Einzelbaumdimensionen vergleichbar zu machen. Es ist deutlich erkennbar, daß das flexible Modell (Pain-Funktion) über den gesamten Bereich der Einzelbaumvariablen BHD und H/D-Wert eine gleichbleibende Schätzgenauigkeit aufweist. 


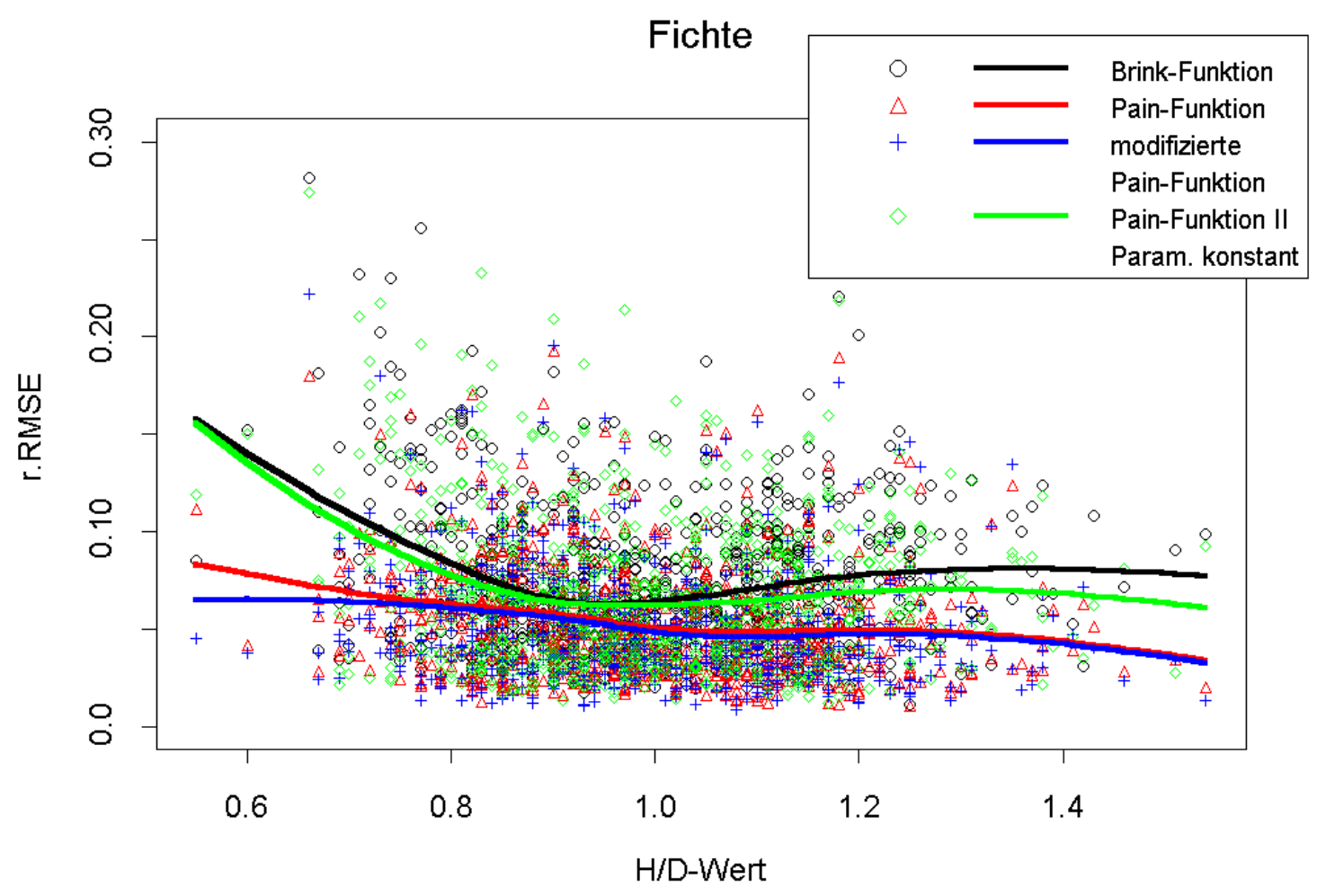

Abb. 69: Einzelbaumweise berechnete relative RMSE über den zugehörigen H/D-Werten der Einzelbäume für die Pain-Funktion (flexibles Schaftformmodell) sowie für die modifizierte Brink-Funktion und die Pain-Funktion II (konstante Parameter) (mittleres Einzelbaummodell). Der Ausgleich der Fehlerwerte erfolgte mit einem GleitendenDurchschnitts-Modell (Kapitel 8.1). Weiterhin sind die relativen RMSE der in Kapitel 3.7.3 beschriebenen Modifikation der Pain-Funktion dargestellt.

Demgegenüber treten beim mittlere Einzelbaummodell auf Basis der Pain-Funktion II an den Randbereichen der erklärenden Variablen und damit des Formenspektrums deutlich höhere Fehlerwerte auf. Die modifizierte Brink-Funktion weist einen dem mittleren Einzelbaummodell ähnlichen Fehlerverlauf über dem H/D-Wert auf, obwohl beide Einzelbaumvariablen BHD und Höhe in das Modell eingehen. Somit beschreibt auch die modifizierte BrinkFunktion lediglich eine mittlere Schaftform des zur Parametrisierung verwendeten Kollektivs. Dabei steigen die Fehlerwerte für das mittlere Einzelbaummodell und die Brink-Funktion mit sinkenden H/D-Werten deutlich stärker an als mit steigenden H/D-Werten. Eine mögliche Erklärung wäre, daß die Abweichungen von der mittleren Schaftform bei Bäumen mit hohen $\mathrm{H} / \mathrm{D}-$ Werten weniger deutlich ausgeprägt sind als bei Bäumen mit niedrigen $\mathrm{H} / \mathrm{D}-$ Werten und daß ab einem bestimmten H/D-Wert keine weitere Zunahme der Vollholzigkeit mehr auftritt. Demgegenüber steigen die Fehlerwerte für das mittlere Einzelbaummodell und die modifizierte Brink-Funktion mit sinkenden H/D-Werten stetig an, was auf eine stetige Zunahme der Abholzigkeit bzw. Abweichung von der mittleren Schaftform schließen läßt. Bei der Interpretation ist die geringe Datengrundlage im Bereich geringer H/D-Werte zu beachten. 


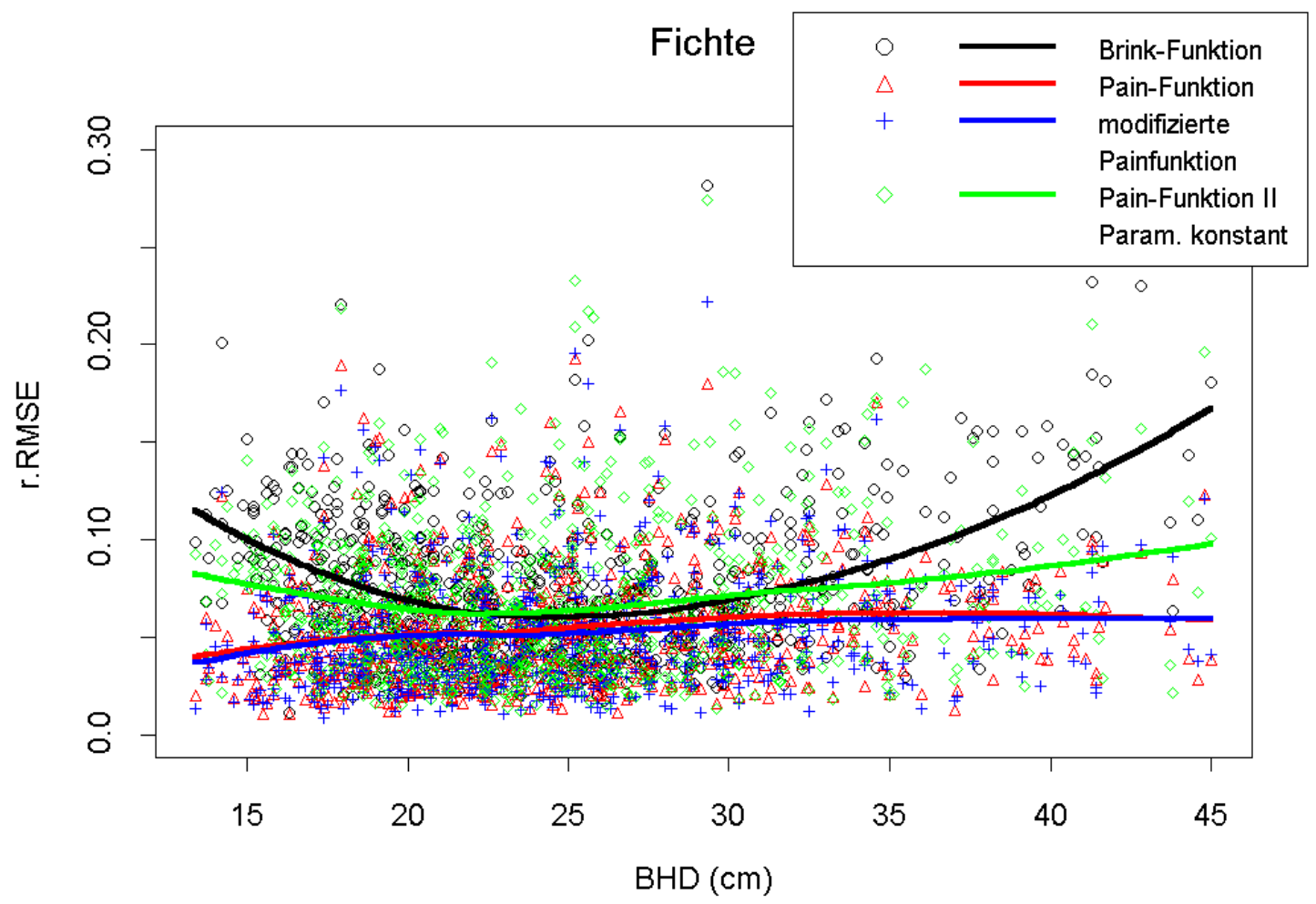

Abb. 70: Einzelbaumweise berechnete relative RMSE über den zugehörigen BHD-Werten der Einzelbäume für die Pain-Funktion (flexibles Schaftformmodell) sowie für die modifizierte Brink-Funktion und die Pain-Funktion II (konstante Parameter) (mittleres Einzelbaummodell). Der Ausgleich der Fehlerwerte erfolgte mit einem GleitendenDurchschnitts-Modell (Kapitel 8.1). Weiterhin sind die relativen RMSE der in Kapitel 3.7.3 beschriebenen Modifikation der Pain-Funktion dargestellt.

Bezüglich der Variablen BHD steigen die einzelbaumweise berechneten Fehlerwerte, ausgehend von einem BHD mit etwa $26 \mathrm{~cm}$, in Richtung schwacher und starker Dimensionen für das mittlere Einzelbaummodell (Pain-Funktion II; Param. konstant) und besonders für die modifizierte Brink-Funktion deutlich an. Als Erkärung kann wieder die stetig zunehmende Abweichung der Schaftform von der mittleren Schaftform mit steigenden und sinkenden Dimensionen angeführt werden. Die Brink-Funktion weist dabei eine deutlich stärkere Zunahme der Fehler zu den Randbereichen auf, während der Fehler des mittleren Einzelbaummodells im mittleren Durchmesserbereich etwas über dem der Brink-Funktion liegt. Das flexible Schaftformmodell erfaßt und beschreibt auch die dimensionsbedingten Schaftformunterschiede, wie an der gleichbleibenden Schätzgenauigkeit über den gesamten Dimensionsbereich zu erkennen ist.

Zur Überprüfung der Verzerrung von Schaftformmodellen sind mittlere Residuen ungeeignet, da sich systematische Über- und Unterschätzungen entlang des Stammes ausgleichen können und damit keine eindeutige Interpretation des Ergebnisses möglich ist. Daher war eine Berechnung der mittleren Residuen in relativen Schafthöhen notwendig. In Abb. 71 und Abb. 72 sind die RMSE $(\mathrm{cm})$ und die mittleren Residuen (Bias) $(\mathrm{cm})$ getrennt nach relativen 
Stammhöhenklassen dargestellt. Für die Zuordnung zu einer relativen Stammhöhenklasse wurde die relative Stammhöhe jeder Schätzung auf eine Nachkommastelle gerundet. Es fällt auf, daß das mittlere Einzelbaummodell (Pain-Funktion II; Param. konstant) und die BrinkFunktion über den ganzen Stammhöhenbereich höhere Fehlerwerte als das flexible Schaftformmodell (Pain-Funktion) aufweisen. Zum Vergleich sind die Fehlerwerte der einzelbaumweisen Anpassung der Pain-Funktion an die Sektionsmessungen (Pain-Funktion (direkt)) dargestellt (Abb. 71). Die direkte einzelbaumweise Parametrisierung anhand der Sektionsmessungen wäre die bestmögliche Anpassung mit Hilfe der Pain-Funktion, wenn sich die formund dimensionsbeschreibenden Parameter $\alpha$ und $\beta$ ohne Fehler schätzen (verallgemeinern) ließen. Beim Vergleich der Verläufe der Bias-Werte sind die Ergebnisse der direkten Anpassung an die Sektionsmessungen der Einzelbäume nicht dargestellt, da eine große Übereinstimmung mit den zugehörigen Modellen bei Schätzung (Verallgemeinerung) der Parameter besteht.

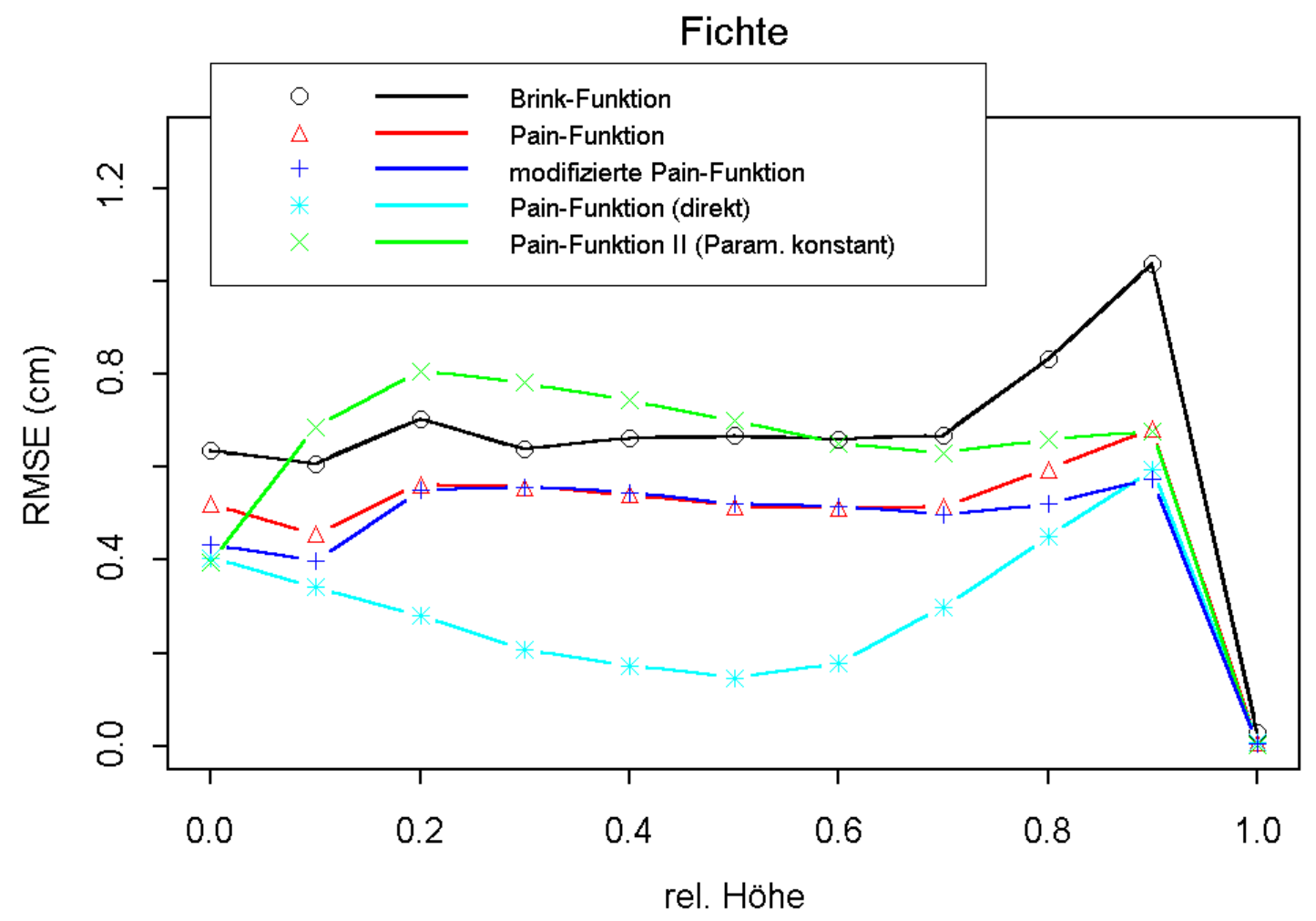

Abb. 71: Verläufe des Fehlers (RMSE) über relativen Schafthöhen für unterschiedliche Schaftformmodelle.

Beim Vergleich der Bias-Werte fällt der sehr viel günstigere Verlauf des flexiblen Modells gegenüber der Brink-Funktion auf (Abb. 72). Während der Bias bei der Brink-Funktion mehrfach zwischen Unter- und Überschätzung wechselt, weist das flexible Modell, bis auf die Überschätzung im Stammfuß- und Zopfbereich, von 20 bis 70\% der Baumhöhe nur geringe Verzerrungen auf. Die Unterschätzung im Stammfußbereich durch die Pain-Funktion ist allerdings deutlich geringer als bei der Brink-Funktion. Das mittlere Schaftformmodell auf Ba- 
sis der Pain-Funktion II weist ebenfalls deutlich geringere Verzerrungen als die BrinkFunktion auf. Um die Schätzung im Stammfußbereich zu verbessern, wurde eine Modifikation der Pain-Funktion entwickelt (Kapitel 3.7.3), die den Meßwert des BHD berücksichtigt. Die modifizierte Brink-Funktion verläuft im Gegensatz zur Pain-Funktion auf $1.3 \mathrm{~m}$ Höhe zwingend durch den Meßwert des BHD.

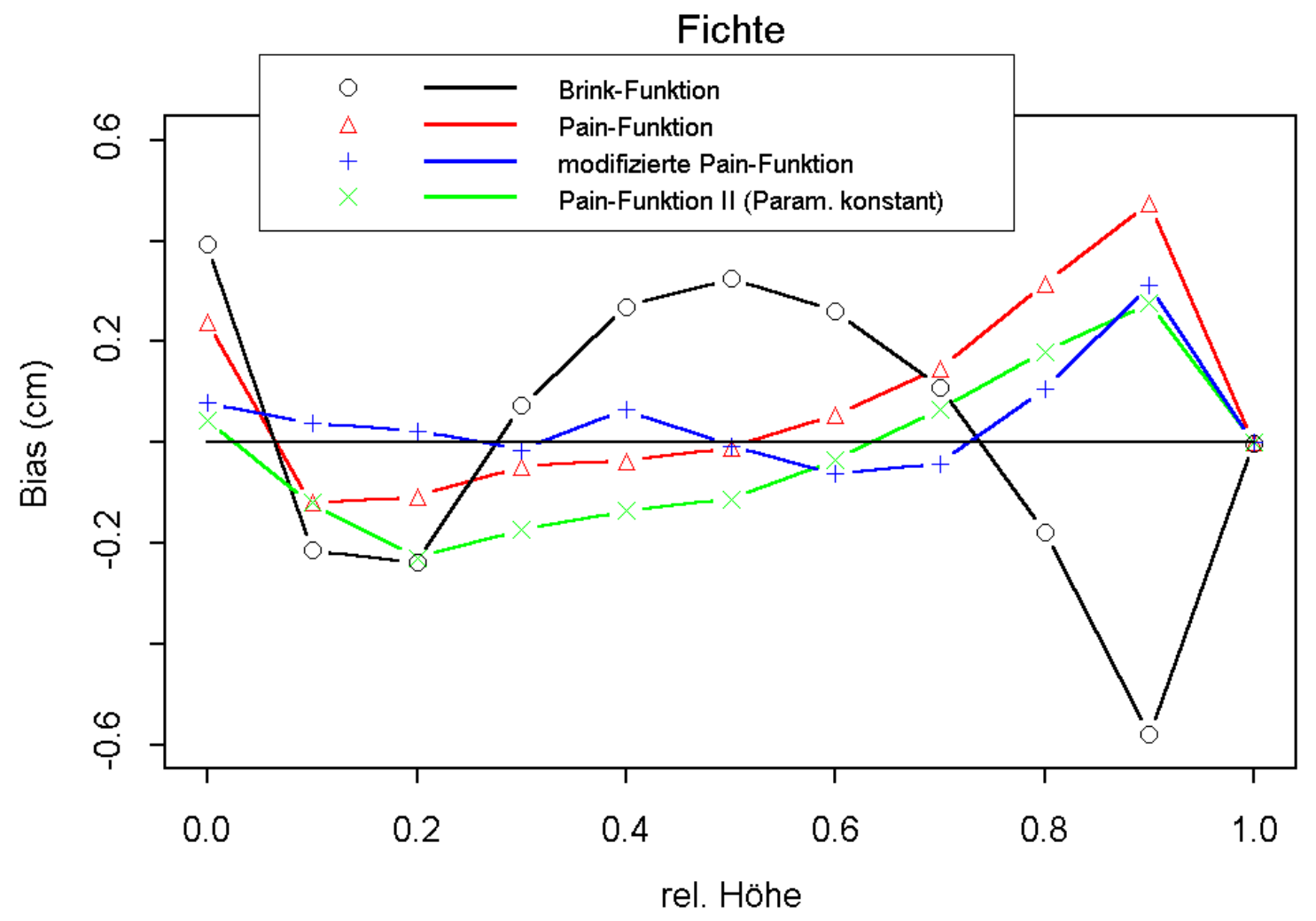

Abb. 72: Verläufe des Bias über relativen Schafthöhen für unterschiedliche Schaftformmodelle.

\subsubsection{Modifikation der Pain-Funktion}

Die modifizierte Pain-Funktion (Formeln $38 \mathrm{a}$ und b) verläuft mathematisch nicht exakt durch den Meßwert des BHD, jedoch sind die Abweichungen gegenüber der Originalversion auf ein Minimum reduziert. Das Modell kann wie die Originalversion in eine Funktion zur Anpassung an Einzelbäume (vgl. Formel (33)) und zwei Funktionen zur Schätzung der dimensionsund formbeschreibenden Parameter untergliedert werden (vgl. Formeln (34) u. (35)). Die Formeln (38 a und b) stellen die modifizierte Version nach Einsetzen der Funktionen zur Verallgemeinerung des Modells in die eigentliche Schaftformfunktion dar. Die Funktionen zur Schätzung der dimensions- und formbeschreibenden Parameter stimmen mit den Funktionen überein, die für die Originalversion verwendet wurden (Formeln (34) u. (35)).

Da durch die Bedingung, daß der Funktionswert auf 1.3 m Höhe mit dem Meßwert des BHD übereinstimmen soll, ein Verlust an Flexibilität verbunden war, wurde die modifizierte Pain-Funktion als segmentierte Funktion konzipiert. Es wurden zwei unterschiedliche Funk- 
tionen für einen unteren und einen oberen Stammteil entwickelt (Formeln (38 a und b)), für die jeweils die Funktionen zur Schätzung der form- und dimensionsbeschreibenden Parameter angepaßt wurden (Formeln (34) u. (35)).

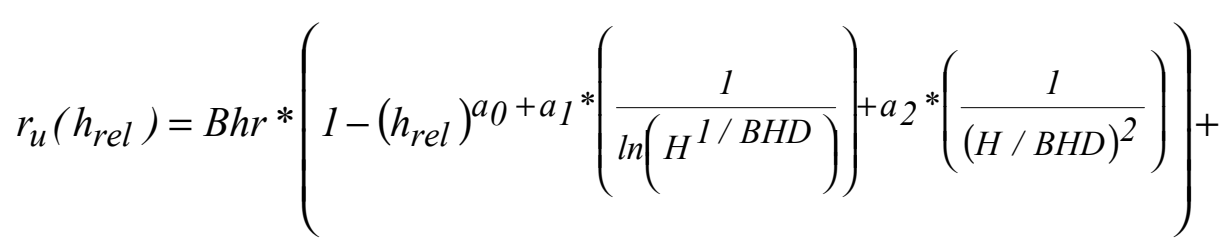

$$
\begin{aligned}
& \ln (h / 1.3) *\left(b_{0}+b_{1} *\left(\frac{1}{\ln \left(H^{1 / B H D}\right)}\right)+b_{2} *\left(\frac{1}{(H / B H D)^{2}}\right)\right) * \ln \left(h_{r e l}\right)
\end{aligned}
$$

, falls $h<=h_{\text {Wendepunkt }}$

$$
\begin{aligned}
r_{O}\left(h_{\text {rel }}\right)= & r_{u}\left(h_{\text {Wendepkt }}\right) *\left(1-\left(\frac{h-h_{\text {Wendepkt }}}{H-h_{\text {Wendepkt }}}\right)^{a_{0}+a_{1} *}\left(\frac{1}{\ln \left(H^{1 / B H D}\right)}\right)+a_{2} *\left(\frac{1}{(H / B H D)^{2}}\right)\right)+ \\
& \ln \left(h / h_{\text {Wendepkt }}\right) *\left(b_{0}+b_{1} *\left(\frac{1}{\ln \left(H^{1 / B H D}\right)}\right)+b_{2} *\left(\frac{1}{(H / B H D)^{2}}\right)\right) * \ln \left(h_{\text {rel }}\right)
\end{aligned}
$$

falls $h>h_{\text {Wendepunkt }}$

wobei:

$r\left(h_{r e l}\right)=$ Schaftradius in der mit der Gesamthöhe relativierten Schafthöhe $h_{r e l}(\mathrm{~cm})$;

$h \quad=$ Schafthöhe $h$ für die der Schaftradius geschätzt werden soll;

$H \quad=$ Baumhöhe $(\mathrm{m})$;

$B H D=$ Brusthöhendurchmesser $(\mathrm{cm})$;

$B h r=$ Schaftradius auf $1.3 \mathrm{~m}$ Schafthöhe;

$a_{0}, a_{1}, a_{2}, b_{0}, b_{1}, b_{2}=$ Parameter.

und:

$h_{\text {Wendepunkt }}=H * \sqrt[3]{\frac{-\beta_{\text {est }}}{6 * \alpha_{\text {est }}}}$ mit der Bedingung: $-6^{*} \alpha_{\text {est }} * h_{\text {rel }}+2 * \beta_{\text {est }} / h_{r e l}{ }^{3} \neq 0$,

wobei:

$\alpha_{e s t}, \beta_{e s t}=$ über Einzelbaumvariablen geschätzte Dimensions- und Formparameter der Pain-Funktion in der Originalversion (Formel (33)).

Die Segmentierung erfolgt im Wendepunkt der Schaftform, an dem der untere konkave Verlauf in den oberen konvexen Verlauf übergeht. Der Wendepunkt wurde näherungsweise mit Hilfe der zweiten bzw. dritten Ableitung der Originalversion der Pain-Funktion ermittelt. Dabei wurde die Stelle bestimmt, für die der zugehörige Funktionswert der zweiten Ableitung der Pain-Funktion gleich Null ist. Anschließend wird überprüft, ob der Funktionswert der 
dritten Ableitung der Pain-Funktion an derselben Stelle ungleich Null ist. Durch die Verwendung der Parameter $\alpha_{e s t}$ und $\beta_{\text {est }}$ in der Pain-Funktion, die über die Formeln (34) und (35) ermittelt wurden, wurde der Schätzfehler bei der Bestimmung des Wendepunktes berücksichtigt und der Wendepunkt daher nur näherungsweise bestimmt.

Der Wendepunkt erscheint als biologisch sinnvollste Segmentierungsstelle, da konkav und konvex gewölbter Schaftformverlauf getrennt beschrieben werden und sich die Steigungen von unterem und oberen Schaftteil zum Wendepunkt hin annähern, was die Modellierung im Übergangsbereich erleichtert. In Tab. 33 sind die Parameterschätzungen und statistischen Kenngrößen für die modifizierte Pain-Funktion für die Baumart Fichte angegeben.

\begin{tabular}{|c|c|c|c|}
\hline Koeffizient & Wert & Std. Fehler & t-Wert \\
\hline Unterer Schaftteil $a_{0}$ & 2.727 & 0.139 & 19.597 \\
\hline Unterer Schaftteil $a_{1}$ & -0.0635 & 0.0226 & -2.809 \\
\hline Unterer Schaftteil $a_{2}$ & -0.00833 & 0.0954 & -0.0874 \\
\hline Unterer Schaftteil $b_{0}$ & -0.298 & 0.0449 & -6.637 \\
\hline Unterer Schaftteil $b_{1}$ & 0.0770 & 0.00983 & 7.832 \\
\hline Unterer Schaftteil $b_{2}$ & 0.354 & 0.0467 & 7.573 \\
\hline Oberer Schaftteil $a_{0}$ & 2.434 & 0.0495 & 49.132 \\
\hline Oberer Schaftteil $a_{1}$ & 0.0197 & 0.0103 & 1.910 \\
\hline Oberer Schaftteil $a_{2}$ & -0.299 & 0.0498 & -6.002 \\
\hline Oberer Schaftteil $b_{0}$ & -0.487 & 0.180 & -2.702 \\
\hline Oberer Schaftteil $b_{1}$ & 0.314 & 0.0459 & 6.845 \\
\hline Oberer Schaftteil $b_{2}$ & 0.501 & 0.236 & 2.124 \\
\hline \multicolumn{2}{|c|}{ Residual Std. Fehler (mm) } & \multicolumn{2}{|c|}{0.481 bei 9757 Freiheitsgraden } \\
\hline
\end{tabular}

Tab. 33: Koeffizienten und statistische Kenngrößen zur Schaftdurchmesserschätzung mit Hilfe der modifizierten Pain-Funktion für die Baumart Fichte. 


\section{Validierung und Bewertung der modifizierten Pain-Funktion:}

In Abb. 73 sind die Verteilungen der Residuen auf $1.3 \mathrm{~m}$ Höhe der 827 untersuchten Fichten für die Pain-Funktion und die modifizierte Pain-Funktion dargestellt. Während bei der Originalversion häufiger Abweichungen von mehreren Millimetern auftreten, liegen bei der modifizierten Version 99.7\% der Residuen deutlich unterhalb von einem Millimeter.

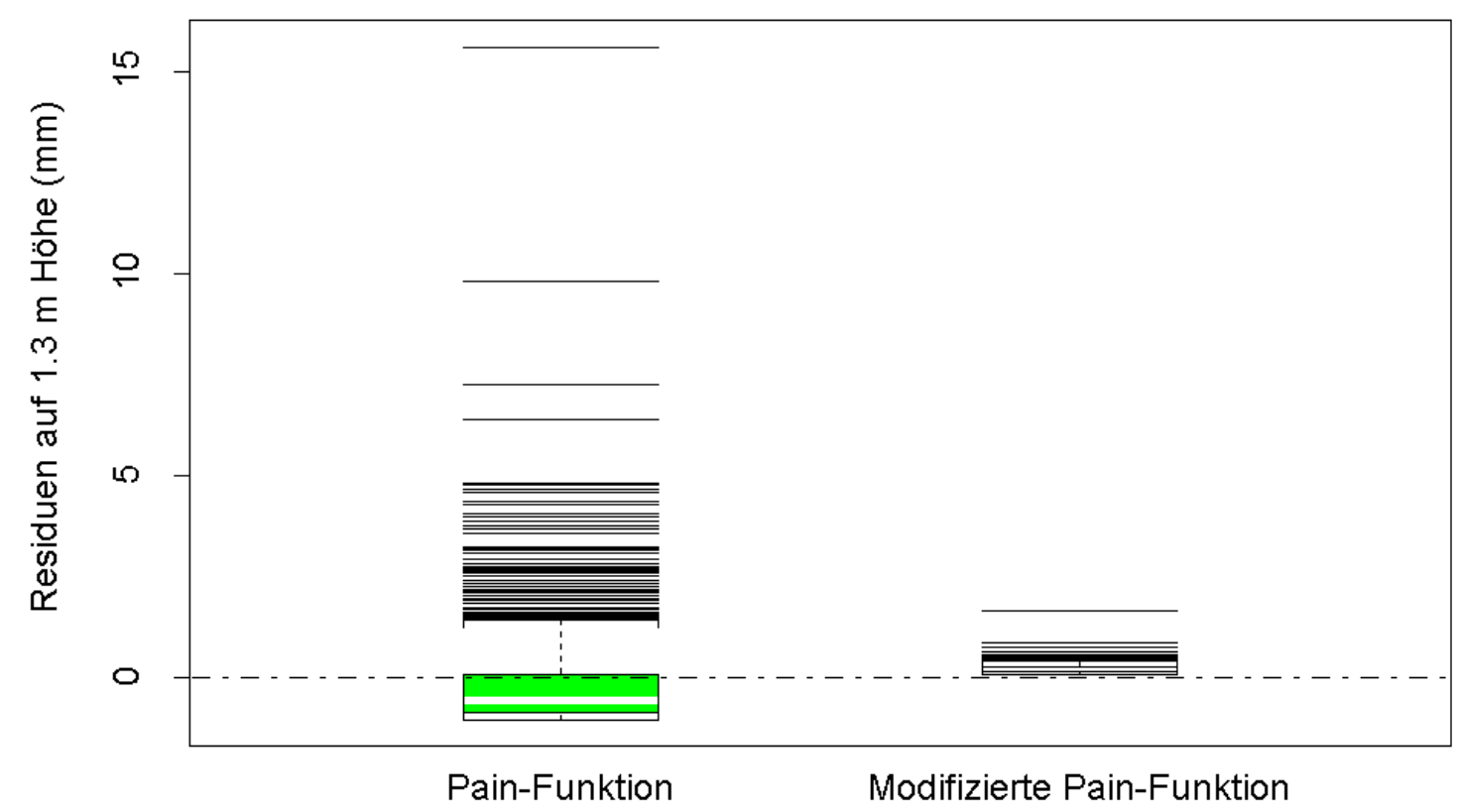

Abb. 73: Box-Plots der Residuen auf 1.3 m Höhe der Pain-Funktion und der modifizierten Pain-Funktion für die Baumart Fichte.

In Abb. 74 sind die Funktionsverläufe der modifizierten Pain-Funktion und der Terme, aus denen sich die Gesamtfunktion zusammensetzt, dargestellt. Im Vergleich zur Originalversion (Abb. 68) sind die beiden rot dargestellten Terme für den unteren und oberen Schaftteil weniger variabel, um den Verlauf der Funktion in 1.3 m Höhe auf den Meßwert des BHD bzw. auf den Funktionswert der unteren Segmentfunktion am Wendepunkt festzulegen. Daher mußten die blau dargestellten Terme im Vergleich zur Originalversion so verändert werden, daß auch negative Werte beschrieben werden können. Trotz der Segmentierung im Wendepunkt mit den bereits erläuterten Vorteilen kommt es zu unplausiblen Knicken am gemeinsamen Funktionswert von unterem und oberem Schaftsegment (schwarze Punkte). Der Übergang kann ohne jeglichen Knick beschrieben werden, wenn an die berechneten Funktionswerte der modifizierten Pain-Funktion eine kubische Spline-Funktion angepaßt wird. Dabei werden die Funktionswerte der modifizierten Pain-Funktion aus dem Bereich des Wendepunktes nicht in die Spline-Interpolation mit einbezogen (grüne Linie). 


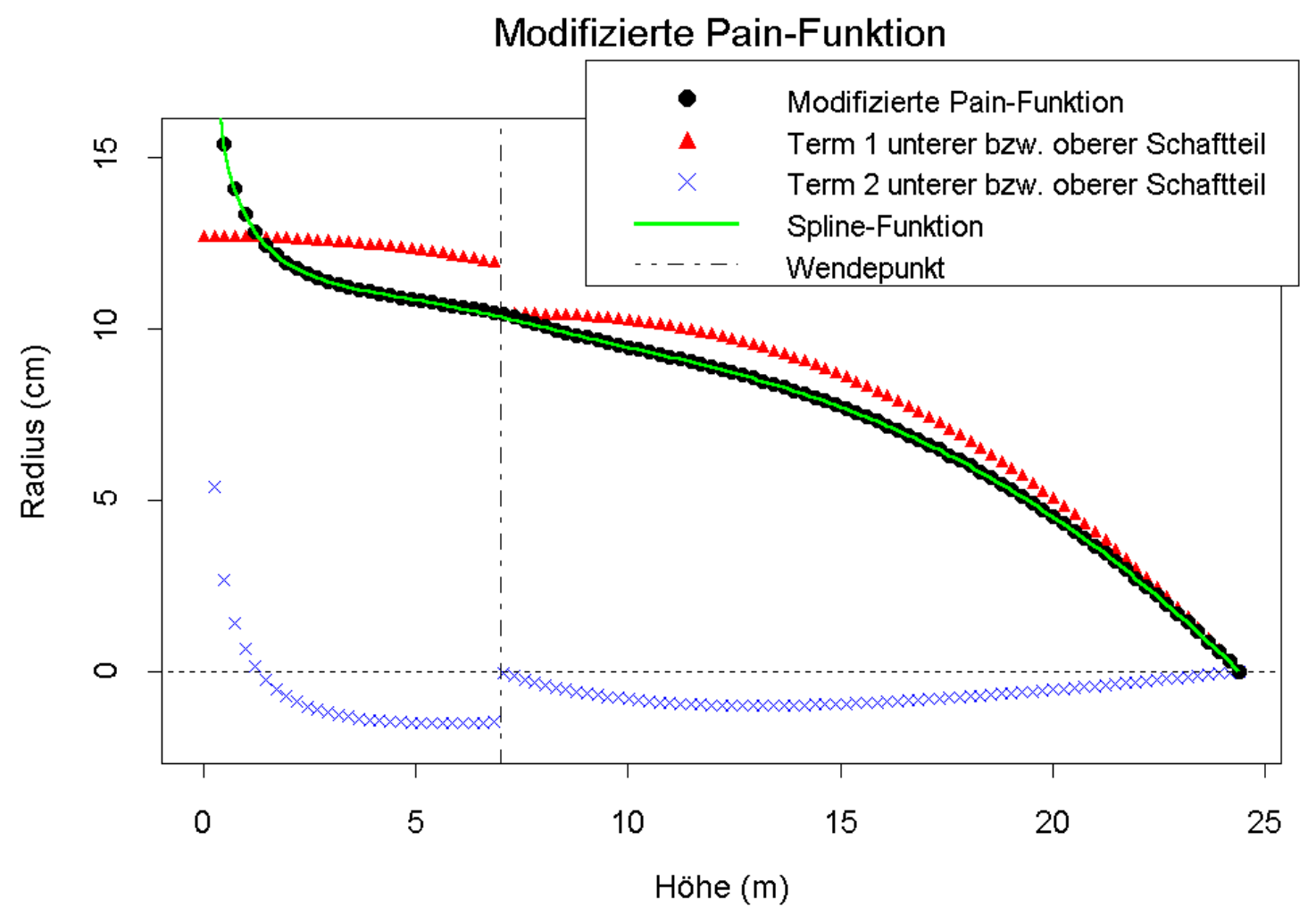

Abb. 74: Schaftform einer Fichte, beschrieben durch die modifizierte Pain-Funktion, mit dem BHD und der Höhe, die den Medianen der BHD- und der Höhenverteilung des untersuchten Datenmaterials entsprechen sowie Zerlegung in die Terme, aus denen sich die Gesamt-Funktion zusammensetzt. Zur Glättung des Übergangsbereiches von unterem und oberem Schaftteil wurde eine kubische Spline-Funktion verwendet.

Die Ergebnisse der Validierung der modifizierten Pain-Funktion sind in Abb. 69 bis Abb. 72 im Vergleich zu den bisher beschriebenen Modellen dargestellt. Bei den einzelbaumweisen Fehlerberechnungen (Abb. 69 und Abb. 70) bestehen nur geringfügige Vorteile gegenüber der Originalversion der Pain-Funktion. Es gibt jedoch für die Baumart Fichte keinen BHD- bzw. H/D-Wert-Bereich, in dem die modifizierte Version nicht einen Vorteil gegenüber der Originalversion aufweist.

Dieser Vorteil, der auch in Abb. 73 zu erkennen ist, wird bei der Berechnung des Fehlers und der Verzerrung in unterschiedlichen relativen Stammhöhen deutlicher (Abb. 71 und Abb. 72). So kann die Schätzung für den Erdstammbereich und den Bereich der Baumspitze durch die Beseitigung systematischer Abweichungen verbessert werden. Für den restlichen Höhenbereich weist die modifizierte Version keine Vorteile gegenüber der Originalversion auf. Bei der Interpretation der Erhöhung der Genauigkeit ist der bereits erläuterte Einfluß der Datenstruktur mit einem hohen Anteil an mittleren Schaftformen zu beachten. Wird als Vergleichsbasis das Modell ohne Schätzfehler für die form- und dimensionsbeschreibenden Parameter, d. h. die direkte Anpassung der Pain-Funktion an die Sektionsmessungen von Einzelbäumen verwendet, so sind die Vorteile der modifizierten Version im Erdstammbereich und für den Bereich der Baumspitze deutlich. Für die Baumarten Douglasie und Kiefer sind 
die Vorteile der modifizierten Version bei der Berechnung von einzelbaumweisen Schätzfehlern in den Randbereichen der BHD- und H/D-Wert-Verteilung teilweise deutlicher als bei der Baumart Fichte (Kapitel 9.9). Die Verbesserungen bei der Betrachtung von Schätzfehlern und Verzerrungen in unterschiedlichen relativen Stammhöhen sind dagegen für Douglasie und Kiefer geringer. Im Gegensatz zur Fichte weist die Originalversion für die Kiefer und Douglasie allerdings auch geringere Verzerrungen für den Erdstammbereich auf. Die Verbesserungen mit Hilfe der modifizierten Pain-Funktion für die Baumarten Buche und Eiche sind deutlich (Kapitel 9.9). Für die untersuchten Laubholzarten wurde aber keine Verbesserung durch die Anwendung der Originalversion der Pain-Funktion gegenüber der Brink-Funktion erreicht. Im Gegensatz dazu ist bei den Nadelholzarten der Vorteil der Pain-Funktion gegenüber der Brink-Funktion sehr viel größer als der Vorteil der modifizierten Version gegenüber der Originalversion der Pain-Funktion.

Insgesamt stellt die neu entwickelte modifizierte Pain-Funktion für alle untersuchten Baumarten das Modell mit der höchsten Schätzgenauigkeit dar. Die modifizierte Version hat gegenüber der Originalversion zusätzlich den Vorteil, daß der Meßwert des BHD nur geringfügig vom zugehörigen Funktionswert abweicht.

Abschließend wird in Abb. 75 anhand der Baumart Fichte der Einfluß der unabhängigen Variablen H/D-Wert sowie der Einzelbaumdimension (BHD und Höhe) auf die mit den unterschiedlichen Schaftformmodelle beschriebene Schaftform verdeutlicht. Das GleitendeDurchschnitts-Modell beschreibt die mittlere Schaftform der zur Parametrisierung verwendeten Bäume und kann daher als Vergleichsbasis dienen. Es fällt auf, daß die durch die PainFunktion und die modifizierte Pain-Funktion beschriebenen Schaftformen eine sehr viel gröBere Variation aufweisen als das Gleitende-Durchschnitts-Modell und die modifizierte BrinkFunktion. Die größten Abweichungen von der mittleren Schaftform treten an den Randbereichen der Verteilungen der unabhängigen Variablen und hier vor allem im Bereich niedriger H/D-Werte und großer Baumdimensionen auf. Die Abweichungen von der mittleren Schaftform in diesem Bereich sind biologisch plausibel, da Bäume mit niedrigen H/D-Werten und großen Dimensionen abholziger als die mittlere Schaftform beschrieben werden. Aber auch bei sehr hohen H/D-Werten prognostizieren die Pain-Funktion und die modifizierte PainFunktion vollholzigere Schaftformen als die mittlere Schaftform.

Die modifizierte Brink-Funktion weist eine relativ geringe Variabilität bei der Beschreibung des Schaftformenspektrums auf. Im Bereich mittlerer Werte der formbeeinflussenden Variablen H/D-Wert bzw. BHD und Höhe beschreiben alle Schaftformmodelle erwartungsgemäß ähnliche Schaftformverläufe. Die in Abb. 75 verdeutlichte höhere Flexibilität der PainFunktion und der modifizierten Pain-Funktion erklärt die höhere Schätzgenauigkeit an den Randbereichen des Formenspektrums und die niedrigeren Gesamtfehler bzw. Gesamtfehler in relativen Höhenstufen. In Abb. 75 sind bewußt auch Schaftformen von Bäumen beschrieben, 
die sich im Extrapolationsbereich (vgl. auch Abb. 63) der zur Parametrisierung verwendeten Datensätze befinden. An diesen Beispielbäumen wird deutlich, daß die Anwendung flexibler Schaftformmodelle auf den Parametrisierungsbereich beschränkt bleiben sollte, da insbesondere im Bereich sehr niedriger H/D-Werte eine unplausibel starke Abholzigkeit prognostiziert wird. Schaftformmodelle, die nicht oder nur geringfügig auf formbeeinflussende Variablen reagieren, sind im Extrapolationsbereich robuster. Da die modifizierte Pain-Funktion im Gegensatz zur Originalversion der Pain-Funktion näherungsweise durch den gemessenen BHD verläuft, ist sie im Extrapolationsbereich bedeutend stabiler. Probleme treten bei der modifizierten Pain-Funktion vor allem im Extrapolationsbereich beim Übergang vom unterem zum oberen Schaftformsegment auf. Dabei werden zu starke Steigungsunterschiede im Übergangsbereich beschrieben. Zur Korrektur kann bei geringfügig unplausiblen Verläufen der Übergangsbereich mit Hilfe von kubischen Splines beschrieben und damit geglättet werden (Abb. 74). Mittelfristiges Ziel muß es sein, die modifizierte Pain-Funktion anhand von Datensätzen, die ein breiteres Formenspektrum abdecken, zu parametrisieren. Die Originalversion der Pain-Funktion führt zumindest in Teilbereichen des Extrapolationsbereiches schneller zu unplausiblen Werten als die modifizierte Pain-Funktion. Da die Bestimmung des Wendepunktes (Grenze von oberem und unterem Schaftsegment) mit Hilfe der Originalversion der Pain-Funktion erfolgt, ist die Bestimmung im Extrapolationsbereich problematisch. Eine Verbesserung könnte durch eine iterative Bestimmung des optimalen gemeinsamen Funktionswertes bei der Parametrisierung und eine anschließende Modellierung des Punktes mit Hilfe von Einzelbaumvariablen erreicht werden. 
H/D-Wert: 50

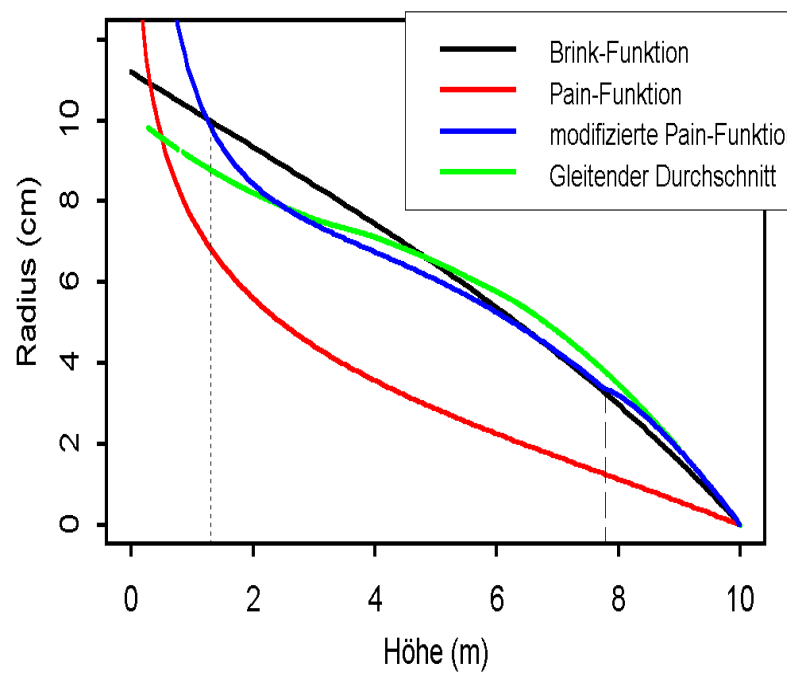

H/D-Wert: 50

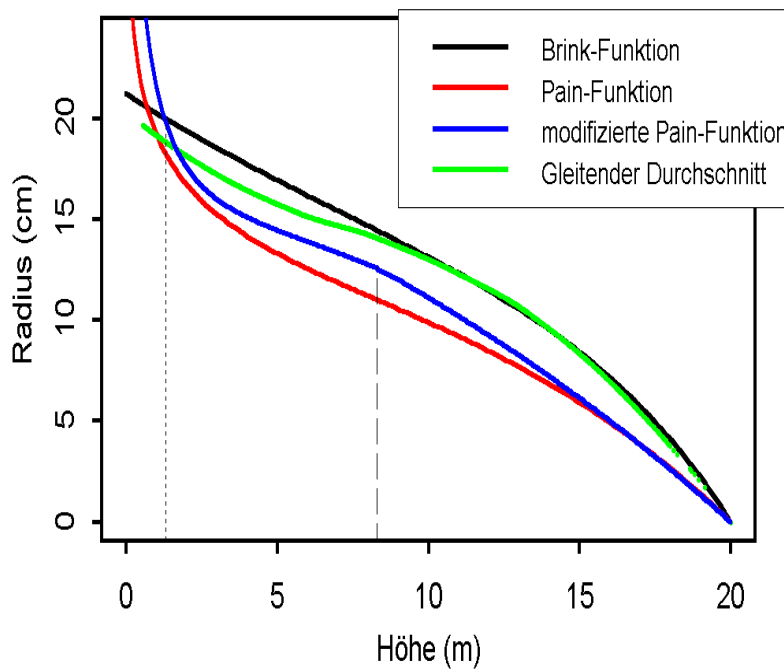

H/D-Wert: 100

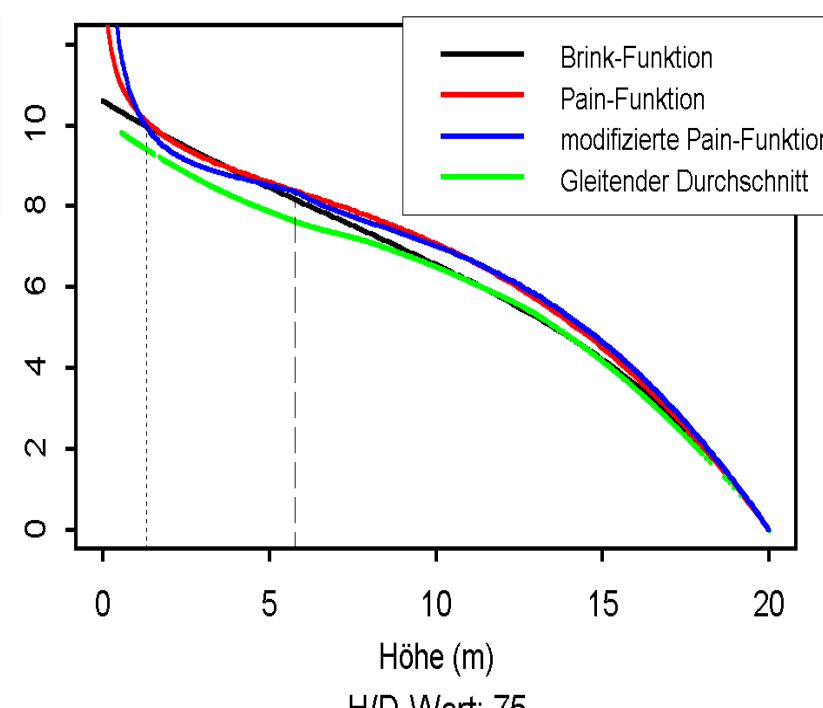

H/D-Wert: 75

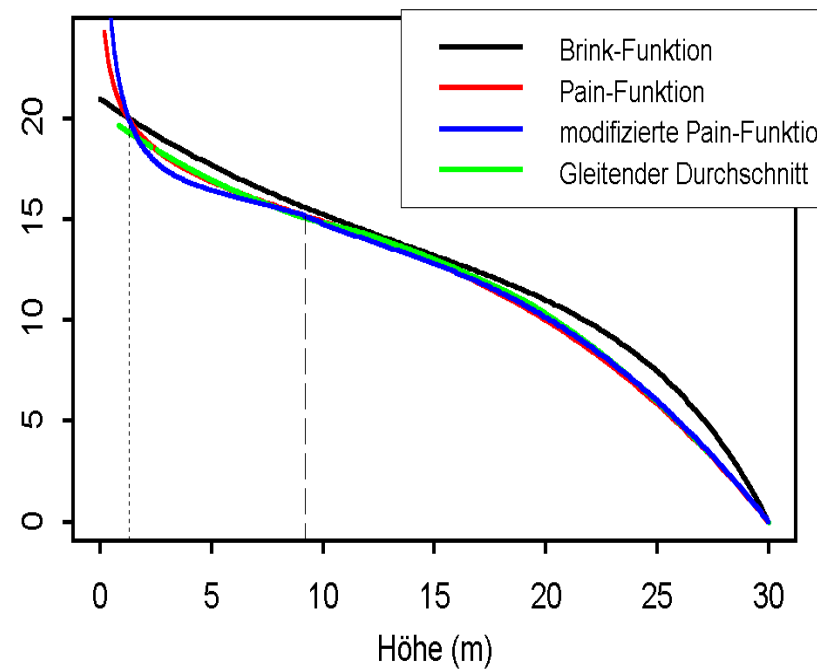

H/D-Wert: 150
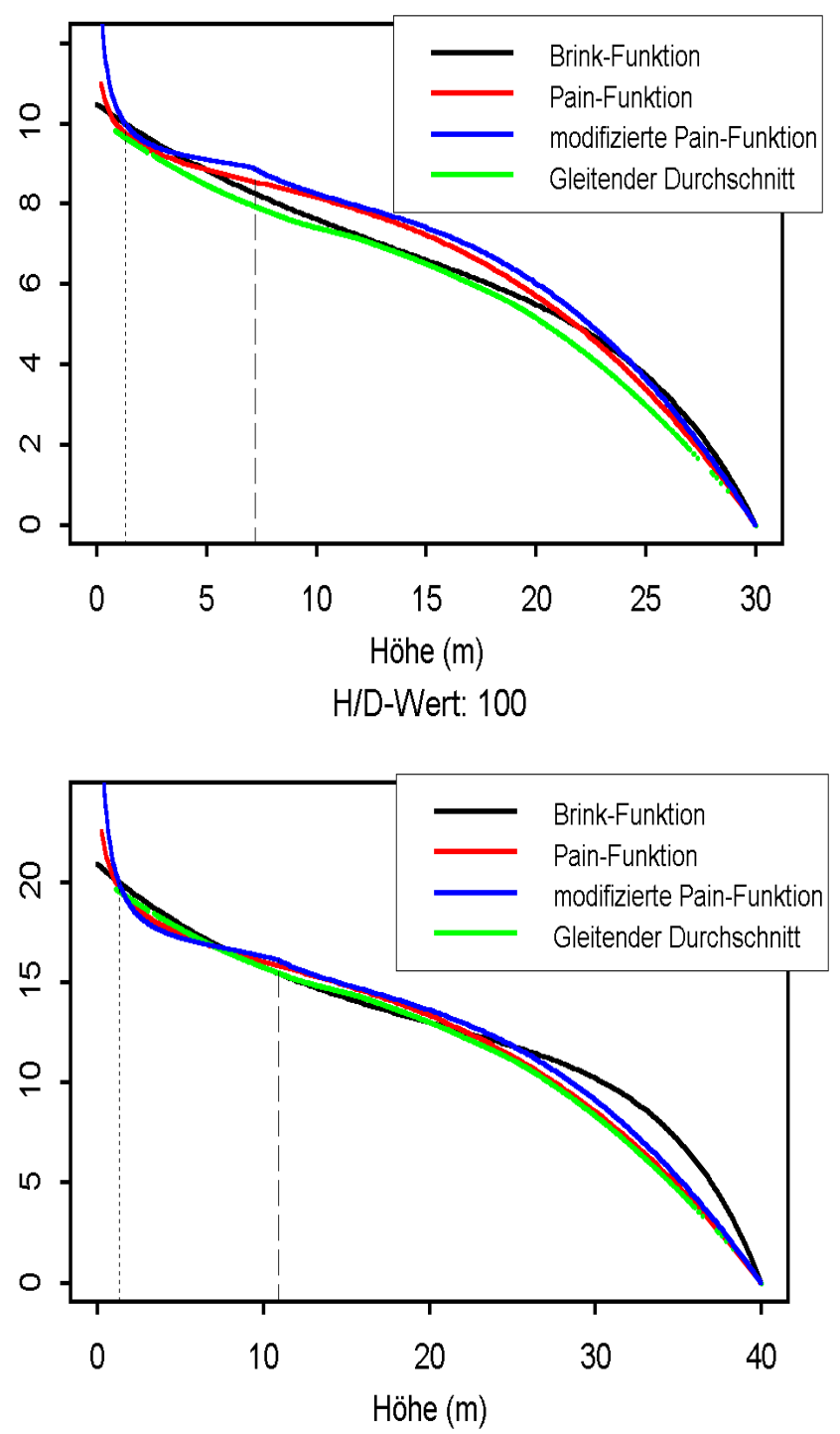
H/D-Wert: 42

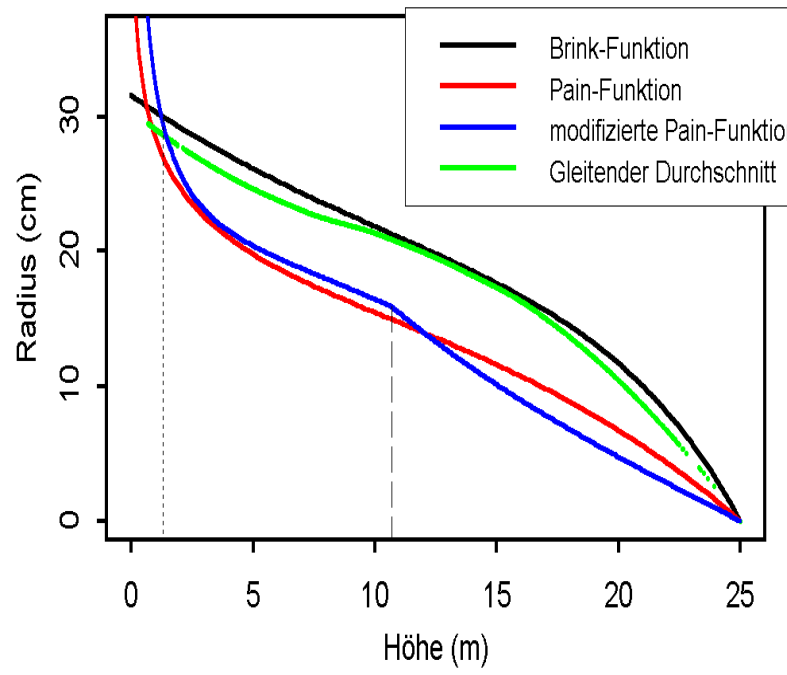

H/D-Wert: 50

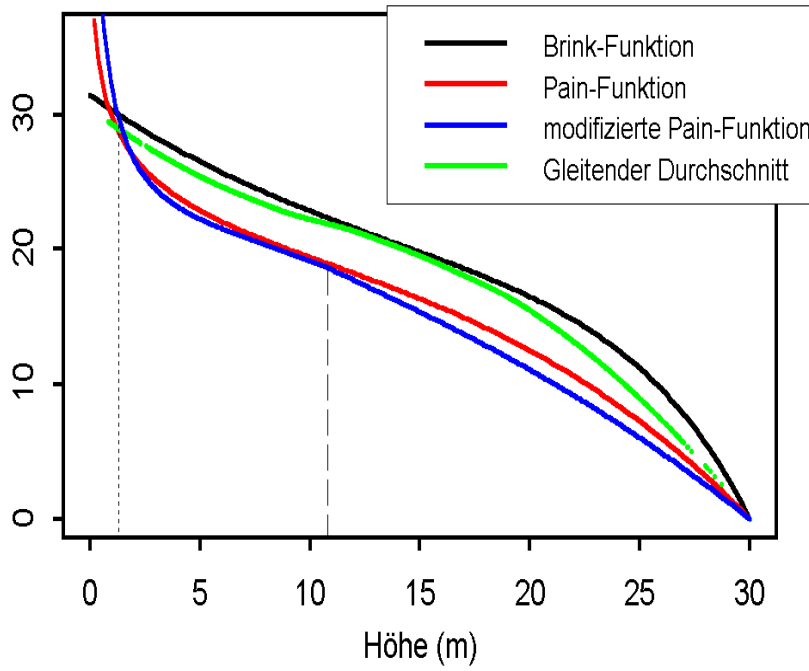

Abb. 75: Beschreibung der Schaftform verschiedener Bäume mit Hilfe der modifizierten BrinkFunktion (37), der Pain-Funktion (33), der modifizierten Pain-Funktion (38 a und b) sowie mit Hilfe eines Gleitenden-Durchschnitts-Modells (Kapitel 8.1). Mit kurz gestrichelter Linie ist die Höhe 1.3 m, mit lang gestrichelter Linie der Wendepunkt der Pain-Funktion bzw. der gemeinsame Funktionswert der Segmente der modifizierten Pain-Funktion gekennzeichnet. Das Gleitende-Durchschnitts-Modell beschreibt die mittlere Schaftform des zur Parametrisierung verwendeten Kollektivs.
H/D-Wert: 67
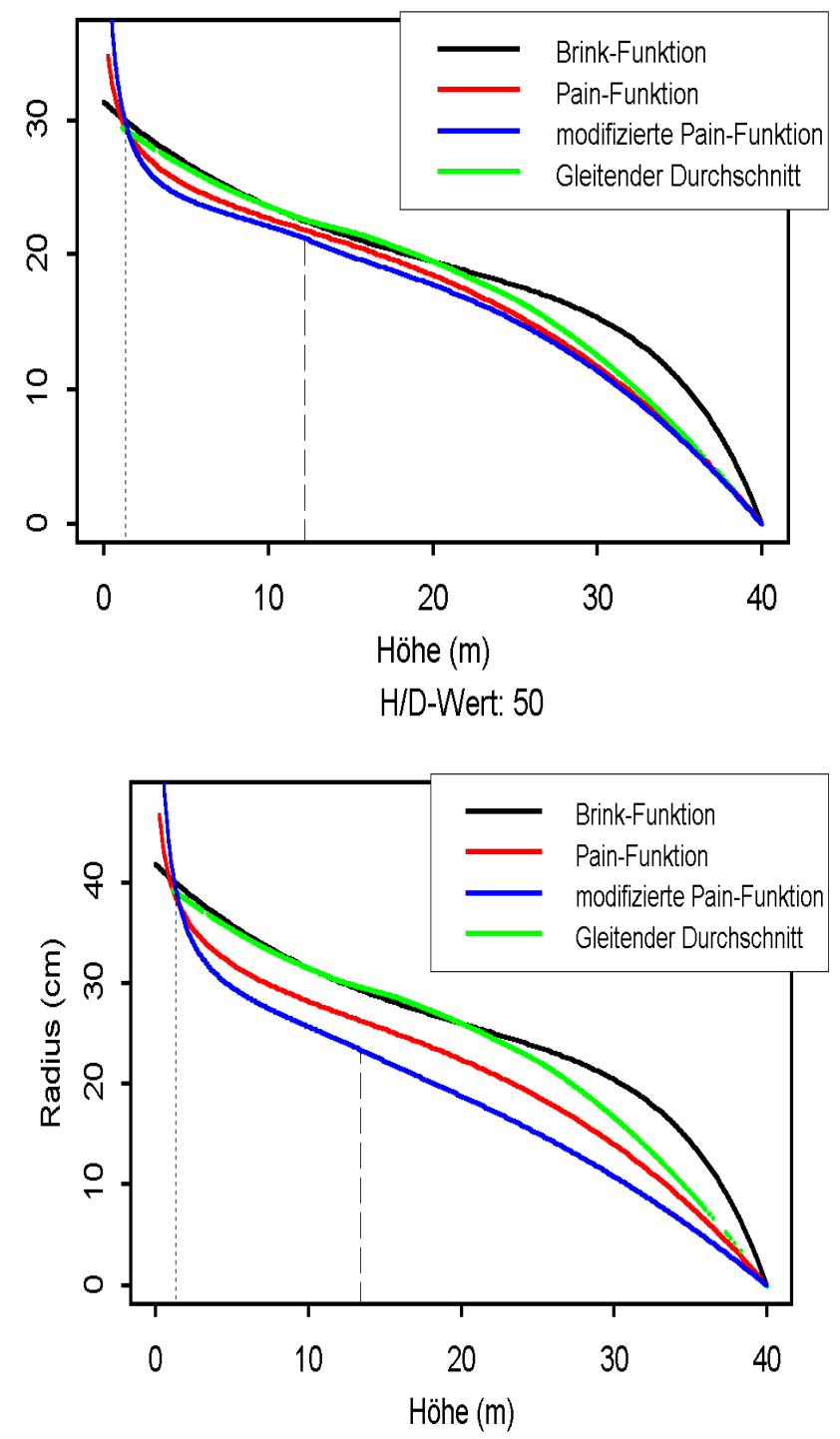


\subsubsection{Einfluß von Variablen höherer Hierarchiestufen}

Wie im Kapitel 3.7.1 beschrieben wurde, können den einzelnen Forstorten zum jetzigen Zeitpunkt keine Variablen höherer Hierarchiestufen (Bestandes- und Standortsvariablen) zugeordnet werden. Während die Zuordnung von Bestandesvariablen auch in Zukunft nicht möglich sein wird, da die Bäume nicht direkt aus Versuchsparzellen stammen, besteht für Standortsvariablen die Möglichkeit der Identifizierung und Zuordnung.

Auch wenn der mögliche Einfluß dieser Variablen hier nicht quantifiziert werden konnte, konnte jedoch der Einfluß des Forstortes als kategorische Variable auf die formbeschreibenden Parameter $\alpha_{r e l}$ und $\beta_{\text {rel }}$ der Pain-Funktion II untersucht werden. Die PainFunktion II wurde verwendet, um die Dimensionsunterschiede der Bäume aus verschiedenen Unterabteilungen aus dem Modell zu beseitigen. In Abb. 76 und Abb. 77 wird deutlich, daß zwischen den Unterabteilungen, aus denen die zur Parametrisierung verwendeten Stämme stammen, Unterschiede bei den Verteilungen der formbeschreibenden Parameter bestehen. Da die kategorische Variable Unterabteilung bzw. Forstort erklärende Variablen aller Hierarchiestufen aggregiert, muß jedoch geklärt werden, ob die in den Abb. 76 und Abb. 77 erkennbaren Formunterschiede nicht lediglich das Resultat des Einflusses der Einzelbaumvariablen BHD und Höhe bzw. H/D-Wert sind (Kapitel 3.7.2).

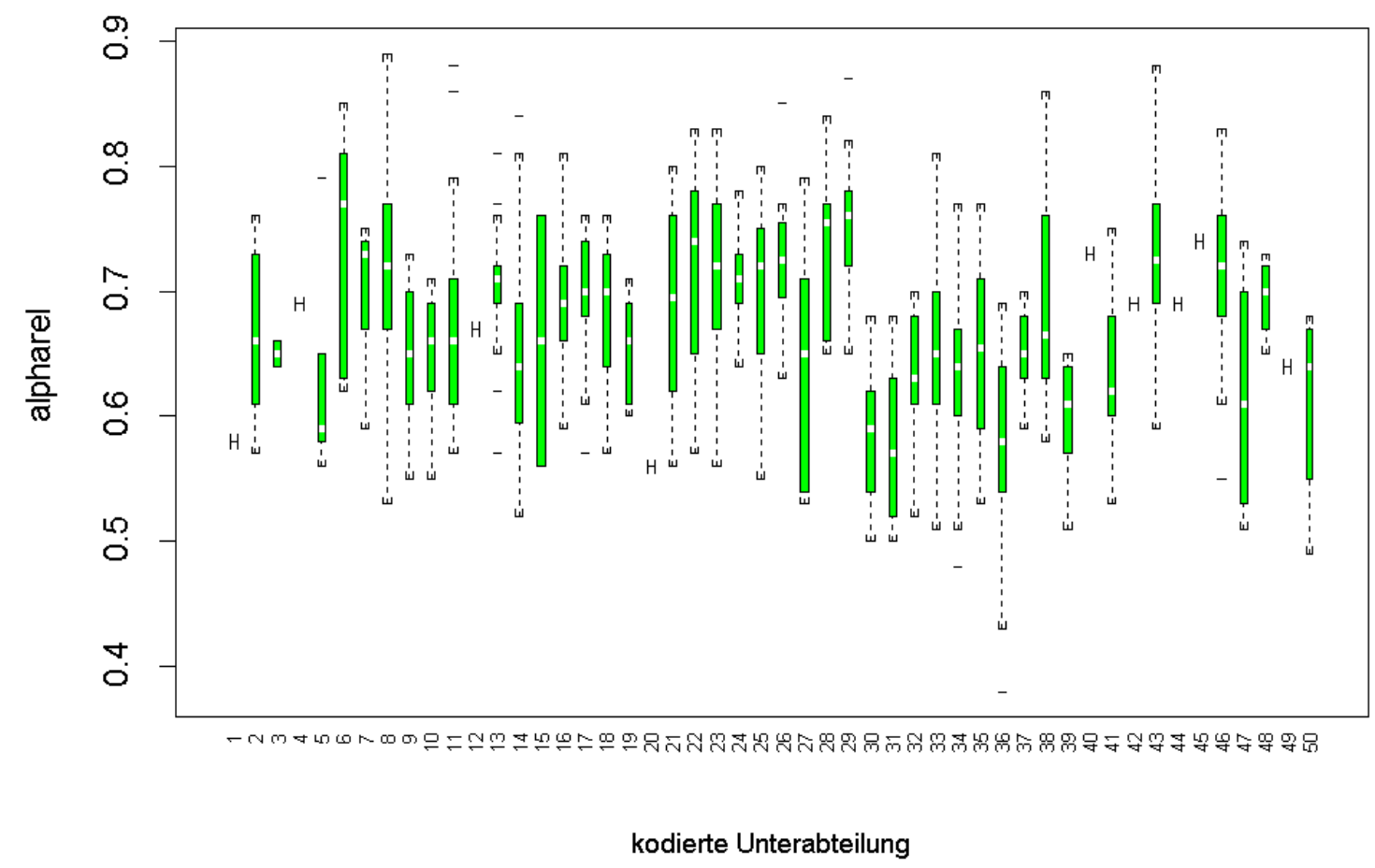

Abb. 76: Getrennt nach Unterabteilungen berechnete Boxplots des ausschließlich formbeschreibenden Parameters $\alpha_{\text {rel }}$ der Pain-Funktion II (36) für die Baumart Fichte. 


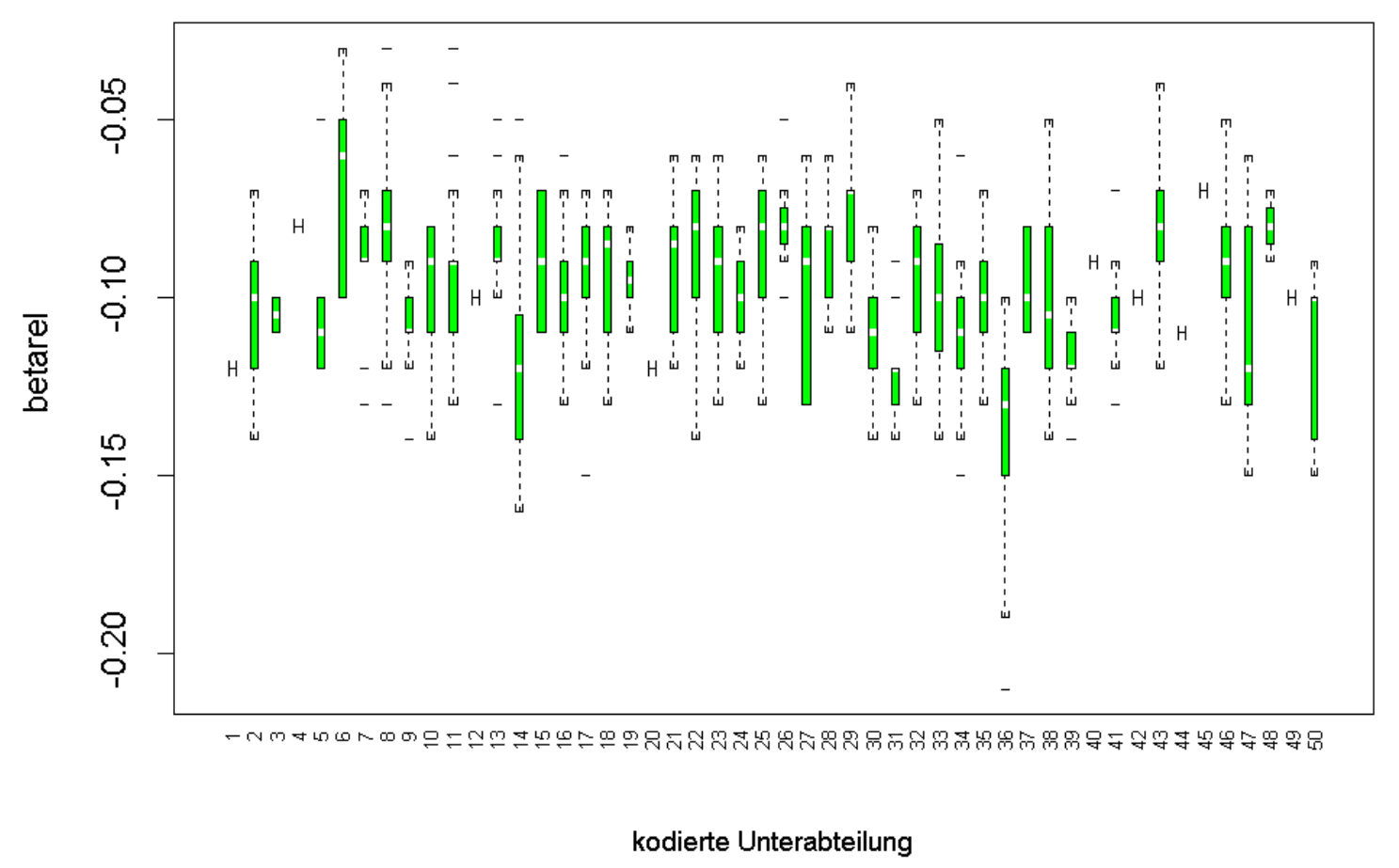

Abb. 77: Getrennt nach Unterabteilungen berechnete Boxplots des ausschließlich formbeschreibenden Parameters $\beta_{\text {rel }}$ der Pain-Funktion II (36) für die Baumart Fichte.

Um zu ermitteln, inwieweit der Forstort über die untersuchten Einzelbaumvariablen hinaus einen Einfluß auf die Stammform hat, wurden Modelle zur Schätzung der Parameter $\alpha_{\text {rel }}$ und $\beta_{\text {rel }}$ parametrisiert, die im Vergleich zu den Modellen (34) und (35) zusätzlich den Forstort als kategorische Variable enthalten. Jeder Forstort erhält einen individuellen Koeffizienten, der als Abweichung vom mittleren Intercept des Modells interpretiert werden kann (Formeln (39) u. (40) bzw. (41)). Dagegen werden in dieser Modellformulierung für die metrischen Variablen, die aus dem BHD und der Höhe eines Baumes berechnet werden, einheitliche Steigungen für alle Unterabteilungen geschätzt. In Formel (40) ist der Einfluß des Terms, der den Einfluß der Einzelbaumdimension auf die Stammform beschreibt, nicht signifikant. Im Gegensatz dazu ist der Koeffizient $b_{1}$ zur Schätzung des Formparameters $\beta_{\text {rel }}$ ohne Berücksichtigung des Forstortes noch signifikant (Tab. 31). Hierin kommt die bereits beschriebene aggregierende Eigenschaft der kategorischen Variablen Forstort zum Ausdruck, die auch die Einzelbaumdimension, speziell in mehr oder weniger einschichtigen Beständen, so stark berücksichtigt, daß die Einzelbaumvariablen BHD und Höhe keinen zusätzlichen signifikanten Einfluß auf die Schaftform haben. 
In Tab. 34 sind die Koeffizienten und statistischen Kennziffern zu den Schätzfunktionen (39) und (40) unter Berücksichtigung des Forstortes angegeben.

$$
\begin{aligned}
& \alpha_{r e l}=a_{0}+a_{1}\left(\frac{1}{\ln \left(H^{1 / D}\right)}\right)+a_{2}\left(\frac{1}{(H / D)^{2}}\right)+a_{3} \text { (Forstort) } \\
& \left.\beta_{\text {rel }}=b_{0}+b_{1}\left(\frac{1}{\ln \left(H^{1 / D}\right)}\right)+b_{2}\left(\frac{1}{(H / D)^{2}}\right)+b_{3} \text { (Forstort }\right),
\end{aligned}
$$

wobei:

$\alpha_{\text {rel }}, \beta_{\text {rel }}=$ formbeschreibende Parameter aus Formel (33);

$H \quad=$ Baumhöhe (m);

$D \quad=$ Brusthöhendurchmesser $(\mathrm{cm})$;

Forstort $=$ Unterabteilung, aus der der durch die Formparameter beschriebene Baum stammt;

$a_{0}, a_{1}, a_{2}, a_{3}, b_{0}, b_{1}, b_{2}, b_{3}=$ Parameter.

die mathematisch korrekte Formulierung für den allgemeinen Fall ist in Formel (41) dargestellt:

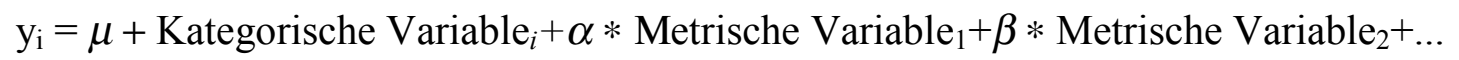

Werden nicht nur die Interzepte, sondern auch die Steigungen der metrischen Variablen bestandesindividuell geschätzt, so lautet die allgemeine Form des zugehörigen Modells:

$\mathrm{y}_{\mathrm{i}}=\mu+$ Kategorische Variable $_{i}+\alpha{ }_{\mathrm{i}} *$ Metrische Variable $_{1}+\beta_{\mathrm{i}} *{\text { Metrische } \text { Variable }_{2}+\ldots}$

wobei:

$i=1 \ldots k=$ Anzahl der untersuchten Bestände.

mit:

\begin{tabular}{|r|r|}
\hline $\begin{array}{c}\text { Schätzung des Parameters } \alpha_{\text {rel }} \text { mit Hilfe von } \\
\text { Formel (39) bzw. (41) }\end{array}$ & $\begin{array}{c}\text { Schätzung des Parameters } \beta_{\text {rel }} \text { mit Hilfe von } \\
\text { Formel (40) bzw. (41) }\end{array}$ \\
\hline $\mathrm{a}_{0}=0.835$ & $\mathrm{~b}_{0}=-0.0617$ \\
\hline $\mathrm{a}_{1}=-0.0124$ & $\mathrm{~b}_{1}$ nicht signifikant \\
\hline $\mathrm{a}_{2}=-0.0572$ & $\mathrm{~b}_{2}=-0.0311$ \\
\hline $\mathrm{a}_{3}=$ variabel in Abhängigkeit vom Forstort & $\mathrm{b}_{3}=$ variabel in Abhängigkeit vom Forstort \\
\hline Multiples Bestimmtheitsmaß: 0.472 & Multiples Bestimmtheitsmaß: 0.470 \\
\hline Adjust. Multipl. Bestimmtheitsma: 0.438 & Adjust. Multipl. Bestimmtheitsmaß: 0.436 \\
\hline $\begin{array}{c}\text { Multiples Bestimmtheitsmaß bei der Schätzung } \\
\text { von } \alpha_{\text {reI }} \text { mit Formel (34): } 0.2772\end{array}$ & $\begin{array}{c}\text { Multiples Bestimmtheitsmaß bei der Schätzung } \\
\text { von } \beta_{\text {rel }} \text { mit Formel (35): 0.3032 }\end{array}$ \\
\hline
\end{tabular}

Tab. 34: Funktionsparameter und Bestimmtheitsmaße für die zur Schätzung der ausschließlich formbeschreibenden Parameter $\alpha_{\text {rel }}$ und $\beta_{\text {rel }}$ der Pain-Funktion II verwendeten Modelle (39) und (40) bzw. (41). 


\begin{tabular}{|c|c|}
\hline $\begin{array}{c}\text { Schätzung des Parameters } \alpha_{\text {rel }} \text { mit Hilfe von } \\
\text { Formel (42) }\end{array}$ & $\begin{array}{c}\text { Schätzung des Parameters } \beta_{\text {rel }} \text { mit Hilfe von } \\
\text { Formel (42) }\end{array}$ \\
\hline Multiples Bestimmtheitsmaß: 0.567 & Multiples Bestimmtheitsmaß: 0.566 \\
\hline Adjust. Multipl. Bestimmtheitsmaß: 0.487 & Adjust. Multipl. Bestimmtheitsmaß: 0.485 \\
\hline
\end{tabular}

Tab. 35: Funktionsparameter und Bestimmtheitsmaße für die zur Schätzung der ausschließlich formbeschreibenden Parameter $\alpha_{\text {rel }}$ und $\beta_{\text {rel }}$ der Pain-Funktion II verwendeten Modelle (42) für die Baumart Fichte.

Anhand des Anstiegs der multiplen Bestimmtheitsmaße auf die adjustierten Bestimmtheitsmaße ( $\alpha_{\text {rel }}: 0.277$ auf $0.438 ; \beta_{\text {rel }}: 0.303$ auf 0.436$)$ zeigt sich, daß die Variable Forstort, über die Verwendung der Einzelbaumvariablen BHD, Höhe und H/D-Wert hinaus, die Schaftformvariabilität beeinflußt. Das adjustierte Bestimmtheitsmaß wurde zur Berücksichtigung der höheren Modellunsicherheit verwendet, die aus der Integration einer hohen Anzahl von Ausprägungen einer kategorischen Variablen resultiert. Werden nicht nur die Interzepte (Formel (41)), sondern auch die Koeffizienten der metrischen Variablen bestandesindividuell geschätzt (Formel (42)), erhöhen sich die adjustierten Bestimmtheitsmaße auf 0.487 für $\alpha_{\text {rel }}$ bzw. auf 0.485 für $\beta_{\text {rel. }}$.

Damit erscheint eine Indentifizierung von Variablen höherer Hierarchiestufen wie Standortsvariablen als ein vielversprechender Ansatz, um sowohl das Modell der PainFunktion (33) als auch die modifizierte Form (38 a und b) in Richtung eines "echten" Einheitsschaftformmodells weiterzuentwickeln. Die Quantifizierung des Einflusses der kategorischen Variablen Forstort ist beispielhaft nur für die Baumart Fichte dargestellt. Für die Baumart Kiefer liegt die Verknüpfung der Sektionsmessungen mit dem Forstort bereits vor, während sie für die Baumarten Buche, Eiche und Douglasie noch nicht existiert, aber prinzipiell möglich ist. 


\section{Diskussion und Ausblick}

Im Rahmen dieses Kapitels soll das entwickelte Modellsystem zur Prognose ausgewählter Gütemerkmale gesamtheitlich bewertet werden. Aufgrund der hohen Anzahl an Modellen, die selbst zur Beschreibung von Teilaspekten der Holzqualität notwendig ist, erfolgte eine detaillierte Einzelbewertung bereits in Kombination mit der Modellentwicklung (Kapitel 3). Zusätzlich zur Gesamtbewertung wird auf Modellbereiche innerhalb des Wachstumssimulators Bwinpro (NAGEL ET AL., 2001) hingewiesen, die indirekt von der Entwicklung der Qualitätsmodelle profitieren bzw. in denen Erkenntnisse aus der Qualitätsmodellierung zur realitätsnäheren Beschreibung und Fortschreibung von Waldbeständen genutzt werden können. Abschließend werden Teilaspekte der bearbeiteten Gütemerkmale genannt, für die weiterer Forschungsbedarf besteht.

Vorrangiges Ziel der Arbeit war es, Modelle für ausgewählte Gütemerkmale auf einer breiten Datenbasis zu parametrisieren. Dieses Ziel wurde für die Mehrzahl der entwickelten Qualitätsmodelle erreicht, deren Geltungsbereich ganz Nordwestdeutschland und eine Vielzahl unterschiedlicher Bestandesbehandlungen umfaßt, wobei jedoch Extremvarianten selten sind. Die Kriterien, die die Auswahl der Gütemerkmale bestimmten, sind in Kapitel 1.2 beschrieben. Das Ziel der Entwicklung neuer Modelltypen bestand darin, im Vergleich zu bestehenden Modellansätzen die Treffgenauigkeit (Abweichungen zwischen Wirklichkeit und Prognose) unter Berücksichtigung von systematischen (Bias) und zufälligen (Präzision) Fehlerkomponenten zu erhöhen. Eine weitere Intention, die indirekt auch zu einer Erhöhung der Modellgenauigkeit führen kann, war die Entwicklung von behandlungssensitiven Modellen. Eine Behandlungssensitivität der Modellkomponenten ist die Voraussetzung für den Ver-

gleich von waldbaulichen Szenarien mit Hilfe von Wachstumssimulationen, wobei es vor allem auf eine möglichst genaue Prognose der Relationen zwischen den Behandlungsvarianten ankommt.

Ein allgemeines Problem der statischen Modelle besteht darin, plausible Zeitreihenwerte zu gewährleisten. Deshalb wurden biologisch plausible und eindeutige Wirkungsrichtungen der unabhängigen Variablen in den statischen Modellen angestrebt, die die Anwendung der Modelle im Rahmen von dynamischen Simulationen erleichtern. Entsprechend der im Rahmen dieser Arbeit verwendeten Gliederung erfolgt die Diskussion getrennt für die Kronenansatz-, Astparameter- und Schaftformmodelle.

\subsection{Kronenansatzmodelle}

\subsubsection{Hierarchisches Modell und einfaches statisches Modell}

Alle neu entwickelten Kronenansatzmodelle weisen für die untersuchten Nadelholzarten gegenüber den statischen Vergleichsmodellen (Kronenansatzmodelle in: Bwin (NAGEL, 1999 a); 
Silva (Pretzsch U. KAHN, 1998); Forest (modifiziert) (Hasenauer, 1994); CEP (COLIN U. HOULLIER, 1992)) eine höhere Treffgenauigkeit auf. Das hierarchische (Kapitel 3.2.1) und das einfache statische Kronenansatzmodell (Kapitel 3.2.2) stellen dabei etwas niedrigere bzw. die gleichen Anforderungen an die Qualität der Eingangsinformationen wie die Vergleichsmodelle (vgl. auch Kapitel 3.2.5). Das hierarchische Modell ist durch seinen zweistufigen Aufbau gekennzeichnet, wobei ein bestandesindividuelles Einzelbaummodell mit Hilfe von Bestandesvariablen verallgemeinert wird. Das einfache statische Modell orientiert sich konzeptionell an den Modellen von NAGEL (1999 a) sowie PRETZSCH und KAHN (1998).

Die Erhöhung der Schätzgenauigkeit wird durch die Berücksichtigung der Spitzenhöhe erreicht, über die der zu beobachtende bestandesübergreifende Dimensionstrend des Kronenverhältnisses erfaßt wird. Die Integration der Spitzenhöhe erhöht die Treffgenauigkeit vor allem durch eine Reduktion der in den Vergleichsmodellen mehr oder weniger stark auftretenden systematischen Unterschätzung der Kronenverhältnisse niedriger Bäume. Der bestandesübergreifende Dimensionstrend einer Abnahme des Kronenverhältnisses mit steigender Spitzenhöhe tritt in den untersuchten Buchen- und Eichenbeständen im Gegensatz zu den Nadelholzarten nicht bzw. nur sehr schwach auf. Infolgedessen weisen das hierarchische (9) (12) (Kapitel 3.2.1) und das einfache statische Kronenansatzmodell (13) (Kapitel 3.2.2) für die Baumart Buche keine bzw. für die Eiche nur geringfügige Verbesserungen auf.

Im Gegensatz zu den Vergleichsmodellen haben die Koeffizienten der Einzelbaumvariablen in den neu entwickelten Modellen ausschließlich die Wirkungsrichtungen (Vorzeichen), die bei einer bestandesweisen Parametrisierung zu beobachten sind. Durch die zusätzliche Verwendung der Spitzenhöhe dient der BHD nicht mehr der Beschreibung des bestandesübergreifenden Dimensionstrends eines abnehmenden Kronenverhältnisses mit fortschreitendem Alter bzw. Höhe eines Bestandes (s. dazu KrAMER, 1988, S. 21, Abb. 7), sondern erfaßt den positiven Zusammenhang zum Kronenverhältnis innerhalb von Beständen (Kapitel 3.2.1 u. 3.2.2). Da der Einzelbaum-H/D-Wert sowohl innerhalb von Beständen als auch bestandesübergreifend einen negativen Einfluß auf das Kronenverhältnis hat, ist auch die Wirkungsrichtung dieser Variablen innerhalb der neu entwickelten Modelle biologisch plausibel.

Im Gegensatz zur allgemeinen Erhöhung der Modellgenauigkeit sind die neuen Modelle, wie auch die Vergleichsmodelle, nur wenig sensitiv gegenüber verschiedenen Bestandesbehandlungen. Das einfache statische Kronenansatzmodell (13) und verschiedene Vergleichsmodelle verwenden mit dem H/D-Wert eine unabhängige Variable, die - sensitive Zuwachsfunktionen für den Durchmesser und die Höhe vorausgesetzt - eine Behandlungssensitivität gewährleisten kann. Es kann jedoch gezeigt werden, daß der H/D-Wert die Höhe des Kronenansatzes nur wenig beeinflußt, wenn der Dimensionstrend über die Variablen BHD und Spitzenhöhe umfassend beschrieben wird. Dies zeigt die nur geringfügige Verbesserung des einfachen statischen Kronenansatzmodells (13) bei Aufnahme des H/D-Wertes in die Mo- 
dellgleichung. In den Vergleichsmodellen ist der Einfluß des H/D-Wertes deutlicher, was aber in der nicht ausreichenden Berücksichtigung des Dimensionstrends in diesen Modellen begründet ist. Eine Behandlungssensitivität der Kronenansatzmodelle setzt die Identifikation signifikanter Zusammenhänge zu konkurrenzbeschreibenden Variablen bzw. zu Variablen voraus, die durch die Konkurrenzbedingungen des Einzelbaumes beeinflußt werden. Zusätzliche Untersuchungen haben jedoch gezeigt, daß die Variablen H/D-Wert und "Summe der Kronenschirmflächen stärkerer Bäume als der Bezugsbaum“ innerhalb von bestandesindividuell parametrisierten Modellen (9) zu keiner Erhöhung der Schätzgenauigkeit führten (Kapitel 3.2.1). Als mögliche Ursache für die fehlenden Zusammenhänge kommen mehrere Faktoren in Frage:

- Konkurrenzindizes beeinflussen eher eine Veränderungsrate als einen Zustand, der das Resultat einer längeren Wuchsperiode ist. Konkurrenzindizes lassen sich somit aufgrund ihrer zeitlich begrenzten Aktualität besser in dynamischen Modellen berücksichtigen (HASENAUER, 1994; s. auch Kapitel 2.3 u. 3.2.4). Versuche, ein dynamisches Modell auf Einzelbaumebene zu entwickeln, führten jedoch zu keinen Verbesserungen. Zudem beinhaltet der H/D-Wert im Gegensatz zu Konkurrenzindizes Informationen über die Wuchsbedingungen einer längeren Wachstumsperiode, so daß seine Verwendung auch in statischen Modellen möglich ist und eine Behandlungssensitivität gewährleisten könnte.

- Als weiterer Grund ist die problematische, mit einem großen Fehler behaftete Messung des Kronenansatzes zu nennen, die insbesondere die Berechnung von Veränderungsraten erschwert.

- Die aktuelle Datenlage mit Informationen überwiegend aus gleichaltrigen Rein- und Mischbeständen führt dazu, daß der H/D-Wert für einzelne Bestände zusätzlich zu den dimensionsbeschreibenden Variablen keinen Einfluß auf das Kronenverhältnis hat (vgl. Kapitel 3.2.1, Abb. 11 u. Abb. 16). Das einfache statische Kronenansatzmodell ermöglicht im Gegensatz zum hierarchischen Modell auch die Überprüfung, inwieweit der Einzelbaum-H/D-Wert das Kronenverhältnis zusätzlich zu den Dimensionsvariablen bestandesübergreifend beeinflußt. Da das einfache statische Kronenansatzmodell keine Verbesserung gegenüber dem hierarchischen Modell aufweist (Kapitel 3.2.2), muß vermutet werden, daß Bäume mit gleichen Dimensionen, die aus Beständen mit gleicher Spitzenhöhe stammen, innerhalb des verwendeten Datenmaterials keine starke Variation in ihren Kronenverhältnissen aufweisen.

Die vorangegangenen Überlegungen setzen voraus, daß der H/D-Wert grundsätzlich einen Einfluß auf das Kronenverhältnis hat, wenn die Bäume unter deutlich unterschiedlichen Wuchsbedingungen erwachsen sind. Die ähnlichen Kronenverhältnisse der Bäume mit ähnlichen Dimensionen aus Beständen mit ähnlicher Spitzenhöhe innerhalb der Datenstrukturen 
haben jedoch zur Folge, daß die Berücksichtigung des H/D-Wertes zum jetzigen Zeitpunkt noch zu keinen Verbesserungen bei der Schätzung der Kronenansatzhöhe führt (Tab. 2 u. Tab. 36). In strukturreichen Wäldern ist mit einer stärkeren Variation der Wuchskonstellationen innerhalb von Beständen zu rechnen (vgl. auch Kapitel 3.2.1). Es kann daher vermutet werden, daß der H/D-Wert in Zukunft einen größeren Einfluß auf die Kronenansatzhöhe haben wird.

Kommen in Zukunft vermehrt Bäume mit ähnlichen Dimensionen aber unterschiedlichen Kronenverhältnissen bzw. H/D-Werten innerhalb von Beständen vor, kann das bestandesindividuelle Modell (9) des hierarchischen Modellansatzes (Kapitel 3.2.1) um die Variable H/D-Wert ergänzt werden. Sind Bäume mit den oben genannten Eigenschaften innerhalb der Datenstrukturen nur in verschiedenen Beständen oder sowohl getrennt als auch gemischt in Beständen vorhanden, bietet sich das einfache statische Kronenansatzmodell als geeignetes Modell an.

Bei der zuletzt skizzierten Datenlage wäre der Einzelbaum-H/D-Wert innerhalb von Beständen teilweise nicht signifikant, so daß seine Integration im hierarchischen Modell problematisch erscheint. Gegenüber den jetzigen Validierungsergebnissen kann bei der Verwendung des einfachen statischen Kronenansatzmodells in strukturreichen Beständen mit einer deutlichen Verbesserung der Schätzung durch die Verwendung des H/D-Wertes gerechnet werden.

Zur Überprüfung dieser Hypothese ist eine spezielle Unterstichprobe aus den gesamten zur Verfügung stehenden Fichtendaten erzeugt worden, die in Abb. 78 dargestellt ist.

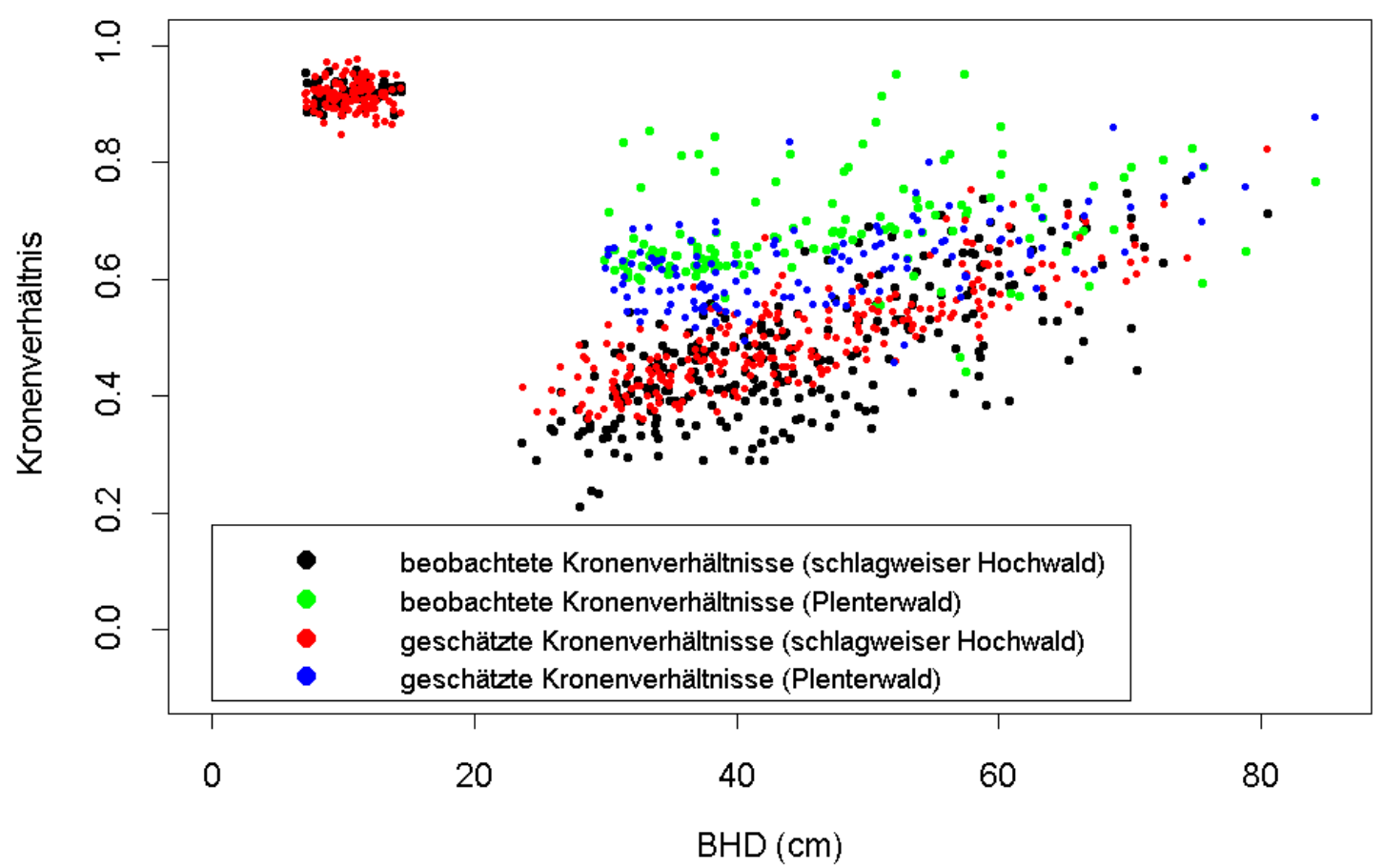

Abb. 78: Parametrisierung des einfachen statischen Kronenansatzmodells an einer heterogenen Unterstichprobe der Gesamtdaten für die Baumart Fichte mit unter sehr unterschiedlichen Bedingungen erwachsenen Bäumen der Oberschicht. 
Die Unterstichprobe zeichnet sich dadurch aus, daß der prozentuale Anteil an Bäumen der Oberschicht, die unter solitärähnlichen Bedingungen erwachsen sind, sehr viel höher ist, als innerhalb der Gesamtdaten. Die solitärähnlichen Bäume mit niedrigen H/D-Werten und hohen Kronenverhältnissen stammen aus der Plenterwaldversuchsfläche der Niedersächsischen Forstlichen Versuchsanstalt im Nationalpark Harz (NIEDERSÄCHSISCHE ForstLiche Versuchsanstalt, 1994; SpellmanN, 1995). Gleichzeitig sind auch Kronenverhältnisse von Oberschichtbäumen aus einem einschichtigen Bestand in der Unterstichprobe enthalten. Weitere Datensätze stammen aus einer gleichförmigen Dickung mit den für sie typischen hohen Kronenverhältnissen nahe dem Wert eins.

Die Unterstichprobe dient der Veranschaulichung der in Zukunft zu erwartenden Datenlage. In jedem Fall haben extremere Behandlungsvarianten in der Unterstichprobe einen größeren Einfluß bei der Parametrisierung des einfachen statischen Kronenansatzmodells. Zur weiteren Ausdehnung des Geltungsbereiches wäre die Berücksichtigung von Oberschichtbäumen aus schwach oder nicht durchforsteten Beständen sinnvoll. Doch bereits mit der erzeugten Unterstichprobe kann verdeutlicht werden, daß der H/D-Wert im Gegensatz zu den bisherigen Erkenntnissen zur behandlungssensitiven Schätzung der Kronenansatzhöhe benutzt werden kann. Bei der Verwendung aller Fichtendaten wird durch die Berücksichtigung des H/D-Wertes fast keine Erhöhung der Modellgenauigkeit und damit auch keine Behandlungssensitivität erreicht (Tab. 36). Dagegen wird für die Unterstichprobe durch die Berücksichtigung des H/D-Wertes erstens der Fehler um $40 \mathrm{~cm}$ reduziert bzw. zweitens eine Behandlungssensitivität gewährleistet.

Wird der H/D-Wert durch die Einzelbaumhöhe ersetzt, so kann der Fehler nochmals um $10 \mathrm{~cm}$ gesenkt werden. Auch die Kombination der unabhängigen Variablen BHD und Einzelbaumhöhe bei gleichzeitiger Beschreibung des bestandesübergreifenden Dimensionstrends mit Hilfe der Spitzenhöhe gewährleistet eine behandlungssensitive Schätzung. Die in Abb. 78 dargestellte Schätzung der Kronenverhältnisse erfolgte mit dem einfachen statischen Kronenansatzmodell, wobei anstelle des H/D-Wertes die Einzelbaumhöhe verwendet wurde. Der Einfluß des H/D-Wertes bzw. der Einzelbaumhöhe bei Bäumen mit gleichem BHD und gleicher zugehöriger Spitzenhöhe ist deutlich zu erkennen und biologisch plausibel, auch wenn die Auswirkungen der Bestandesbehandlung unterschätzt werden (Abb. 78). Die Schätzungen für die Kronenverhältnisse im Plenterwald (blau) und im schlagweisen Hochwald (rot) sind klar voneinander getrennt und geben tendenziell die in den Meßwerten zu beobachtenden Unterschiede (Plenterwald: grün, Altersklassenwald: schwarz) wieder. Es fällt weiterhin auf, daß die Unterschiede zwischen Bäumen mit unterschiedlichen Dimensionen (BHD) innerhalb von Beständen mit sinkendem mittleren HD-Wert abnehmen (vgl. auch Abb. 22). 


\begin{tabular}{|l|r|c|r|r|r|}
\hline Datenbasis & \multicolumn{2}{|c|}{ Gesamtdatenmaterial der Fichte } & \multicolumn{2}{|c|}{$\begin{array}{c}\text { Künstlicher Bestand mit stark unterschiedlich er- } \\
\text { wachsenen Bäumen in der Oberschicht }\end{array}$} \\
\hline Modelltyp & $\begin{array}{c}\text { einfaches stati- } \\
\text { sches Kronen- } \\
\text { ansatzmodell } \\
\text { (mit H/D-Wert) }\end{array}$ & $\begin{array}{c}\text { einfaches stati- } \\
\text { sches Kronen- } \\
\text { ansatzmodell } \\
\text { ohne Verwen- } \\
\text { dung des H/D- } \\
\text { Wertes }\end{array}$ & $\begin{array}{c}\text { einfaches stati- } \\
\text { sches Kronen- } \\
\text { ansatzmodell } \\
\text { (mit H/D-Wert) }\end{array}$ & $\begin{array}{c}\text { einfaches stati- } \\
\text { sches Kronen- } \\
\text { ansatzmodell } \\
\text { ohne Verwen- } \\
\text { dung des H/D- } \\
\text { Wertes }\end{array}$ & $\begin{array}{c}\text { einfaches stati- } \\
\text { sches Kronen- } \\
\text { ansatzmodell } \\
\text { mit der Einzel- } \\
\text { baumhöhe an- } \\
\text { stelle des H/D- } \\
\text { Wertes }\end{array}$ \\
\hline RMSE (m) & 1.817 & 1.837 & 2.416 & 2.867 & 2.339 \\
\hline
\end{tabular}

Tab. 36: Vergleich der Auswirkungen einer Berücksichtigung des H/D-Wertes (der Einzelbaumhöhe) im neu entwickelten, einfachen statischen Kronenansatzmodell bei Anwendung auf die gesamte Datenbasis und bei Anwendung auf eine Unterstichprobe mit unter sehr unterschiedlichen Bedingungen erwachsenen Bäumen der Oberschicht.

Abschließend kann das einfache statische Kronenansatzmodell aufgrund seiner Konzeption als geeignet beurteilt werden, die Kronenansatzhöhe behandlungssensitiv zu prognostizieren. Voraussetzung dafür ist jedoch, daß innerhalb der zur Parametrisierung verwendeten Datenstruktur unterschiedliche waldbauliche Behandlungen gleichmäßig repräsentiert sind. Die aktuelle Datenstruktur weist eine breite regionale Repräsentativität auf. Auch wird die Bandbreite von standardmäßig definierten Durchforstungsarten und -stärken in gleichaltrigen Reinund Mischbeständen erfaßt. Problematisch sind jedoch die geringen Anteile an Extremvarianten. Für eine behandlungssensitive Schätzung der Kronenansatzhöhe sollten daher die Möglichkeiten einer gewichteten Regressionsanalyse für eine stärkere Berücksichtigung von Bäumen mit extremen H/D-Werten untersucht werden. Eine Eingrenzung der Parametrisierungsgrundlage wie im Beispiel in Abb. 78 erscheint aufgrund der Auswirkungen auf die regionale Repräsentativität des Modells nicht ratsam zu sein.

Weiterhin muß getestet werden, ob in heterogenen Datenstrukturen die Einzelbaumhöhe anstelle des H/D-Wertes die bessere Variablenwahl zusätzlich zur Spitzenhöhe und zum BHD darstellt. Die in Kapitel 3.2.1 (Validierung und Bewertung) ausführlich diskutierte Verwendung von nach Ober- und Unterschicht getrennt berechneten Spitzenhöhen ist weiterhin zwingend notwendig für eine zuverlässige Prognose des Kronenverhältnisses.

Eine weitere Möglichkeit zur sensitiven Schätzung der Kronenansatzhöhe besteht in einer stärker ökophysiologisch ausgerichteten Modellierung der Absterbeprozesse der unteren Grünäste. $\mathrm{Zu}$ unterscheiden sind dabei einfache Regeln der Absterbereihenfolge - wie sie in Kapitel 2.3 anhand der Ansätze von KELLOMÄKI und VÄISÄNEN (1988) sowie KELLOMÄKI ET AL. (1989) dargestellt wurden - von Modellen, in denen der Absterbeprozeß über ein Strahlungsmodell gesteuert wird (KELLOMÄKI ET AL., 1999). Strahlungsmodelle weisen im Gegensatz zu starren Regeln der Absterbereihenfolge eine hohe potentielle Behandlungssensitivität durch die Berücksichtigung der Kronenstruktur bzw. der gegenseitigen Beeinflussung der 
Bäume auf. Ein weiteres Beispiel eines Strahlungsmodells, das allerdings zur Wachstumsprognose eingesetzt wird, stellt BRUNNER (1998) vor. Ein Vorteil gegenüber dem einfachen statischen Kronenansatzmodell (13) könnte in einer höheren Sensitivität der ökophysiologischen Modelle schon bei weniger ausgeprägten Behandlungsunterschieden bestehen. Die Entwicklung von ökophysiologischen Modellen zur Modellierung der Kronenansatzhöhe setzt voraus, daß die vertikalen und nach Himmelsrichtungen untergliederten Verteilungsmuster von Grünund Totästen durch das zur Verfügung stehende Strahlungsangebot beschrieben werden können. Da mit Strahlungsmodellen der Absterbezeitpunkt der Einzeläste direkt geschätzt wird, entfällt die für die statistischen Kronenansatzmodelle notwendige Formulierung von einfachen Regeln zur Festlegung der Absterbereihenfolge.

Selbst wenn es gelingt, Absterbeprozesse über ökophysiologische Modelle zu steuern, erscheint es notwendig, die Kronenansatzhöhen zu Beginn einer Wachstumssimulation mit Hilfe statistischer Modelle zu initialisieren. Da eine ökophysiologisch gesteuerte Veränderung der Kronenansatzhöhe mittelfristig nur auf einer relativ eng begrenzten Datenbasis entwickelt werden kann, kommt den statistischen Modellen eventuell auch eine Kontrollfunktion durch die Definition von plausiblen Grenzwerten zu.

\subsubsection{Einheitskronenansatzmodell / Dynamisierung des Einheitskronenansatzmodells}

Zusätzlich zum hierarchischen (9)-(12) und einfachen statischen Kronenansatzmodell (13) wurde ein Einheitskronenansatzmodell (14) entwickelt, das hochwertige Eingangsinformationen wie einige gemessene Kronenansatzhöhen oder die Schätzung des Kronenverhältnisses des Spitzenhöhenstammes bei der Prognose berücksichtigt (Kapitel 3.2.3). Das Modell führt bei allen Baumarten, d. h. auch bei den Laubholzarten, zu einer nochmaligen Erhöhung der Schätzgenauigkeit.

Um die hochwertigen Eingangsinformationen über den Zeitpunkt ihrer Erhebung hinaus verwenden zu können, wurde zusätzlich eine Möglichkeit zur Fortschreibung des Kronenverhältnisses des Spitzenhöhenstammes bzw. zur Dynamisierung des Einheitskronenansatzmodells entwickelt (Kapitel 3.2.4). Das dynamisierte Einheitskronenansatzmodell weist bei kurzund mittelfristigen Simulationszeiträumen Vorteile gegenüber den statischen Modellansätzen ohne Berücksichtigung von Zusatzinformationen auf. Einsatzmöglichkeiten für das dynamische Einheitskronenansatzmodell bestehen daher vor allem in der Fortschreibung von Kontrollstichproben- und Weiserflächendaten sowie von Daten aus ertragskundlichen Versuchsflächen und Naturwäldern.

Weitergehende Untersuchungen bezüglich der Fortschreibung des Kronenverhältnisses des Spitzenhöhenstammes konnten nur für die Baumart Fichte durchgeführt werden. Es besteht daher weiterer Forschungsbedarf, inwieweit der Vorteil des dynamischen Modells durch die Komponenten Schätzung des Ausgangskronenverhältnisses, Schätzung der Veränderungs- 
rate, Länge der Prognosedauer und der in einem Simulationsschritt beschriebenen Periodenlänge beeinflußt wird (Kapitel 3.2.4, Validierung und Bewertung). Das Einheitskronenansatzmodell weist in der statischen und dynamischen Version ebenfalls die theoretische Möglichkeit einer behandlungssensitiven Beschreibung des Kronenverhältnisses auf. Auch hier ist es jedoch notwendig, das Modell auf einer Datengrundlage zu parametrisieren, die neben typischen waldbaulichen Behandlungen auch Extremvarianten repräsentiert.

Weiterer Forschungsbedarf besteht bei der Überprüfung, inwieweit genauere Prognosen der Kronenansatzhöhe über die Berechnung von Konkurrenzindizes und Kronendimensionsmaßen wie der Kronenmantelfläche zu einer exakteren Schätzung des Zuwachses führen (NAGEL, 2001). Die genauere Beschreibung der Kronenansatzhöhe ermöglicht zusätzlich eine größere Realitätsnähe bei der Visualisierung.

\subsection{Astparametermodelle (Nadelholzarten und Laubholzarten mit starker Apikalkon- trolle)}

\subsubsection{Astdurchmessermodell für die Quirläste in der grünen Krone}

Ziel war es, auch für die Schätzung qualitätsrelevanter Astparameter Modelle mit einer hohen allgemeinen Prognosegenauigkeit und Behandlungssensitivität zu entwickeln. Für die Nadelholzarten konnte ein behandlungssensitives Modell (20) zur Beschreibung der Durchmesser der stärksten Quirläste in der grünen Krone (bis zum untersten Grünast) formuliert werden (Kapitel 3.3.1). Die Behandlungssensitivität wird im Modellansatz über die unabhängigen Variablen H/D-Wert und die Höhe des untersten Grünastes berücksichtigt. Eine behandlungssensitive Prognose der Astdurchmesser wird damit maßgeblich von einer behandlungssensitiven Beschreibung des H/D-Wertes beeinflußt. Das Astdurchmessermodell für die Nadelholzarten orientiert sich in seiner allgemeinen Konzeption an parameterkargen Schaftformfunktionen (Hui U. GAdow, 1997; s. Kapitel 3.7). Das bedeutet, daß der typische Verlauf der Astdurchmesserstruktur bereits durch die Funktionsform vorgegeben ist und nur noch eine Variation dieser Form in Abhängigkeit von Einzelbaumvariablen erfolgt. Der Vorteil dieses Ansatzes gegenüber den häufig verwendeten segmentierten und unsegmentierten Polynomialgleichungen (Colin U. Houllier, 1991; Maguire et Al., 1994; Vestöl et AL., 1996) sind eindeutige, biologisch plausible Wirkungsrichtungen der verwendeten unabhängigen Variablen (Kapitel 3.3.1).

Die Vorgabe eines typischen Funktionsverlaufes erscheint auch modelltheoretisch als vorteilhaft, da dadurch die Korrelation der Beobachtungen an einem Baum berücksichtigt wird. Weiterhin konnte anhand der Baumart Fichte gezeigt werden, daß die Schätzungen in den Randbereichen des Spektrums der verwendeten unabhängigen Variablen eine höhere Genauigkeit aufweisen als ein Vergleichsmodell von MAGUIRE ET AL. (1994) (Abb. 38). Der Vergleich des verallgemeinerten Modells mit einem einzelbaumweise angepaßten Gleitenden- 
Durchschnitts-Modell verdeutlicht, daß das neu entwickelte Modell eine gute Sensitivität bezüglich der verwendeten unabhängigen Variablen besitzt (Kapitel 3.3.1). Die hohe Sensitivität des Modells gegenüber dem H/D-Wert (Abb. 36) erlaubt theoretisch auch eine Prognose der Auswirkung einer Überschirmung auf die Astdurchmesserstruktur. Voraussetzung dafür ist jedoch eine realitätsnahe Prognose der H/D-Werte von unterständigen Bäumen und die Erweiterung der Parametrisierungsgrundlage.

Die parameterkarge Konzeption des Modells hat zusätzlich den Vorteil, daß bei der Prognose der Durchmesser der nächststärkeren Quirläste - mit Ausnahme von Schätzungen im Extrapolationsbereich - stabile, biologisch plausible Verläufe innerhalb der Krone prognostiziert werden (Kapitel 3.3.2, Abb. 41 u. Abb. 42). Bei der nach Astrangfolgestraten getrennten Parametrisierung eines Modells ist es keinesfalls selbstverständlich, daß für alle Kombinationen von unabhängigen Variablen biologisch plausible Verläufe der Quirläste zueinander prognostiziert werden. In der ersten Version des neu entwickelten Modells wiesen lediglich untypische Variablenkombinationen im Extrapolationsbereich biologisch unplausible Überschneidungen der Quirlastverläufe verschiedener Rangstufen auf. Durch einen Parameterausgleich über der Astrangfolge wurde die endgültige Modellversion bei gleichzeitig vernachlässigbaren Verlusten der Schätzgenauigkeit so stabilisiert, daß auch für untypische Variablenkombinationen biologisch plausible Verläufe prognostiziert werden (Abb. 44). Für die Anwendung dieser heuristischen Methode zur Gewährleistung plausibler Astdurchmesserverläufe werden die Parameterkargheit des Modells bzw. die eindeutigen Wirkungsrichtungen der unabhängigen Variablen ebenfalls als vorteilhaft beurteilt.

Im Gegensatz zu anderen Astdurchmessermodellen weist das neu entwickelte Modell nur einen Geltungsbereich für die grüne Krone auf. Auf eine Extrapolation in die Totastzone soll mit Blick auf den Einsatz in einem Wachstumssimulator bewußt verzichtet werden, da vor allem die Astdurchmesser in der grünen Krone in einem direkten Zusammenhang zu den aktuellen Variablen bzw. Eigenschaften eines Baumes stehen. Allerdings würde die verwendete Modellkonzeption auch eine Prognose der Astdurchmesser in der Totastzone zulassen. Vielfach kann die Astdurchmesserstruktur in der Totastzone auch über aktuelle Variablen beschrieben werden, wobei jedoch keine extremen Wechsel in den Wuchsbedingungen des Baumes aufgetreten sein dürfen. Beispielsweise kann das feinastige Erdstammstück eines Plenterwaldbaumes aus der Oberschicht nicht aus den aktuellen, solitärähnlichen Baumattributen prognostiziert werden.

Für eine Prognose der gesamten äußeren Astigkeit durch Quirläste ist es jedoch notwendig, die Astdurchmesserstruktur in der Totastzone zum Simulationsbeginn direkt in Abhängigkeit von den aktuellen Baumvariablen zu schätzen. Die Prognose ist dabei umso unsicherer, je höher der Baum bzw. je länger die Totastzone zum Simulationsbeginn bereits ist. $\mathrm{Ab}$ dem Simulationsbeginn wird die Durchmesserstruktur in der Totastzone über die Durch- 
messer beschrieben, die die untersten Grünäste zum Zeitpunkt ihres Absterbens aufweisen, so daß keine Extrapolation in den Totastzonenbereich mehr notwendig ist. Das Modell zur Beschreibung der Quirlastdurchmesser wurde im Rahmen dieser Arbeit ausschließlich mit Daten aus dem Bereich der grünen Krone parametrisiert. Daher sollte das Modell für die direkte Prognose der Durchmesserstruktur in der Totastzone mit allen zur Verfügung stehenden Daten, d. h. auch mit Totastdurchmessern parametrisiert werden. Im streng mathematischen Sinn handelt es sich damit nicht mehr um eine Extrapolation. Das Problem, daß die Astdurchmesser in Teilbereichen der Totastzone unter Umständen nicht durch die aktuellen Baumeigenschaften beschrieben werden können, kann durch die Erweiterung der Parametrisierungsgrundlage jedoch nicht beseitigt werden.

Die Möglichkeit zur direkten Prognose der Astdurchmesser in der Totastzone kann trotz der damit verbundenen Probleme als Vorteil gegenüber bestimmten statistischbiomechanischen Modellen genannt werden (Kapitel 2.4.1, Abb. 3). Diese Modelle beschreiben den Astdurchmesser in Abhängigkeit von der in die Ebene projizierten Astlänge (MEILBY, 1999). Somit ist auch eine konkurrenzsensitive Prognose des Astdurchmessers möglich (SEIFERT, 1999), wofür allerdings plastische Kronenmodelle zur Beschreibung der Astlänge notwendig sind. Die Zusammenhänge zwischen Astlänge und -durchmesser gelten jedoch nur für Grünäste und die Astlängen können nur über ein Kronenmodell geschätzt werden, so daß eine direkte Anwendung auf die Totastzone nicht möglich ist.

Für die Baumart Fichte wurde der Trend beobachtet, daß unterhalb von Kronenbrüchen stärkere Astdurchmesser auftreten als an Quirlen mit sonst übereinstimmenden Eigenschaften von gesunden Bäumen. Daher wurde für diese Baumart ein Modell entwickelt, das die Auswirkungen von Kronenbrüchen auf die Astdurchmesserstruktur des unterhalb des Kronenbruches liegenden und verwertbaren Stammstücks erfaßt (Kapitel 3.3.3). Voraussetzung für die Anwendung des Modells ist, daß der Zeitpunkt des ersten und für die Stammholzaushaltung entscheidenden Kronenbruchs eines Baumes bekannt ist.

Die Prognose der inneren Astigkeit wird noch stärker als die Prognose der gesamten äuBeren Astigkeit durch das physiologische Alter eines Baumes zum Simulationsbeginn beeinflußt. Während die direkte Anwendung des Astdurchmessermodells in der Totastzone modelltechnisch möglich ist, ist die Beschreibung der radialen Durchmesserentwicklung von Ästen in Richtung des Marks über den Simulationsstartpunkt zurückgehend nicht direkt realisierbar. Ähnliches gilt für den vertikalen Astabgangswinkel. Die innere Astigkeit im Stammbereich, der radial außerhalb der Stammform zum Simulationsbeginn liegt, wird dagegen durch Fortschreibung mit Hilfe des Astdurchmessermodells im Rahmen der Wachstumssimulation erfaßt. Auf der Basis dieser Astdurchmesserschätzungen muß auch eine Extrapolation des Astdurchmessers in radialer Richtung in den inneren Stammbereich erfolgen, wenn die gesamte innere Astigkeit erfaßt werden soll. Zukünftig sollte auch hier ein statistisches Mo- 
dell entwickelt werden, das Zusammenhänge zum radialen Durchmesserverlauf von Ästen identifiziert und beschreibt. Für den vertikalen Astabgangswinkel ist die Extrapolation unproblematisch, da das zugrundeliegende Modell nur eine Abhängigkeit des Winkels von der Quirlrangfolge ausgehend von der Baumspitze unterstellt und somit relativ starr ist (Kapitel $3.5)$.

Langfristig sollen die im Rahmen dieser Arbeit entwickelten Modelle auch um eine Komponente ergänzt werden, die die Auswirkungen der Raumstruktur auf die Aststärke berücksichtigt. Eine Voraussetzung hierfür ist die realitätsnahe Generierung von Baumverteilungsplänen, da nur in seltenen Fällen eingemessene Baumstandpunktkoordinaten vorliegen (Pretzsch, 1993; LewandowsKi U. Gadow, 1997).

Die prognostizierten Astdurchmesserverläufe der fünf stärksten Quirläste können als die mittleren Verläufe für die jeweilige Kombination unabhängiger Variablen aufgefaßt werden. Es ist zu vermuten, daß ein Teil der nicht erklärten Streuung des Astdurchmessers über die Raumstruktur des Baumes bzw. seine Konkurrenzsituation erklärt werden kann. Für die Erfassung der Auswirkungen der Konkurrenz auf einen speziellen Astdurchmesser ist die Berechnung von gerichteten Konkurrenzindizes notwendig. Beispielsweise könnte der kleinräumige $\mathrm{C}_{66}$-Konkurrenzindex (NAGEL, 1999 a, S. 20) so modifiziert werden, daß er nur in Teilsegmenten des sonst als Flächenbezug verwendeten Einflußkreises berechnet wird. Das Teilsegment könnte durch die Aufteilung der Horizontalwinkel zu den beiden Nachbarästen eines Astes ermittelt werden (Abb. 8). Als Schnittebene würde anstelle der 66\% Kronenlänge von der Baumspitze aus die jeweilige Höhe des betrachteten Astes verwendet. Der berechnete Konkurrenzindex würde dazu eingesetzt werden, den über das Astdurchmessermodell (20) prognostizierten Astdurchmesser zu modifizieren. Der Modifikationsfaktor könnte ermittelt werden, indem die beobachteten Abweichungen von den Schätzwerten des Modells (20) als Funktion des gerichteten Konkurrenzindizes beschrieben würden. Um Wechsel in der Aststärkenrangfolge innerhalb eines Quirls zu vermeiden, sollten die Astdurchmesser vor ihrer Modifizierung umgekehrt zu ihrer Stärke den ansteigenden Konkurrenzindizes zugeordnet werden. Ein ähnlicher Ansatz wird von SEIFERT (1999) für die Berechnung eines gerichteten bzw. modifizierten Konkurrenzindizes auf der Basis des KKL-Index (PRETZSCH U. KAHN, 1998, S. 79 ff.) zur konkurrenzsensitiven Beschreibung von Kronenbreiten bzw. Astlängen verwendet, die anschließend eine Prognose des Astdurchmessers zulassen.

Es kann vermutet werden, daß die Berücksichtigung der Raumstruktur insbesondere bei der Prognose des Einflusses von stark asymetrischen Konkurrenzbedingungen, wie sie bei bestimmten Ausgangsverbänden sowie an Bestandes- und Gassenrändern auftreten, zu einer deutlichen Erhöhung der Schätzgenauigkeit führen würde. Beispielsweise können an Bäumen mit stark asymetrischen Konkurrenzbedingungen mehrere, jeweils ähnlich starke und schwache Astdurchmesser in einem Quirl auftreten. Dieses Astdurchmessermuster würde von dem 
in Kapitel 3.3.2 dargestellten Modell für die fünf stärksten Quirläste nicht ausreichend beschrieben.

Voraussetzung für die Entwicklung des skizzierten Modifikationsmaßes ist, daß die Himmelsrichtung eines Astes nach der Fällung des Baumes rekonstruiert werden kann. Weiterhin müssen die Astmessungen von Bäumen aus Versuchsflächen mit eingemessenen Baumfußpunktkoordinaten stammen, damit die Nachbarschaftsverhältnisse erfaßt werden können. Der Vorteil einer Modifikation des entwickelten Astdurchmessermodells besteht darin, daß durch die weniger hohen Anforderungen an die Eingangsdaten eine schnelle Erweiterung der Datenbasis bzw. des Geltungsbereiches des nicht modifizierten Modells (20) möglich ist. Gleichzeitig wird die, durch die höheren Anforderungen an die Datenqualität bedingte, geringere Parametrisierungsgrundlage für den Modifikationsfaktor weniger kritisch als bei der Entwicklung eines eigenständigen Modellansatzes beurteilt.

Die Anwendung des für Nadelholzarten entwickelten Astdurchmessermodells kann auch für Laubholzarten erfolgen. Voraussetzung dafür ist jedoch, daß eine Baumart fast ausschließlich wipfelschäftige Stammformen und durch eine starke Apikalkontrolle (Abb. 4) geprägte typische Astdurchmesserstrukturen ausbildet (excurrente Kronenform ${ }^{21}$ ). Das entwikkelte Modell kann folglich zur Beschreibung der Astigkeit von Baumarten wie Erle, Esche und Ahorn eingesetzt werden. Diese Baumarten weisen in der Jugend überwiegend wipfelschäftige Stammformen auf, so daß in dieser Phase eine Prognose der gesamten Astdurchmesserstruktur in der grünen Krone erfolgen kann. Die Prognose der Astigkeit in der wipfelschäftigen Wachstumsphase reicht aus, da die Wertschöpfung bei diesen Baumarten zum großen Anteil auf das Erdstammstück konzentriert ist.

Eine Stratifizierung nach der Astrangfolge zur Streuungsreduktion wie bei den Nadelholzarten erscheint infolge der fehlenden Anordnung der Äste in Quirlen nicht möglich. Durch die gegenständige Anordnung der Äste bei den Baumarten Esche und Ahorn könnte jedoch eine Unterteilung in zwei Straten untersucht werden, wobei immer die Äste an Knoten und nicht in Quirlen stratifiziert würden. Prinzipiell könnte auch für diese Baumarten das einfachere Astdurchmessermodell (24) - (27) verwendet werden, das für die Baumart Buche entwickelt wurde (Kapitel 3.4.1). Das Modell für die Baumart Buche prognostiziert jedoch nur die Astdurchmesser im Kronenansatzbereich, so daß eine Erfassung von Astdurchmesserstrukturen für den gesamten Kronenbereich nicht möglich ist. Die genannten Laubholzarten weisen aber durch die starke Apikalkontrolle in jüngerem Alter eine strengere Gliederung der Kronenarchitektur in eine Schaftachse und mehr oder weniger horizontale Äste 1. Ordnung

\footnotetext{
${ }^{21}$ GRUBER (1987, S. 185) spricht bei fehlender Zentralachse bzw. mehreren steilen Mischachsen von einer decurrenten und bei vorhandener Zentralachse mit mehr oder weniger horizontal abzweigenden Seitenachsen von einer excurrenten Kronenform.
} 
auf. Daher ist während der wipfelschäftigen Phase eine Ausbildung typischer Astdurchmesserstrukturen wie bei den Nadelholzarten zu erwarten. Die Erfassung von bestimmten Astdurchmesserverläufen in der grünen Krone kann jedoch nur mit den für die Nadelholzarten entwickelten Modellen erfolgen. Auch die Möglichkeiten zur direkten Beschreibung der Astdurchmesserstruktur in der Totastzone durch die Erweiterung der Parametrisierungsgrundlage (s. o.) ist als Vorteil des Modells zu nennen.

Das Astdurchmessermodell müßte für die Laubholzarten um ein Modell zur Prognose von Astabständen ergänzt werden, da die Äste nicht in Quirlen angeordnet sind und qualitätsrelevante Äste nicht nur am oberen Ende eines Jahrestriebes auftreten. Die Astabstände können somit nicht über das Höhenwachstum prognostiziert werden (Kapitel 3.4.2). Wie bei der Baumart Buche beschrieben (Roloff, 1986, S. 84 ff.; Kapitel 3.4.2), tritt auch bei den genannten Laubholzarten eine Veränderung der initial angelegten Astabstände durch die partielle Mortalität und Astreinigung in der grünen Krone auf. Im Gegensatz zu den Nadelholzarten bleibt die wipfelschäftige Wuchsform in den meisten Fällen nicht über die gesamte Lebenszeit eines Baumes erhalten, so daß bei Wachstumssimulationen die Stammhöhe bzw. der Zeitpunkt prognostiziert werden muß, ab dem sich die Schaftachse auflöst. Im Mittel kann von einer Auflösung der Schaftachse ausgegangen werden, wenn das verwendete Kronenansatzmodell nur noch geringfügige Veränderungen der Kronenansatzhöhe prognostiziert.

Die Erkenntnisse aus der Beschreibung der Astdurchmesserstruktur können zusätzlich für eine genauere Beschreibung der Kronenform verwendet werden. Eine wirklichkeitsnähere Beschreibung der Kronenform kann für die Visualisierung und die genauere Berechnung von Konkurrenzindizes und der Kronenmantelfläche bei der Wachstumsprognose eingesetzt werden (NAGEL, 1999, S. 28).

Für die Beschreibung der Krone mit Hilfe einfacher geometrischer Formen hat die Höhe der maximalen Kronenbreite neben der Kronenansatzhöhe eine entscheidende Bedeutung (Kändler, 1986; PretzSCH, 1992). So definiert die Höhe der maximalen Kronenbreite den Übergang von Licht- und Schattenkrone, die mit Hilfe unterschiedlicher geometrischer Modelle beschrieben werden. Allerdings werden aufgrund mangelnder empirischer Grundlage häufig baumartenspezifische, sonst aber konstante Licht- und Schattkronenanteile unterstellt (Burger, 1939; Badoux, 1939; Pretzsch, 1992). Demgegenüber konnte Guericke (2001, S. 105 ff.) für die Baumarten Buche und Lärche einen Zusammenhang zwischen der relativen Position der maximalen Kronenbreite innerhalb der Krone und der Baumhöhe nachweisen. Die Erfassung der Astdurchmesserstruktur innerhalb der grünen Krone erlaubt darüber hinaus eine behandlungssensitive Prognose der Höhe der maximalen Kronenbreite, da der Astdurchmesser eng mit der in die Ebene projizierten Astlänge korreliert ist (SEIFERT, 1999). Aufgrund der in Abb. 41, Abb. 42 und Abb. 44 dargestellten Ergebnisse würden bei gleichem BHD für 
solitärähnliche Bäume maximale Kronenbreiten nah der Kronenbasis, d. h. deutlich unter den Werten für unter Konkurrenz erwachsene Bäume geschätzt.

Die Kenntnis der vertikalen Astdurchmesserstruktur läßt verschiedene noch weitreichendere Modifikationen der Kronenform zu. Die über ein Kronenbreitenmodell (SCHMIDT ET AL., 2001) geschätzte maximale Kronenbreite kann mit Hilfe der zu den Astdurchmessern eines Astranges zugehörigen projizierten Astlängen reduziert werden. Der Astrang definiert die Stellung des Astes innerhalb des Quirls entsprechend seines Durchmessers. Dabei wird die projizierte Astlänge des stärksten Astes eines Astranges zur Normierung aller anderen Astlängen verwendet. Die relativ starre Beschreibung mit Hilfe einfacher geometrischer Formen könnte damit entfallen. Wird ein rotationssymetrischer Kronenaufbau unterstellt, sollte der vertikale Verlauf des mittleren Quirlastdurchmessers zur Beschreibung der Kronenform verwendet werden.

Prinzipiell erlaubt der enge Zusammenhang zwischen der projizierten Astlänge und dem Astdurchmesser auch eine Beschreibung der Kronenform ohne die Berücksichtigung eines Kronenbreitenmodells. Die Unterschiede der Astdurchmesser innerhalb eines Quirls, die eventuell zusätzlich über Konkurrenzfaktoren modifiziert werden (s. o.), könnten sogar zur Erfassung des Einflusses der Raumstruktur auf die Kronenform verwendet werden. Dabei muß allerdings beachtet werden, daß die Parametrisierungsgrundlage des Kronenbreitenmodells sehr viel umfangreicher als die der Astdurchmessermodelle ist. Die Kombination eines Kronenbreiten- mit dem Astdurchmessermodell wird daher bei der Prognose der Kronenform stabiler eingeschätzt als eine Prognose ausschließlich auf der Grundlage von Astdurchmessern.

\subsubsection{Astanzahlen pro Quirl und vertikaler Astabgangswinkel}

Neben der Prognose der Astdurchmesser von Quirlästen erfordert die Beschreibung der qualitätsrelevanten Astigkeit eine Prognose der Astanzahl pro Quirl und des vertikalen Astabgangswinkels. Während die Astanzahl pro Quirl beispielsweise für die Berechnung moderner Kriterien der Schnittholzsortierung wie dem "Knot Area Ratio" (KAR) notwendig ist, wirkt sich der Astabgangswinkel durch die Störung der Faserstruktur auf die innere Astigkeit aus (Kapitel 2.4.6, Abb. 8 u. Kapitel 3.5). In Übereinstimmung mit anderen Untersuchungen konnten auch in dieser Arbeit nur schwache Zusammenhänge zwischen der Quirlastanzahl und Variablen auf Einzelbaum- und Quirlebene identifiziert werden. Die Modellierung erfolgt daher wie häufig üblich über die Verwendung des Mittelwertes (KELLOMÄKI ET AL., 1988) (Kapitel 3.5). In die Untersuchung wurden nur Äste mit einem Durchmesser von mehr als $5 \mathrm{~mm}$ einbezogen, da schwächere Äste kaum qualitätsrelevant sind.

Um weitergehende Informationen bei der Modellierung zu berücksichtigen, können die Astanzahlen mit stochastischer Streuung auf der Basis der zugehörigen Häufigkeitsverteilung 
generiert werden. Für den Astabgangswinkel wurde im Rahmen dieser Arbeit kein neues Modell entwickelt (Kapitel 3.5). Wenn man davon ausgeht, daß der Astabgangswinkel im Gegensatz zu Wachstumsprozessen vor allem durch die statischen Eigenschaften eines Astes beeinflußt wird, können bereits entwickelte Modelle eventuell ohne erneute Parametrisierung für die Prognose dieses Gütemerkmals eingesetzt werden. Für die Baumart Fichte wäre beispielsweise zu überprüfen, inwieweit ein von COLIN und HoulliER (1992; s. Kapitel 2.4.6) entwickeltes Modell für nordwestdeutsche Verhältnisse eingesetzt werden kann.

\subsection{Astparametermodelle (Laubholzarten ohne starke Apikalkontrolle)}

\subsubsection{Astdurchmessermodell}

Infolge der durch zusätzliche Gütemerkmale stärker beeinflußten Holzqualität und der uneinheitlicheren Kronenarchitektur sind bisher kaum statistische Astparametermodelle für die Laubholzarten entwickelt worden. Für die Laubholzarten ohne starke Apikalkontrolle (z B. Buche), die unterschiedliche Kronentypen mit überwiegend decurrenter Kronenform (RolofF, 1986, S. 90) ausbilden, eignen sich die Modelle zur Beschreibung der Astdurchmesser in der gesamten Krone nicht, da keine typische, allgemeingültige Struktur unterstellt werden kann.

Um die qualitätsrelevante Astigkeit von Bäumen mit unterschiedlichen Kronenformen erfassen zu können, wurde ein Modell entwickelt, das dem Ansatz von MAGUIRE ET. AL. (1991) zur Beschreibung des stärksten Astes am Baum ähnelt (Kapitel 2.4.2). Der Modellnachteil bei der Anwendung auf Nadelholzarten, daß die Astdurchmesserstruktur nicht für den gesamten Kronenbereich erfaßt werden kann (Kapitel 2.4.2), spielt für die Buche keine Rolle. Durch den hohen Anteil des Stammholzes an der Wertschöpfung reicht die statische Prognose der Astdurchmesser im Kronenansatzbereich aus, die im Rahmen einer Wachstumssimulation die Prognose der Astigkeit für den gesamten späteren Stammholzbereich zuläßt. Infolge der schnellen Astreinigung der Buche ist die direkte Beschreibung der äußeren Astigkeit des gesamten Stammbereiches, die zu großen Anteilen durch Astnarben bestimmt wird, ohnehin nicht möglich. Auf die Erweiterung des Parametrisierungsbereiches in die Totastzone, die aus methodischen Gründen auch für die Nadelholzarten nur für den Startpunkt einer Simulation von Bedeutung ist (Kapitel 4.2.1), wurde aufgrund der schnellen Astreinigung der Buche verzichtet.

Infolge der hohen, nicht erklärten Reststreuung bei Anwendung eines regressionsanalytischen Ansatzes (23) (Abb. 48) wurde jedoch ein verallgemeinertes Verteilungsmodell ausgewählt (24) - (27). Ein solches Modell kann die Information, die in der nicht erklärten Reststreuung eines Regressionsmodells enthalten ist, bei der Generierung von Durchmesserwerten berücksichtigen. Das hier entwickelte Modell beschreibt die Durchmesserverteilung für Äste aus dem Kronenansatzbereich in Abhängigkeit von der mittleren Tiefe der Äste in der Krone 
und dem BHD des zugehörigen Baumes. Durch die Eingrenzung des Parametrisierungsbereiches auf den Kronenansatzbereich konnten auch Werte von nicht wipfelschäftigen Bäumen berücksichtigt werden. Astdurchmesser, die zu einer Auflösung der Schaftachse führten sowie Äste oberhalb der Auflösung der Schaftachse, wurden nicht verwendet. Die gutachterliche Ansprache der Auflösung der Schaftachse erfolgte im Gelände.

Um Unterschiede in der Astdurchmesserstruktur von Bäumen zu erfassen, die über den Trend hinausgehen, daß starke Bäume stärkere Äste als dünnere Bäume aufweisen, wurden die Astdurchmesser mit dem zugehörigen BHD normiert. Der Wertebereich wird durch die Normierung auf das Intervall [0,1] begrenzt. Als Verteilungsfunktion wurde die BetaVerteilung verwendet, die implizit auf das Intervall [0,1] beschränkt ist und sich daher besonders zur Beschreibung von biologischen Phänomenen mit natürlichen oberen und unteren Grenzen eignet (Kapitel 3.4.1; HAHN U. SHAPIRO, 1967).

Für eine einzelbaumweise Parametrisierung der Beta-Verteilung stehen pro Baum zuwenig Werte zur Verfügung, so daß Straten für Bäume mit ähnlichen Kombinationen des BHD und der mittleren Tiefe in der Krone gebildet wurden. Die Verteilungsparameter der Beta-Verteilung konnten anschließend über den Stratenmittelwerten ausgeglichen werden, wobei mit zunehmender Tiefe in der Krone und sinkendem BHD im Mittel höhere normierte Astdurchmesser geschätzt werden. Die Streuung der normierten Astdurchmesser steigt ebenfalls mit zunehmender mittlerer Tiefe in der Krone und sinkendem BHD (Abb. 52). Für Buchen mit längeren Kronen aus stark durchforsteten Beständen werden somit stärkere normierte Astdurchmesser prognostiziert, was biologisch plausibel ist. Der negative Einfluß des BHD auf den normierten Astdurchmesser führt dazu, daß die absoluten Astdurchmesser mit steigendem BHD nur degressiv ansteigen. Hierbei ist zu beachten, daß das Modell nicht nur biologische Eigenschaften der Äste, sondern auch die Beschränkung auf Äste beinhaltet, die eine Aushaltung von Stammholz zulassen. Es kann daher unterstellt werden, daß ein normierter Astdurchmesser umso eher zur Auflösung der Schaftachse führt, je stärker der Baum ist. Aufgrund der Datengrundlage weist das verallgemeinerte Verteilungsmodell einen Geltungsbereich bis zu einem BHD von $30 \mathrm{~cm}$ auf, was zukünftig eine Erweiterung der Parametrisierungsgrundlage erfordert. So werden bereits für Bäume mit $40 \mathrm{~cm}$ BHD und verhältnismäßig kurzen zugehörigen Kronen (Kapitel 3.4.1, Abb. 53) biologisch unplausible Werte generiert. Bei der Extrapolation wird in diesem Fall eine zu starke Abnahme der normierten Astdurchmesser prognostiziert.

Durch die Verwendung der Variablen mittlere Tiefe der Äste in der Krone besitzt das Astdurchmessermodell eine Reaktionsfähigkeit gegenüber waldbaulichen Eingriffen, die jedoch eine behandlungssensitive Prognose des Kronenansatzes voraussetzt. In diesem Zusammenhang ist als problematisch zu bewerten, daß die Kronenansatzmodelle für die Baumarten Buche und Eiche die höchsten Schätzfehler aufweisen (Kapitel 9.2, Tab. 54 u. Tab. 52). Auch 
muß geprüft werden, inwieweit durch gewichtete Regressionsanalysen oder Veränderungen der Parametrisierungsgrundlage (vgl. Kapitel 4.1.1) eine behandlungssensitive Schätzung des Kronenansatzes für Laubholzarten entwickelt werden kann.

Für die Laubholzarten mit schwacher Apikalkontrolle muß ebenfalls vorhergesagt werden, ab welchem Zeitpunkt bzw. welcher Stammhöhe eine Auflösung der Schaftachse erfolgt. Auch hier kann vereinfachend die Stammhöhe unterstellt werden, bei der das verwendete Kronenansatzmodell nur noch geringfügige Veränderungen der Kronenansatzhöhe prognostiziert.

Das verallgemeinerte Astdurchmessermodell kann aufgrund der kombinierten Erfassung des Einflusses unabhängiger Variablen und der nicht erklärten Reststreuung als gut geeignet zur Prognose der Astigkeit der Buche beurteilt werden. Eine vergleichende Validierung mit bereits existierenden, statistischen Modellen konnte aufgrund fehlender Modellansätze nicht erfolgen. So dienen die in letzter Zeit zur Beschreibung von Gütemerkmalen bei Laubholzarten entwickelten Modelle (z. B. NePVEU ET AL., 1999) der Prognose der Jahrringbreite, der Holzdichte und des Splintholzanteils nicht aber der Beschreibung der Astigkeit. Im Gegensatz zu den Nadelholzarten besteht daher großer Forschungsbedarf, die Astigkeit von Baumarten, die überwiegend decurrente Kronenformen ausbilden, mit einfachen statistischen, aber behandlungssensitiven Modellen zu beschreiben. In diesem Zusammenhang sollten auch vergleichende Validierungen mit Kronenarchitekturmodellen (KURTH, 1994) erfolgen, die die qualitätsrelevanten Astparameter ebenfalls beinhalten. Ein spezieller Schwerpunkt muß dabei auf der Beurteilung der Behandlungssensitivität liegen. Auch sollte eine Erweiterung der Parametrisierungsgrundlage für die Baumart Buche und die Entwicklung eines Modells für die Baumart Eiche erfolgen.

\subsubsection{Astabstandsmodell}

Durch die fehlende Anordnung der Äste in Quirlen sowie die partielle Astreinigung in der grünen Krone ist für eine umfassende Modellierung der Astigkeit der Buche zusätzlich eine Prognose der Astabstände notwendig (Kapitel 3.4.2). Der Abstand zwischen zwei Ästen wird dabei als Entfernung eines Astes zu einem bereits unterhalb plazierten Ast geschätzt (29). Der Durchmesser und die Tiefe in der Krone des bereits plazierten Astes haben einen positiven Einfluß auf den Astabstand, während die Durchmesserdifferenz beider Äste und der BHD einen negativen Einfluß auf den Astabstand haben. Infolge der Definition des zu schätzenden Astabstandes wurde ein Modelltyp gewählt, der nur positive Schätzungen zuläßt. Eine umfassendere Beurteilung der biologischen Plausibilität des Modells sowie die Darstellung der programmtechnischen Umsetzung bzw. des Zusammenwirkens von Astdurchmesser- und Astabstandsmodell für die Baumart Buche ist in Kapitel 3.4 und Abb. 59 dargestellt. Besonders zu beachten ist dabei die Verwendung des statischen Verteilungsmodells zur Fortschreibung von 
Variablen sowie der begrenzte räumliche Geltungsbereich der Modelle. So müssen bestimmte Einschränkungen bezüglich der zur Generierung von Astdurchmessern verwendeten Zufallszahlen bzw. äquidistant verteilten Zahlen beachtet werden, um plausible Zeitreihenwerte zu erzeugen.

Im Gegensatz zum Anstieg von Astdurchmessern im Rahmen einer Wachstumssimulation ist die prognostizierte Vergrößerung von Astabständen nur plausibel, wenn Äste absterben. Die Durchmesser- und Astabstandsmodelle wurden daher um einfache Regeln zur Definition der Mortalitäts- und Astreinigungreihenfolge von Einzelästen ergänzt. Die Aktualisierung der Astabstände im Rahmen einer Waldwachstumssimulation kann somit dazu verwendet werden, die partielle Astreinigung im unteren Teil der grünen Krone nachzubilden.

Die Anwendung des komplexen Systems von statischen Modellen und Regeln im Rahmen von Wachstumsprognosen (Kapitel 3.4, Abb. 59 ) erfordert zusätzlich zur Validierung der Einzelmodelle die Überprüfung im Simulationsbetrieb. Prinzipiell gilt diese Forderung für alle neu entwickelten Modellgruppen, wobei jedoch auf der Prüfung der Astparametermodelle der Buche ein besonderer Schwerpunkt liegen sollte, da neben den eigentlichen Modellen relativ viele Zusatzregeln formuliert werden mußten. Unter die Zusatzregeln fallen die Festlegung der Astmortalität und -reinigung sowie die nachgeschalteten Plausibilitätskontrollen bei der Erzeugung von Astabständen. Darüber hinaus weisen die Modelle im Vergleich zu den Astparametermodellen für die Nadelholzarten eine geringere Treffgenauigkeit auf. Auch ist das verallgemeinerte Verteilungsmodell das einzige Modell, das einen über die jeweilige Verteilungsform gesteuerten Zufallseffekt in die Schätzung integriert.

\subsection{Modelle zur Beschreibung des Astzustandes}

Die in dieser Arbeit verwendeten Modelle prognostizieren den Astzustand (Grünast, Totast, äußerlich nicht mehr vorhandener Ast) im Gegensatz zu neueren ökophysiologischen Modellen (COURBAud ET AL., 1996; FourCAUd ET AL., 1996; MÄKELÄ ET AL., 1996; KellOMÄKI ET AL., 1999) nicht direkt auf der Ebene des Einzelastes.

Die Modellierung des Astzustandes erfolgt zweistufig, indem Schätzungen für die Astzonenlängen mit einfachen Regeln zur Reihenfolge der Mortalität und Astreinigung kombiniert werden. Die Prognose von Astzonenlängen ermöglicht im Gegensatz zum Ansatz von COLIN und Houllier (1992) zur Schätzung der Höhe des untersten Tot- und Grünastes eine Berücksichtigung der Kronenansatzschätzung (Kapitel 3.2, 3.6). Somit können die Modelle problemlos mit den verschiedenen Typen von neu entwickelten Kronenansatzmodellen (Kapitel 3.2.5) kombiniert werden und eine unterschiedliche Güte der Eingangsinformationen berücksichtigen. Gleichzeitig werden unplausible Schätzungen verhindert, indem die verschiedenen Astzonenlängen im Verhältnis ihres theoretischen Maximalwertes angegeben werden und das Modell nur Schätzungen im Intervall [0,1] zuläßt. Die Maximalwerte sind für 
die Übergangsastzone durch die Höhe des Kronenansatzes und für die Totastzone durch die Höhe des untersten Grünastes vorgegeben. Die Grünastzone ergibt sich als Differenz von Baumhöhe und Kronenansatzhöhe, und die astfreie Schaftlänge resultiert aus den Schätzungen für die übrigen Astzonen.

Bei der Baumart Buche entfällt die Prognose einer Übergangsastzone, da der Kronenansatz durch den untersten lebenden Primärast definiert ist und gleichzeitig angenommen wird, daß oberhalb des Kronenansatzes keine Totäste auftreten. Für die Übergangsastzone konnte bei keiner der Nadelholzarten ein Zusammenhang zu den untersuchten unabhängigen Variablen identifiziert werden. Die Modellierung erfolgt daher lediglich über den Mittelwert. Die in Formel (31) dargestellte Funktionalisierung dient somit ausschließlich der Erfassung des durch die Normierung verursachten Trends.

Die normierte Totastzonenlänge wurde für die Buche infolge fehlender Zusammenhänge und für die Kiefer infolge der eingeschränkten Datengrundlage lediglich mit Verteilungsmodellen zur Erfassung der stochastischen Streuung beschrieben (Kapitel 3.6.2). Aufgrund der fehlenden statistischen Zusammenhänge erfolgt die Modellierung der stochastischen Streuung ohne Verallgemeinerung des Verteilungsmodells, so daß sich eine flexible, nichtparametrische Kernel-Verteilungsfunktion zur optimalen Beschreibung anbietet (SILVERMAN, 1986; HÄRDLE, 1989; Abb. 61 u. Abb. 62).

Für die Fichte und Douglasie konnte ein Regressionsmodell zur Schätzung der normierten Totastzonenlänge über der Baumhöhe parametrisiert werden (Formel (32)), wobei ein Absinken der normierten Totastzonenlänge mit steigender Baumhöhe prognostiziert wird (Abb. 60, Abb. 117). Dieser Trend kann aufgrund der ab einer bestimmten Baumhöhe einsetzenden Astreinigung und der sich verlangsamenden Verlagerung der Kronenansatzhöhe bzw. Höhe des untersten Grünastes als plausibel beurteilt werden. Die nicht erklärte Streuung der Modelle nimmt mit steigender Baumhöhe stark zu. Für die Douglasie mußte infolge der begrenzten Datengrundlage zusätzlich zur Beschränkung der Schätzwerte auf das Intervall [0,1] eine Übereinstimmung mit den bei der Fichte beobachteten Modelleigenschaften unterstellt werden. Im Mittel weist die Fichte etwas höhere normierte Totastzonenlängen als die Douglasie auf.

Behandlungssensitive Strahlungsmodelle (KRANIGK, 1996; BRUNNER, 1998; KellomÄKI ET AL., 1999), die eine Alternative zu den statistischen Kronenansatzmodellen darstellen, erlauben eine Schätzung des Strahlungsangebotes auf der Ebene des Einzelastes. Werden auf der Basis dieser Schätzungen kritische Strahlungsmengen für das Überleben eines Astes definiert, kann der Zeitpunkt des Absterbens für jeden Ast prognostiziert werden. Die ökophysiologischen Strahlungsmodelle liefern damit die Grundlage für die Prognose des Astzustandes für jeden Einzelast und damit für die detaillierte Beschreibung der Grünast- und Übergangsastzone. Die Astreinigung kann dagegen nicht mit Hilfe der Strahlungsmodelle 
beschrieben werden. Als Alternative können einfache, biologisch plausible Regeln zur Beschreibung empirischer Astzustands- bzw. Astreinigungsmuster formuliert werden, wie sie in den beschriebenen Astpopulationsmodellen verwendet werden (KELLOMÄKI U. VÄISÄNEN, 1988; KellomÄKI ET AL., 1989; Kapitel 2.3 u. 2.4.4). Im Gegensatz zu den Strahlungsmodellen weisen diese Modelle jedoch ein geringes Erklärungspotential bzw. eine geringe Behandlungssensitivität auf.

\subsection{Flexible Schaftformmodelle}

Mit Hilfe normierter Sektionsmessungen konnten für die untersuchten Nadelholzarten deutliche und für die untersuchten Laubholzarten nur schwache Formvariationen über den Einzelbaumvariablen BHD und H/D-Wert nachgewiesen werden (beispielhaft für Fichte: Abb. $64 \mathrm{u}$. Abb. 65 und für Buche: Abb. 134 u. Abb. 135). Ein von PAIN und BOYER (1996) entwickeltes paramterkarges Schaftformmodell (33) - (35) (Pain-Funktion) kann über eine Verallgemeinerung bzw. Flexibilisierung dazu genutzt werden, den formbeeinflussenden Effekt dieser Einzelbaumvariablen bei der Schaftformprognose zu berücksichtigen. Eine vergleichende Validierung mit der modifizierten Brink-Funktion (37) (RIEMER ET AL., 1995) und einer nicht flexibilisierten Form der Pain-Funktion (36) zeigt, daß die Pain-Funktion speziell an den Rändern des Formenspektrums deutliche Vorteile aufweist. Diese Vorteile werden auch anhand der getrennt nach Höhenstufen berechneten Gesamtfehler und systematischen Fehleranteile deutlich.

Durch die Erfassung des formbeschreibenden Effektes des H/D-Wertes wird wie bei den Kronenansatz- und Astdurchmessermodellen eine behandlungssensitive Beschreibung der Schaftform gewährleistet. Damit wird erneut die zentrale Bedeutung einer behandlungssensitiven Prognose des H/D-Wertes für die Modellierung der untersuchten Qualitätsaspekte deutlich. Allerdings nimmt der Vorteil der Pain-Funktion beispielsweise gegenüber der modifizierten Brink-Funktion von der Fichte über die Douglasie zur Kiefer ab, während für die Laubholzarten keine Modellverbesserungen zu beobachten sind. Diese Unterschiede stehen in direktem Zusammenhang $\mathrm{zu}$ den Formvariationen über den beschreibenden Variablen (Abb. 64 u. Abb. 65). D. h., nur wenn die Variablen BHD und H/D-Werte einen Einfluß auf das beobachtete Formenspektrum haben, kann dieser Einfluß auch vom Modell beschrieben werden.

Ein methodischer Nachteil der Pain-Funktion besteht darin, daß der durch die Schaftformfunktion prognostizierte BHD nicht mit dem gemessenen Durchmesser in $1.3 \mathrm{~m}$ Höhe übereinstimmt, wie es beispielsweise bei der modifizierten Brink-Funktion gewährleistet ist. Auch wenn die Abweichungen in den weitaus überwiegenden Fällen lediglich im Millimeterbereich liegen, wird damit die Information des gemessenen BHD nicht optimal im Modell verwendet. Es wurde daher eine Modifikation der Pain-Funktion entwickelt (38 a und b), die 
nur noch vernachlässigbare Abweichungen vom gemessenen BHD-Wert zuläßt (Abb. 73). So liegen für die Fichte 99.7\% der Schätzwerte weniger als einen Millimeter vom Meßwert entfernt. Weiterhin weist die modifizierte Pain-Funktion eine Segmentierung in zwei Funktionsteile zur getrennten Beschreibung des oberen und unteren Schaftteils auf. Die Segmentierung erfolgt im Wendepunkt der entsprechenden Schätzung mit Hilfe des verallgemeinerten Modells der Pain-Funktion. Die modifizierte Pain-Funktion führt bei allen untersuchten Baumarten zu Verbesserungen gegenüber der Standardversion. Dabei werden bei den Nadelholzarten nur relative geringe Verbesserungen durch die Reduktion von systematischen Fehlern im oberen Stammbereich und bei der Fichte im Wurzelanlaufbereich erreicht. Bei den Laubholzarten sind die Unterschiede zwischen der Standardversion und der modifizierten Pain-Funktion sowohl in der einzelbaumweisen Betrachtung als auch bei der Fehlerberechnung in Höhenstufen deutlicher.

Die verallgemeinerten Schaftformmodelle Pain-Funktion und modifizierte PainFunktion sollten aufgrund ihrer größeren Flexibilität nur im durch die Datengrundlage repräsentierten Bereich eingesetzt werden. Im Extrapolationsbereich werden bei den flexiblen Modellen im Gegensatz zu relativ starren Modellen schneller unplausible Verläufe prognostiziert, wobei besonders der Bereich niedriger H/D-Werte beachtet werden muß (Abb. 75). Speziell bei den Nadelholzarten mit einer größeren Schaftformflexibilität über dem H/D-Wert sollte die Datengrundlage langfristig um solitärähnlich erwachsene Bäume ergänzt werden.

Im Extrapolationsbereich ergeben sich teilweise ungünstige Segmentierungstellen für die modifizierte Pain-Funktion, da die über die Pain-Funktion geschätzten unplausiblen Schaftformen zur Bestimmung realitätsnaher Wendepunkte ungeeignet sind (Abb. 75). Als modelltechnische Verbesserung sollte daher eine iterative Bestimmung optimaler Segmentierungspunkte und eine anschließende Modellierung des Punktes mit Hilfe von Einzelbaumvariablen geprüft werden. Speziell bei untypischen Variablenkombinationen treten durch zu starke Steigungsdifferenzen verursachte unplausible Übergänge von unterem und oberem Schaftsegment bei der modifizierten Pain-Funktion auf. Der Übergangsbereich kann jedoch mit Hilfe einer Spline-Funktion so beschrieben werden, daß durch eine Glättung ein plausibler Übergang prognostiziert wird (Abb. 74). Auch muß geprüft werden, ob durch die Erweiterung der Datengrundlage und die alternative Bestimmung des Segmentierungspunktes für untypische Variablenkombinationen direkt plausible Übergänge von oberem und unterem Schaftteil prognostiziert werden können.

Die Schaftformmodelle sollen zukünftig auch dazu eingesetzt werden, Anteile an juvenilem Holz und Kernholzteile bei den obligaten Kernholzarten zu erfassen. Dabei wird die Prognosegenauigkeit allerdings weniger von der Art der Schaftformmodelle als vielmehr von der Identifizierung von Zusammenhängen zu den Kernholzanteilen abhängen (HAPLA U. SABOROWSKi, 1994; OJANSUU U. MALTAMO, 1995). 
In Kapitel 3.7.4 konnte nachgewiesen werden, daß die Berücksichtigung der Variablen Forstort, die Standorts- und Bestandesvariablen aggregiert, in einem verallgemeinerten Schaftformmodell zu einer deutlich verbesserten Schätzgenauigkeit der formbeschreibenden Funktionsparameter und damit der Schaftform führt. Für eine Anwendung in einem Einheitschaftformmodell bzw. für eine Regionalisierung müssen jedoch metrische Standorts- oder Bestandesvariablen identifiziert werden, die ebenfalls zu einer verbesserten Schätzung der formbeschreibenden Paramter verwendet werden können. Da die Sektionsmessungen nur Unterabteilungen nicht aber einzelnen Versuchsparzellen zugeordnet werden können, ist die Zuordnung von Bestandesvariablen nur sehr eingeschränkt möglich. Ein Schwerpunkt zukünftiger Forschung zur Verbesserung der Schätzgenauigkeit von Schaftformfunktionen sollte daher in der Erfassung des Einflusses von Standortsvariablen (KoRHONEN, 1993) wie z. B. der Seehöhe liegen, von denen angenommen werden kann, daß sie die Schätzgenauigkeit zusätzlich zu Einzelbaumvariablen erhöhen.

Wie bereits erläutert (Kapitel 3.2.1 u. 3.4.1), können bei der Parameterschätzung im Rahmen der Verallgemeinerung von Modellen dadurch Probleme auftreten, daß unterschiedliche Parameterkombinationen ähnliche Funktionsverläufe beschreiben. Die Zusammenhänge der formbeschreibenden Parameter zu unabhängigen Variablen werden somit durch die Korrelation der Parameter untereinander überlagert, was die Prognose der Parameter erschwert. Ein Indiz für die gegenseitige Beeinflussung der Parameter sind hohe Korrelationskoeffizienten zwischen den Funktionsparameter, wie sie auch bei der Pain-Funktion auftreten. Weitergehende Möglichkeiten, um eine effektivere Verallgemeinerung von Modellen zu erreichen, können daher darin bestehen, Modelltypen auf der untergeordneten Ebene (Einzelbaum, Stratum, Bestand) zu konzipieren, in denen die Koeffizienten statistisch unabhängig voneinander sind bzw. in denen die Koeffizientenabhängigkeiten nur aus den Dateneigenschaften resultieren. Ein Beispiel hierfür wurde bei der Transformation der Formparameter der BetaVerteilung zur Entwicklung eines Modells für die Beschreibung von Astdurchmessern beschrieben (Kapitel 3.4.1). Auch bei der Entwicklung des hierarchischen Kronenansatzmodells wurde die statistische Unabhängigkeit der Koeffizienten als vorteilhaft für eine Verallgemeinerung des bestandesindividuellen Modells beurteilt.

Für die Schaftformfunktion sollten daher Methoden zur Transformation der unabhängigen Variablen untersucht werden, die eine Beseitigung der Korrelation zwischen den einzelnen Koeffizienten ermöglichen. Auch die sogenannten Random Coefficient Modelle bieten Möglichkeiten, die Abhängigkeiten von Regressionskoeffizienten bei der Verallgemeinerung zu erfassen bzw. zu berücksichtigen (LAPPI, 1986). 


\subsection{Gesamtbetrachtung des Modellsystems}

Das Gesamtsystem der beschriebenen Modelle eignet sich zur Prognose der qualitätsrelevanten äußeren und inneren Astigkeit unter Berücksichtigung verschiedener Astparameter sowie zur Prognose der Stammform bzw. Abholzigkeit der Baumarten Fichte, Douglasie, Kiefer und Buche. Die entwickelten Schaftformmodelle erlauben darüber hinaus für die genannten Baumarten und die Eiche Sortimentsschätzungen. Die genaueren Prognosen der neu entwikkelten Kronenansatzmodelle wirken sich sowohl bei der Einschätzung von Qualitätsaspekten als auch auf die Wachstumsprognose positiv aus. Aus den Astdurchmessermodellen können zusätzlich Informationen für die Modellierung realitätsnäherer Kronenformen abgeleitet werden.

Die Astparametermodelle weisen momentan eine regional stark eingeschränkte Datenbasis auf. Trotz der vergleichsweise geringen Datenumfänge erfassen aber insbesondere die Astdurchmessermodelle für die Baumarten Fichte und Douglasie bereits deutlich unterschiedliche Konkurrenzsituationen der Einzelbäume und damit unterschiedliche waldbauliche Bestandesbehandlungen. Für die Erweiterung des Geltungsbereiches müssen jedoch für alle Baumarten speziell an den Randbereichen der Verteilungen der verwendeten Variablen weitere Datenerfassungen erfolgen. Insbesondere sind dabei Astmessungen an Bäumen mit extremen H/D-Werten wie Solitären und überschirmten Bäumen sowie Astmessungen an sehr starken und schwachen Bäumen notwendig.

Die Kronenansatzmodelle weisen dagegen eine sehr umfangreiche Datenbasis auf, wobei jedoch der zukünftigen Berücksichtigung von Bäumen mit solitärähnlichen Eigenschaften eine große Bedeutung zukommt. Für die Modelle zur Prognose der Übergangs- und Totastzone sind zusätzliche Datenerhebungen notwendig. Allerdings reichen aufgrund der geringen Flexibilität der Modelle bzw. fehlender statistischer Zusammenhänge relativ wenige ergänzende Messungen aus. Die Datengrundlage der Schaftformmodelle weist ebenfalls eine breite regionale Repräsentativität auf und beinhaltet ein breites Dimensionsspektrum. Da vor allem in Richtung der solitärähnlichen Bäume mit niedrigen H/D-Werten eine starke Formveränderung zu beobachten ist (Abb. 75), sollten Sektionsmessungen von Solitären zu einer Stabilisierung der flexiblen Schaftformmodelle verwendet werden.

Das entwickelte Modellsystem kann, abgesehen von den notwendigen Ergänzungen der Parametrisierungsgrundlage und einzelnen modelltechnischen Verbesserungsmöglichkeiten, bereits als ausreichend zur Entwicklung eines Qualitätsmoduls für EinzelbaumWachstumsmodelle beurteilt werden. Die Einsatzmöglichkeiten von Qualitätsmodulen wurden bereits in Kapitel 1.2 (Zielsetzung) umfassend beschrieben. Momentan ist die Prognose der erfaßten Gütemerkmale ausreichend genau für die Modellierung der äußeren Astigkeit für die Rohholzsortierung und der inneren Astigkeit für die Schnittholzsortierung. Auch läßt die 
flexible Schaftformmodellierung die Beurteilung des Merkmals Abholzigkeit bei der rechnerischen Rohholzsortierung und in Bezug auf die Schnittholzausbeute zu. Für die Modellierung spezieller Holzeigenschaften im Rahmen unterschiedlicher Holzverwendungen (s. auch Kapitel 1.2) können die entwickelten Qualitätsmodelle nur Teilaspekte bereitstellen. So würden etwa für die Festigkeitsprüfungen von Schnittholz lediglich die durch die Astparameter, nicht aber die durch die Jahrringbreite bzw. die Holzdichte bestimmten Festigkeitsmaße eingeschätzt werden können.

Durch die Kombination mit virtuellen Sägewerken ${ }^{22}$ wie Seesaw (GARCIA, 1987; PARK, 1989), Autosaw (PARK U. Todoroki, 1992; BARbour ET AL., 1999) sowie mit Algorithmen zur Optimierung der Aushaltung des Roh- und Schnittholzes (BRIGgS, 1980; MendozA U. BARE, 1986; Sessions ET AL., 1989; PuUMALAINEN, 1997) können Szenarien der optimalen Wertschöpfung bei unterschiedlichen Ausgangsqualitäten des Rohholzes ermittelt werden. Auch für solche nachgeschaltete, komplexe Auswertungen sowie Visualisierungen stellt das Modellsystem bereits eine ausreichende Grundlage dar.

Neben den mittelfristig zusätzlich im Modellsystem integrierbaren Modellen zur Erfassung der Anteile an juvenilem Holz, der Kernholzanteile bei obligaten Kernholzbildnern sowie von Holzdichteverteilungen auf der Basis der Jahrringbreite sollten zukünftig vor allem zwei weitere Gütemerkmale im Qualitätsmodul integriert werden. Es handelt sich dabei um den Buchenrotkern und die Rotfäule bei Fichte (Kapitel 2.6). Der Buchenrotkern führt bei Überschreitung bestimmter Stammdurchmesseranteile zu einer Sortierung in die Güteklassen BK bzw. C von äußerlich in die Güteklasse B oder A (Furnier) eingestuften Stämmen. Die Rotfäule bei Fichte ist neben der Astigkeit das entscheidende Kriterium für eine Einstufung in die Güteklasse $\mathrm{C}$ und dominierendes Kriterium für die Einstufung in die Güteklassen CGW bzw. D. In Kapitel 2.2 sind die besonderen Schwierigkeiten bei der Modellierung der fakultativen Gütemerkmale bereits beschrieben worden.

Für die Modellierung der Rotfäule und des Buchenrotkerns werden daher Modellentwicklungen angestrebt, die Informationen über die Merkmalsverteilung in Abhängigkeit von der Einzelbaumdimension aus den vorhergehenden Eingriffen bei der Prognose berücksichtigen bzw. fortschreiben. Zusätzlich muß überprüft werden, inwieweit allgemeingültige Zusammenhänge beispielsweise zum Bestandesalter, zu Standortsvariablen, zur Rückeintensität etc. in den Modellen berücksichtigt werden können (vgl. Kapitel 2.6).

Die in digitaler Form vorliegenden Einschlagsergebnisse stellen dabei die einzig praktikable Möglichkeit für eine umfangreiche Datenerfassung dar. Voraussetzung für die Verwendung der Einschlagsergebnisse ist jedoch, daß der Grund für die Herabstufung eines Stamm-

\footnotetext{
${ }^{22}$ Unter virtuellen Sägewerken werden hier Benutzerprogramme verstanden, die die Simulation von unterschiedlichen Einschnittsmustern und -verfahren zur Prognose der Schnittholzausbeute und -qualität ermöglichen.
} 
stücks erfaßt wird. D. h., daß eine Buche, die aufgrund ihrer Astigkeit oder Stammkrümmung in die Güteklasse C sortiert wird, von einer Buche zu unterscheiden ist, die aufgrund von Rotkern in die Güteklasse C sortiert wird. Das gleiche gilt für eine Einstufung von Fichtenstammholz in die Güteklasse C aufgrund seiner Astigkeit bzw. des rotfaulen Stammdurchmessers.

Liegen zusätzlich subjektive Einschätzungen von Stammzahlanteilen für weitere Gütemerkmale vor, können diese nach der Anwendung des entwickelten Modellsystems berücksichtigt werden, indem die jeweiligen Attribute zufällig auf die Einzelbäume verteilt werden. In Abb. 79 ist zusammenfassend der Einsatz des hier entwickelten Modellsystems zur Prognose der äußeren und inneren Astigkeit sowie der Stammform bzw. Abholzigkeit im Rahmen von Wachstumssimulationen dargestellt. 

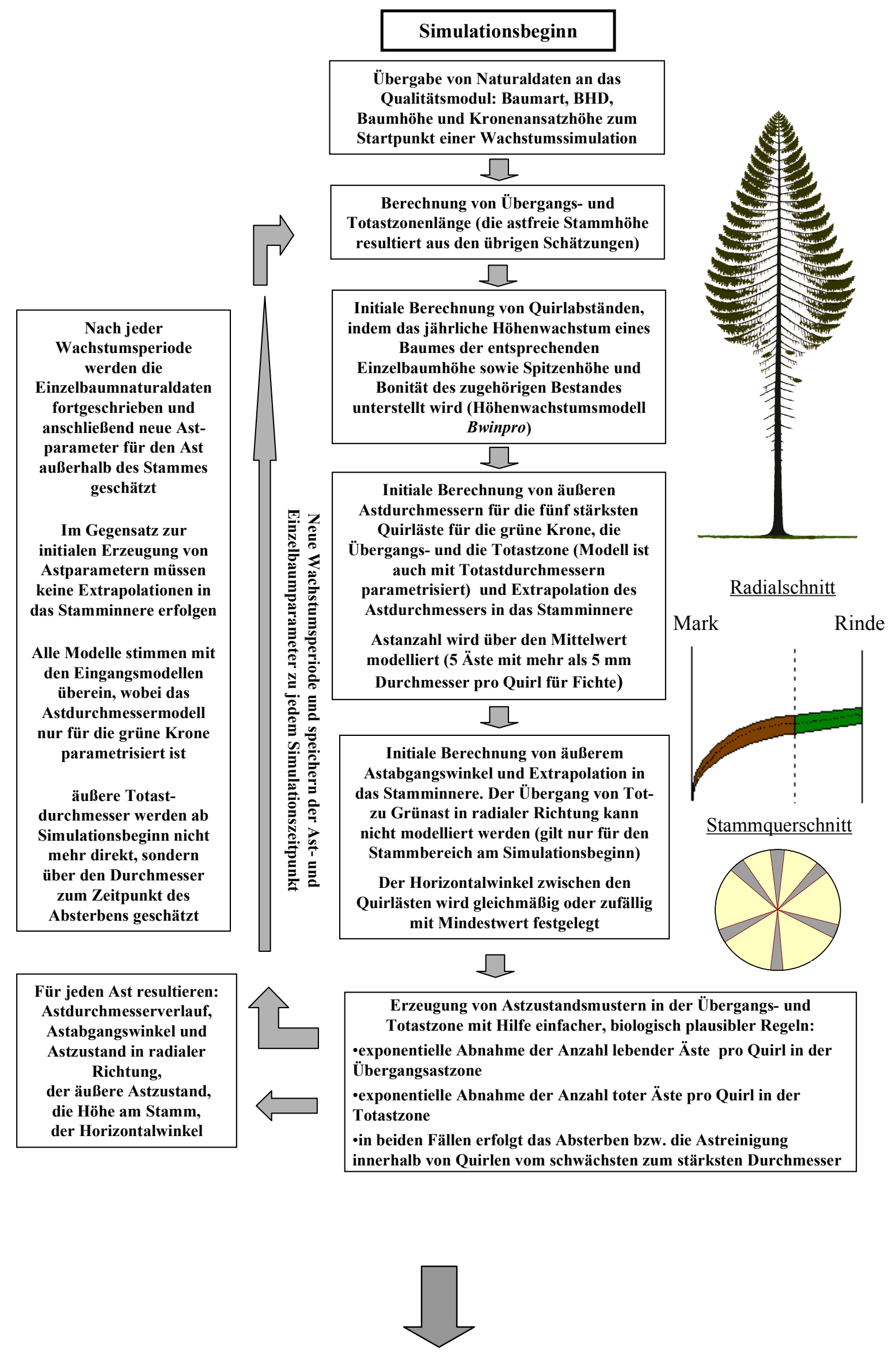


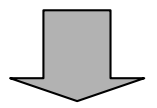

Simulationsende: Prognose der inneren und äußeren Astigkeit

Behandlungssensitive Beschreibung der Schaftformentwicklung über der Zeit auf der Basis der zu jedem Simulationsschritt abgespeicherten BHD- und Höhen-Werte

Berechnung von Volumen- und Durchmesseranteilen für den Grün- und Totastbereich sowie den äußerlich astfreien Bereich, Prognose an astfreien Stammradien auf beliebigen Höhen

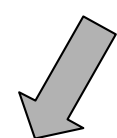

Rohholzqualität

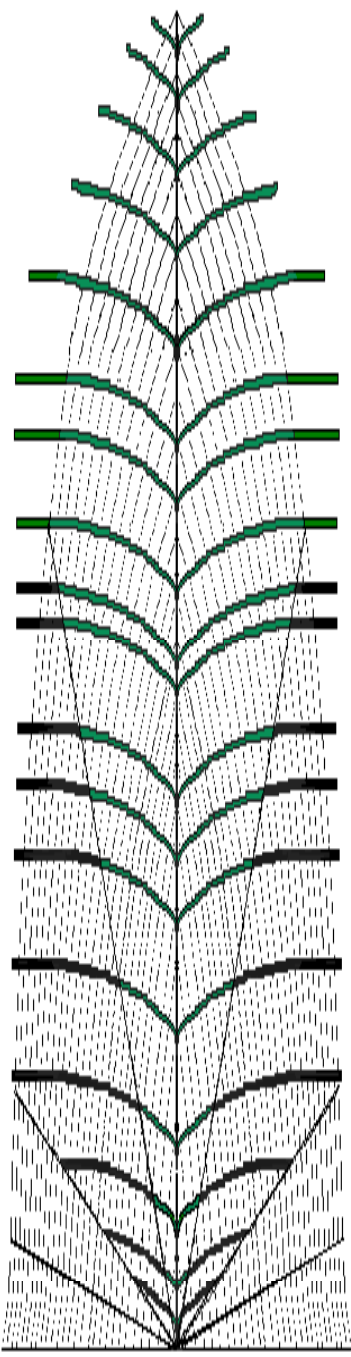

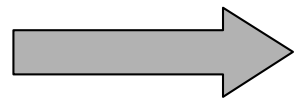

Grünastzone

Übergangsastzone

Totastzone

Narben bzw. Beulenzone

Zone ohne äußere Astmerkmale

\section{$\underline{\text { Stammquerschnitt }}$}

Simulation von Einschnittsmustern

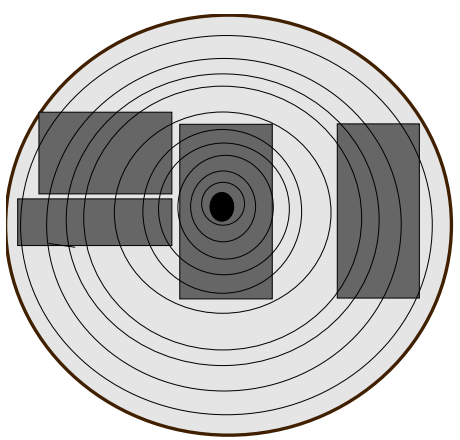

Prognose der durch die Astigkeit beeinflußten Schnittholzqualität und der von der Schaftform beeinflußten Schnittholzausbeute

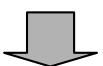

$\underline{\text { Schnittholz mit Ästen }}$

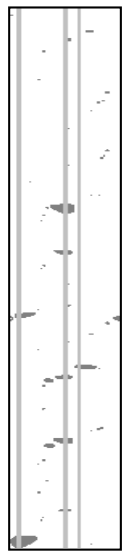

Abb. 79: Schematische Darstellung der Modellierung der äußeren und inneren Astigkeit sowie der Schaftform auf der Basis von Naturaldaten, die von einem Einzelbaumwachstumssimulator zur Verfügung gestellt werden und mit Hilfe von verschiedenen Astparametermodellen und einem Schaftformmodell. 


\section{$5 \quad$ Zusammenfassung}

In den 1990er Jahren haben in den deutschsprachigen Ländern Europas statistische Einzelbaumwachstumssimulatoren einen Entwicklungsstand erreicht, der ihren Einsatz in der forstlichen Praxis ermöglicht. Diese Simulatoren eröffnen aufgrund ihrer Konzeption die Möglichkeit, die Entwicklung von unterschiedlichsten Bestandesstrukturen sowie die Auswirkungen verschiedener waldbaulicher Behandlungsvarianten zu prognostizieren. Zusätzlich bieten sie die notwendigen Voraussetzungen, um die hohe Datenqualität und -auflösung moderner Inventurkonzepte bei der Wachstumsprognose zu berücksichtigen und die Ergebnisse auf der Einzelbaumebene bereitzustellen.

Diese naturalen Prognoseergebnisse bilden wiederum die Voraussetzung für weitergehende Auswertungen wie die Berechnung von Stärkeklassensortimentierungen zur Bewertung der Nutzfunktion oder von Strukturindizes zur Bewertung der Schutzfunktion von Waldbeständen. Eine realitätsnahe Bewertung der Nutzfunktion erfordert neben der Prognose der Stärkeklassenverteilung auch die Prognose der Güteklassenverteilung bzw. der Rohholzqualität. Programme zur rechnerischen Stärkeklassensortimentierung bieten daher häufig die Möglichkeit, Anteile an verschiedenen Güteklassen gutachterlich vorzugeben. Für bestimmte Gütemerkmale, wie die Aststärke und die Schaftform (Abholzigkeit), bestehen jedoch relativ enge Zusammenhänge zur Dimension und zu den Wachstumsbedingungen des Einzelbaumes. Ziel der Arbeit war deshalb die Entwicklung von behandlungssensitiven, statistischen Modellen zur Prognose der Gütemerkmale Astigkeit (Baumarten: Fichte, Douglasie, Kiefer, Buche) und Schaftform (Baumarten: Fichte, Douglasie, Kiefer, Buche, Eiche). Die Modelle beschreiben den Zusammenhang zwischen den Wachstumsbedingungen und der Holzqualität des Einzelbaumes. Sie schaffen damit die Voraussetzung, um die Auswirkungen waldbaulicher Maßnahmen bzw. der Bestandesstruktur auf die Holzqualität zu quantifizieren. Für die Auswahl der Qualitätskriterien war neben den vorhandenen Zusammenhängen zu den Wachstumsbedingungen eines Baumes auch die Relevanz für die Sortierentscheidung maßgebend.

Im Rahmen dieser Arbeit wurde zunächst eine neue Klassifikation für Gütemerkmale entwickelt, die im Gegensatz zu herkömmlichen Einteilungen grundsätzliche Unterschiede der Modellentwicklung als Klassifikationskriterium verwendet. Es werden lediglich die Klassen obligate und fakultative Gütemerkmale unterschieden. Die Klassifikation dient der Verdeutlichung von spezifischen Problemen der Modellierung für die beiden Merkmalsklassen sowie als Hilfe bei der Modellkonzeption. Die obligaten Gütemerkmale resultieren aus den Wachstumsprozessen eines Baumes (z. B. die Aststärke), während die fakultativen Merkmale weder am Einzelbaum noch im Bestand zwingend auftreten (z. B. die Rotfäule). Aufgrund dieses wesentlichen Unterschiedes sind bei den fakultativen Merkmalen drei, bei den obliga- 
ten Merkmalen lediglich eine Modellebene für die vollständige Erfassung des jeweiligen Merkmals notwendig.

Die Beschreibung der Astigkeit umfaßt die Teilaspekte Aststärke, Astdichte und den Astzustand. Der Astzustand wurde mit Hilfe von Modellen zur Beschreibung der Länge der grünen Krone, der Übergangsastzone ${ }^{23}$ sowie der Totastzone erfaßt. In statistischen Wachstumsmodellen wird die Länge der grünen Krone über Kronenansatzmodelle gesteuert. Neben der Bedeutung für die Prognose des Astzustandes beeinflußt die Kronenansatzschätzung auch die Prognose der maximalen Aststärken eines Baumes, da zwischen der Kronenlänge und der Lebensdauer von Ästen enge Zusammenhänge bestehen. Aufgrund der großen Bedeutung der Kronenansatzhöhe für eine behandlungssensitive Prognose der Astparameter lag ein weiterer Schwerpunkt der Arbeit in der Entwicklung neuer Kronenansatzmodelle. Es wurden mehrere Typen von Kronenansatzmodellen für die Baumarten Fichte, Douglasie, Kiefer, Europäische Lärche, Buche und Eiche entwickelt, die eine an der Auflösung der Eingangsdaten orientierte Prognose erlauben. Die Modelle sind im einzelnen:

I. Ein zweistufiges hierarchisches Modell, das aus einem bestandesindividuellen Modell und einem Modell zur Verallgemeinerung dieses Modells mit Hilfe von Bestandesvariablen besteht.

II. Ein einfaches statisches Modell, das auf einem von Pretzsch und KAHN (1998) bzw. WeNSEL ET AL. (1987) verwendeten Modellansatz basiert, zusätzlich jedoch die Spitzenhöhe als unabhängige Variable verwendet.

III. Ein Einheitskronenansatzmodell, das auf dem Einheitshöhenkurvenmodell von SLOBODA ET AL. (1993) basiert, jedoch zur Einhängung eine Schätzung des Kronenverhältnisses des Spitzenhöhenstammes benötigt.

IV. Ein Modell zur Dynamisierung des Einheitskronenansatzmodells über die Fortschreibung des Kronenverhältnisses des Spitzenhöhenstammes.

Die Modelle (I) und (II) weisen gegenüber mehreren Vergleichsmodellen zum Teil deutliche Verbesserungen bei der Schätzgenauigkeit auf, die durch die Beseitigung systematischer Fehlerkomponenten erreicht werden. Dabei ist die zusätzliche Verwendung der Spitzenhöhe entscheidend, die der Beschreibung des bestandesübergreifenden Dimensionstrends ${ }^{24}$ dient. Gleichzeitig wird durch die Verwendung der Spitzenhöhe erreicht, daß die Variablen BHD und H/D-Wert die gleichen Wirkungsrichtungen haben, die bei einer bestandesindividuellen

\footnotetext{
${ }^{23}$ Als Übergangsastzone wird in dieser Arbeit der Stammbereich bezeichnet, in dem sowohl grüne als auch Totäste auftreten.

${ }^{24}$ Der bestandesübergreifende Dimensionstrend beschreibt den Zusammenhang, daß Bäume mit gleichen Einzelbaumdimensionen, um so geringere Kronenverhältnisse aufweisen, je höher der Bestand (Spitzenhöhe $\mathrm{H}_{100}$ ) ist, aus dem sie stammen.
} 
Parametrisierung beobachtet werden können. Das einfache statische Modell (II) besitzt gegenüber dem hierarchischen Modell (I) bei etwas höheren Anforderungen an die Eingangsdaten konzeptionelle Vorteile bei der Beschreibung unterschiedlicher Datenstrukturen.

Das Einheitskronenansatzmodell (III) dient der Berücksichtigung hochwertiger Informationen, wie einiger gemessener Kronenansatzhöhen oder des Kronenverhältnisses des Spitzenhöhenstammes, die aber für die Anpassung eines bestandesindividuell parametrisierten Regressionsmodells nicht ausreichen. Derartige Informationen können beispielsweise im Rahmen von Waldbegängen bei der Höhenmessung zur Bonitierung erhoben werden und stehen für Stichprobenpunkte der Betriebsinventur zur Verfügung. Um diese hochwertigen Informationen über den Startpunkt einer Wachstumssimulation hinaus verwenden zu können, wurde mit dem dynamisierten Einheitskronenansatzmodell (IV) ein Ansatz zur Fortschreibung des Kronenverhältnisses des Spitzenhöhenstammes entwickelt. Dieses Modell weist im Vergleich zum einfachen statischen Modell (II) eine deutlich höhere Schätzgenauigkeit bei der Prognose für kurz- bis mittelfristige Zeiträume auf. Es eignet sich damit hervorragend für die Fortschreibung der Kronenansatzhöhe im Rahmen der Forsteinrichtung.

Da die aktuellen Datenstrukturen von gleichaltrigen Rein- und Mischbeständen dominiert werden, hat der Einzelbaum-H/D-Wert zusätzlich zu den verwendeten Dimensionsvariablen BHD und Spitzenhöhe keinen deutlichen Einfluß auf die Schätzung des Kronenverhältnisses. Anhand einer sehr heterogenen Unterstichprobe kann jedoch gezeigt werden, daß die Modelle behandlungssensitive Schätzungen erlauben, wenn die gesamte Bandbreite möglicher Bestandesstrukturen bei der Parametrisierung ausreichend repräsentiert wird. Weiterhin setzt eine realitätsnahe Beschreibung der Kronenansatzhöhe die behandlungssensitive Prognose des H/D-Wertes im verwendeten Wachstumssimulator voraus.

Wichtigster Astparameter für die Prognose der Holzqualität ist der Astdurchmesser. Für die Baumarten Fichte, Douglasie und Kiefer wurden behandlungssensitive Modelle zur Prognose der stärksten Quirlastdurchmesser in der grünen Krone entwickelt. Die Behandlungssensitivität wird durch den deutlichen Einfluß des H/D-Wertes gewährleistet, der bei einer Erweiterung der Datengrundlage auch die Prognose für überschirmte Bäume möglich erscheinen läßt.

Für die Fichte wurden zusätzlich Modelle zur Prognose der fünf stärksten Astdurchmesser pro Quirl entwickelt, die konzeptionell auf die anderen Nadelholzarten übertragen werden können. Bei der Buche werden aufgrund der uneinheitlichen Kronentypen nur Äste im Kronenansatzbereich beschrieben. Äste in der Totastzone werden für alle Baumarten über die Kombination mit einem Wachstumsmodell eingeschätzt, wobei die zum Zeitpunkt der Astmortalität geltenden Astparameter übernommen werden. Der Geltungsbereich der Astdurchmessermodelle wird auf die grüne Krone beschränkt, da vor allem dieser Bereich durch die 
aktuellen Baumeigenschaften beeinflußt wird. Eine Ausnahme ist die initiale Astdurchmesserschätzung für die Totastzone zum Simulationsbeginn.

Das neu entwickelte Astdurchmessermodell zeichnet sich neben der Behandlungssensitivität durch eindeutige, biologisch plausible Wirkungsrichtungen der unabhängigen Variablen aus. Diese Modelleigenschaft wird durch eine an parameterkargen Schaftformmodellen orientierte Konzeption erreicht. Auch das Astdurchmessermodell kann als zweistufig betrachtet werden, da einzelbaumweise Parametrisierungen auf der Basis von Asteigenschaften mit Hilfe von Einzelbaumattributen verallgemeinert werden.

Für die Baumart Buche tritt bei der Verwendung eines regressionsanalytischen Ansatzes zur Prognose der Astdurchmesser im Kronenansatzbereich eine hohe Residualstreuung auf. Daher wurde als Modelltyp ein verallgemeinertes Verteilungsmodell auf der Basis der BetaVerteilung verwendet, das Informationen über die Form der Residualstreuung bei der Generierung von Astdurchmesserwerten berücksichtigen kann. Die (standardisierte) BetaVerteilung wurde verwendet, da sie implizit auf das Intervall $[0,1]$ beschränkt ist und sich daher modelltheoretisch gut zur Beschreibung der mit dem BHD normierten Astdurchmesser eignet. Die Form und Lage der spezifischen Astdurchmesserverteilungen werden über die Variablen BHD und "mittlere Tiefe in der Krone" der betrachteten Äste beschrieben. Letztere ist eng mit der Kronenlänge korreliert und gewährleistet damit ebenfalls eine Behandlungssensitivität, was allerdings eine möglichst exakte Prognose der Kronenansatzhöhe voraussetzt.

Neben der Aststärke muß für eine realitätsnahe Holzqualitätsprognose auch die Astdichte erfaßt werden. Für die Nadelholzarten wurde daher die Anzahl der Äste pro Quirl bestimmt, die einen Mindestdurchmesser von $5 \mathrm{~mm}$ aufwiesen. Es konnten jedoch, in teilweiser Übereinstimmung mit ähnlichen Untersuchungen, keine Zusammenhänge zu Quirl- oder Einzelbaumparametern identifiziert werden, so daß die Beschreibung mit Hilfe des Mittelwertes erfolgt. Der Quirlabstand, der die Astdichte ebenfalls beeinflußt, wird über das Höhenwachstumsmodell des jeweiligen Wachstumssimulators geschätzt.

Die qualitätsbestimmenden Äste der Baumart Buche sind im Gegensatz zu den Nadelholzarten nicht in Quirlen angeordnet. Für die Bestimmung der Astdichte wurde daher ein Modell für die Prognose der Astabstände im Kronenansatzbereich entwickelt. Das Modell beinhaltet unter anderem die Tiefe in der Krone als unabhängige Variable, so daß auch hier eine behandlungssensitive Schätzung möglich ist.

Die Modelle zur Beschreibung des Astzustandes schätzen nicht die Höhe des untersten Grün- und Totastes, sondern die Länge der Übergangs- und Totastzone. Dadurch ist eine Kombination mit der Schätzung der Kronenansatzhöhe möglich, die als Ausgangswert für die Astzonenlängen verwendet wird. Damit kann die unterschiedliche Prognosegenauigkeit der Kronenansatzschätzung (s. o.) auch bei der Schätzung der Höhe des untersten Grün- und To- 
tastes berücksichtigt werden. Für die Länge der Übergangsast- und Totastzone konnte keine Behandlungssensitivität festgestellt werden.

Ein weiterer Schwerpunkt der Arbeit ist die Entwicklung von flexiblen Schaftformmodellen. Die Schaftform (Abholzigkeit) ist ein Gütemerkmal für die Einschätzung der Rohholzqualität. Weiterhin erfordert die Prognose der inneren Astigkeit inklusive der Einschätzung von qualitativ unterschiedlichen Stammholzanteilen die Kombination der Astmodelle mit Schaftformmodellen und einem Wachstumssimulator. Auf der Basis eines von PAIN und BOYER (1996) vorgestellten Modells (Pain-Funktion) wurde ein neues Schaftformmodell (Modifizierte Pain-Funktion) entwickelt.

Beide Versionen gehören zur Gruppe der parameterkargen Modelle (HUI U. GADOW, 1997). Der Modellansatz ist zweistufig, d. h. ein Modell, das in einem ersten Schritt einzelbaumweise parametrisert wurde, wird in einem zweiten Schritt mit Hilfe von Einzelbaumvariablen verallgemeinert. Weitergehende eigene Untersuchungen deuten an, daß auch eine Verallgemeinerung bzw. Regionalisierung auf der Basis von Standortsvariablen, wie der Höhenlage, möglich erscheint.

Das Modell von PAIN und BOYER (1996) weist gegenüber mehreren Vergleichsmodellen eine größere Flexibilität gegenüber dem H/D-Wert auf. Wie bei den Astdurchmessermodellen ist damit eine behandlungssensitive Prognose möglich. Die größere Flexibilität wird in der präziseren Prognose für Bäume an den Rändern des Formenspektrums, insbesondere stark abholziger, Bäume deutlich. Eine Verbesserung konnte allerdings nur für die untersuchten Nadelholzarten erreicht werden, da nur diese deutliche Zusammenhänge zwischen dem H/DWert und der Stammform aufweisen. Für die Laubholzarten besitzt die als Vergleichsmodell verwendete modifizierte Brink-Funktion (RIEMER ET AL., 1995) eine ähnliche Modellgüte.

Das Modell von PAIN und BOYER (1996) besitzt den methodischen Nachteil, daß der durch die Funktion prognostizierte Wert in $1.3 \mathrm{~m}$ Höhe nicht mit dem Eingangswert des BHD übereinstimmt. Die neu entwickelte modifizierte Pain-Funktion weist demgegenüber nur noch geringfügige Abweichungen vom Eingangswert auf (99.7\% der untersuchten Fichten hatten eine Abweichung von unter $1 \mathrm{~mm}$ ). Durch die Vorgabe, daß der Funktionswert auf $1.3 \mathrm{~m}$ Höhe mit dem Eingangswert für den BHD übereinstimmen soll, wird die Flexibilität der Funktion eingeschränkt. Die modifizierte Pain-Funktion ist daher ein segmentierter Modelltyp, der den unteren und oberen Schaftabschnitt getrennt beschreibt. Die Vorteile gegenüber der Originalversion sind für die Douglasie und die Kiefer geringfügig. Bei der Fichte wird eine Verbesserung der Schätzung für den untersten Stammbereich erreicht. Die Vorteile für die Baumarten Buche und Eiche sind am deutlichsten. Somit kann die modifizierte Pain-Funktion abschließend als gut geeignet zur Prognose der Schaftform bei allen Baumarten beurteilt werden, wobei die Schaftform der Nadelholzarten behandlungssensitiv beschrieben wird. 
Das entwickelte Modellsystem aus Kronenansatz-, Ast- und Schaftformmodellen erlaubt eine umfassende Prognose der äußeren Astigkeit und Schaftform für die Baumarten Fichte, Douglasie, Kiefer und Buche und ermöglicht damit eine kombinierte rechnerische Stärke- und Güteklassensortimentierung für Rohholz im Rahmen von waldbaulichen Szenariensimulationen. Die überwiegende Anzahl der verwendeten Modelle ist behandlungssensitiv bzw. weist konzeptionell eine theoretische Behandlungssensitivität für zukünftig heterogenere Datenstrukturen auf.

Die Kombination der Astmodelle mit Schaftformmodellen und Wachstumssimulatoren ermöglicht zusätzlich eine umfassende Prognose der inneren Astigkeit und liefert daher Eingangsdaten für weitergehende Auswertungen, wie die Prognose der Schnittholzqualität und die Optimierung der Schnittholzausbeute. Die flexible, am einzelnen Gütemerkmal orientierte Modellierung erlaubt eine Prognose auch bei sich ändernden Sortiergrenzwerten und speziellen Qualitätsanforderungen sowie die Ergänzung mit gutachterlichen Schätzungen oder Informationen aus Holzeinschlägen für zusätzliche Qualitätsmerkmale.

Das Modellsystem ist die Grundlage für die Entwicklung eines Programmoduls zur kombinierten rechnerischen Stärke- und Güteklassensortimentierung auf der Basis naturaler Prognosergebnisse von Einzelbaumsimulatoren sowie der Ergebnisse von Betriebs- und Bestandesinventuren. Ein solches Programmodul ermöglicht eine umfassende Beschreibung der Nutzfunktion von Waldbeständen und ist damit eine Voraussetzung für die multikriterielle Bewertung waldbaulicher Szenariensimulationen sowie realer Waldbestände und Bestandesstraten. 


\section{Summary}

In the 1990s, statistical single-tree growth models reached a developmental standard allowing their implementation in forest management. Due to their conception, these growth simulators provide the possibility predicting the growth of varying stand structures, as well as the effects of silvicultural treatments. In addition, they provide the necessary prerequisites of taking the high-quality data and resolution of modern inventories for growth prognoses into consideration and to providing the results at the single-tree level.

The prognoses results allow for advanced analyses such as calculation of size class assortment distribution for validating the utility benefit, or of structural indices for validating the proctective role of forest stands. A realistic assessment of the utility benefit demands among the assortment prognosis for size classes, a prognosis of the quality class distribution or the single-timber quality. Application programs for calculative grading of size classes therefore offer the possibility of defining rates of different quality classes. However, for certain quality criterion such as diameter of nodal branches and taper form (tapering), close connections to dimensions and growth conditions of a tree exist. The purpose of this thesis was therefore to develop treatment sensitive, statistical models for the prognosis of the wood quality criterion branchiness (tree species: Norway spruce, Douglas fir, Scots pine, European beech) and taper form (tree species: Norway spruce, Douglas fir, Scots pine, European beech and oak / sessile and common oak combined). The derived models describe the connection between growth conditions and the wood quality of a single tree. For this reason, they lay the foundations of quantifying the effects of silvicultural treatments and stand structure on wood quality. Apart from existing connections of a quality criterion to the growth condition of a tree, the relevance for the grading decision was also decisive in selecting quality criteria.

In the context of this thesis, initially a new classification for quality criteria was developed that, in contrast to conventional classifications, uses principle differences in modelling. Only the classes obligatory and facultative quality criteria are differentiated. The classification points up specific problems in modelling for both classes and supports model conception. The obligatory quality criteria results from growth processes of a tree (e.g. branch diameter), whereas facultative criteria, neither at tree level nor at stand level, invariably occur (e.g., root rot). Due to this essential difference, three modelling levels for facultative criteria and merely one level for obligatory criteria are necessary for a total gathering of the respective characteristic.

The description of branchiness covers the aspects: branch diameter, branch density and branch status. The branch status was assessed using models for estimating the length of the 
living crown, the transition zone $\mathrm{e}^{25}$ as well as the dead branch zone. In statistical growth models, the length of the living crown is often affected by the model for estimating height to crown base. Apart from the importance for prognosis of branch status, the estimation of height to crown base influences the prognosis of maximum branch diameter of a tree, since a strong connection between crown length and life span of branches exists. Due to the strong relevance of the height to crown base for a treatment sensitive prognosis of branchiness, a further emphasis of this thesis lays on the development of new models for estimating height to crown base. Several types of height to crown base models have been derived for Norway spruce, Douglas fir, Scots pine, European larch, European beech and oak (sessile and common oak combined), that allow for different resolution of raw data. Specified, the models are:

I. A two-stage hierarchical model consisting of an individual model for single stands and a model for generalizing the individual model on the basis of stand variables.

II. A simple statistical model based on an approach from PRETZSCH and KAHN (1998) and WENSEL ET AL. (1987), but additionally uses dominant height $\left(\mathrm{H}_{100}\right)$ as a predictor variable.

III. A generalized height to crown base model based on an approach by SLOBODA ET AL. (1993), but which for calibration needs an estimation of the crown ratio of the dominant height stem $\left(\mathrm{H}_{100}\right)$.

IV. A model for periodic adjustment of the generalized height to crown base model (III) by updating the crown ratio of the dominant height stem $\left(\mathrm{H}_{100}\right)$.

The models (I) and (II) compared to several existing models, feature explicit improvements for estimation accuracy by removal of systematic error components. Thereby the additional use of dominant height is important, that integrates an overall dimension trend ${ }^{26}$. Simultaneously, the use of dominant height effects coefficient signs for the variables dbh and height/dbh ratio that can be observed for an individual parameterization of single stands. The simple statistical model (II), when compared with the hierarchical model (I), shows slightly higher requirements for the raw data, but shows conceptional advantages for considering different data structures.

The generalized height to crown base model (III) considers high quality raw data such as some measured crown base heights or a measured crown ratio of the dominant height stem that are not allowed for a parameterization of an individual stand regression model. Such in-

\footnotetext{
${ }^{25}$ The stem area is indicated as a transition zone, which contains living as well as dead branches.

${ }^{26}$ The overall dimension trend describes the fact that trees which feature the same single tree height, show the lower crown ratios the higher the stand (dominant height) they belong to.
} 
formation can, for instance, be derived within standard standwise inventories at the height measurement for site index calculation and often yet exist for sample plots of permanent inventories. To use this high quality information beyond the starting point of a growth simulation, a dynamic Version (IV) of the generalized height to crown base model that updates the crown ratio of the dominant height stem was developed. This model, when compared with the simple statistically approach (II), shows an obviously higher precision for short- and mid-term prognoses. For this reason, the model is well-suited for updating height to crown base data for forest management purposes.

Since the current data base for parameterization is dominated by even-aged pure and mixed species stands, the height/dbh-ratio of single trees, in addition to the applied size variables dbh and dominant height, has only weak influence on the estimation of crown ratio. However, an approach using a quite heterogeneous sub-sample as the data base shows sensitive estimation with the developed models, because of a better representation of the wide range of possible silvicultural management strategies and results. Furthermore, a realistic description of height to crown base assumes a sensitive prognosis of height/dbh-ratio in the applied growth simulator.

The most important branchiness parameter for prognosis of wood quality is the diameter of nodal branches. For Norway spruce, Douglas fir and Scots pine, treatment-sensitive models for estimating maximum branch diameter for each whorl in the living crown have been developed. The sensitivity against silvicultural treatments is ensured by the distinct influence of height/dbh ratio, so that by enlarging the data base, a prognosis for understory trees should also be possible.

For Norway spruce, models for prognosis of the five thickest branches per whorl have been additionally developed, that conceptionally can be transferred to the other conifer species as well. For European beech, only branches near the height of crown base are described, because of the irregular crown structures in these broadleafed species. Branches within the dead branch zone are prognosed in combination with a growth model, whereas branch diameter at death of a branch is used. The scope of the branch diameter models is limited to the living crown, since primarily this zone is influenced through current properties of a tree. An exception from this conception is the initial estimation of branch diameters at the starting point of a growth simulation.

Apart from treatment sensitivity, the new branch diameter model features explicit, biologically realistic effects of the predictor variables. These properties are a result of the model conception that is oriented at parameter parsimonious taper functions. The branch diameter model could also be considered as a two-stage approach, since individual single tree parameterization on the basis of branch properties is generalized using tree variables. 
For European beech, a high residual variance occurs when using a regression approach for prognosis of diameter of branches located near the crown base. Thus, a generalized distribution model on the basis of the Beta distribution was used to take information about residual variance into account when generating branch diameters. The (standardized) Beta distribution was used since it is implicitly limited to the interval [0.1] and thus, is theoretically well-suited for a description of standardized (on the basis of $\mathrm{dbh}$ ) branch diameters. Form and location of specific branch diameter distributions are estimated with the help of the predictor variables dbh and "mean depth into crown" of the considered branches. The latter is strongly correlated with crown length and thus, its use ensures a treatment sensitivity, which however requires the most exact estimation of height to crown base as possible.

Apart from branch diameter, density of branches had to be estimated for a realistic prognosis of wood quality. Thus for conifer species, the number of branches per whorl was investigated that reached at least a minimum diameter of $5 \mathrm{~mm}$. Consistent with similar investigations, no connections to whorl or tree variables could be detected, so that the mean is used for modelling. The interwhorl distance that also influences branch density is estimated with the help of the height growth model of the used growth simulator.

In contrast to the conifer species, the branches relevant for the wood quality of European beech are not located in whorls. Thus for estimating branch density, a model for prognosis of branch distances in the crown base zone was derived. Among other things, the model includes "depth into crown" as a predictor variable, so that a sensitive estimation is also possible for this aspect.

The models for predicting branch status do not estimate the heights to the lowest living and dead branch, but the length of branch transition and dead branch zone. Thus a combination with the estimation of height to crown base is possible, whereby height to crown base is used as a reference value. Thus, different estimation accuracies of height to crown base (see above) can also be regarded at estimation of the height to the lowest dead and living branch. For the length of branch transition and dead branch zone, no sensitivity against treatments could be detected.

Another emphasis of this thesis was the development of flexible taper functions. Stem form (tapering) is a criterion for assessing timber quality. Furthermore, the prognosis of inner branchiness, including estimation of rates of different wood quality, demands the combination of branchiness models with taper functions and a growth simulator. On the basis of a model presented by PAIN and BOYER (1996) (Pain function), a new taper model was derived (modified Pain function).

Both versions belong to the category of parameter parsimonious models (HUI and GADOw, 1997). The approach is two-staged, i.e., a model that is parameterized for single trees in a first step, is generalized on the basis of tree variables. Advanced investigations indicate 
that a generalization and regionalization on the basis of site variables such as altitude might be possible.

The model developed by PAIN and BOYER (1996) shows a high flexibility against height/dbh ratio, compared with other models. As for branch diameter models, a treatment sensitive prognosis is thus possible. The higher flexibility is obvious when looking at the prognosis for trees at the edges of the form range, particularly for trees with a heavy tapering of stem form. Indeed, an improvement only for the investigated conifer species could be derived, since only these show explicit connection between height/dbh ratio and taper form. For the explored broadleafed species, the modified Brink function (RIEMER ET AL., 1995) that was used in comparison, showed a similar model accuracy.

The model developed by PAIN and BOYER (1996) feature the methodological disadvantage that the prognosed value at $1.3 \mathrm{~m}$ height does not equal the measured or default value for dbh. In contrast, the modified Pain function shows only slight deviations from the default value $(99.7 \%$ of the explored Norway spruce trees had a deviation of less than $1 \mathrm{~mm})$. The specification to adjust the estimated value for dbh with the default one, restricts the flexibility of the resulting function. The modified Pain function is therefore conceived as a segmented model type that describes the lower and upper part of the stem separately. The improvements against the original version of the Pain function for Douglas fir and Scots pine are slight. For Norway spruce, an improvement for the lowest part of the stem is derived. The advantages for European beech and oak (sessile and common oak combined) are the most obvious. Thus, the modified Pain function could be assessed as well-suited for prognosis of taper form for all investigated tree species, whereas the taper form of conifer trees is described as treatment sensitive.

The developed model system, including models for height to crown base, branch properties and taper form, allows for an extensive prognosis of outer branchiness and stem form for Norway spruce, Douglas fir, Scots pine and European beech and thus, for a combined calculative grading of timber in silvicultural scenario simulation concerning size and quality aspects. The vast majority of the derived models shows a sensitivity against silvicultural treatments or is conceptionally constructed for sensitive estimation, if a more heterogeneous data structure will arise.

The combination of branch and taper models in the framework of a growth simulator additionally allows for an extensive prognosis of inner branchiness and supplies therefore data for advanced analyses as a prognosis of trimmed timber or an optimization of recovery of trimmed timber. The flexible modelling that is based on the single quality criteria allows for a prognosis, even if threshold values for grading are changing or special quality requirements are desired. A supplementation with expert knowledge from inventories or with information from previous harvests for additional quality criteria is also possible. 
The model framework is the base for the development of a module for a combined calculative grading regarding size and quality aspects using data that can either be supplied by a growth simulator or extracted from inventories at the enterprise or stand level. Such a module enables an extensive gathering of utility benefits of forest stands and thus, it is an essential tool for a multi-objective evaluation of silvicultural scenario simulation, as well as of real forest stands and stand stratums. 


\section{$7 \quad$ Literatur}

A

AKৎA, A., 1993: Zur Methodik und Bedeutung der kontinuierlichen Forstinventuren. AFJZ 164. Jg. Nr. 11: S. 193 - 198.

Albert, M. UND GADOW, K. V., 1998: Strukturparameter als Kontrollinstrumente für Überführungsvorhaben. In: HANEWINKEL, M. (Hrsg.) 1998: Überführung von Altersklassenwäldern in Dauerwälder. Freiburger Forstliche Forschung Heft 8: S. 14 - 37.

AlBERT, M., 2000: Ein funktionalisierter Höhenergänzungsalgorithmus für Einzelbaumwachstumsmodelle. Jahrestagung der Sektion Ertragskunde des DVFFA in Kaiserslautern, 05.-07.06.2000: S. 32 - 43.

Altherr, E., Unfried, P., Hradetzky, J. Und Hradetzky, V., 1978: Statistische Rindenbeziehungen als Hilfsmittel zur Ausformung und Aufmessung unentrindeten Stammholzes. Teil IV: Fichte, Tanne, Douglasie und Sitka-Fichte. Mitteilungen der Forstlichen Versuchs- und Forschungsanstalt Baden-Württemberg Heft 90: S. 294.

ANDERSON, D. J. UND BARE, B. B., 1994: A dynamic programming algorithm for optimization of uneven-aged forest stands. Canadian Journal of Forest Research Vol. 24: S. 1758 1765 .

\section{B}

Badoux, A., 1939: De l'influence de diverse modes et degrés d'éclairicie dans les hetraies pures. Mitteilungen der Schweizerischen Anstalt für das forstliche Versuchswesen Bd. 21: S. 58 - 146.

Barbour, J., Lowell, E. C., Todoroki, C. L., Parry, D. L. und Christensen, G. A., 1999: Simulating North american lumber grade recovery with AUTOSAW using externally visible branch and stem form characteristics. In: NEPVEU, G. (ed.), 1999: Proceedings of the Third IUFRO-Workshop on the "Connection between Silviculture and Wood Quality through Modelling Approaches and Simulation Softwares". La Londe-les-Maures, France, September 5-12, 1999: 621 S.: 493 - 504.

BARTEls, H., 1993: Gehölzkunde: Einführung in die Dendrologie. Ulmer, Stuttgart: 336 S. BeCKER, G. UND SeEling, U., 1998: Holzqualität der Fichte. AFZ 53. Jg. Nr. 8: S. 434 - 435. Beimgraben, T., Kirsch, E. Und BeCKer, G., 1998: Wachstumsspannungen im Buchenstammholz. AFZ /DerWald 199. Jg. Nr. 26: S. 1593 - 1594.

BI, H., 2000: Trigonometric variable-form taper equations for australian eucalyptus. Forest Science Vol. 46 No. 3: S. 397 - 409. 
BIBER, P., 1999: Ein Verfahren zum Ausgleich von Randeffekten bei der Berechnung von Konkurrenzindizes. Jahrestagung 1999 der Sektion Ertragskunde des DVFFA in Kevelaer, Tagungsbericht: S. 189 - 202.

Biging, G. S. UND DobBertin, M., 1995: Evaluation of competition indices in individual tree growth models. Forest Science Vol. 41 No. 2: S. 360 - 377.

BML, 1969: Verordnung über gesetzliche Handelsklassen für Rohholz.

BöcKmanN, T., SpellmanN, H. UND HÜSING, F., 1998: Neukonzeption und Weiterentwicklung der Forsteinrichtung in Niedersachsen. Forst und Holz 53. Jg. Nr. 10: S. 298 - 302.

BÖCKMANN, T. UND HÜSING, F., 1999: Weiserflächenkonzeption in der Niedersächsischen Landesforstverwaltung. Forst und Holz 54. Jg. Nr. 10: S. 299 - 302.

BÖRNER, M., 1997: Zur Rotkernigkeit der Buche- Prüfung der Zusammenhänge mit Einzelbaumparametern und Modellierung der Auswirkungen auf den Rohholzerlös. Deutscher Verband Forstlicher Forschungsanstalten - Sektion Ertragskunde, 12.-14. Mai, 1997 Grünberg: S. 126 - 138.

Bos, F., Chiorescu, S., Constant, T., Jaeger, M., Mothe, F. und Thibaut, B., 1999: Simulating the rotary-cutting of a softwood: Modelling the variations of the veneer properties and the consequences on the plywood quality. In: NePVEU, G. (ed.), 1999: Proceedings of the Third IUFRO-Workshop on the "Connection between Silviculture and Wood Quality through Modelling Approaches and Simulation Softwares". La Londe-les-Maures, France, September 5-12, 1999: 621 S.: S. 290-293.

Bossel, H., 1996: TREEDYN3 forest simulation model. Ecol. Modelling 90: S. 187 - 227.

BRAun, H. J., 1988: Bau und Leben der Bäume. Verlag Rombach Wissenschaft, Freiburg: $295 \mathrm{~S}$.

BRINK, C. UND GADOW, K. V., 1986: On the use of growth and decay functions for modelling stem profiles. EDV in Medizin und Biologie 17, 1/2: S. 20 - 27.

BRIGGS, D. G., 1980: A dynamic programming approach to optimizing stem conversion. $\mathrm{Ph}$. D. dissertation. College of Forest Resources, University of Washington. Seattle, WA: $397 \mathrm{~S}$.

BRUNNER, A., 1998: A light Model for spatially explicit forest stand models. Forest Ecology and Management Vol. 107 No. 1-3: S. 19 - 46

Burger, H., 1939: Kronenaufbau gleichaltriger Nadelholzbestände. Mitt. Schweiz. Anst. f. d. forstl. Vers.-Wesen Bd. 21: S. 5 - 58.

Burschel, P., 1981: Neue Erziehungskonzepte für Fichtenbestände. AFZ 152. Jg. Nr. 36: S. $1386-1395$. 
C

Chadoeuf, J., Pierrat, J. C., Nandris, D., Geiger, J. P. und Nicole, M., 1993: Modeling Rubber Tree Root Disease Epidemics with a Markov Spatial Process. Forest Science Vol. 39. No. 1: S. 41 - 54.

Clark, P. J. UND Evans, F.C., 1954: Distance to nearest neighbor as a measure of spatial relationships in populations. Ecology Vol. 35: S. 445 - 453.

Colin, F. Und Houllier, F., 1991: Branchiness of Norway spruce in north-eastern France: modelling vertical trends in maximum nodal branch size. Annales des Sciences Forestieres 48: S. 679 - 693.

Colin, F. Und Houllier, F., 1992: Branchiness of Norway spruce in north-eastern France: predicting the main crown characteristics from usual tree measurements. Annales des Sciences Forestieres 49: S. 511 - 538.

Courbaud, B., Houllier, F. und Sinoquet, H., 1996: Modelling the growth of Norway spruce (Picea abies) in mountain heterogeneous forests. In: NEPVEU, G. (ed.), 1996: Proceedings of the Second Workshop "Connection between Silviculture and Wood Quality through Modelling Approaches and Simulation Softwares“. Berg-en-Dal, Kruger National Park, South Africa, August 26-31, 1996. Publication Equipe de Recherches sur la Qualité des Bois 1997/7, December. INRA-Nancy, France: 450 S.: S. 313 - 320.

Cown, D. J., 1973: Comparison or the effects of two thinning regimes on some properties of radiata pine. NZ Journal of Forestry Science Vol. 4 No. 3: S. 540 - 551.

COWn, D. J., HÉBERT, J. UND BALl, R., 1999: Modelling Radiata pine lumber characteristics. Part 1: Mechanical properties of small clears. In: NEPVEU, G. (ed.), 1999: Proceedings of the Third IUFRO-Workshop on the "Connection between Silviculture and Wood Quality through Modelling Approaches and Simulation Softwares". La Londe-lesMaures, France, September 5-12, 1999: 621 S.: S. 294 - 302.

CuRTIS, R. O., 1982: A simple index of stand density for Douglas-fir. Forest Science. Vol. 28 No. 1: S. 92 - 94.

\section{D}

Dahlblom, O., Persson, K., Ormarsson, S. und Pettersson H., 1999: Stiffness and shape stability analysis of sawn timber based on experimentally found variations of wood properties. In: NEPVEU, G. (ed.), 1999: Proceedings of the Third IUFRO-Workshop on the "Connection between Silviculture and Wood Quality through Modelling Approaches and Simulation Softwares". La Londe-les-Maures, France, September 5-12, 1999: 621 S.: S. $363-375$. 
Delorme, A., 1999: Naturnahe Forstwirtschaft und ihre Auswirkungen auf die Holzverwendung. Forst und Holz 54. Jg. Nr. 9: S. 264 - 268.

DEUTSCHES INSTITUT FÜR NORMUNG, 1989: Sortierung von Nadelholz nach der Tragfähigkeit; Nadelschnittholz ; DIN 4074-1, 1989; Beuth Verlag, Berlin. (In Vorbereitung [NormEntwurf] befindet sich eine überarbeitete Norm für Nadelschnittholz und eine Norm für Laubschnittholz).

DEUTSCHES INSTITUT FÜR NORMUNG, 1997 a: Laub-Rundholz - Qualitäts-Sortierung - Teil 1: Eiche und Buche; DIN EN 1316-1:1997; Beuth Verlag, Berlin.

DeUTSCHES INSTITUT FÜR NORMUNG, 1997 b: Laub-Rundholz - Qualitäts-Sortierung - Teil 2: Pappel; DIN EN 1316-2:1997; Beuth Verlag, Berlin.

DEUTSCHES INSTITUT FÜR NORMUNG, 1997 c: Laub-Rundholz - Qualitäts-Sortierung - Teil 3: Esche und Ahorn; DIN EN 1316-3:1997; Beuth Verlag, Berlin.

Deutsches Institut FÜr NoRmung, 1997 d: Rund- und Schnittholz - Messung der Merkmale; DIN EN 1310:1997; Beuth Verlag, Berlin.

DEUTSCHES INSTITUT FÜR NORMUNG, 1999 a: Qualitäts-Sortierung von Nadel-Rundholz Teil 1: Fichten und Tannen; DIN ENV (Vornorm) 1927-1:1998; Beuth Verlag, Berlin.

DEUTSCHES INSTITUT FÜR NORMUNG, 1999 b: Qualitäts-Sortierung von Nadel-Rundholz -

Teil 2: Kiefern; DIN ENV (Vornorm) 1927-2:1998; Beuth Verlag, Berlin.

DEUTSCHES INSTITUT FÜR NORMUNG, 1999 c: Qualitäts-Sortierung von Nadel-Rundholz -

Teil 3: Lärchen und Douglasien; DIN ENV (Vornorm) 1927-3:1998; Beuth Verlag, Berlin.

Deutsches InstituT FÜr NoRmung, 1999 d: Schnittholz - Sortierung nach dem Aussehen von Nadelholz - Teil 1: Europäische Fichten, Tannen, Kiefern und Douglasie; DIN EN 1611-1: 1999; Beuth Verlag, Berlin.

DÜSER, R., 1978: Programmierte Berechnung stehenden Holzes mit dem Taschenrechner nach der Düser-Flori-Formel. Beilage der AFJZ 33. Jg. Nr. 36: ohne Paginierung.

$\mathbf{E}$

EFron B., 1979: Bootstrap methods: another look at the jackknife. Annals of Statistics Vol. 7.: S. 1 - 26.

EFron, B. Und Tibshirani, R. J., 1993: An Introduction into the Bootstrap. Monographs on statistics and applied probability 57. Chapman \& Hall, New York: 436 S.

EK, A. R., 1974: Dimensional relationships of forest and open-grown stems in Wisconsin. Univ. Of Wisconsin. Forestry Res. Note 181: $7 \mathrm{~S}$.

EK, A. R. Und Monserud, R. A., 1974: Forest: A computer model for simulating the growth and reproduction of mixed species stands. Univ. Wisconsin, College of Forestry, Dep. of Forest Resources Staff Paper Series 20: 25 S. 
EllenberG, H., 1986:Vegetation Mitteleuropas mit den Alpen in ökologischer Sicht. Ulmer, Stuttgart, 4. verb. Aufl.: 989 S.

\section{$\mathbf{F}$}

FISCHER, H. W., 1994: Untersuchungen der Qualitätseigenschaften, insbesondere der Festigkeit von Douglasien-Schnittholz, erzeugt aus nicht-wertgeästeten Stämmen. Dissertation, Forstliche Fakultät der Universität Göttingen. S.

Forster, R. B. Und CALlahan, J. C., 1968: Allocating indiana hardwood timber in multiple product situations. Purdue University Agriculture Exp. Sta., Research Bulletin 843 Lafayette, IN.: $18 \mathrm{~S}$.

Fourcaud, T., Blaise, F., Barthelemy, D., Houllier, F. und De Reffye, Ph., 1996: A physiological approach for tree growth modelling in the software AMAPpara. In: NePveU, G. (ed.), 1996: Proceedings of the Second Workshop "Connection between Silviculture and Wood Quality through Modelling Approaches and Simulation Softwares“. Berg-en-Dal, Kruger National Park, South Africa, August 26-31, 1996. Publication Equipe de Recherches sur la Qualité des Bois 1997/7, December. INRA-Nancy, France: 450 S.: S. 321 - 334.

\section{G}

Gadow, K. V., Hui, G.Y. UND AlBert, M., 1998: Das Winkelmaß - ein Strukturparameter zur Beschreibung der Individualverteilung in Waldbeständen. Centralblatt $\mathrm{f}$. d. gesamte Forstwesen 115. Jg. Heft 1 : S. 1 - 10.

GADOW, K. V., 1984: Die Erfassung von Durchmesserverteilungen in gleichaltrigen Kiefernbeständen. Forstw. Cbl. 103. Jg. : S. 360 - 374.

GADOW, K. V., 1987: Untersuchungen zur Konstruktion von Wuchsmodellen für schnellwüchsige Plantagenbaumarten. Forstliche Forschungsberichte München Nr. 77: $147 \mathrm{~S}$.

GADOW, K. V. UND SCHMIDT, M., 1998: Periodische Inventuren und Eingriffsinventuren. Forst und Holz 53. Jg. Nr. 22: S. 667 - 671.

Gadow, K. V. und PuUmalainen, J., 1998: Neue Herausforderungen für die Waldökosystemplanung. AFZ / Der Wald 53. Jg. Nr. 20: S. 1248 - 1250.

GAFFREY, D., 1996: Sortenorientiertes Bestandeswachstums-Simulationsmodell auf der Basis intraspezifischen, konkurrenzbedingten Einzelbaumwachstums -insbesondere hinsichtlich des Durchmessers- am Beispiel der Douglasie. Berichte des Forschungszentrums Waldökosysteme Reihe A Bd. 133. Fachbereiche Geowissenschaften und Biologie und Forstwissenschaftlicher Fachbereich, Universität Göttingen: 413 S. 
GARCIA, O., 1987: A visual sawing simulator; Part 2: The SEESAW computer program. In Proceedings of the Conversion Planning Conference. J. A. Kininmonth (Comp.) Ministry of Forestry. FRI Bulletin 128: S. 97 - 106.

GAFFREY, D. UND NAGEL, J., 1998: Lineares Schaftformmodell für Douglasie für das nördliche und mittlere Westdeutschland. AFJZ 169. Jg. Nr. 8: S. 144 - 148.

GiLl, S. J., Biging, G. S. Und MurPhy, E. C., 2000: Modeling conifer tree crown radius and estimating canopy cover. Forest Ecology and Management 126: S. 405 - 416.

Gove, J. H. Und FAIRWEATHER, S. E., 1992: Optimizing the Management of Uneven-Aged Forest Stands: A Stochastic Approach. Forest Science Vol. 38: S. 623 - 640.

Grace, J. C. Und PonT, D., 1997: Modelling Branch Development in Radiata Pine. In: Amaro, A., Tomé, M. (ed.), Empirical and process-based models for forest tree and stand growth simulation, 21-27 September 1997, Oeiras, Portugal: S. 173-184.

Grammel, R., 1989: Forstbenutzung. Verlag Paul Parey, Hamburg und Berlin: 193 S.

GRAMMEL, R., 1990: Zusammenhänge zwischen Wachstumsbedingungen und holztechnologischen Eigenschaften der Fichte. Forstw. Cbl. 109. Jg.: 119-129.

GRIESEL, F. H. UND GADOW, K. V., 1995: Waldbauliche Zielsetzungen in Mitteleuropa (Begriffsbestimmungen, Konzepte und Richtlinien, Beispielhafte Anwendungen). Arbeitspapier, Institut für Forsteinrichtung und Ertragskunde der Universität Göttingen: 16 S.

GRUBER, F., 1987: Beiträge zum morphogenetischen Zyklus der Knospe, zur Phyllotaxis und zum Triebwachstum der Fichte (Picea abies [L.] Karst.) auf unterschiedlichen Standorten. Berichte des Forschungszentrums Waldökosysteme/Waldsterben Reihe A Bd. 25. Fachbereiche Geowissenschaften und Biologie und Forstwissenschaftlicher Fachbereich, Universität Göttingen: $215 \mathrm{~S}$.

Grünebaum, M., Teutenberg-Raupach, A. und Paul, C., 1993: Ein Weitverband bei Traubeneiche - Auswirkungen auf Wachstum, Schnittholzqualität und Furniereignung. Forst und Holz 48. Jg. Nr. 1: S. 3 - 7.

GUERICKE, M., 2001: Untersuchungen zur Wuchsdynamik von Mischbeständen aus Buche und Europ. Lärche (Larix decidua, Mill.) als Grundlage für ein abstandsabhängiges Einzelbaumwachstumsmodell. Dissertation, Forstliche Fakultät der Universität Göttingen. $269 \mathrm{~S}$.

\section{H}

HaAra, A., Maltamo, M. und Tokola, T., 1997: The k-nearest neighbour method for estimating basal-area distribution. Scandinavian Journal of Forest Research Vol. 12 No. 2: S. $200-208$. 
HAHN, G. J., UND SHAPIRO, S. S., 1967: Statistical models in engineering. New York: Wiley.

HANN, D. W., 1999: A adjustable predictor of crown profile for stand-grown Douglas-Fir trees. Forest Science Vol. 45 No. 2: S. 217 - 225.

HAPLA, F., 1981: Untersuchung der Auswirkung verschiedener Pflanzverbandsweiten auf die Holzeigenschaften der Douglasie (Pseudotsuga menziesii [Mirb.] Franco). Forstarchiv 52. Jg. Nr. 4 und 5.: S. 134 - 143 und S. 186 - 190.

HAPLA, F., 1986: Beeinflussen unterschiedliche Durchforstungsmaßnahmen die Holzeigenschaften der Douglasie? Forstarchiv 57. Jg. Nr. 3: S. 99 - 104.

HAPLA, F. UND SAUTER, U. H., 1987: Auswirkungen der Hoch- und Niederdurchforstung auf Zuwachs, Rohdichte, und Festigkeit von Douglasien aus 100-jährigen Beständen. Der Forst- und Holzwirt 42. Jg. Nr. 12: S. 310 - 314.

Hapla, F., KNigge, W. und Rommerskirchen, 1987: Physikalische Holzeigenschaften und Zuwachs von schadsymptomfreien und immissionsgeschädigten Kiefern. Forstarchiv 58. Jg. Nr. 5: S. 211 - 216.

HAPLA, F. UND SABOROWSKI, J., 1994: Quantifizierung der Kernvolumina von Nadelbäumen mit obligatorischer Farbkernholzbildung. Holz als Roh- und Werkstoff 52: S. 205 - 209.

HÄRDLE, W., 1989: Applied nonparametric regression. Cambridge University: 323 S.

HASENAUER, H., 1994: Ein Einzelbaumwachstumssimulator für ungleichaltrige Fichten, Kiefern- und Buchen-Fichtenmischbestände. Forstliche Schriftenreihe Universität für Bodenkultur, Wien Bd. 8: $152 \mathrm{~S}$.

Hasenauer, H., 2001: Konzepte der Waldökosystemmodellierung. Centralblatt f. d. gesamte Forstwesen 117. Jg. Nr. 3/4: S. 137 - 164.

HeCKeR, M. UND BECKER, G., 1997: Möglichkeiten zur Beeinflußung der Oberflächenrauhigkeit von Douglasien-Furnieren durch waldbauliche Maßnahmen. Forstw. Cbl. 116: S. $45-52$.

HöIbÖ, O. A., Vestöl, G. I., Sundby, H. J. und MolteberG, D. E., 1996: Modelling knottiness and knot characteristics of Norway spruce (Picea abies (L.) Karst): the influence of diameter and height growth on knot characteristics. In: NEPVEU, G. (ed.), 1996: Proceedings of the Second Workshop "Connection between Silviculture and Wood Quality through Modelling Approaches and Simulation Softwares“،. Berg-en-Dal, Kruger National Park, South Africa, August 26-31, 1996. Publication Equipe de Recherches sur la Qualité des Bois 1997/7, December. INRA-Nancy, France: 450 S.: S. 45 - 48.

Houllier, F., LEBAN, J. M. UND COLIN, F., 1995: Linking growth modelling to timber quality assessment for Norway spruce. Forest Ecology and Management 74: S. 91-102.

HrAdETZKY, J., 1981: Spline Funktionen und ihre Anwendung in der forstlichen Forschung. Forstw. Cbl. 100: S. 45 - 49. 
HuI, G. Y. UND GADOw, K. V., 1993: Zur Entwicklung von Einheitshöhenkurven am Beispiel der Baumart Cunninghamia lanceolata. AFJZ 164. Jg. Nr. 12: S. 218 - 220.

HUI, G. Y. UND GADOW, K. V., 1997: Entwicklung und Erprobung eines Einheitsschaftmodells für die Baumart Cunninghamia lanceolata. Forstw. Cbl. 116: S. 315 - 321.

Hussein, K. A., 2001: Parameter-parsimonious models for crown and stem profiles. Dissertation, Fakultät für Forstwissenschaften und Waldökologie der Universität Göttingen: 118 $\mathrm{S}$.

\section{I}

INSTITUT FÜR DEN WISSENSCHAFTLICHEN FILM, 1998: Ökosystemforschung Wald. Göttingen, CD-ROM. ISBN 3-540-14670-9.

\section{$\mathbf{K}$}

KAHN, M., 1995: Quasikausale Modellierung des Standort-Leistung-Bezuges als Voraussetzung zum Aufbau flexibler Mischbestandsmodelle. Forstw. Cbl. 114. Jg. : S. 175 - 187.

KÄNDLER, G., 1986: Die Ermittlung von Bestandesparametern als Eingangsgröße für Interzeptionsmodelle mit Hilfe aerophotogrammetrischer Verfahren. Mitteilungen der Forstlichen Versuchs- und Forschungsanstalt Baden-Württemberg Heft 127: $129 \mathrm{~S}$.

KELLOMÄKI, S., 1981: Effect of the within-stand light conditions on the share of stem, branch and needle growth in a twenty-year-old scots pine stand. Silva Fennica Vol. 15 No. 2: S. $130-139$.

KELlOMÄKI, S. UND VÄISÄNEN, H., 1988: Dynamics of branch population in the canopy of young scots pine stands. Forest Ecology and Management 24 : S. 67 - 83.

KellomäKi, S., OKer Blom, P., VAltonen, E. Und VÄIsÄnen, H., 1989: Structural development of Scots pine stands with varying initial density: effect of pruning on branchiness of wood. Forest-Ecology-and-Management 27, 3-4: S. 219 - 233.

KellomäKi, S., Peltola, H. UND IKonen, V. P, 1999: Modelling the structural growth and timber quality of Scots pine related to silvicultural management. In: NEPVEU, G. (ed.), 1999: Proceedings of the Third IUFRO-Workshop on the "Connection between Silviculture and Wood Quality through Modelling Approaches and Simulation Softwares". La Londe-les-Maures, France, September 5-12, 1999: 621 S.: S. 565 - 570.

KENK, G., 1990: Fichtenbestände aus Weitverbänden - Entwicklung und Folgerungen. Forstw. Cbl. 109. Jg. : S. 86 - 100.

Kershaw, J. A. JR., Maguire, D. A. und Hann, D. W., 1990: Longevity and duration of radial growth in Douglas-fir branches. Canadian Journal of Forest Research Vol. 20 No. 11: S. 1690 - 1695. 
Kleinman, J. C., 1973: Proportions with Extraneous Variance: Single and Independent Samples. JASA 68. Jg.: S. 46 - 54.

Knigge, W. und Schulz, H., 1996: Grundriss der Forstbenutzung. Verlag Paul Parey, Hamburg und Berlin: $584 \mathrm{~S}$.

KNIGGE, W., 1970: Probleme der Gütesortierung nach der Verordnung über gesetzliche Handelsklassen für Rohholz. Der Forst- u. Holzwirt 25. Jg. Nr. 12: S. 273 - 276.

KNOKE, T., 1999: Ökonomische Aspekte der Holzproduktion in ungleichaltrigen Wäldern; Auswirkungen großflächiger Überführungsmaßnahmen. In: HANEWINKEL, M. (Hrsg.), 1999: Überführung von Altersklassenwäldern in Dauerwälder. Berichte Freiburger Forstl. Forschung 8: S. 175 - 189.

KöHL, M., ScOTT, C. T. UND ZINGG, A., 1995: Evaluation of permanent sample surveys for growth and yield studies: a Swiss example. Forest Ecology and Management 71: S. 187 $-194$.

Korhonen, K. T., 1993: Mixed estimation in calibration of volume functions of Scots pine. Silva Fennica Vol. 27 No. 4: S. 269 - 76.

KoZAK, A. UND YANG, R. C., 1978: Height-diameter curves, another application of the Weibull function in forestry. In: Mitteilungen der Forstlichen Versuchs- und Forschungsanstalt Baden-Württemberg Heft 91 Nr. 28: S. 171 - 185.

Kramer, H., Dong, P. H. und Rusack, H. J., 1971: Untersuchung der Baumqualität in weitständig begründeten Fichtenbeständen. AFJZ 142. Jg. Nr. 2: S. 33 - 46.

KRAMER, H., 1977: Zur Qualitätsentwicklung junger Kiefernbestände in Abhängigkeit vom Ausgangsverband. Der Forst- und Holzwirt 32. Jg. Nr. 23: S. 469 - 476.

Kramer, H., 1988: Waldwachstumskunde. Verlag Paul Parey, Hamburg und Berlin: 374 S.

KRANIGK, J., UND GRAVENHORST, G., 1993: Ein dreidimensionales Modell für Fichtenkronen AFJZ 164. Jg. Nr. 8: S. $146-149$.

KRANIGK, J., 1996: Ein Modell für den Strahlungstransport in Fichtenbeständen. Dissertation, Mathematisch-Naturwissenschaftlicher Fachbereich der Universität Göttingen. Cuvillier, Göttingen: $124 \mathrm{~S}$.

KREMPL, H. UND MARK, E., 1962: Untersuchungen über den Kern der Rotbuche. Allgemeine Forstzeitschrift (Wien) 73 Jg. Nr. 15-16: S. 186 - 191.

Kublin, E. und Scharnagl, G., 1988: Verfahrens- und Programmbeschreibung zum BWIUnterprogramm BDAT. Abschlußbericht, Forstliche Versuchs- und Forschungsanstalt Baden-Württemberg, 7800 Freiburg i. Br.: $87 \mathrm{~S}$.

Kupper, L. L., Portier, C., Hogan, M. D. und Yamamoto, E., 1986: The Impact of Litter Effects on Dose - Response Modeling in teratology. Biometrics Vol. 42. : S. 85 - 98. 
KURTH, W., 1994: Growth grammar interpreter GROGRA 2.4 : a software tool for the 3dimensional interpretation of stochastic, sensitive growth grammars in the context of plant modelling; introduction and reference manual. Berichte des Forschungszentrums Waldökosysteme der Universität Göttingen Reihe B Bd. 38: 192 S.

\section{$\mathbf{L}$}

VAN LAAR, A. UND MOSANDL, R., 1989: Durchmesserverteilungen in Eichenjungbeständen. AFJZ 160. Jg. Nr. 9-10: S. 189 - 194.

LAASASENAHO, J., 1982: Taper curve and volume functions for pine, spruce and birch. Comm. Inst. For. Fenn. 108: S. 1 - 74.

LaAsasenaho, J., 1987: Use of Taper Curve in Determining Tree Branchiness and Preparing Instructions on Stand Densities. Paper presented at the IUFRO Forest Growth Modelling and Prediction Conference, Minneapolis, MN, August 24-28, 1987: S. 1014 - 1020.

LÄMSÄ, P., KellomÄKI, S. UND VÄISÄNEN, H., 1990: Branchiness of young Scots Pine as related to stand structure and site fertility (in Finnish). Folia For. Helsinki 746: S. 1 - 22.

LAPPI, J., 1986: Mixed linear Models for Analysing and Predicting Stem Form Variation of Scots Pine. Communicationes Instituti Forestalis Feniae 134, Helsinki: 69 S.

Leban, J. M., Daquitaine, R., Houllier, F. Und SAINT-AndRÉ, L., 1996: Linking models of tree growth and wood quality in Norway spruce. Part I: validation. In: NEPVEU, G. (ed.), 1996: Proceedings of the Second Workshop "Connection between Silviculture and Wood Quality through Modelling Approaches and Simulation Softwares“. Berg-en-Dal, Kruger National Park, South Africa, August 26-31, 1996. Publication Equipe de Recherches sur la Qualité des Bois 1997/7, December. INRA-Nancy, France: 450 S.: S. $220-228$.

LEWANDOWSKI, A. UND GADOW, K. V., 1997:Ein heuristischer Ansatz zur Reproduktion von Waldbeständen. AFJZ 168. Jg. Nr. 9 : S. 170 - 174.

LIU, S., BAO, F. UND JIANG Z., 1999: Modelling the relationships between wood properties and quality of veneer and plywood in plantations Poplar. In: NEPVEU, G. (ed.), 1999: Proceedings of the Third IUFRO-Workshop on the "Connection between Silviculture and Wood Quality through Modelling Approaches and Simulation Softwares". La Londe-les-Maures, France, September 5-12, 1999: 621 S.: S. 334 - 340.

LÖFFLER, H., 1965: Kriterien der Bewertung von Nadelstammholz. Schriftenreihe der forstl. Abteilung der Universität Freiburg Bd. 4. 


\section{M}

MaguiRe, D. A. und HanN, D. W., 1990: Constructing models for direct prediction of 5-year crown recession in southwestern Oregon Douglas-fir . Canadian Journal of Forest Research Vol. 20: S. 1044 - 1052.

Maguire, D. A., Moeur, M. und Bennett, W. S., 1990: Simulating branch diameter and branch distribution for Douglas-fir. Publ. FWS-2-90, Virginia Polytechnic Institute and State University, School of Forestry and Wildlife Ressources, Blacksburg VA: S. 85 94.

Maguire, D. A., Kershaw, J. A. JR. und Hann, D. W., 1991: Predicting the effects of silvicultural regime on branch size and crown wood core in Douglas-fir. Forest Science Vol. 37 No. 5: S. 1409 - 1428.

Maguire, D. A., Moeur, M. und Bennett, W. S., 1994: Models for describing basal diameter and vertical distribution of primary branches in young Douglas-Fir. Forest Ecology and Management 63: S. 23 - 55.

MAHLER, G. UND HöWECKE, B., 1991: Verkernungserscheinungen bei der Buche in BadenWürttemberg in Abhängigkeit von Alter, Standort und Durchmesser. Schweizer Zeitschrift für das Forstwesen 142. Jg. Nr. 5: S. 375 - 390.

MÄKelä, A., VAnNinen, P. Und IKOnEN, V. P., 1996: PipeQual - a process-based model of tree form developement in different stocking densities. In: NePVEU, G. (ed.), 1996: Proceedings of the Second Workshop "Connection between Silviculture and Wood Quality through Modelling Approaches and Simulation Softwares“. Berg-en-Dal, Kruger National Park, South Africa, August 26-31, 1996. Publication Equipe de Recherches sur la Qualité des Bois 1997/7, December. INRA-Nancy, France: 450 S.: S. 304 - 312.

MÄKELÄ, A., 1997: A carbon balance model of growth and self-pruning in trees based on structural relationships. Forest Science Vol. 43. No. 1: S. 7 - 24.

Maltamo, M., Puumalainen, J. Und PÄIVInen, R., 1995: Comparison of beta and Weibull functions for modelling the basal area diameter distribution in stands of Pinus sylvestris and Picea abies. Scandinavian Journal of Forest Research Vol. 10: S. 284 - 295.

Maltamo, M. Und KANGAS, A., 1998: Methods based on k-nearest neighbour regression in the prediction of basal area diameter distribution. Canadian Journal of Forest Research Vol. 28. No. 8: S. 1107 - 1115

MAthsoft, InC., 1995: S-Plus for Windows, Version 3.3, Release 1, Copyright 1988.

MeILBY, H., 1999: Modelling the Growth and Development of Crown, Step, and Nodal Branches in Norway Spruce (Picea abies [L.] Karst.). Ph. D. thesis, Department of Economics and Natural Resources, The Royal Veterinary and Agricultural University, Copenhagen: $252 \mathrm{~S}$. 
MendozA, G. A. und Bare, B. B., 1986: A two-stage decision model for log bucking and allocation. Forest. Prod. Journal Vol. 36 No. 10: S. 70 - 74.

Meyer, P., 2001: Untersuchung der Waldstruktur und ihrer Dynamik in Naturwaldreservaten. IHW-Verlag, Eching: 107 S.

Mitchell, K. J., 1975: Dynamics and simulated Yield of Douglas-fir. Forest Science Monographs No. 17, Supplement to Forest Science Vol. 21 No. 4: 39 S.

\section{$\mathbf{N}$}

Nagel, J. Und Biging, G. S., 1995: Schätzung der Parameter der Weibullfunktion zur Generierung von Durchmesserverteilungen. AFJZ 166. Jg. Nr. 9/10: S. 185 - 189.

NAGEL, J., 1999 a: Konzeptionelle Überlegungen zum schrittweisen Aufbau eines waldwachstumskundlichen Simulationssystems für Nordwestdeutschland. Schriften aus der Forstlichen Fakultät der Universität Göttingen und der Niedersächsischen Forstlichen Versuchsanstalt Bd. 128: 122 S.

NAGEL, J., 1999 b: Volumenermittlung von stehendem und liegendem Totholz. NUASeminarbericht Bd. 4, Natur- und Umweltschutz Akademie des Landes NordrheinWestfalen (NUA): S. 311-314.

Nagel, J., Albert, M. Und Schmidt, M., 2001: Online-Dokumentation zum Prognose- und Entscheidungsmodell BWINPro 6.0. http://www.nfv.gwdg.de/nfvabw01.htm

NAGEL, J., 2001: Zur Genauigkeit von Ertragstafeln und dem Einzelbaummodell BWinPro. In: AKৎA, A, Hoffmann, B., Schumann, K. Und Staupendahl, K. (Hrsg.), 2001: Waldinventur, Waldwachstum und Forstplanung - Moderne Technologien, Methoden und Verfahrensweisen. Festschrift zum 60. Geburtstag von Prof. Dr. K. v. Gadow, Zohab Verlag, Göttingen: S. 145 - 152.

Nepveu, G., Constant, T., Colin, F., Dhôte, J-F., Guilley, E. und Le Moguédec, G., 1999: Along the chain from the plant to the plank: The experience recently gained in Sessile oak (Quercus petraea Liebl.) by the French connection. In: NePVEU, G. (ed.), 1999: Proceedings of the Third IUFRO-Workshop on the "Connection between Silviculture and Wood Quality through Modelling Approaches and Simulation Softwares". La Londe-les-Maures, France, September 5-12, 1999: 621 S.: S. 514 - 526.

NiEDERSÄCHSISCHE FORSTLICHE VERSUCHSANSTALT, 1994: Exkursionsführer: FichtenPlenterwald (Andreasberg, Abt. 396).

NiEDERSÄCHSISCHE FORSTLICHE VerSUCHSANSTALT, 1996: Arbeitsanweisung Außenarbeiten. NigGEMEYER, P. UND SCHMIDT, M., 1999: Estimating Diameter Distributions using nonparametric Methods. Proceeding at the $3^{\text {rd }}$ INCO meeting in Mombasa Kenya. 
NutTO, L., 1999: Neue Perspektiven in der Begründung und Pflege von jungen Eichenbeständen: Ergebnisse einer Untersuchung zur Kronenentwicklung, Astreinigung und Dickenwachstum junger Stiel- und Traubeneichen in Europa (Quercus robur L. und Quercus petraea (Matt.) Liebl.). Schriftenreihe Freiburger Forstliche Forschung, Band 5. Freiburg i. Brsg.: $190 \mathrm{~S}$.

NuTTO, L., 2000: Wachstum und Qualität von femelartig bewirtschafteten Eichen. AFZ / Der Wald. 55. Jg. Nr. 8: S. 399-400.

\section{$\mathbf{O}$}

OjansuU, R. Und Maltamo, M., 1995: Sapwood and heartwood taper in Scots pine stems. Canadian Journal of Forest Research Vol. 25: S. 1928 - 1943.

OKer Blom P., KellomÄKi, S., Valtonen, E. Und VÄIsÄnen, H., 1988: Structural development of Pinus sylvestris stands with varying initial density: a simulation model. Scandinavian Journal of Forest Research Vol. 3 No. 2: S. 185-200.

Отто, Н. J., 1989: Langfristige, ökologische Waldbauplanung für die Niedersächsischen Landesforsten. Bd. 1. Aus dem Walde Heft 42. Mitteilungen aus der Niedersächsischen Landesforstverwaltung: $442 \mathrm{~S}$.

Отто, Н. J., 1991: Langfristige, ökologische Waldbauplanung für die Niedersächsischen Landesforsten. Bd. 2. Aus dem Walde Heft 43. Mitteilungen aus der Niedersächsischen Landesforstverwaltung: $527 \mathrm{~S}$.

\section{$\mathbf{P}$}

PAIN, O. UND BOYER., E., 1996: A whole individual tree growth model for norway spruce. In: NePVeU, G. (ed.), 1996: Proceedings of the Second Workshop "Connection between Silviculture and Wood Quality through Modelling Approaches and Simulation Softwares“. Berg-en-Dal, Kruger National Park, South Africa, August 26-31, 1996. Publication Equipe de Recherches sur la Qualité des Bois 1997/7, December. INRA-Nancy, France: 450 S.: S. 13 - 23.

PARK, J. C., 1989: Comparison, via the Seesaw simulator, of three sawing systems for pruned logs. NZ Journal of Forestry Science Vol. 19 No. 1: S. 54 - 67.

PARK, J. C. UND TODOROKI, C. L., 1992: Autosaw sawing simulator \& the pruned resource: Pruned log index sets value of silvicultural practice. NZ Forest Industries, April: S. 30 32.

Pelz, S. Und SAuter, U. H., 1998: Marktchancen von schwachem Douglasienrundholz. AFZ/DerWald 53. Jg. Nr. 26: S. 1602 - 1604. 
Peters, S. UND BeCKeR, G., 1995: Die Holzqualität weitständig und engständig erwachsener Stieleichen. AFZ 50. Jg. Nr.13: S. 716 - 717.

POMMERENING, A., 1998: Forstschreibung von Stichprobendaten mit positionsabhängigen

Wuchsmodellen. Jahrestagung 1998 der Sektion Ertragskunde des DVFFA in Kevelaer, Tagungsbericht: S. 40-51.

PretzSCH, H., 1992: Konzeption und Konstruktion von Wuchsmodellen für Rein- und Mischbestände. Forstliche Forschungsberichte München Bd. 115: 332 S.

PretzsCH, H., 1993: Analyse und Reproduktion räumlicher Bestandesstrukturen. Versuche mit dem Strukturgenerator STRUGEN. Schriften aus der Forstlichen Fakultät der Universität Göttingen und der Niedersächsischen Forstlichen Versuchsanstalt Bd. 114, J. D. Sauerländer's Verlag, Frankfurt am Main: 87 S.

PretzSCH, H., 1995: Analyse und Reproduktion räumlicher Bestandesstrukturen. Methodische Überlegungen am Beispiel niedersächsischer Buchen-Lärchen-Mischbestände. Centralblatt f. d. gesamte Forstwesen 112. Jg. Nr. 2: S. 91 - 117.

PRETZSCH, H. UND KaHN, M., 1998: Forschungsvorhaben „Konzeption und Konstruktion“ von Wuchs- und Prognosemodellen für Mischbestände in Bayern. Abschlußbericht Projekt W 28, Teil 2 - Methodische Grundlagen: 279 S.

Pretzsch, H., Biber, P., Dursky, J., Gadow, K. V., Hasenauer, H., Hradetzky, J., Kändler G., Kublin, E., Nagel, J., Pommerening, A. und Sterba, H., 2000: Empfehlungen zur Einführung und Weiterentwicklung von Waldwachstumssimulatoren. AFJZ 171. Jg. Nr. 3: S. 52 - 57.

PuUmalainen, J., 1997: Marktorientierte Vorratsschätzung und terrestrische Vorinformationen. Dissertation, Forstliche Fakultät der Universität Göttingen: 176 S.

\section{$\mathbf{R}$}

Racz, J., Schulz, H. Und Knigge, W.; 1961: Untersuchungen über das Auftreten des Buchenrotkerns. Der Forst- und Holzwirt 16. Jg. Nr. 19: S. 413 - 417.

ReAL, P. UND MoORE, J., 1987: An individual tree taper system for douglas- fir in the inland northwest. Proceeding at the IUFRO forest growth modelling and prediction conference, Minneapolis, 1987: S. 1037 - 1044.

RIEMER, T., GADOW, K. v. UND SLOBODA, B., 1995: Ein Modell zur Beschreibung von Baumschäften. AFJZ 166. Jg. Nr. 7: S. 144 - 147.

RITTER, U., 1998: Rundholzsortierung und -vermarktung in der europäischen Normung. AFZ 53. Jg. Nr. 19: S. 1001 - 1004.

Roloff, A., 1983: Morhologie und Phänologie der Rotbuche unter besonderer Berücksichtigung des Verzweigungssystems. Diplom-Arbeit, Forstliche Fakultät der Universität Göttingen: $105 \mathrm{~S}$. 
Roloff, A., 1986: Morphologie der Kronenentwicklung von Fagus sylvatica (L.) Rotbuche unter besonderer Berücksichtigung möglicherweise neuartiger Veränderungen. Berichte des Forschungszentrums Waldökosysteme/Waldsterben Bd. 18: 177 S.

\section{$\mathbf{S}$}

Saborowski, J., Sloboda, B. Und Junge, A., 1981: Darstellung von Schaftformen durch kubische Spline-Interpolation und Reduktion der Stützstellenanzahl. Forstarchiv 4: S. $127-130$.

SACHSSE, H. UND GRÜNEBAUM, M., 1990: Untersuchung der Holzqualität von Traubeneichen aus unterschiedlich dicht begründeten Beständen. Holz als Roh- und Werkstoff $48 \mathrm{Jg}$. Nr. 7-8: S. 255 - 260.

SaChSSE, H., 1991: Das Holz der Douglasie - Bau, Eigenschaften, und Verwendung. Forst und Holz 46. Jg. Nr. 12: S. 326 - 331.

SAUTER, U. H. UND FAHRBACH, M., 1993: Ästigkeitsverhältnisse und Schnittholzqualität in einem weitständig begründeten Fichtenbestand. AFZ 48. Jg. Nr. 17: S. 875 - 878.

SCHMALTZ, J., 1989: Aststärkenentwicklung und Schaftqualität in zwei Kiefernverbandsversuchen. AFJZ 160. Jg. Nr. 50: S. 1337 - 1339.

Schmid-HAAs, P., BAumann, E. Und Werner, J., 1993: Kontrollstichproben: Aufnahmeinstruktion. Berichte der Eidgenössischen Forschungsanstalt für Wald, Schnee und Landschaft Nr. 186: 143 S.

SCHMIDT, M. U. GADOW, K. V., 1999: Baumhöhenschätzung mit Hilfe der bivariaten Johnson's $S_{B B}$-Funktion. Forstw. Cbl. 118. Jg. Nr. 6: S. 355 - 367.

Schmidt, M., Spellmann, H. Und Nagel, J., 2001: Waldwachstumskundliche Entscheidungshilfen zur Ästung der Douglasie. AFJZ: im Druck.

SCHOBER, R. IN KRAMER, H., 1988: Waldwachstumskunde, Kapitel 7 Einfluß der Provenienz auf die Ertragsleistung. Verlag Paul Parey, Hamburg und Berlin: S. 129 - 153.

SCHÖNHAR, S., 1988: Zur Ausbreitung von Heterobasidion annosum in Fichtenbeständen. Forst und Holz 43. Jg. Nr. 7: S. 156-158.

SCHÖNHAR, S., 1994: Untersuchungen über die Infektion der Fichte (Picea abies Karst.) durch Heterobasidion annosum (Fr.) Bref. AFJZ 166. Jg. Nr.1: S. 14 - 17.

SCHÖPFER W. UND DAUBER E., 1989: Bestandessortentafeln, '82/85. Mitteilungen der Forstlichen Versuchs- und Forschungsanstalt Baden-Württemberg Heft 147: $51 \mathrm{~S}$.

Schulz, H., 1959: Güteklassen des Stammholzes und ihre Abgrenzung gegeneinander. HolzZentralblatt 85. Jg. Nr. 57: S. 753 - 754. 
Schumacher, P., Ritter, U., Schulz, H. Und Wegener, G., 1997 a: Qualitätssortierung von Fichtenstammholz nach dem Europäischen Normentwurf prEN 1927-1 (1). HolzZentralblatt 123. Jg. Nr. 74: S. 1137 - 1138.

Schumacher, P., Ritter, U., Schulz, H. Und Wegener, G., 1997 b: Qualitätssortierung von Fichtenstammholz nach dem europäischen Normentwurf prEN 1927-1 (2). Holzzentralblatt 123. Jg. Nr. 76: S. 1191.

SchÜtZ, J. PH., 1999: Die Plenterung und ihre unterschiedlichen Formen. Skript zur Vorlesung Waldbau II und Waldbau IV, ETH Zürich: $126 \mathrm{~S}$.

SEELING, U., 1991: Abnorme Kernbildung bei Rotbuche (Fagus sylvatica L.) und ihr Einfluß auf holzbiologische und holztechnologische Kenngrößen. Dissertation, Forstliche Fakultät der Universität Göttingen: $167 \mathrm{~S}$.

SEELING, U., 1995: Zielstärkendurchforstung Olper Fichtenjungbestände. AFZ 50. Jg. Nr. 13: S. $711-714$.

SEELING, U. UND DAQUITAINE R., 1999: Lumber and timber quiality of Norway spruce border trees (Picea abies, Karst.). Results of measurements and simulations. In: NEPVEU, G. (ed.), 1999: Proceedings of the Third IUFRO-Workshop on the "Connection between Silviculture and Wood Quality through Modelling Approaches and Simulation Softwares". La Londe-les-Maures, France, September 5-12, 1999: 621 S.: S. 526 - 534.

SEIFERT, T., 1999: Modelling wood quality of Norway spruce (Picea abies) depending on silvicultural treatment. In: NePveU, G. (ed.), 1999: Proceedings of the Third IUFROWorkshop on the "Connection between Silviculture and Wood Quality through Modelling Approaches and Simulation Softwares". La Londe-les-Maures, France, September 5-12, 1999: 621 S.: S. 534 - 540.

Sessions, J., Olsen, E., Und Garland, J., 1989: Tree bucking for optimal stand value with $\log$ allocation constraints. Forest Science Vol. 35 No. 1: S. 271 - 276.

Short, E. und Burkhart, H. 1992: Predicting crown-height increment for thinned and unthinned lobolly pine plantations. Forest Science Vol. 38: S. 594 - 610.

Silverman, B. W., 1986: Density Estimation for Statistics and Data Analysis. Chapman and Hall, London: $175 \mathrm{~S}$.

Sloboda, B., Gaffrey, D. Und Matsumara, N., 1993: Regionale und lokale Systeme von Höhenkurven für gleichaltrige Waldbestände. AFJZ 164. Jg. Nr. 12: S. 225 - 228.

Sloboda, B., GafFrey, D. Und Matsumara, N., 1998: Erfassung individueller Baumschaftformen und ihrer Dynamik durch Spline-Funktionen und Verallgemeinerung durch lineare Schaftformmodelle. AFJZ 169. Jg. Nr. 2: S. 29 - 39.

Sloboda, B. Und Pfreundt, J., 1989: Baum- und Bestandeswachstum. Ein systemanalytischer räumlicher Ansatz mit Versuchsplanungskonsequenzen für die Durchforstung und 
Einzelbaumentwicklung. Deutscher Verband Forstlicher Forschungsanstalten - Sektion

Ertragskunde, Jahrestagung vom 8. bis 10. Mai 1989, Attendorn/Olpe: S. 17/1 - 17/25.

SpEIDEL, G., 1955: Die Wertklasse als Gütemaßstab in der Forsteinrichtung. Forstarchiv 26.

Jg. Nr. 10: S. 217 - 224.

Spellmann, H. Und NAGEl, J., 1992: Auswertung des Nelder-Pflanzverbandsversuches mit Kiefer im Forstamt Walsrode. AFJZ 163. Jg. Nr. 11/12: S. 221 - 229.

SPELlMANN, H., 1995: Vom strukturarmen zum strukturreichen Wald. Forst und Holz 50. Jg.

Nr.2: S. 35 - 44.

Spellmann, H., Wagner, S., Nagel, J., Guericke, M. und Griese, F., 1996: In der Tradition stehend, neue Wege beschreitend. Waldwachstumskundliche Forschung an der Niedersächsischen Forstlichen Versuchsanstalt. Forst und Holz 51. Jg. Nr. 11: S. 363 368.

SpellmanN, H., 1997: Ertragsentwicklung im "LÖWE"-Wald der Niedersächsischen Landesforstverwaltung. Forst und Holz 52. Jg Nr. 24.: S. 711 - 718.

SPIECKER, H., 1991: Steuerung des Dickenwachstums und der Astreinigung von Trauben- und Stieleichen : (Quercus petraea (Matt.) Liebl. und Quercus robur L.). Schriftenreihe der Landesforstverwaltung Baden-Württemberg Bd. 72: $155 \mathrm{~S}$.

S-Plus, Guide to Statistics, 1998: Data Analysis Products Division MathSoft, Inc. Seattle, Washington: $881 \mathrm{~S}$.

SteIngASS, F., 1996: Beschreibung der Schaftprofile von Douglasien. Diplomarbeit, Forst. Fachbereich, Univ. Göttingen: 44 S.

StenLID, J., 1987: Controlling and Predicting the Spread of Heterobasidion annosum from Infected Stumps and Trees of Picea abies. Scandinavian Journal of Forest Research Vol. 2 No. 2: S. 187 - 198.

STERBA, H., 1995: Prognaus - ein abstandsunabhängiger Wachstumssimulator für gleichaltrige Mischbestände. Deutscher Verband Forstlicher Forschungsanstalten - Sektion Ertragskunde, 29.-31. Mai 1995, Joachimsthal: S. 173 - 183.

STEUeR, W., 1985: Vom Baum zum Holz. DRW-Verlag, Stuttgart: 256 S.

Swindel, B. F., Smith, H. D. und Grosenbaugh, L. R., 1987: Fitting diameter distributions with a hand-held prorammable calculator. Scandinavian Journal of Forest Research Vol. 2 No. 2: S. 325 - 334.

\section{$\mathbf{T}$}

Thren, M, 1983: Erste Ergebnisse eines Kiefernverbandsversuchs im Fbz. Karlsruhe-Hardt. AFJZ 156. Jg. Nr. 1-2: S. 24 - 37. 
Trincado, G., Gadow, K. v. und Tewari, V. P., 1996: Comparison of three stem profile equations for Quercus robur L.. Suid-Afrikaanse Bosboutydskrif Nr. 177: S. 23 - 29.

$\mathbf{U}$

UseniUs, A., 1999: Wood conversion chain optimization. In: NePVEU, G. (ed.), 1999: Proceedings of the Third IUFRO-Workshop on the "Connection between Silviculture and Wood Quality through Modelling Approaches and Simulation Softwares". La Londeles-Maures, France, September 5-12, 1999: 621 S.: S. 541 - 549.

\section{V}

VALSTA, L., 1992: An optimization model for Norway spruce management based on individual-tree growth models. Acta Forestalia Fennica 232: $20 \mathrm{~S}$.

Vestöl, G. I., HöIbö, O. A., Molteberg, D. E. und Sundby H. J., 1996: Modelling knottiness and knot characteristics of Norway spruce (Picea abies (L.) Karst): differences in the distribution of sound and dead knots between "suppressed" and "dominant" trees. In: NePVEU, G. (ed.), 1996: Proceedings of the Second Workshop "Connection between Silviculture and Wood Quality through Modelling Approaches and Simulation Softwares“. Berg-en-Dal, Kruger National Park, South Africa, August 26-31, 1996. Publication Equipe de Recherches sur la Qualité des Bois 1997/7, December. INRA-Nancy, France: 450 S.: S. 40 - 44

Vollbrecht, G., Gemmel, P. und Pettersson, N., 1995: The Effect of Precommercial Thinning on the Incidence of Heterobasidion annosum in Planted Picea abies. Scandinavian Journal of Forest Research Vol. 10: S. 37 - 41.

Volbbrecht, G., Und Agestam, E., 1995: Modelling Incidence of Root Rot in Picea abies Plantations in southern Sweden. Scandinavian Journal of Forest Research Vol. 10: S. 74 $-81$.

\section{W}

WALTER, M., 1997: Auswirkungen der CEN-Normen für Rundholz auf den Sortenanfall bei Fichte und Kiefer in Bayern (I und II). Holz-Zentralblatt Nr. 70/71: S. 1127.

WeIBuLl, W., 1951: "A Statistical Distribution Function of Wide Applicability," Journal of Applied Mechanics.

Wensel, L., Meerschaert, W. und Biging, G. S., 1987: Tree Height and Diameter Growth Models for Northern California Conifers. Hilgardia, University of California Vol. 55 No. 5: $20 \mathrm{~S}$. 
WIEGARD, C., 1998: Untersuchungen zur Entwicklung flexibler Wertinventuren in Waldbeständen. Dissertation, Forstliche Fakultät der Universität Göttingen: 185 S.

White, M. A., Thornton, P. E., Running, S. W. und Nemani, R. R.; 2000: Parametrisation and sensitivity analysis of the BIOME-BGC terrestrial ecosystem model: net primary production controls. Earth Interactions 4. Jg. Nr. 3: S. 1 - 85.

WILL, G. M., 1971: Nitrogen supply, apical dominance and branch growth in Pinus radiata. Plant Soil 34: S. 515 - 517.

WoBst, J., 1995: Auswirkungen von Standortwahl und Durchforstungsstrategie auf verwertungsrelevante Holzeigenschaften der Douglasie (Pseudotsuga menziesii (Mirb.(Franco)). Dissertation, Forstliche Fakultät der Universität Göttingen 210 S.

Wobst, J. Und Becker, G., 1997: Möglichkeiten zur Erweiterung des waldbaulichen Einflusses auf die Holzqualität der Douglasie. Forst und Holz 52. Jg Nr. 19.: S. 555 - 563.

\section{$\mathbf{Z}$}

ZuCChINI, W. UND OHLMER, U., 1997: Die Bootstrap-Methode. Vorlesungsskript: Institut für Statistik und Ökonometrie der Universität Göttingen: 80 S.

ZuCCHINI, W., 2001: Interactive data analysis. Kurs des Instituts für Statistik und Ökonometrie der Universität Göttingen.

ZYCHA, H. UND KATÓ, F., 1967: Untersuchungen über die Rotfäule der Fichte. Schriftenreihe der Forstlichen Fakultät der Universität Göttingen und Mitteilungen der Niedersächsischen Forstlichen Versuchsanstalt Bd. 39, J. D. Sauerländer's Verlag, Frankfurt am Main: $120 \mathrm{~S}$.

ZyCHA, H., 1976: Beitrag in: Der Wurzelschwamm (Fomes annosus) und die Rotfäule der Fichte (Picea abies). Beiheft Nr. 36 zum Forstw. Cbl., Verlag Paul Parey, Hamburg und Berlin.

ZYCHA, H., 1978: Beitrag: "Fomes annosus: infection phenomena“. In: Proceedings of the fifth international conference on problems of root and butt rots in conifers, Kassel, FRG, Aug. 7-12, 1978, IUFRO Working Party. 


\section{Anhang I}

\subsection{Exkurs: Gleitende-Durchschnitts-Regression}

Die zur Verdeutlichung von Zusammenhängen und zur Datenanalyse in Rahmen der Arbeit verwendeten Gleitenden-Durchschnitts-Modelle sollen kurz erläutert werden, da im Bereich der nicht-parametrischen Regressionsmodelle eine Vielzahl ähnlicher Verfahren existiert. Nicht-parametrische Regressionsverfahren weisen eine hohe Flexibilität auf, die daraus resultiert, daß keine explizite Funktionsform bei der Parametrisierung vorgegeben wird. Sie sind daher besonders gut dazu geeignet, Zusammenhänge in Datenstrukturen zu identifizieren und hervorzuheben. Das verwendete Modell ist eine gewichtete lokale kleinste QuadrateSchätzung, die das Statistik-Paket S-PLUS 3.3 (MATHSOFT, 1995) zur Verfügung stellt (SPlus 4 Guide to Statistics 1998, S. 247). Die einzelnen Berechnungsschritte sind:

1. Für jeden X-Wert $X_{0}$ werden die k nächsten Nachbarn gesucht, die die Nachbarschaft $N\left(X_{0}\right)$ von $X_{0}$ definieren. Die Anzahl, der bei der Festlegung der Nachbarschaft $N\left(X_{0}\right)$ berücksichtigten X-Werte $k$, wird durch einen sogenannten Glättungsparameter festgelegt, der den prozentualen Anteil der berücksichtigten X-Werte im Verhältnis aller X-Werte angibt.

2. Innerhalb der Nachbarschaft $N\left(X_{0}\right)$ wird der größte Abstand zwischen dem Wert $X_{0}$ und einem anderen X-Wert innerhalb der Nachbarschaft berechnet:

$$
\Delta\left(X_{0}\right)=\max _{N\left(X_{0}\right)}\left|X_{0}-X 1\right|
$$

3. Jedem X-Wert innerhalb der Nachbarschaft $N\left(X_{0}\right)$ wird ein Gewicht zugeordnet, wobei eine kubische Gewichtungsfunktion verwendet wird:

$$
W\left(\frac{\left|X_{0}-X_{1}\right|}{\Delta\left(X_{0}\right)}\right)
$$

wobei:

$$
W(u)=\left\{\begin{array}{c}
\left(1-u^{3}\right)^{3}, \text { falls } 0 \leq u<1 \\
0, \text { sonst }
\end{array}\right.
$$

4. Berechnung des gewichteten kleinste Quadrate-Schätzers für $Y_{0}$ an der Stelle $X_{0}: \hat{Y}_{0}$

5. Die Berechnung wird für jeden Wert von $X_{0}$ wiederholt. 


\subsection{Exkurs: Bootstrap-Methode}

Die von EFrOn (1979) entwickelte Bootstrap-Methode dient der Beurteilung der Genauigkeit eines Schätzers, beispielsweise des Mittelwertes einer Population (s. auch EFRON U. TIBSHIRANI, 1993). Unter dem Begriff Genauigkeit schließt Efron u. a. den Standardfehler des Schätzers, Konfidenzintervalle, Bias als auch den Test von Hypothesen ein. Die Beurteilung der Genauigkeit eines Schätzers erfolgt anhand der Verteilung eines Schätzers. Die BootstrapMethode schätzt die Verteilung eines Schätzers, indem sie dessen Verteilung unter der Annahme betrachtet, daß die Stichprobe die Grundgesamtheit darstellt (ZUCCHINI U. OHLMER, 1997). Natürlich ist es auch möglich, daß eine zufällig ausgewählte Stichprobe die Population nur schlecht representiert. Dann wird die Bootstrap-Methode, wie jede andere statistische Methode auch, zu ungenauen Schätzungen führen.

Die Methode findet vor allem da Anwendung, wo analytische Herleitungen von Konfidenzintervallen nicht möglich oder sehr kompliziert sind oder Annahmen bzw. Voraussetzungen in der praktischen Anwendung nicht überprüfbar bzw. nachweisbar sind. Die praktische Durchführung einer Bootstrap-Simulation erfolgt, indem aus der vorhandenen Stichprobe der Größe $n$ eine hinreichend große Anzahl Stichproben des gleichen Umfangs $n$ mit Zurücklegen gezogen wird. Beim Bootstrap von Regressionen können die zwei Verfahren "Bootstrap von Residuen" und "Bootstrap von Vektoren" unterschieden werden (siehe ZuCCHINI U. OHLMER, 1997). In der hier vorliegenden Anwendung wurde das Verfahren "Bootstrap von Vektoren“ gewählt, da der Zusammenhang zwischen abhängiger und unabhängiger Variable gewahrt werden soll (inhomogene Varianz der Residuen). Außerdem reagiert dieser Bootstraptyp weniger empfindlich auf Modellannahmen. Für jede der BootstrapStichproben wurde der interessierende Parameter geschätzt. Die Verteilung der einzelnen Parameterschätzungen ist die Bootstrap-Schätzung der Verteilung des Parameters. Als einziger Nachteil der Bootstrap-Methode muß der teilweise große Rechenaufwand bei komplizierteren Problemen genannt werden.

In der vorliegenden Anwendung wurde die Bootstrap-Methode verwendet, da für eine analytische Herleitung von Konfidenzintervallen der Regressionsfunktion (18) von der Annahme ausgegangen werden müßte, daß die Residuen normalverteilt sind und eine homogene Varianz aufweisen. Anhand der Abb. 103 und Abb. 107 ist leicht erkennbar, daß diese Voraussetzungen nicht erfüllt werden. 


\subsection{Exkurs: Modellierung von Dichteverteilungen mit Hilfe nicht-parametrischer Dichte-Funktionen}

Das Verfahren zur Anpassung von nicht-parametrischen Dichte-Funktionen und zur Generierung von Werten auf der Basis dieser Modelle ist in einzelne Schritte unterteilt, für die das Statistik-Paket S-PLUS 3.3 (MATHSOFT, 1995) spezielle Funktionen zur Verfügung stellt:

- Anpassung der nicht-parametrischen Dichtefunktion mit Hilfe einer Kernel-Schätzung sowie die Bestimmung des Glättungsparameters $h$. Je höher der Wert von $h$ ist, desto weniger flexibel wird die empirische Verteilung durch die Dichtefunktion abgebildet. Als Kernel-Funktion wurde die Normalverteilung verwendet. (S-PLUS-Funktion: density). Die geglättete Dichteverteilung wurde mit Hilfe der folgenden Dichtefunktion modelliert:

$$
f(x)=\frac{1}{n h} \sum_{i=1}^{n} K\left(\frac{x-X_{i}}{h}\right)
$$

wobei:

$n$ = Anzahl der normierten Längenwerte;

$h=$ Glättungsparameter;

$K=$ Kernel-Funktion (Normalverteilung);

$\mathrm{x}=$ normierter Längenwert, für den die Dichte geschätzt werden soll;

$\mathrm{X}_{\mathrm{i}}=\mathrm{i}$-te beobachtete normierte Länge.

und:

$$
K(x)=\frac{1}{\sqrt{2 \pi}} e^{-(1 / 2) x^{2}} \text { wobei } \int_{-\infty}^{\infty} K(x) d x=1 \text { gilt. }
$$

Der von Silverman (1986, S. 45 ff.) zur Schätzung des Glättungsparameters vorgeschlagene einfache Algorithmus ("Silverman's Faustregel") lautet bei Verwendung einer Normalverteilung:

$$
h=0.9 A n^{-1 / 5}
$$

mit: $A=\min ($ Standardabweichung, Interquartilbereich/1.34).

Die in Formel (44) dargestellte Schätzfunktion liefert nach SILVERMAN (1986, S. 48) für einen weiten Bereich von Dichteverteilungen gute Anpassungsergebnisse.

- Anpassung einer Funktion mit Hilfe linearer Interpolation zur Approximation der Dichtefunktion. Berechnung der Dichte im Intervall $[0,1]$ mit Hilfe der approximierten Dichtefunktion. Aufgrund der Definition sind nur Werte im Bereich zwischen 0 und 1 für die Werte der normierten Übergangsastzone plausibel. Die über Kernel-Schätzung ermittelte Dichtefunktion ist jedoch nicht auf einen bestimmten Datenbereich begrenzt. Um die Dichtefunktion auf das Intervall $[0,1]$ zu begrenzen, müssen die Funktionswerte so kali- 
briert werden, daß die Dichte im Intervall $[0,1]$ eins beträgt. (S-PLUS-Funktion: approxfun)

- Erneute Approximation der Dichtefunktion an den kalibrierten Funktionswerten mit Hilfe linearer Interpolation. (S-PLUS-Funktion: approxfun). Berechnung von Funktionswerten bei geringer Schrittweite (0.01) für normierte Längenwerte zwischen 0 und 1.

- Aufsummierung der mit der Schrittweite multiplizierten Dichteschätzungen für jeden normierten Längenwert zwischen 0 und 1 und Approximation einer Funktion mit Hilfe linearer Interpolation. Die Approximation stellt eine Schätzung der zur kalibrierten Dichtefunktion zugehörigen Verteilungsfunktion dar (Abb. 80).

kumulierte relative Häufigkeit

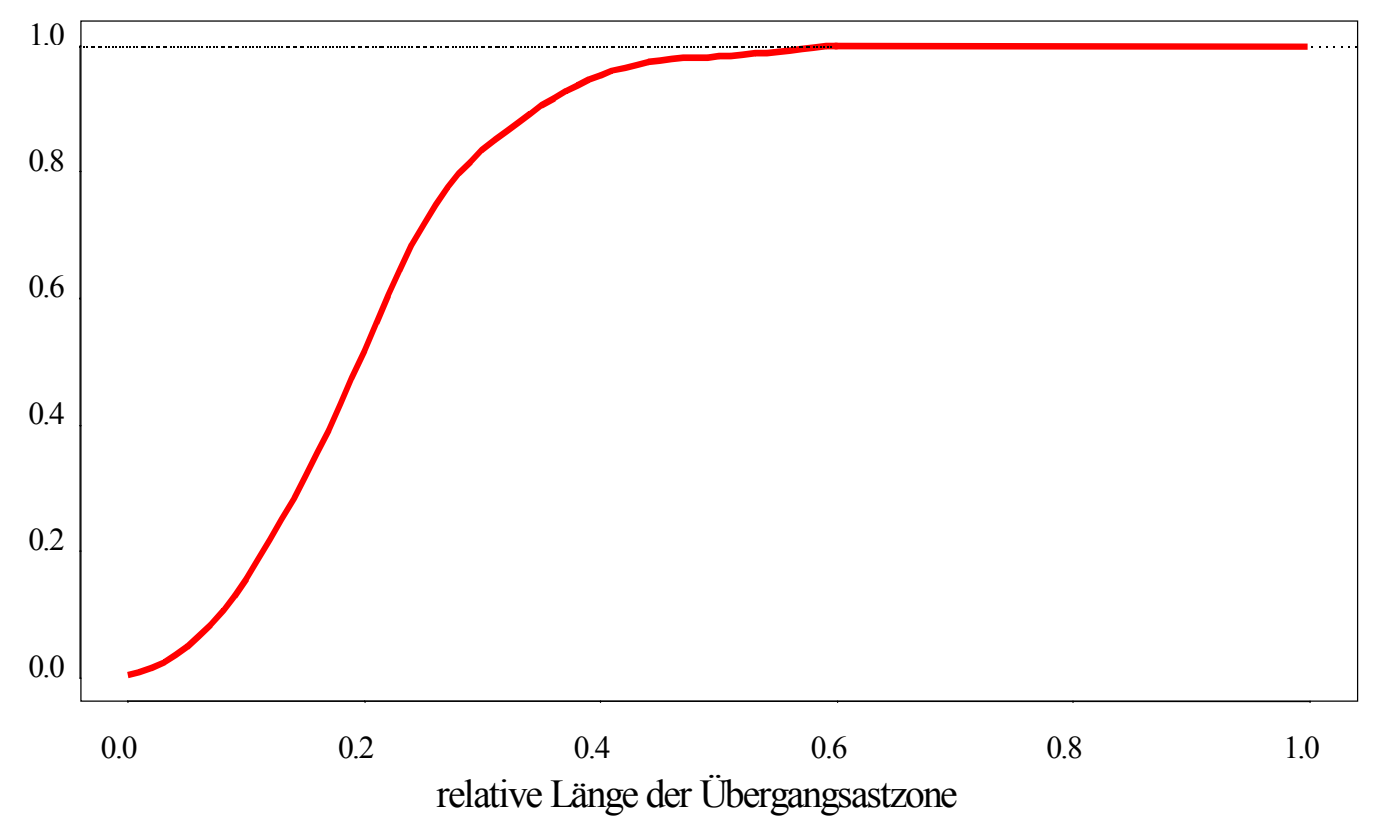

Abb. 80: Durch Approximation ermittelte Verteilungsfunktion der kalibrierten Dichteverteilung für die Baumart Fichte, die mit Hilfe von Kernel-Schätzung ermittelt wurde.

- Approximation einer Funktion an die invertierten Werte der Verteilungsfunktion mit Hilfe linearer Interpolation. Die Approximation stellt eine Schätzung für die sogenannte Quantilsfunktion dar, die auch bei parametrischen Verteilungsfunktionen zur Generierung von Werten verwendet wird (Kapitel 3.4.1, Formel (28), Abb. 53). In Abb. 81 ist die Quantilsfunktion auf der Basis der kalibrierten Dichtefunktion für die Baumart Fichte dargestellt. 
relative Länge der Übergangsastzone

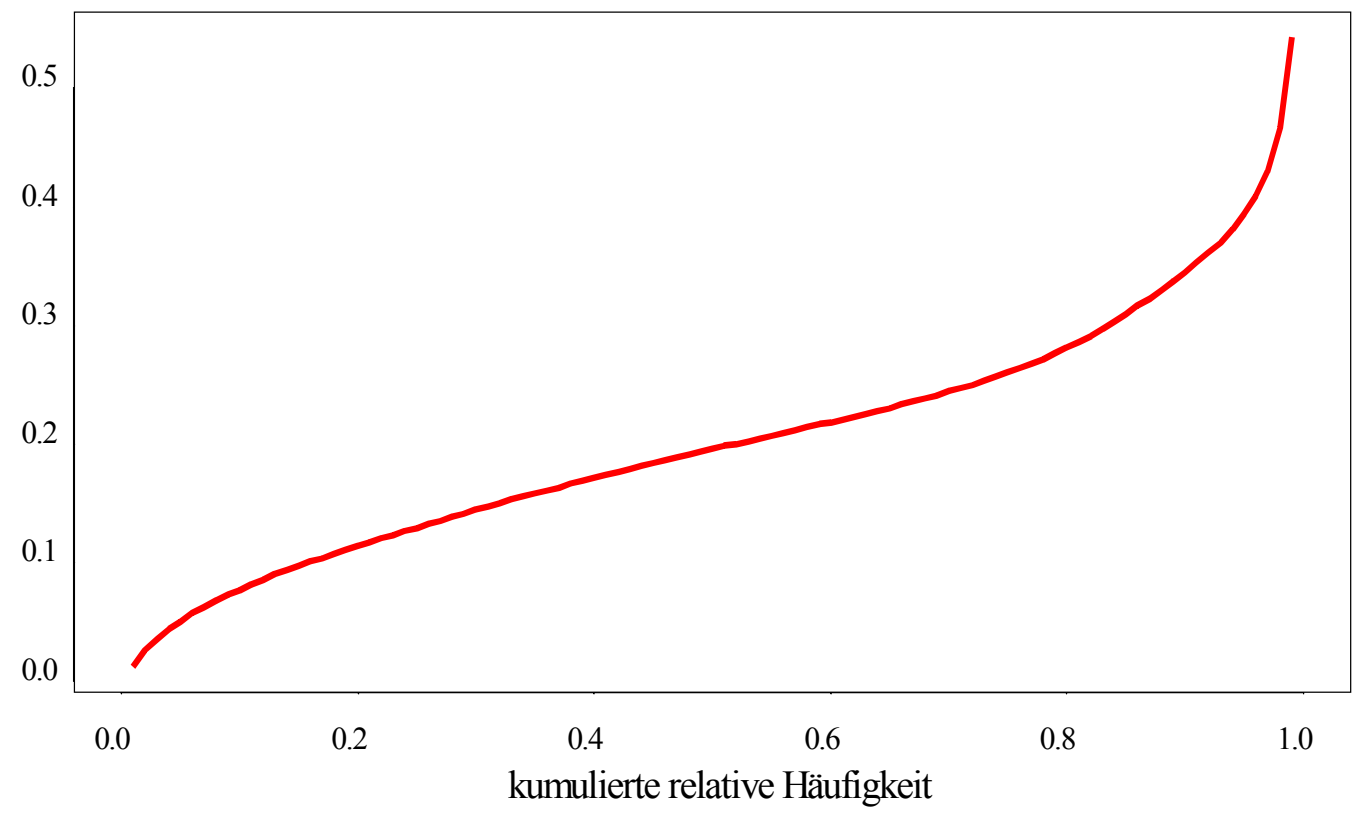

Abb. 81: Durch Approximation ermittelte Quantilsfunktion der kalibrierten Dichteverteilung für die Baumart Fichte, die mit Hilfe von Kernel-Schätzung ermittelt wurde.

- Generierung von normierten Längen der Übergangsastzone durch Einsetzen von im Intervall $[0,1]$ verteilten Zufallszahlen oder äquidistant verteilten Zahlen in die Quantilsfunktion.

Die Verwendung von mit Hilfe linearer Interpolation approximierter Funktionen zur Beschreibung der Dichte-, Verteilungs- und Quantilsfunktion ist notwendig, weil die Schätzung der jeweiligen Funktionswerte nicht wie bei parametrischen Verteilungsfunktionen auf der Basis der zugehörigen Funktionsgleichungen erfolgen kann. Weist die nicht kalibrierte Dichtefunktion auf der Basis der Kernel-Schätzung im Intervall $[0,1]$ bereits eine Dichte nahe dem Wert 1 auf, sind die Unterschiede zwischen kalibrierter und nicht kalibrierter Dichtefunktion geringfügig. 


\section{Anhang II}

\subsection{Hierarchisches Kronenansatzmodell}

\section{Douglasie:}

Für die Berechnung der Kronenansatzmodelle standen 11841 Einzelmessungen aus 313 Parzellenaufnahmen mit mindestens 20 gemessenen Kronenansatzhöhen pro Aufnahme zur Verfügung.
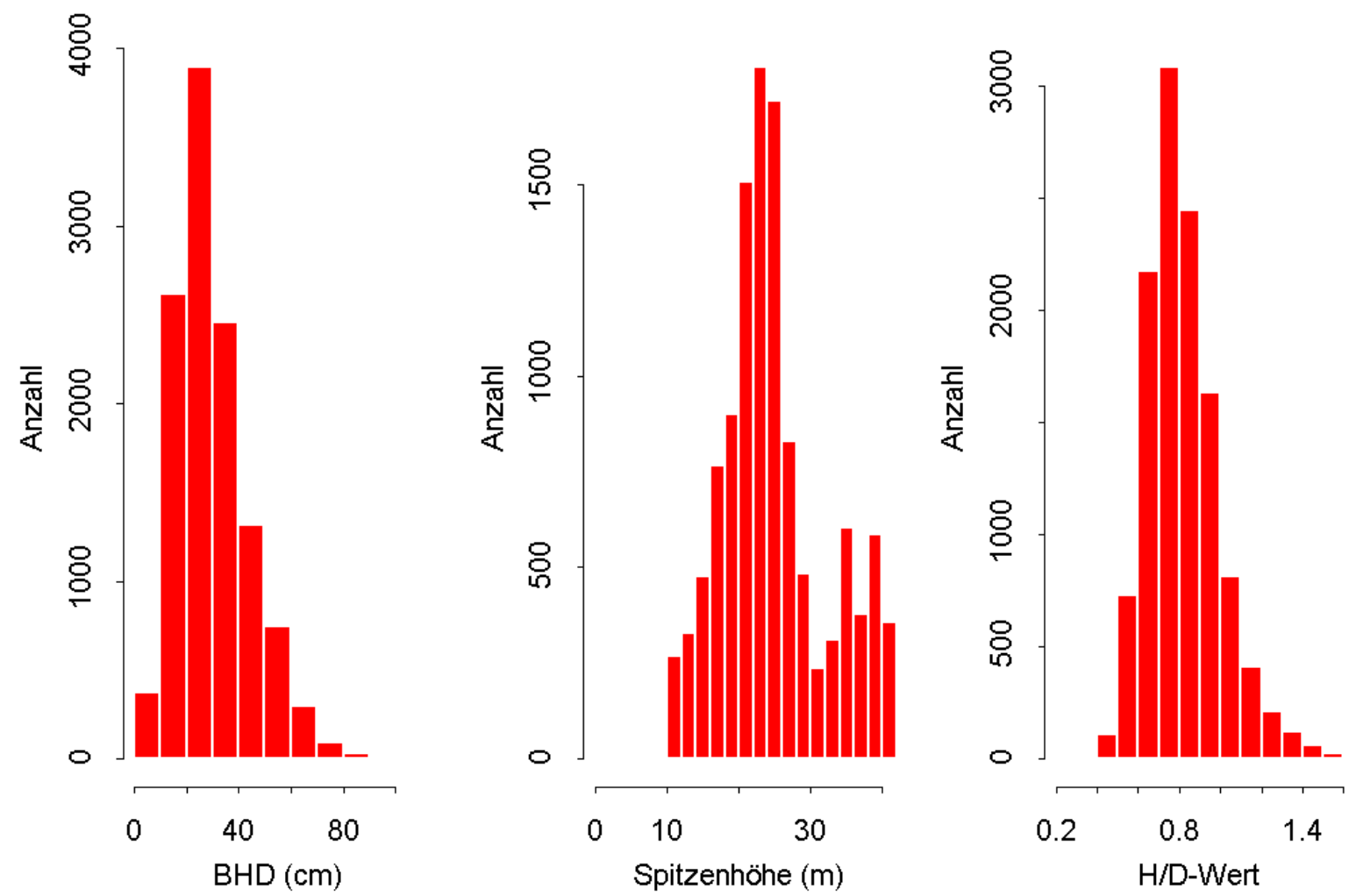

Abb. 82: Verteilungen der Variablen, die in den neu entwickelten Modellen zur Schätzung des Kronenansatzes für die Baumart Douglasie verwendet werden.

\begin{tabular}{|c|r|r|r|r|}
\hline Koeffizienten & Wert & Std. Fehler & t-Wert & \multicolumn{1}{|c|}{$\operatorname{Pr}(>|\mathrm{t}|)$} \\
\hline$\alpha$ & 2.2107 & 0.1018 & 21.7160 & 0.0000 \\
\hline$\beta$ & -0.2898 & 0.0117 & -24.7366 & 0.0000 \\
\hline$\lambda$ & -0.3961 & 0.0314 & -12.6012 & 0.0000 \\
\hline$\delta$ & -0.0533 & 0.0099 & -5.3895 & 0.0000 \\
\hline \multicolumn{4}{|r|}{ Multiples Bestimmtheitsma $\beta$} & 0.05964 bei 308 Freiheitsgraden \\
\hline \multicolumn{4}{r|r}{ F-Statistik } & 232.4 bei 3 und 308 Freiheitsgraden, $p$-Wert=0 \\
\hline
\end{tabular}

Tab. 37: Koeffizienten und statistische Kenngrößen zur Schätzung des Koeffizienten "mittleres Kronenverhältnis" der Bäume einer Baumart im Bestand (verwendet in Formel (9)) mit Hilfe des Modells (10) für die Baumart Douglasie. 


\begin{tabular}{|c|c|c|c|c|}
\hline Koeffizienten & Wert & Std. Fehler & $\mathrm{t}$-Wert & $\operatorname{Pr}(>|t|)$ \\
\hline$\alpha$ & 1.7385 & -0.0039 & 32.1085 & 0.0000 \\
\hline$\beta$ & -0.2968 & 0.0122 & -24.3983 & 0.0000 \\
\hline$\lambda$ & -0.3930 & 0.0328 & -11.9745 & 0.0000 \\
\hline \multicolumn{2}{|c|}{ Residual Std. Fehler } & \multicolumn{3}{|c|}{0.06229 bei 309 Freiheitsgraden } \\
\hline \multicolumn{2}{|c|}{ Multiples Bestimmtheitsmaß } & \multicolumn{3}{|r|}{0.6647} \\
\hline \multicolumn{2}{|r|}{ F-Statistik } & \multicolumn{3}{|c|}{306.3 bei 2 und 309 Freiheitsgraden, $p$-Wert $=0$} \\
\hline
\end{tabular}

Tab. 38: Koeffizienten und statistische Kenngrößen zur Schätzung des Koeffizienten "mittleres Kronenverhältnis " der Bäume einer Baumart im Bestand (verwendet in Formel (9)) mit Hilfe des Modells (11) für die Baumart Douglasie.

\begin{tabular}{|c|c|c|c|c|}
\hline Koeffizienten & Wert & Std. Fehler & $\mathrm{t}$-Wert & $\operatorname{Pr}(>|t|)$ \\
\hline$\alpha$ & -0.000218137 & 0.0000 & -10.7781 & 0.0000 \\
\hline$\beta$ & 0.013892500 & 0.0006 & 21.3890 & 0.0000 \\
\hline \multicolumn{2}{|c|}{ Residual Std. Fehler } & \multicolumn{3}{|c|}{0.003423 bei 310 Freiheitsgraden } \\
\hline \multicolumn{2}{|c|}{ Multiples Bestimmtheitsmaß } & \multicolumn{3}{|r|}{0.7814} \\
\hline & F-Statistik & \multicolumn{3}{|c|}{554 bei 2 und 310 Freiheitsgraden, $p$-Wert $=0$} \\
\hline
\end{tabular}

Tab. 39: Koeffizienten und statistische Kenngrößen zur Schätzung des Steigungskoeffizienten (verwendet in Formel (9)) mit Hilfe des Modells (12) für die Baumart Douglasie.

\begin{tabular}{|l|r|r|r|r|r|}
\hline & \multicolumn{1}{|c|}{ Bwin } & \multicolumn{1}{c|}{ Silva } & \multicolumn{1}{c|}{ Bwinpro } & \multicolumn{1}{c|}{ EKVK } & \multicolumn{1}{c|}{ EKVK (Kor.) } \\
\hline RMSE $(\mathrm{m})$ & 3.0195 & 2.35634 & 2.0646 & 1.60771 & 1.601242 \\
\hline RMSE & 0.138195 & 0.1059616 & 0.09446313 & 0.08510012 & 0.07380325 \\
\hline
\end{tabular}

\begin{tabular}{|l|c|r|r|r|r|}
\hline & $\begin{array}{c}\text { Dyn. } \\
\text { EKVK (Kor.) }\end{array}$ & $\begin{array}{c}\text { Dyn. } \\
\text { EKVK (Kor.) }<=15\end{array}$ & $\begin{array}{c}\text { Dyn. } \\
\text { EKVK (Kor.) }<=5\end{array}$ & $\begin{array}{c}\text { hierarchisches } \\
\text { Modell I }\end{array}$ & $\begin{array}{c}\text { hierarchisches } \\
\text { Modell II }\end{array}$ \\
\hline RMSE (m) & 2.162177 & 1.959785 & 1.728928 & 2.04525300 & 2.485864 \\
\hline RMSE & 0.07777741 & 0.07641482 & 0.06777084 & 0.09338052 & 0.09918276 \\
\hline
\end{tabular}

\begin{tabular}{|l|r|r|r|}
\hline & $\begin{array}{c}\text { Direkte } \\
\text { Anpassung } \\
\text { an die Daten }\end{array}$ & $\begin{array}{c}\text { Forest } \\
\text { modifiziert }\end{array}$ & \multicolumn{1}{c|}{ CEP } \\
\hline RMSE $(\mathrm{m})$ & 1.510076 & 2.27771 & 2.25965 \\
\hline RMSE & 0.06588725 & 0.1120496 & 0.1010027 \\
\hline
\end{tabular}

Tab. 40: Standardfehler der Kronenansatzschätzung (m) und der Kronenverhältnisschätzung für verschiedene Kronenansatzmodelle für die Baumart Douglasie. 


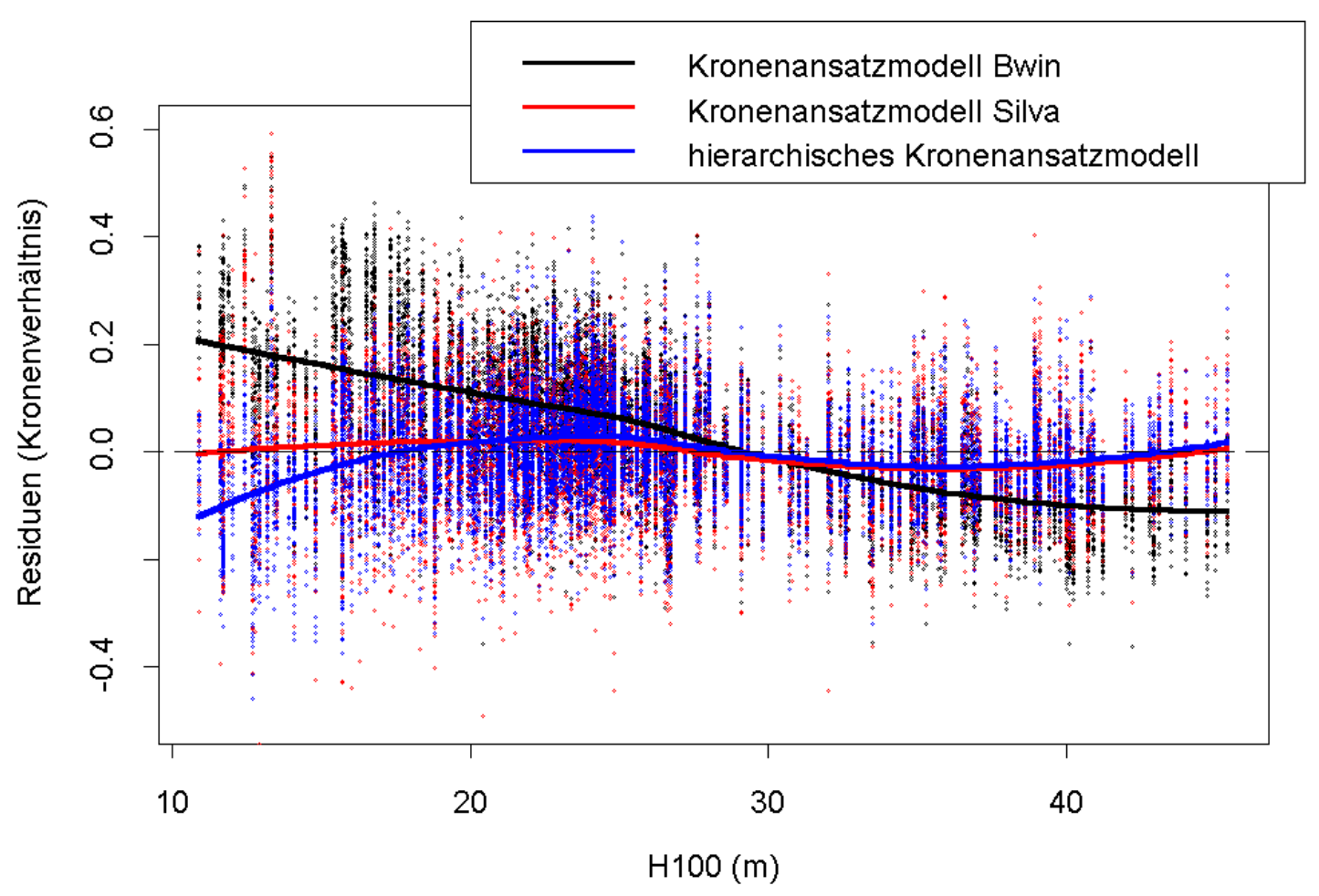

Abb. 83: Residuen der Kronenverhältnisschätzung mit Hilfe der Kronenansatzmodelle in Bwin, in Silva und mit dem neu entwickelten hierarchischen Modell für die Baumart Douglasie. Die Linien sind die zugehörigen Funktionswerte, die mittels eines Gleitenden-Durchschnitts-Modells geschätzt wurden (Kapitel 8.1).

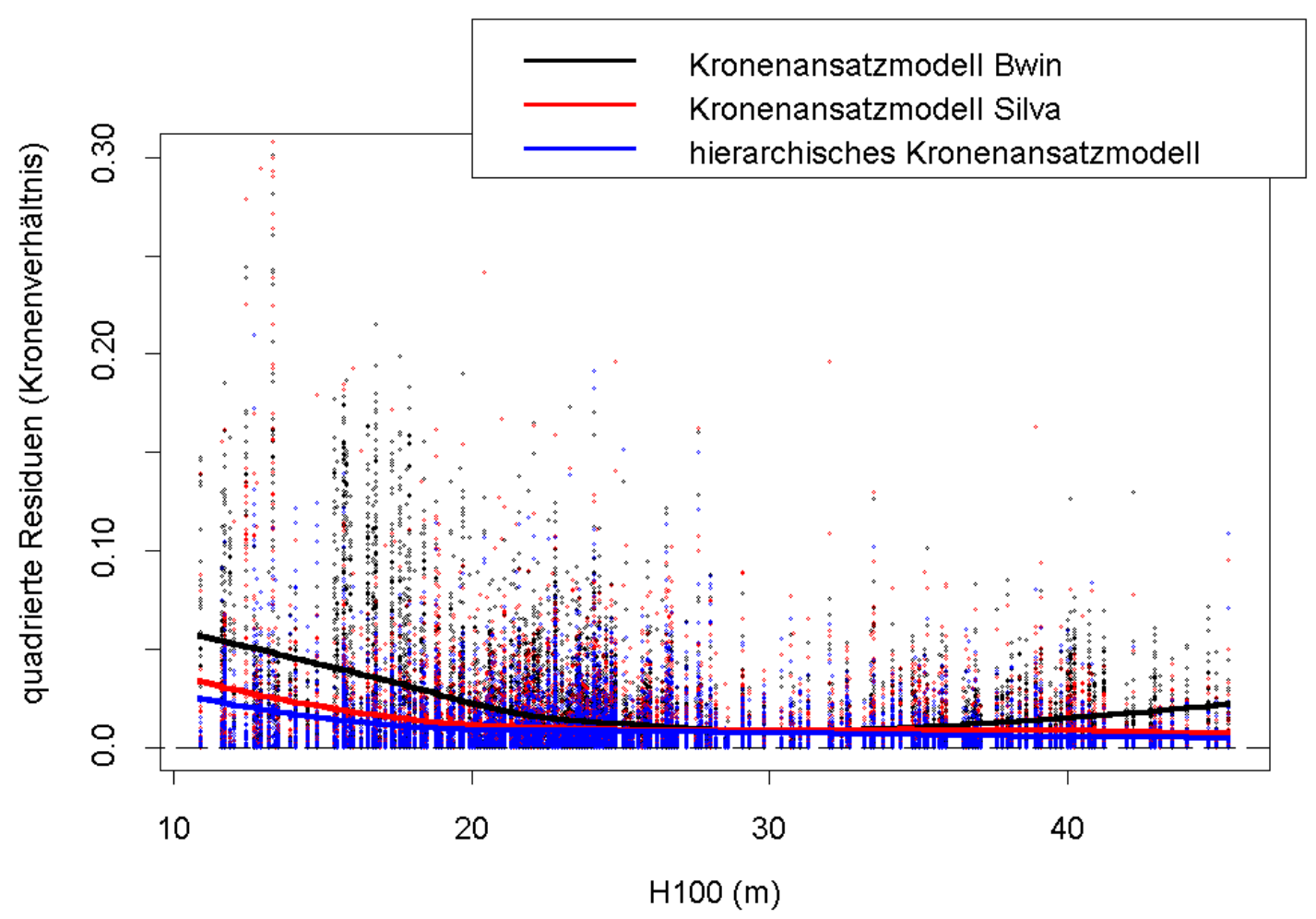

Abb. 84: Quadrierte Residuen der Kronenverhältnisschätzung mit Hilfe der Kronenansatzmodelle in Bwin, in Silva und mit dem neu entwickelten hierarchischen Modell für die Baumart Douglasie. Die Linien sind die zugehörigen Funktionswerte, die mittels eines Gleitenden-Durchschnitts-Modells geschätzt wurden (Kapitel 8.1). 


\section{Kiefer:}

Für die Berechnung der Kronenansatzmodelle standen 7407 Einzelmessungen aus 176 Parzellenaufnahmen mit mindestens 20 gemessenen Kronenansatzhöhen pro Aufnahme zur Verfügung. Die unabhängige Variable Bestandeskronenschirmfläche (Formel (10)) ist für die Schätzung des Parameters “mittleres Kronenverhältnis“ der Bäume einer Baumart im Bestand nicht signifikant.
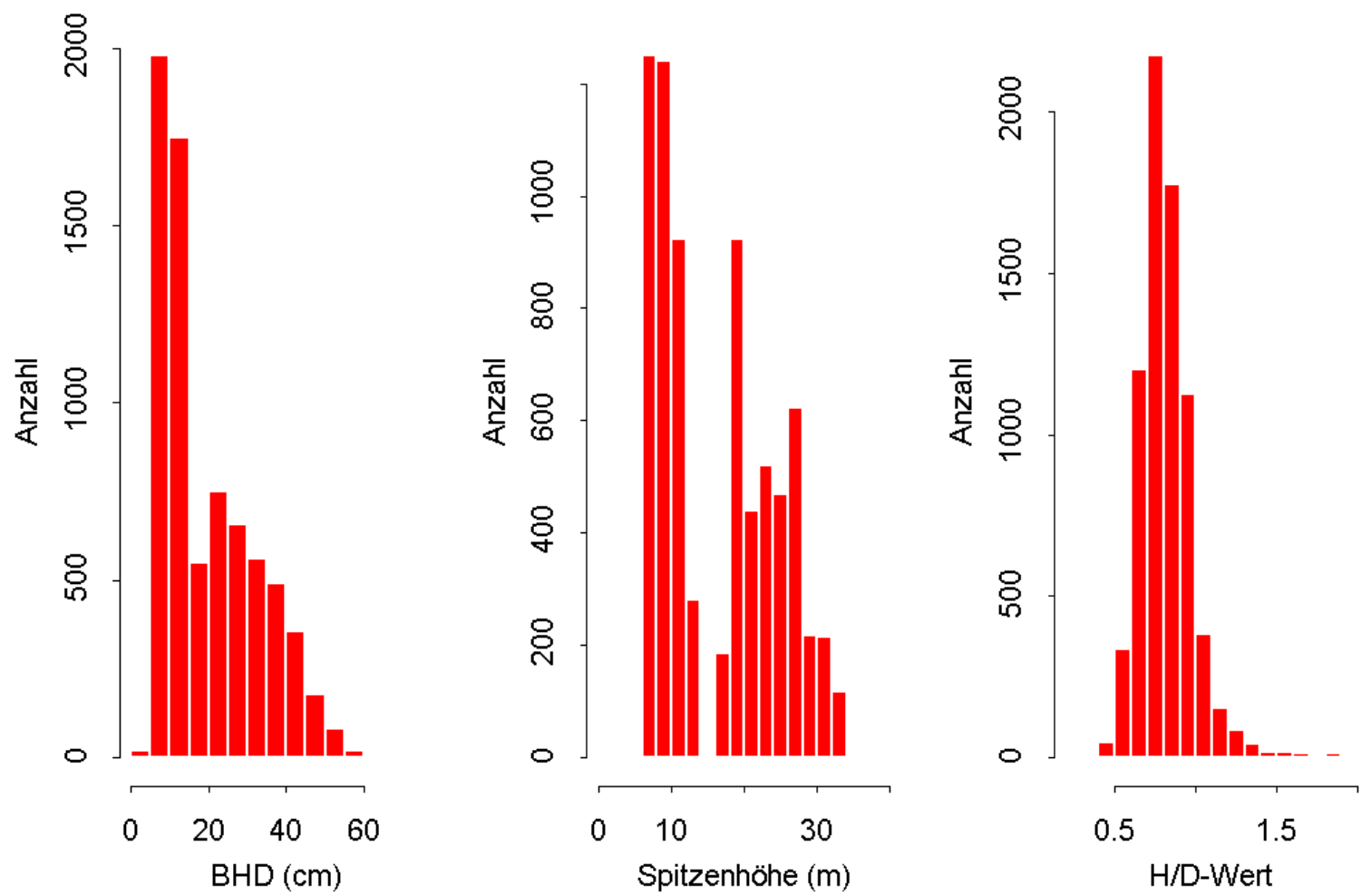

Abb. 85: Verteilungen der Variablen, die in den neu entwickelten Modellen zur Schätzung des Kronenansatzes verwendet werden für die Baumart Kiefer.

\begin{tabular}{|c|c|c|c|c|}
\hline Koeffizienten & Wert & Std. Fehler & t-Wert & $\operatorname{Pr}(>|t|)$ \\
\hline$\alpha$ & 1.2765 & 0.0400 & 31.8757 & 0.0000 \\
\hline$\beta$ & -0.2514 & 0.0081 & 31.0845 & 0.0000 \\
\hline$\lambda$ & -0.2494 & 0.0325 & -7.6661 & 0.0000 \\
\hline \multicolumn{2}{|c|}{ Residual Std. Fehler } & \multicolumn{3}{|c|}{0.0475 bei 173 Freiheitsgrader } \\
\hline \multicolumn{2}{|c|}{ Multiples Bestimmtheitsmaß } & \multicolumn{3}{|r|}{0.8484} \\
\hline \multicolumn{2}{|r|}{ F-Statistik } & \multicolumn{3}{|c|}{484.1 bei 2 und 173 Freiheitsgraden, $p$-Wert $=0$} \\
\hline
\end{tabular}

Tab. 41: Koeffizienten und statistische Kenngrößen zur Schätzung des Koeffizienten "mittleres Kronenverhältnis " der Bäume einer Baumart im Bestand (verwendet in Formel (9)) mit Hilfe des Modells (11) für die Baumart Kiefer. 
Steigungskoeffizient $=\alpha+\beta * H 100+\lambda * \overline{H / D}$

(12) abgewandelt

mit:

\begin{tabular}{|c|c|c|c|c|}
\hline Koeffizienten & Wert & Std. Fehler & $\mathrm{t}$-Wert & $\operatorname{Pr}(>|t|)$ \\
\hline$\alpha$ & 0.0133 & 0.0027 & 5.0047 & 0.0000 \\
\hline$\beta$ & -0.0006 & 0.0000 & -13.4543 & 0.0000 \\
\hline$\lambda$ & 0.0072 & 0.0028 & 2.6173 & 0.0096 \\
\hline \multicolumn{2}{|c|}{ Residual Std. Fehler } & \multicolumn{3}{|c|}{0.003907 bei 173 Freiheitsgraden } \\
\hline \multicolumn{2}{|c|}{ Multiples Bestimmtheitsmaß } & & & 0.5878 \\
\hline & F-Statistik & \multicolumn{3}{|c|}{123.4 bei 2 und 173 Freiheitsgraden, $p$-Wert $=0$} \\
\hline
\end{tabular}

Tab. 42: Koeffizienten und statistische Kenngrößen zur Schätzung des Steigungskoeffizienten (verwendet in Formel (9)) mit Hilfe des Modells (12, abgewandelt) für die Baumart Kiefer.

\begin{tabular}{|l|c|r|r|r|r|}
\hline & Bwin & \multicolumn{1}{c|}{ Silva } & Bwinpro & \multicolumn{1}{c|}{ EKVK } & EKVK (Kor.) \\
\hline RMSE $(\mathrm{m})$ & 1.83043 & 1.40935 & 1.18733 & 0.94663800 & 0.9399308 \\
\hline RMSE & 0.1720927 & 0.0909624 & 0.07528212 & 0.05725069 & 0.05659381 \\
\hline
\end{tabular}

\begin{tabular}{|l|c|c|r|r|r|}
\hline & $\begin{array}{c}\text { Dyn. } \\
\text { EKVK (Kor.) }\end{array}$ & $\begin{array}{c}\text { Dyn. } \\
\text { EKVK (Kor.) }<=15\end{array}$ & $\begin{array}{c}\text { Dyn. } \\
\text { EKVK (Kor.) }<=5\end{array}$ & $\begin{array}{c}\text { hierarchisches } \\
\text { Modell I }\end{array}$ & $\begin{array}{c}\text { hierarchisches } \\
\text { Modell II }\end{array}$ \\
\hline RMSE (m) & 1.258897 & 1.102364 & 0.8117288 & 1.19948400 & 1.36503 \\
\hline RMSE & 0.06413492 & 0.06254063 & 0.06210687 & 0.06965503 & 0.07692277 \\
\hline
\end{tabular}

\begin{tabular}{|l|r|r|r|}
\hline & \multicolumn{1}{|c|}{$\begin{array}{c}\text { Direkte } \\
\text { Anpassung } \\
\text { an die Daten }\end{array}$} & $\begin{array}{c}\text { Forest } \\
\text { modifiziert }\end{array}$ & \multicolumn{1}{|l|}{ CEP } \\
\hline RMSE $(\mathrm{m})$ & 0.8653057 & 1.29314 & 1.5474 \\
\hline RMSE & 0.04909218 & 0.08101073 & 0.1191697 \\
\hline
\end{tabular}

Tab. 43: Standardfehler der Kronenansatzschätzung (m) und der Kronenverhältnisschätzung für verschiedene Kronenansatzmodelle für die Baumart Kiefer. 


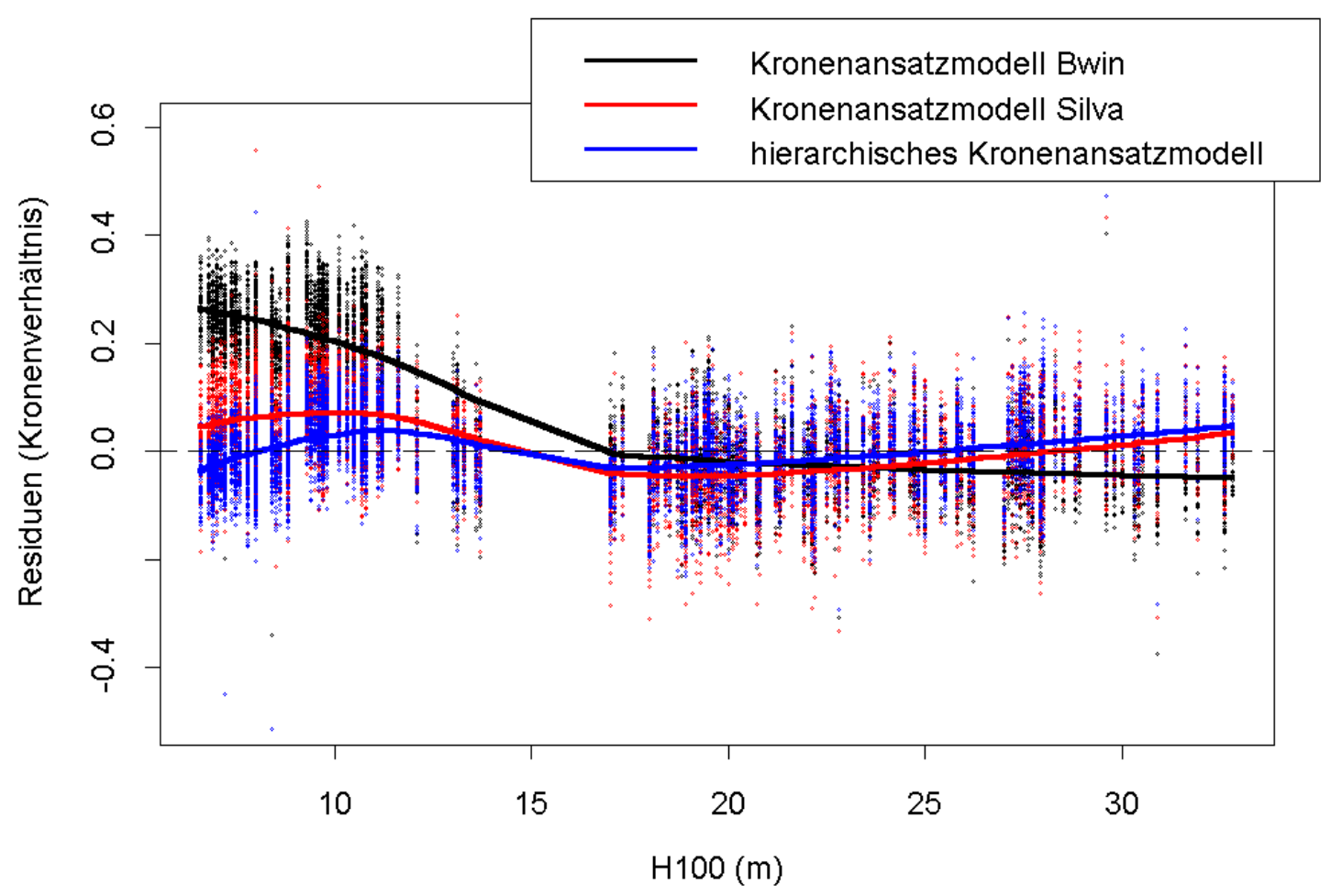

Abb. 86: Residuen der Kronenverhältnisschätzung mit Hilfe der Kronenansatzmodelle in Bwin, in Silva und mit dem neu entwickelten hierarchischen Modell für die Baumart Kiefer. Die Linien sind die zugehörigen Funktionswerte, die mittels eines GleitendenDurchschnitts-Modells geschätzt wurden (Kapitel 8.1).

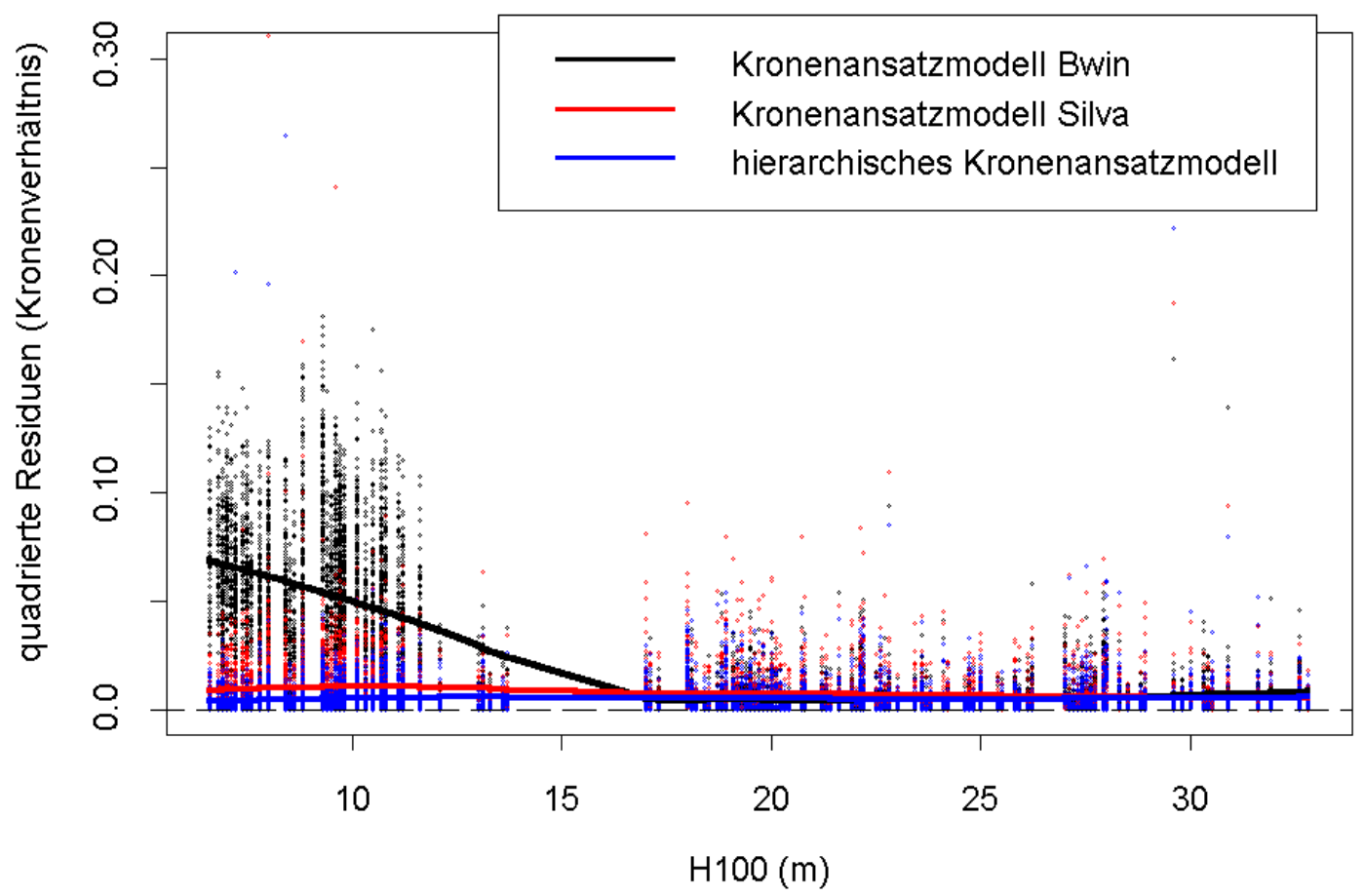

Abb. 87: Quadrierte Residuen der Kronenverhältnisschätzung mit Hilfe der Kronenansatzmodelle in Bwin, in Silva und mit dem neu entwickelten hierarchischen Modell für die Baumart Kiefer. Die Linien sind die zugehörigen Funktionswerte, die mittels eines Gleitenden-Durchschnitts-Modells geschätzt wurden (Kapitel 8.1). 


\section{Europäische Lärche:}

Für die Berechnung der Kronenansatzmodelle standen 3632 Einzelmessungen aus 125 Parzellenaufnahmen mit mindestens 20 gemessenen Kronenansatzhöhen pro Aufnahme zur Verfügung. Für die Europäische Lärche wurde die unabhängige Variable Spitzenhöhe (Formel 10) durch die Variable Alter ersetzt, da dadurch die Schätzgenauigkeit des Modells deutlich verbessert werden konnte.
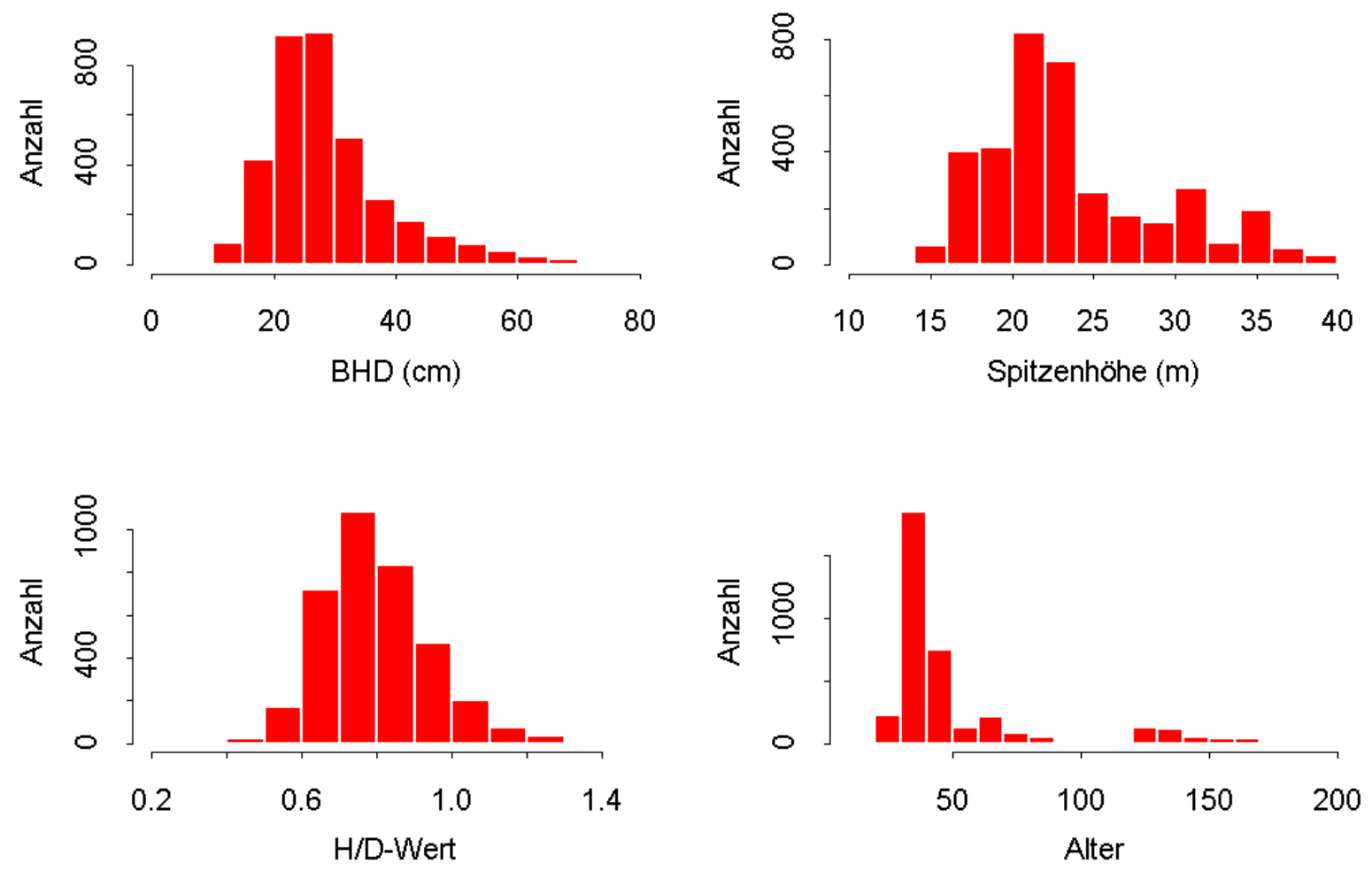

Abb. 88: Verteilungen der Variablen, die in den neu entwickelten Modellen zur Schätzung des Kronenansatzes verwendet werden für die Baumart Europäische Lärche.

$$
\overline{K V}=\alpha+\beta * \text { Alter }+\lambda * \ln (\overline{H / D)}+\delta * \ln (K S F)
$$

(10) abgewandelt

mit:

\begin{tabular}{|r|r|r|r|r|}
\hline Koeffizienten & Wert & Std. Fehler & t-Wert & \multicolumn{1}{|c|}{$\operatorname{Pr}(>|\mathrm{t}|)$} \\
\hline$\alpha$ & 0.7146 & 0.1077 & 6.6345 & 0.0000 \\
\hline$\beta$ & -0.0012 & 0.0002 & -5.8227 & 0.0000 \\
\hline$\lambda$ & -0.1618 & 0.0485 & -3.3378 & 0.0011 \\
\hline$\delta$ & -0.0360 & 0.0124 & -2.9051 & 0.0044 \\
\hline \multicolumn{3}{|c|}{ Residual Std. Fehler } & 0.05098 bei 121 Freiheitsgraden \\
\hline Multiples Bestimmtheitsmaß & F-Statistik & 30.32 bei 3 und 121 Freiheitsgraden, $p$-Wert $=0$ \\
\hline
\end{tabular}

Tab. 44: Koeffizienten und statistische Kenngrößen zur Schätzung des Koeffizienten "mittleres Kronenverhältnis " der Bäume einer Baumart im Bestand (verwendet in Formel (9)) mit Hilfe des Modells (10, abgewandelt) für die Baumart Europäische Lärche. 
$\overline{K V}=\alpha+\beta *$ Alter $+\lambda * \ln (\overline{H / D)}$

(11) abgewandelt

mit:

\begin{tabular}{|c|c|c|c|c|}
\hline Koeffizienten & Wert & Std. Fehler & t-Wert & $\operatorname{Pr}(>|t|)$ \\
\hline$\alpha$ & 0.4033 & 0.0114 & 35.5042 & 0.0000 \\
\hline$\beta$ & -0.0015 & 0.0002 & -8.8170 & 0.0000 \\
\hline$\lambda$ & -0.1927 & 0.0487 & -3.9549 & 0.0001 \\
\hline \multicolumn{2}{|c|}{ Residual Std. Fehler } & \multicolumn{3}{|c|}{0.05251 bei 122 Freiheitsgrader } \\
\hline \multicolumn{2}{|c|}{ Multiples Bestimmtheitsmaß } & \multicolumn{3}{|r|}{0.3894} \\
\hline \multicolumn{2}{|r|}{ F-Statistik } & \multicolumn{3}{|c|}{38.89 bei 2 und 122 Freiheitsgraden, $p$-Wert $=0$} \\
\hline
\end{tabular}

Tab. 45: Koeffizienten und statistische Kenngrößen zur Schätzung des Koeffizienten "mittleres Kronenverhältnis" der Bäume einer Baumart im Bestand (verwendet in Formel (7)) mit Hilfe des Modells (11, abgewandelt) für die Baumart Europäische Lärche.

\begin{tabular}{|c|c|c|c|c|}
\hline Koeffizienten & Wert & Std. Fehler & $\mathrm{t}$-Wert & $\operatorname{Pr}(>|t|)$ \\
\hline$\alpha$ & -0.0002 & 0.0000 & -5.2261 & 0.0000 \\
\hline$\beta$ & 0.0147 & 0.0013 & 11.0787 & 0.0000 \\
\hline \multicolumn{2}{|c|}{ Residual Std. Fehler } & \multicolumn{3}{|c|}{0.003079 bei 123 Freiheitsgrader } \\
\hline \multicolumn{2}{|c|}{ Multiples Bestimmtheitsmaß } & \multicolumn{3}{|r|}{0.8247} \\
\hline & F-Statistik & \multicolumn{3}{|c|}{289.3 bei 2 und 123 Freiheitsgraden, $p$-Wert $=0$} \\
\hline
\end{tabular}

Tab. 46: Koeffizienten und statistische Kenngrößen zur Schätzung des Steigungskoeffizienten (verwendet in Formel (7)) mit Hilfe des Modells (12) für die Baumart Europäische Lärche.

\begin{tabular}{|l|c|c|r|r|r|}
\hline & Bwin & \multicolumn{1}{|c|}{ Silva } & Bwinpro & \multicolumn{1}{|c|}{ EKVK } & \multicolumn{1}{|c|}{ EKVK (Kor.) } \\
\hline RMSE $(\mathrm{m})$ & 2.25074 & 2.00691 & 1.80292 & 1.4052 & 1.323387 \\
\hline RMSE & 0.09309194 & 0.08602158 & 0.07688881 & 0.06001318 & 0.05788788 \\
\hline
\end{tabular}

\begin{tabular}{|l|c|c|r|r|r|}
\hline & $\begin{array}{c}\text { Dyn. } \\
\text { EKVK (Kor.) }\end{array}$ & $\begin{array}{c}\text { Dyn. } \\
\text { EKVK (Kor.) }<=15\end{array}$ & $\begin{array}{c}\text { Dyn. } \\
\text { EKVK (Kor.) }<=5\end{array}$ & $\begin{array}{c}\text { hierarchisches } \\
\text { Modell I }\end{array}$ & $\begin{array}{c}\text { hierarchisches } \\
\text { Modell II }\end{array}$ \\
\hline RMSE (m) & 1.735923 & 1.721559 & 1.501334 & 1.740774 & 1.902174 \\
\hline RMSE & 0.07270074 & 0.07246217 & 0.06896684 & 0.07496598 & 0.0796335 \\
\hline
\end{tabular}

\begin{tabular}{|l|c|r|r|}
\hline & $\begin{array}{c}\text { Direkte } \\
\text { Anpassung } \\
\text { an die Daten }\end{array}$ & $\begin{array}{c}\text { Forest } \\
\text { modifiziert }\end{array}$ & \multicolumn{1}{l|}{ CEP } \\
\hline RMSE $(\mathrm{m})$ & 1.32773 & 1.99451 & 1.83474 \\
\hline RMSE & 0.0557433 & 0.08821068 & 0.07868276 \\
\hline
\end{tabular}

Tab. 47: Standardfehler der Kronenansatzschätzung (m) und der Kronenverhältnisschätzung für verschiedene Kronenansatzmodelle für die Baumart Europäische Lärche. 


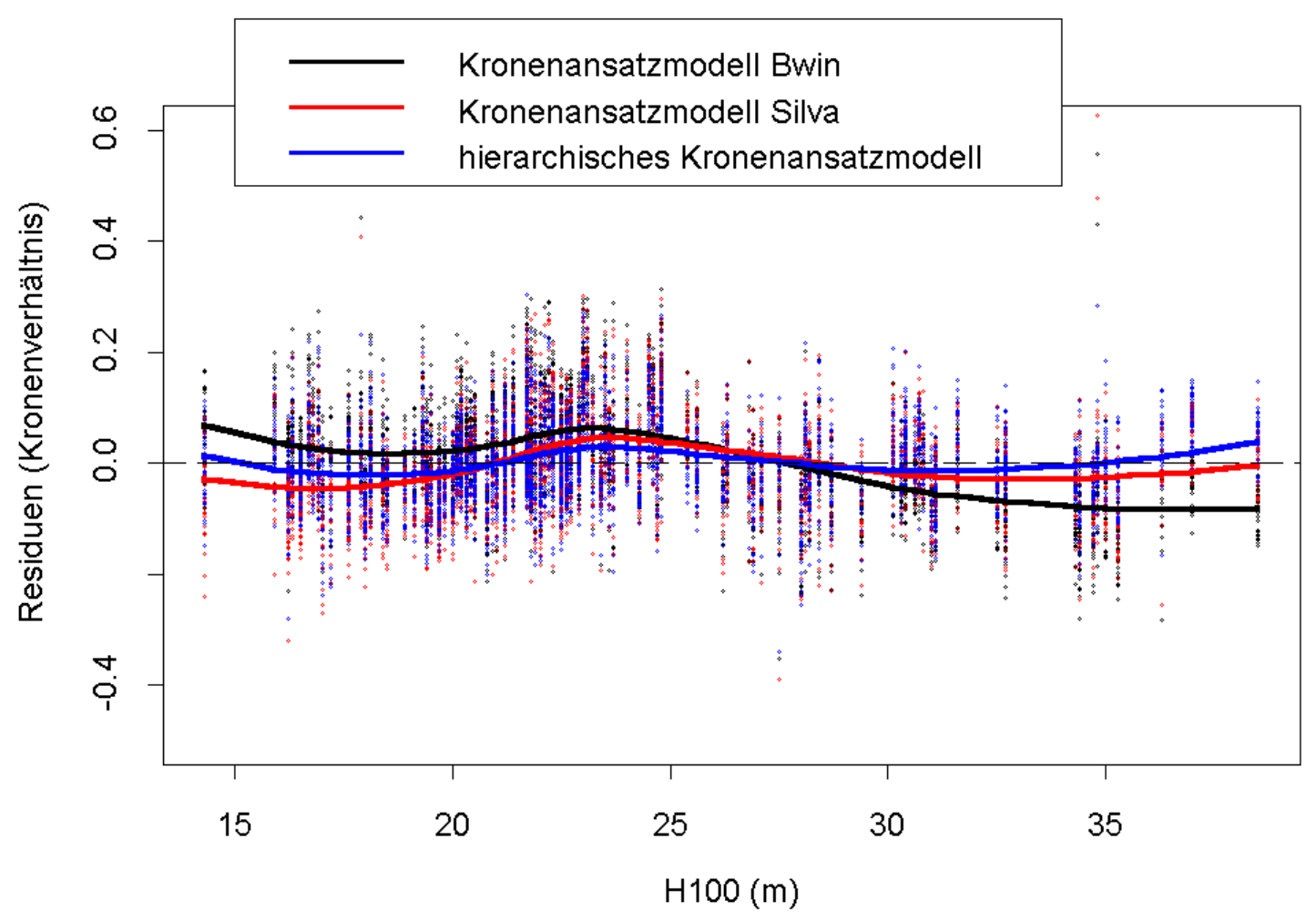

Abb. 89: Residuen der Kronenverhältnisschätzung mit Hilfe der Kronenansatzmodelle in Bwin, in Silva und mit dem neu entwickelten hierarchischen Modell für die Baumart Europäische Lärche. Die Linien sind die zugehörigen Funktionswerte, die mittels eines Gleitenden-Durchschnitts-Modells geschätzt wurden (Kapitel 8.1).

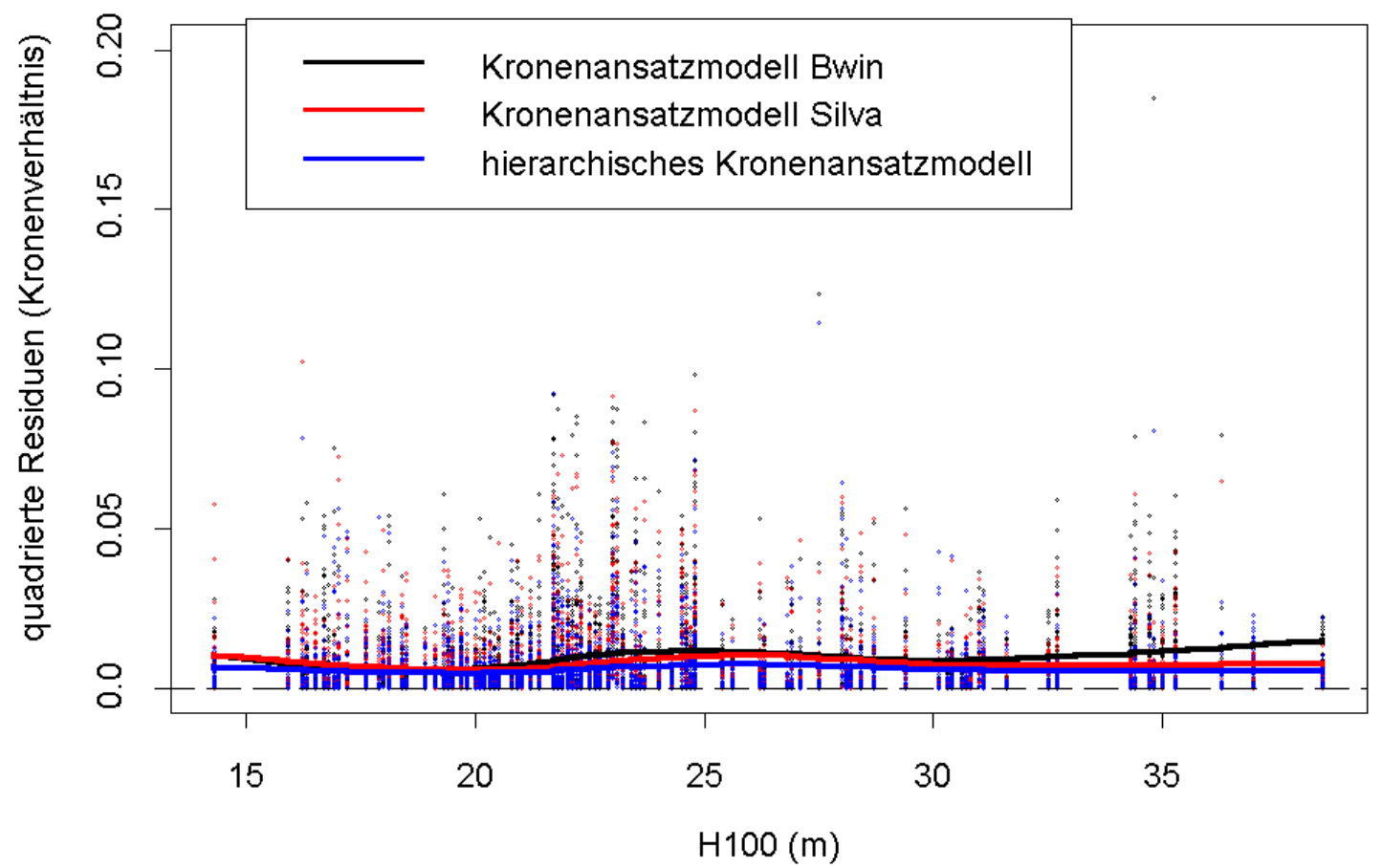

Abb. 90: Quadrierte Residuen der Kronenverhältnisschätzung mit Hilfe der Kronenansatzmodelle in Bwin, in Silva und mit dem neu entwickelten hierarchischen Modell für die Baumart Europäische Lärche. Die Linien sind die zugehörigen Funktionswerte, die mittels eines Gleitenden-Durchschnitts-Modells geschätzt wurden (Kapitel 8.1). 


\subsection{Einfaches statisches Kronenansatzmodell}

\section{Douglasie:}

$$
K A=H *\left(1-e^{-a b s\left(\alpha+\beta^{*} H / D+\gamma^{*} D^{*}+\delta^{*} H 100\right)}\right)
$$

(13) abgewandelt

mit:

\begin{tabular}{|r|r|r|r|}
\hline Koeffizienten & Wert & Std. Fehler & t-Wert \\
\hline$\alpha$ & 0.12895700 & 0.017449600 & 7.39029 \\
\hline$\beta$ & -0.24964500 & 0.021217700 & -11.76590 \\
\hline$\gamma$ & 0.00671424 & 0.000364748 & 18.40790 \\
\hline$\delta$ & -0.03511180 & 0.000562897 & -62.37700 \\
\hline \multicolumn{2}{|c|}{ Residual Std. Fehler (m) } & \multicolumn{2}{c|}{2.0646 bei 11837 Freiheitsgraden } \\
\hline
\end{tabular}

Tab. 48: Koeffizienten und statistische Kenngrößen zur Kronenansatzschätzung mit Hilfe des Modells (13, abgewandelt) für die Baumart Douglasie.
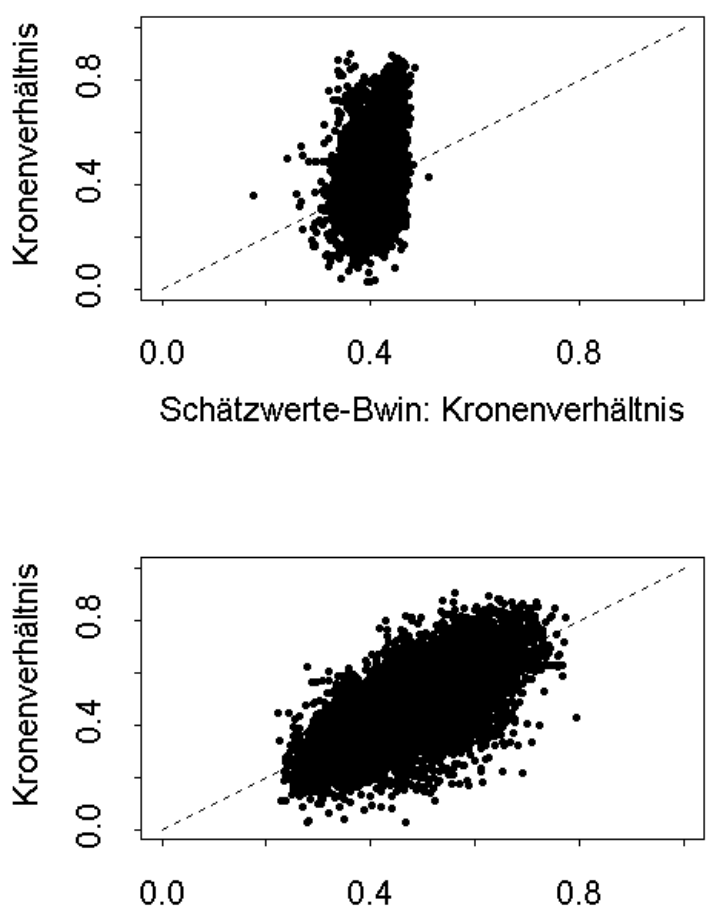

Schätzwerte-Bwinpro: Kronenverhältnis
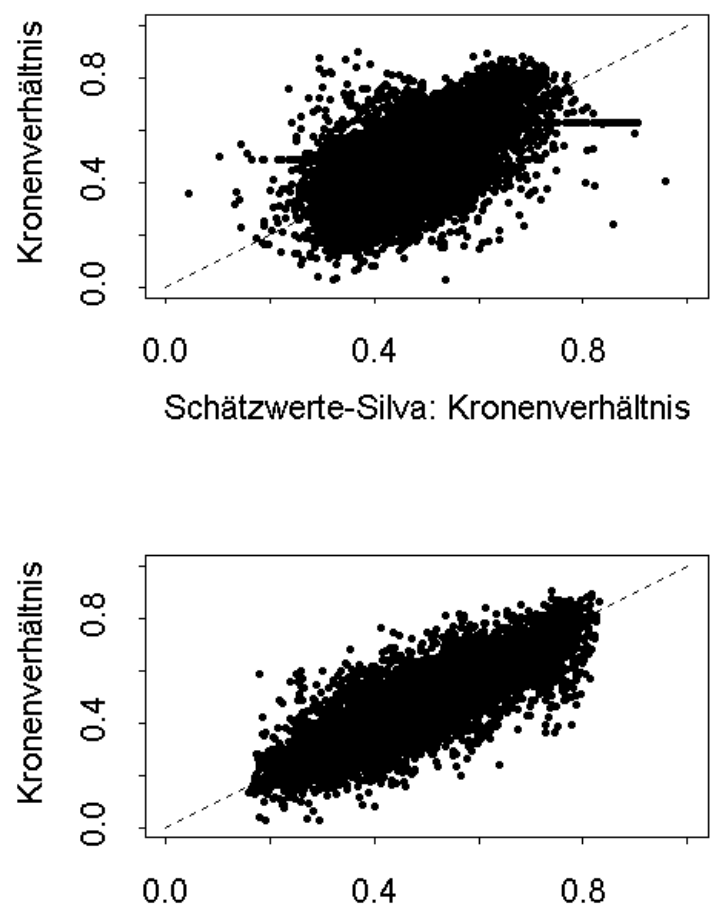

Schätzwerte-(EKVK (Kor.)): Kronenverhältnis

Abb. 91: Darstellung der gemessenen über den geschätzten Kronenverhältnissen der Schätzung der Kronenansatzmodelle in Bwin, in Silva, des neu entwickelten, einfachen statischen Modells (in Bwinpro) und des in Kapitel 3.2.3 beschriebenen Einheitskronenansatzmodells mit Korrektur durch eine Begrenzungsfunktion (EKVK (Kor.)) für die Baumart Douglasie. 
Kiefer:

\begin{tabular}{|r|r|r|r|}
\hline Koeffizienten & Wert & Std. Fehler & t-Wert \\
\hline$\alpha$ & 1.20847000 & 0.023657100 & 51.08250 \\
\hline$\beta$ & -0.23919000 & 0.027364300 & -8.74097 \\
\hline$\gamma$ & 0.00742456 & 0.000584344 & 12.70580 \\
\hline$\delta$ & -0.78971500 & 0.013767300 & -57.36160 \\
\hline \multicolumn{2}{|c|}{ Residual Std. Fehler (m) } & \multicolumn{2}{c|}{1.18733 bei 7403 Freiheitsgraden } \\
\hline
\end{tabular}

Tab. 49: Koeffizienten und statistische Kenngrößen zur Kronenansatzschätzung mit Hilfe des Modells (13) für die Baumart Kiefer.

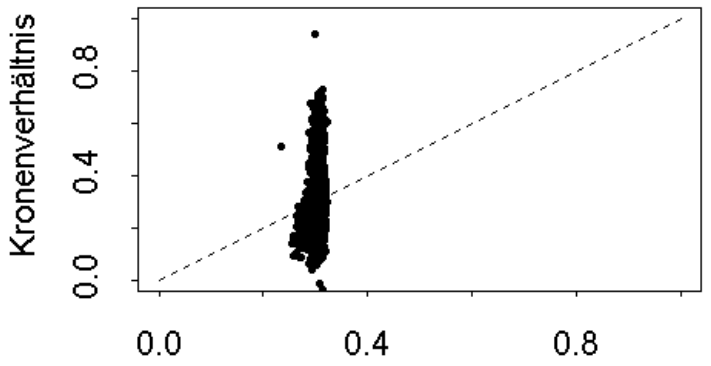

Schätzwerte-Bwin: Kronenverhältnis

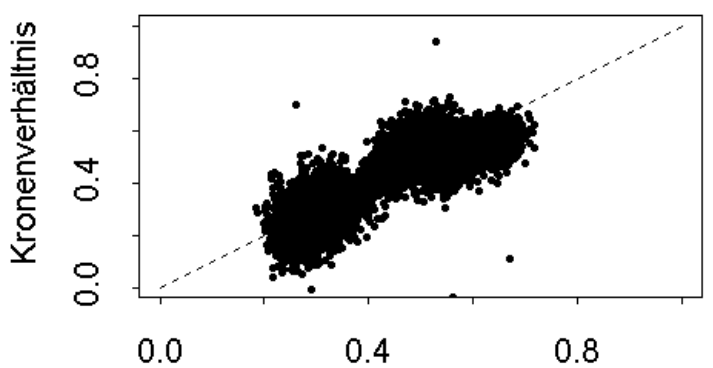

Schätzwerte-Bwinpro: Kronenverhältnis

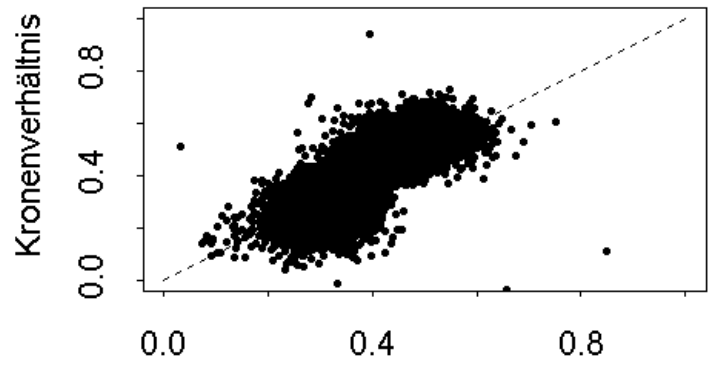

Schätzwerte-Silva: Kronenverhältnis

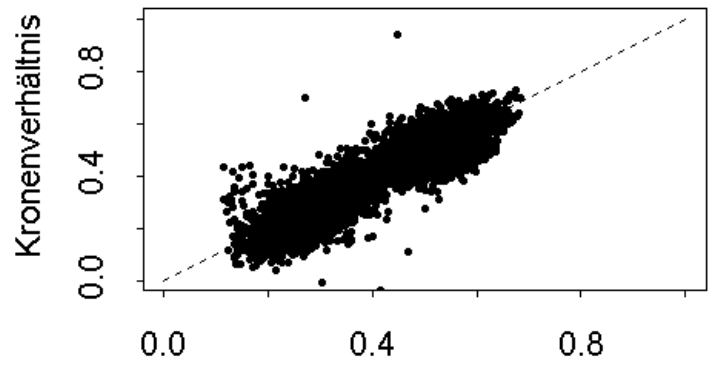

Schätzwerte-(EKVK (Kor.)): Kronenverhältnis

Abb. 92: Darstellung der gemessenen über den geschätzten Kronenverhältnissen der Schätzung der Kronenansatzmodelle in Bwin, in Silva, des neu entwickelten, einfachen statischen Modells (in Bwinpro) und des in Kapitel 3.2.3 beschriebenen Einheitskronenansatzmodells mit Korrektur durch eine Begrenzungsfunktion (EKVK (Kor.)) für die Baumart Kiefer. 


\section{Europäische Lärche:}

$$
K A=H^{*}\left(1-e^{-a b s\left(\alpha+\beta^{*} H / D+\gamma^{*} D^{*}+\delta^{*} H 100\right)}\right)
$$

(13) abgewandelt

mit:

\begin{tabular}{|r|r|r|r|}
\hline Koeffizienten & Wert & Std. Fehler & t-Wert \\
\hline$\alpha$ & -0.30354300 & 0.04556370 & -6.66195 \\
\hline$\beta$ & -0.14515000 & 0.05952030 & -2.43867 \\
\hline$\gamma$ & 0.00493425 & 0.00122238 & 4.03658 \\
\hline$\delta$ & -0.03026960 & 0.00195591 & -15.47600 \\
\hline \multicolumn{2}{|c|}{ Residual Std. Fehler (m) } & \multicolumn{2}{c|}{1.88352 bei 3628 Freiheitsgraden } \\
\hline
\end{tabular}

Tab. 50: Koeffizienten und statistische Kenngrößen zur Kronenansatzschätzung mit Hilfe des Modells (13, abgewandelt) für die Baumart Europäische Lärche.

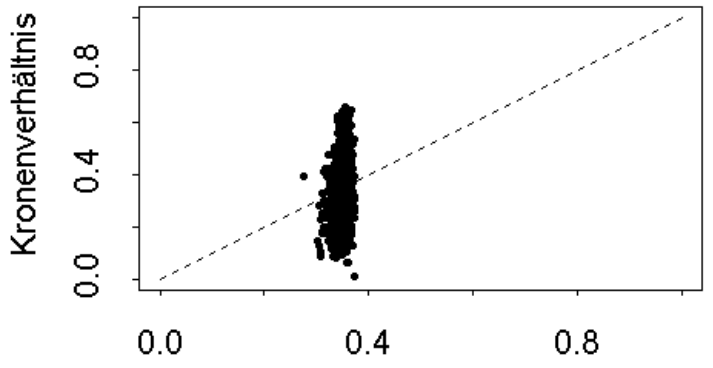

Schätzwerte-Bwin: Kronenverhältnis

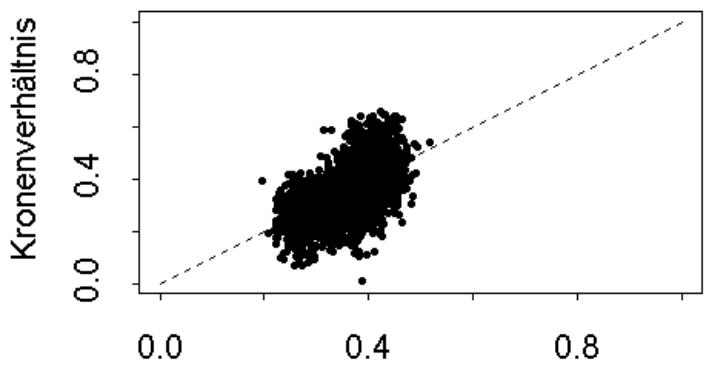

Schätzwerte-Bwinpro: Kronenverhältnis

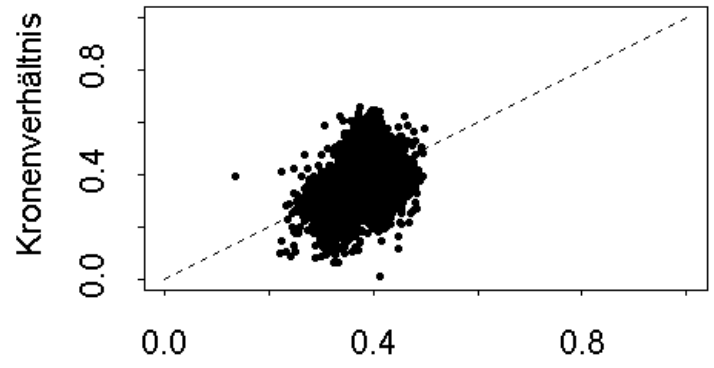

Schätzwerte-Silva: Kronenverhältnis

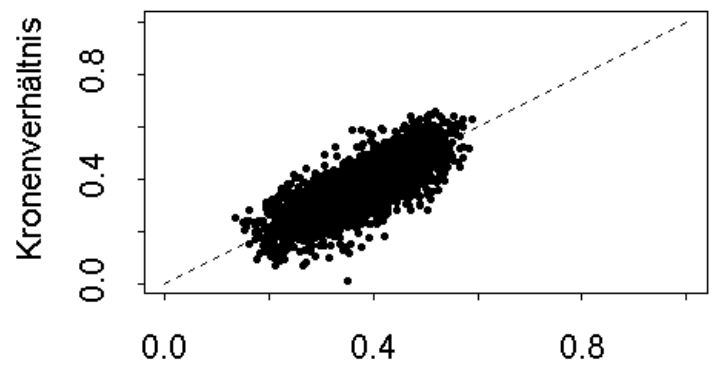

Schätzwerte-(EKVK (Kor.)): Kronenverhältnis

Abb. 93: Darstellung der gemessenen über den geschätzten Kronenverhältnissen der Schätzung der Kronenansatzmodelle in Bwin, in Silva, des neu entwickelten, einfachen statischen Modells (in Bwinpro) und des in Kapitel 3.2.3 beschriebenen Einheitskronenansatzmodells mit Korrektur durch eine Begrenzungsfunktion (EKVK (Kor.)) für die Baumart Europäische Lärche. 


\section{Eiche:}

Für die Berechnung der Kronenansatzmodelle standen 12776 Einzelmessungen aus 375 Parzellenaufnahmen mit mindestens 20 gemessenen Kronenansatzhöhen pro Aufnahme zur Verfügung.
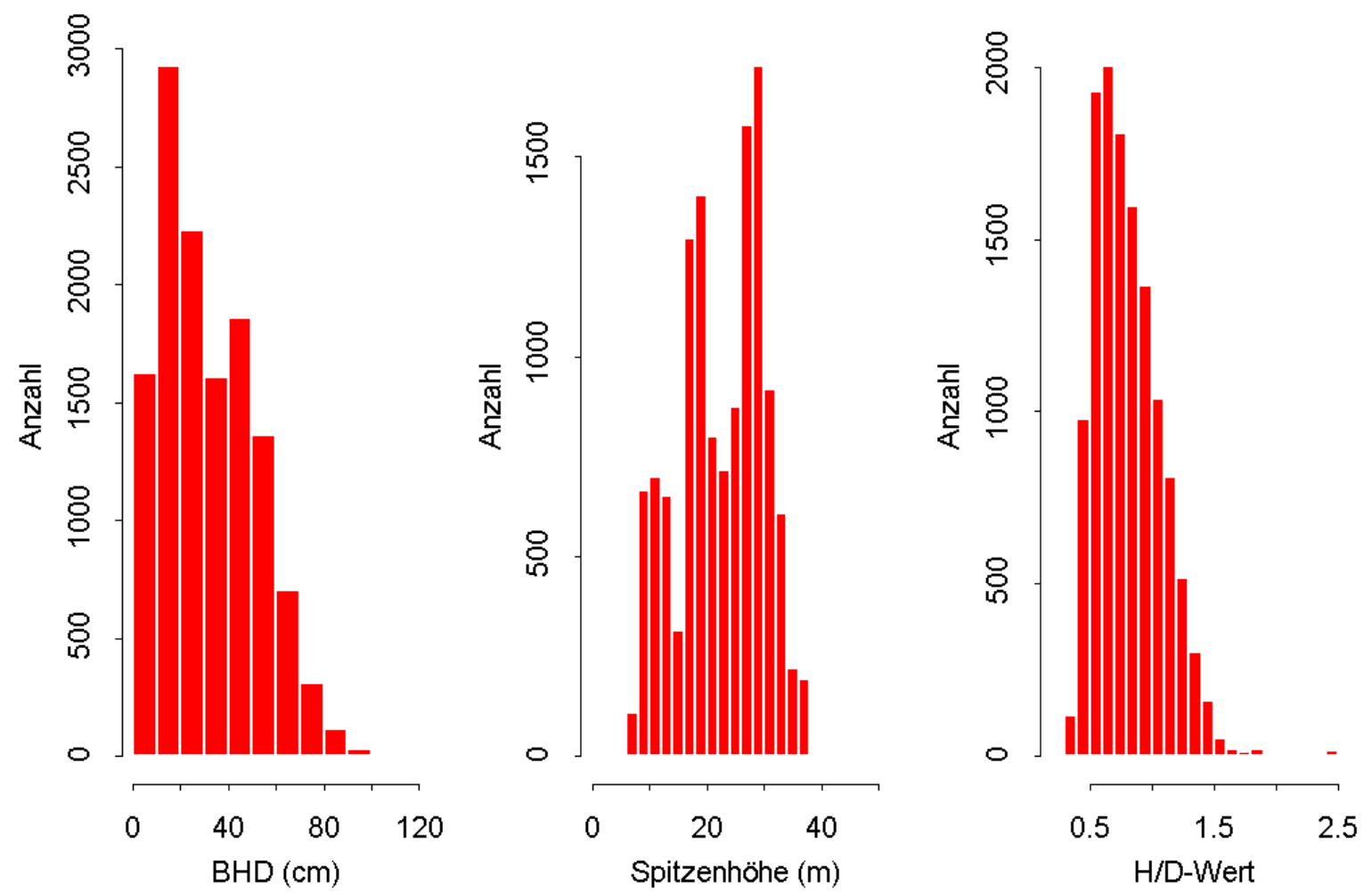

Abb. 94: Verteilungen der Variablen, die in den neu entwickelten Modellen zur Schätzung des Kronenansatzes verwendet werden für die Baumart Eiche.

\begin{tabular}{|r|r|r|r|}
\hline Koeffizienten & Wert & Std. Fehler & \multicolumn{1}{c|}{ t-Wert } \\
\hline$\alpha$ & 0.52682300 & 0.04155020 & 12.6792 \\
\hline$\beta$ & -0.22870500 & 0.02078110 & -11.0054 \\
\hline$\gamma$ & 0.00453026 & 0.00025418 & 17.8230 \\
\hline$\delta$ & -0.47121000 & 0.01238590 & -38.0439 \\
\hline \multicolumn{2}{|c|}{ Residual Std. Fehler (m) } & \multicolumn{2}{c|}{1.97239 bei 12772 Freiheitsgraden } \\
\hline
\end{tabular}

Tab. 51: Koeffizienten und statistische Kenngrößen zur Kronenansatzschätzung mit Hilfe des Modells (13) für die Baumart Eiche. 

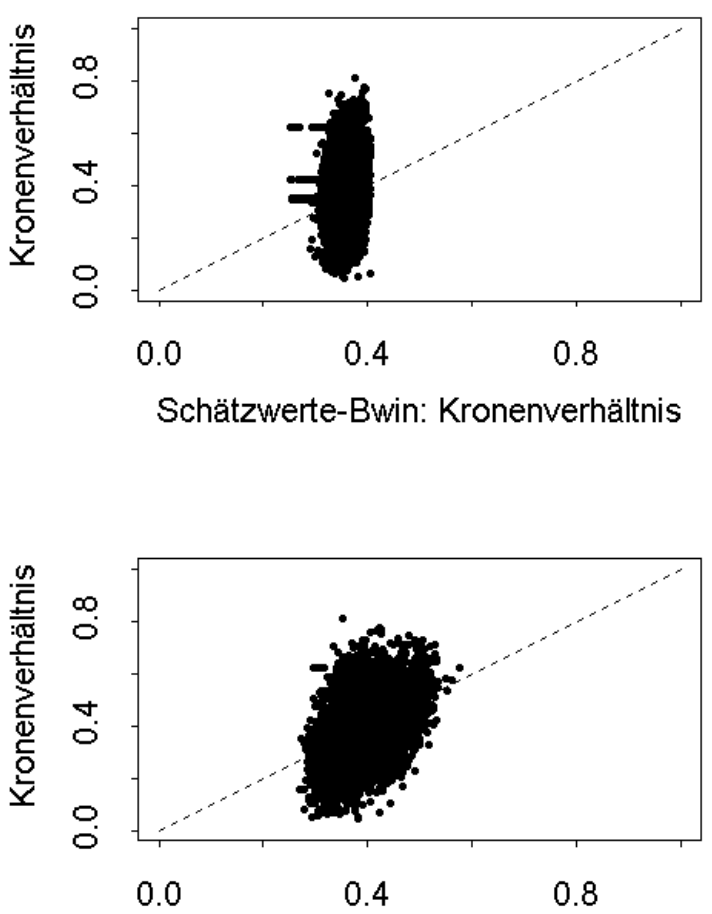

Schätzwerte-Bwinpro: Kronenverhältnis

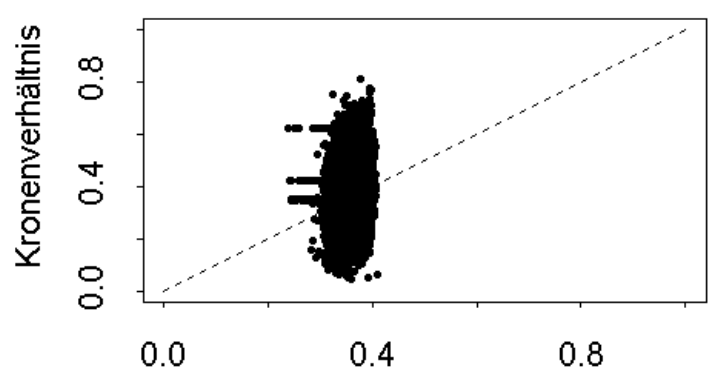

Schätzwerte-Silva: Kronenverhältnis

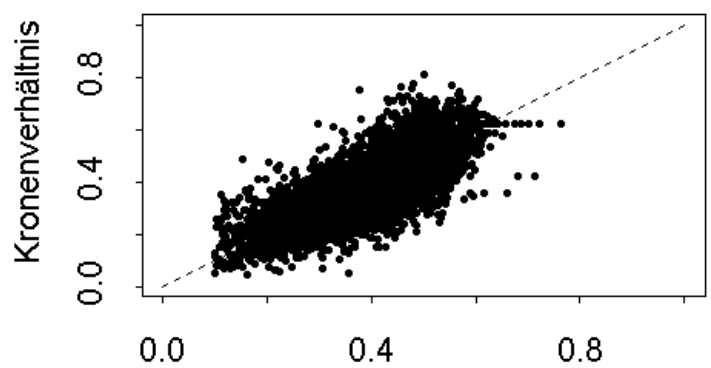

Schätzwerte-(EKVK (Kor.)): Kronenverhältnis

Abb. 95: Darstellung der gemessenen über den geschätzten Kronenverhältnissen der Schätzung der Kronenansatzmodelle in Bwin, in Silva, des neu entwickelten, einfachen statischen Modells (in Bwinpro) und des in Kapitel 3.2.3 beschriebenen Einheitskronenansatzmodells mit Korrektur durch eine Begrenzungsfunktion (EKVK (Kor.)) für die Baumart Eiche.

\begin{tabular}{|l|c|c|c|c|c|}
\hline & Bwin & Silva & Bwinpro & EKVK & \multicolumn{1}{c|}{ EKVK (Kor.) } \\
\hline RMSE $(\mathrm{m})$ & 2.0710800 & 2.0702600 & 1.97239000 & 1.37815000 & 1.37338700 \\
\hline RMSE & 0.1013073 & 0.1011789 & 0.08869202 & 0.06685757 & 0.06559008 \\
\hline
\end{tabular}

\begin{tabular}{|l|c|c|c|c|c|}
\hline & $\begin{array}{c}\text { Dyn. EKVK } \\
\text { (Kor.) }\end{array}$ & $\begin{array}{c}\text { Dyn. EKVK } \\
\text { (Kor.) }<=15\end{array}$ & $\begin{array}{c}\text { Dyn. EKVK } \\
\text { (Kor.) }<=5\end{array}$ & $\begin{array}{c}\text { Forest } \\
\text { modifiziert }\end{array}$ & CEP \\
\hline RMSE $(\mathrm{m})$ & 2.34525800 & 1.76511000 & 1.68173600 & 2.03609000 & 2.0264700 \\
\hline RMSE & 0.09522251 & 0.08292387 & 0.08134027 & 0.09456839 & 0.1032165 \\
\hline
\end{tabular}

\begin{tabular}{|l|c|}
\hline & $\begin{array}{c}\text { Direkte } \\
\text { Anpassung } \\
\text { an die Daten }\end{array}$ \\
\hline RMSE $(\mathrm{m})$ & 1.29647200 \\
\hline RMSE & 0.06101208 \\
\hline
\end{tabular}

Tab. 52: Standardfehler der Kronenansatzschätzung (m) und der Kronenverhältnisschätzung für verschiedene Kronenansatzmodelle für die Baumart Eiche. 


\section{Buche:}

Für die Berechnung der Kronenansatzmodelle standen 23812 Einzelmessungen aus 485 Parzellenaufnahmen mit mindestens 20 gemessenen Kronenansatzhöhen pro Aufnahme zur Verfügung.
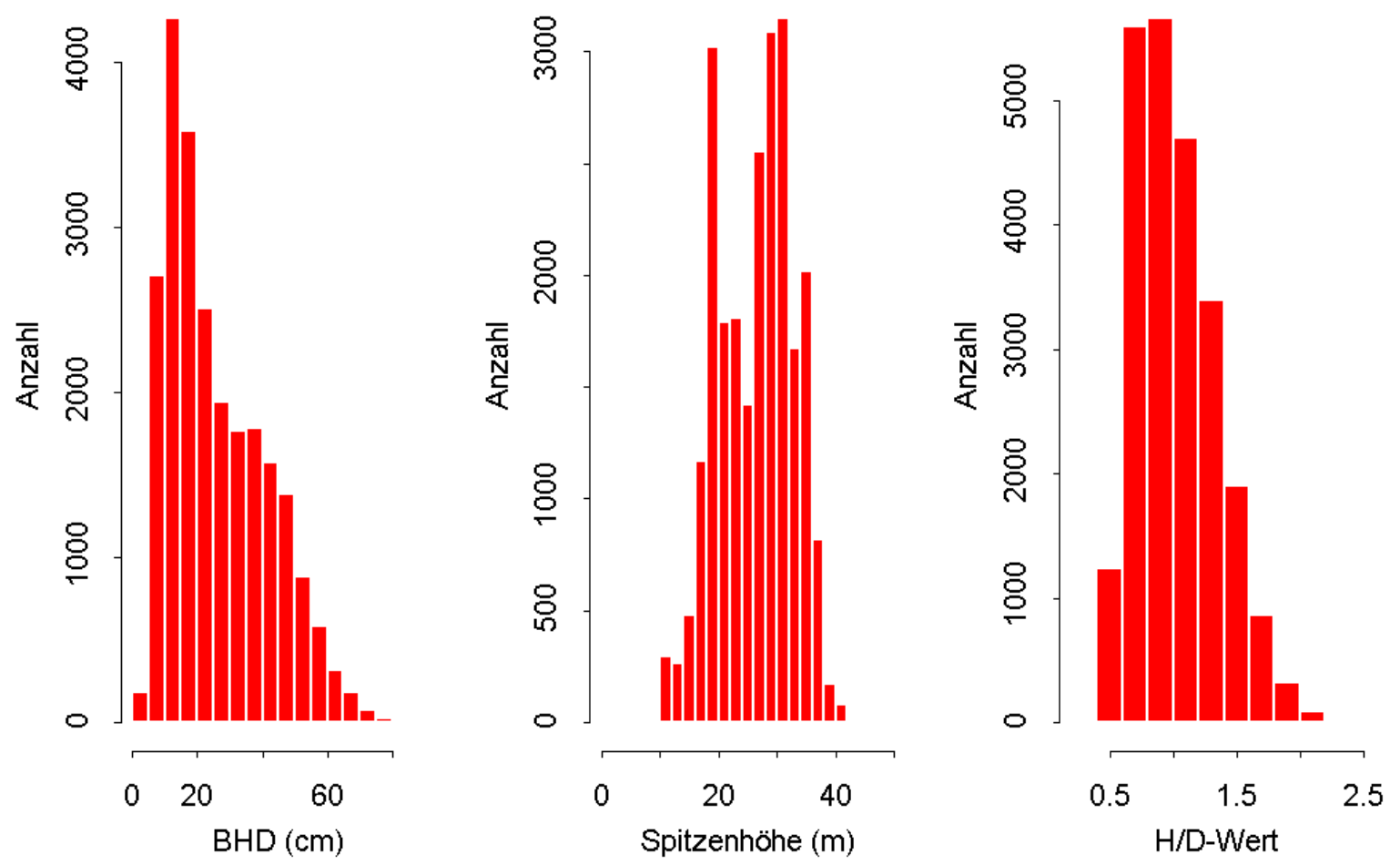

Abb. 96: Verteilungen der Variablen, die in den neu entwickelten Modellen zur Schätzung des Kronenansatzes verwendet werden für die Baumart Buche.

\begin{tabular}{|r|r|r|r|}
\hline Koeffizienten & Wert & Std. Fehler & \multicolumn{1}{c|}{ t-Wert } \\
\hline$\alpha$ & 0.25703700 & 0.037525100 & 6.84972 \\
\hline$\beta$ & 0.11818900 & 0.013734100 & 8.60548 \\
\hline$\gamma$ & -0.00206248 & 0.000281607 & -7.32396 \\
\hline$\delta$ & 0.13830700 & 0.012396200 & 11.15720 \\
\hline \multicolumn{3}{|c|}{ Residual Std. Fehler (m) } & \multicolumn{2}{c|}{3.25173 bei 23808 Freiheitsgraden } \\
\hline
\end{tabular}

Tab. 53: Koeffizienten und statistische Kenngrößen zur Kronenansatzschätzung mit Hilfe des Modells (13) für die Baumart Buche. 

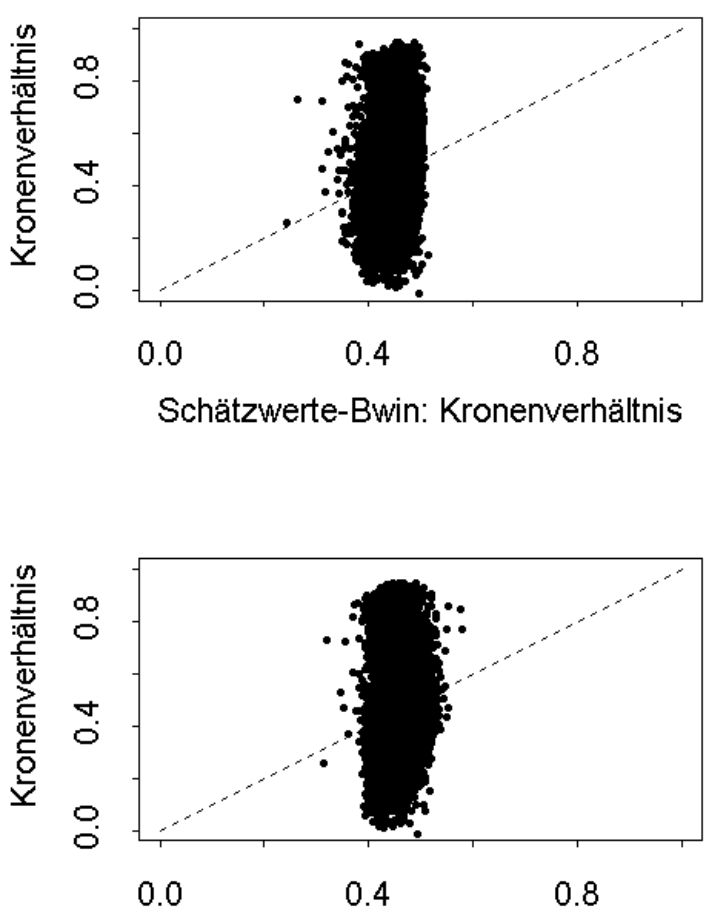

Schätzwerte -Bwinpro: Kronenverhältnis

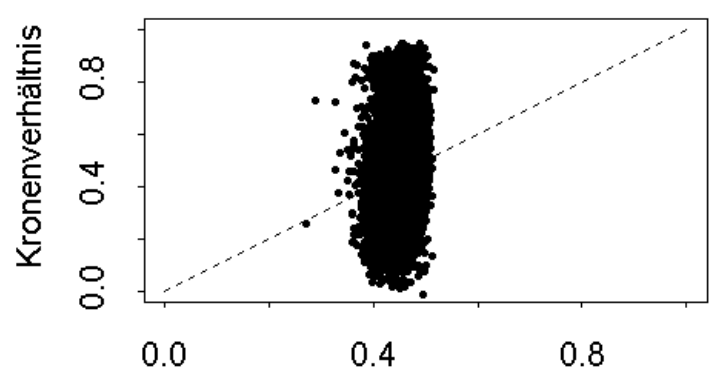

Schätzwerte -Silva: Kronenverhältnis

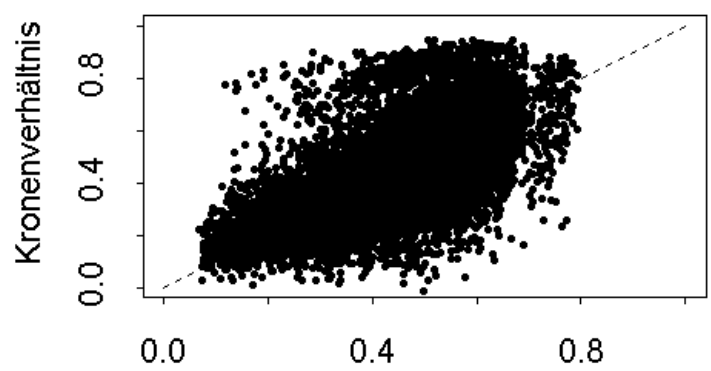

Schätzwerte-(EKVK (Kor.)): Kronenverhältnis

Abb. 97: Darstellung der gemessenen über den geschätzten Kronenverhältnissen der Schätzung der Kronenansatzmodelle in Bwin, in Silva, des neu entwickelten, einfachen statischen Modells (in Bwinpro) und des in Kapitel 3.2.3 beschriebenen Einheitskronenansatzmodells mit Korrektur durch eine Begrenzungsfunktion (EKVK (Kor.)) für die Baumart Buche.

\begin{tabular}{|l|l|l|r|r|r|}
\hline & Bwin & Silva & \multicolumn{1}{c|}{ Bwinpro } & \multicolumn{1}{c|}{ EKVK } & EKVK (Kor.) \\
\hline RMSE $(\mathrm{m})$ & 3.2600500 & 3.2594900 & 3.251190 & 2.3418700 & 2.3372310 \\
\hline RMSE & 0.1491195 & 0.1491533 & 0.149311 & 0.1213766 & 0.1187773 \\
\hline
\end{tabular}

\begin{tabular}{|l|c|c|c|c|c|}
\hline & $\begin{array}{c}\text { Dyn. EKVK } \\
\text { (Kor.) }\end{array}$ & $\begin{array}{c}\text { Dyn. EKVK } \\
\text { (Kor.) }<=15\end{array}$ & $\begin{array}{c}\text { Dyn. EKVK } \\
\text { (Kor.) }<=5\end{array}$ & $\begin{array}{c}\text { Forest } \\
\text { modifiziert }\end{array}$ & CEP \\
\hline RMSE $(\mathrm{m})$ & 3.2961170 & 2.9096920 & 2.6667350 & 3.2522500 & 3.2239400 \\
\hline RMSE & 0.1293703 & 0.1204719 & 0.1087179 & 0.1487272 & 0.1481009 \\
\hline
\end{tabular}

\begin{tabular}{|l|c|}
\hline & $\begin{array}{c}\text { Direkte } \\
\text { Anpassung } \\
\text { an die Daten }\end{array}$ \\
\hline RMSE (m) & 2.1377380 \\
\hline RMSE & 0.1030478 \\
\hline
\end{tabular}

Tab. 54: Standardfehler der Kronenansatzschätzung (m) und der Kronenverhältnisschätzung für verschiedene Kronenansatzmodelle für die Baumart Buche. 


\subsection{Einheitskronenansatzmodell}

\section{Douglasie:}

\begin{tabular}{|r|r|r|r|}
\hline Koeffizienten & Wert & Std. Fehler & \multicolumn{1}{c|}{ t-Wert } \\
\hline$\alpha$ & 3.59953 & 0.996836 & 3.61096 \\
\hline$\beta$ & -4.99455 & 0.276972 & -18.03270 \\
\hline$\gamma$ & 5.05041 & 0.337989 & 14.94250 \\
\hline \multicolumn{2}{|c|}{ Residual Std. Fehler (m) } & \multicolumn{2}{c|}{1.60939 bei 11838 Freiheitsgraden } \\
\hline
\end{tabular}

Tab. 55: Koeffizienten und statistische Kenngrößen zur Kronenansatzschätzung mit Hilfe des Modells (14) für die Baumart Douglasie.

\begin{tabular}{|r|r|r|r|}
\hline Koeffizienten & Wert & Std. Fehler & \multicolumn{1}{c|}{ t-Wert } \\
\hline$\alpha$ & 59.73460 & 12.1402000 & 4.92040 \\
\hline$\beta$ & 49.43000 & 13.0790000 & 3.77935 \\
\hline$\delta$ & 1.22671 & 0.0916096 & 13.39060 \\
\hline \multicolumn{2}{|c|}{ Residual Std. Fehler (m) } & \multicolumn{2}{|c|}{1.35627 bei 44 Freiheitsgraden } \\
\hline
\end{tabular}

Tab. 56: Koeffizienten und statistische Kenngrößen der Begrenzungsfunktion (2-parametrige Verteilungsfunktion der Weibull-Funktion; Formel (15)) der Kronenansatzschätzung mit Hilfe des Modells (14) für die Baumart Douglasie.

\section{Kiefer:}

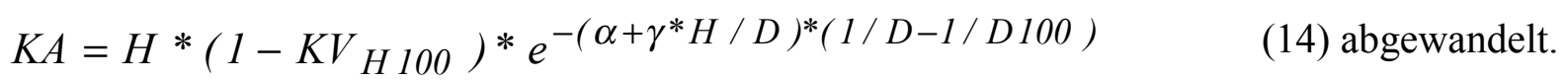

mit:

\begin{tabular}{|r|r|r|r|}
\hline Koeffizienten & Wert & Std. Fehler & t-Wert \\
\hline$\alpha$ & -7.05500 & 0.198055 & -35.6214 \\
\hline$\gamma$ & 2.82681 & \multicolumn{2}{|c|}{0.155426} \\
\hline \multicolumn{2}{|c|}{ Residual Std. Fehler (m) } & \multicolumn{2}{c|}{0.946587 bei 7405 Freiheitsgraden } \\
\hline
\end{tabular}

Tab. 57: Koeffizienten und statistische Kenngrößen zur Kronenansatzschätzung mit Hilfe des Modells (14, abgewandelt) für die Baumart Kiefer.

\begin{tabular}{|r|r|r|r|}
\hline Koeffizienten & Wert & Std. Fehler & \multicolumn{1}{c|}{ t-Wert } \\
\hline$\alpha$ & 44.99430 & 4.6248000 & 9.72892 \\
\hline$\beta$ & 33.57090 & 4.4171200 & 7.60019 \\
\hline$\delta$ & 1.31329 & 0.0637397 & 20.60390 \\
\hline \multicolumn{2}{|c|}{ Residual Std. Fehler (m) } & \multicolumn{2}{|c|}{0.698466 bei 33 Freiheitsgraden } \\
\hline
\end{tabular}

Tab. 58: Koeffizienten und statistische Kenngrößen der Begrenzungsfunktion (2-parametrige Verteilungsfunktion der Weibull-Funktion; Formel (15)) der Kronenansatzschätzung mit Hilfe des Modells (14, abgewandelt) für die Baumart Kiefer. 


\section{Europäische Lärche:}

$K A=H *\left(1-K V_{H 100}\right) * e^{-\left(\beta * \ln (H 100)+\gamma^{*} H / D\right) *(1 / D-1 / D 100)} \quad$ (14) abgewandelt mit:

\begin{tabular}{|r|r|r|r|}
\hline Koeffizienten & Wert & Std. Fehler & t-Wert \\
\hline$\beta$ & -4.74502 & 0.207469 & -22.8710 \\
\hline$\gamma$ & 7.88157 & 0.583772 & 13.5011 \\
\hline \multicolumn{2}{|c|}{ Residual Std. Fehler (m) } & \multicolumn{2}{c|}{1.41037 bei 3629 Freiheitsgraden } \\
\hline
\end{tabular}

Tab. 59: Koeffizienten und statistische Kenngrößen zur Kronenansatzschätzung mit Hilfe des Modells (14, abgewandelt) für die Baumart Europäische Lärche.

\begin{tabular}{|r|r|r|r|}
\hline Koeffizienten & Wert & Std. Fehler & t-Wert \\
\hline$\alpha$ & 36.73910 & 2.83988 & 12.9369 \\
\hline$\beta$ & 26.87640 & 2.62519 & 10.2379 \\
\hline$\delta$ & 1.53527 & 0.11658 & 13.1692 \\
\hline \multicolumn{2}{|c|}{ Residual Std. Fehler $(\mathrm{m})$} & \multicolumn{2}{c|}{1.23591 bei 32 Freiheitsgraden } \\
\hline
\end{tabular}

Tab. 60: Koeffizienten und statistische Kenngrößen der Begrenzungsfunktion (2-parametrige Verteilungsfunktion der Weibull-Funktion; Formel (15)) der Kronenansatzschätzung mit Hilfe des Modells (14, abgewandelt) für die Baumart Europäische Lärche.

Eiche:

\begin{tabular}{|r|r|r|r|}
\hline Koeffizienten & Wert & Std. Fehler & t-Wert \\
\hline$\alpha$ & -7.86466 & 0.857934 & -9.16698 \\
\hline$\beta$ & -1.48221 & 0.227018 & -6.52904 \\
\hline$\gamma$ & 6.03096 & 0.277253 & 21.75250 \\
\hline \multicolumn{2}{|c|}{ Residual Std. Fehler (m) } & \multicolumn{2}{c|}{1.37815 bei 12773 Freiheitsgraden } \\
\hline
\end{tabular}

Tab. 61: Koeffizienten und statistische Kenngrößen zur Kronenansatzschätzung mit Hilfe des Modells (14) für die Baumart Eiche.

\begin{tabular}{|r|r|r|r|}
\hline Koeffizienten & Wert & Std. Fehler & t-Wert \\
\hline$\alpha$ & 28.09940 & 0.9200250 & 30.5420 \\
\hline$\beta$ & 19.99650 & 0.9973660 & 20.0493 \\
\hline$\delta$ & 1.48362 & 0.0889247 & 16.6840 \\
\hline \multicolumn{2}{|c|}{ Residual Std. Fehler (m) } & \multicolumn{2}{|c|}{1.09062 bei 36 Freiheitsgraden } \\
\hline
\end{tabular}

Tab. 62: Koeffizienten und statistische Kenngrößen der Begrenzungsfunktion (2-parametrige Verteilungsfunktion der Weibull-Funktion; Formel (15)) der Kronenansatzschätzung mit Hilfe des Modells (14) für die Baumart Eiche. 


\section{Buche:}

$K A=H *\left(1-K V_{H 100}\right) * e^{-(\alpha+\gamma * H / D-W e r t) *(1 / D-1 / D 100)}$

(14) abgewandelt

mit:

\begin{tabular}{|r|r|r|r|}
\hline Koeffizienten & Wert & Std. Fehler & t-Wert \\
\hline$\alpha$ & -7.03902 & 0.232942 & -30.2179 \\
\hline$\gamma$ & 2.59732 & 0.157035 & 16.5398 \\
\hline \multicolumn{2}{|c|}{ Residual Std. Fehler (m) } & \multicolumn{2}{c|}{2.34133 bei 23810 Freiheitsgraden } \\
\hline
\end{tabular}

Tab. 63: Koeffizienten und statistische Kenngrößen zur Kronenansatzschätzung mit Hilfe des Modells (14, abgewandelt) für die Baumart Buche.

\begin{tabular}{|r|r|r|r|}
\hline Koeffizienten & Wert & Std. Fehler & t-Wert \\
\hline$\alpha$ & 27.84620 & 0.879152 & 31.6740 \\
\hline$\beta$ & 19.01680 & 0.971708 & 19.5705 \\
\hline$\delta$ & 1.48322 & \multicolumn{2}{|c|}{0.100339} \\
\hline \multicolumn{2}{|c|}{ Residual Std. Fehler (m) } & \multicolumn{2}{|c|}{1.34541 bei 40 Freiheitsgraden } \\
\hline
\end{tabular}

Tab. 64: Koeffizienten und statistische Kenngrößen der Begrenzungsfunktion (Verteilungsfunktion der Weibull-Funktion; Formel (15)) der Kronenansatzschätzung mit Hilfe des Modells (14, abgewandelt) für die Baumart Buche. 


\subsection{Dynamisierung des Einheitskronenansatzmodells}

\section{Douglasie:}

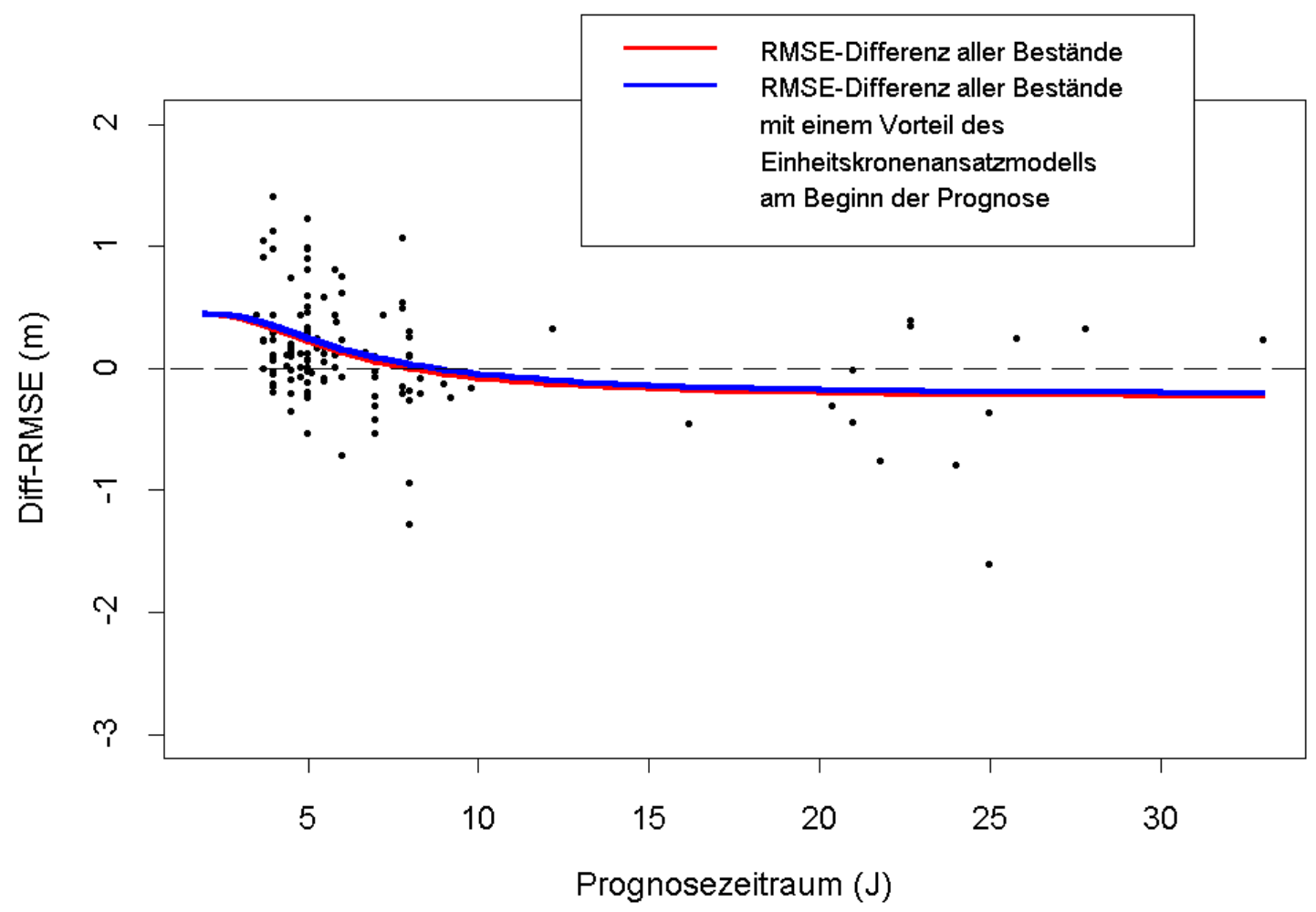

Abb. 98: Fehlerdifferenz der nach Parzellen und Aufnahmezeitpunkten berechneten RMSE des statischen und dynamischen Modellansatzes zur Kronenansatzschätzung in Abhängigkeit vom Prognosezeitraum für die Baumart Douglasie. Die dynamische Schätzung wurde in einem Schritt ohne Zwischenberechnungen der unabhängigen Variablen durchgeführt. Das zum Ausgleich verwendete Modell ist in Formel (18) dargestellt.

\begin{tabular}{|l|r|rr|}
\hline & Alle Bestände & \multicolumn{2}{|c|}{$\begin{array}{c}\text { Nur Bestände mit guter Anpassung des } \\
\text { Einheitskronenansatzmodells } \\
\text { (Startpunktvorteil besteht) }\end{array}$} \\
\hline $\begin{array}{l}\text { Kritischer Prognosezeitraum der } \\
\text { Kronenansatzschätzung (Jahre) }\end{array}$ & 8 & & 8.9 \\
\hline $\begin{array}{l}\text { Maximaler Modellvorteil (m) im } \\
\text { Durchschnitt über alle Bestände }\end{array}$ & 0.449 & & 0.449 \\
\hline $\begin{array}{l}\text { Kritischer Prognosezeitraum der } \\
\text { Kronenverhältnisschätzung (Jahre) }\end{array}$ & 8.4 & & 0.6189 \\
\hline $\begin{array}{l}\text { Maximaler Modellvorteil im } \\
\text { Durchschnitt über alle Bestände } \\
\text { (Kronenverhältnis) }\end{array}$ & 0.0189 & & 0.018 \\
\hline
\end{tabular}

Tab. 65: Kritische Prognosezeiträume und durchschnittliche maximale Vorteile (aller Prognosen und der Prognosen mit günstigem Startpunkt) des dynamischen Kronenansatzmodells für die Baumart Douglasie. Die Werte wurden über das Modell zur Einschätzung der Unterschiede der Genauigkeit von statischem und dynamischem Modellansatz bestimmt. Die Berechnung erfolgte anhand der Fehlerdifferenzen für die Kronenansatzund die Kronenverhältnisschätzung (Formel (18)). 

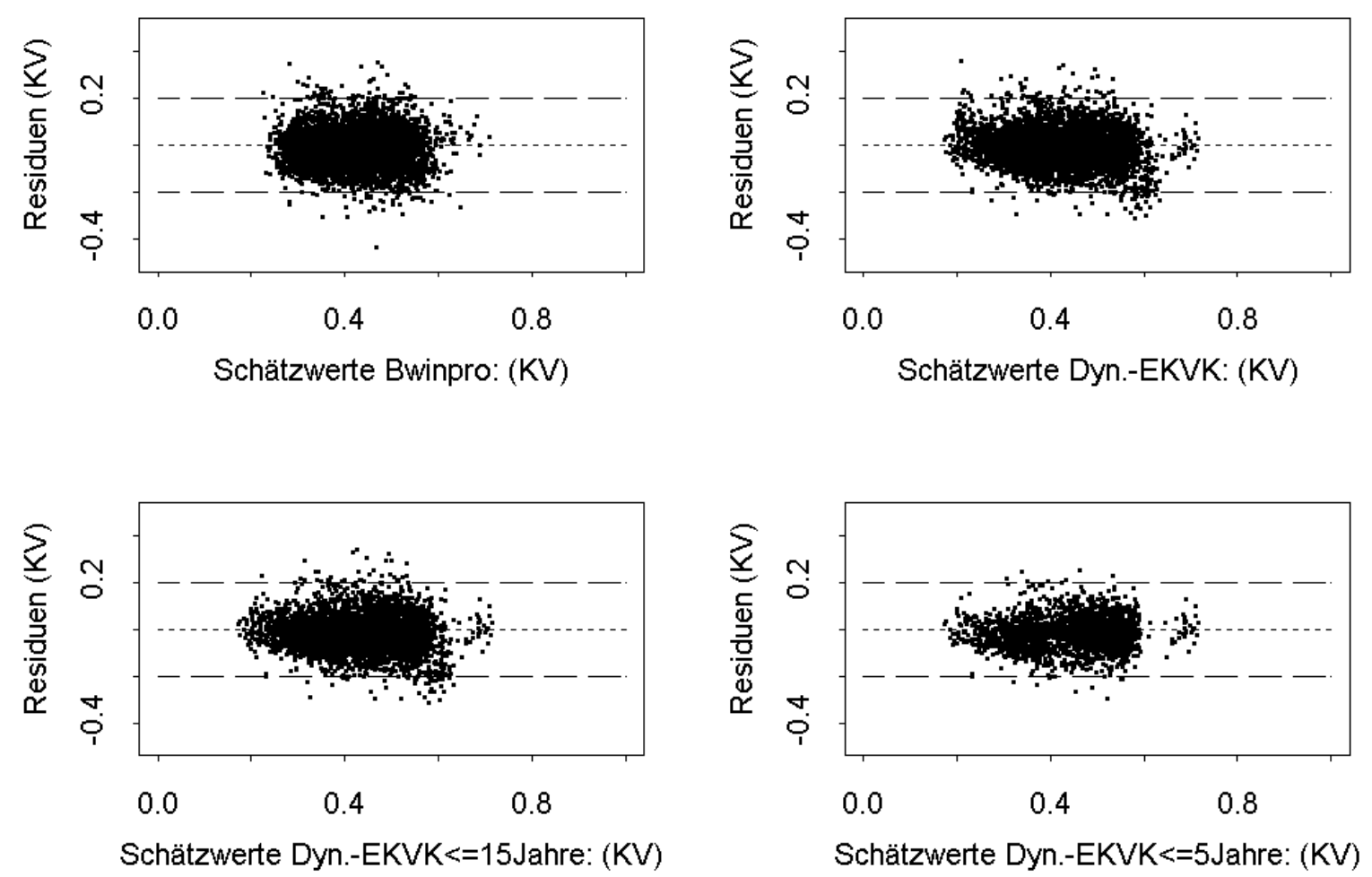

Abb. 99: Residuenplots der Kronenverhältnisschätzung mit Hilfe des neu entwickelten, einfachen statischen Modells (in Bwinpro, Formel (13, abgewandelt)) und des dynamischen Modellansatzes (14) und (16) für die Baumart Douglasie. Für den dynamischen Modellansatz sind neben den Ergebnissen der Schätzung für alle Prognosezeiträume auch die Ergebnisse der Begrenzung der Prognosezeiträume auf maximal 15 und 5 Jahre dargestellt (Dyn. EKVK, Dyn. EKVK <=15, Dyn. EKVK <=5). Die Schätzungen des dynamischen Modells sind mit Hilfe der Begrenzungsfunktion (15) überprüft und gegebenenfalls korrigiert worden. 
Kiefer:

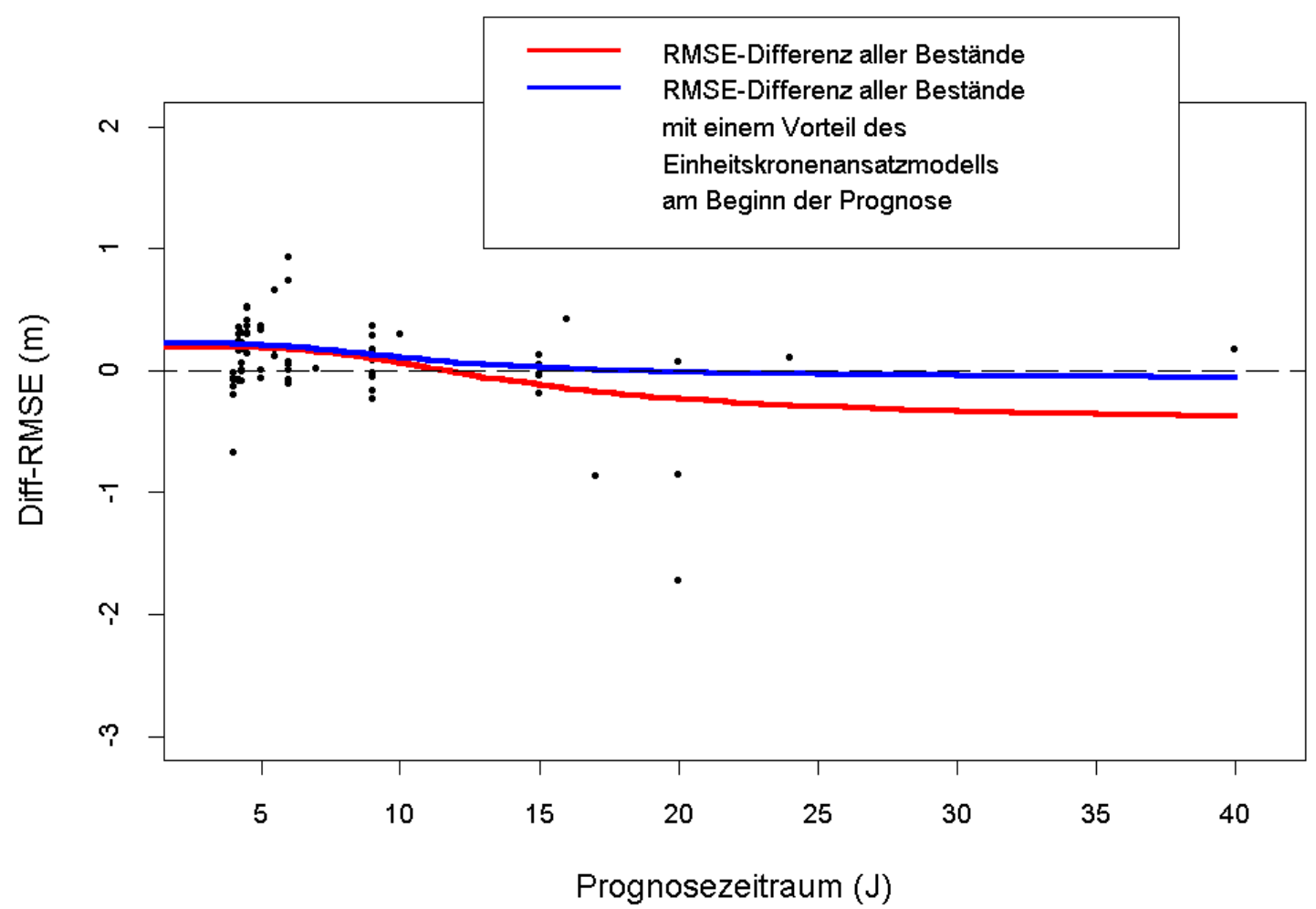

Abb. 100: Fehlerdifferenz der nach Parzellen und Aufnahmezeitpunkten berechneten RMSE des statischen und dynamischen Modellansatzes zur Kronenansatzschätzung in Abhängigkeit vom Prognosezeitraum für die Baumart Kiefer. Die dynamische Schätzung wurde in einem Schritt ohne Zwischenberechnungen der unabhängigen Variablen durchgeführt. Das zum Ausgleich verwendete Modell ist in Formel (18) dargestellt.

\begin{tabular}{|l|r|rr|}
\hline & Alle Bestände & \multicolumn{2}{|c|}{$\begin{array}{c}\text { Nur Bestände mit guter Anpassung des } \\
\text { Einheitskronenansatzmodells } \\
\text { (Startpunktvorteil besteht) }\end{array}$} \\
\hline $\begin{array}{l}\text { Kritischer Prognosezeitraum der } \\
\text { Kronenansatzschätzung (Jahre) }\end{array}$ & 11.6 & & 18.6 \\
\hline $\begin{array}{l}\text { Maximaler Modellvorteil (m) im } \\
\text { Durchschnitt über alle Bestände }\end{array}$ & 0.188 & & 0.224 \\
\hline $\begin{array}{l}\text { Kritischer Prognosezeitraum der } \\
\text { Kronenverhältnisschätzung (Jahre) }\end{array}$ & 11.4 & & 0.0222 \\
\hline $\begin{array}{l}\text { Maximaler Modellvorteil im } \\
\text { Durchschnitt über alle Bestände } \\
\text { (Kronenverhältnis) }\end{array}$ & 0.0163 & & \\
\hline
\end{tabular}

Tab. 66: Kritische Prognosezeiträume und durchschnittliche maximale Vorteile (aller Prognosen und der Prognosen mit günstigem Startpunkt) des dynamischen Kronenansatzmodells für die Baumart Kiefer. Die Werte wurden über das Modell zur Einschätzung der Unterschiede der Genauigkeit von statischem und dynamischem Modellansatz bestimmt. Die Berechnung erfolgte anhand der Fehlerdifferenzen für die Kronenansatzund die Kronenverhältnisschätzung (Formel (18)). 

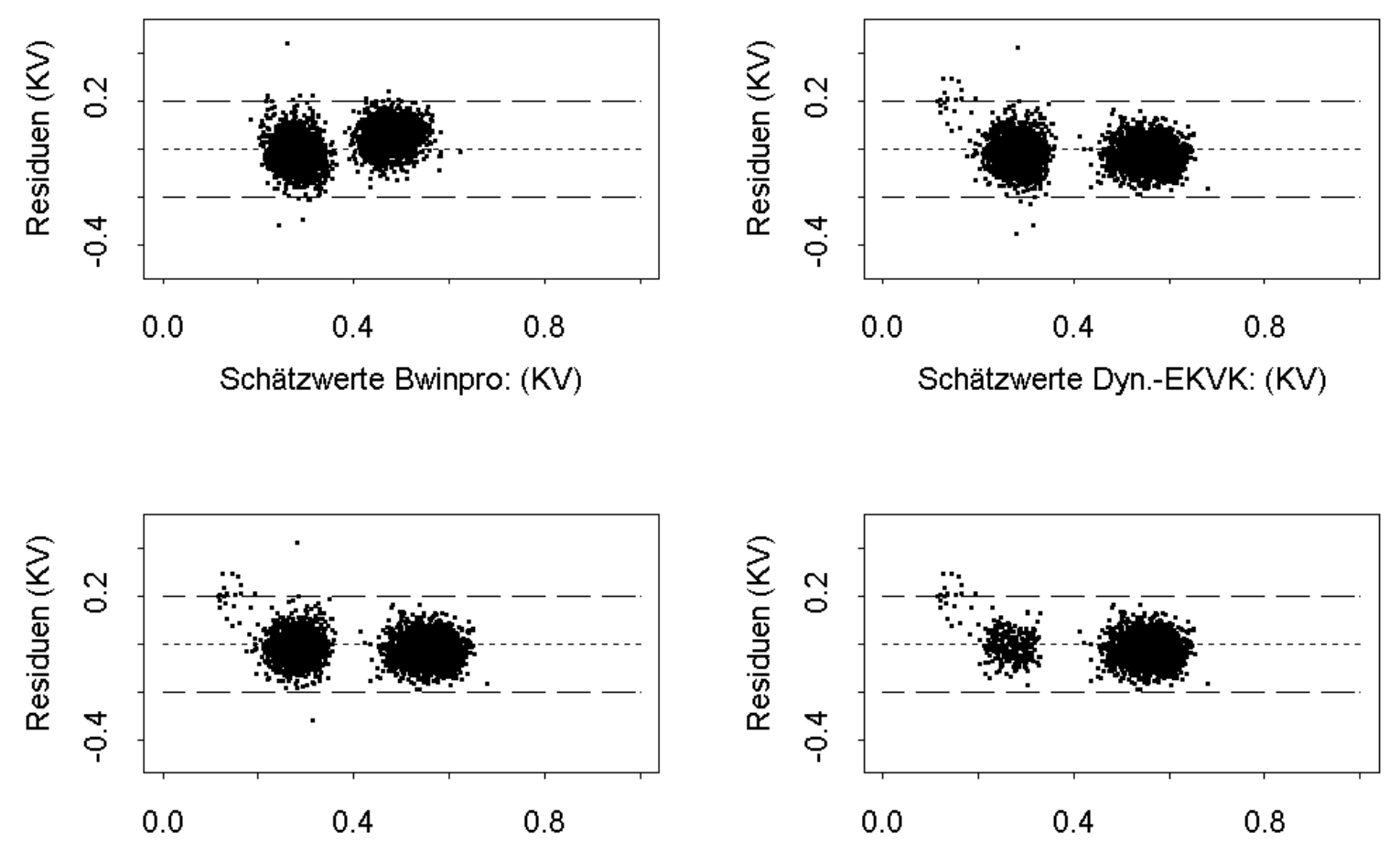

Schätzwerte Dyn.-EKVK<=15Jahre: (KV)

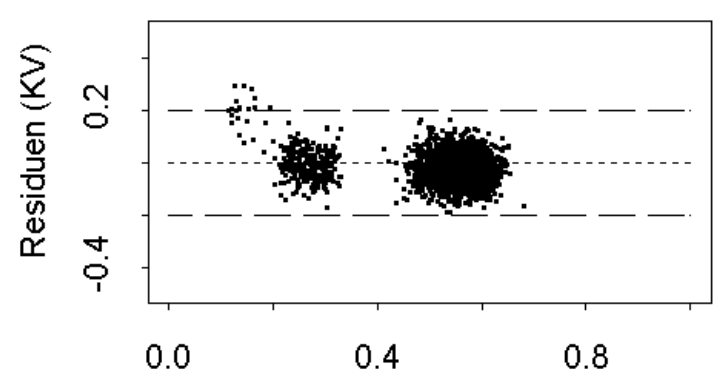

Abb. 101: Residuenplots der Kronenverhältnisschätzung mit Hilfe des neu entwickelten, einfachen statischen Modells (in Bwinpro, Formel (13)) und des dynamischen Modellansatzes (14, abgewandelt) und (16) für die Baumart Kiefer. Für den dynamischen Modellansatz sind neben den Ergebnissen der Schätzung für alle Prognosezeiträume auch die Ergebnisse der Begrenzung der Prognosezeiträume auf maximal 15 und 5 Jahre dargestellt (Dyn. EKVK, Dyn. EKVK $<=15$, Dyn. EKVK $<=5$ ). Die Schätzungen des dynamischen Modells sind mit Hilfe der Begrenzungsfunktion (15) überprüft und gegebenenfalls korrigiert worden.

\section{Europäische Lärche:}

\begin{tabular}{|l|c|cc|}
\hline & Alle Bestände & \multicolumn{2}{|c|}{$\begin{array}{c}\text { Nur Bestände mit guter Anpassung des } \\
\text { Einheitskronenansatzmodells } \\
\text { (Startpunktvorteil besteht) }\end{array}$} \\
\hline $\begin{array}{l}\text { Maximaler Modellvorteil (m) im } \\
\text { Durchschnitt über alle Bestände }\end{array}$ & 0.190 & & 0.229 \\
\hline $\begin{array}{l}\text { Maximaler Modellvorteil im } \\
\text { Durchschnitt über alle Bestände } \\
\text { (Kronenverhältnis) }\end{array}$ & 0.00723 & & 0.00883 \\
\hline
\end{tabular}

Tab. 67: Durchschnittliche maximale Vorteile (aller Prognosen und der Prognosen mit günstigem Startpunkt) des dynamischen Kronenansatzmodells für die Baumart Europäische Lärche. Die Werte wurden über das Modell zur Einschätzung der Unterschiede der Genauigkeit von statischem und dynamischem Modellansatz bestimmt. Die Berechnung erfolgte anhand der Fehlerdifferenzen für die Kronenansatz- und die Kronenverhältnisschätzung (Formel (18)). 

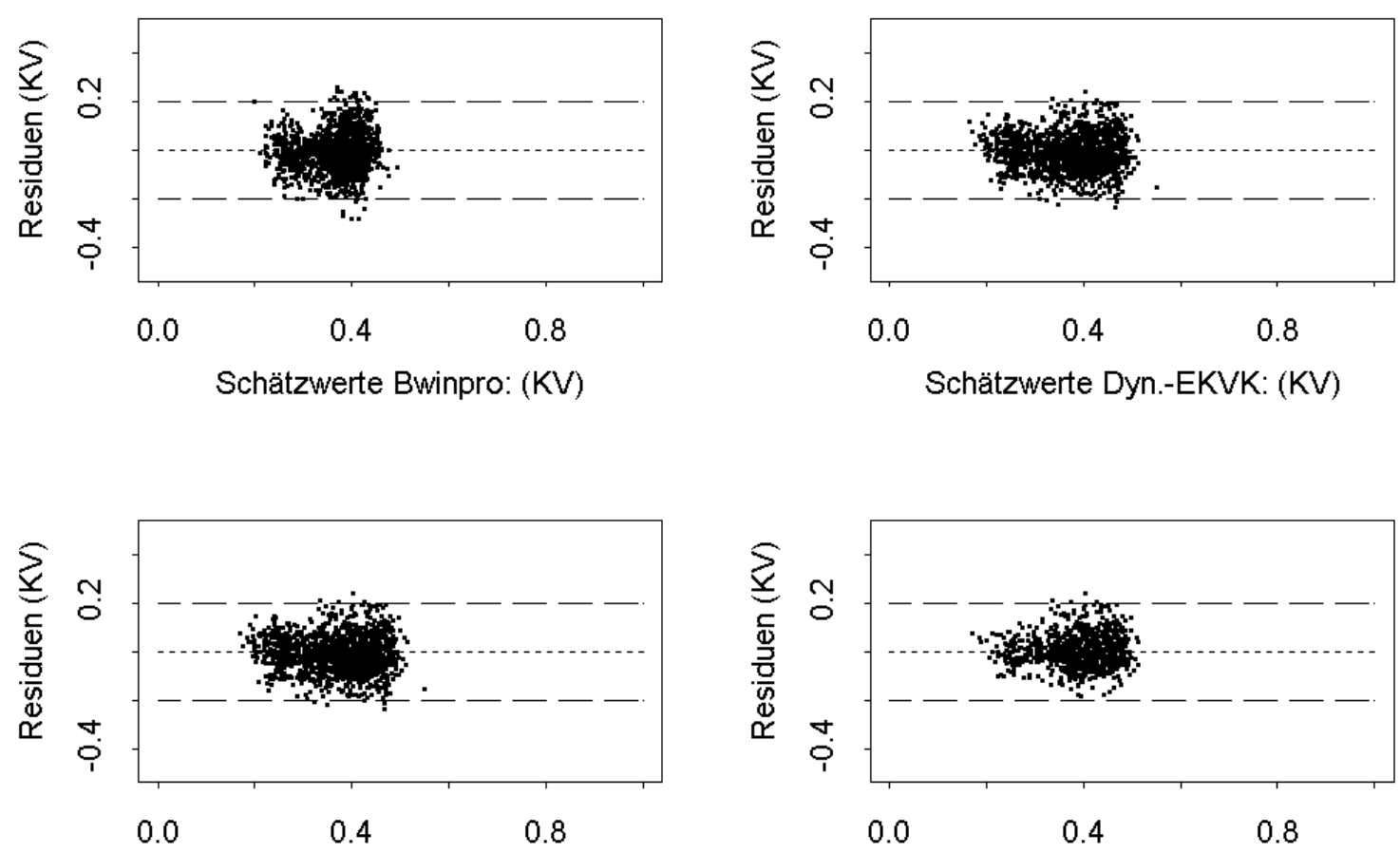

Schätzwerte Dyn.-EKVK<=15Jahre: (KV)

Schätzwerte Dyn.-EKVK<=5Jahre: (KV)

Abb. 102: Residuenplots der Kronenverhältnisschätzung mit Hilfe des neu entwickelten, einfachen statischen Modells (in Bwinpro, Formel (13, abgewandelt)) und des dynamischen Modellansatzes (14, abgewandelt) und (16) für die Baumart Europäische Lärche. Für den dynamischen Modellansatz sind neben den Ergebnissen der Schätzung für alle Prognosezeiträume auch die Ergebnisse der Begrenzung der Prognosezeiträume auf maximal 15 und 5 Jahre dargestellt (Dyn. EKVK, Dyn. EKVK $<=15$, Dyn. EKVK $<=$ 5). Die Schätzungen des dynamischen Modells sind mit Hilfe der Begrenzungsfunktion (15) überprüft und gegebenenfalls korrigiert worden. 


\section{Eiche:}

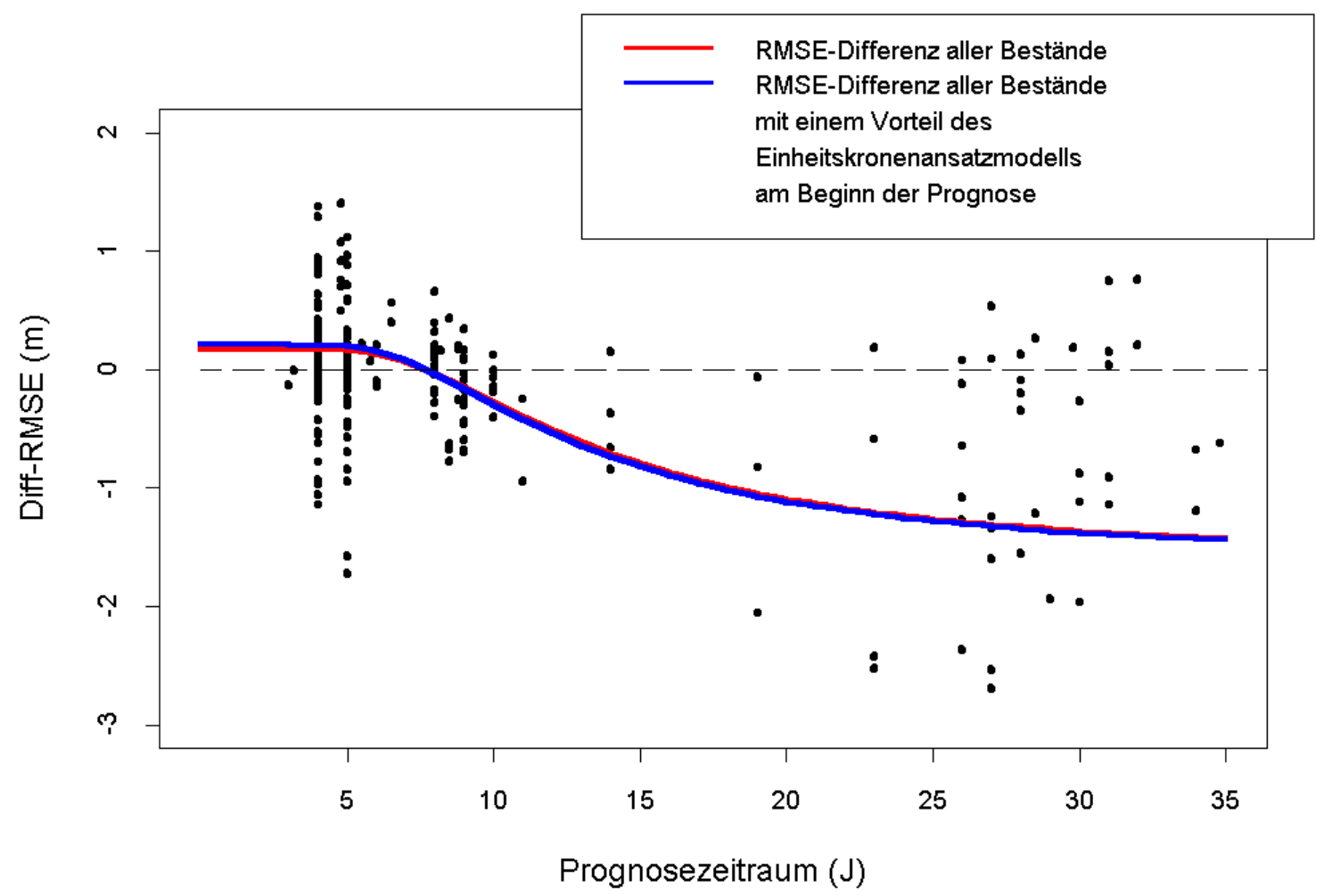

Abb. 103: Fehlerdifferenz der nach Parzellen und Aufnahmezeitpunkten berechneten RMSE des statischen und dynamischen Modellansatzes zur Kronenansatzschätzung in Abhängigkeit vom Prognosezeitraum für die Baumart Eiche. Die dynamische Schätzung wurde in einem Schritt ohne Zwischenberechnungen der unabhängigen Variablen durchgeführt. Das zum Ausgleich verwendete Modell ist in Formel (18) dargestellt.

\begin{tabular}{|l|r|rr|}
\hline & Alle Bestände & \multicolumn{2}{|c|}{$\begin{array}{l}\text { Nur Bestände mit guter Anpassung des } \\
\text { Einheitskronenansatzmodells } \\
\text { (Startpunktvorteil besteht) }\end{array}$} \\
\hline $\begin{array}{l}\text { Kritischer Prognosezeitraum der } \\
\text { Kronenansatzschätzung (Jahre) }\end{array}$ & 7.8 & \multicolumn{2}{|c|}{7.8} \\
\hline $\begin{array}{l}\text { Maximaler Modellvorteil (m) im } \\
\text { Durchschnitt über alle Bestände }\end{array}$ & 0.175 & & 0.207 \\
\hline $\begin{array}{l}\text { Kritischer Prognosezeitraum der } \\
\text { Kronenverhältnisschätzung (Jahre) }\end{array}$ & 7.8 & & 0.0099 \\
\hline $\begin{array}{l}\text { Maximaler Modellvorteil im } \\
\text { Durchschnitt über alle Bestände } \\
\text { (Kronenverhältnis) }\end{array}$ & 0.0076 & & \\
\hline
\end{tabular}

Tab. 68: Kritische Prognosezeiträume und durchschnittliche maximale Vorteile (aller Prognosen und der Prognosen mit günstigem Startpunkt) des dynamischen Kronenansatzmodells für die Baumart Eiche. Die Werte wurden über das Modell zur Einschätzung der Unterschiede der Genauigkeit von statischem und dynamischem Modellansatz bestimmt. Die Berechnung erfolgte anhand der Fehlerdifferenzen für die Kronenansatzund die Kronenverhältnisschätzung (Formel (18)). 


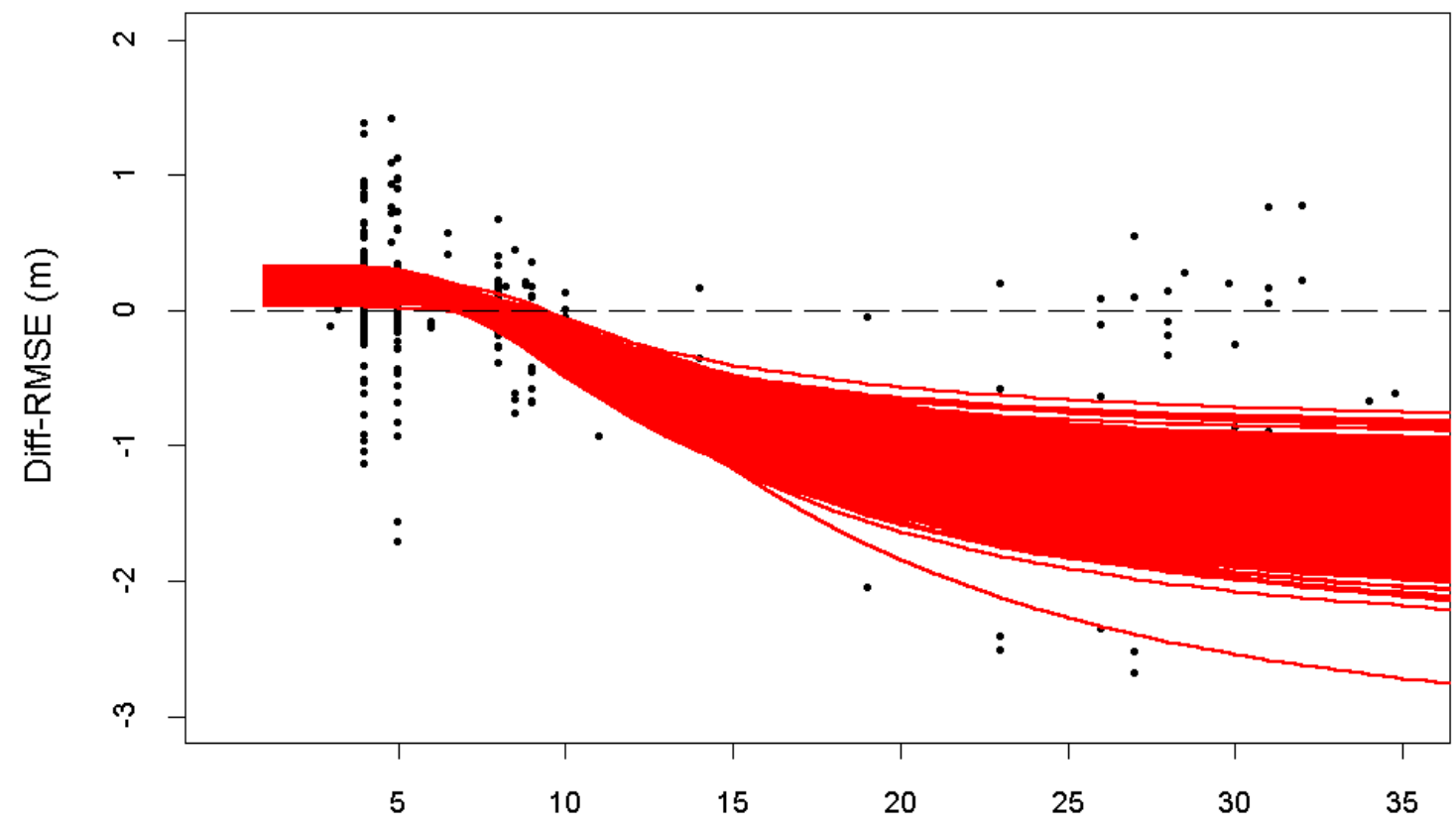

Prognosezeitraum $(\mathrm{J})$

Abb. 104: Schätzung der Verteilung von Realisationen der Regressionsfunktion (18) zur Schätzung der Unterschiede von statischem und dynamischem Modellansatz mit Hilfe von 1000 Bootstrap-Simulationen (Kapitel 8.2) für die Baumart Eiche.

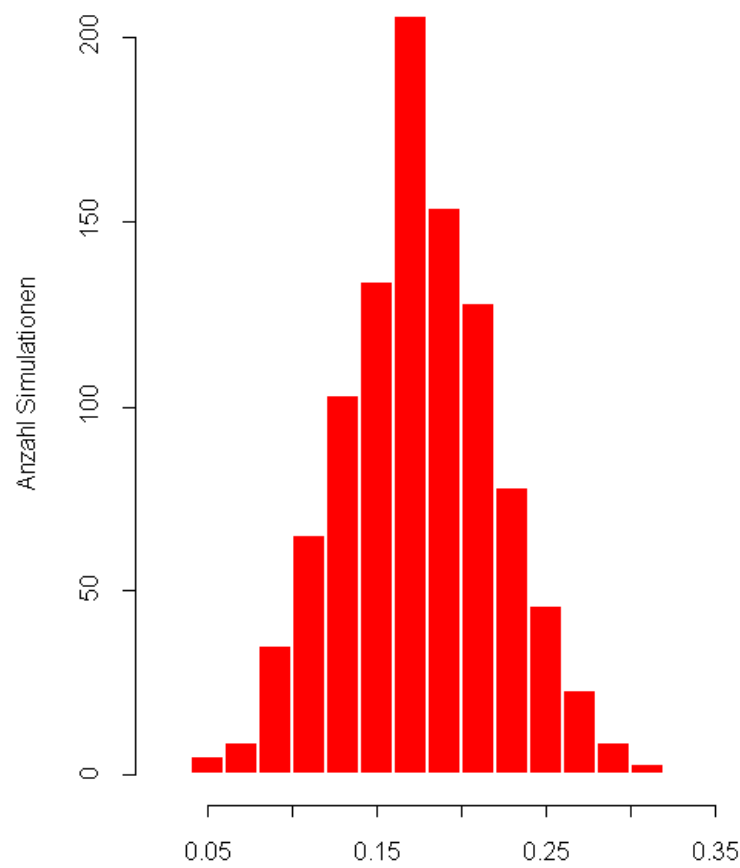

durchschnittl. max. Vorteil des dyn. Modells (Diff-RMSE (m))

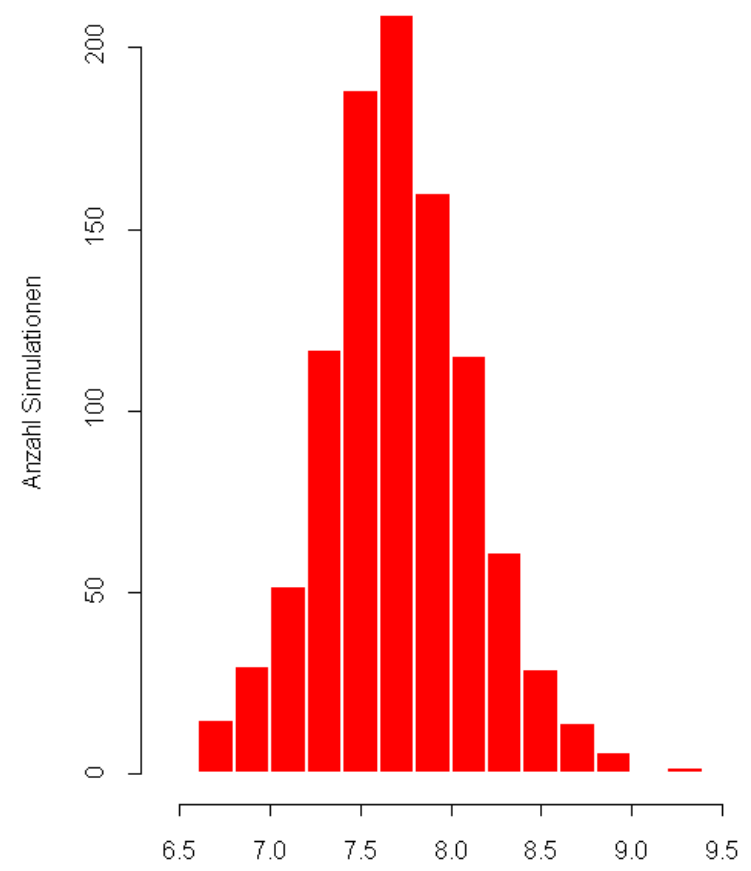

Prognosezeitraum (J) ab dem das dyn. Modell ungünstiger wird

Abb. 105: Mit Hilfe der 1000 Bootstrap-Simulationen (Kapitel 8.2) des Modells zur Schätzung der Unterschiede von statischem und dynamischem Modellansatz geschätzte Verteilungen des durchschnittlichen maximalen Vorteils und des kritischen Prognosezeitraumes des dynamischen Modells für die Baumart Eiche. 

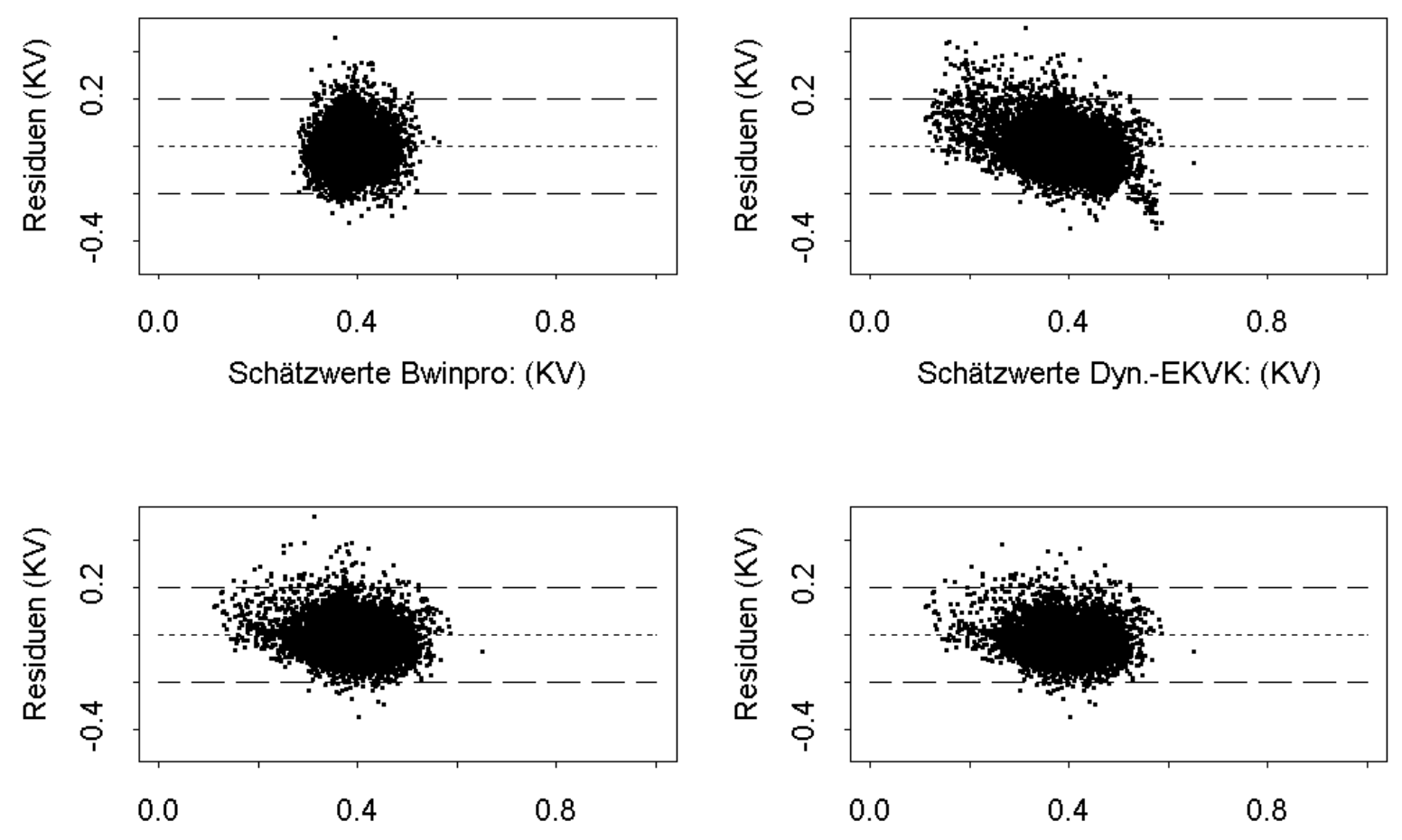

Schätzwerte Dyn.-EKVK<=15Jahre: (KV) Schätzwerte Dyn.-EKVK<=5Jahre: (KV)

Abb. 106: Residuenplots der Kronenverhältnisschätzung mit Hilfe des neu entwickelten, einfachen statischen Modells (in Bwinpro, Formel (13)) und des dynamischen Modellansatzes (14) und (16) für die Baumart Eiche. Für den dynamischen Modellansatz sind neben den Ergebnissen der Schätzung für alle Prognosezeiträume auch die Ergebnisse der Begrenzung der Prognosezeiträume auf maximal 15 und 5 Jahre dargestellt (Dyn. EKVK, Dyn. EKVK <= 15, Dyn. EKVK <= 5). Die Schätzungen des dynamischen Modells sind mit Hilfe der Begrenzungsfunktion (15) überprüft und gegebenenfalls korrigiert worden. 


\section{Buche:}

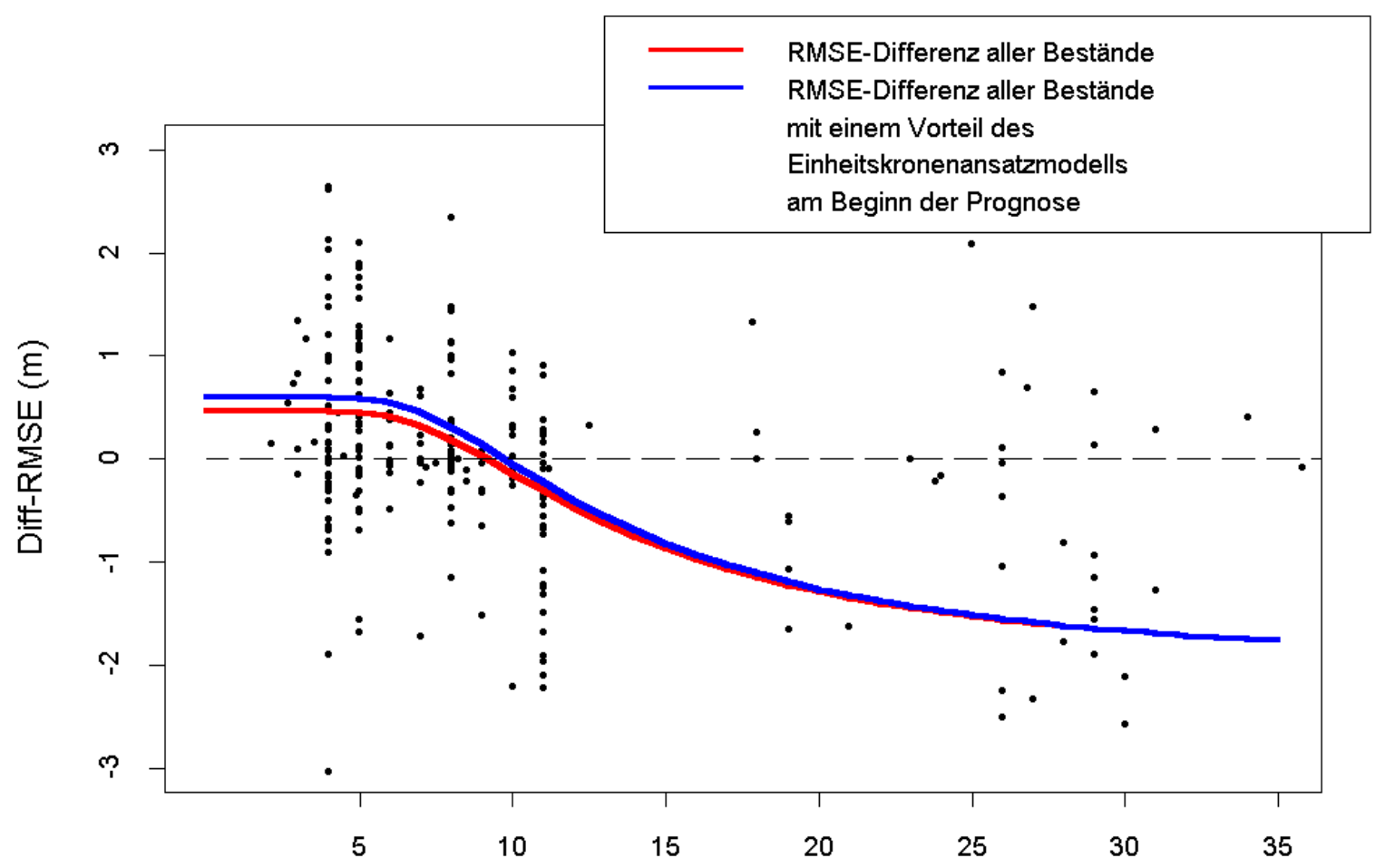

Prognosezeitraum (J)

Abb. 107: Fehlerdifferenz der nach Parzellen und Aufnahmezeitpunkten berechneten RMSE des statischen und dynamischen Modellansatzes zur Kronenansatzschätzung in Abhängigkeit vom Prognosezeitraum für die Baumart Buche. Die dynamische Schätzung wurde in einem Schritt ohne Zwischenberechnungen der unabhängigen Variablen durchgeführt. Das zum Ausgleich verwendete Modell ist in Formel (18) dargestellt.

\begin{tabular}{|l|r|rr|}
\hline & Alle Bestände & \multicolumn{2}{|c|}{$\begin{array}{l}\text { Nur Bestände mit guter Anpassung des } \\
\text { Einheitskronenansatzmodells } \\
\text { (Startpunktvorteil besteht) }\end{array}$} \\
\hline $\begin{array}{l}\text { Kritischer Prognosezeitraum der } \\
\text { Kronenansatzschätzung (Jahre) }\end{array}$ & 9.2 & & 9.8 \\
\hline $\begin{array}{l}\text { Maximaler Modellvorteil (m) im } \\
\text { Durchschnitt über alle Bestände }\end{array}$ & 0.457 & & 0.593 \\
\hline $\begin{array}{l}\text { Kritischer Prognosezeitraum der } \\
\text { Kronenverhältnisschätzung (Jahre) }\end{array}$ & 9.1 & & 0.3 \\
\hline $\begin{array}{l}\text { Maximaler Modellvorteil im } \\
\text { Durchschnitt über alle Bestände } \\
\text { (Kronenverhältnis) }\end{array}$ & 0.020 & & 0.026 \\
\hline
\end{tabular}

Tab. 69: Kritische Prognosezeiträume und durchschnittliche maximale Vorteile (aller Prognosen und der Prognosen mit günstigem Startpunkt) des dynamischen Kronenansatzmodells für die Baumart Buche. Die Werte wurden über das Modell zur Einschätzung der Unterschiede der Genauigkeit von statischem und dynamischem Modellansatz bestimmt. Die Berechnung erfolgte anhand der Fehlerdifferenzen für die Kronenansatzund die Kronenverhältnisschätzung (Formel (18)). 


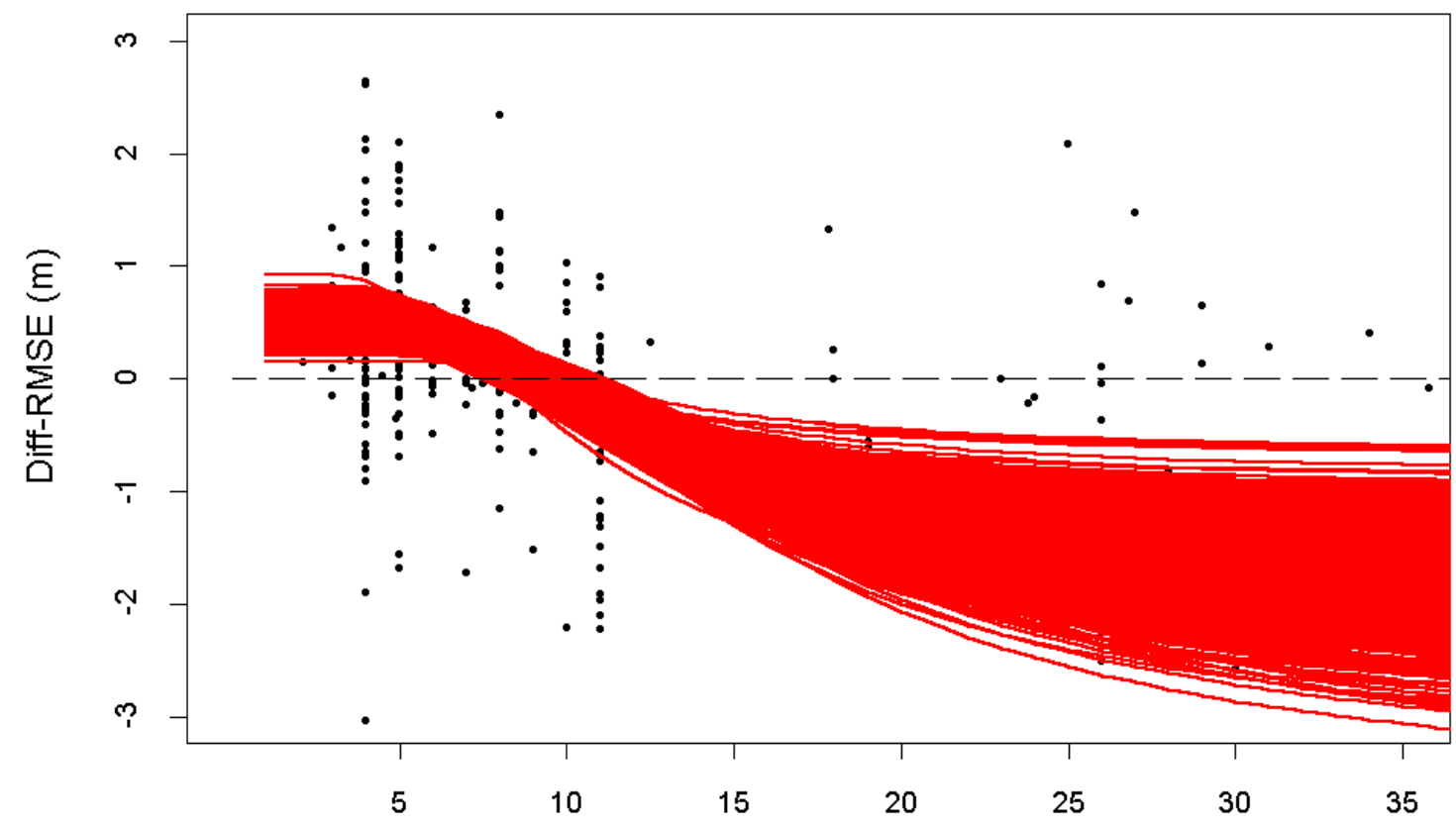

Prognosezeitraum (J)

Abb. 108: Schätzung der Verteilung von Realisationen der Regressionsfunktion (18) zur Schätzung der Unterschiede von statischem und dynamischem Modellansatz mit Hilfe von 1000 Bootstrap-Simulationen (Kapitel 8.2) für die Baumart Buche.

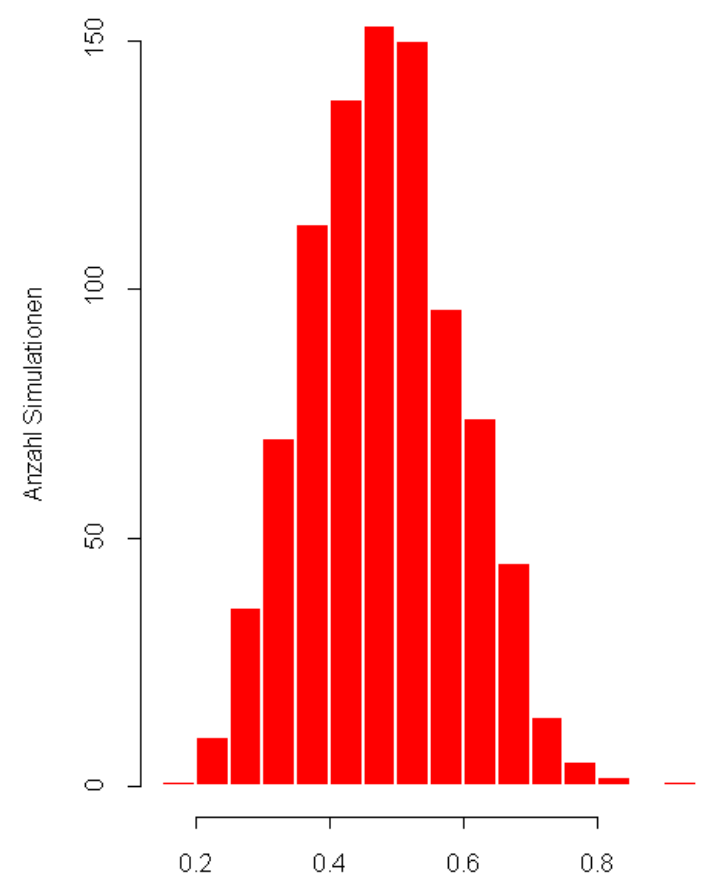

durchschnittl. max. Vorteil des dyn. Modells (Diff-RMSE (m))

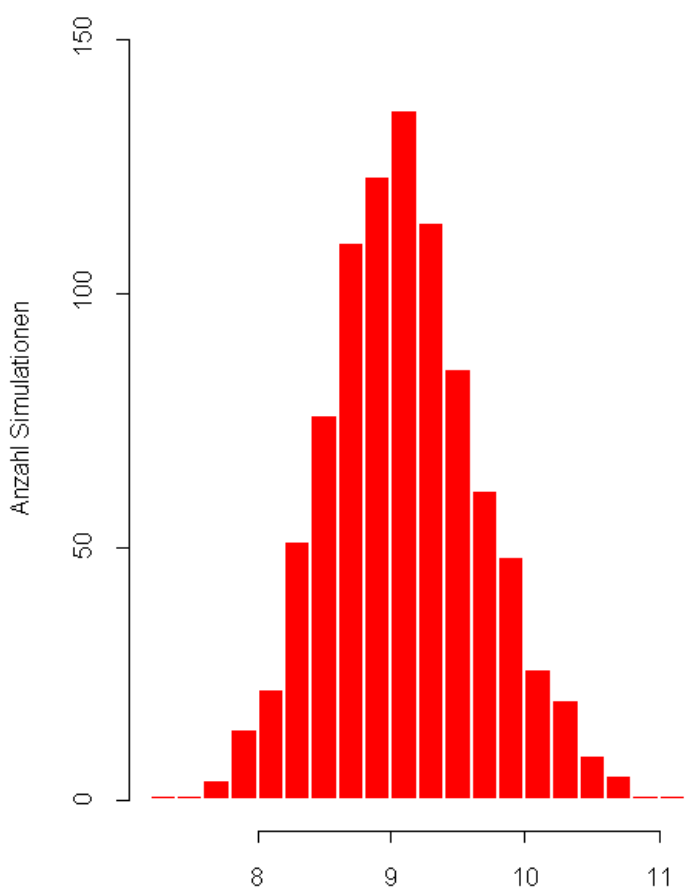

Prognosezeitraum (J.) ab dem das dyn. Modell ungünstiger wird

Abb. 109: Mit Hilfe der 1000 Bootstrap-Simulationen (Kapitel 8.2) des Modells zur Schätzung der Unterschiede von statischem und dynamischem Modellansatz geschätzte Verteilungen des durchschnittlichen maximalen Vorteils und des kritischen Prognosezeitraumes des dynamischen Modells für die Baumart Buche. 

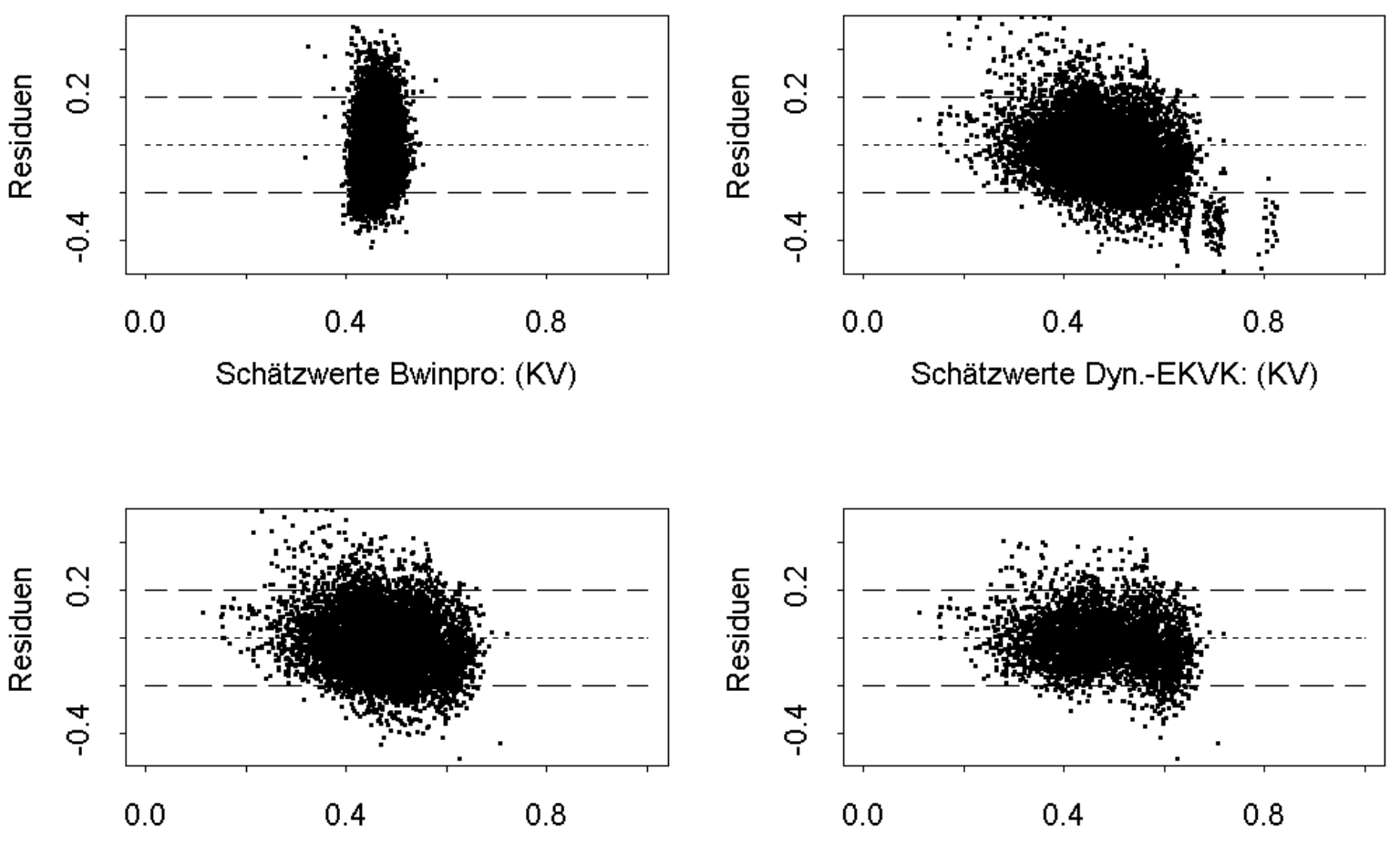

Schätzwerte Dyn.-EKVK<=15Jahre: (KV)

Schätzwerte Dyn.-EKVK<=5Jahre: (KV)

Abb. 110: Residuenplots der Kronenverhältnisschätzung mit Hilfe des neu entwickelten, einfachen statischen Modells (in Bwinpro, Formel (13)) und des dynamischen Modellansatzes (14, abgewandelt) und (16) für die Baumart Buche. Für den dynamischen Modellansatz sind neben den Ergebnissen der Schätzung für alle Prognosezeiträume auch die Ergebnisse der Begrenzung der Prognosezeiträume auf maximal 15 und 5 Jahre dargestellt (Dyn. EKVK, Dyn. EKVK <=15, Dyn. EKVK <=5). Die Schätzungen des dynamischen Modells sind mit Hilfe der Begrenzungsfunktion (15) überprüft und gegebenenfalls korrigiert worden. 


\subsection{Astdurchmesser der stärksten Quirläste}

\section{Douglasie:}
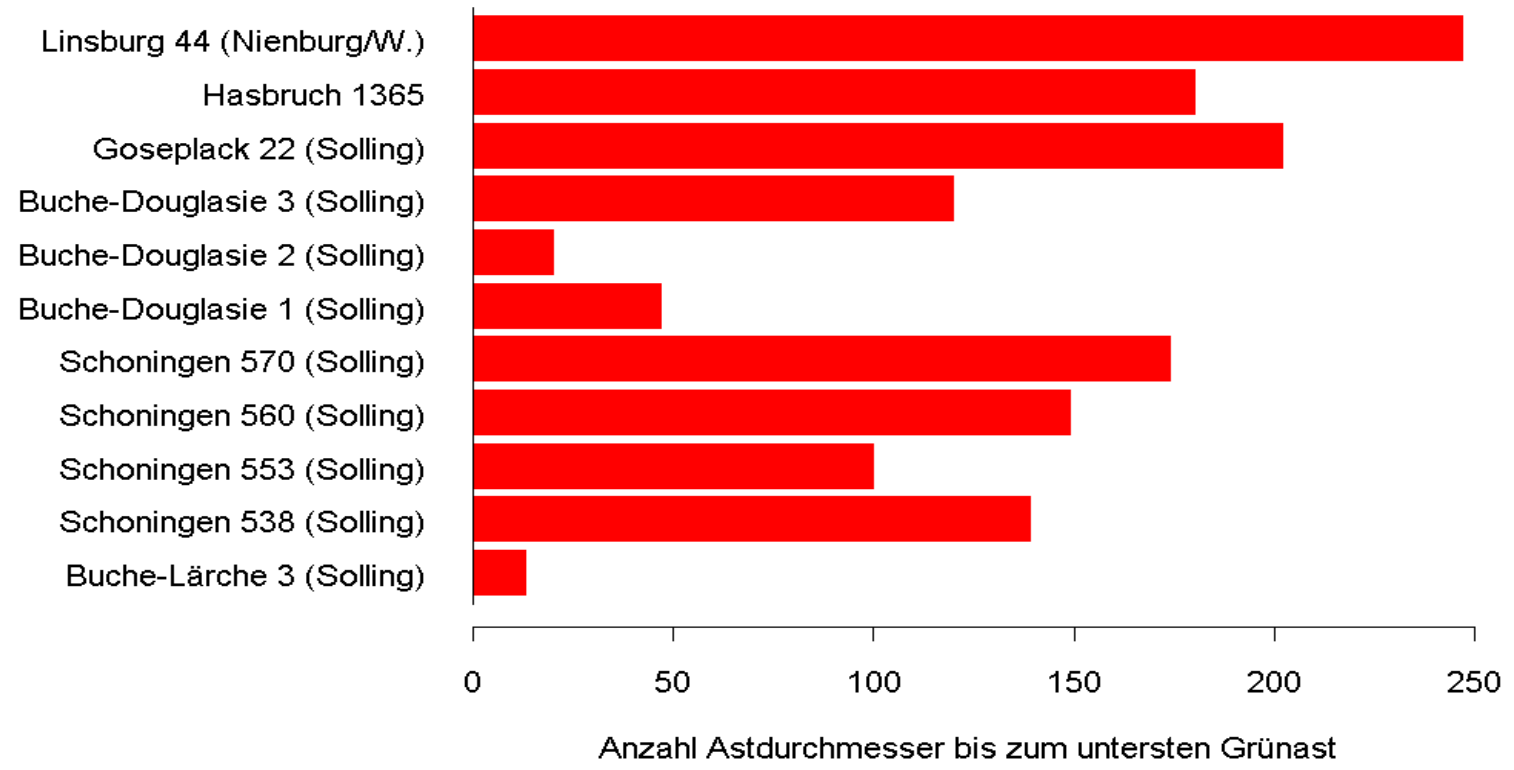

Abb. 111: Verteilung der Astdurchmessermessungen der stärksten Quirläste innerhalb der grünen Krone gegliedert nach Versuchsflächen bzw. Abteilungen für die Baumart Douglasie.
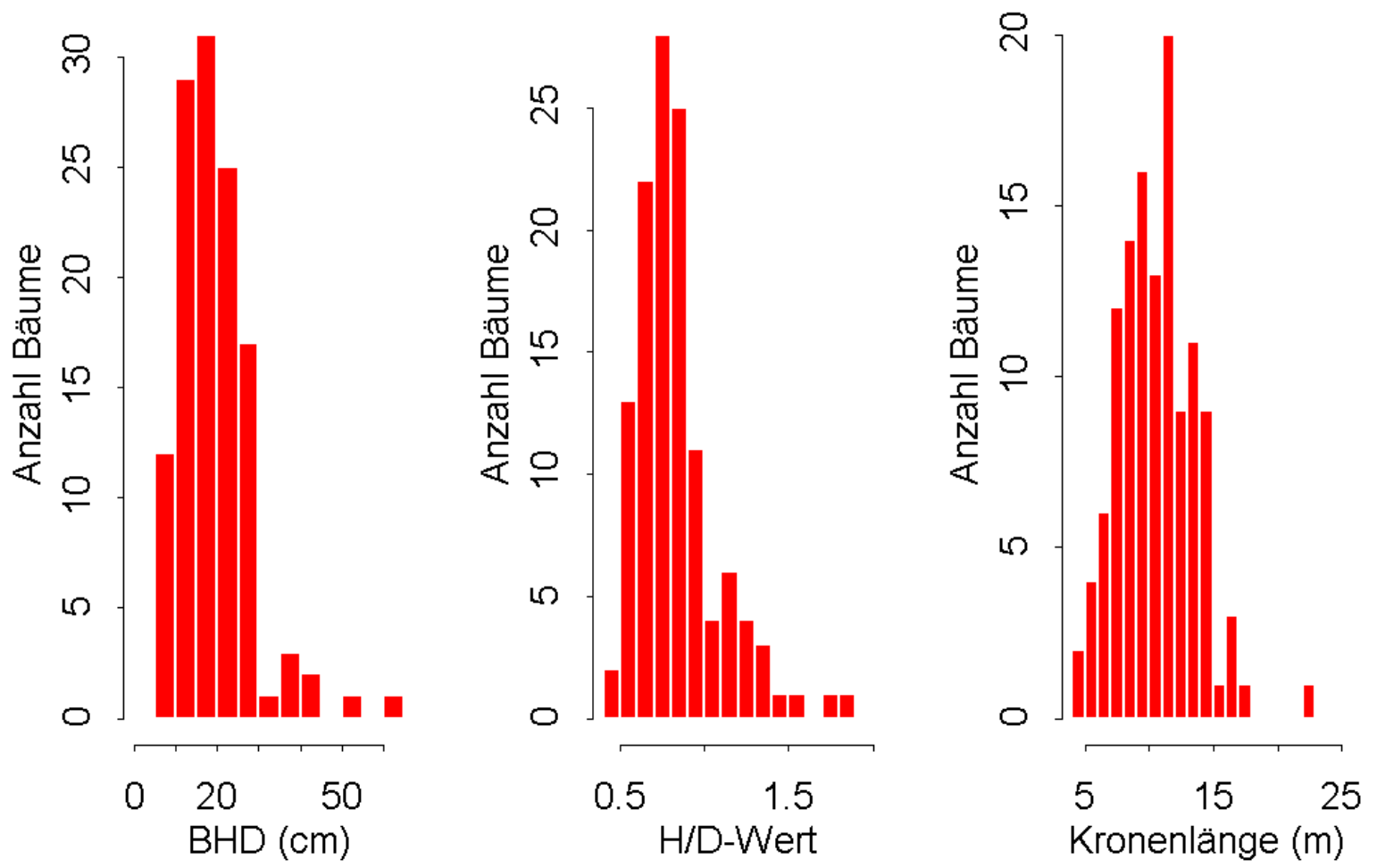

Abb. 112: BHD- und H/D-Wert-Verteilungen für 122 Douglasien, die zur Entwicklung des Modells zur Durchmesserschätzung der stärksten Quirläste verwendet wurden. 


\begin{tabular}{|rr|r|r|r|}
\hline Koeffizient & \multicolumn{1}{c|}{ Wert } & Std. Fehler & \multicolumn{1}{c|}{ t-Wert } \\
\hline & $a_{1}$ & 0.4338440 & 0.01868840 & 23.21460 \\
\hline$a_{3}$ & -0.4502310 & 0.01693630 & -26.58380 \\
\hline$a_{4}$ & 4.2322200 & 0.38175000 & 11.08640 \\
\hline$b_{0}$ & 0.0107444 & 0.00174862 & 6.14452 \\
\hline$b_{2}$ & 0.0642220 & 0.00276615 & 23.21710 \\
\hline \multicolumn{2}{|c|}{$b_{3}$} & 11.4740000 & 0.40541000 & 28.30220 \\
\hline \multicolumn{2}{|c|}{ Residual Std. Fehler (mm) } & & 4.21529 bei 1385 Freiheitsgraden \\
\hline
\end{tabular}

Tab. 70: Koeffizienten und statistische Kenngrößen zur Durchmesserschätzung der stärksten Quirläste innerhalb der grünen Krone mit Hilfe des neu entwickelten Modells (20) für die Baumart Douglasie.

\begin{tabular}{|r|r|r|r|}
\hline Koeffizient & \multicolumn{1}{|c|}{ Wert } & \multicolumn{1}{c|}{ Std. Fehler } & \multicolumn{1}{c|}{ t-Wert } \\
\hline$b_{0}$ & 6.48528 & $3.52505^{*} 10^{-1}$ & 18.39770 \\
\hline$b_{1}$ & $3.34054 * 10^{-1}$ & $1.30970^{*} 10^{-2}$ & 25.50610 \\
\hline$b_{2}$ & $1.99328 * 10^{-3}$ & $1.03284 * 10^{-4}$ & 19.29910 \\
\hline$b_{3}$ & $-8.87176^{*} 10^{-6}$ & $9.41288^{*} 10^{-7}$ & -9.42513 \\
\hline$b_{4}$ & $-2.36802 * 10^{-3}$ & $1.85089^{*} 10^{-4}$ & -12.79400 \\
\hline$b_{5}$ & $1.39293 * 10^{-5}$ & $1.69018^{*} 10^{-6}$ & -20.13140 \\
\hline$b_{6}$ & $-2.53235^{-5} 10^{-5}$ & $1.25791 * 10^{-6}$ & 2.134734 \\
\hline \multicolumn{5}{|c|}{ Residual Std. Fehler (mm) } & 4.78765 bei 1384 Freiheitsgraden \\
\hline $\begin{array}{r}\text { Residual Std. Fehler bei einzelbaumweiser Anpassung mit } \\
\text { einem Gleitenden-Durchschnitts-Modell (mm) }\end{array}$ \\
\hline
\end{tabular}

Tab. 71: Koeffizienten und statistische Kenngrößen zur Durchmesserschätzung der stärksten Quirläste innerhalb der grünen Krone mit Hilfe des von MAGUIRE ET AL. (1994) entwikkelten Modells (7) und der einzelbaumweisen Anpassung mit Hilfe eines GleitendenDurchschnitts-Modells für die Baumart Douglasie. Der iterativ ermittelte gemeinsame Funktionswert beider Segmente liegt bei einer Tiefe in der Krone von 10\% der Kronenlänge. 


\section{Kiefer:}

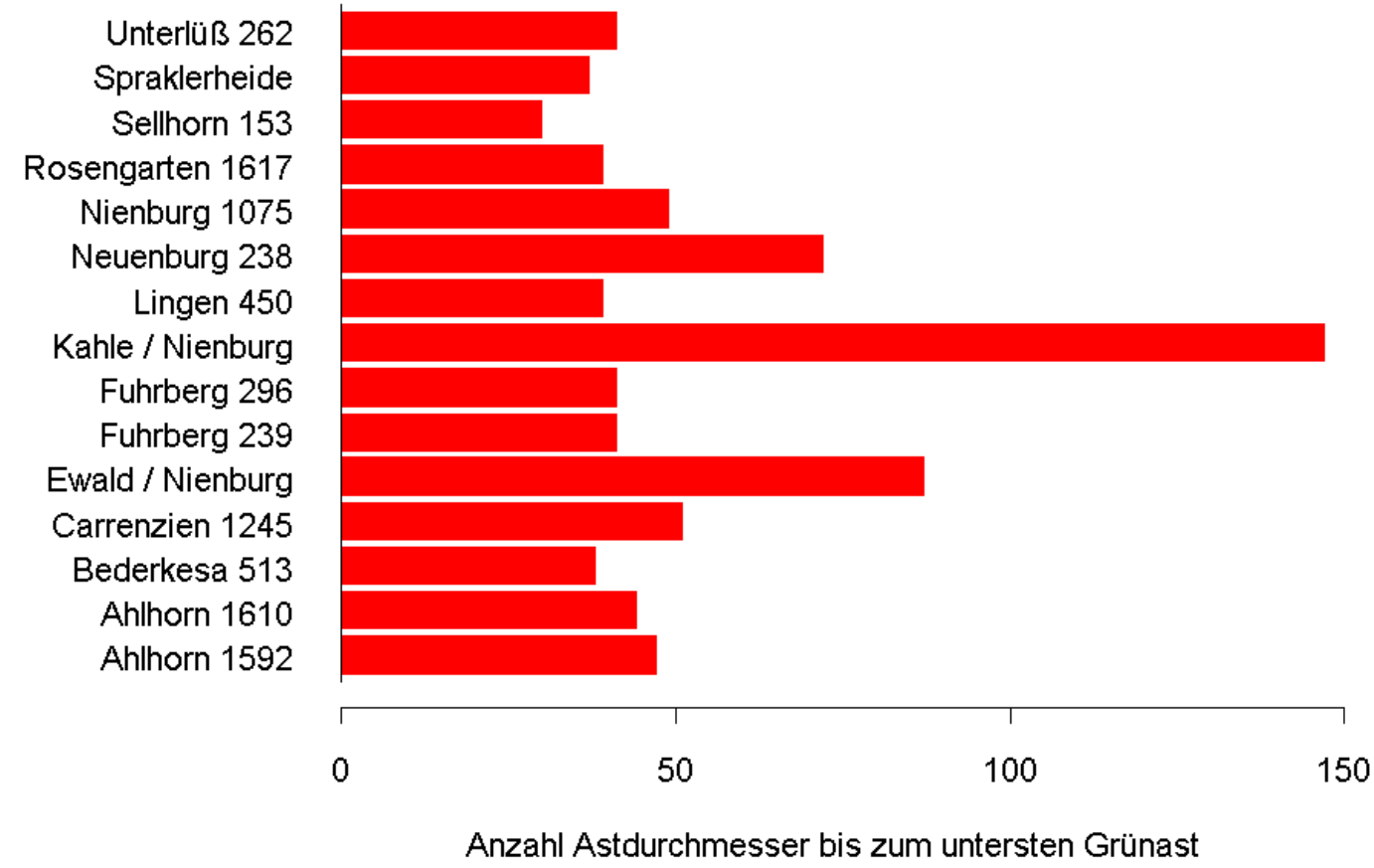

Abb. 113: Verteilung der Astdurchmessermessungen der stärksten Quirläste innerhalb der grünen Krone gegliedert nach Versuchsflächen bzw. Abteilungen für die Baumart Kiefer.
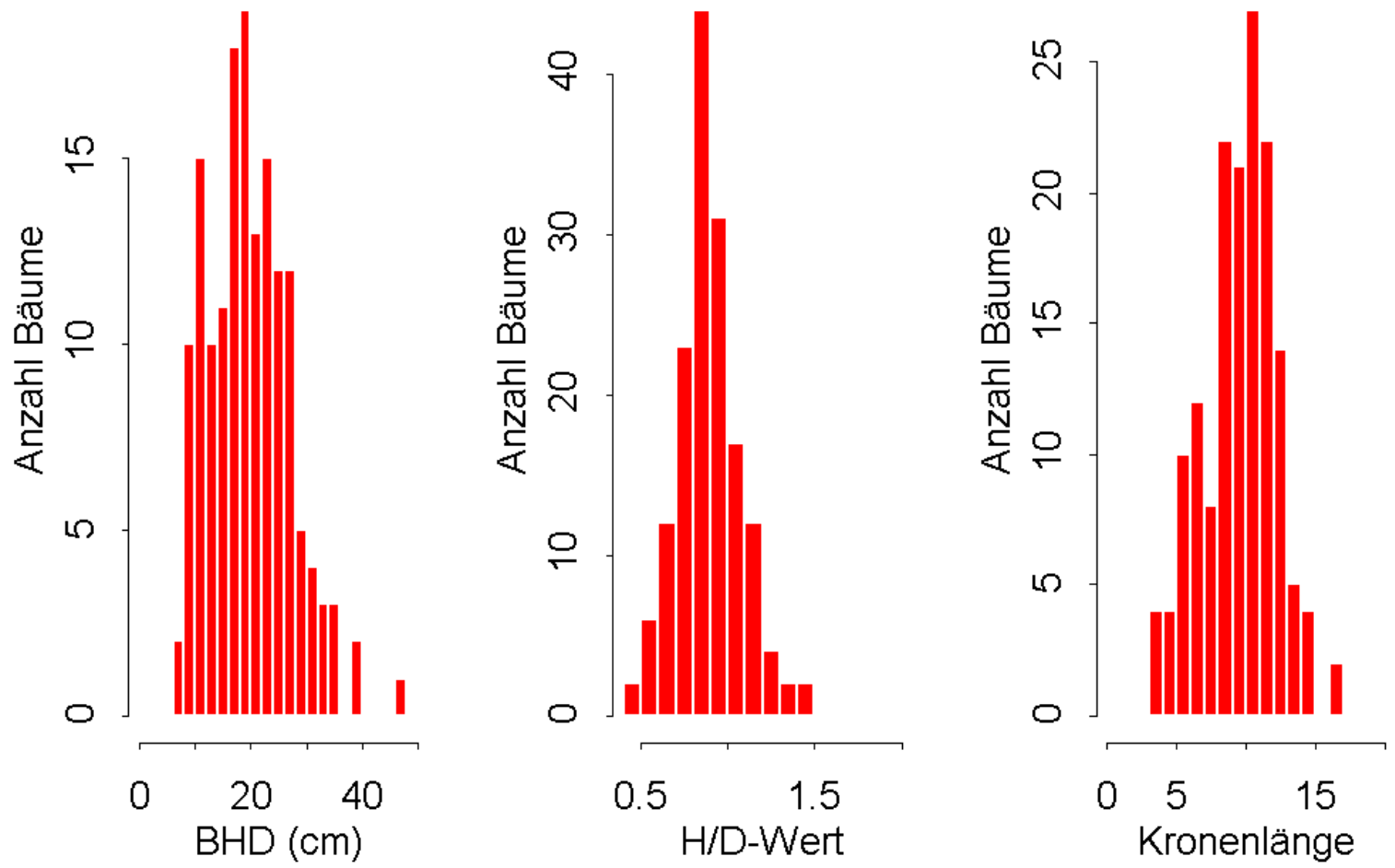

Abb. 114: BHD- und H/D-Wert-Verteilungen für 73 Kiefern, die zur Entwicklung des Modells zur Durchmesserschätzung der stärksten Quirläste verwendet wurden. 


\begin{tabular}{|c|c|c|c|}
\hline Koeffizient & Wert & Std. Fehler & $\mathrm{t}$-Wert \\
\hline$a_{1}$ & 0.8848900 & 0.04093230 & 21.61840 \\
\hline$a_{3}$ & -0.8235200 & 0.03656370 & -22.52290 \\
\hline$a_{4}$ & 2.4905100 & 0.24224900 & 10.28080 \\
\hline$b_{0}$ & 0.0177383 & 0.00459411 & 3.86111 \\
\hline$b_{2}$ & 0.0560909 & 0.00860936 & 6.51511 \\
\hline$b_{3}$ & 11.9693000 & 1.25016000 & 9.57426 \\
\hline Residual Std. Fehle & \multicolumn{3}{|c|}{4.5511 bei 797 Freiheitsgraden } \\
\hline
\end{tabular}

Tab. 72: Koeffizienten und statistische Kenngrößen zur Durchmesserschätzung der stärksten Quirläste innerhalb der grünen Krone mit Hilfe des neu entwickelten Modells (20) für die Baumart Kiefer.

\begin{tabular}{|r|r|r|r|}
\hline Koeffizient & \multicolumn{1}{|c|}{ Wert } & Std. Fehler & \multicolumn{1}{c|}{ t-Wert } \\
\hline$b_{0}$ & 6.48630 & $5.19050^{*} 10^{-1}$ & 12.496500 \\
\hline$b_{1}$ & $4.66245^{*} 10^{-1}$ & $3.21588^{*} 10^{-2}$ & 14.498200 \\
\hline$b_{2}$ & $3.77116^{*} 10^{-3}$ & $5.21566^{*} 10^{-4}$ & 7.230470 \\
\hline$b_{3}$ & $-8.39702 * 10^{-6}$ & $9.49112^{*} 10^{-6}$ & -0.884724 \\
\hline$b_{4}$ & $-2.15647 * 10^{-3}$ & $6.34309^{*} 10^{-4}$ & -3.399720 \\
\hline$b_{5}$ & $-1.57984 * 10^{-6}$ & $1.29203 * 10^{-5}$ & -0.122276 \\
\hline$b_{6}$ & $-6.16211 * 10^{-5}$ & $6.96225^{*} 10^{-6}$ & -8.850740 \\
\hline \multicolumn{5}{|c|}{ Residual Std. Fehler (mm) } & 4.71472 bei 796 Freiheitsgraden \\
\hline $\begin{array}{r}\text { Residual Std. Fehler bei einzelbaumweiser Anpassung mit } \\
\text { einem Gleitenden-Durchschnitts-Modell (mm) }\end{array}$ \\
\hline
\end{tabular}

Tab. 73: Koeffizienten und statistische Kenngrößen zur Durchmesserschätzung der stärksten Quirläste innerhalb der grünen Krone mit Hilfe des von MAGUIRE ET AL. (1994) entwikkelten Modells (7) und der einzelbaumweisen Anpassung mit Hilfe eines GleitendenDurchschnitts-Modells für die Baumart Kiefer. Der iterativ ermittelte gemeinsame Funktionswert beider Segmente liegt bei einer Tiefe in der Krone von 10\% der Kronenlänge.

\subsection{Astdurchmesser weiterer Quirläste für Fichte}

\begin{tabular}{|r|r|r|r|r|r|}
\hline Koeffizient & \multicolumn{1}{|c|}{$\begin{array}{l}\text { stärkster } \\
\text { Quirlast }\end{array}$} & $\begin{array}{c}\text { 2.-stärkster } \\
\text { Quirlast }\end{array}$ & $\begin{array}{c}\text { 3.-stärkster } \\
\text { Quirlast }\end{array}$ & $\begin{array}{c}\text { 4.-stärkster } \\
\text { Quirlast }\end{array}$ & $\begin{array}{c}\text { 5.-stärkster } \\
\text { Quirlast }\end{array}$ \\
\hline$a_{1}$ & 0.4309 & 0.3973 & 0.3852 & 0.3703 & 0.3166 \\
\hline$a_{3}$ & -0.4466 & -0.4170 & -0.3828 & -0.3723 & -0.3142 \\
\hline$a_{4}$ & 1.8443 & 1.9703 & 2.0605 & 2.1973 & 2.5132 \\
\hline$b_{0}$ & 0.0276 & 0.0243 & 0.0211 & 0.0159 & 0.0123 \\
\hline$b_{2}$ & 0.0399 & 0.0340 & 0.0192 & 0.0133 & 0.0026 \\
\hline$b_{3}$ & 10.3519 & 9.9497 & 9.2845 & 9.4771 & 10.202 \\
\hline Residual Std. Fehler $(\mathrm{mm})$ & 3.817 & 3.685 & 3.775 & 3.894 & 4.027 \\
\hline
\end{tabular}

Tab. 74: Koeffizienten und statistische Kenngrößen zur Durchmesserschätzung der stärksten Quirläste innerhalb der grünen Krone mit Hilfe des neu entwickelten Modells (20) ohne Koeffizientenausgleich für die Baumart Fichte. 


\begin{tabular}{|r|r|r|r|r|r|}
\hline \multicolumn{1}{|c|}{ Koeffizient } & \multicolumn{1}{c|}{$\begin{array}{c}\text { stärkster } \\
\text { Quirlast }\end{array}$} & $\begin{array}{c}\text { 2.-stärkster } \\
\text { Quirlast }\end{array}$ & $\begin{array}{c}\text { 3.-stärkster } \\
\text { Quirlast }\end{array}$ & $\begin{array}{c}\text { 4.-stärkster } \\
\text { Quirlast }\end{array}$ & $\begin{array}{c}\text { 5.-stärkster } \\
\text { Quirlast }\end{array}$ \\
\hline$a_{1}$ & 0.4312 & 0.4057 & 0.3801 & 0.3546 & 0.3289 \\
\hline$a_{3}$ & -0.4485 & -0.4176 & -0.3866 & -0.3557 & -0.3247 \\
\hline$a_{4}$ & 1.8042 & 1.9607 & 2.1172 & 2.2737 & 2.4301 \\
\hline$b_{0}$ & 0.0281 & 0.0242 & 0.0203 & 0.0164 & 0.0125 \\
\hline$b_{2}$ & 0.0409 & 0.0314 & 0.0218 & 0.0123 & 0.0028 \\
\hline$b_{3}$ & 10.0076 & 9.9303 & 9.8530 & 9.7758 & 9.6986 \\
\hline Residual Std. Fehler $(\mathrm{mm})$ & 3.821 & 3.688 & 3.781 & 3.900 & 4.034 \\
\hline
\end{tabular}

Tab. 75: Koeffizienten und statistische Kenngrößen zur Durchmesserschätzung der stärksten Quirläste innerhalb der grünen Krone mit Hilfe des neu entwickelten Modells (20) mit Koeffizientenausgleich für die Baumart Fichte.

\begin{tabular}{|c|c|c|c|c|c|c|}
\hline & $\mathrm{a}_{1}$ & $\mathrm{~b}_{0}$ & $\mathrm{~b}_{2}$ & $\mathrm{~b}_{3}$ & $\mathrm{a}_{3}$ & $\mathrm{a}_{4}$ \\
\hline$\alpha$ & 0.4568 & 0.0320 & 0.0504 & 10.0848 & -0.4795 & 1.6478 \\
\hline$\beta$ & -0.0256 & -0.0039 & -0.0095 & -0.0772 & 0.0310 & 0.1565 \\
\hline
\end{tabular}

Tab. 76: Koeffizienten für das Modell (21) zum Parameterausgleich des Modells (20) zur Schätzung der fünf stärksten Quirlastdurchmmesser in der grünen Krone für die Baumart Fichte. 


\subsection{Modelle zur Beschreibung der Astdurchmesser bei Buche}
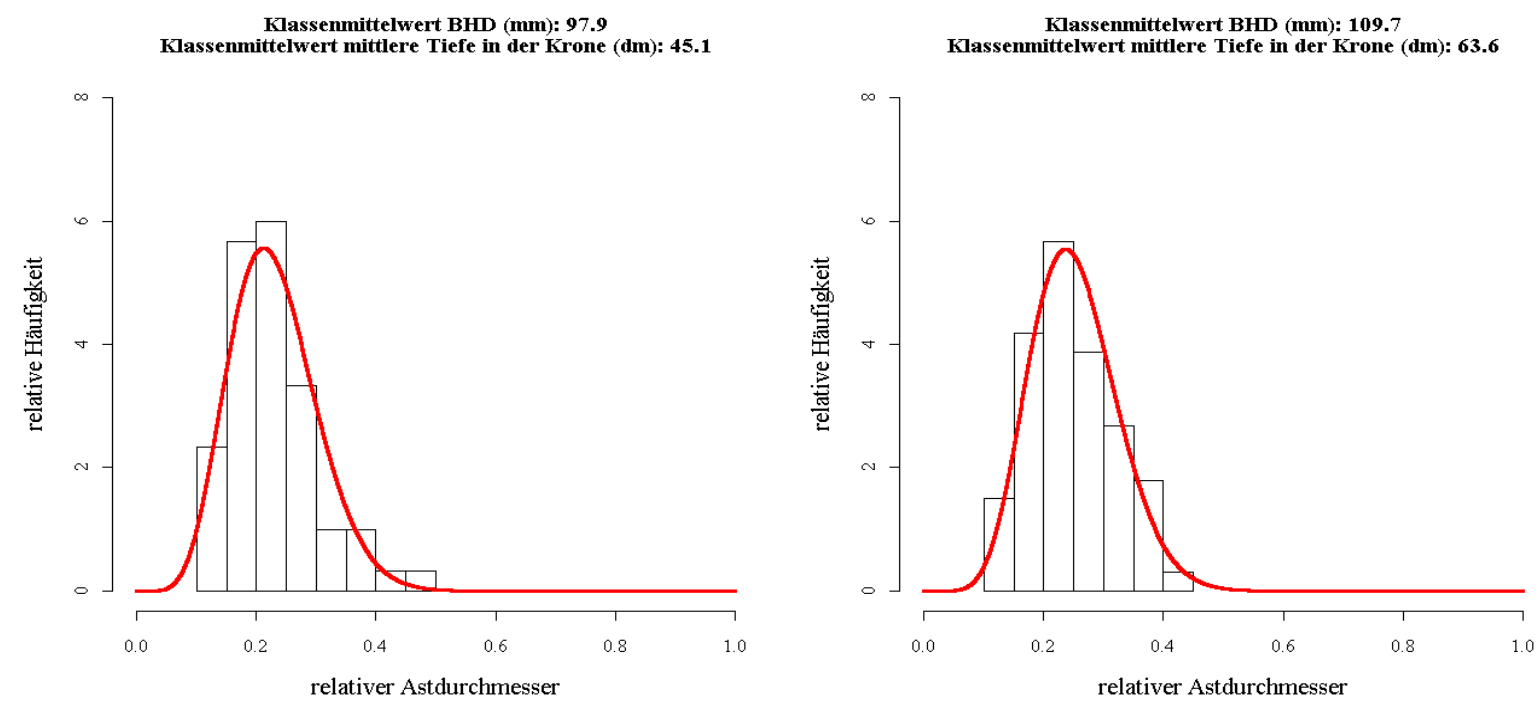

Klassenmittelwert BHD (mm): 102.4
Klassenmittelwert mittlere Tiefe in der Krone (dm): 81.8

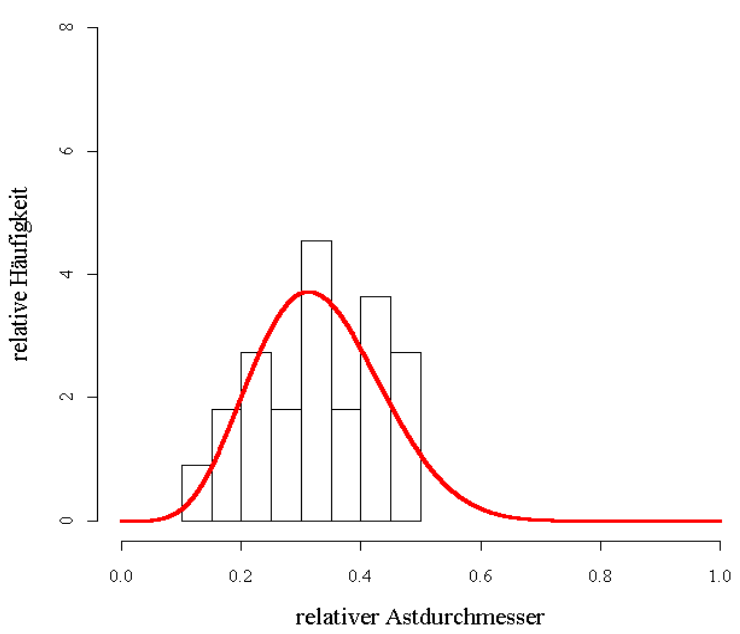

Klassenmittelwert BHD (mm): 140.5

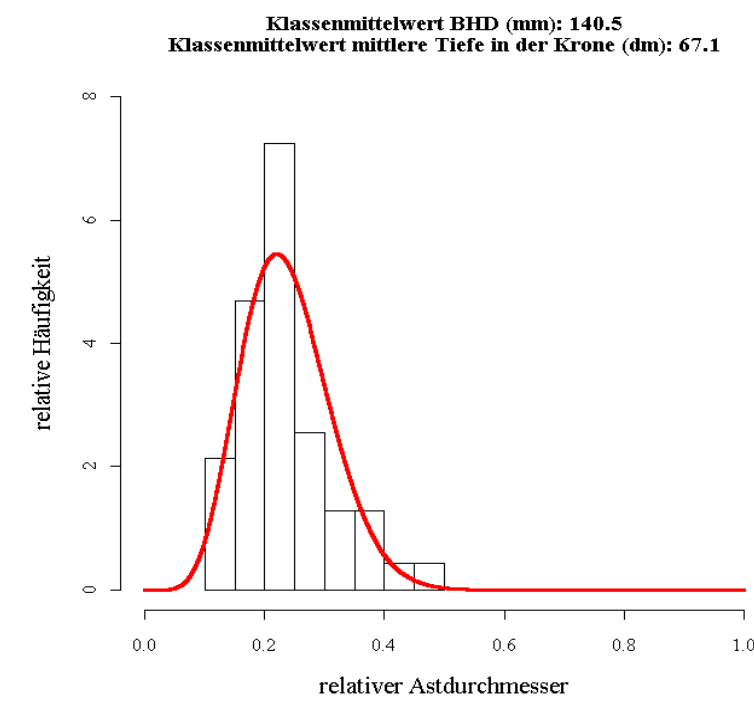

Klassenmittelwert BHD (mm): 148 Klassenmittelwert mittlere Tiefe in der Krone $(\mathrm{dm})$ : 81.6
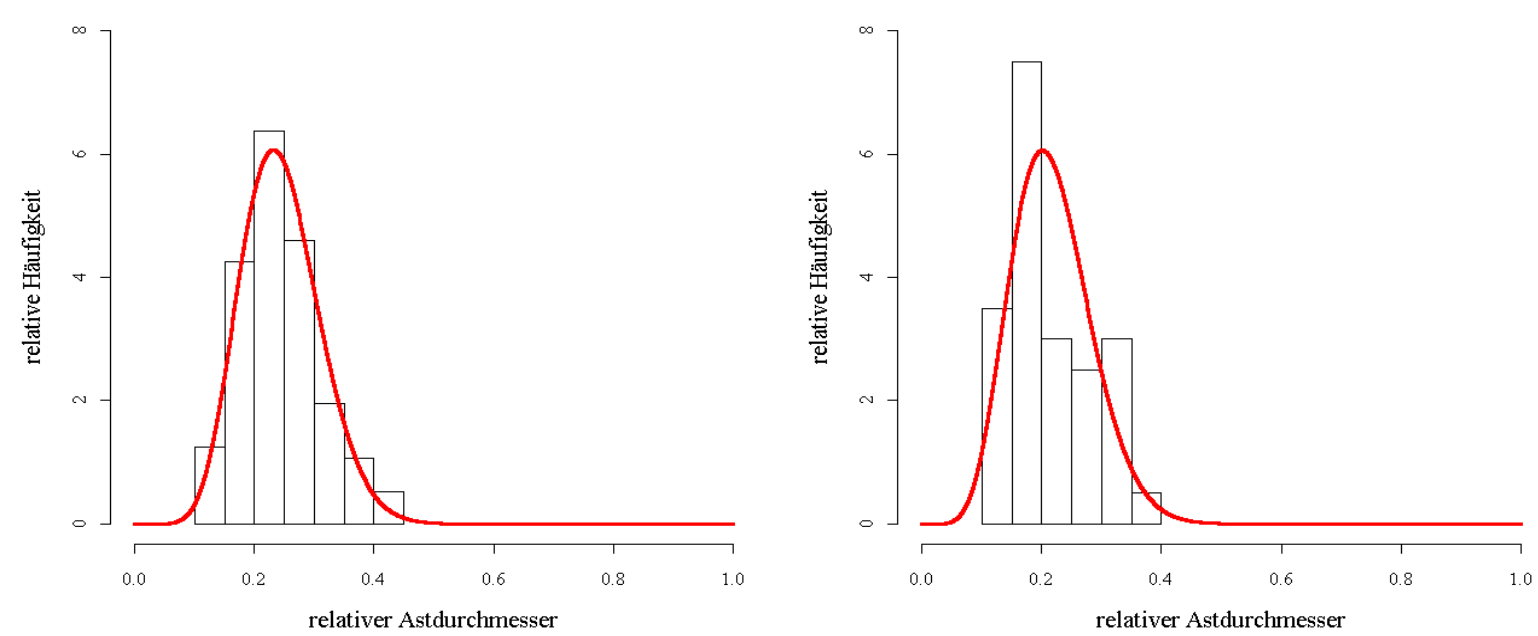
Klassenmittelwert BHD (mm): 182.8

Klassenmittelwert mittlere Tiefe in der Krone (dm): 84.2

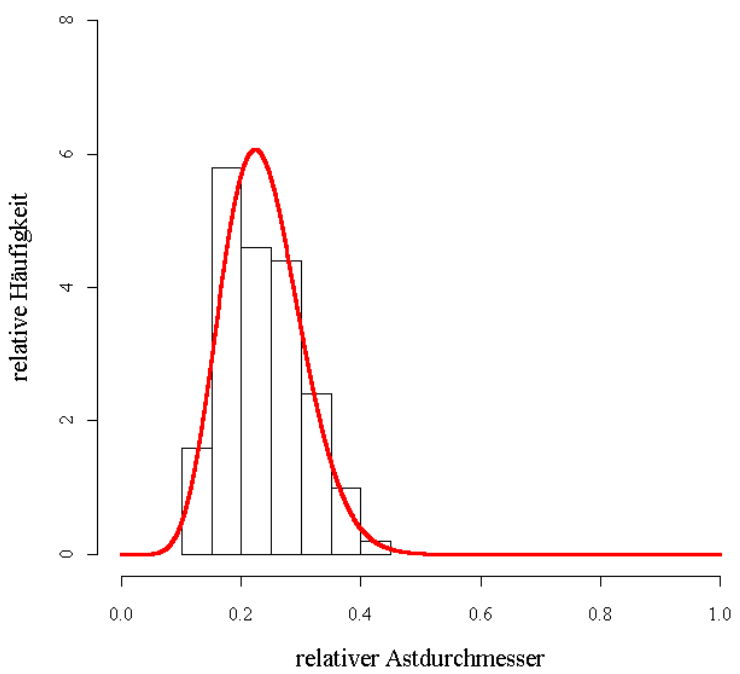

Klassenmittelwert BHD (mm): 140 Klassenmittelwert mittlere Tiefe in der Krone (dm): 97.9

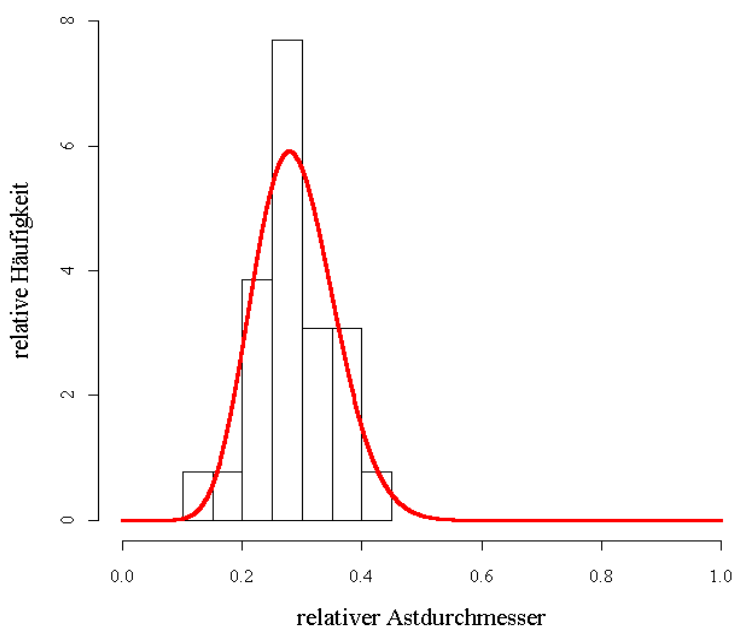
Klassenmittelwert mittlere Tiefe in der Krone (dm): 98.5

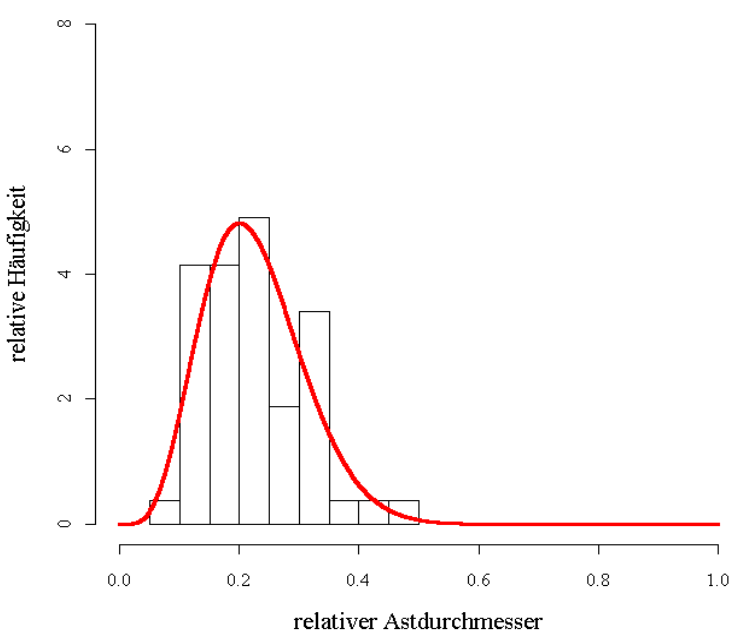

Klassenmittelwert BHD (mm): 217.1

Klassenmittelwert mittlere Tiefe in der Krone (dm): 79.1

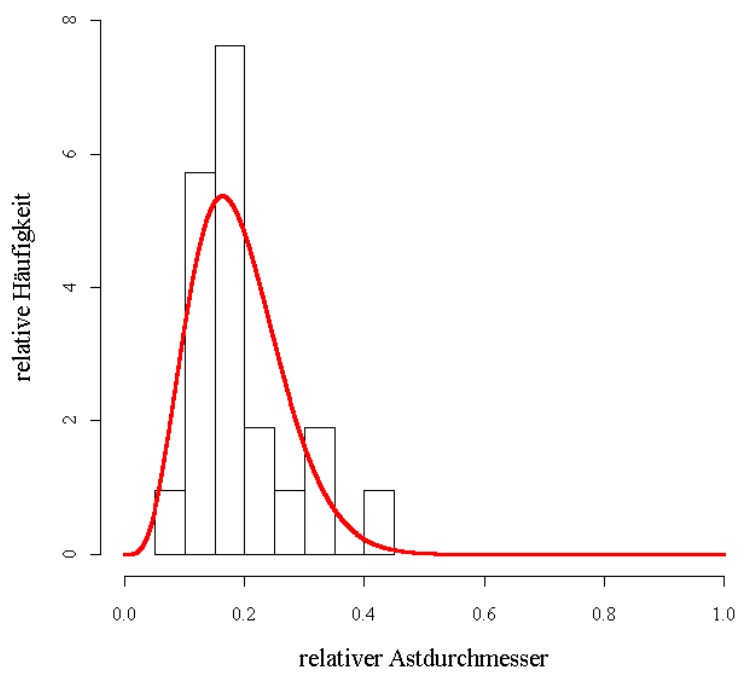

Klassenmittelwert BHD (mm): 186.8 Klassenmittelwert mittlere Tiefe in der Krone (dm): 98.1

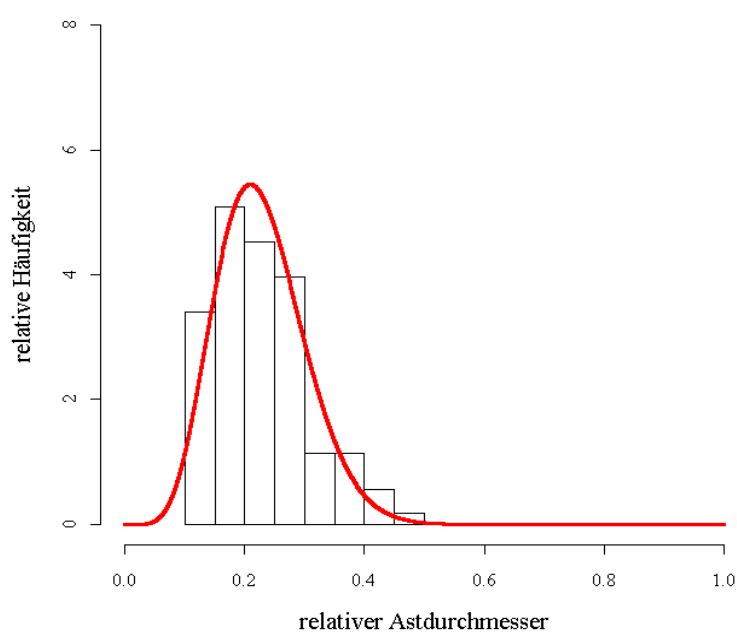

Klassenmittelwert BHD (mm): 260.6 Klassenmittelwert mittlere Tiefe in der Krone (dm): 103.7

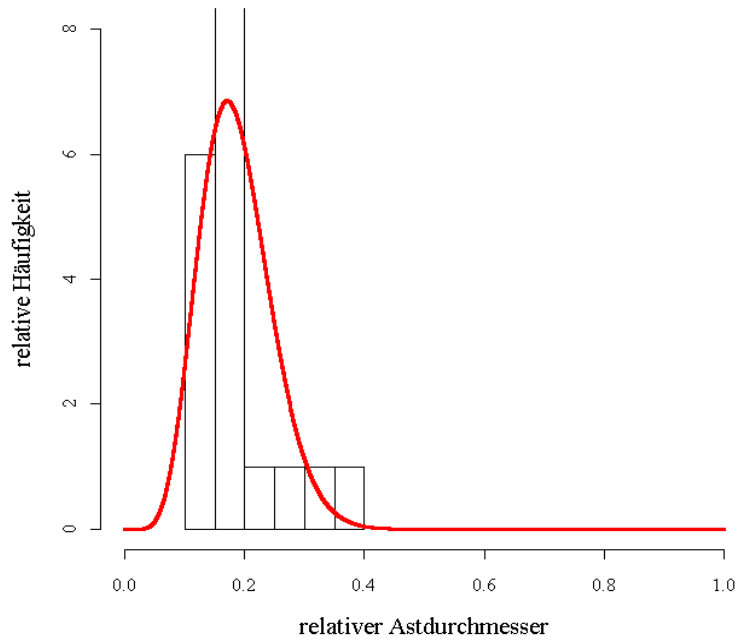




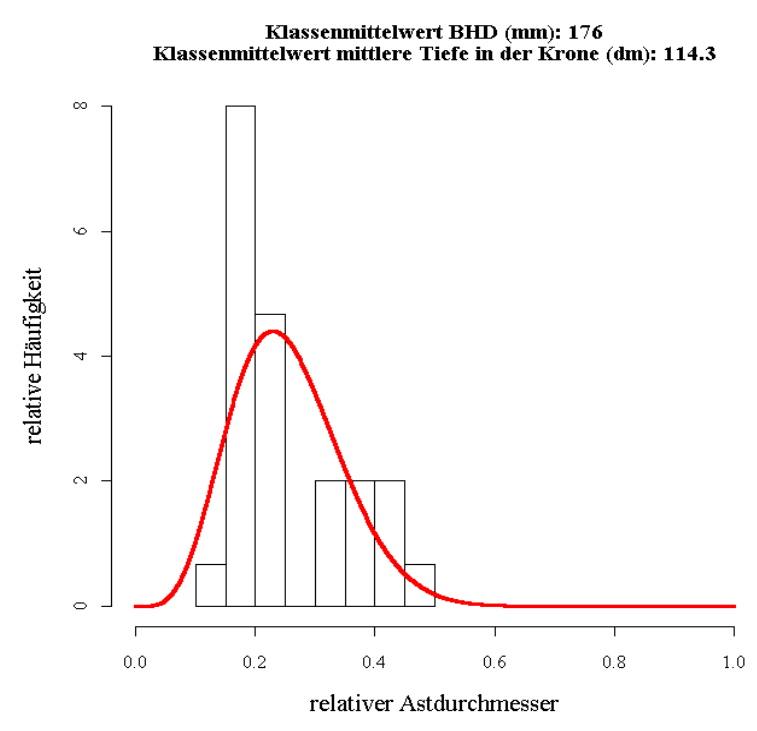

Klassenmittelwert BHD (mm): 265.2
Klassenmittelwert mittlere Tiefe in der Krone (dm): 119.9

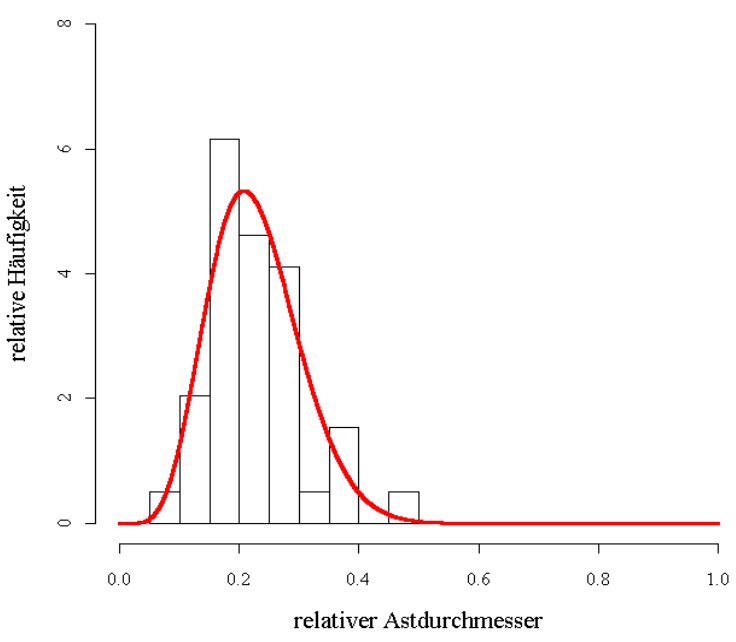

Klassenmittelwert BHD (mm): 341.5
Klassenmittelwert mittlere Tiefe in der Krone (dm): 155.4

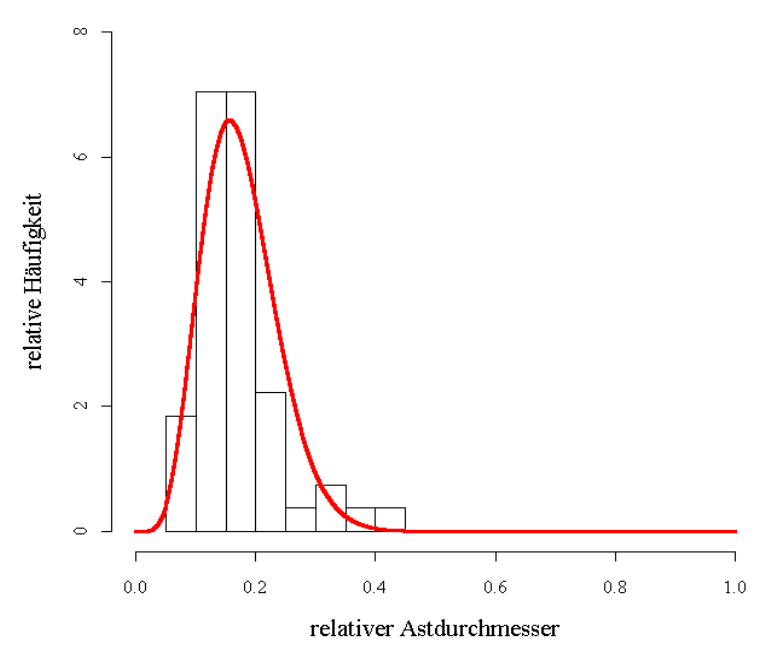

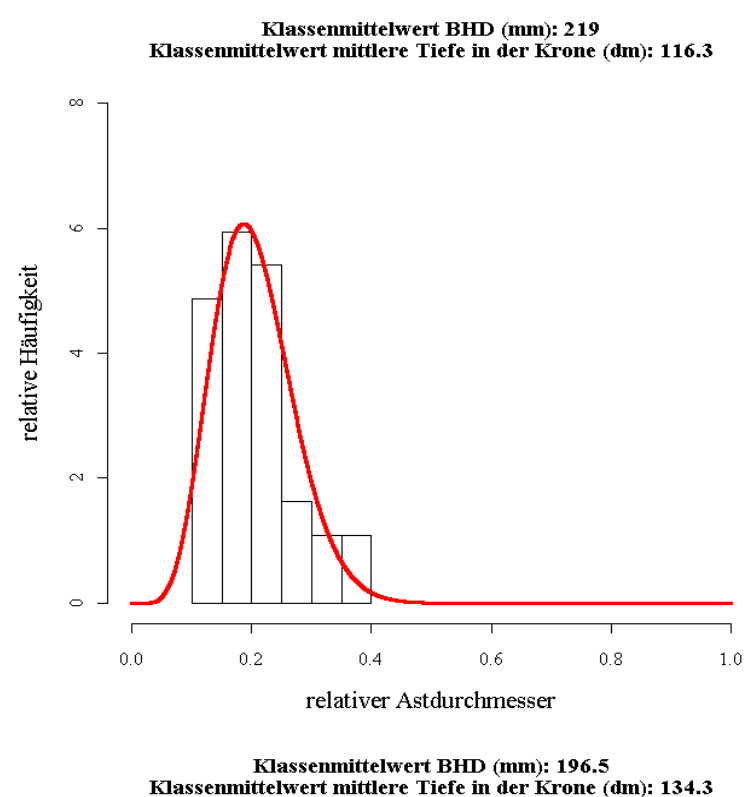

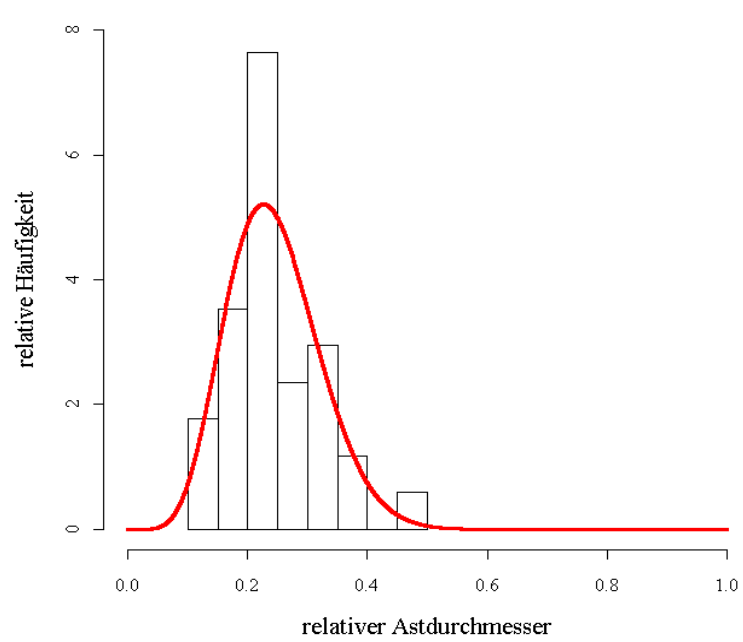

Abb. 115: Getrennt nach Straten berechnete empirische Verteilungen der normierten Astdurchmesser aus dem Bereich des Kronenansatzes von Buchen und die zugehörige Modellierung der Dichteverteilung mit Hilfe der Beta-Verteilung. Zusätzlich sind die Mittelwerte der Variablen BHD und mittlere Tiefe in der Krone für die einzelnen Straten angegeben. Zur Abgrenzung der einzelnen Straten siehe Tab. 19. 

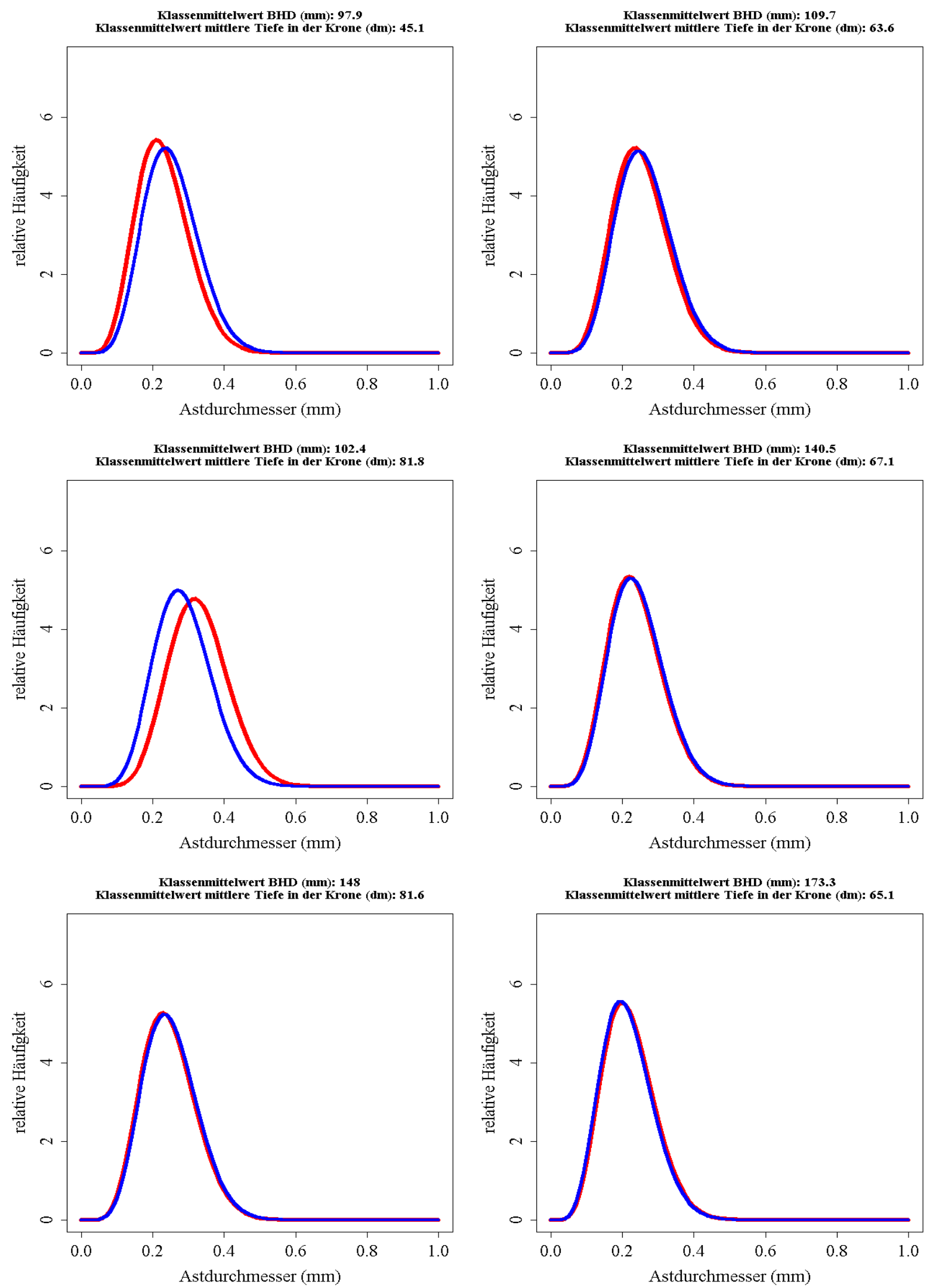

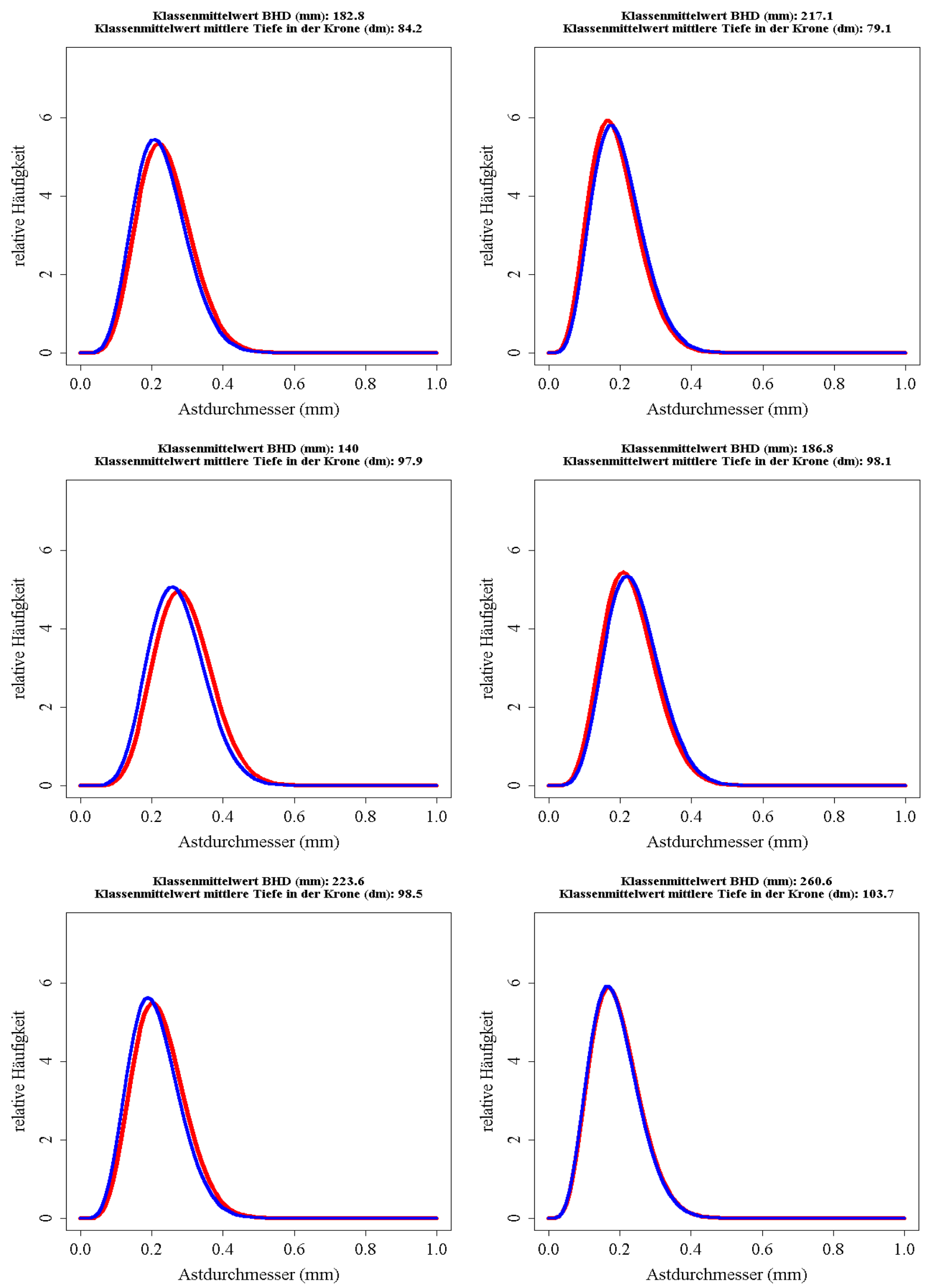

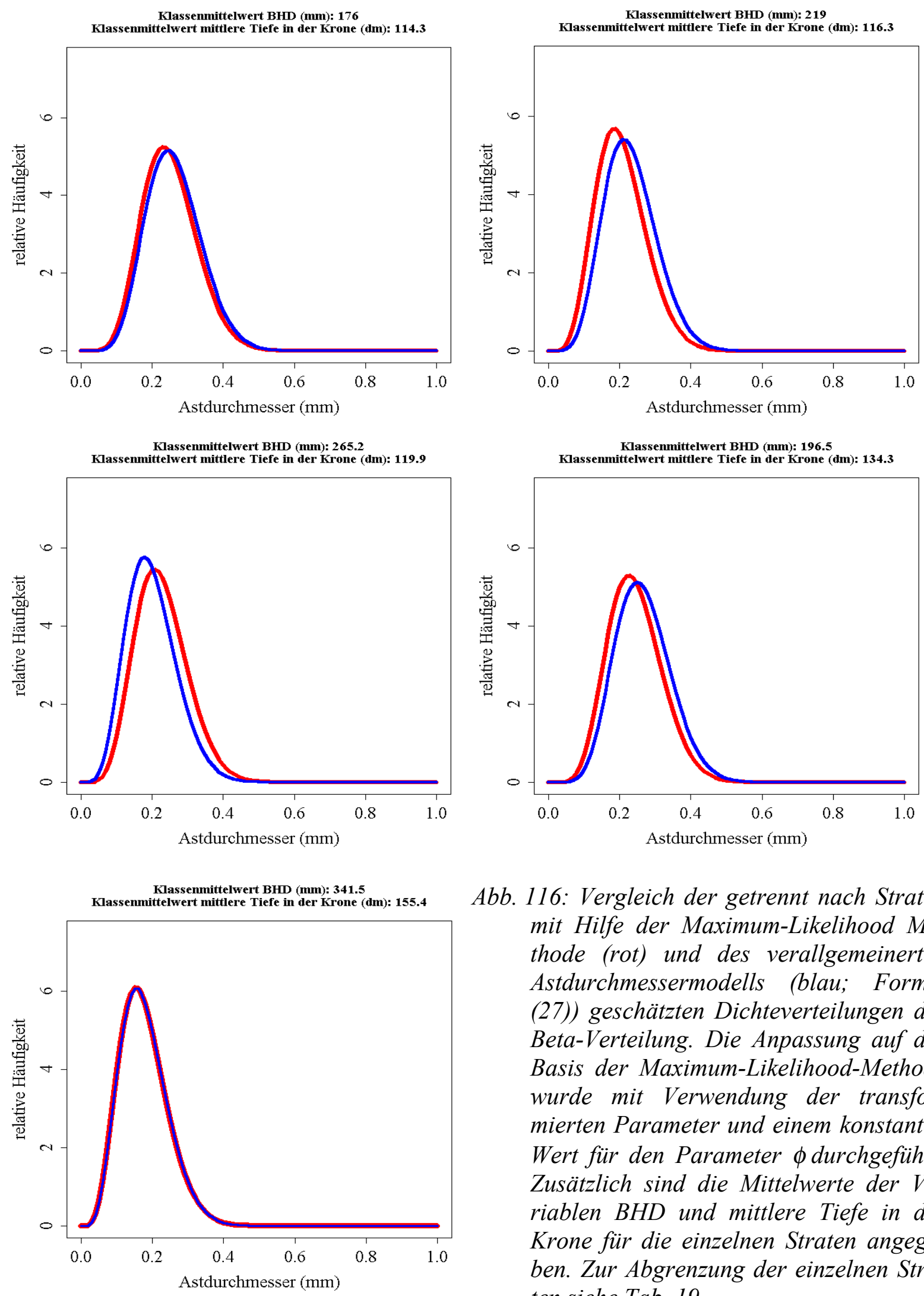

Abb. 116: Vergleich der getrennt nach Straten mit Hilfe der Maximum-Likelihood Methode (rot) und des verallgemeinerten Astdurchmessermodells (blau; Formel (27)) geschätzten Dichteverteilungen der Beta-Verteilung. Die Anpassung auf der Basis der Maximum-Likelihood-Methode wurde mit Verwendung der transformierten Parameter und einem konstanten Wert für den Parameter $\phi$ durchgeführt. Zusätzlich sind die Mittelwerte der Variablen BHD und mittlere Tiefe in der Krone für die einzelnen Straten angegeben. Zur Abgrenzung der einzelnen Straten siehe Tab. 19. 


\subsection{Astmortalitäts- und Astreinigungsmodelle}

\section{Douglasie:}

$H D I F F \ddot{U}_{r e l}=\alpha * e^{\beta^{*} K A}$

mit:

\begin{tabular}{|r|c|c|c|}
\hline Koeffizient & Wert & Std. Fehler & t-Wert \\
\hline$\alpha$ & 0.35293100 & 0.03758410 & 9.39043 \\
\hline$\beta$ & -0.00658461 & 0.00216769 & -3.03762 \\
\hline & Residual Std. Fehler & \multicolumn{2}{c|}{0.16109 bei 87 FG bzw. 9.65 dm } \\
\hline
\end{tabular}

Tab. 77: Koeffizienten und statistische Kenngrößen für das Modell zur Schätzung des vertikalen Abstandes zwischen der Kronenansatzhöhe und der Höhe zum untersten Grünast für die Baumart Douglasie.

\section{Kiefer:}

$H D I F F \ddot{U}_{r e l}=\alpha * e^{\beta^{*} K A}$

mit:

\begin{tabular}{|c|c|c|c|}
\hline Koeffizient & Wert & Std. Fehler & $\mathrm{t}$-Wert \\
\hline$\alpha$ & 0.3901710 & 0.05846710 & 6.67334 \\
\hline$\beta$ & -0.0120233 & 0.00269934 & -4.45415 \\
\hline \multicolumn{2}{|c|}{ Residual Std. Fehler } & \multicolumn{2}{|c|}{0.09869 bei $71 \mathrm{FG}$ bzw. $6.05 \mathrm{dm}$} \\
\hline
\end{tabular}

Tab. 78: Koeffizienten und statistische Kenngrößen für das Modell zur Schätzung des vertikalen Abstandes zwischen der Kronenansatzhöhe und der Höhe zum untersten Grünast für die Baumart Kiefer. 


\section{Douglasie:}

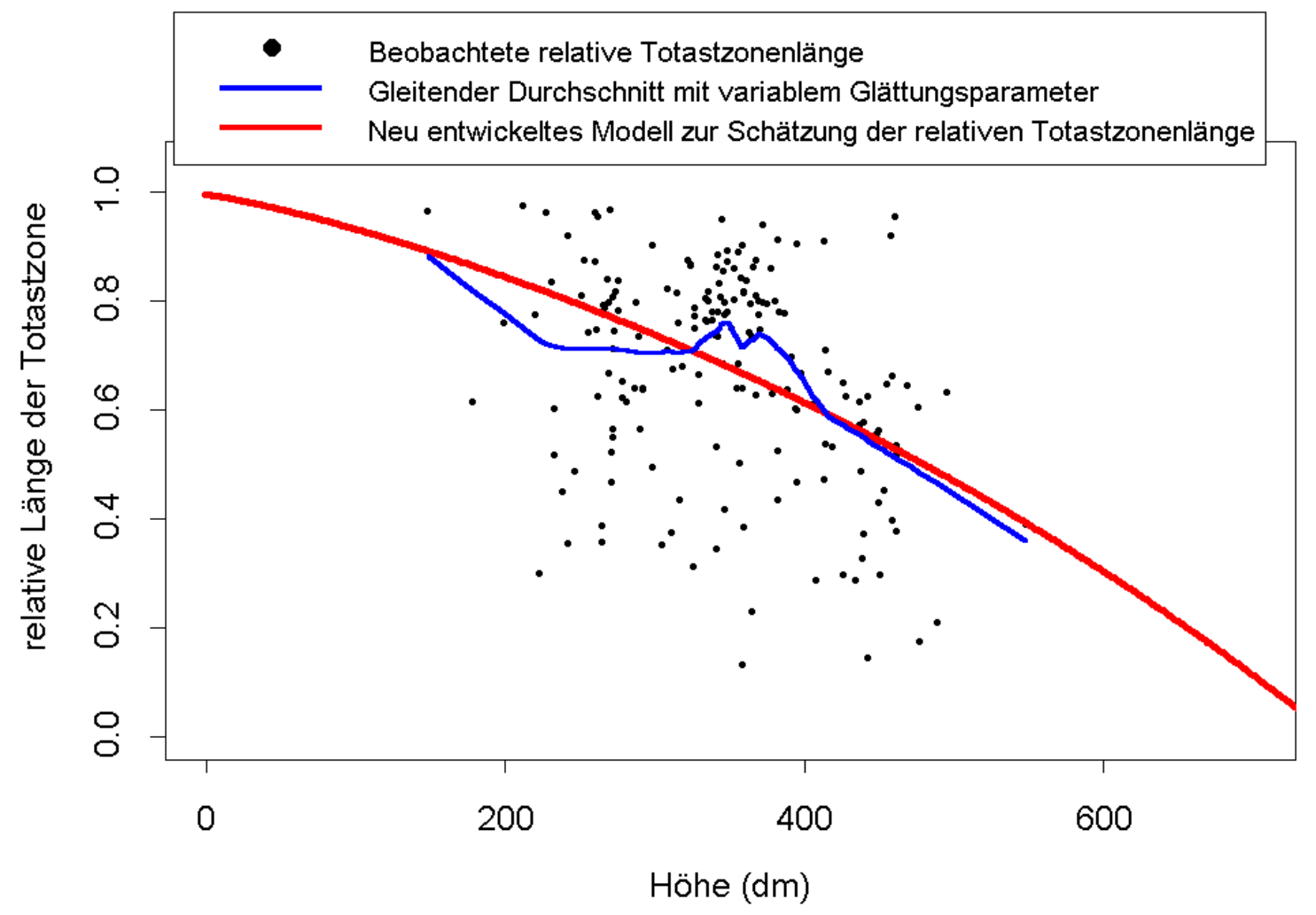

Abb. 117: Modell zur Beschreibung der relativen Länge der Totastzone in Abhängigkeit von der Baumhöhe für die Baumart Douglasie. Zur Verdeutlichung des Trends wird ein Gleitendes-Durchschnitts-Modell mit variablem Glättungsparameter verwendet (Kapitel 8.1).

HDIFFTOT $_{r e l}=2-e^{\left(\alpha^{*} H\right)^{1.2} / 1000}$

(32) abgewandelt

mit:

\begin{tabular}{|c|c|c|c|}
\hline Koeffizient & Wert & Std. Fehler & $\mathrm{t}$-Wert \\
\hline$\alpha$ & 0.307989 & 0.0091694 & 33.5888 \\
\hline & al Std. Fehler & \multicolumn{2}{|c|}{0.18368 bei 180 bzw. $33.643 \mathrm{dm}$} \\
\hline
\end{tabular}

Tab. 79: Koeffizienten und statistische Kenngrößen für das Modell zur Schätzung des vertikalen Abstandes zwischen der Höhe zum untersten Grünast und der Höhe zum untersten Totast für die Baumart Douglasie (Totastzone). 


\subsection{Flexible Schaftformmodelle}

\section{Douglasie:}
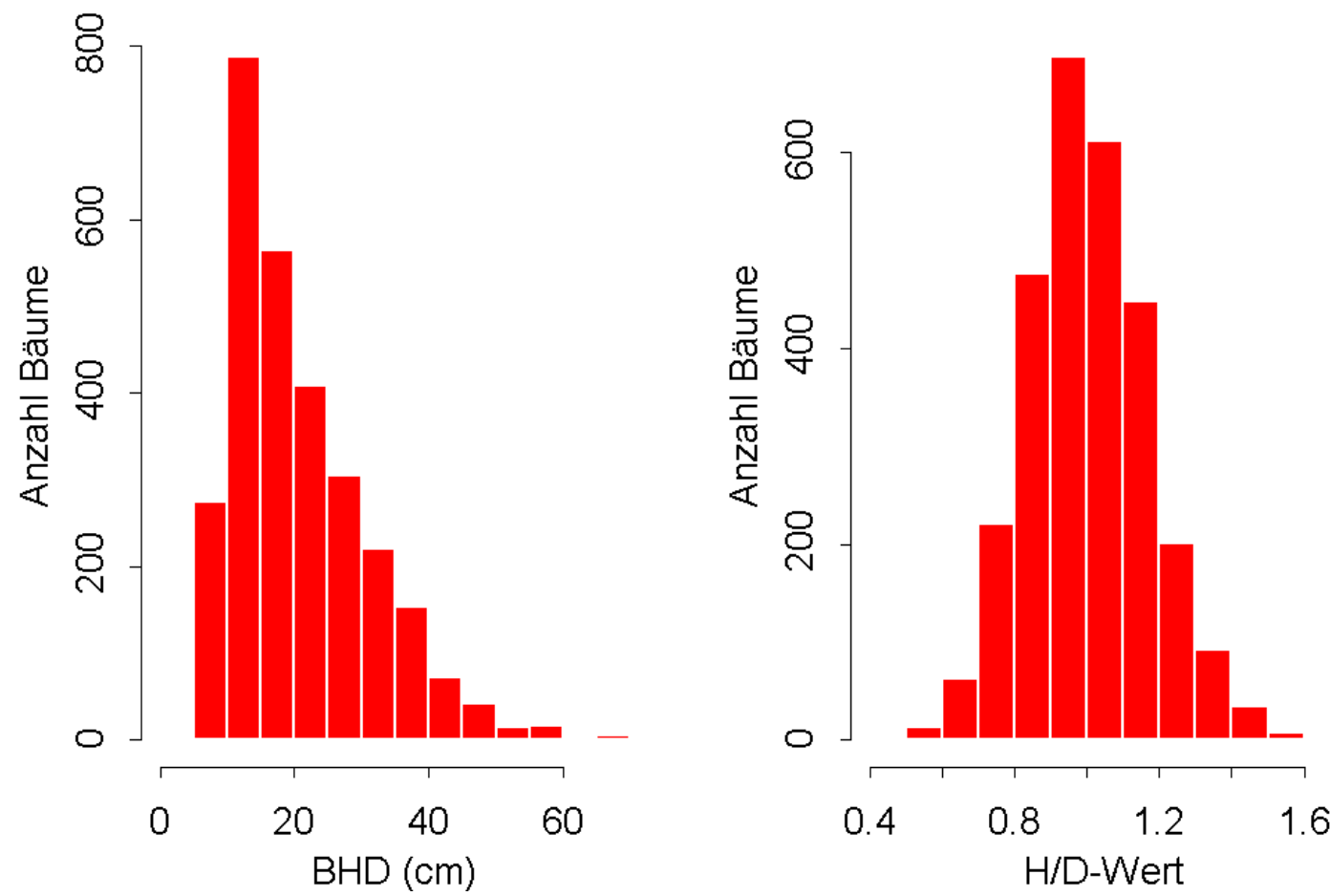

Abb. 118: BHD- und H/D-Wert-Verteilungen für 2870 Douglasien, die zur Entwicklung eines Einheitsschaftformmodells verwendet wurden.

\section{Douglasie}

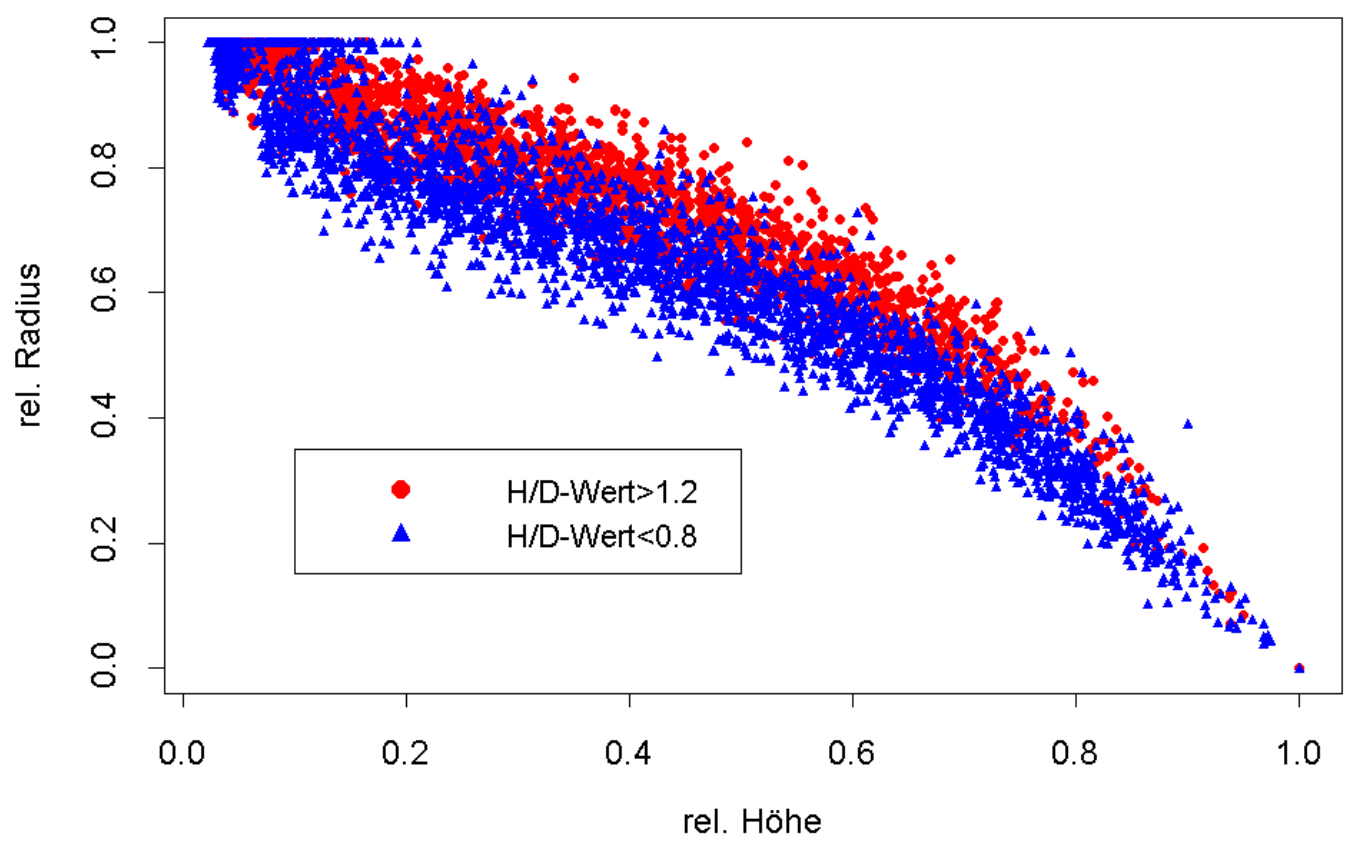

Abb. 119: Relativierte Sektionsmessungen für die Teilkollektive von Douglasien mit einem H/D-Wert von über 1.2 bzw. unter 0.8 zur Darstellung des Formenspektrums und des Einflusses der Variablen H/D-Wert. 


\section{Douglasie}

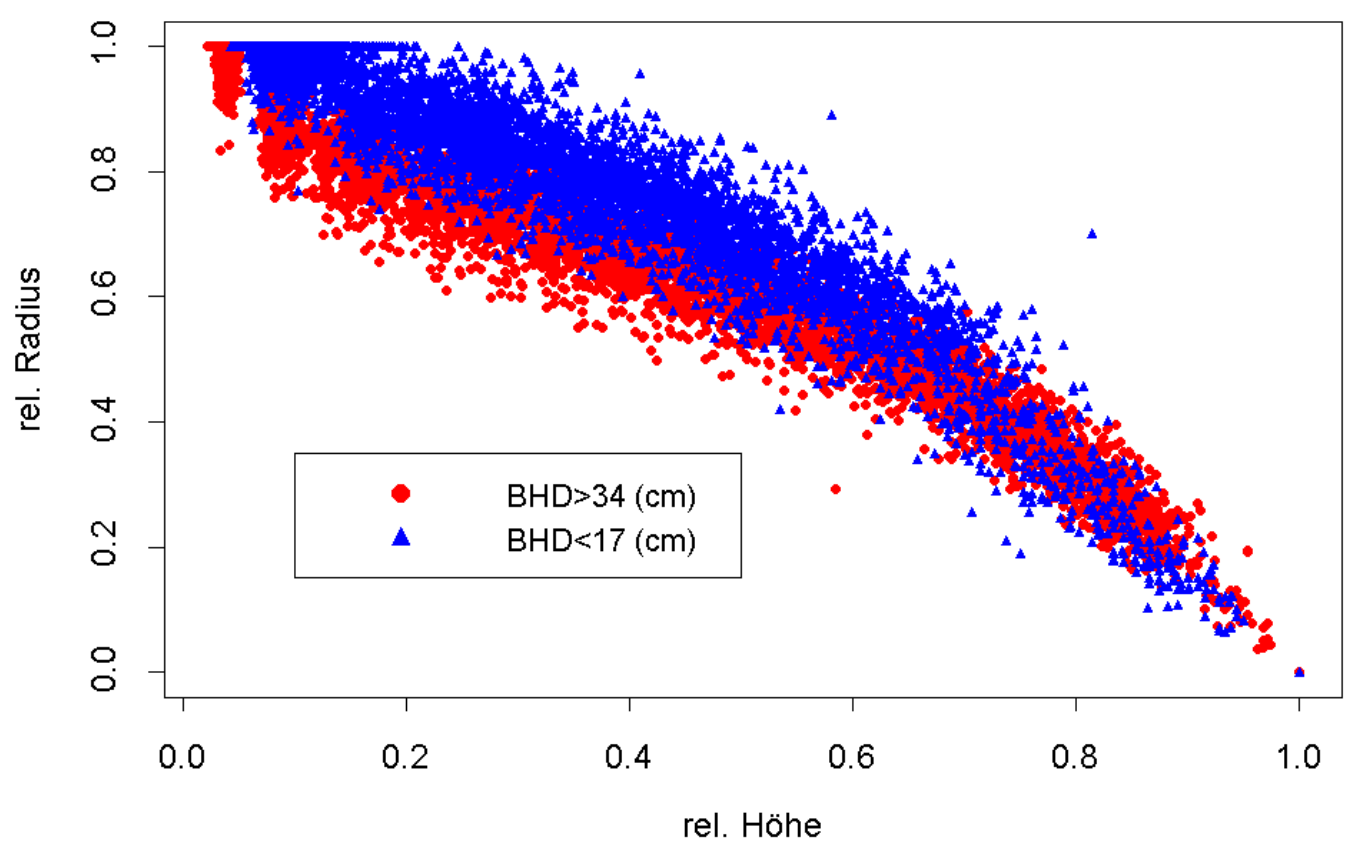

Abb. 120: Relativierte Sektionsmessungen für die Teilkollektive von Douglasien mit einem BHD von über $34 \mathrm{~cm}$ bzw. unter $17 \mathrm{~cm}$ zur Darstellung des Formenspektrums und des Einflusses der Variablen BHD.

\begin{tabular}{|r|r|r|r|r|}
\hline \multicolumn{1}{|c|}{ Koeffizient } & Wert & Std. Fehler & \multicolumn{1}{c|}{$\mathrm{t}$-Wert } & \multicolumn{1}{c|}{$\operatorname{Pr}(>|\mathrm{t}|)$} \\
\hline$a_{0}$ & -0.5828 & 0.0251 & -23.2380 & 0.0000 \\
\hline$a_{1}$ & 1.4423 & 0.0046 & 315.5793 & 0.0000 \\
\hline$a_{2}$ & -2.1807 & 0.0301 & -72.4895 & 0.0000 \\
\hline$b_{0}$ & 0.4369 & 0.0135 & 32.2455 & 0.0000 \\
\hline$b_{1}$ & -0.2008 & 0.0025 & -79.1233 & 0.0000 \\
\hline$b_{2}$ & -0.2836 & 0.0167 & -17.0032 & 0.0000 \\
\hline \multicolumn{2}{|r|}{ Residual Std. Fehler (mm) } & \multicolumn{3}{c}{0.5274 bei 28350 Freiheitsgraden } \\
\hline \multicolumn{2}{r|r}{ Multiples Bestimmtheitsmaß } & F-Statistik & 1575000 bei 6 und 28350 Freiheitsgr., $p$-Wert $=0$ \\
\hline
\end{tabular}

Tab. 80: Koeffizienten und statistische Kenngrößen zur Schaftdurchmesserschätzung mit Hilfe des Gesamtmodells der Pain-Funktion (33) bzw. (34) und (35) für die Baumart Douglasie.

\begin{tabular}{|r|r|r|r|}
\hline Koeffizient & \multicolumn{1}{c|}{ Wert } & Std. Fehler & \multicolumn{1}{c|}{ t-Wert } \\
\hline$k$ & 0.4135470 & 0.00524202 & 78.8908 \\
\hline$p$ & 0.0390303 & 0.00105840 & 36.8766 \\
\hline$q$ & 0.2030370 & 0.00324901 & 62.4920 \\
\hline \multicolumn{2}{|r|}{ Residual Std. Fehler (mm) } & \multicolumn{3}{|c}{0.646661 bei 28353 Freiheitsgraden } \\
\hline
\end{tabular}

Tab. 81: Koeffizienten und statistische Kenngrößen zur Schaftdurchmesserschätzung mit Hilfe der modifizierten Brink-Funktion (37) für die Baumart Douglasie. 


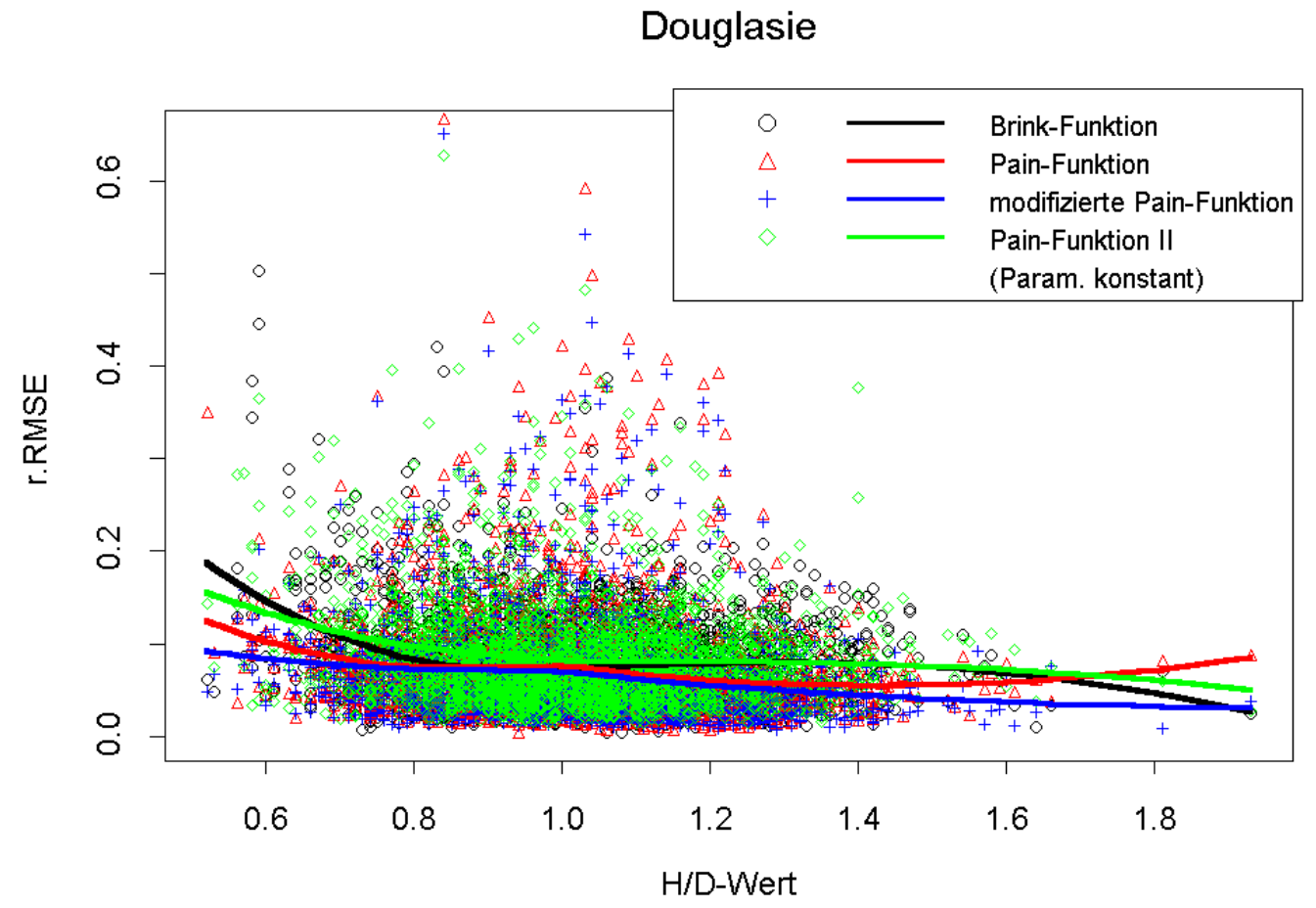

Abb. 121: Einzelbaumweise berechnete relative RMSE über den zugehörigen $H / D$-Werten der Einzelbäume für die Pain-Funktion (flexibles Schaftformmodell) sowie für die modifizierte Brink-Funktion und die Pain-Funktion II (konstante Parameter) (mittleres Einzelbaummodell). Der Ausgleich der Fehlerwerte erfolgte wie auch in Abb. 122 mit einem Gleitenden-Durchschnitts-Modell (Kapitel 8.1). Weiterhin sind die relativen RMSE der in Kapitel 3.7.3 beschriebenen Modifikation der Pain-Funktion dargestellt.

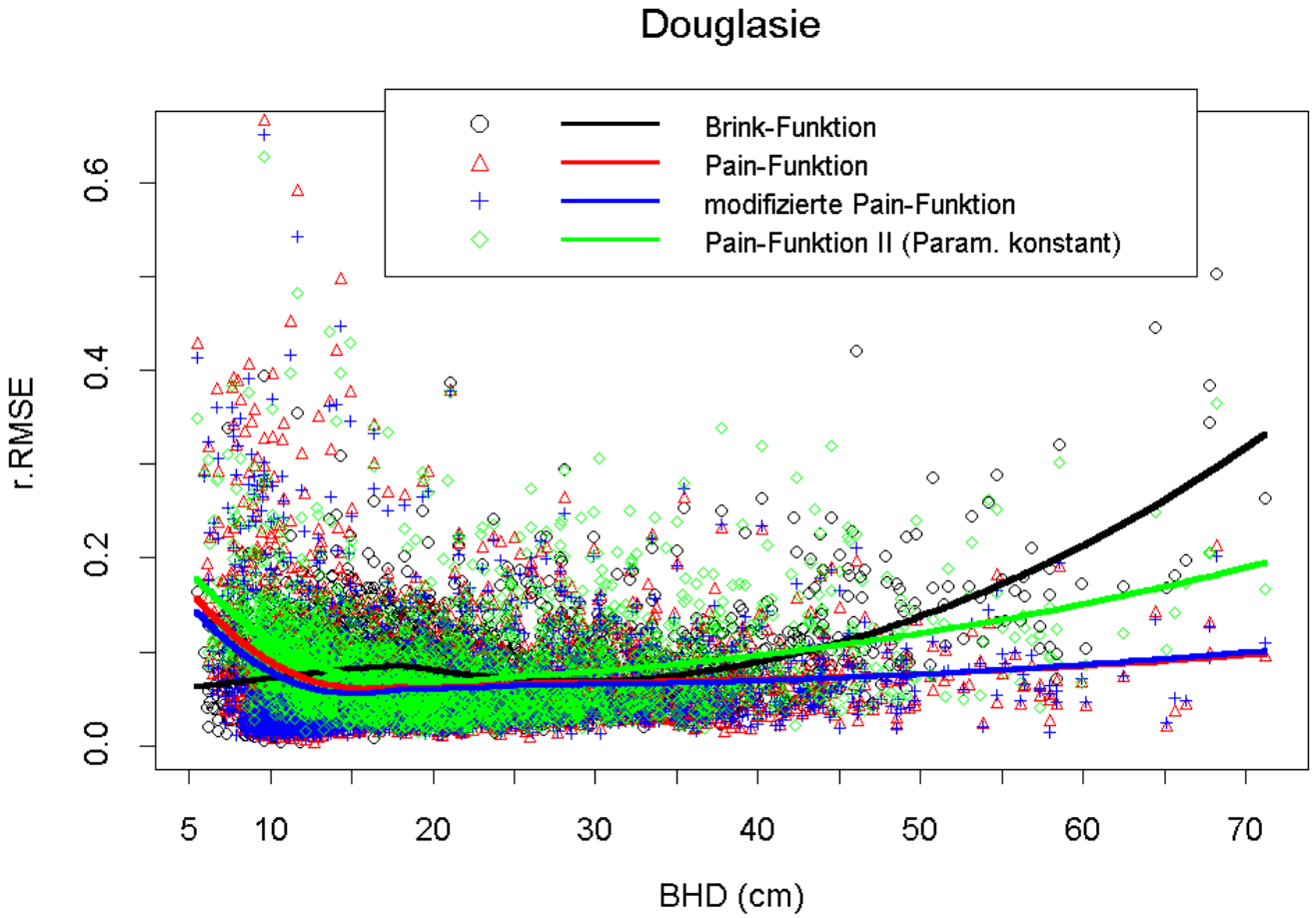

Abb. 122: Einzelbaumweise berechnete relative RMSE über den zugehörigen BHD-Werten der Einzelbäume für die Pain-Funktion (flexibles Schaftformmodell) sowie für die modifizierte Brink-Funktion und die Pain-Funktion II (konstante Parameter) (mittleres Einzelbaummodell). Weiterhin sind die relativen RMSE der in Kapitel 3.7.3 beschriebenen Modifikation der Pain-Funktion dargestellt. 


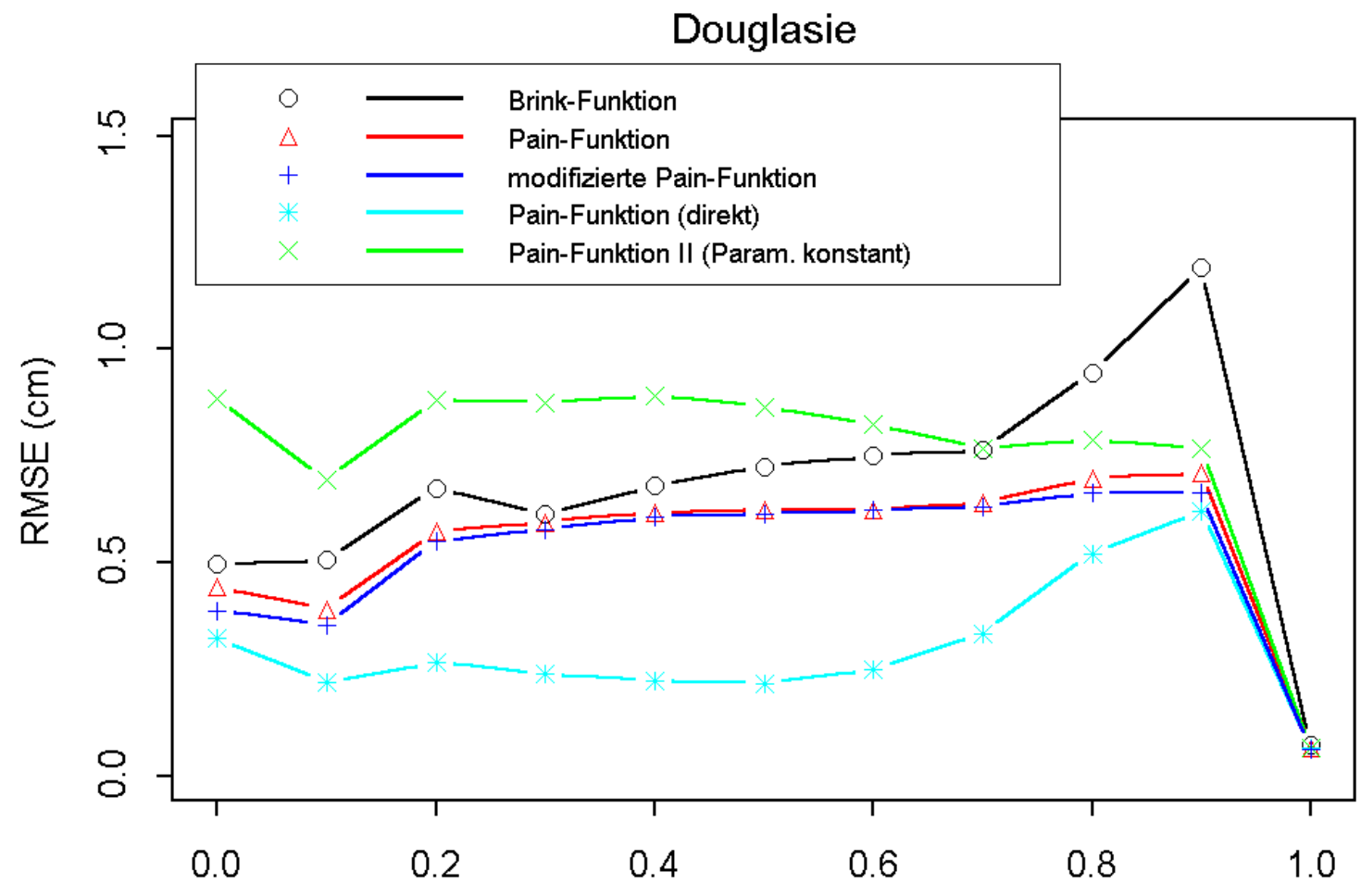

rel. Höhe

Abb. 123: Verläufe des Fehlers (RMSE) über relativen Schafthöhen für unterschiedliche Schaftformmodelle.

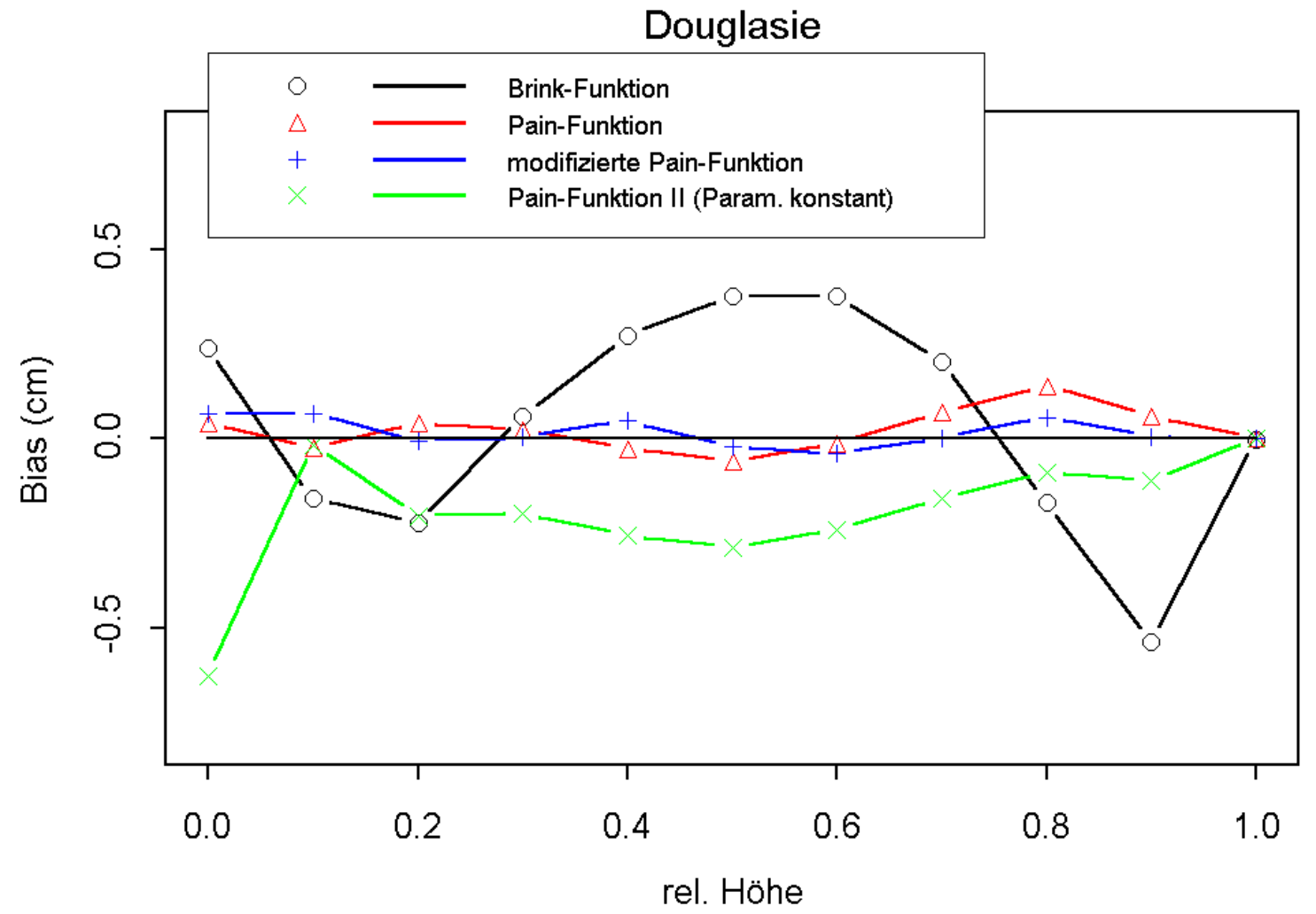

Abb. 124: Verläufe des Bias über relativen Schafthöhen für unterschiedliche Schaftformmodelle. 


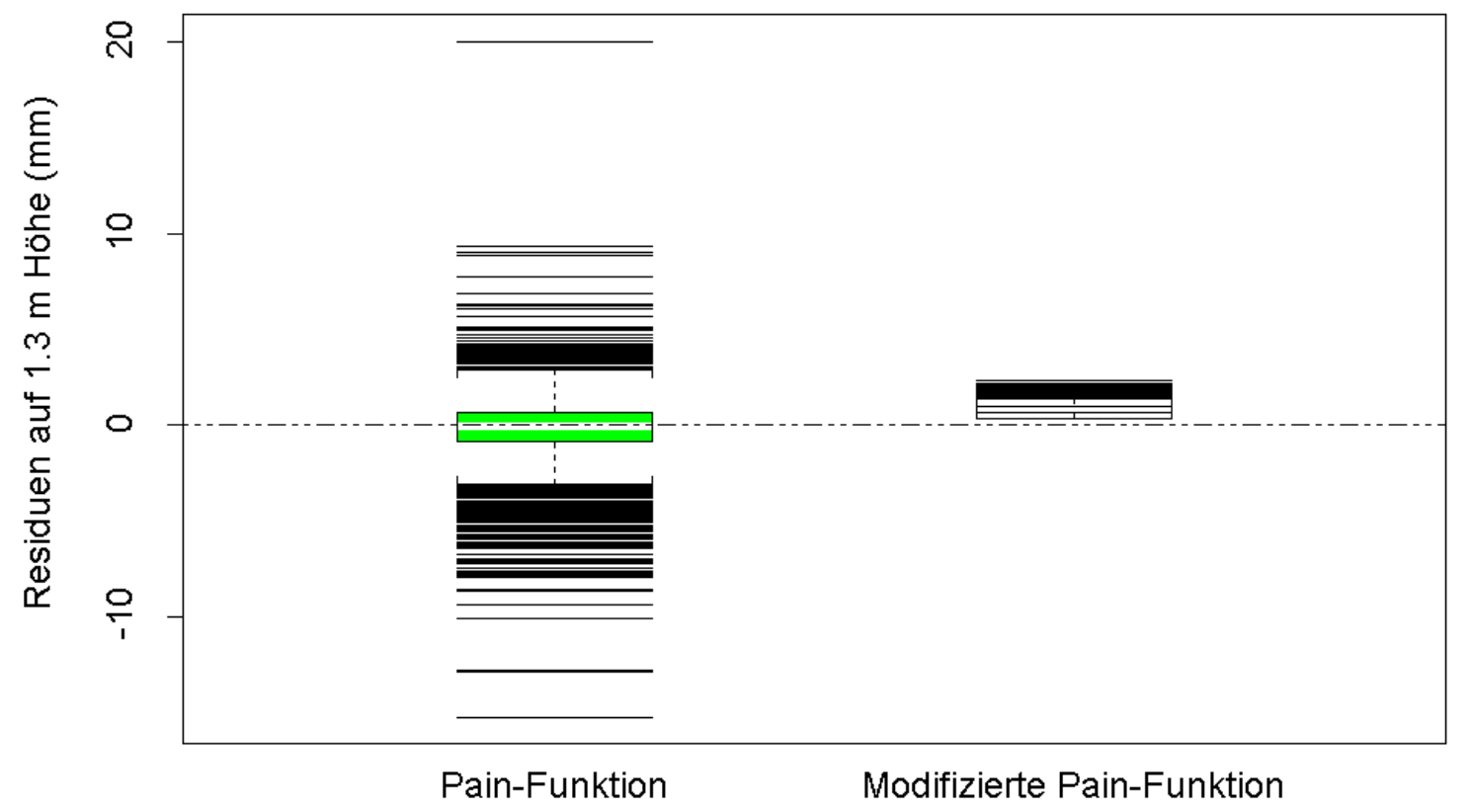

Abb. 125: Box-Plots der Residuen auf 1.3 m Höhe der Pain-Funktion und der modifizierten Pain-Funktion für die Baumart Douglasie.

\begin{tabular}{|c|c|c|c|}
\hline Koeffizient & Wert & Std. Fehler & $\mathrm{t}$-Wert \\
\hline Unterer Schaftteil $a_{0}$ & 1.9875800 & 0.02135960 & 93.05320 \\
\hline Unterer Schaftteil $a_{1}$ & -0.0457369 & 0.00364692 & -12.54130 \\
\hline Unterer Schaftteil $a_{2}$ & 0.1129080 & 0.02288480 & 4.93373 \\
\hline Unterer Schaftteil $b_{0}$ & -0.2049750 & 0.01563850 & -13.10710 \\
\hline Unterer Schaftteil $b_{1}$ & 0.0776066 & 0.00308172 & 25.18290 \\
\hline Unterer Schaftteil $b_{2}$ & 0.1104870 & 0.02017180 & 5.47729 \\
\hline Oberer Schaftteil $a_{0}$ & 2.0346900 & 0.02121890 & 95.89010 \\
\hline Oberer Schaftteil $a_{1}$ & 0.0478614 & 0.00391065 & 12.23870 \\
\hline Oberer Schaftteil $a_{2}$ & -0.3112880 & 0.02326600 & -13.37950 \\
\hline Oberer Schaftteil $b_{0}$ & -0.9480640 & 0.08971550 & -10.56740 \\
\hline Oberer Schaftteil $b_{1}$ & 0.4241100 & 0.01935150 & 21.91610 \\
\hline Oberer Schaftteil $b_{2}$ & 0.2300130 & 0.13344200 & 1.72369 \\
\hline \multicolumn{2}{|c|}{ Residual Std. Fehler (mm) } & \multicolumn{2}{|c|}{0.5178946 bei 28344 Freiheitsgraden } \\
\hline
\end{tabular}

Tab. 82: Koeffizienten und statistische Kenngrößen zur Schaftdurchmesserschätzung mit Hilfe des in Formel (38 a und b) dargestellten Schaftformmodells (modifizierte PainFunktion) für die Baumart Douglasie. 
Kiefer:
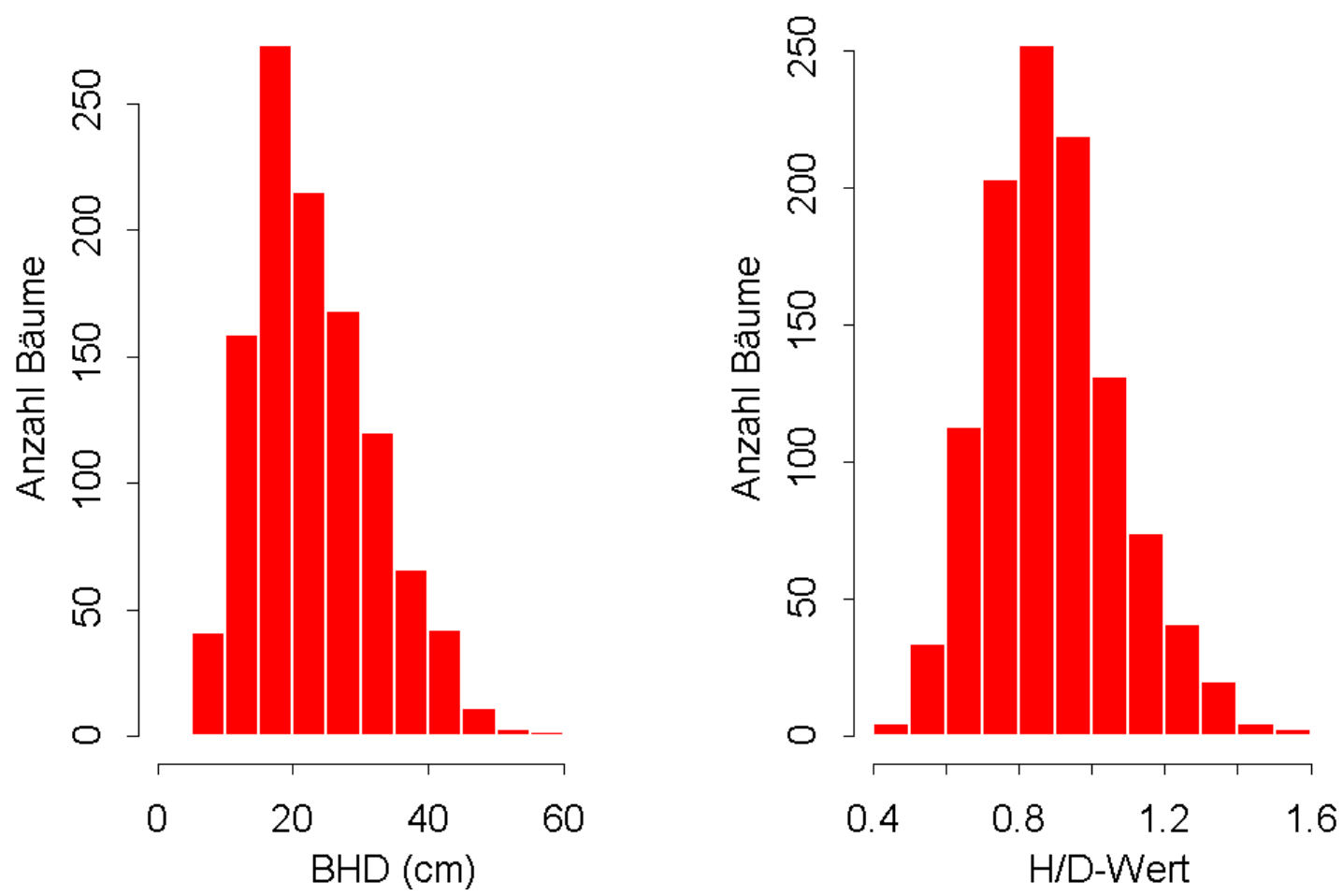

Tab. 83: BHD- und H/D-Wert-Verteilungen für 1100 Kiefern, die zur Entwicklung eines Einheitsschaftformmodells verwendet wurden.

\section{Kiefer}

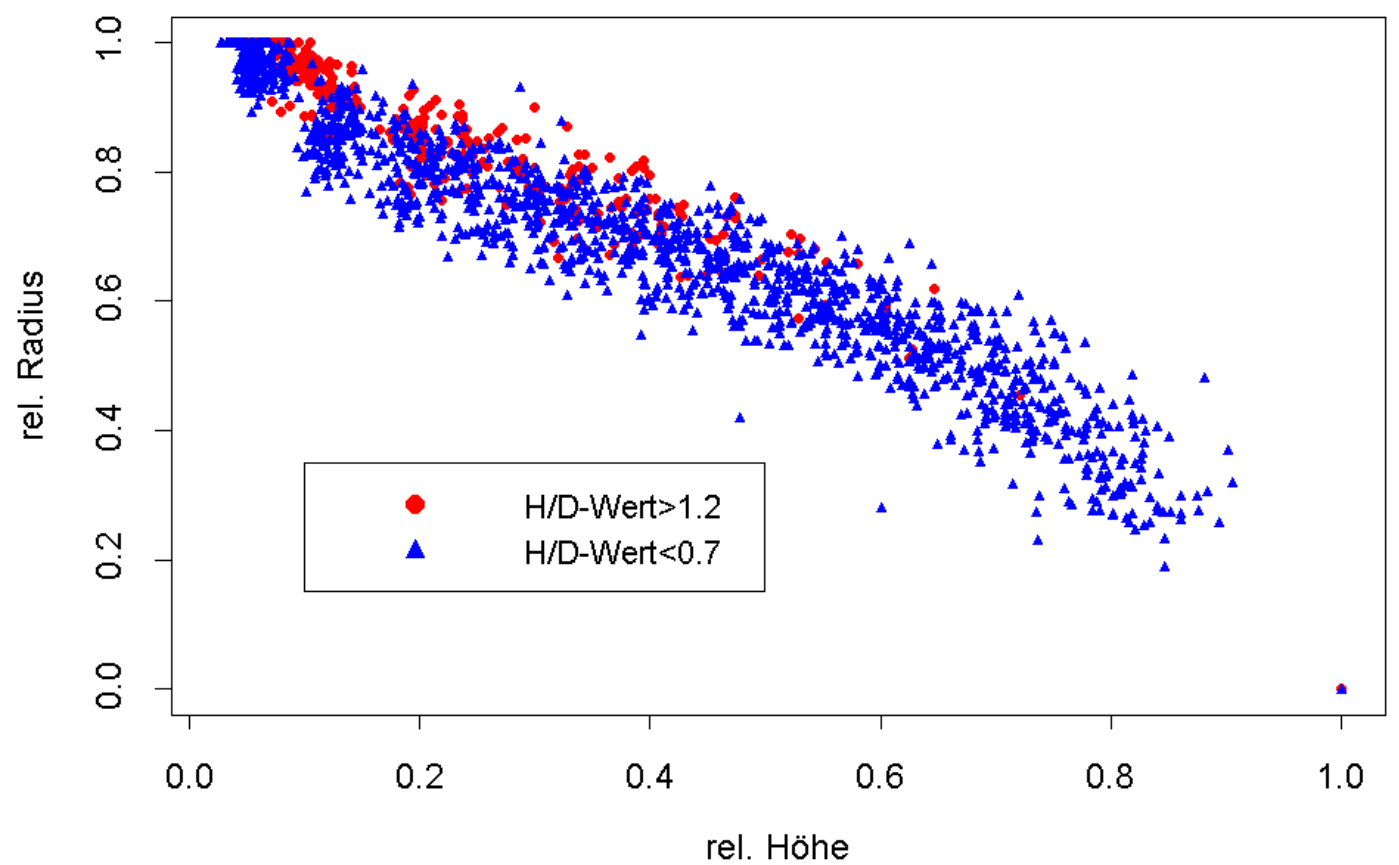

Abb. 126: Relativierte Sektionsmessungen für die Teilkollektive von Kiefern mit einem H/DWert von über 1.2 bzw. unter 0.7 zur Darstellung des Formenspektrums und des Einflusses der Variablen H/D-Wert. 


\section{Kiefer}

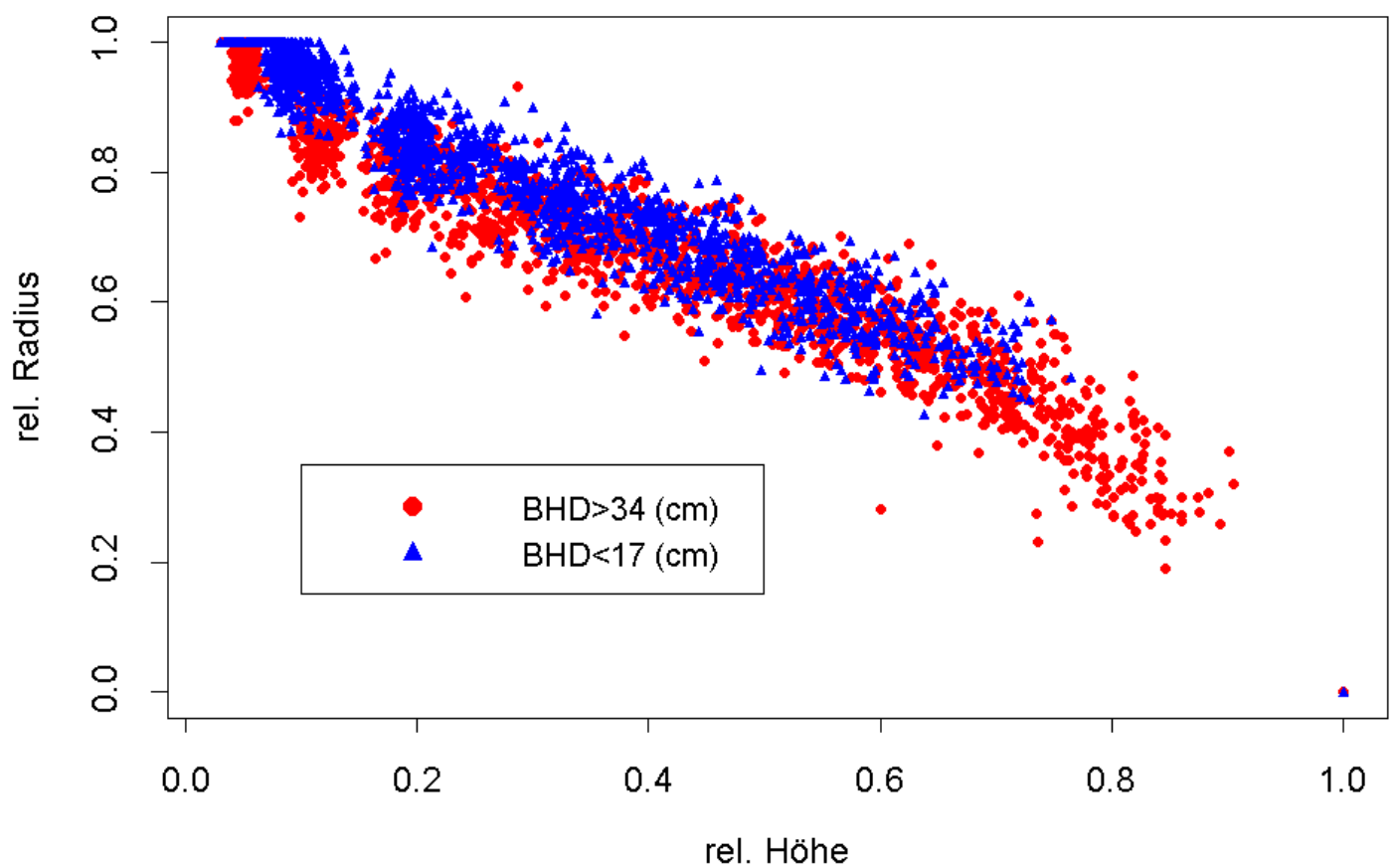

Abb. 127: Relativierte Sektionsmessungen für die Teilkollektive von Kiefern mit einem BHD von über $34 \mathrm{~cm}$ bzw. unter $17 \mathrm{~cm}$ zur Darstellung des Formenspektrums und des Einflusses der Variablen BHD.

\begin{tabular}{|c|c|c|c|c|}
\hline Koeffizient & Wert & Std. Fehler & $\mathrm{t}$-Wert & $\operatorname{Pr}(>|t|)$ \\
\hline$a_{0}$ & -1.7258 & 0.0194 & -88.9947 & 0.0000 \\
\hline$a_{1}$ & 1.3311 & 0.0072 & 185.5373 & 0.0000 \\
\hline$a_{2}$ & -0.7016 & 0.0350 & -20.0722 & 0.0000 \\
\hline \multicolumn{5}{|l|}{$b_{0} \mathrm{n}$. signifikant } \\
\hline$b_{1}$ & -0.2142 & 0.0035 & -60.5993 & 0.0000 \\
\hline$b_{2}$ & 0.1306 & 0.0188 & 6.9432 & 0.0000 \\
\hline \multicolumn{2}{|c|}{ Residual Std. Fehler (mm) } & \multicolumn{3}{|c|}{0.4822 bei 10723 Freiheitsgraden } \\
\hline \multicolumn{2}{|c|}{ Multiples Bestimmtheitsmaß } & \multicolumn{3}{|r|}{0.9976} \\
\hline \multicolumn{2}{|r|}{ F-Statistik } & \multicolumn{3}{|c|}{882000 bei 5 und 10723 Freiheitsgraden, $p$-Wert $=0$} \\
\hline
\end{tabular}

Tab. 84: Koeffizienten und statistische Kenngrößen zur Schaftdurchmesserschätzung mit Hilfe des Gesamtmodells der Pain-Funktion (33) bzw. (34) und (35) für die Baumart Kiefer.

\begin{tabular}{|c|c|c|c|}
\hline Koeffizient & Wert & Std. Fehler & t-Wert \\
\hline$k$ & 0.5125230 & 0.00706113 & 72.5837 \\
\hline$p$ & 0.0793867 & 0.00245392 & 32.3510 \\
\hline$q$ & 0.2263740 & 0.00426382 & 53.0919 \\
\hline Residual Std. Fehler & \multicolumn{3}{|c|}{0.540235 bei 10725 Freiheitsgraden } \\
\hline
\end{tabular}

Tab. 85: Koeffizienten und statistische Kenngrößen zur Schaftdurchmesserschätzung mit Hilfe der modifizierten Brink-Funktion (37) für die Baumart Kiefer. 


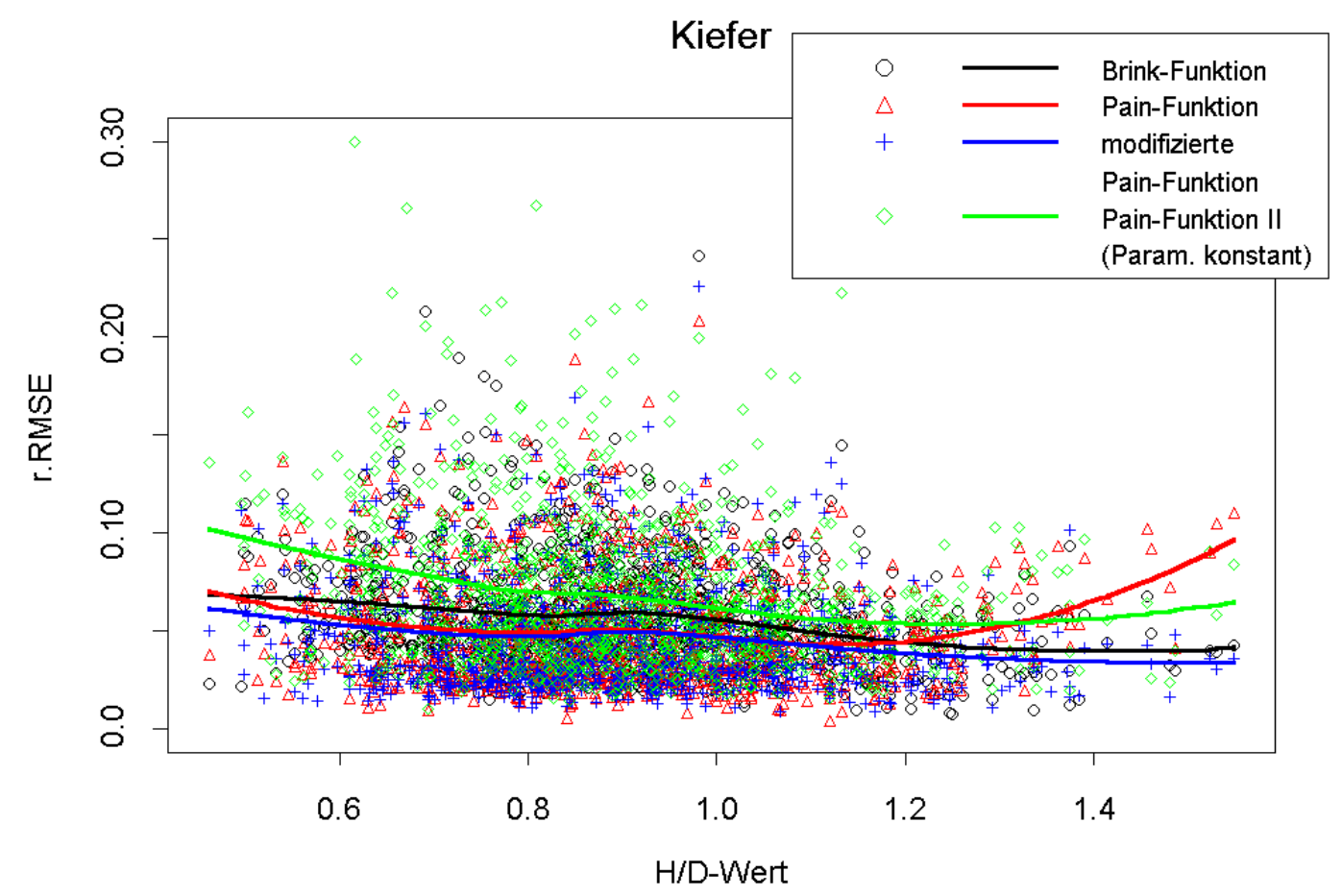

Abb. 128: Einzelbaumweise berechnete relative RMSE über den zugehörigen $H / D$-Werten der Einzelbäume für die Pain-Funktion (flexibles Schaftformmodell) sowie für die modifizierte Brink-Funktion und die Pain-Funktion II (konstante Parameter) (mittleres Einzelbaummodell). Der Ausgleich der Fehlerwerte erfolgte wie auch in Abb. 129 mit einem Gleitenden-Durchschnitts-Modell (Kapitel 8.1). Weiterhin sind die relativen RMSE der in Kapitel 3.7.3 beschriebenen Modifikation der Pain-Funktion dargestellt.

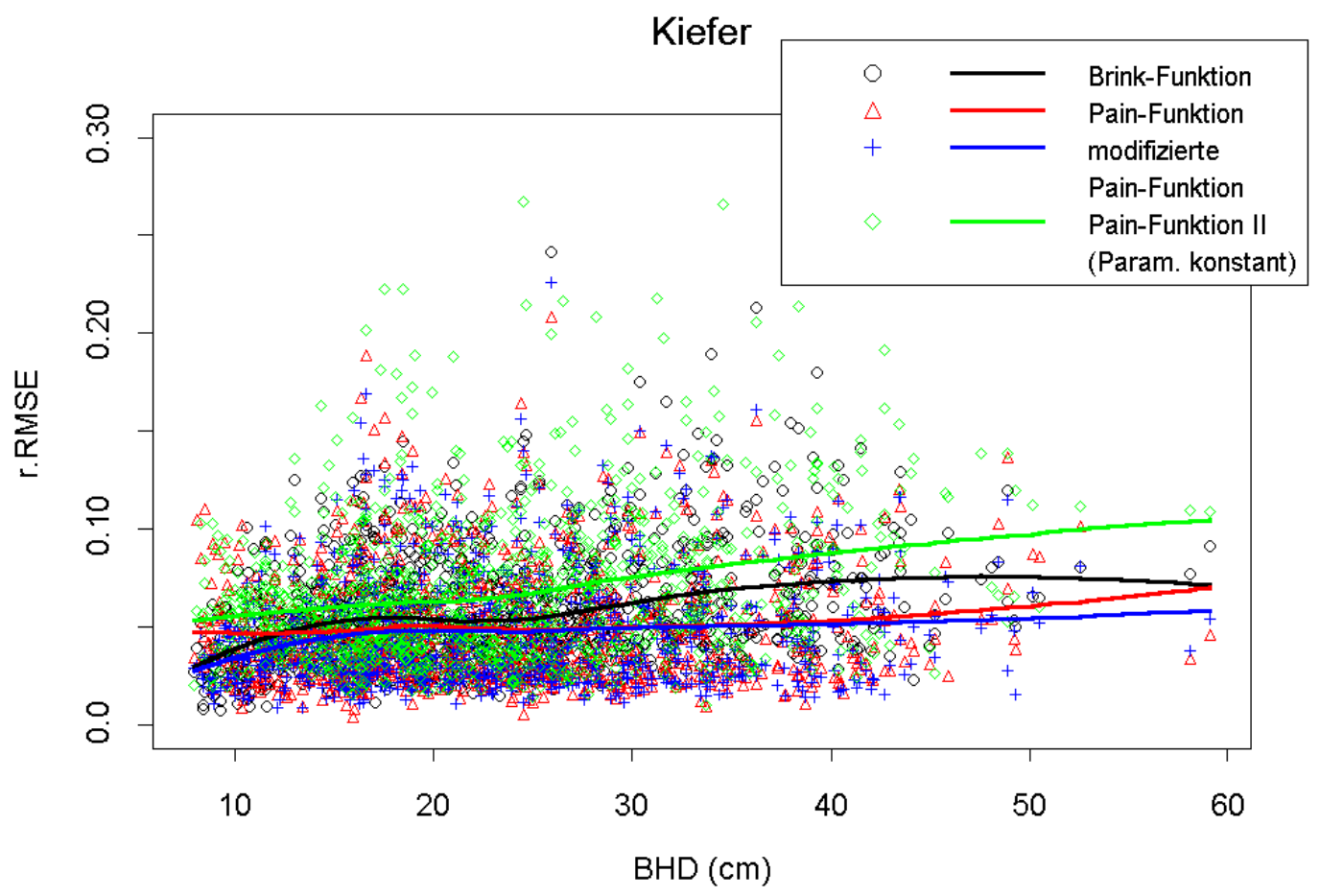

Abb. 129: Einzelbaumweise berechnete relative RMSE über den zugehörigen BHD-Werten der Einzelbäume für die Pain-Funktion (flexibles Schaftformmodell) sowie für die modifizierte Brink-Funktion und die Pain-Funktion II (konstante Parameter) (mittleres Einzelbaummodell). Weiterhin sind die relativen RMSE der in Kapitel 3.7.3 beschriebenen Modifikation der Pain-Funktion dargestellt. 


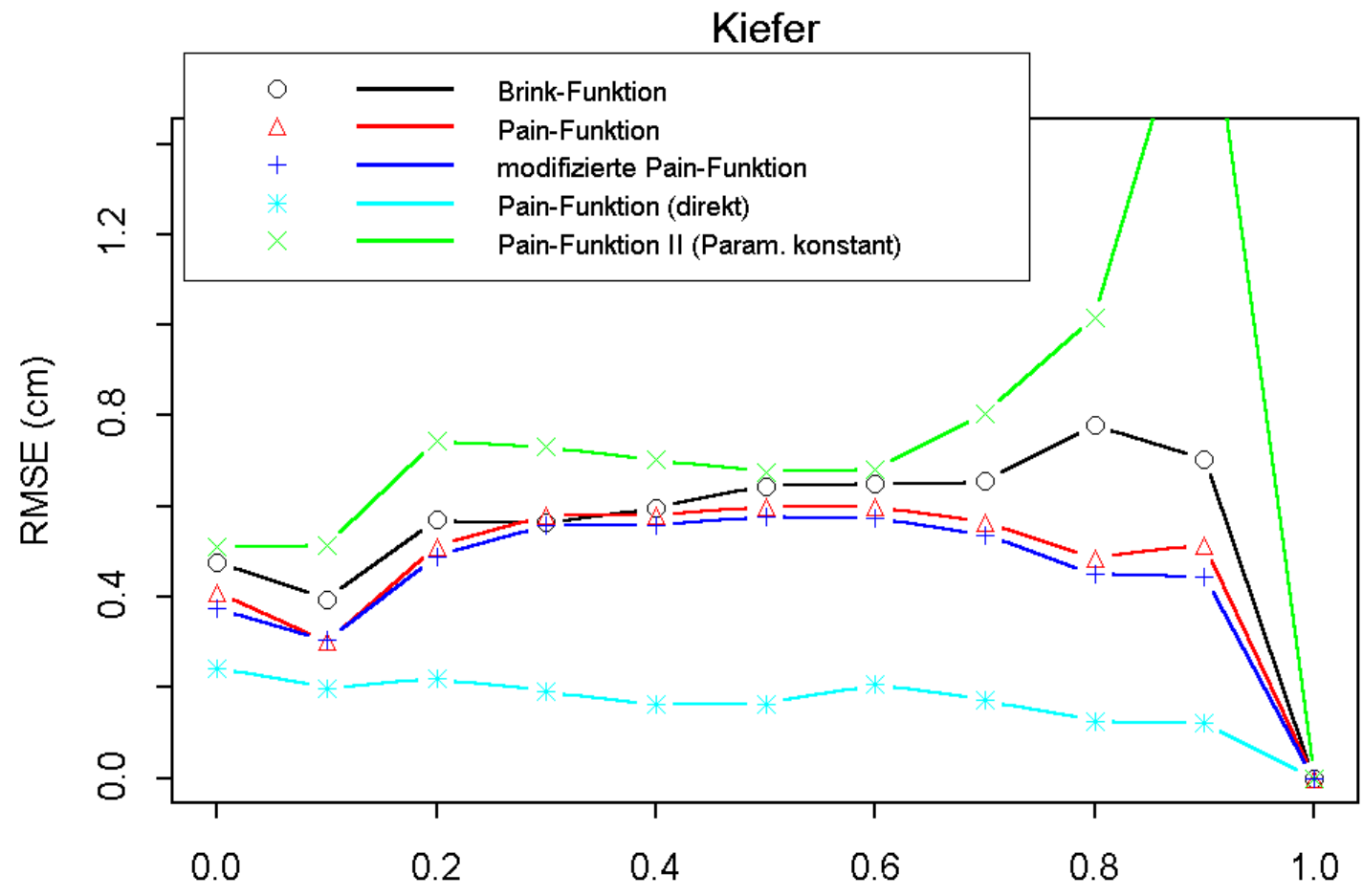

rel. Höhe

Abb. 130: Verläufe des Fehlers (RMSE) über relativen Schafthöhen für unterschiedliche Schaftformmodelle.

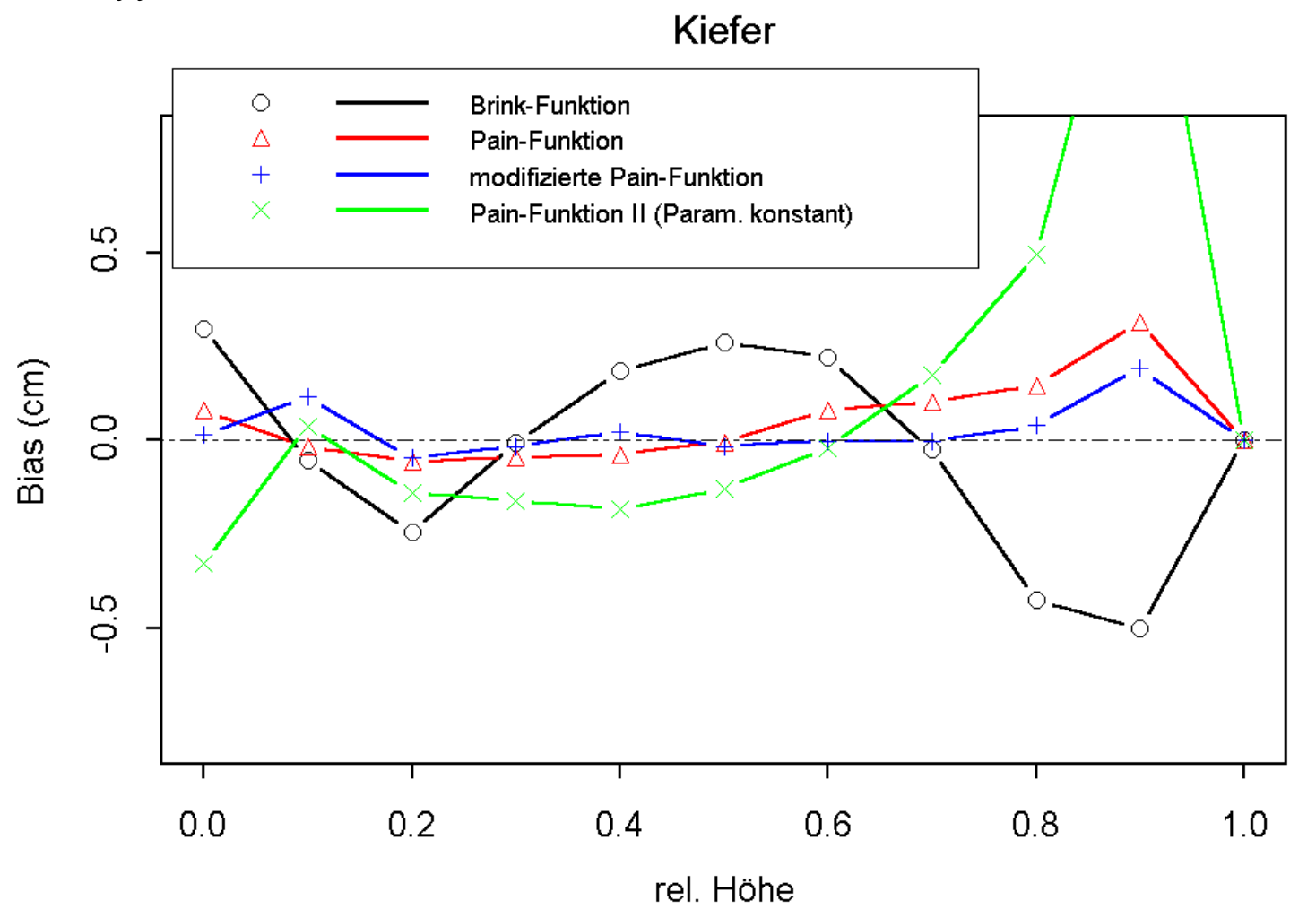

Abb. 131: Verläufe des Bias über relativen Schafthöhen für unterschiedliche Schaftformmodelle. 


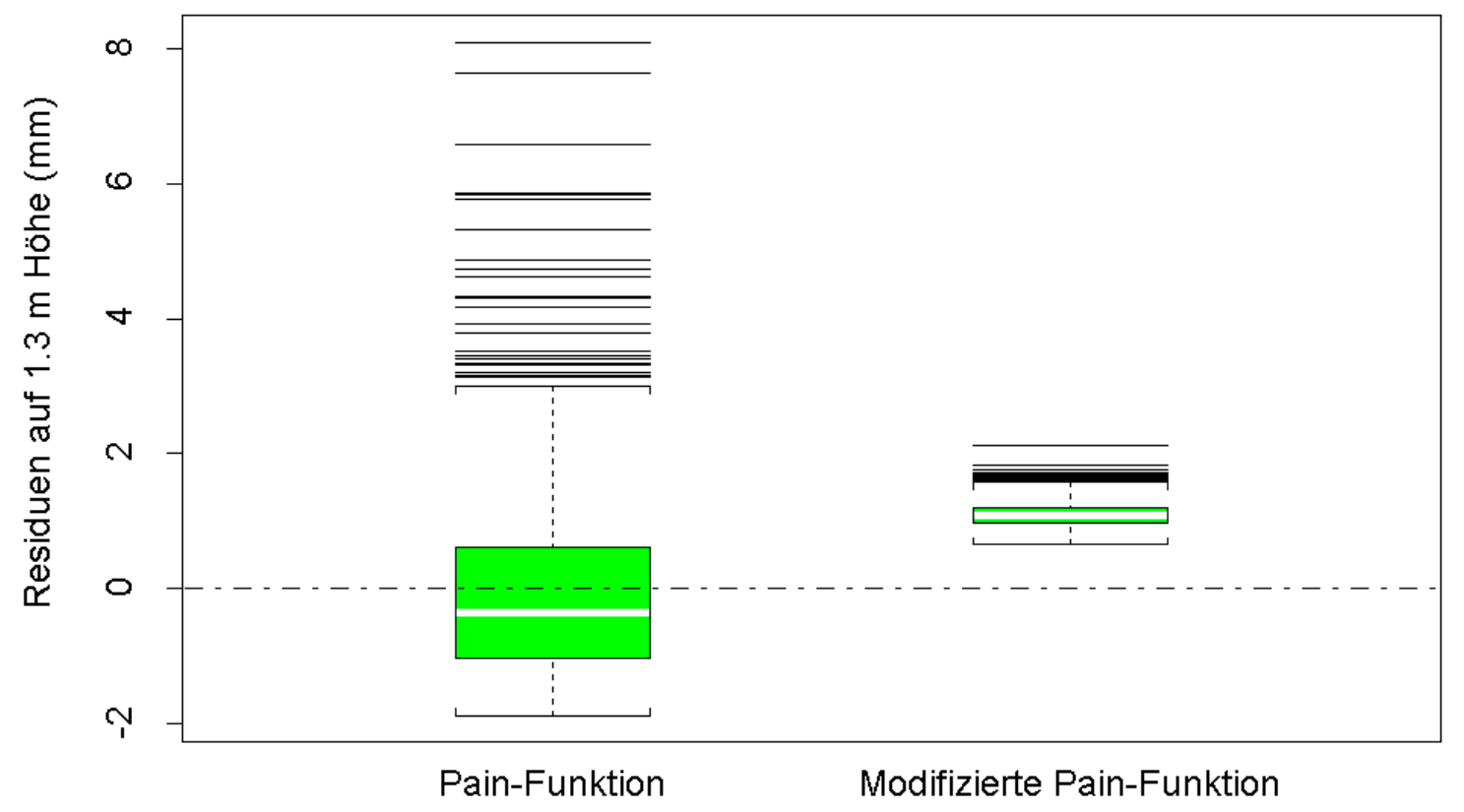

Abb. 132: Box-Plots der Residuen auf 1.3 m Höhe der Pain-Funktion und der modifizierten Pain-Funktion für die Baumart Kiefer.

\begin{tabular}{|c|c|c|c|}
\hline Koeffizient & Wert & Std. Fehler & t-Wert \\
\hline Unterer Schaftteil $a_{0}$ & 1.9246100 & 0.03173830 & 60.64010 \\
\hline Unterer Schaftteil $a_{1}$ & -0.0635714 & 0.00662266 & -9.59908 \\
\hline Unterer Schaftteil $a_{2}$ & 0.2056370 & 0.02728310 & 7.53717 \\
\hline Unterer Schaftteil $b_{0}$ & -0.1870630 & 0.02273610 & -8.22759 \\
\hline Unterer Schaftteil $b_{1}$ & 0.1052080 & 0.00586434 & 17.94020 \\
\hline Unterer Schaftteil $b_{2}$ & -0.0777224 & 0.02438490 & -3.18732 \\
\hline Oberer Schaftteil $a_{0}$ & 2.1020800 & 0.05026580 & 41.81940 \\
\hline Oberer Schaftteil $a_{1}$ & -0.0367694 & 0.00891831 & -4.12291 \\
\hline Oberer Schaftteil $a_{2}$ & 0.1686570 & 0.03090550 & 5.45718 \\
\hline Oberer Schaftteil $b_{0}$ & 1.1679200 & 0.20762000 & 5.62526 \\
\hline Oberer Schaftteil $b_{1}$ & -0.0825875 & 0.04581160 & -1.80277 \\
\hline Oberer Schaftteil $b_{2}$ & 1.3003900 & 0.17232400 & 7.54620 \\
\hline \multicolumn{2}{|c|}{ Residual Std. Fehler (mm) } & \multicolumn{2}{|c|}{0.4612912 bei 10716 Freiheitsgraden } \\
\hline
\end{tabular}

Tab. 86: Koeffizienten und statistische Kenngrößen zur Schaftdurchmesserschätzung mit Hilfe des in Formel (38 a und b) dargestellten Schaftformmodells (modifizierte PainFunktion) für die Baumart Kiefer. 


\section{Buche:}
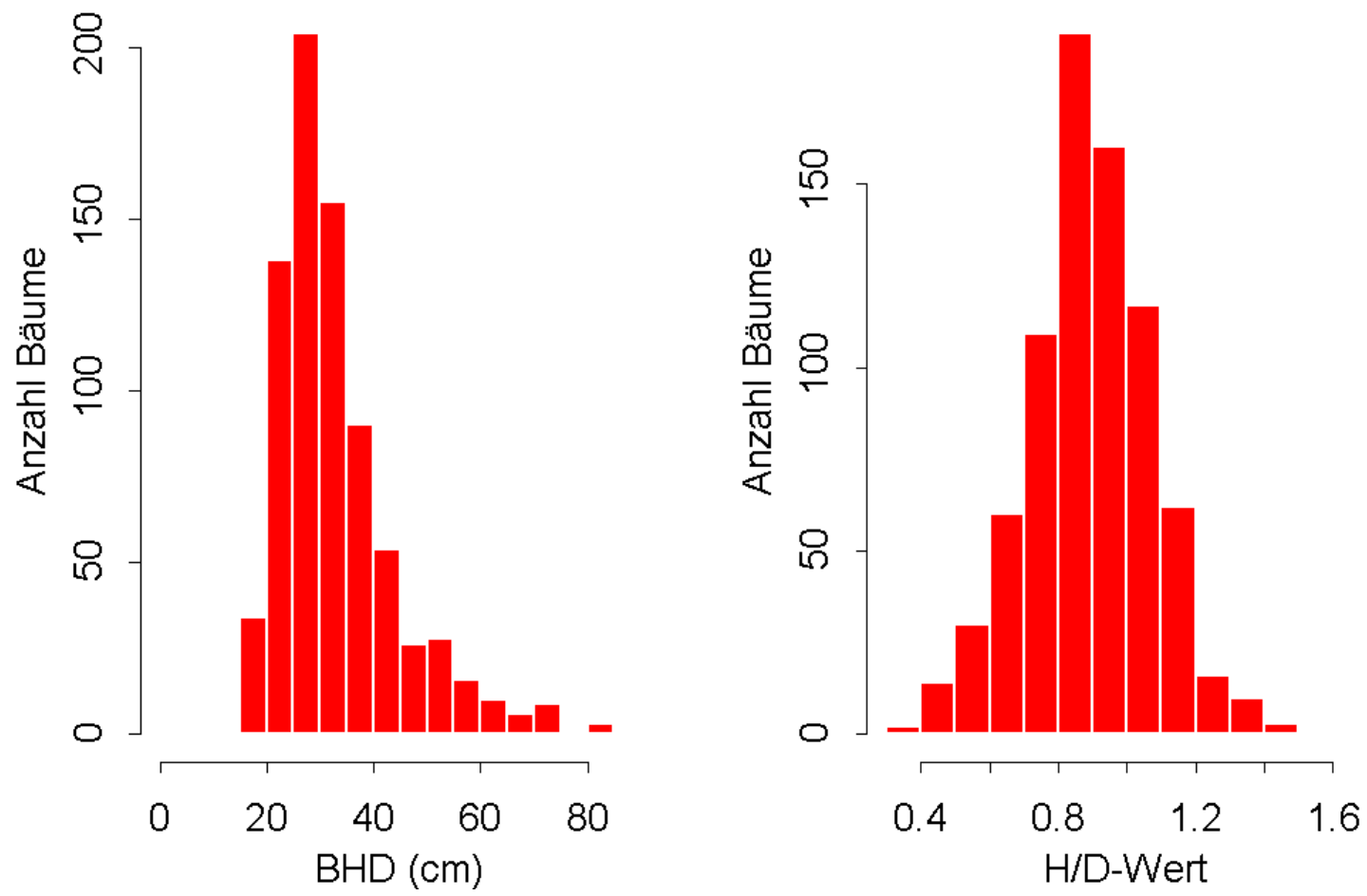

Abb. 133: BHD- und H/D-Wert-Verteilungen für 774 Buchen, die zur Entwicklung eines Einheitsschaftformmodells verwendet wurden.

\section{Buche}

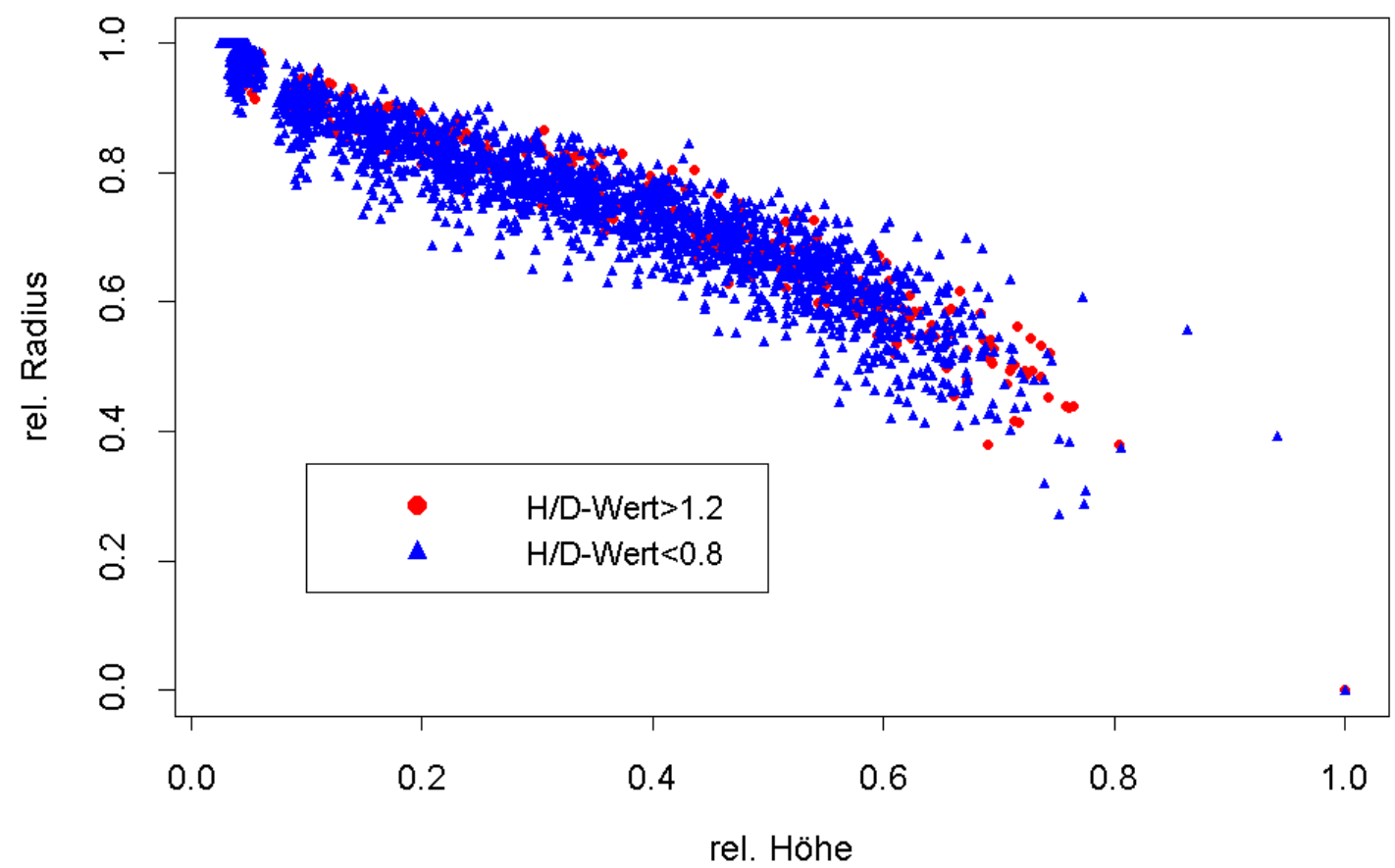

Abb. 134: Relativierte Sektionsmessungen für die Teilkollektive von Buchen mit einem H/DWert von über 1.2 bzw. unter 0.8 zur Darstellung des Formenspektrums und des Einflusses der Variablen H/D-Wert. 


\section{Buche}

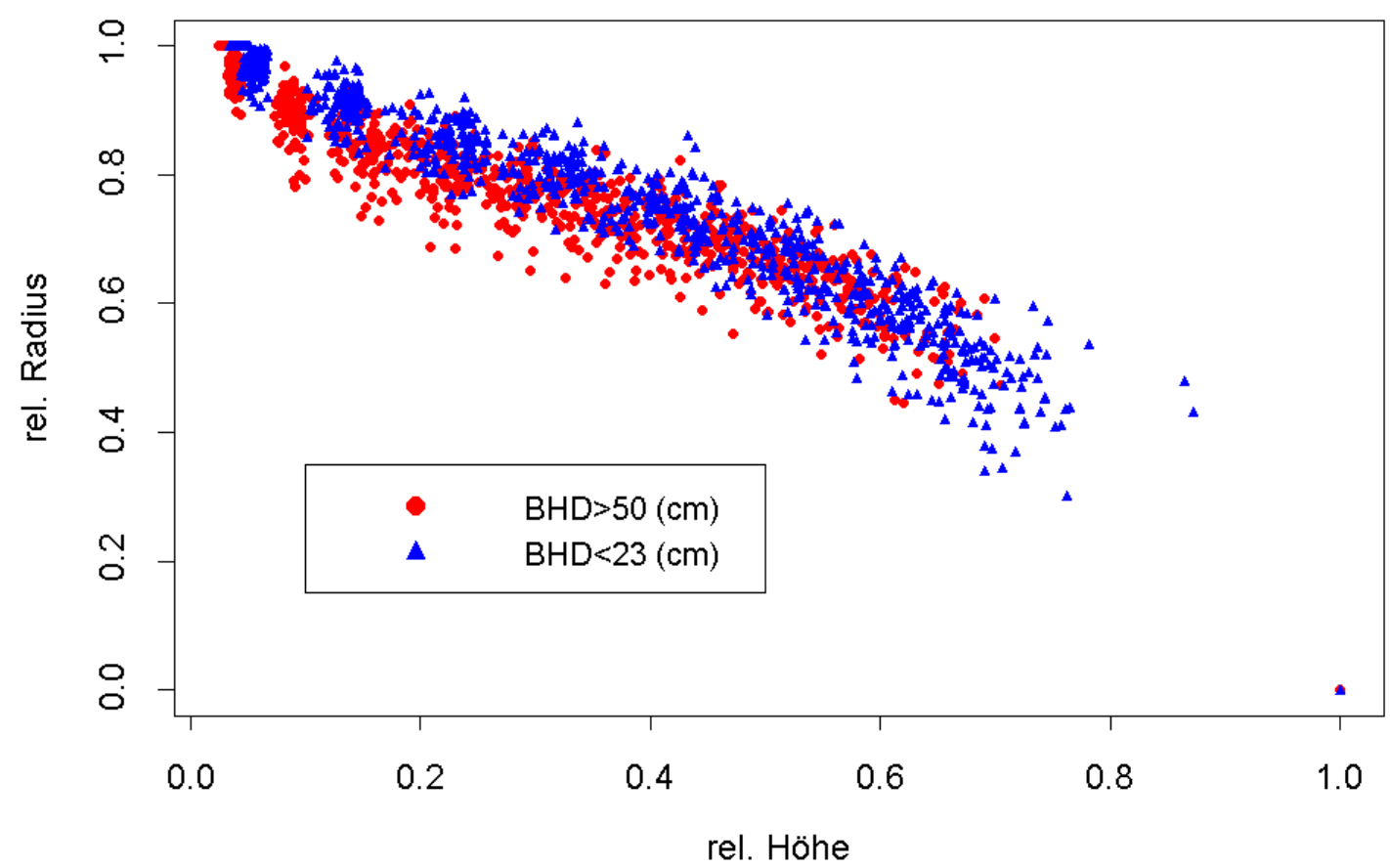

Abb. 135: Relativierte Sektionsmessungen für die Teilkollektive von Buchen mit einem BHD von über $50 \mathrm{~cm}$ bzw. unter $23 \mathrm{~cm}$ zur Darstellung des Formenspektrums und des Einflusses der Variablen BHD.

\begin{tabular}{|c|c|c|c|c|}
\hline Koeffizient & Wert & Std. Fehler & t-Wert & $\operatorname{Pr}(>|t|)$ \\
\hline$a_{0}$ & -2.5902 & 0.0385 & -67.2870 & 0.0000 \\
\hline$a_{1}$ & 1.7416 & 0.0092 & 188.2916 & 0.0000 \\
\hline$a_{2}$ & -1.7516 & 0.0458 & -38.2321 & 0.0000 \\
\hline \multicolumn{5}{|l|}{$b_{0}$ n. signifikant } \\
\hline$b_{1}$ & -0.1248 & 0.0033 & -37.8255 & 0.0000 \\
\hline$b_{2}$ & -0.1749 & 0.0208 & -8.3913 & 0.0000 \\
\hline \multicolumn{2}{|c|}{ Residual Std. Fehler (mm) } & \multicolumn{3}{|c|}{0.6656 bei 9036 Freiheitsgraden } \\
\hline \multicolumn{2}{|c|}{ Multiples Bestimmtheitsmaß } & \multicolumn{3}{|r|}{0.9978} \\
\hline & F-Statistik & \multicolumn{3}{|c|}{808600 bei 5 und 9036 Freiheitsgraden, $p$-Wert $=0$} \\
\hline
\end{tabular}

Tab. 87: Koeffizienten und statistische Kenngrößen zur Schaftdurchmesserschätzung mit Hilfe des Gesamtmodells der Pain-Funktion (33) bzw. (34) und (35) für die Baumart Buche.

\begin{tabular}{|c|c|c|c|}
\hline Koeffizient & Wert & Std. Fehler & t-Wert \\
\hline$k$ & 0.6946140 & 0.00975345 & 71.2173 \\
\hline$p$ & 0.0862735 & 0.00427386 & 20.1863 \\
\hline$q$ & 0.1359840 & 0.00304893 & 44.6007 \\
\hline Residual Std. Fehler & \multicolumn{3}{|c|}{0.683244 bei 6331 Freiheitsgraden } \\
\hline
\end{tabular}

Tab. 88: Koeffizienten und statistische Kenngrößen zur Schaftdurchmesserschätzung mit Hilfe der modifizierten Brink-Funktion (37) für die Baumart Buche. 


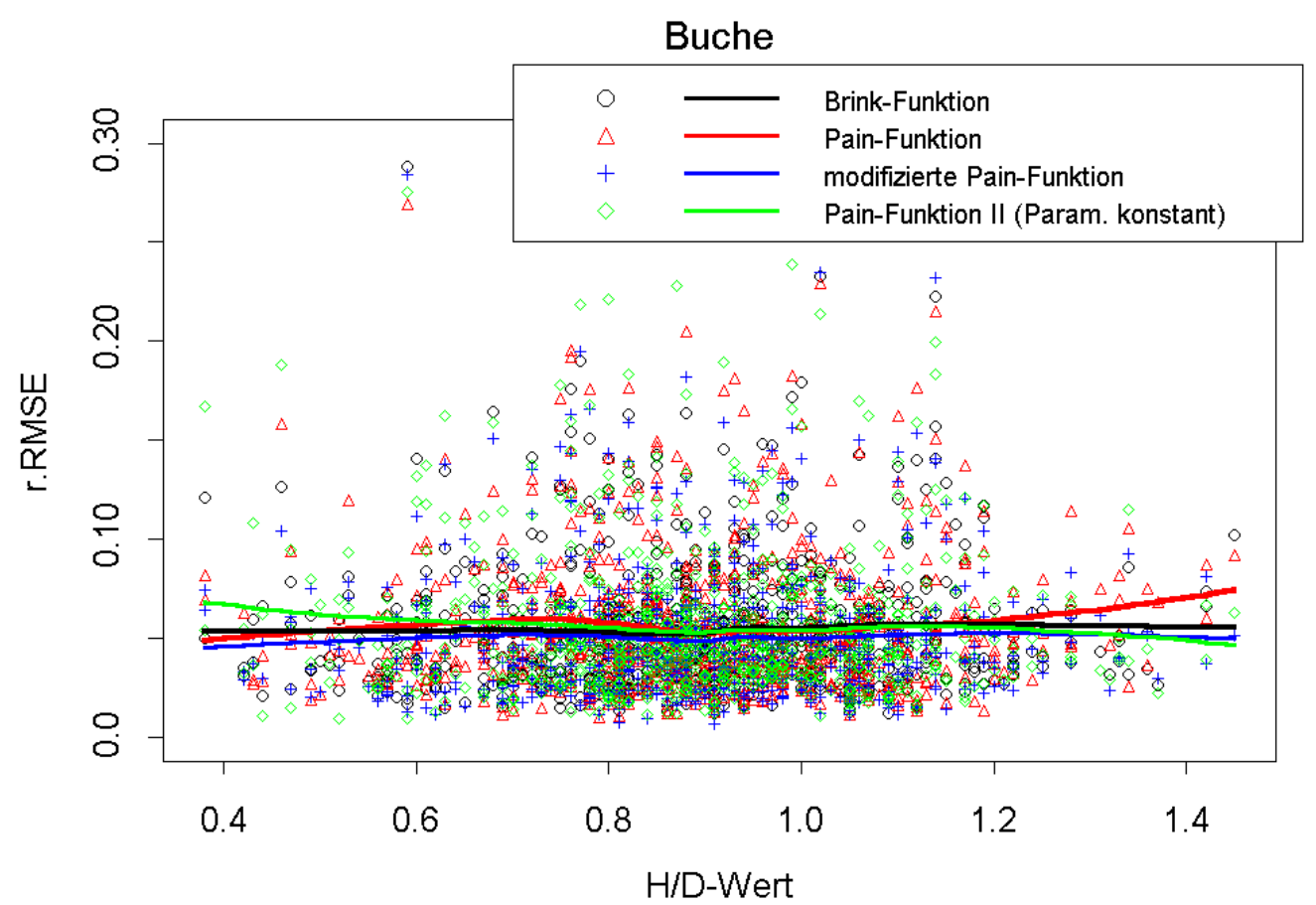

Abb. 136: Einzelbaumweise berechnete relative RMSE über den zugehörigen $H / D$-Werten der Einzelbäume für die Pain-Funktion (flexibles Schaftformmodell) sowie für die modifizierte Brink-Funktion und die Pain-Funktion II (konstante Parameter) (mittleres Einzelbaummodell). Der Ausgleich der Fehlerwerte erfolgte wie auch in Abb. 137 mit einem Gleitenden-Durchschnitts-Modell (Kapitel 8.1). Weiterhin sind die relativen RMSE der in Kapitel 3.7.3 beschriebenen Modifikation der Pain-Funktion dargestellt.

\section{Buche}

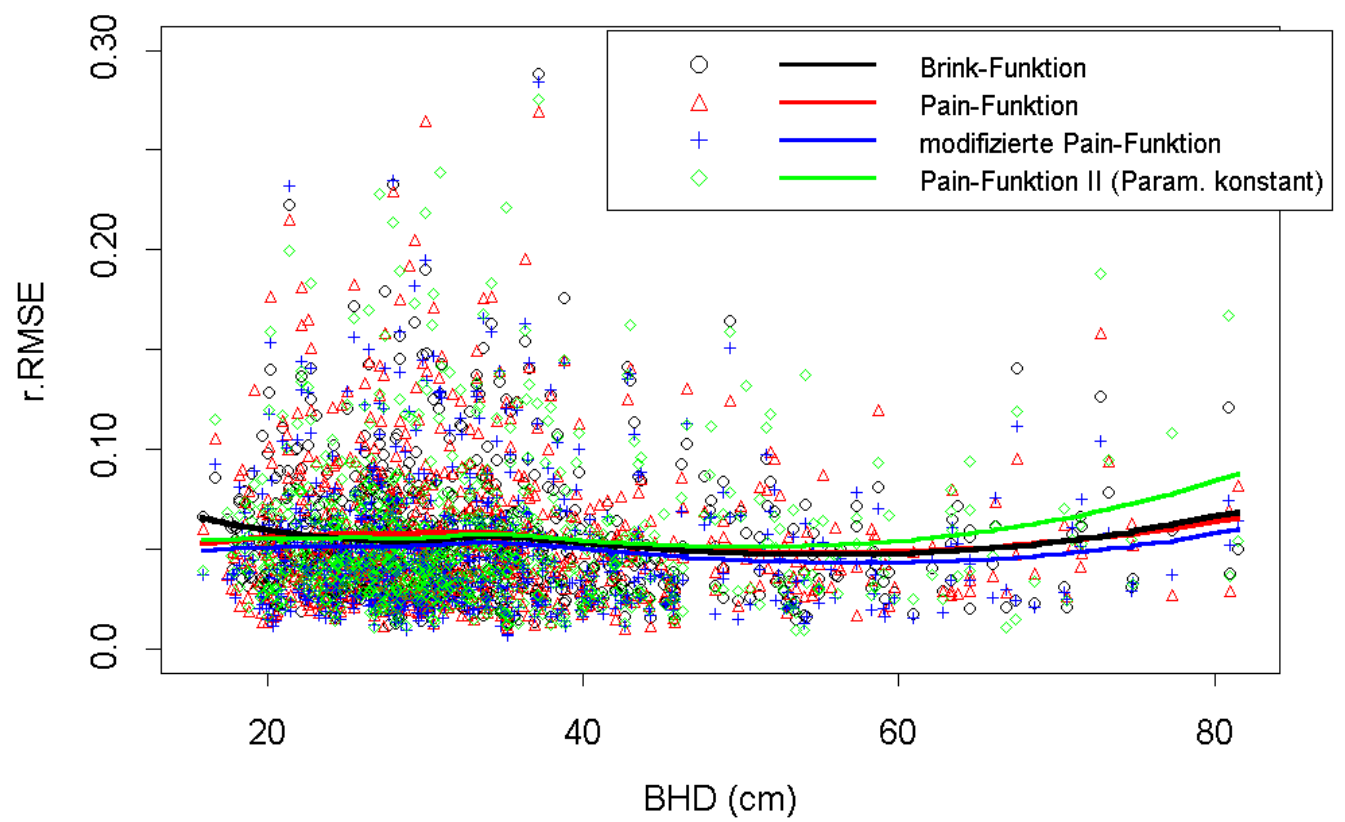

Abb. 137: Einzelbaumweise berechnete relative RMSE über den zugehörigen BHD-Werten der Einzelbäume für die Pain-Funktion (flexibles Schaftformmodell) sowie für die modifizierte Brink-Funktion und die Pain-Funktion II (konstante Parameter) (mittleres Einzelbaummodell). Weiterhin sind die relativen RMSE der in Kapitel 3.7.3 beschriebenen Modifikation der Pain-Funktion dargestellt. 


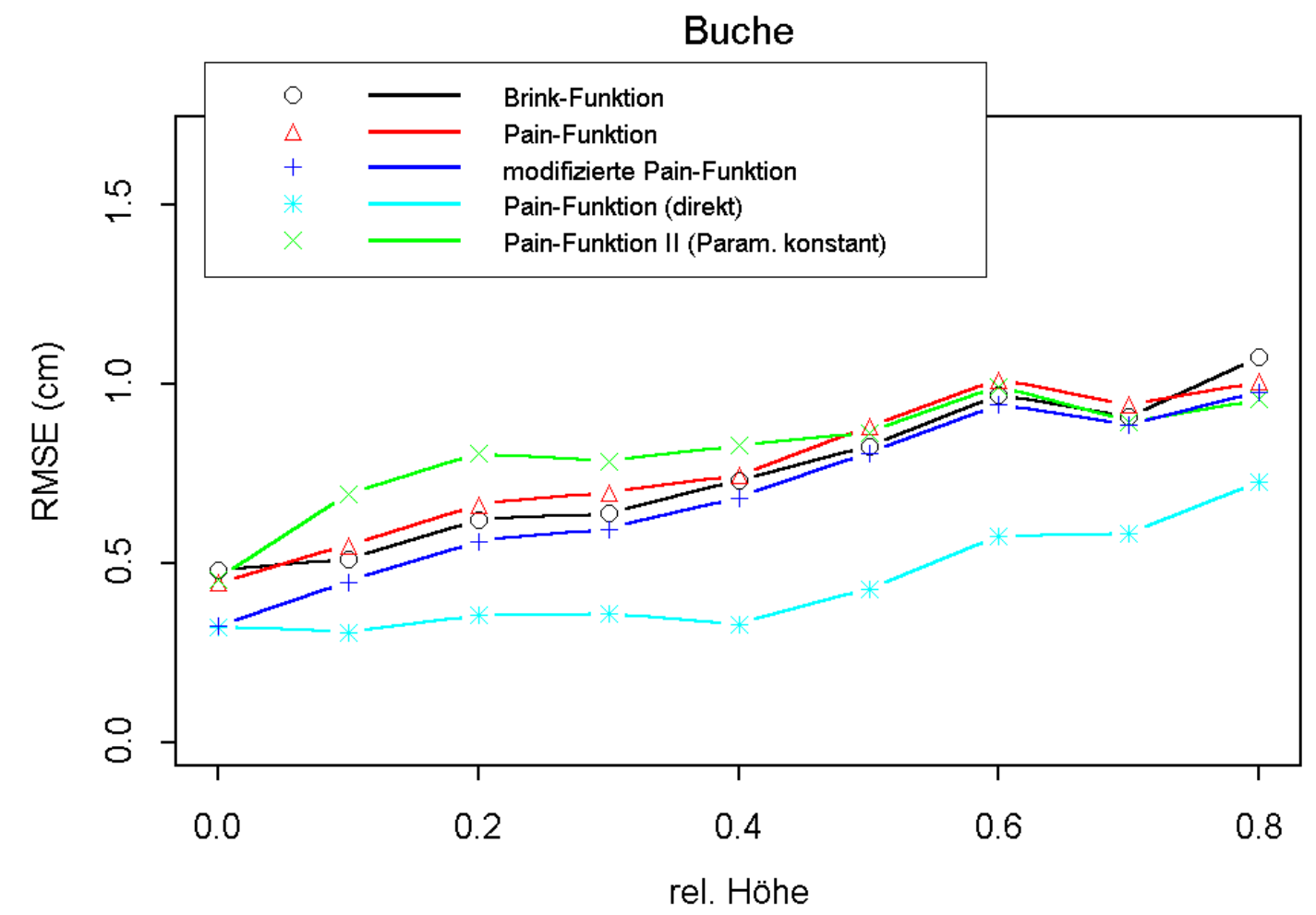

Abb. 138: Verläufe des Fehlers (RMSE) über relativen Schafthöhen für unterschiedliche Schaftformmodelle.

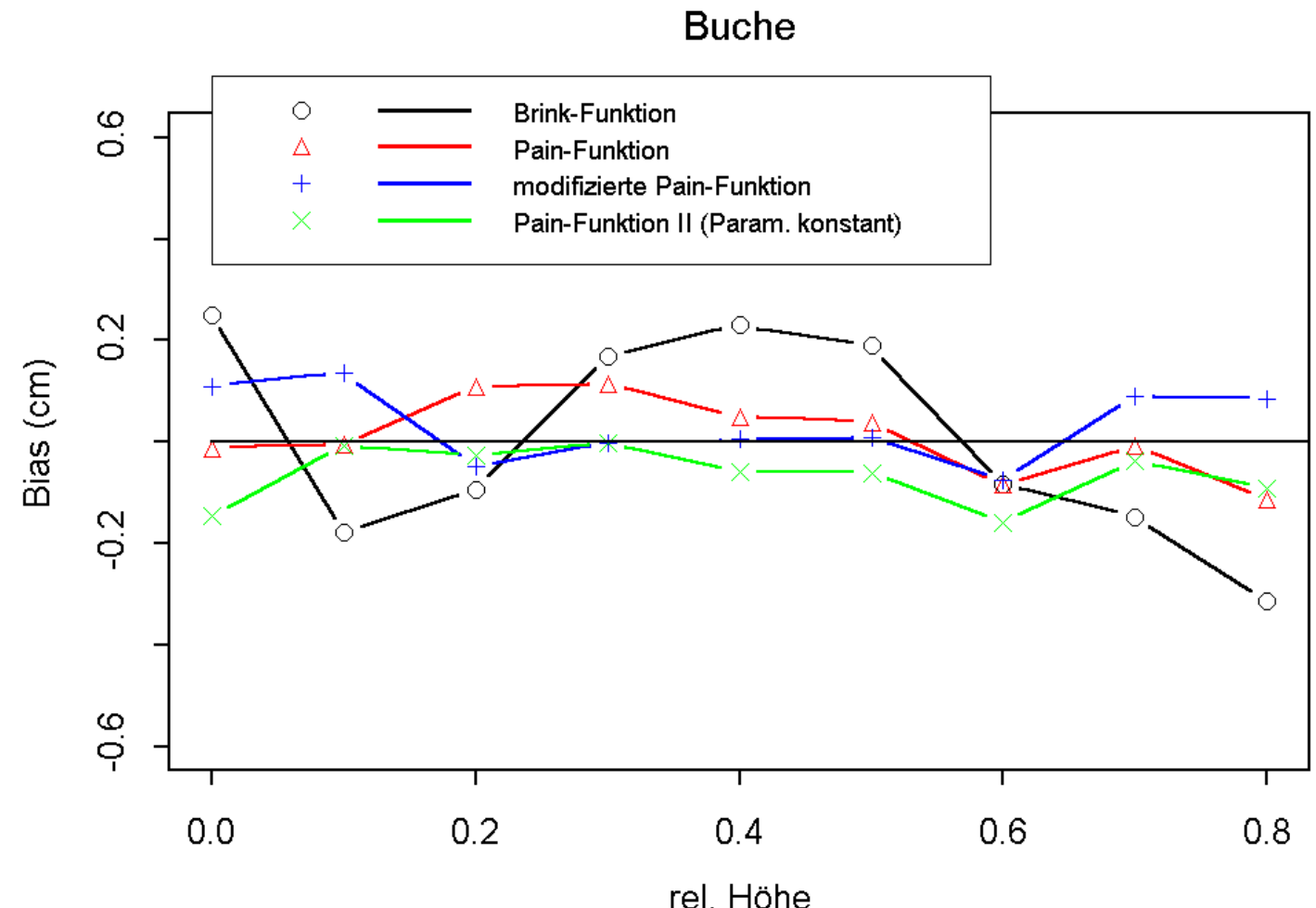

Abb. 139: Verläufe des Bias über relativen Schafthöhen für unterschiedliche Schaftformmodelle. 


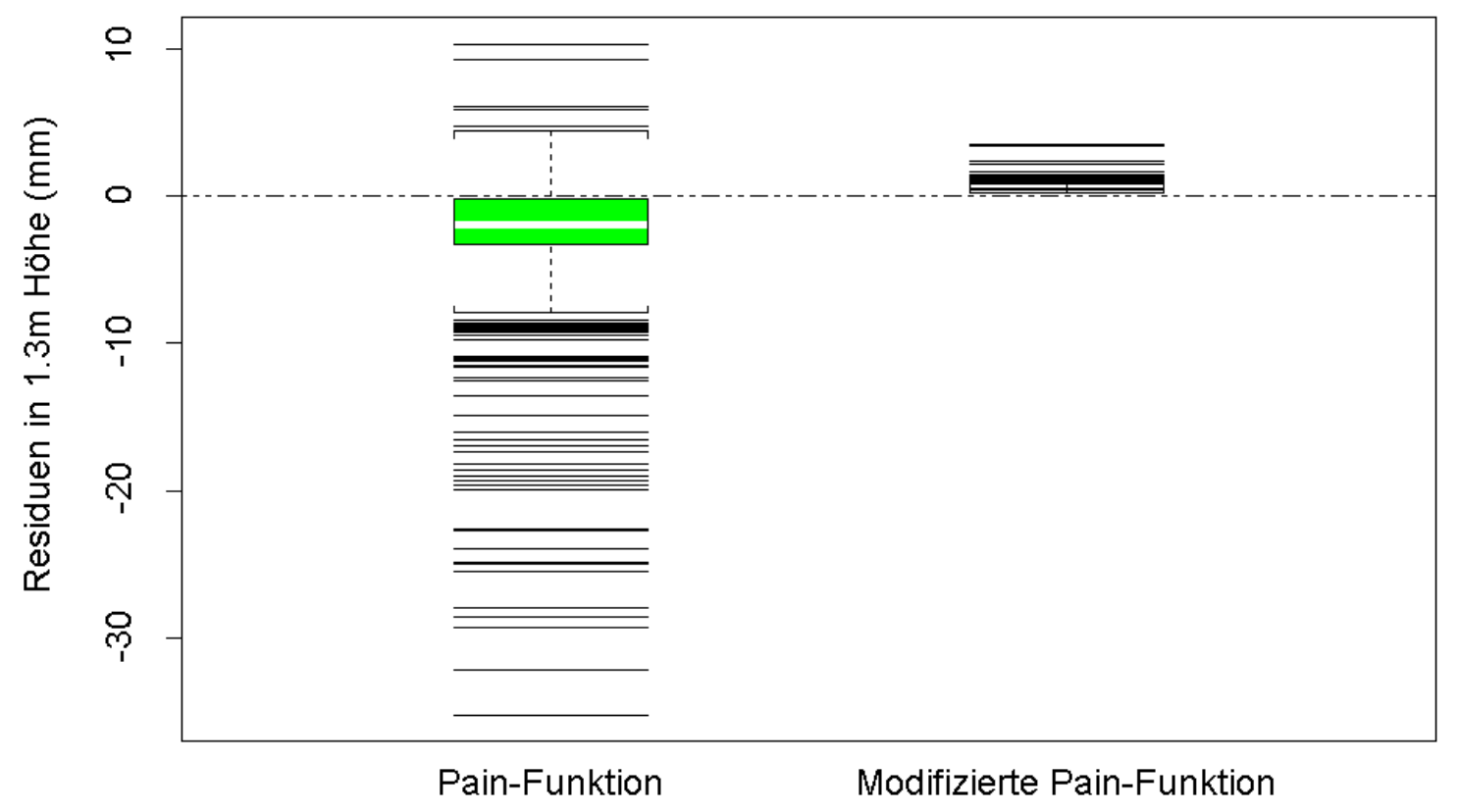

Abb. 140: Box-Plots der Residuen auf 1.3 m Höhe der Pain-Funktion und der modifizierten Pain-Funktion für die Baumart Buche.

\begin{tabular}{|c|c|c|c|}
\hline Koeffizient & Wert & Std. Fehler & $\mathrm{t}$-Wert \\
\hline Unterer Schaftteil $a_{0}$ & 1.9166800 & 0.07239910 & 26.473800 \\
\hline Unterer Schaftteil $a_{1}$ & 0.0081528 & 0.00983102 & 0.829294 \\
\hline Unterer Schaftteil $a_{2}$ & -0.0687322 & 0.02807070 & -2.448540 \\
\hline Unterer Schaftteil $b_{0}$ & -0.1295680 & 0.04152680 & -3.120100 \\
\hline Unterer Schaftteil $b_{1}$ & 0.0580784 & 0.00695798 & 8.347020 \\
\hline Unterer Schaftteil $b_{2}$ & 0.0539410 & 0.02526760 & 2.134790 \\
\hline Oberer Schaftteil $a_{0}$ & 1.7300700 & 0.0738141 & 23.4382000 \\
\hline Oberer Schaftteil $a_{1}$ & 0.0322571 & 0.0128356 & 2.5130900 \\
\hline Oberer Schaftteil $a_{2}$ & -0.0437421 & 0.0498922 & -0.8767320 \\
\hline Oberer Schaftteil $b_{0}$ & 0.0276928 & 0.3499050 & 0.0791437 \\
\hline Oberer Schaftteil $b_{1}$ & 0.2637900 & 0.0675473 & 3.9052600 \\
\hline Oberer Schaftteil $b_{2}$ & -0.2228300 & 0.2747220 & -0.8111110 \\
\hline \multicolumn{2}{|c|}{ Residual Std. Fehler (mm) } & \multicolumn{2}{|c|}{0.6232363 bei 9029 Freiheitsgraden } \\
\hline
\end{tabular}

Tab. 89: Koeffizienten und statistische Kenngrößen zur Schaftdurchmesserschätzung mit Hilfe des in Formel (38 a und b) dargestellten Schaftformmodells (modifizierte PainFunktion) für die Baumart Buche. 


\section{Eiche:}
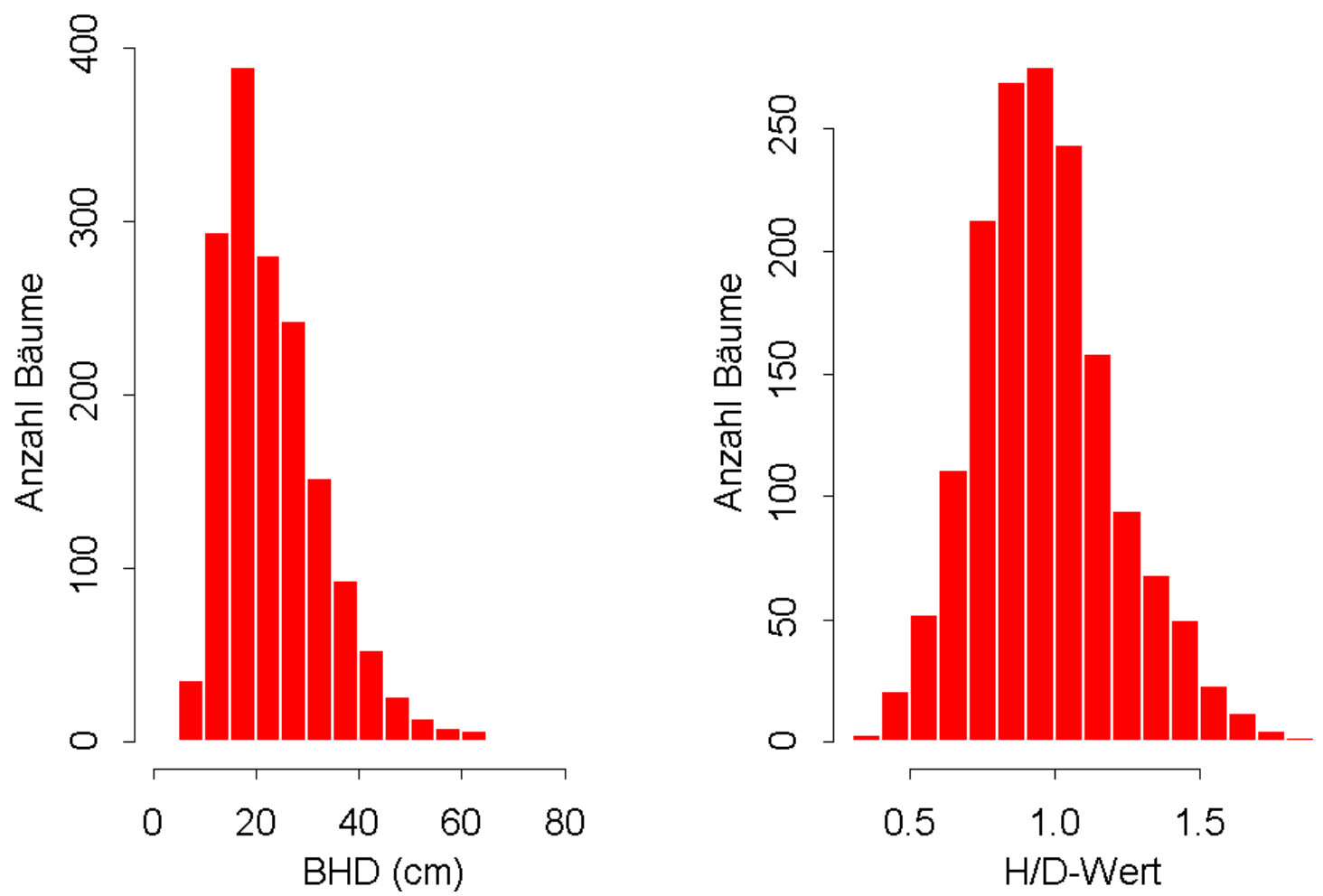

Abb. 141: BHD- und H/D-Wert-Verteilungen für 1599 Eichen, die zur Entwicklung eines Einheitsschaftformmodells verwendet wurden.

\section{Eiche}

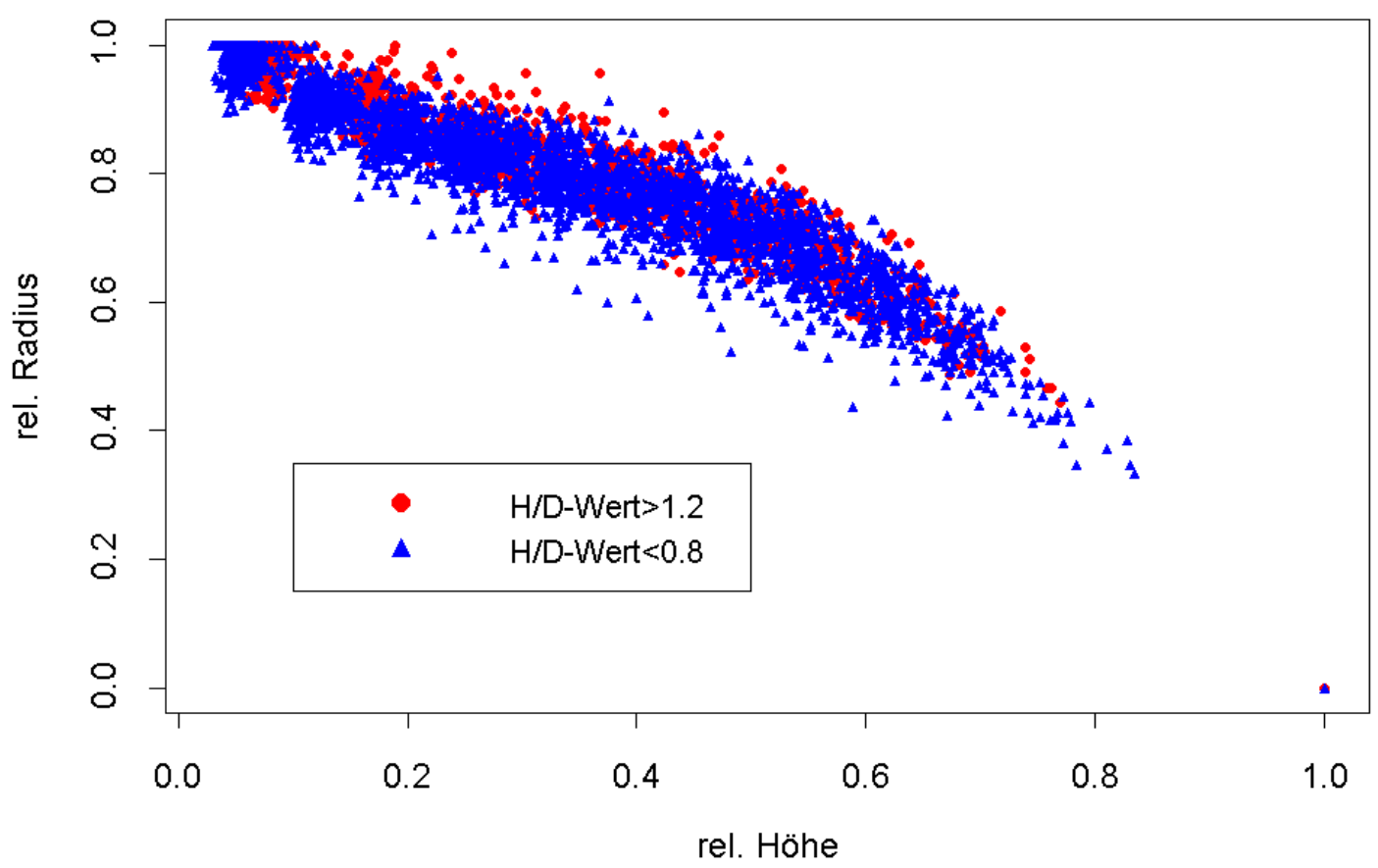

Abb. 142: Relativierte Sektionsmessungen für die Teilkollektive von Eichen mit einem H/DWert von über 1.2 bzw. unter 0.8 zur Darstellung des Formenspektrums und des Einflusses der Variablen H/D-Wert. 


\section{Eiche}

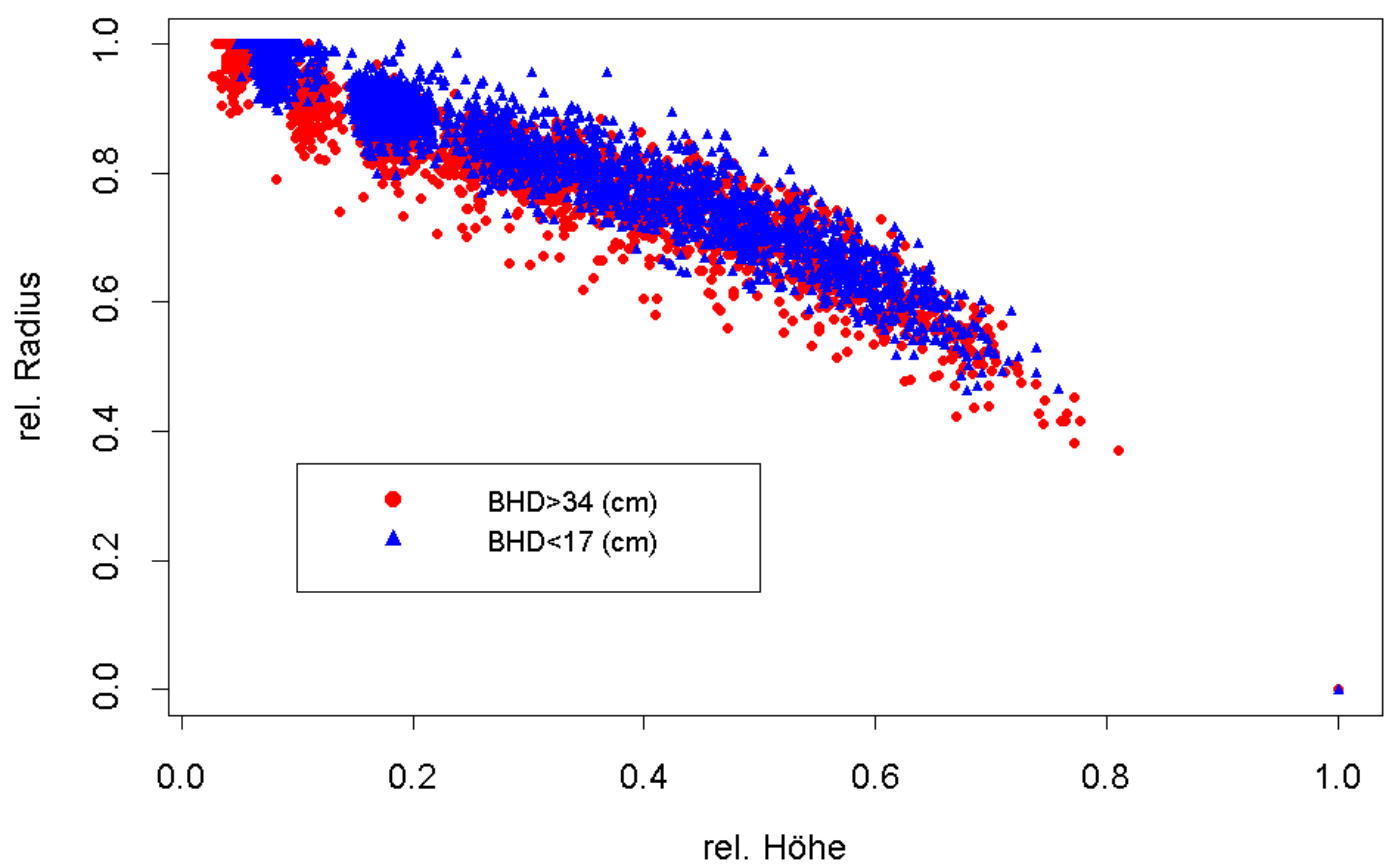

Abb. 143: Relativierte Sektionsmessungen für die Teilkollektive von Eichen mit einem BHD von über $34 \mathrm{~cm}$ bzw. unter $17 \mathrm{~cm}$ zur Darstellung des Formenspektrums und des Einflusses der Variablen BHD.

\begin{tabular}{|r|r|r|r|r|}
\hline \multicolumn{1}{|c|}{ Koeffizient } & Wert & Std. Fehler & t-Wert & $\operatorname{Pr}(>|\mathrm{t}|)$ \\
\hline$a_{0}$ & -2.0611 & 0.0167 & -123.0709 & 0.0000 \\
\hline$a_{1}$ & 1.7254 & 0.0061 & 283.5864 & 0.0000 \\
\hline$a_{2}$ & -1.6759 & 0.0293 & -57.2877 & 0.0000 \\
\hline$b_{0}$ n. signifikant & & & & 0.0000 \\
\hline$b_{1}$ & -0.0947 & 0.0026 & -36.3773 & 0.0000 \\
\hline$b_{2}$ & -0.2128 & 0.0144 & -14.8244 & 0.0000 \\
\hline \multicolumn{2}{|r|}{ Residual Std. Fehler (mm) } & \multicolumn{3}{c|}{0.4763 bei 14068 Freiheitsgraden } \\
\hline \multicolumn{2}{r|r}{ Multiples Bestimmtheitsmaß } & F-Statistik & 1461000 bei 5 und 14068 Freiheitsgraden, $p$-Wert $=0$ \\
\hline
\end{tabular}

Tab. 90: Koeffizienten und statistische Kenngrößen zur Schaftdurchmesserschätzung mit Hilfe des Gesamtmodells der Pain-Funktion (33) bzw. (34) und (35) für die Baumart Eiche.

\begin{tabular}{|c|c|c|c|}
\hline Koeffizient & Wert & Std. Fehler & $\mathrm{t}$-Wert \\
\hline$k$ & 0.5698770 & 0.01181670 & 48.2263 \\
\hline$p$ & 0.0450652 & 0.00354560 & 12.7102 \\
\hline$q$ & 0.2452940 & 0.00724047 & 33.8782 \\
\hline Residual Std. Fehler & \multicolumn{3}{|c|}{0.504138 bei 9421 Freiheitsgraden } \\
\hline
\end{tabular}

Tab. 91: Koeffizienten und statistische Kenngrößen zur Schaftdurchmesserschätzung mit Hilfe der modifizierten Brink-Funktion (37) für die Baumart Eiche. 
Eiche

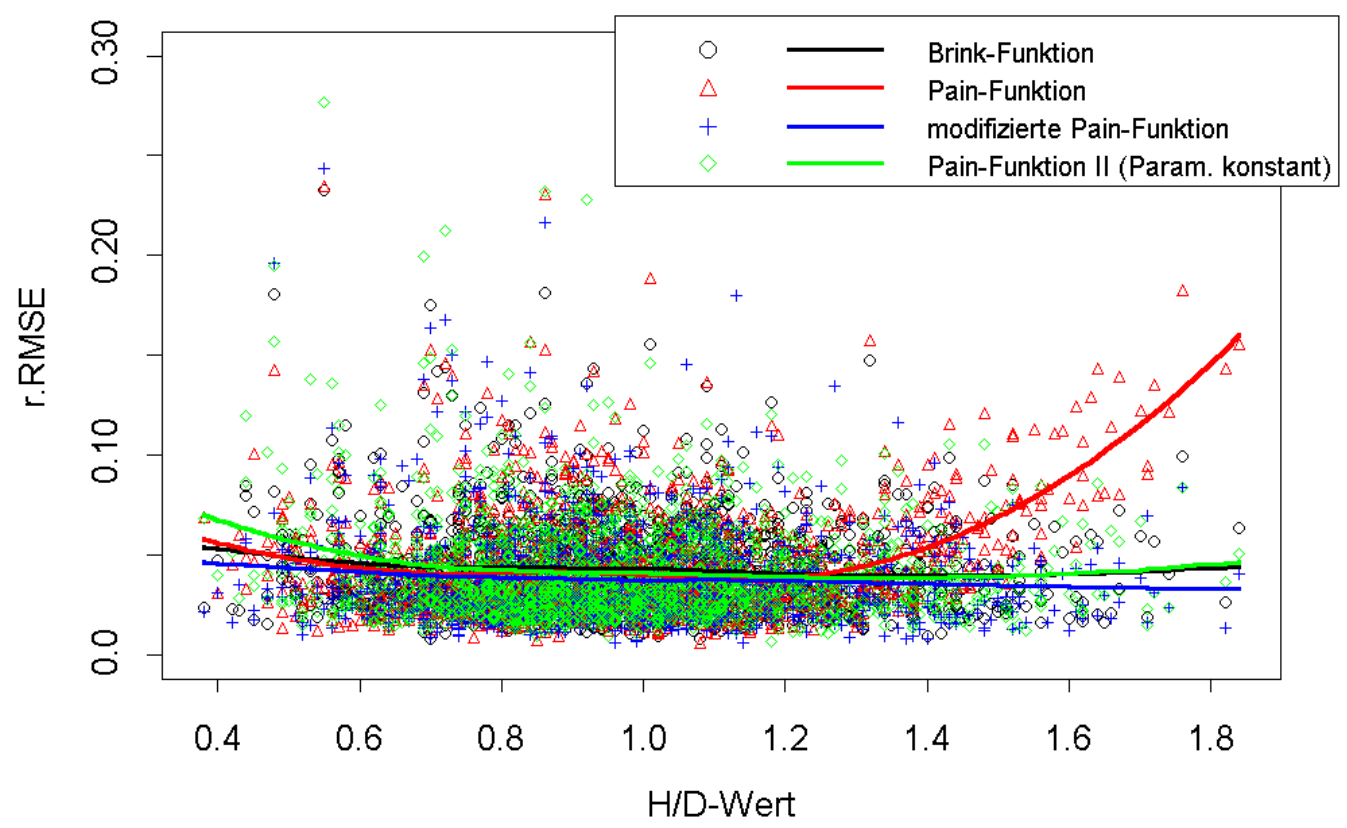

Abb. 144: Einzelbaumweise berechnete relative RMSE über den zugehörigen $H / D$-Werten der Einzelbäume für die Pain-Funktion (flexibles Schaftformmodell) sowie für die modifizierte Brink-Funktion und die Pain-Funktion II (konstante Parameter) (mittleres Einzelbaummodell). Der Ausgleich der Fehlerwerte erfolgte wie auch in Abb. 145 mit einem Gleitenden-Durchschnitts-Modell (Kapitel 8.1). Weiterhin sind die relativen RMSE der in Kapitel 3.7.3 beschriebenen Modifikation der Pain-Funktion dargestellt.

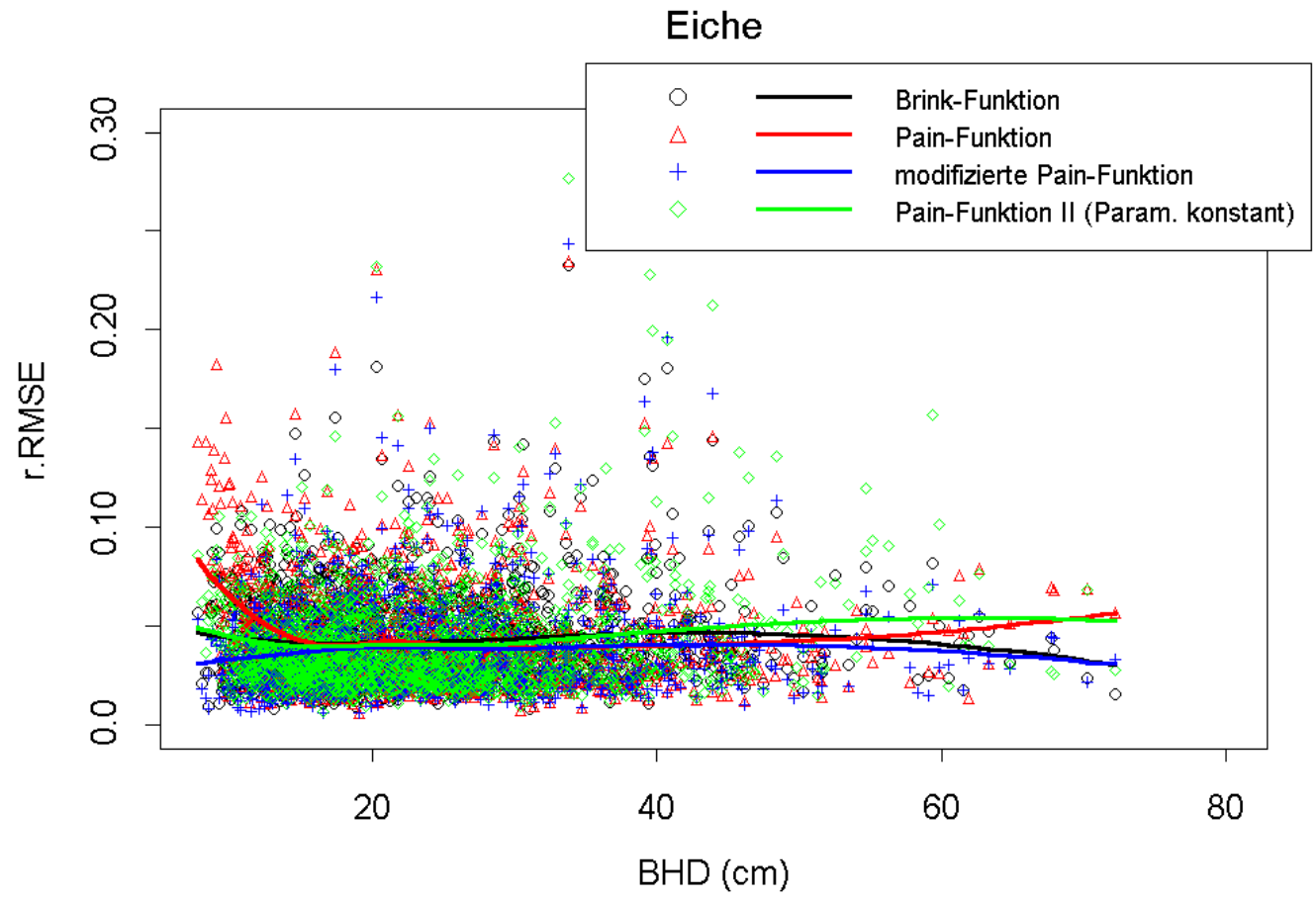

Abb. 145: Einzelbaumweise berechnete relative RMSE über den zugehörigen BHD-Werten der Einzelbäume für die Pain-Funktion (flexibles Schaftformmodell) sowie für die modifizierte Brink-Funktion und die Pain-Funktion II (konstante Parameter) (mittleres Einzelbaummodell). Weiterhin sind die relativen RMSE der in Kapitel 3.7.3 beschriebenen Modifikation der Pain-Funktion dargestellt. 


\section{Eiche}

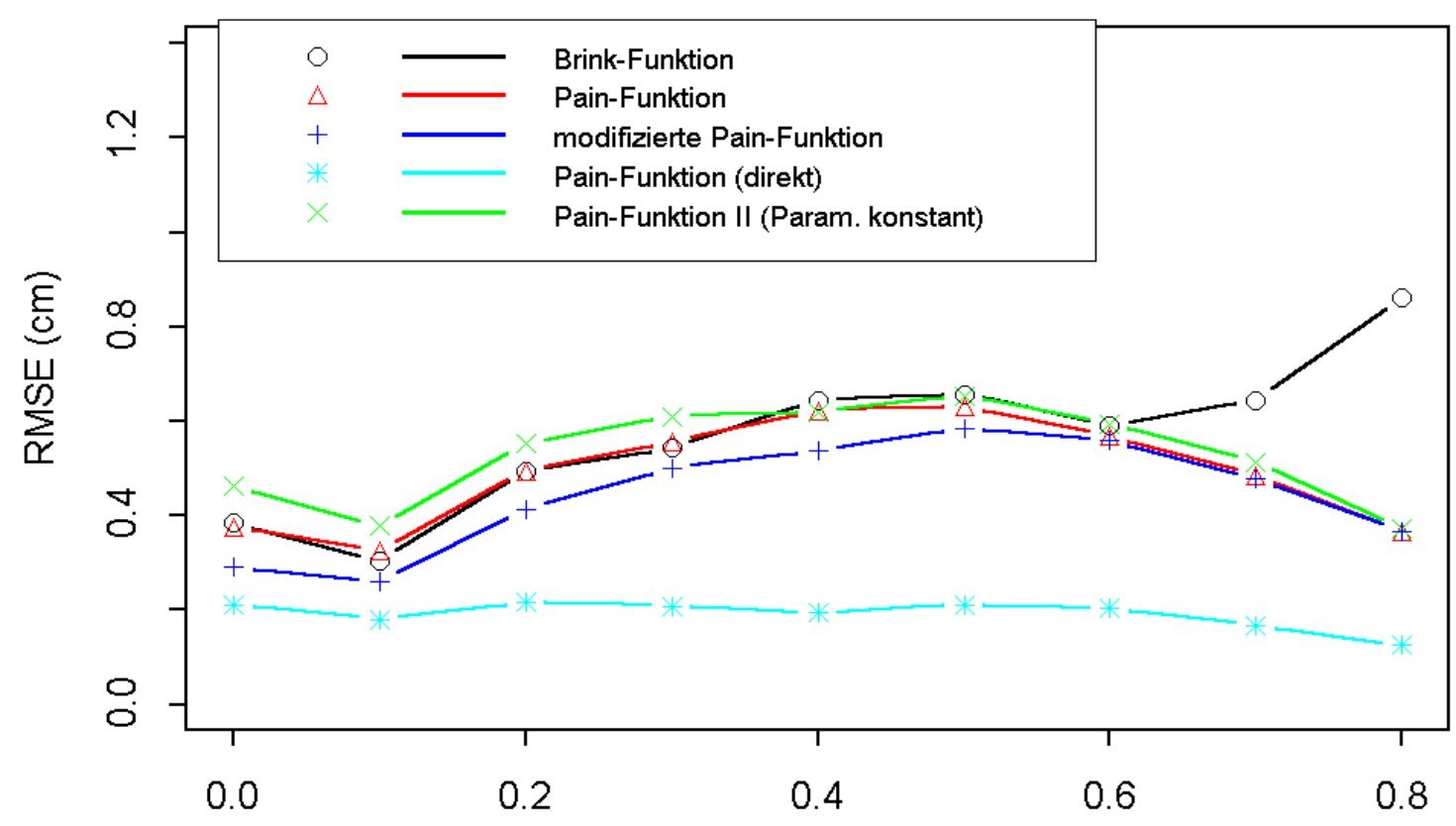

rel. Höhe

Abb. 146: Verläufe des Fehlers (RMSE) über relativen Schafthöhen für unterschiedliche Schaftformmodelle.

\section{Eiche}

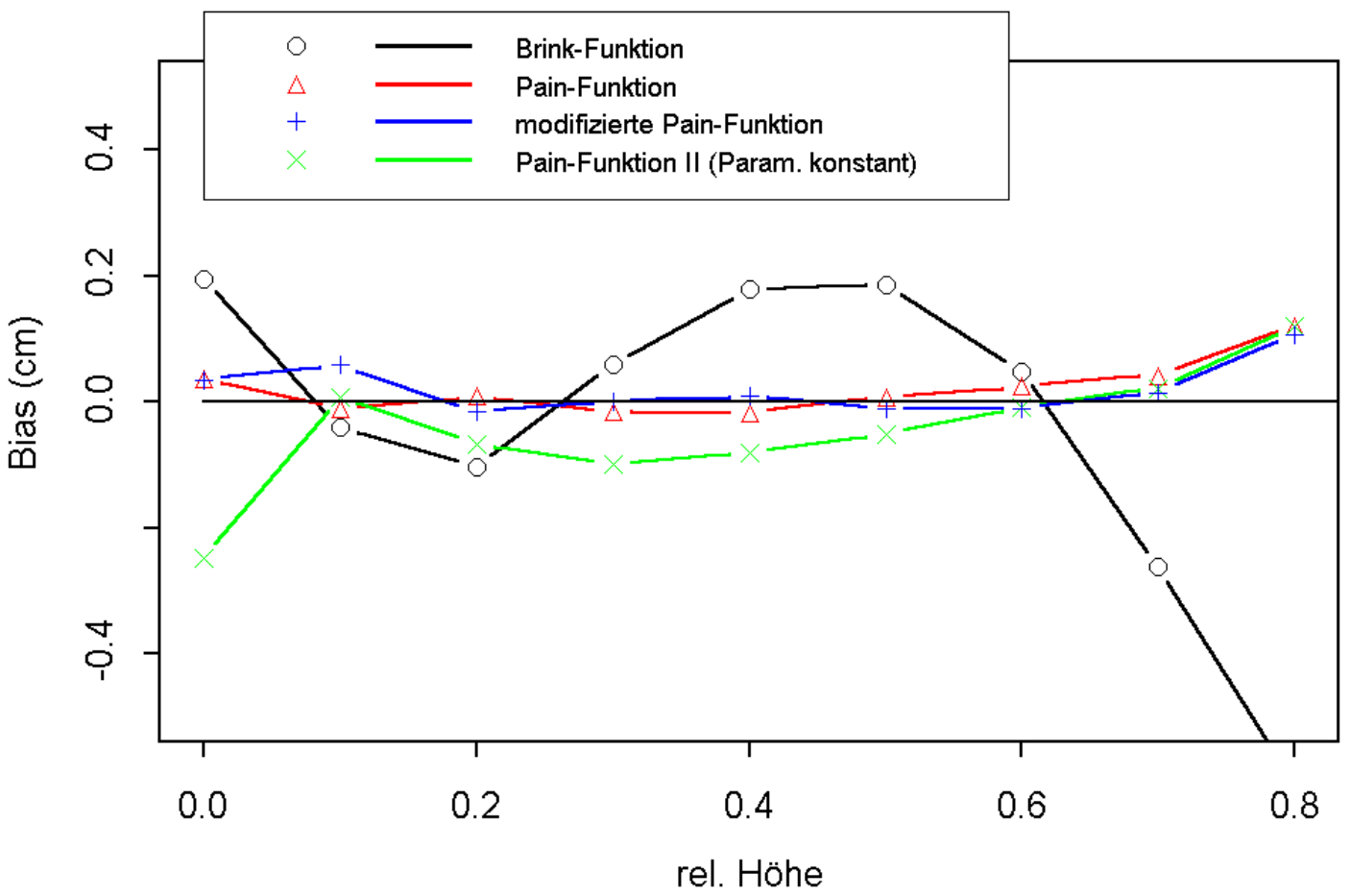

Abb. 147: Verläufe des Bias über relativen Schafthöhen für unterschiedliche Schaftformmodelle. 


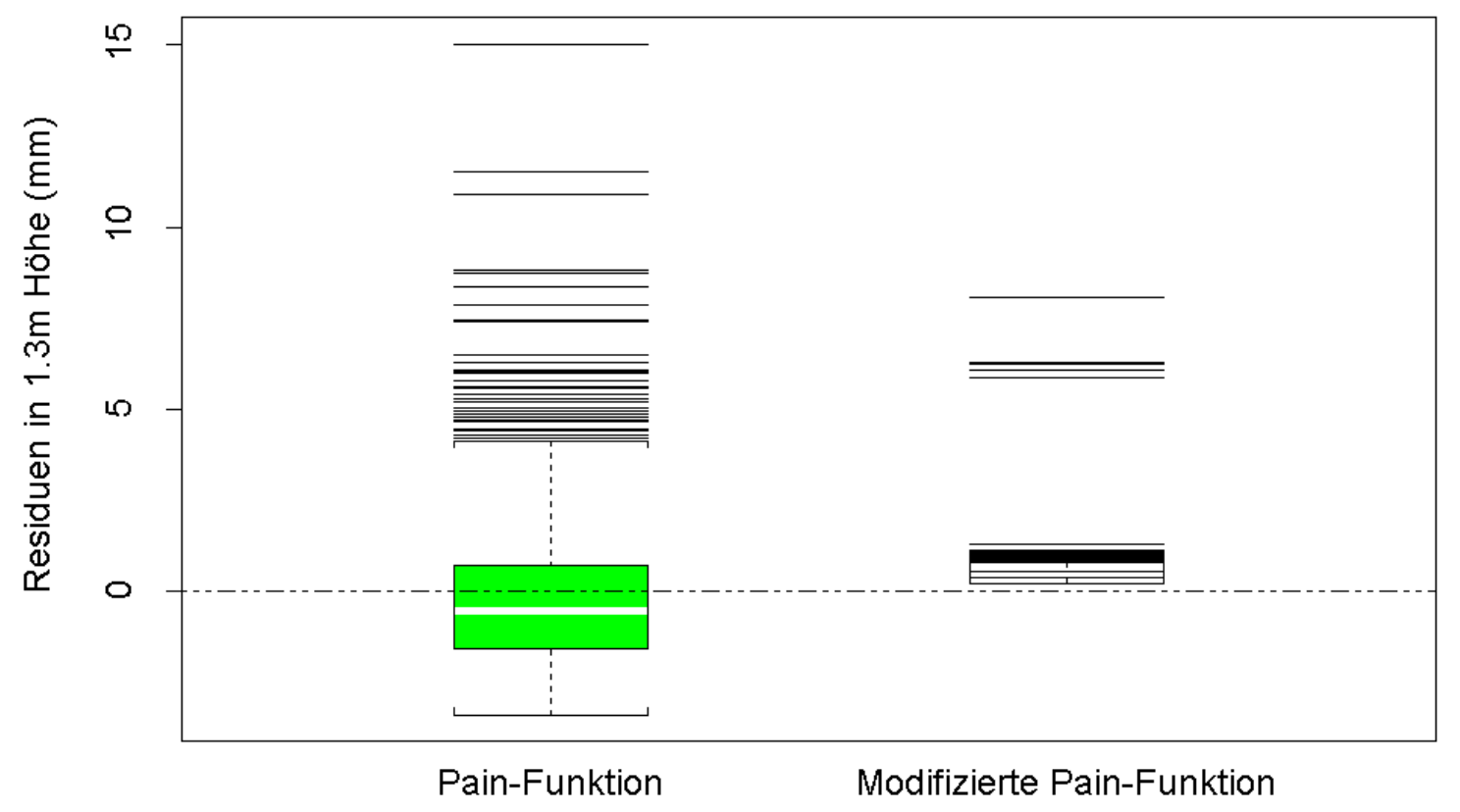

Abb. 148: Box-Plots der Residuen auf 1.3 m Höhe der Pain-Funktion und der modifizierten Pain-Funktion für die Baumart Eiche.

\begin{tabular}{|c|c|c|c|}
\hline Koeffizient & Wert & Std. Fehler & $\mathrm{t}$-Wert \\
\hline Unterer Schaftteil $a_{0}$ & 2.1042300 & 0.04670050 & 45.058100 \\
\hline Unterer Schaftteil $a_{1}$ & -0.0208067 & 0.00852220 & -2.441470 \\
\hline Unterer Schaftteil $a_{2}$ & 0.0340243 & 0.02702050 & 1.259200 \\
\hline Unterer Schaftteil $b_{0}$ & -0.0183653 & 0.02121000 & -0.865877 \\
\hline Unterer Schaftteil $b_{1}$ & 0.0376793 & 0.00497195 & 7.578390 \\
\hline Unterer Schaftteil $b_{2}$ & 0.0802658 & 0.01836060 & 4.371620 \\
\hline Oberer Schaftteil $a_{0}$ & 2.0284900 & 0.04197960 & 48.320800 \\
\hline Oberer Schaftteil $a_{1}$ & 0.0426441 & 0.00766806 & 5.561260 \\
\hline Oberer Schaftteil $a_{2}$ & -0.1810540 & 0.02452650 & -7.381960 \\
\hline Oberer Schaftteil $b_{0}$ & -0.1044610 & 0.11333000 & -0.921744 \\
\hline Oberer Schaftteil $b_{1}$ & 0.2870950 & 0.02863010 & 10.027700 \\
\hline Oberer Schaftteil $b_{2}$ & -0.4369570 & 0.11450500 & -3.816060 \\
\hline \multicolumn{2}{|c|}{ Residual Std. Fehler (mm) } & \multicolumn{2}{|c|}{0.416936 bei 14051 Freiheitsgraden } \\
\hline
\end{tabular}

Tab. 92: Koeffizienten und statistische Kenngrößen zur Schaftdurchmesserschätzung mit Hilfe des in Formel (38 a und b) dargestellten Schaftformmodells (modifizierte PainFunktion) für die Baumart Eiche. 


\title{
L E B E N S L A U F
}

\author{
M A T TH I A S C H M I T
}

Geburtstag: $\quad$ 17. Oktober 1969

Geburtsort: $\quad$ Wunstorf/Luthe

Eltern: $\quad$ Jürgen Schmidt, Dipl. Ingenieur (FH)

Gisela Schütz-Schmidt, Bürokauffrau

Schulabschluß: $\quad$ Abitur 1989, Gymnasium Hindenburgschule, Nienburg/Weser

Grundwehrdienst: 1989-1990, Nienburg/Weser

Berufsausbildung: 1990-1991, Hochschul-Praktikum im ehemaligen Staatlichen Forstamt Hardegsen

1991-1996, Studium der Forstwissenschaften, Georg-August-Universität Göttingen, Abschluß: Diplom-Forstwirt

1994, Studium der Forstwissenschaften, ETH Zürich

Berufsausübung: 1997-1998, Wissenschaftlicher Mitarbeiter am Institut für Forsteinrichtung und Ertragskunde der Universität Göttingen: Durchführung des LÖBFForschungsprojektes "Forsteinrichtung auf Basis von Stichprobeninventuren im Forstbetrieb der Stadt Brilon".

1998-2001, Wissenschaftlicher Mitarbeiter an der Niedersächsischen Forstlichen Versuchsanstalt (NFV) im Rahmen des DBU-Forschungsprojektes "Aufbau eines waldbaulichen Prognose- und Entscheidungsmodells für den norddeutschen Kleinprivatwald“".

Seit 2001, Wissenschaftlicher Mitarbeiter an der Niedersächsischen Forstlichen Versuchsanstalt (NFV) im Rahmen des EU-Forschungsprojektes "Implementing Tree Growth Models as Forest Management Tools“ (ITM). 